KADIO

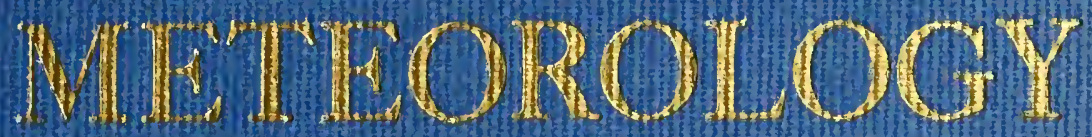

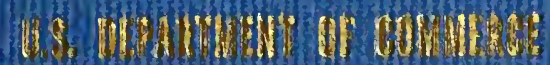

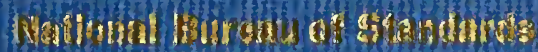

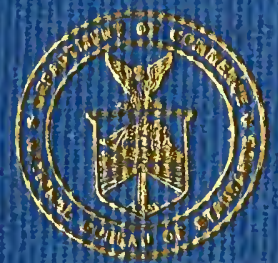




$$
\begin{aligned}
& \text { G. } Q \\
& 4=-9 p 027,964
\end{aligned}
$$



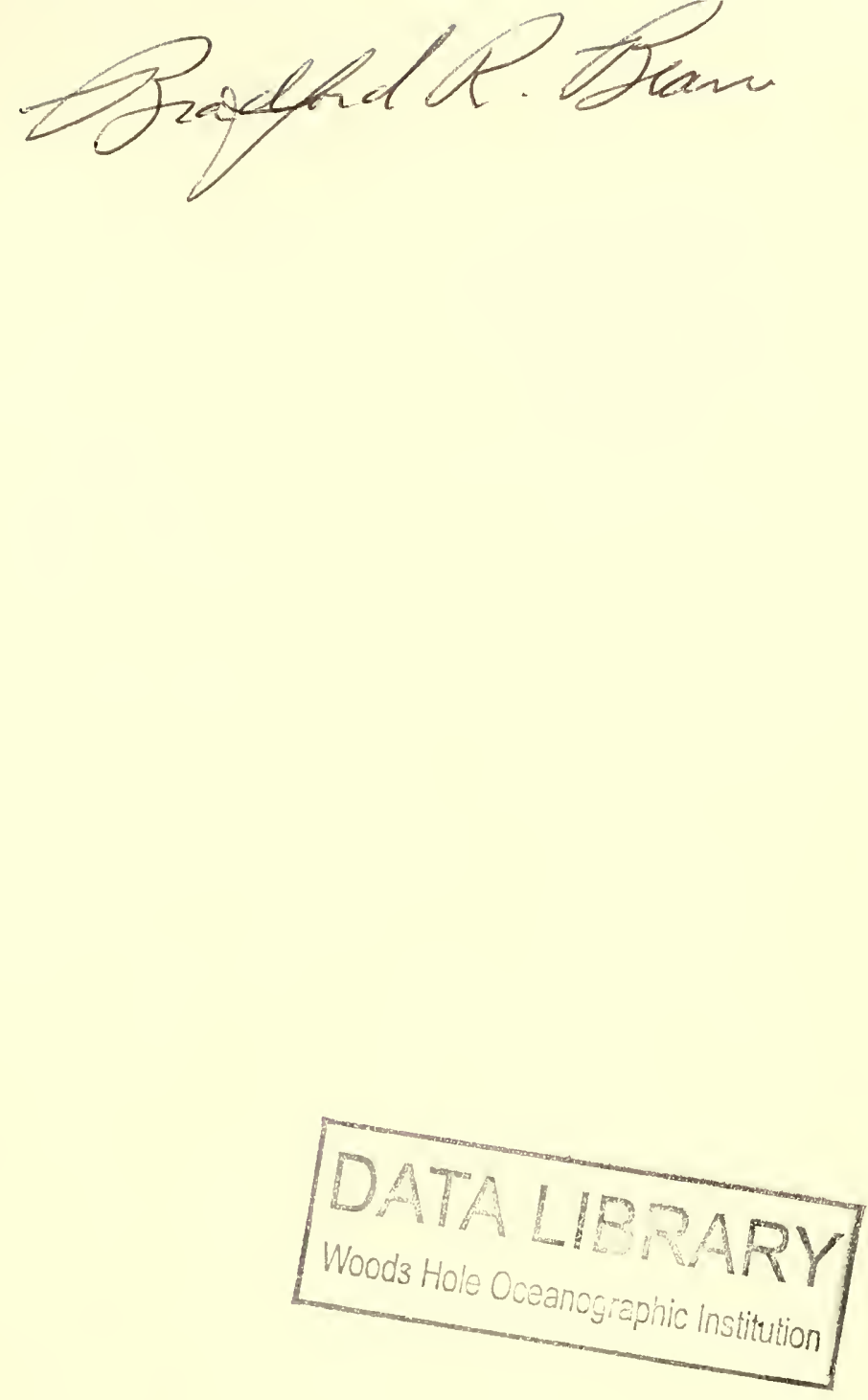





\title{
Radio Meteorology
}

\author{
B. R. Bean and E. J. Dutton \\ Central Radio Propagation Laboratory* \\ National Bureau of Standards \\ Boulder, Colorado
}

*The CRPL was transferred to the

Enviromental Science Services Administration

while this Monograph was in press.

National Bureau of Standards Monograph 92

Issued March 1, 1966

For sale by the Superintendent of Documents, U. S. Government Printing Office Washington, D. C. 20402 Price $\$ 2.75$ 
Library of Congress Catalog Card No. 65-60033 


\section{Foreword}

This volume brings together the work done in radio meteorology over the past ten years at the National Bureau of Standards' Central Radio Propagation Laboratory (CRPL). This decade has seen the development, on an international scale, of great emphasis upon the effects of the lower atmosphere on the propagation of radio waves. The CRPL group has concentrated upon the refraction of radio waves as well as the refractive index structure of the lower atmosphere on both synoptic and climatic scales. These are the areas of radio meteorology that are treated in this volume, with additional chapters on radio-meteorological parameters and the absorption of radio waves by the various constituents of the lower atmosphere. An effort has been made to include results obtained in other laboratories both in the United States and abroad.

A. V. Astin, Director. 


\section{Preface}

The authors express their gratitude and appreciation to the many members of the Central Radio Propagation Laboratory staff and others with whom they have had the pleasure of working. This volume would not have been possible without their assistance, encouragement, and stimulation. Particular acknowledgements are due: R. L. Abbott, Mrs. L. Bradley, Miss B. A. Cahoon, C. B. Emmanuel, L. Fehlhaber,* V. R. Frank, Miss S. Gerkin, Mrs. B. J. Gibson, J. Grosskopf, * J. W. Herbstreit, J. D. Horn, J. A. Lane, $\dagger$ J. R. Lebsack, R. E. MeGavin, Miss F. M. Meaney, C. M. Miller, K. A. Norton, A. M. Ozanich, Jr., the late Mrs. G .E. Richmond, L. P. Riggs, P. L. Rice, C. A. Samson, W. B. Sweezy, G. D. Thayer, Mrs. B. J. Weddle, E. R. Westwater, Mrs. P. C. Whittaker and W. A. Williams.

B. R. BEAN

E. J. Dutton

*Fernmeldetechnisches, Darmstadt, Germany.

†Radio Research Station, Slough, England. 


\section{Contents}

Foreword . .

Preface

Chapter 1. The radio refractive index of air

1.1. Introduction...... 1

1.2. Dielectric constant of moist air ........................ 2

1.3. Constants in the equation for $N \ldots \ldots \ldots$

1.4. Errors in the practical use of the equation for $N \ldots \ldots$

1.5. Presentation of $N$ data 13

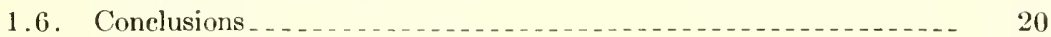

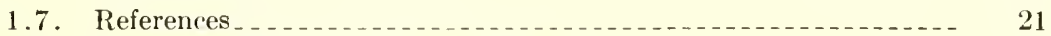

Chapter 2. Measuring the radio refractive index $\ldots \ldots \ldots$

2.1. The measurement of the radio refractive index _......... 23

2.2. Indirect measurement of the radio refractive index . _._._. 23

2.3. Direct measurement of the refractive index . . . . . . . . . 30

2.4. Comparison between the direct and indirect methods of measurement. . .

2.5. Radiosonde lag constants

2.5.1. Introduction

2.5 .2 . Theory of sensor time lags

2.5.3. Radiosonde profile analysis $\ldots \ldots \ldots \ldots \ldots$

2.5.4. Conclusion

2.6. References _._._._. 45

Chapter 3. Tropospheric refraction

3.1. Introduction _......... 49

3.2. Limitations to radio ray tracing

3.3. An approximation for high initial elevation angles........... 53

3.4. The statistical method .................................... 54

3.5. Schulkin's method

3.6. Linear or effective earth's radius model _._._._._._._._._. 56

3.7. Modified effective earth's radius model _._._._._._._._. 59

3.8. The exponential model

3.9. The initial gradient correction method

3.10. The departures-from-normal method $\ldots \ldots \ldots \ldots \ldots$

3.11. A graphical method .

3.12. Derivations 82

3.13. References $\ldots$

Chapter 4. $N$ climatology

4.1. Introduction

4.2. Radio-refractive-index climate near the ground _............ 89

4.2.1. Introduction _............... 89

4.2.2. Presentation of basic data____._._. 89

4.2.3. Worldwide values of $N_{0}$

4.2.4. Climatic classifieation by $N_{\text {s._._._._._._._._. }} 102$

4.2.5. Applications _._.

4.2.6. Critical appraisal of results _. _ _ _ _ _ _.

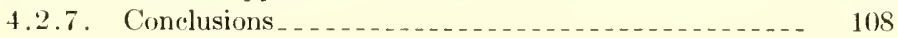


4.3. On the average atmospheric radio refractive index structure over

North America

4.3.1. Introduction _...

4.3.2. Meteorological data and reduction techniques

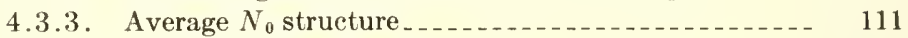

4.3.4. Continental cross sections .................. 122

4.3.5. Delineation of climatic eharacteristics______-_._. 129

4.4. The elimatology of ground-based radio ducts _._._._._._. 132

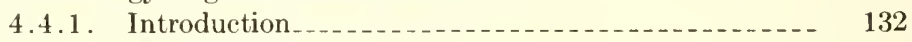

4.4.2. Meteorological conditions associated with radio refractive index profiles . . . . . . . . . . . . . . . . . 132

4.4.3. Refractive conditions due to local meteorological phenomena

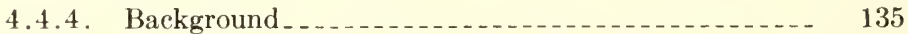

4.4.5. Description of observed ground-based atmospheric

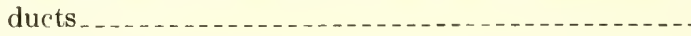

4.5. A study of fading regions within the horizon caused by a surface

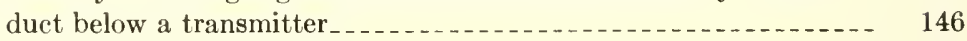

4.5.1. Introduction

4.5.2. Regions and extent of fading within the horizon in the presence of superrefraction

4.5.3. Theory and results _........... 149

4.5.4. Sample computations _... 157

4.6. Air mass refractive properties _._. 163

4.6.1. Introduction

4.6.2. Refraction of radio rays .............. 164

4.6.3. Conclusions _...

4.7. References _........ 170

Chapter 5. Synoptic radio meteorology

5.1. Introduction

5.2. Background ........... 173

5.3. Refractive index parameters ....... 185

5.4. A synoptic illustration__._. 195

5.5. Surface analysis in terms of $N_{0} \ldots \ldots$

5.6. Constant pressure chart analysis _._._._._. 205

5.7. Vertical distribution of the refractive index using $A$ units_..._. 211

5.8. Summary _....... 223

5.9. References......... 224

Chapter 6. Transhorizon radio-meteorological parameters _._._._._. 229

6.1. Existing radio-meteorological parameters _._. . . .

6.1.1. Introduction

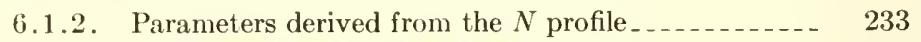

6.1.3. Parameters involving thermal stability _.......... 238

6.1.4. Composite parameter..._._._................ 238

6.1.5. Potential refractive index (or modulus), $K \ldots \ldots \ldots . .239$

6.1.6. Vertical motion of the atmosphere

6.1.7. Discussion of the parameters................ 241

6.1.8. Comparison of some parameters

6.1.9. Some exceptions and anomalies _._........ 243

6.1.10. Conclusions _._. 246 
6.2. An analysis of $\mathrm{VHF}$ field strength variations and refractive index profiles

6.2.1. Introduction

6.2.2. Radio and meteorological data_.............. 247

6.2.3. Classification of radio field strengths by profile types_ 248

6.2.4. Prediction of field strength for unstratified conditions_ 250

6.2.5. The effect of elevated layers on the Illinois paths _... 251

6.2.6. Elevated layers at temperature inversions........ 252

6.2.7. The influence of small layers

6.2.7. Conclusions _...

6.3. A new turbulence parameter

6.3.1. Introduction

6.3.2. The concept of thermal stability _............. 258

6.3.3. The adiabatic lapses of $N_{\ldots} \ldots \ldots \ldots$

6.3.4. The turbulence parameter, II _............... 262

6.3.5. Comparison of radio-meteorological parameters _... 263

6.3.6. Conclusion _._._._. 266

6.4. References................... 266

Chapter 7. Attenuation of radio waves

7.1. Introduction ................ 269

7.2. Background

7.3. Attenuation by atmospheric gases..................... 270

7.4. Estimates of the range of total gaseous absorption

7.5. Total radio path absorption. . .

7.6. Derivation of absorption estimate for other areas............ 286

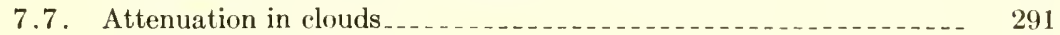

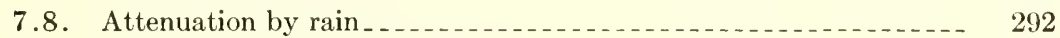

7.9. Rainfall attenuation climatology _._._._._._._._._._._. 297

7.10. Rain attenuation effects on radio systems engineering ..... 298

7.11. Attenuation by hail _........... 302

7.12. Attenuation by fog

7.13. Thermal noise emitted by the atmosphere $\ldots \ldots \ldots \ldots 4$

7.14. References _........ 308

Chapter 8. Applications of tropospheric refraction and refractive index models

8.1. Concerning the bi-exponential nature of the tropospheric radio

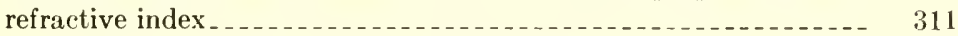

8.1.1. Introduction and background ............ 311

8.1.2. $N$ structure in the I.C.A.O. atmosphere $\ldots \ldots \ldots+\ldots$

8.1.3. Properties of the dry and wet term scale heights _. . $\quad 314$

8.1.4. Refraction in the bi-exponential model ......... 321

8.1.5. Extension to other regions _._._._._. 321

8.2. Effect of atmospheric horizontal inhomogeneity upon ray tracing - 322

8.2.1. Introduction and background _.............. 322

8.2.2. Canterbury

8.2.3. Cape Kennedy _...

8.2.4. Ray bending $\ldots \ldots \ldots \ldots$

8.2.5. Comparisons

8.2.6. Extension to other regions _...

8.2.7. Conclusions ....... 333 
8.3. Comparison of observed atmospheric radio refraction effects with values predicted through the use of surface weather observations

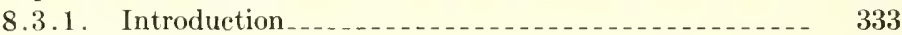

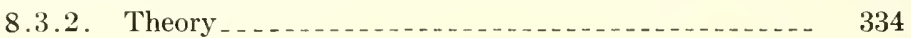

8.3.3. The CRPL standard atmospheric radio refractive index profile sample............ 340

8.3.4. Comparison with independent data ............... 342

8.3.5. Comparison with experimental results_.......... 344

8.3.6. Discussion of results _._.

8.4. Correction of atmospheric refraction errors in radio height finding- 356

8.4.1. Introduction _...

8.4.2. Refractive index $\ldots$

8.4.3. Ray theory

8.4.4. Use of the effective earth's radius _.......... 360

8.4.5. Meteorological parameters _.................. 362

8.4.6. Calculation and correlation of height errors _._._._ 363

8.4.7. Estimation of the average gradient._._._. 363

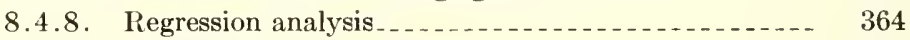

8.4.9. Height error equations _... 372

8.4.10. Conclusions _... 373

8.5. References._._._._._._. 373

Chapter 9. Radio-meteorological charts, graphs, tables, and sample computations..._.

9.1. Sample computations of atmospheric refraction_..._._._._._. 375

9.2. Tables of refraction variables for the exponential reference

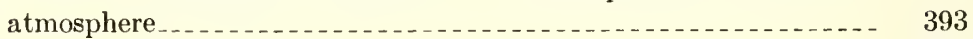

9.3. Refractivity tables _._._. 396

9.4. Climatological data of the refractive index for the United States - $\quad 404$

9.5. Statistical prediction of elevation angle error

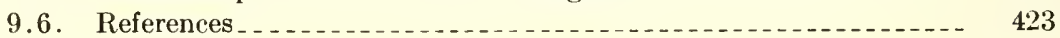




\section{Chapter 1. The Radio Refractive Index of Air}

\subsection{Introduction}

The last few decades have seen a remarkable inerease in the practical utilization of the radio spectrum above $30 \mathrm{Mc} / \mathrm{s}$. This, in turn, has focussed attention upon the mechanisms by which these radio waves are propagated. Since radio energy at these frequencies is not normally reflected by the ionosphere, variability in the characteristics of the received fields is attributed to variations in the lower atmosphere and, in particular, to the radio refractive index.

The radio refractive index is central to all theories of radio propagation through the lower atmosphere. The atmosphere causes a downward eurvature of horizontally launched radio waves which is normally about one quarter of that of the earth. Under unusual meteorological conditions, however, the radio energy may be confined to thin layers near the earth's surface with resultant abnormally high field strengths being observed beyond the normal radio horizon. At other times a transition layer between differing air masses will give rise to the reflection of radio energy. In addition to these gross profile effects, the atmosphere is always more or less turbulent, with the result that radio energy is scattered out of the normal antenna pattern.

It is not the purpose of the present discussion to emphasize the interplay of various propagation mechanisms, as has been done, for example, in a classic paper by Saxton [1] ${ }^{1}$, but rather to emphasize that the refractive index of the troposphere is of central concern in the propagation of radio waves at frequencies above $30 \mathrm{Me} / \mathrm{s}$. In what follows, then, the classical equation for the radio refractive index will be considered, a summary of the recent determinations of the constants in this equation given, the errors in the practical use of the equation will be analy zed, and, finally, the normal gross features of atmospheric refractive index structure will be described.

\subsection{Dielectric Constant of Moist Air}

Debye [2] has considered the effect of an impressed electric field upon the dielectric constant of both non-polar and polar molecules, a polar

\footnotetext{
${ }^{1}$ Figures in brackets indicate the literature references on p. 21.
} 
molecule being one with a strong permanent dipole moment. He derives the same result from both classical and quantum mechanical methods; i.e., the polarizability is composed of two effects: one due to the distortion of all of the molecules by the impressed field and the second arising from an orientation effect exerted upon polar molecules. The polarization, $P$, of a polar liquid under the influence of a high-frequency radio field is given by

$$
P(\omega)=\frac{\epsilon-1}{\epsilon+2} \frac{M}{\rho}=\frac{4 \pi N}{3}\left[\alpha_{0}+\frac{\mu^{2}}{3 k T} \frac{1}{1+i \omega \tau}\right]
$$

where: $\epsilon$ is the dielectric constant,

$M$ is the molecular weight,

$\rho$ is the density of the liquid,

$N$ is Avogadro's number,

$\alpha_{0}$ is the average polarizability of the molecules in the liquid, assuming no interaction between molecules,

$\mu$ is the permanent dipole moment,

$k$ is Boltzmann's constant,

$T$ is the absolute temperature,

$\tau$ is the relaxation time required for external field-induced orientations of the molecules to return to random distribution after the field is removed,

$\omega=2 \pi f$ where $f$ is the frequency of the external field.

One concludes from Debye's analysis that for external fields with frequencies less than $100 \mathrm{Gc} / \mathrm{s}, \omega \tau \ll 1$ with the result that $(1.1)$ is written ${ }^{2}$

$$
\frac{\epsilon-1}{\epsilon+2} \frac{M}{\rho}=\frac{4 \pi N}{3}\left[\alpha_{0}+\frac{\mu^{2}}{3 k T}\right]
$$

The dispersive effect of the $22.5 \mathrm{Gc} / \mathrm{s}$ water-vapor absorption line is not expected to be important below $30 \mathrm{Gc} / \mathrm{s}$ although, as shall be seen, experimental evidence indicates some dispersion due to the $60 \mathrm{Gc} / \mathrm{s}$ absorption

\footnotetext{
2 The effect of the relaxation time upon interpretation of measurements of dielectric constant has been discussed by Saxton [1]; he has also given a description of measurement techniques involved in the determination of dielectric constant during the war years [3].
} 
line of oxygen. For non-polar gases $(\mu=0)$, this equation becomes

$$
\frac{\epsilon-1}{\epsilon+2} \frac{M}{\rho}=\frac{4 \pi N \alpha_{0}}{3}
$$

which is well approximated by

$$
\epsilon-1 \approx \frac{\rho}{M} 4 \pi N \alpha_{0}
$$

for gases at low pressures.

This equation may be rewritten, by assuming the perfect gas law, as

$$
\epsilon-1=K_{1}^{\prime} \frac{P}{T}
$$

where $K_{1}^{\prime}$ is a constant. The result for polar gases, (1.2), may be written

$$
\epsilon-1 \approx \frac{\rho}{M} 4 \pi N\left[\alpha_{0}+\frac{\mu^{2}}{3 k T}\right]
$$

which, assuming (1.5), can be rewritten as

$$
\epsilon-1=K_{2}^{\prime} \frac{P}{T}\left(A+\frac{B}{T}\right)
$$

where $K_{2}^{\prime}, A$, and $B$ are constants. For a mixture of gases, Dalton's law of partial pressures is assumed to hold with the result that we can sum the effects of polar and non-polar gases and hence obtain

$$
\epsilon-1=\sum_{i} K_{1 i}^{\prime} \frac{P_{i}}{T}+\sum_{g} K_{2 g}^{\prime} \frac{P_{g}}{T}\left(A_{g}+\frac{B_{g}}{T}\right) .
$$

For the troposphere, however, we need only consider the effects of $\mathrm{CO}_{2}$, dry air (non-polar gases), and water vapor (a polar gas) such that

$$
\epsilon-1=K_{11}^{\prime} \frac{P_{d}}{T}+K_{21}^{\prime} \frac{e}{T}\left(A+\frac{B}{T}\right)+K_{12}^{\prime} \frac{P_{c}}{T}
$$

where $P_{d}$ is the pressure of dry air, $e$ is the partial pressure of water vapor, and $P_{c}$ the partial pressure of $\mathrm{CO}_{2}$. The equation for refractive index, $n$, is obtained using the expression $n=\sqrt{\mu \epsilon}$, where $\mu$, the permeability, may 
be assumed to be approximately unity for air. Since

$$
n=\sqrt{1+(\mu \epsilon-1)},
$$

one may employ the approximation

$$
n-1 \simeq \frac{\mu \epsilon-1}{2},
$$

and obtain the familiar form of practical application ${ }^{3}$

$$
N=(n-1) 10^{6}=K_{1} \frac{P_{d}}{T}+K_{2} \frac{e}{T}+K_{3} \frac{e}{T^{2}}+K_{4} \frac{P_{c}}{T}
$$

where $K_{1}, K_{2}, K_{3}$, and $K_{4}$ are constants.

\subsection{Constants in the Equation for $N$}

A survey of the various determinations of $K_{1}, K_{2}$, and $K_{3}$ was recently made by Smith and Weintraub [4] to arrive at a set of "best values" to represent the mean of previous' independent determinations. In radio work one is interested in propagation through the troposphere, therefore, the composition of air should be taken to include an average amount of carbon dioxide. However, laboratory measurements usually are made on $\mathrm{CO}_{2}$-free air due to variable concentrations of $\mathrm{CO}_{2}$ in the laboratory. Hence Smith and Weintraub have adjusted the the values of $\epsilon-1$ originally published for $\mathrm{CO}_{2}$-free air by raising them to 0.02 percent to correspond to a 0.03 percent $\mathrm{CO}_{2}$ content. It should be noted that this correction of 0.02 percent is essentially of academic interest, since as shall be seen, the final equation for $N$ will be considered accurate to 0.5 percent. The value of 0.02 percent was obtained by noting that the value of dielectric constant for $\mathrm{CO}_{2}$-free air, $\epsilon^{\prime}$, in the expression

$$
\epsilon^{\prime}-1=\frac{K_{1} P_{t}}{T}
$$

could be utilized for applications in the real atmosphere by expressing total atmospheric pressure, $P_{t}$, as

$$
P_{t}=P_{d}+P_{c}
$$

${ }^{3}$ Henceforth, $N$ will be used to denote $(n-1) 10^{6}$ and will not be used again for Avogadro's number. This slight inconsistency was adopted to maintain notational agreement with both Debye's early work and the later work in radio meteorology. 
By assuming a constant $\mathrm{CO}_{2}$ content of 0.03 percent one obtains, from Dalton's law of partial pressures,

$$
P_{d}=\left(1-3 \times 10^{-4}\right) P_{t}
$$

and

$$
P_{c}=3 \times 10^{-4} P_{t}
$$

with the result that the dielectric constant for the real, dry atmosphere, $\epsilon$, may be written

$$
\epsilon-1=\frac{K_{1}\left(1-3 \times 10^{-4}\right) P_{\ell}}{T}+\frac{K_{4}\left(3 \times 10^{-4}\right) P_{t}}{T} .
$$

One may then adjust measurements of $\mathrm{CO}_{2}$-free air upwards by

$$
\left[\frac{\epsilon-1}{\epsilon^{\prime}-1}-1\right] 10^{2}=3 \times 10^{-2}\left(\frac{K_{4}}{K_{1}}-1\right) \text { percent } \cong 0.02 \text { percent }
$$

since $K_{4} / K_{1} \simeq 5 / 3$. Such values are given in table 1.1 on a real, rather than an ideal, gas basis. The first determination shown, that of Barrell [5], is an average of the constant term ( $n$ for $\lambda=\infty$ ) of the optical Cauchy dispersion equations for standard air used in three of the principal measurement laboratories of the world. Theoretical considerations indicate that the dielectric constant for dry air will be the same for optical and radio frequencies. Barrell's value is converted to dielectric constant from the relationship $\sqrt{\mu \epsilon}$ with $\mu$, the permeability, taken as unity at

\begin{tabular}{|c|c|c|c|c|}
\hline Referenee & $\begin{array}{l}\text { Frequency of } \\
\text { measurement }\end{array}$ & $\underset{N}{\text { Measured }}$ & $(\epsilon-1) 10^{6}$ & Year \\
\hline Barrell [5] - & Optieala ${ }^{\mathrm{a}} \ldots$ & $287.7 \pm 0.0$ & $575.6 \pm 0.1$ & 1951 \\
\hline Birnbaum, Kryder, and Lyons[40] & $9,000 \mathrm{Mc} / \mathrm{s}_{\ldots} \ldots$ & 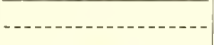 & $575.8 \pm 0.3$ & 1951 \\
\hline Essen and Froome $[11] \ldots . .$. & $24,000 \mathrm{Me} / \mathrm{s} \ldots \ldots$ & $288.2 \pm 0.1$ & $576.1 \pm 0.2$ & 1951 \\
\hline
\end{tabular}
optical frequencies. Unless otherwise noted, the standard error is used here and throughout the remainder of this discussion.

TABLE 1.1. Dry air refractive index and dielectric constant at $0^{\circ} \mathrm{C}$ and 1 atm

288.0. 0 . 0.0

a Maryott and Buck]ey [6] have determined a mean value for the dielectrie constant from the simple average of eight different determinations that, when adjusted to the pressure, temperature, and value of $\mu$ assumed in table 1.1, yield a value of $N=287.7 \pm 0.15$, whieh is in agreement with liarrell's value.

b Derived froni $n=\sqrt{\mu \epsilon}$ where $\mu-1=0.4 \times 10^{-6}$ is taken for radio frequeneies to aceount for the permeability. 
The statistical mean of the dielectric constant is then converted to refractive index. The constant $K_{1}$ is evaluated from

$$
N=K_{1} \frac{P_{d}}{T},
$$

which stems from (1.10) when $e=0$. Setting $N=288.04, P_{d}=1013.25$ mbar, $T=273^{\circ} \mathrm{K}$ and solving for $K_{1}$ : then ${ }^{4}$

$$
K_{1}=77.607 \pm 0.13 \frac{{ }^{\circ} \mathrm{K}}{\mathrm{mbar}} .
$$

The constants $K_{2}$ and $K_{3}$ have been evaluated from a survey of determinations of the dielectric constant of water vapor in the microwave region by Birnbaum and Chatterjee [7]. These values, determined from the data of table 1.2 , are

$$
K_{2}=71.6 \pm 8.5^{\circ} \mathrm{K} / \mathrm{mbar}
$$

and

$$
K_{3}=(3.747 \pm 0.031) 10^{5}\left({ }^{\circ} \mathrm{K}\right)^{2} / \mathrm{mbar}
$$

and were obtained as the weighted means of the various determinations, the weights being taken inversely proportional to the square of the probable errors. Although Birnbaum and Chatterjee considered only those data that would allow a determination of $K_{2}$ and $K_{3}$ over a wide range of temperature, they conclude their results are in satisfactory agreement

\begin{tabular}{|c|c|c|c|}
\hline Observer & $K_{2}$ & $K_{3} \times 10^{-5}$ & Temperature range \\
\hline $\begin{array}{l}\text { Birnbaum and Chatterjee }[7] \\
\text { Groves and Sugden }[12] \\
\text { Hurdis and Smyth }[13] \\
\text { Stranathan }[8]\end{array}$ & $\begin{array}{l}69.43 \pm 13.02 \\
77.75 \pm 21.70 \\
61.47 \pm 21.70 \\
72.86 \pm 7.05\end{array}$ & $\begin{array}{l}\text { 3. } 774 \pm 0.043 \\
\text { 3.742 } 0.097 \\
\text { 3. } 765 \pm 0.096 \\
\text { 3. } 736 \pm 0.025\end{array}$ & $\begin{array}{r}{ }^{\circ} \mathrm{C} \\
25-103 \\
110-211 \\
111-249 \\
21-189\end{array}$ \\
\hline
\end{tabular}
with those of Stranathan [8] at $1 \mathrm{Mc} / \mathrm{s}$, Phillips [9] at $3000 \mathrm{Mc} / \mathrm{s}$, Crain [10] at $9000 \mathrm{Mc} / \mathrm{s}$, and Essen and Froome [11] at 24,000 Mc/s.

TABLE 1.2. Values of the constants $K_{2}$ and $K_{3}$ used by Birnbaum and Chatterjee

${ }^{4}$ The development here follows that of Smith and Weintraub. Crain's [10] determination of the dielectric constant of dry air $(\epsilon=1.000572)$ indicates that $K_{1}$ may be somewhat lower than the "best value" of Smith and Weintraub, since his value, $K_{1}=77.10$, is about 40 standard deviations lower than that of Smith and Weintraub. 
The full equation for the refractive index is now

$$
N=77.6 \frac{P_{d}}{T}+72 \frac{e}{T}+3.75 \times 10^{5} \frac{e}{T^{2}},
$$

after reducing the values to three figures where significant. These are the constants recommended by Smith and Weintraub to yield an overall accuracy of \pm 0.5 percent in $N{ }^{5}$ Equation (1.15) may be simplified by assuming $P_{t}=P=P_{d}+e$ and obtaining

$$
N=77.6 \frac{P}{T}-5.6 \frac{e}{T}+3.75 \times 10^{5} \frac{e}{T^{2}} .
$$

For practical work in radio-meteorological studies, (1.16) may be simplified to the two-term expression

$$
N=77.6 \frac{P}{T}+3.73 \times 10^{5} \frac{e}{T^{2}},
$$

which yields values of $N$ within 0.02 percent of those obtained by (1.16) for the temperature range of -50 to $40{ }^{\circ} \mathrm{C}$; i.e., a maximum error of 0.1 $N$ unit, and a standard error of 0.5 percent of about $4.5 \mathrm{~N}$ units.

This is accomplished by dividing the second and third terms of (1.16) by $e / T$ and solving for the composite constant, $K_{5}$, in relation

$$
\frac{3.75 \times 10^{5}}{T}-5.6=\frac{K_{5}}{T}
$$

which, for $T^{\prime}=273^{\circ} \mathrm{K}$, results in:

$$
K_{5}=3.73 \times 10^{5}\left({ }^{\circ} \mathrm{K}^{2} / \mathrm{mbar}\right) .
$$

Equation (1.17) is commonly written as

$$
N=\frac{77.6}{T}\left(P+\frac{4810 e}{T}\right) .
$$

Although this last equation is in widespread use by radio scientists throughout the world, it is by no means adopted on an international seale.

${ }^{5}$ The values of $K_{2}$ and $K_{3}$ given in (1.13) and (1.14) are those of Birnbaum and Chatterjee as reported by Smith and Weintraub. A consideration of the ratio of $K_{2} / K_{3}$ shows the Smith-Weintraub value to be about 0.2 percent greater than that obtained from the original data, indicating an arithmetic conversion error. This error is, however, totally absorbed in the rounding to three significant figures in (1.15). 
Another set of values in common use is that of Essen and Froome (11) which is listed in table 1.3 along with values determined by several other authors. Subsequent microwave refractometer determinations of these constants by Saito [14] in Japan yielded a value of $K_{1}$ lower than that of either Smith and Weintraub or of Essen and Froome and nearer to Crain's determination. Saito's values of $K_{2}$ and $K_{3}$ lie between those of Smith and Weintraub, and Essen and Froome. A recent French microwave determination of these constants by Battaglie, Boudouris, and Gozzini [15], has found the Smith and Weintraub values of $K_{1}, K_{2}$, and $K_{3}$ given by (1.16) fit their experimental data within measurement error. The use of the Essen and Froome values in radio geodesy appears to be based upon Wadley's [16] comparison of optical and radio surveys and his conclusion that the best (radio) consistency is obtained when using Essen and Froome's constants in conjunction with their determination of the velocity of light.

TABLE 1.3. Table of constants used by different authors

$$
N=(n-1) 10^{6}=K_{1} \frac{P_{d}}{T}+K_{2} \frac{e}{T}+K_{3} \frac{e}{T^{2}}
$$

\begin{tabular}{|c|c|c|c|c|}
\hline Reference & Date & $K_{1}$ & $K_{2}$ & $K_{3}$ \\
\hline $\begin{array}{l}\text { Schelleng, Burrows, and Ferrell [20] } \\
\text { Englund, Crawford, and Mumford [41]]- } \\
\text { Waynick [35] } \\
\text { Smith-Rose and Stickland [36] } \\
\text { Burrows and Attwood N RDC }[37] \\
\text { Metcorol. Factors in Prop. [40] } \\
\text { Crain }[10] \\
\text { Craig et al. [32] } \\
\text { Essen and Froome [11] } \\
\text { Smith and Weintraub [4] } \\
\text { Essen [38] } \\
\text { Saito [14]. } \\
\text { Battaglia, Boudouris, and Gozzini }[15] \\
\text { Magee and Crain [42] }\end{array}$ & $\begin{array}{l}1933 \\
1935 \\
1940 \\
1943 \\
1946 \\
1946 \\
1948 \\
1951 \\
1951 \\
1952 \\
1953 \\
1955 \\
1957 \\
1958\end{array}$ & $\begin{array}{l}79.0 \\
79.1 \\
79.0 \\
79.0 \\
79.0 \\
79.0 \\
77.10 \\
79.0 \\
77.64 \\
77.6 \\
77.26 \\
77.6 \\
77.5\end{array}$ & $\begin{array}{l}67.5 \\
68.3 \\
68.5 \\
68.0 \\
68.0 \\
68.0 \\
(79) \\
64.68 \\
72.0 \\
75.0 \\
67.5 \\
72.0 \\
65.0\end{array}$ & $\begin{array}{l}1.35 \\
3.81 \\
3.72 \\
3.8 \\
3.8 \\
3.8 \\
3.8 \\
3.718 \\
3.75 \\
3.68 \\
3.77 \\
3.75 \\
3.70\end{array}$ \\
\hline
\end{tabular}

The particular constants in the equation for $N$ given by Smith and Weintraub are considered to be good to 0.5 percent in $N$ for frequencies up to $30,000 \mathrm{Mc} / \mathrm{s}$ and normally encountered ranges of pressure, temperature, and humidity. Experimental determinations of the variability of the radio refractivity, $(n-1) 10^{6}$, with frequency have been carried out by Essen and Froome [11] and are summarized in table 1.4.

Remembering that the dispersion of refractive index would be expected to be greatest at frequencies slightly off the water vapor resonance at $22 \mathrm{Gc} / \mathrm{s}$ and the oxygen resonance at $60 \mathrm{Gc} / \mathrm{s}$ [17] one concludes that for the normally used frequencies, $f<30 \mathrm{Gc} / \mathrm{s}$, the various gases give a frequency variation of $N$ well within the limits of accuracy given by Smith 
TABLE 1.4. Refractivities of water vapor and dry gases

(Dry gases at $0{ }^{\circ} \mathrm{C}, 760 \mathrm{~mm} \mathrm{Hg}$; water vapor at $20^{\circ} \mathrm{C}, 10 \mathrm{~mm} \mathrm{Hg}$ )

\begin{tabular}{|c|c|c|c|}
\hline Gas & $9 \mathrm{Gc} / \mathrm{s}$ & $24 \mathrm{Gc} / \mathrm{s}$ & $72 \mathrm{Gc} / \mathrm{s}$ \\
\hline $\begin{array}{l}\text { Water vapor } \\
\text { Dry air } \\
\text { Oxygen }\end{array}$ & $\begin{array}{l}60.7 \pm 0.2 \\
288.10 \pm 0.10 \\
266.2 \pm 0.2\end{array}$ & $\begin{array}{l}60.7 \pm 0.2 \\
288.15 \pm 0.10 \\
266.4 \pm 0.2\end{array}$ & $\begin{array}{l}61.0 \pm 0.2 \\
287.66 \pm 0.11 \\
263.9 \pm 0.2\end{array}$ \\
\hline
\end{tabular}

and Weintraub. With the exception of oxygen the same is true up to $72 \mathrm{Gc} / \mathrm{s}$. This conclusion is somewhat strengthened by the results of Hughs and Armstrong [18] at $3000 \mathrm{Mc} / \mathrm{s}$ which agree within 1.5 percent of those of Essen and Froome. It is important to note that the differences in formulas are greater than the frequency variation and, further, as shall be seen in the next section, the errors of determining $P, T$, and $e$ are sufficiently large to mask even the differences in formulas.

\subsection{Errors in the Practical Use of the Equation for $N$}

A high degree of accuracy of temperature, pressure, and water vapor pressure measurements is necessary for precise determinations of the refractive index from (1.20). If one assumes that the formula for $N$ is exact, then a relation between small changes in $N$ and small changes in temperature, pressure, and vapor pressure may be evaluated from

$$
d N=\frac{\partial N}{\partial T} d T+\frac{\partial N}{\partial e} d e+\frac{\partial N}{\partial P} d P
$$

assuming that the errors in $P, T$, and $e$ are unrelated.

The partial derivatives may be evaluated by reference to some standard atmosphere to yicld the approximate expression

$$
\Delta N^{\prime}=a \Delta T+b \Delta e+c \Delta P .
$$

The root-mean-square (rms) error is then

$$
\Delta N=\left\{(a \Delta T)^{2}+(b \Delta e)^{2}+(c \Delta P)^{2}\right\}^{1 / 2} .
$$

Typical values of the constants $a, b$, and $c$, based upon the International Civil Aviation Organization (ICAO) standard atmosphere and assuming 60 percent relative humidity, are given in table 1.5 for various altitudes. 
It was mentioned earlier that the differences in refractive index obtained by the two most commonly used formulas, those of Essen and Froome and of Smith and Weintraub, are small compared to the error made in observing $P, T$, and $e$. For example, by assuming errors of \pm 2 mbar in $P, \pm 1^{\circ} \mathrm{C}$ in $T$, and \pm 5 percent in relative humidity $(\mathrm{RH})$ common in radiosonde measurements with sea-level values of $P=1013$ mbar, $T=15^{\circ} \mathrm{C}$ and 60 percent $\mathrm{RH}$, a standard error of $4.1 \mathrm{~N}$ units may be obtained compared to the difference of about $0.5 \mathrm{~N}$ units between the values obtained from the two formulas. It is seen from table 1.6 that (over the normal range of sea-level temperatures, $-50{ }^{\circ} \mathrm{C}$ to $+40{ }^{\circ} \mathrm{C}$ ) the differences in the formulas are comparable to those associated with surface meteorological measurements but are significantly less than the errors that may arise in radiosonde observations.

In addition to these errors, there is an additional source of error in the uncertainty of the constants in the equation for $N$. Equation (1.22) may be written to include this additional source of error as

$$
\Delta N^{\prime}=a \Delta T+b \Delta e+c \Delta P+d \Delta K_{1}+f \Delta K_{2}+g \Delta K_{3},
$$

and, again assuming that the errors are uncorrelated, the rms error may be evaluated as before. Table 1.7 lists the percentage error arising from errors in the various surface meteorological observations as well as those of the constants. It is seen that the errors in constants constitute no more than 30 percent of the total error. All sources of error combined will yield errors no larger than 1.5 percent in $N$. Thus, although the Smith and Weintraub equation has a stated accuracy of 0.5 percent due to the constants alone, total errors of nearly twice that figure may occur under conditions of extremely high humidity.

Currently one must use radiosonde data for estimation of $N$ gradients, with the result that the overall accuracy is determined much more by the errors in the meteorological sensors than by errors between constants in the equation for $N$. Until such time as better measurement methods for $T$ and $e$ are developed, there appears to be little or no need for more accurate determinations of the constants in (1.10). The different determinations of the constants now available are in essential agreement. For example, the use of the Essen and Froome expression will give values of $N$ that generally lie within the standard error of \pm 0.5 percent of the Smith-Weintraub expression. It appears desirable for our purposes to use the Snith-Weintraub constants since they represent the weighted mean of several independent determinations, noting also that the value of $K_{2}$ given by Essen and Froome was obtained by extrapolation from optical measurements rather than direct measurements at radio frequencies. 
TABLE 1.5. Values of the constants $\mathrm{a}, \mathrm{b}$, and $\mathrm{c}$ in the expression $\Delta \mathrm{N}=\mathrm{a} \Delta \mathrm{T}+\mathrm{b} \Delta \mathrm{e}$ $+\mathrm{c} \mathrm{P}$, for the ICAO Standard Atmosphere and 60 percent relative humidity

\begin{tabular}{|c|c|c|c|c|c|c|c|}
\hline Altitude & $N$ & $T$ & $P$ & $\ell$ & $a$ & $b$ & $c$ \\
\hline $\begin{array}{r}0 \\
1 \\
3 \\
10 \\
20 \\
50\end{array}$ & $\begin{array}{c}319 \\
27 \\
216 \\
92 \\
20 \\
0.2\end{array}$ & $\begin{array}{r}{ }^{\circ} C \\
15.0 \\
8.2 \\
-4.5 \\
-50.3 \\
-56.5 \\
9.5\end{array}$ & $\begin{array}{c}\text { mbar } \\
1013 \\
893 \\
701 \\
262 \\
55 \\
0.8\end{array}$ & $\begin{array}{c}m b a r \\
10.2 \\
\text { 6. } \\
2.6 \\
0.04 \\
0 \\
0\end{array}$ & $\begin{array}{l}{ }^{\circ} K^{-1} \\
-1.27 \\
-1.09 \\
-0.86 \\
-0.50 \\
-0.09 \\
-0.0005\end{array}$ & $\begin{array}{r}m b a r^{-1} \\
4.50 \\
4.72 \\
5.17 \\
7.52 \\
7.96 \\
4.67\end{array}$ & $\begin{array}{r}\text { mbar-1 } \\
0.27 \\
0.28 \\
0.29 \\
0.30 \\
0.35 \\
0.27\end{array}$ \\
\hline
\end{tabular}

TABLE 1.6. Comparison of errors in determining $\mathrm{N}$ from meteorological measurements assuming no error in the equation for $\mathrm{N}$

$[p=1013$ mbar and $\mathrm{R} 11=60$ percent $]$

\begin{tabular}{|c|c|c|c|c|c|c|}
\hline \multicolumn{3}{|c|}{ Source of error } & $-50^{\circ} \mathrm{C}$ & $0{ }^{\circ} \mathrm{C}$ & $+15^{\circ} \mathrm{C}$ & $+40^{\circ} \mathrm{C}$ \\
\hline \multirow[t]{2}{*}{ Difference in formulas: } & \multicolumn{2}{|c|}{$\begin{array}{l}\text { Smith-W Wintraub [4] (three term) } \\
\text { Essen and Froome [11] (three term). }\end{array}$} & $\begin{array}{l}352.61 \\
352.79\end{array}$ & $\begin{array}{l}306.18 \\
306.07\end{array}$ & $\begin{array}{l}318.79 \\
318.28\end{array}$ & $\begin{array}{l}419.55 \\
417.19\end{array}$ \\
\hline & \multicolumn{2}{|c|}{$\begin{array}{l}\text { Difference from } \mathrm{Smith} \text {-Weintraub } \\
\text { value in } N \text { units........ }\end{array}$} & +0.18 & -0.11 & -0.51 & -2.34 \\
\hline \multicolumn{2}{|c|}{$\begin{array}{l}\text { Surface weather observations ( } \pm 1 \text { mbar of pressure, } \\
\pm 0.10^{\circ} \mathrm{C} \text { of } T \text {, and } \pm 1 \text { percent } \mathrm{R} 11 \text { ), (all errors in } \\
N \text { units). }\end{array}$} & $\begin{array}{l}\mathrm{rms} \\
\text { error }\end{array}$ & \pm 0.38 & \pm 0.43 & \pm 0.82 & \pm 2.83 \\
\hline \multicolumn{2}{|c|}{$\begin{array}{l}\text { Radiosonde observations ( } \pm 2 \text { mbar of pressure, } \pm 11^{\circ} \mathrm{C} \\
\text { of } T \text {, and } \pm 5 \text { pereent } \mathrm{R} I 1 \text { ) (atl errors in } N \text { units). }\end{array}$} & $\underset{\text { error }}{\mathrm{rms}}$ & \pm 1.73 & \pm 2.02 & \pm 4.07 & \pm 14.19 \\
\hline
\end{tabular}

TABLE 1.7. Percentage contribution of errors in surface meteorological measurements and constants in the equation for $\mathrm{N}$

\begin{tabular}{|c|c|c|c|c|c|c|c|}
\hline \multirow{2}{*}{ Temp $\left({ }^{\circ} \mathrm{C}\right)$} & \multicolumn{6}{|c|}{ Pereent of total variance due to: } & \multirow{2}{*}{$\begin{array}{c}\text { Total rms } \\
\text { error ( } N \text {-units) }\end{array}$} \\
\hline & $\Delta T$ & se & $\Delta P$ & $\Delta K_{1}$ & $\Delta K_{2}$ & $\Delta K_{3}$ & \\
\hline $\begin{array}{r}-50 \\
0 \\
15 \\
40\end{array}$ & $\begin{array}{r}16 . \overline{1} \\
6.2 \\
1.8 \\
0.3\end{array}$ & $\begin{array}{r}0.0 \\
41.3 \\
64.1 \\
69.3\end{array}$ & $\begin{array}{r}81.0 \\
35.6 \\
7.9 \\
0.5\end{array}$ & $\begin{array}{l}2.3 \\
1.0 \\
0.2 \\
0.0\end{array}$ & $\begin{array}{r}0.0 \\
5.7 \\
10.0 \\
12.7\end{array}$ & $\begin{array}{r}0.0 \\
10.2 \\
16.0 \\
17.2\end{array}$ & $\begin{array}{r}0.387 \\
.476 \\
955 \\
3.378\end{array}$ \\
\hline \multicolumn{8}{|c|}{ rms error in $N$-units } \\
\hline $\begin{array}{r}-50 \\
0 \\
15 \\
40\end{array}$ & $\begin{array}{r}0.158 \\
.119 \\
.127 \\
.188\end{array}$ & $\begin{array}{r}0.005 \\
.306 \\
.765 \\
2.811\end{array}$ & $\begin{array}{r}0.348 \\
.284 \\
.269 \\
.248\end{array}$ & $\begin{array}{r}0.059 \\
.048 \\
.045 \\
.040\end{array}$ & $\begin{array}{r}0.001 \\
.114 \\
.302 \\
1.202\end{array}$ & $\begin{array}{r}0.002 \\
.152 \\
.382 \\
.399\end{array}$ & $\begin{array}{r}0.387 \\
.476 \\
.955 \\
3.378\end{array}$ \\
\hline
\end{tabular}

This opinion is particularly reinforeed by Essen's subsequent determination of $K_{2}$ and $K_{3}$ at $9200 \mathrm{Mc} / \mathrm{s}$, where closer agreement was obtained with the Smith-Weintraub values (note items 9, 10, 11 in table 1.3). The difference in $K_{2}$ and $K_{3}$ accounts for most of the final difference in $N$ obtained from the two formulas. Although the great precision of Essen and Froome's work is reflected in their standard deviation of experimental 
values being about one-tenth that of other workers, one must recognize that systematic error's undoubtedly contribute to the disagreement in values obtained by different workers. It appears that there exists a real need for a new determination carried out with the greatest possible care and over as large a pressure and temperature range as possible.

The need for further measurements is particularly evident by the several Essen and Froome determinations. Their values of the dielectric constant have been determined with impressive precision and absolute accuracy over a wide range of frequencies, but their concomitant determinations of the constants in the equation for $N$ differ from one another as much as the Smith-Weintraub and the 1951 Essen and Froome values. It is clear that the use of either set of Essen and Froome values permits the calculation of either relative or absolute values of $N$ with equal precision. However, the question of comparative absolute accuracy with the results of other workers remains.

As a further example, drawn from radio geodesy, consider the electrical path length

$$
R=\int_{0}^{S} n d S
$$

where $S$ indicates the radio path. If we assume that the measurements are made in a stratum of constant $n$, then (1.23) becomes

$$
R=n \int_{0}^{S} d s=n S=S\left(1+N \times 10^{-6}\right)
$$

and

$$
S=R /\left(1+N \times 10^{-6}\right) \simeq R\left(1-N \times 10^{-6}\right) .
$$

An error in $N$ thus directly produces an error in radio distance determination. In typical radio geodesic field work (see, for example, Wadley, [16]) $N$ would be determined from pressure, dry-bulb temperature, and wet-bulb temperature readings. The partial pressure of water vapor may be obtained from Sprung's [19] (psychrometric) formula

$$
e=e^{\prime}{ }_{s}-0.00067\left(T-T^{\prime}\right) P
$$

where $e^{\prime}$ denotes the saturation vapor pressure at the wet-bulb temperature, $T^{\prime}$. For sea-level conditions of $P=1013 \mathrm{mbar}, T=15^{\circ} \mathrm{C}$ and $T-T^{\prime}=4.1{ }^{\circ} \mathrm{C}(60$ percent $\mathrm{RH})$ one finds, neglecting errors in the constants, that

$$
\Delta N^{\prime} \sim 0.28 \Delta P-4.32 \Delta T-6.96 \Delta T^{\prime} .
$$


Assuming errors of $\pm 0.1 \mathrm{mbar}$ for $P$ and $\pm 0.25^{\circ} \mathrm{C}$ for $T$ and $T^{\prime}$ (derived from considering Wadley's field data as being accurate to \pm 5 units of the next significant figure beyond the tabulated whole degrees Fahrenheit for wet and dry bulb temperatures and hundredths of an inch of mercury for total air pressure) one finds that errors in $N$ may be as large as $2.75 \mathrm{~N}$ units, which is much greater than the $0.5 \mathrm{~N}$ unit difference given by the two formulas for $N$. Again it is seen that the difference in the constants used in the two most widely accepted expressions for $N$ yields an error of the same size or smaller than that produced by errors of measurement of the necessary meteorological parameters.

\subsection{Presentation of $N$ Data}

There is now, in the literature, an almost bewildering choice of modifications to basic $N$ data when presented as a function of height, The underlying principle is always to remove the systematic decrease of $N$ with height, $h$, in an assumed standard atmosphere. This arises from the fact that the curvature of a radio ray, $C$, is given by ${ }^{6}$

$$
C=-\frac{1}{n} \frac{d n}{d h} \cos \theta
$$

where $\theta$ is the local elevation angle of the ray. Since the curvature of a radio ray is proportional to the gradient of the refractive index, specification of a model for $d n / d h$ specifies the curvature of radio rays in that model atmosphere. For example, it is customary for radio engineers to think in terms of effective earth radius factors [20]. This convenient fiction, discussed in chapter 3, makes straight the actual curved path of a radio ray in the atmosphere by presenting it relative to an imaginary earth larger in radius by a factor $k$ than the radius of the real earth, $a$, thus maint aining the relative curvature bet ween earth and radio ray.

This is expressed as

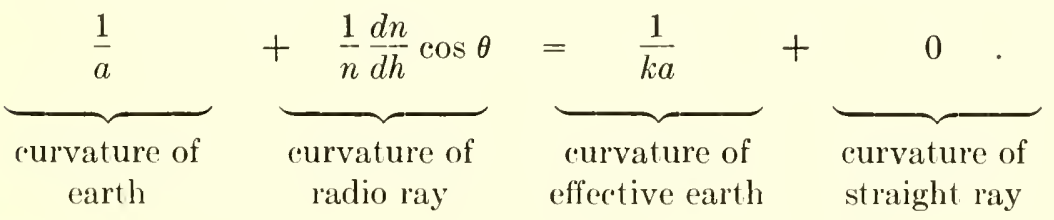

${ }^{6}$ Equation (1.27) as well as general ray curvature considerations are elegantly derived by Millington [21] for the effective earth's radius model. The same equation is also derived in chapter 3. 
Thus $k$ is defined by

$$
k=\frac{1}{1+\frac{a}{n} \frac{d n}{d h} \cos \theta}
$$

which, for rays tangential to the earth $\left(\theta_{0}=0\right)$ and assuming $n$ in unity, is approximated by

$$
k \simeq \frac{1}{1+a d n / d h}
$$

It is customary to set

$$
d n / d h=-\frac{1}{4 a},
$$

and thus obtain $k=4 / 3$. Since $1 / 4 a$ is close to the observed gradients of $n$, this represents a "standard atmosphere" gradient and one may remove the "standard" decrease of $N$ with height by adding a quantity, $\delta N$

$$
\delta N=(h / 4 a) 10^{6}
$$

to $N(h)$ thus obtaining the " $B$ unit" or

$$
B(h)=[(n-1)+h / 4 a] 10^{6}=N(h)+(h / 4 a) 10^{6} .
$$

This method of presenting refractive index profiles has been shown to be very useful in emphasizing the departure from standard of the atmosphere over southern California [22]. The principal advantage of the $B$ unit is that any departure from a vertical line, and thus constant $n$ gradient of $d n / d h=-1 / 4 a$, equals a departure in refractive bending from that in the effective earth's radius at mosphere with $k=4 / 3$.

A very similar approach is used to aid in the study of extended ranges of radio waves. The most pronounced case of this phenomena occurs when $d n / d h=-(1 / a)$ which, from (1.29), gives $k=\infty$ or an effective earth of infinite radius. This then implies, for radio purposes, the earth is flat and communication is between "radio-visible" terminals. For this special study the modified index of refraction, $M$, is defined by

$$
M(h)=[(n-1)+h / a] 10^{6}=N(h)+(h / a) 10^{6} .
$$

A gradient of $d M / d h=0$ implies that $k=\infty$ and also implies a theoretically infinite range of radio signals. $M$ units were used to present the 
meteorological data gathered by the Canterbury Project [23] in their intensive study of ranges of over-water radar signals. Both $B$ and $M$ units assume a standard atmosphere with a linear decrease of $N$ with height and thus introduce a correction which increases linearly with height. If the actual atmosphere had in fact the assumed $N$ distribution then, for example, $B(h) \equiv B(0)$. Recent studies [24, 25, 26] have shown than an exponential decrease of $N$ with height is a more realistic model of the true atmosphere.

For example, the data given on figure 3.3 were chosen to represent the extremes of average $N$ profile conditions over the United States. The Miami, Fla., profile is typical of warm, humid, sea-level stations that tend to have maximum refraction effects while the Portland, Me., profile is associated with nearly minimum sea-level refraction conditions. Although Ely, Nev., has a much smaller surface $N$ value than either Miami or Portland, its $N$ profile falls within the limits of the maximum and minimum sea-level profiles. The $N$ distribution for the $4 / 3$ effective earth's radius atmosphere is also shown on figure 3.3 .

It is quite evident that the $4 / 3$ earth distribution has about the correct slope in the first kilometer above the earth's surface but decreases much too rapidly above that height. It is also seen that the observed refractivity distribution is more nearly an exponential function of height than a linear function as assumed by the effective earth's radius model. The exponential decrease of $N$ with height is sufficiently regular as to permit a first approximation of average $N$ structure from surface conditions alone. Consider that

$$
N(h)=N_{s} \exp (-h / H),
$$

where $H$ is a scale height appropriate to the value of $N$ at zero height, $N_{s}$. Average values of $N_{s}$ and $H$ for the United States are approximately 313 and $7 \mathrm{~km}$ respectively. As used here, scale height is simply that height at which $N(h)$ is $1 / e$ of $N_{s}$ under the assumption of (1.34).

One would expect the gradient

$$
\frac{d N(h)}{d h}=-\left(N_{s} / H\right) \exp (-h / H)
$$

also to be sufficiently well-behaved as to allow prediction of at least its general features. In fact a high correlation between $\Delta N$, the simple difference between $N$ at the earth's surface and at $1 \mathrm{~km}$ above the earth's surface, and $N_{s}$ has been observed in a number of regions. This is 
particularly true of climatological data such as 5-year averages for given months. In the United States the expression [26]

$$
\begin{gathered}
-\overline{\Delta N}=7.32 \exp \left(0.005577 \bar{N}_{s}\right), \\
r_{\ln \overline{\Delta N}} \cdot \bar{N}_{s}=0.93
\end{gathered}
$$

where $r$ denotes the standard correlation coefficient, has been found, while in Germany [27] the expression

$$
\begin{aligned}
-\overline{\Delta N}= & 9.30 \exp \left(0.004565 \bar{N}_{s}\right), \\
& r_{\ln \bar{\Delta} \bar{N}} \cdot \bar{N} s=0.6,
\end{aligned}
$$

has been reported. A recent article by Lane [28] indicates that a similar correlation, $r_{\ln \Delta N} \cdot \vec{N} s=0.85$, is found over the British Isles. Although the slopes and intercepts of this relation between $\Delta N$ and $N_{s}$ obviously vary with climate, one concludes, for the purposes of the present discussion, that the observational evidence corroborates the assumption of an average exponential decrease of $N$ with height. The general application of the exponential model must await considerable work upon the part of the radio climatologists.

This has naturally led to the definition of a new unit that adds a correction factor to account for this exponential decrease $[29,30]$. This new unit, $A$, is given by

$$
A(h)=N(h)+N_{s}[1-\exp (-h / H)]
$$

It is possible to calculate a theoretical value of the scale of $N$ by assuming a distribution of water vapor. This is, however, quite a complex procedure. Futhermore, the value obtained depends upon the model of the water vapor distribution, and no definite conclusion can be justified considering the extreme variation of water vapor concentration with season, geographic location, and height above the earth's surface. A convenient and simple alternative is to adopt a value for $H$ from the average $(n-1)$ variation with height in the free atmosphere. Several such values of $H$ were determined by reference to the National Advisory Committee for Aeronautics ( $\mathrm{NACA}$ ) standard atmosphere and recent climatological stuclies of atmospheric refractive index structure [26]. It is seen in table 1.8 that $H$ varies from 6.56 to $7.63 \mathrm{~km}$ in the NACA standard atmosphere, depending on the value of the relative humidity assumed. The value of $H=7.01 \mathrm{~km}$ for $\$ 0$ percent relative humidity is 
in close agreement with $H=6.95 \mathrm{~km}$ obtained from climatological studies of $(n-1)$.

TABLE 1.8. Determination of the effective scale height, $\mathrm{H}$, for the radio refractive index

\begin{tabular}{|c|c|c|}
\hline Souree & Humidity & $H$ \\
\hline $\begin{array}{l}\text { NACA standard atmosphere. } \\
\text { Climatological data } \\
\text { adopted for this discussion. }\end{array}$ & $\begin{array}{l}\text { percent } \\
100 \\
80 \\
60 \\
\text { As observed }\end{array}$ & $\begin{array}{l}6.56 \\
7.01 \\
7.63 \\
6.95 \\
7.0^{*}\end{array}$ \\
\hline
\end{tabular}

*The value of $H=7.0 \mathrm{~km}$ was arbitrarily adopted for cse in the present discussion. Note that this value is smaller than the usual seale height near sea level of $8.0 \mathrm{~km}$ for the density distribution of the air. Although the value $H$ is quite arbitrary, it is evident from table 1.8 that $H$ should lie between 6.5 and $7.5 \mathrm{~km}$.

A comparison of $B, M, N$, and $A$ units is shown on figure 1.1 for aretic, temperate, and tropical climates. It is quite evident that both $M$ and $B$ over-correct the profile to produce increasing values with height while the $A$ unit tends to yield a constant value at heights in excess of $2 \mathrm{~km}$. It is noted, however, that important departures of the $N(h)$ profile from normal within the first few kilometer's are emphasized by both the $A$ and $B$ profiles.

In radio meteorology, as in other branches of meteorology, it is often convenient to express the potential value of the refractive index referred to some standard pressure level. In practice this is found by adding to the values at any height the product of the $N$ lapse rate and the height. This has already been done in the case of the $B$ unit, while the $A$ unit simply adds the total decrease of $N$ in an exponential atmosphere from the surface to the height under consideration. Yet another approach is to replace the values of pressure, temperature, and vapor pressure in the expression for $N$ with their values at some desired pressure level. This unit, ealled the potential refractive modulus, $\phi$, is then defined by

$$
\phi=\frac{77.6}{\theta}\left[P_{0}+4810 \frac{e_{0}}{\theta}\right]
$$

where $\theta$ is the potential temperature and $e_{0}$ the potential partial pressure of water vapor, both referred to the reference pressure $P_{0}$. This formula is that of Katz [31] with the Smith-Weintraub constants.

The potential temperature is defined as

$$
\theta=T\left(P_{0} / p\right)^{R / m c_{p}},
$$



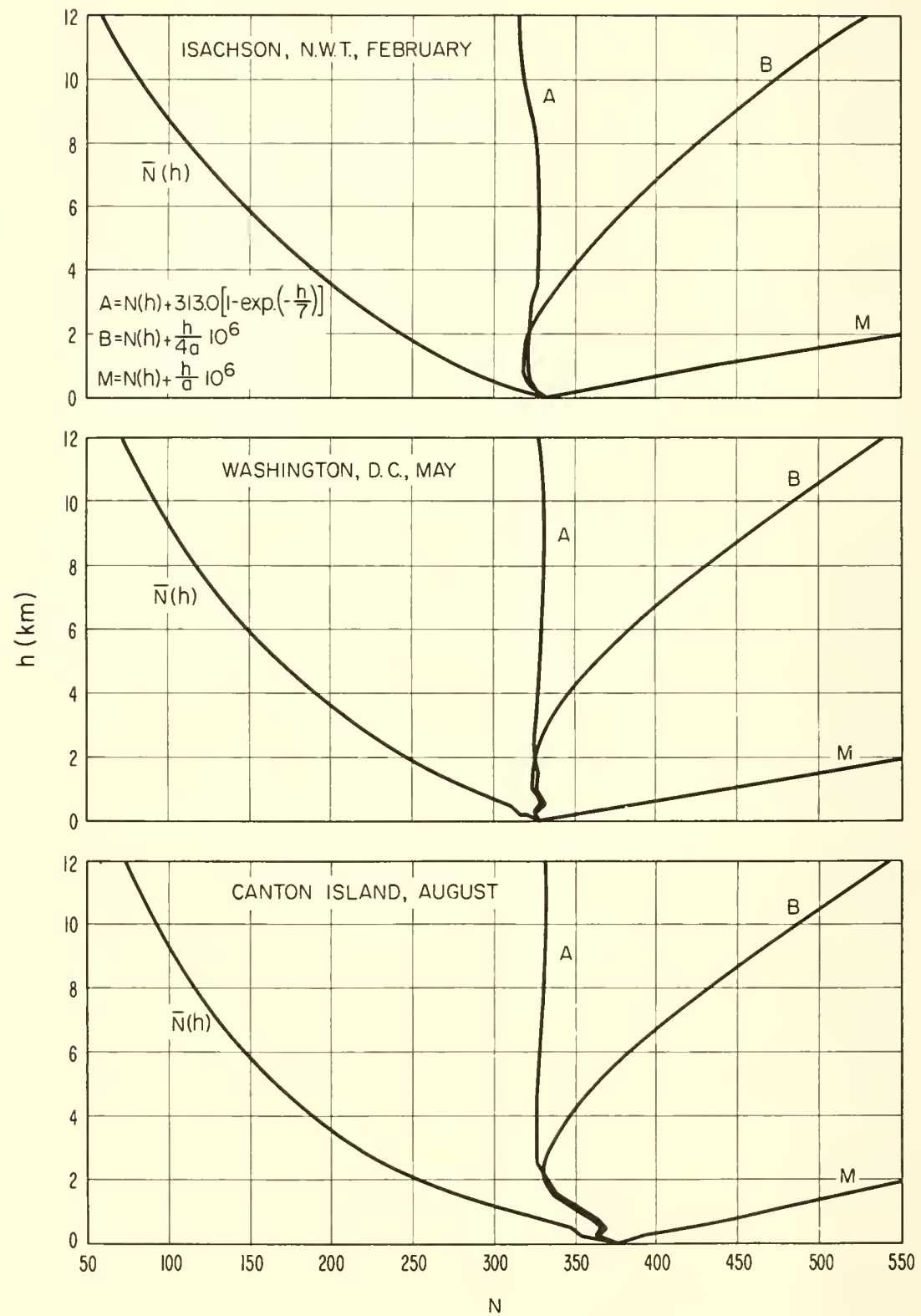

Figure 1.1. Variation of $\mathrm{N}, \mathrm{A}, \mathrm{B}$, and $\mathrm{M}$ profiles with climate. 
and $e_{0}$ as

$$
e_{0}=e\left(P_{0} / p\right)
$$

where $R$ is the universal gas constant, $m$ the molecular weight of air, and $c_{x}$ the specific heat of air at constant pressure. By setting $P_{0}=1000$ mbar and noting that $R / m c_{p}=0.286$, one obtains

$$
\phi=\frac{77.6 P}{T}\left(\frac{1000}{p}\right)^{0.714}+3.73 \times 10^{5} \frac{e}{T^{2}}\left(\frac{1000}{p}\right)^{0.428} .
$$

Thus, it is seen that $\phi$ is obtained by correcting the dry air term of $N$, $77.6 p / T$, by the factor $(1000 / p)^{0.714}$ and the water vapor term by the factor $(1000 / p)^{0.428}$. In well-mixed air, $\theta$ and $e_{0}$ are independent of height and consequently $\phi$ is independent of height. If the temperature lapse rate is less than the dry adiabatic rate, the potential temperature increases with height and, consequently, the first term of $\phi$ decreases with height. If the lapse of the partial pressure of water vapor is less than than given by (1.41) then $e_{0}$ and the second term of $\phi$ increase with height, conversely, if $e$ decreases more rapidly with height than the decrease given by (1.41), $e_{0}$ and the second term of $\phi$ decrease with height. Abundant examples of the vertical distribution of $\phi$ under various meteorological conditions are given throughout volume 13 of the Radiation Laboratory Series [32]. The potential refraction modulus has recently been used by Jehn [33] to illustrate the variability of $N$ about a classic polar-front wave.

A conceptually similar approach to that above is to arbitrarily adopt a reduced-to-sea-level value of refractivity

$$
N_{0}=N_{s} \exp (+h / 7.0)
$$

where $h$ is in kilometers, that effectively removes the station elevation dependence of $N_{s}$ [34] and allows the emphasis of air-mass differences.

Since the effects of the atmosphere upon the propagation of radio waves are dependent upon either the absolute value of $n$ or its gradient, we may use this as a criterion for the ehoice of appropriate units to represent the $N$ profile. One may recover both $n$ and its gradient from a knowledge of the height structure alone. The corrections are additive in the rase of $B, M$, and $A$ and multiplicative for $N_{0}$. The values $B, M$, and $A$ are in convenient form for refraction calculations since it is the gradient above the surface of the earth that is needed. The value, $N_{0}$, removes the effect of station elevation and thus is convenient for mapping of surface conditions in a fashion similar to sea-level pressure rather than station pressure. One cannot obtain the gradient and absolute value of $n$ from a $\phi(h)$ distribution, although $\phi(h)$ may be graphically calculated from the familiar 
characteristic diagram of the meteorologist; even for that process one must first calculate the potential values of both temperature and water vapor pressure. The relative merits of the various units are summarized in table 1.9 .

\subsection{Conclusions}

The above discussion has emphasized the following points:

(a) The differences in constants in the expression for $N$ are small compared with the error inherent in the formula.

(b) The refractive index is effectively nondispersive for frequencies below, say, $50 \mathrm{Gc} / \mathrm{s}$.

(c) The choice of atmospheric model for $N$, and concomitant units, depends upon the application at hand.

(d) The atmosphere, on the average, yields an exponential d'stribution of $N$ with leight.

(e) The use of an exponential model facilitates the preparation of climatic maps of $N$ and makes clear the effect of non-normal $N$ structure upon the bending of radio rays.

TABLE 1.9. Comparison of various units used in radio meteorology

\begin{tabular}{|c|c|c|c|c|}
\hline Unit & Gradient & $\begin{array}{l}\text { Referred } \\
\text { to: }\end{array}$ & Advantages & Disadvantages \\
\hline$B(h)=N(h)+(h / 4 a) 10^{6}$, & $\frac{d B(h)}{d h}=\frac{d N(h)}{d h}+(1 / 4 a) 10^{6}$ & Surface... & $\begin{array}{l}\text { Vertical distribution } \\
\text { identifies " } 4 / 3 \text { earth" } \\
\text { conditions; absolute } \\
\text { value and gradient of n } \\
\text { easily recovered. }\end{array}$ & $\begin{array}{l}\text { Over-corrects } \\
N(h) \text { above } \\
\text { the first few } \\
\text { kilometers. }\end{array}$ \\
\hline$M(h)=N^{\top}(h)+(h / a) 10^{6}$ & $\frac{d M(h)}{d h}=\frac{d N^{\top}(h)}{d h}+(1 / a) 10^{6}$ & Surface... & $\begin{array}{l}\text { Trapping or ducting } \\
\text { layers identified by } \\
(d M / d h) \leq 0, \text { absolute } \\
\text { value and gradient of } \\
\text { n easily recovered. }\end{array}$ & $\begin{array}{l}\text { Grossly over- } \\
\text { corrects } N(h) \\
\text { above the } \\
\text { trapping } \\
\text { layer. }\end{array}$ \\
\hline$\Lambda_{0}(h)=\Lambda^{\top}(h) \exp (h / \bar{\tau}, 0)$ & $\begin{aligned} \frac{d N_{0}(h)}{d h} & =\frac{d N^{\top}(h)}{d h} \exp \left\{\frac{h}{7.0}\right\} \\
& +\frac{N^{\top}(h)}{7} \exp \left\{\frac{h}{7.0}\right\}\end{aligned}$ & Sea-level. & $\begin{array}{l}\text { Removes effects of both } \\
\text { height and station ele- } \\
\text { vation; facilitates map- } \\
\text { ping since } N(h) \text { is } \\
\text { easily recovered. }\end{array}$ & $\begin{array}{l}\text { Gradient on } n \\
\text { not easily re- } \\
\text { covered. }\end{array}$ \\
\hline $\begin{array}{l}1(h)=N^{\top}(h) \\
\quad+313[1-\exp (-h / \pi .0)]\end{array}$ & $\begin{array}{l}\frac{d \cdot 1(h)}{d h}=\frac{d N(h)}{d h}+\frac{313}{7} \\
\quad \exp (-h / 7.0)\end{array}$ & Surface_.. & $\begin{array}{l}\text { Removes effects of height } \\
n \text { and gradient easily } \\
\text { recovered. }\end{array}$ & $\begin{array}{l}\text { Does not re- } \\
\text { move the ef- } \\
\text { fect of station } \\
\text { elevation. }\end{array}$ \\
\hline$\phi(h)=\frac{\pi 7.6}{\theta}\left(1000+\frac{4810 e_{0}}{\theta}\right)$ & $\begin{aligned} \frac{d \phi(h)}{d h}=\frac{\partial \phi(h)}{\partial \theta} & \frac{d \theta}{d h} \\
& +\frac{\partial \phi(h)}{\partial \ell_{0}} \frac{d e_{0}}{d h}\end{aligned}$ & $1000 \mathrm{mbar}$ & $\begin{array}{l}\text { Removes the effects of } \\
\text { height and station ele- } \\
\text { vation, may be de- } \\
\text { rived from character- } \\
\text { istic metcorological } \\
\text { diagram. }\end{array}$ & $\begin{array}{l}\text { Neither } n \text { or } \\
d n / d h \text { may } \\
\text { be recovered } \\
\text { from } \phi(h) ; \\
\text { must calcu- } \\
\text { late both } \\
\text { from basic } p \text {, } \\
T, \text { and } e \text { data }\end{array}$ \\
\hline
\end{tabular}




\subsection{References}

[1] Saxton, J. A. (Sept. 1951), Propagation of metre radio waves beyond the normal horizon, Proc. IEE 98, 360-369.

[2] Debye, P. (1957), Book, Polar Molecules, pp. 89-90 (I)over Publ. Co., New York, N.Y.).

[3] Saxton, J. A. (1947), The anomalous dispersion of water vapour at very high frequencies, Parts I-IV, Book, Meteorological Factors in Radio Wave Propagation, pp. 278-316 (The Physical Society, London, England).

[4] Smith, E. K., and S. Weintraub (Aug. 1953), The constants in the equation for atmospheric refractive index at radio frequencies, Proc. IRE 41, 1035-1037.

[5] Barrell, H. (May 1951), The dispersion of air between $2500 \AA$ and $6500 \AA$, J. Opt. Soc. Am. 41, 295-299.

[6] Maryott, A. A., and F. Buckley (1953), Table of dielectric constants and electric dipole moments of substances in the gaseous state, NBS Circular 537.

[7] Birnbaum, G., and S. K. Chatterjee (Feb. 1952), The dielectric constant of water vapor in the microwave region, J. App. Phys. 32, 220-223.

[8] Stranathan, J. J. (Sept. 1935), Dielectric constant of water vapor, Phys. Rev. 48, $538-544$.

[9] Phillips, W. C. (July 1950), The permittivity of air at a wave-length of 10 centimeters, Proc. IRE 38, 786-790.

[10] Crain, C. M. (Sept. 1948), The dielectric constant of several gases at a wavelength of 3.2 centimeters, Phys. Rev. 74, No. 6, 691-693.

[11] Essen, L., and K. I). Froome (Oct. 1951), The refractive indices and dielectric constants of air and its principal constituents at 24,000 Mc/s, Proc. Phys. Soc. (London, England) 64, 862-875.

[12] Groves, L. G., and S. Sugden (1935), Dipole moments of vapors II, J. Chen. Soc. England 30, 971-974.

[13] Hurdis, E. C., and C. P. Smyth (1942), Dipole moment, induction and resonance in nitroethane and some chloronitroparaffins, J. Am. Chem. Soc. 64, 28-29.

[14] Saito, S. (Aug. 1955), Measurement at 9,000 Mc of the dielectric constant of air containing various quantities of water vapor, Proc. IRE 43, No. 8, 1009.

[15] Battaglia, A., G. Boudouris, et A. Gozzini (Mlay 1957), Sur l'indice de refraction de l'air humide en microondes, Ann. de Telecomm. 12, 181-184.

[16] Wadley, T. L. (1957), The tellurometer system of distance neasurement, Empire Survey Review 14, No. 105, 100-111, and 14, No. 106, 1232-1239.

[17] Born, M., and E. Wolf (1959), Book, Principles of Opties, p. 89 (Pergamon Press, New York, N.Y., and London, England).

[18] Hughs, J. V., and H. L. Armstrong (May 1952), The dielectric constant of dry air, J. Appl. Phys. 23, 501-504.

[19] Sprung, A. (1888), Bestimm. d. Luftfeuchtigk. mit Hülfe d. Assmann'schen Asperationspsychrometers, 1)as Wetter 5, 105-109.

[20] Shelleng, J. C., C. R. Burrows, and E. B. Ferrell (Mar. 1933), Ultra-short-wave propagation, Proc. IlRE 21, 427-463.

[21] Millington, G. (Jan. 1957), The concept of the equivalent radius of the earth in tropospheric propagation, Marconi Rev. 20, No. 126, 79-93.

[22] Sinyth, J. B., and 1. G. 'Trolese (Nov. 1947), Propagation of radio waves in the lower atmosphere, Proc. IRE 35, 1198-1202.

[23] Report of Factual Data from the Canterbury Project (1951), Vols. I-I1I (1)ept. Sci. Indus. Research, Wellington, New Zealand).

[24] Anderson, L. J. (Apr. 1958), Tropospheric bending of radio waves, Trans. Am. Geophys. Union 39, 208-212. 
[25] Bauer, J. R., W. C. Mason, and F. A. Wilson (27 Aug. 1958), Radio refraction in a cool exponential atmosphere, Tech. Rept. No. 186, Lincoln Laboratory, Massachusetts Institute of Technology, Cambridge, Mass.

[26] Bean, B. R., and G. D. Thayer (May 1959), On models of the atmospheric refractive index, Proc. IRE 47, No. 5, 740-755.

[27] Bean, B. R., L. Fehlhaber, and J. Grosskopf (Jan. 1962), Die Radiometeorologie und ihre Bedeutung für die Ausbreitung der $\mathrm{m}-, \mathrm{dm},-$ und $\mathrm{cm}-$ Wellen auf grosse Entfernungen, 15, 9-16, Nachrichtentechnische Zeitschrift.

[28] Lane, J. A. (1961), The radio refractive index gradient over the British Isles, J. Atmospheric Terrest. Phys. 21, Nos. 2/3, 157-166.

[29] Bean, B. R., and L. P. Riggs (July-Aug. 1959), Synoptic variation of the radio refractive index, J. Res. NBS 63D (Radio Prop.), No. 1, 91-97.

[30] Bean, B. R., I. P. Riggs, and J. D. Horn (Sept.-Oct. 1959), Synoptic study of the vertical distribution of the radio refractive index, J. Res. NBS $63 \mathrm{D}$ (Radio Prop.), No. 2, 249-258.

[31] Craig, R. A., I. Katz, R. B. Montgomery, and P. J. Rubenstein (1951), Gradient of refractive modulus in homogeneous air, potential modulus, Book, Propagation of Short Radio Waves, ed. D. E. Kerr, pp. 198-199 (McGraw-Hill Book Co., Inc., New York, N.Y.).

[32] Craig, R. A., I. Katz, R. B. Montgomery, and P. J. Rubenstein (1951), Gradient of refractive modulus in homogeneous air, potential modulus, Book, Propagation of Short Radio Waves, ed. D. E. Kerr, p. 189 (McGraw-Hill Book Co., Inc., New York, N.Y.).

[33] Jehn, K. H. (June 1960), The use of potential refractive index in synoptic scale radio meteorology, J. Meteorol. 17, 264.

[34] Bean, B. R., and R. M. Gallet (Oct. 1959), Applications of the molecular refractivity in radio meteorology, J. Geophys. Res. 64, No. 10, 1439-1444.

[35] Waynick, A. H. (Oct. 1940), Experiments on the propagation of ultra-short-wave Proc. IRE, 28, 468-475.

[36] Smith-Rose, R. L., and A. C. Stickland (Mar. 1943), A study of propagation over the ultra-short-wave radio link between Guernsey and England on wavelengths of 5 and 8 meters, J. IEE 90, No. III, 12-24.

[37] Burrows, C. R., and S. S. Atwood (1949), Radio wave propagation, Consolidated Summary Technical Report of the Committee on Propagation, NDRC, p. 219 (Academic Press, Inc., New York, N.Y.).

[38] Essen, L. (Mar. 1953), The refractive indices of water vapour, air, oxygen, nitrogen, hydrogen, deuterium, and helium, Proc. Phys. Soc. B 66, 189-193.

[39] Birnbaum, G., S. J. Kryder, and H. Lyons, (Aug. 1951), Microwave measurements of the dielectric properties of gases, J. Appl. Phys. 22, 95-102.

[40] Meteorological Factors in Radio Wave Propagation (1946), (The Physical Society, London, England) Foreword.

[41] Englund, C. R., A. B. Crawford, and W. W. Mumford (July 1935), Further results of a study of ultra-short-wave transmission phenomena, Bell Sys. Tech. J. 13, 369-387.

[42] Magee, J. B., and C. M. Crain (Jan. 1958), Recording microwave hygrometer, Rev. Sci. Instr. 29, 51-54. 


\section{Chapter 2. Measuring the Radio Refractive Index}

\subsection{The Measurement of the Radio Refractive Index}

The radio refractive index is defined as the ratio of the speed of propagation of radio energy in a vacuum to the speed in a specified medium. The radio refractive index may be measured directly if the measuring instrument is sensitive to the speed of propagation. Refractive index is measured indirectly by measuring temperature, pressure, and humidity with subsequent conversion to the refractive index as indicated in the previous chapter. The direct method employs radio frequency refractometers to determine the refractive index; the indirect method, standard weather observations. The direct method appears preferable because accuracy is dependent upon a single sensor, rather than three and, of course, because $N$ values are obtained directly. However, refractometers are relatively complex and expensive devices requiring no small degree of skill to maintain. Hence, as yet, refractometers are not in general use, or even available in sufficient quantity to permit large-scale mapping of refractive index structures. The bulk of the synoptic and climatological mapping of refractive index is still based on the indirect method of measurement. The laborious task of converting the measured parameters to refractive index has been somewhat alleviated by the development of special analog computers $[1]^{1}$ and digital computers.

\subsection{Indirect Measurement of the Radio Refractive Index}

The accuracy of the determination of the refractive index from standard weather observations has been discussed in chapter 1 . Figure 2.1 illustrates the degree of accuracy to be expected in the measurement of the refractive index as a function of the accuracies of the sensors for sea-level conditions. It is assumed that the errors are additive. Planes of equal

${ }^{1}$ Figures in brackets indicate the literature references on p. 45. 


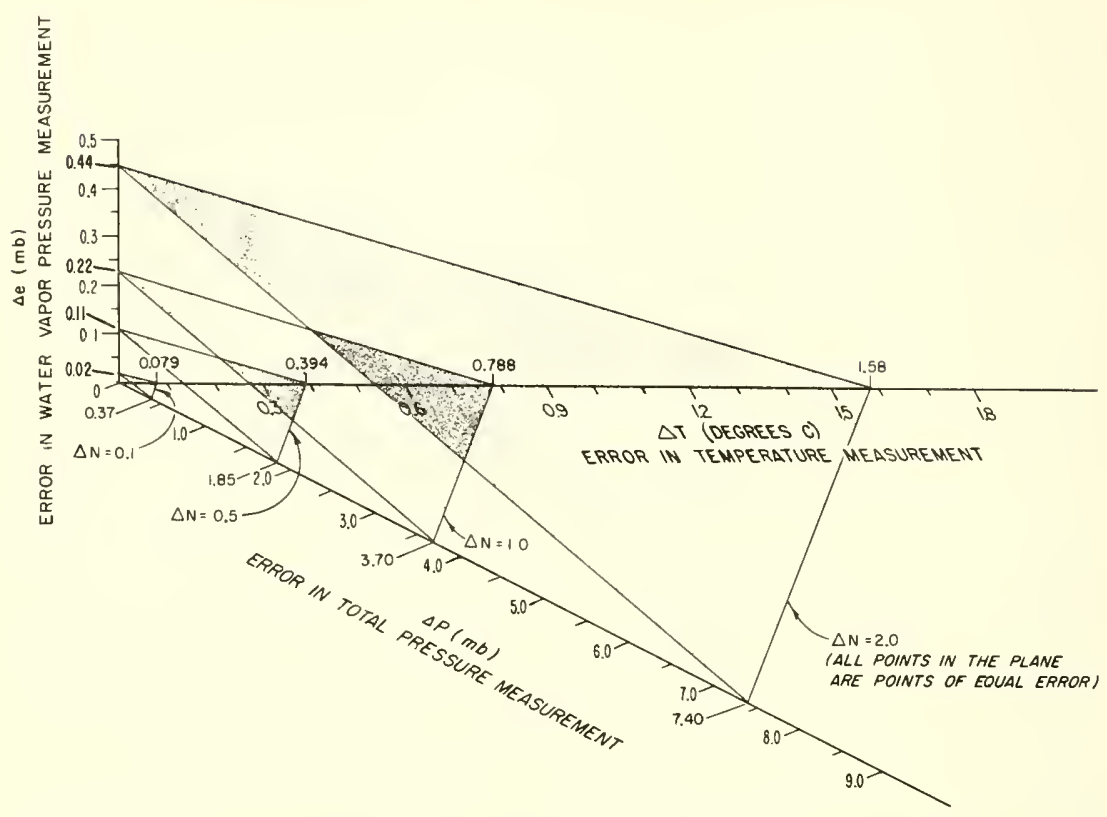

Figure 2.1. Accuracy of the determination of refractivity as a function of the accuracies of the meteorological sensors.

accuracy are shown for accuracies of $\pm 0.1, \pm 0.5, \pm 1.0$, and $\pm 2.0 \mathrm{~N}$. It can be seen that a measurement error of $\pm 1.0 \mathrm{~N}$ can be contributed by each sensor if the sensor errors exceed $\pm 0.8^{\circ} \mathrm{C}$ for temperature, \pm 3.7 mbar for total pressure, and \pm 0.22 mbar for vapor pressure measurements. It is apparent that extreme accuracy is required in the measurement of the water pressure. The temperature can be measured easily to within several tenths of a degree, the total pressure to within several millibars, but the humidity is considerably less responsive to the same degree of relative accuracy. If the wet and dry bulb hygrometer technique is used to measure the humidity with subsequent conversion using either the psychrometric formula of Sprung ${ }^{2}$ [2] or List ${ }^{3}$ [3], the degree of accuracy is seen to be a function of temperature. For an error of no greater that $\pm 1.0 \mathrm{~N}$, the relative humidity must be accurate to within 0.33 percent at $35^{\circ} \mathrm{C}$, while at $0{ }^{\circ} \mathrm{C}$ an accuracy of 3.0 percent is necessary.

$$
\begin{aligned}
& { }^{2} e=e_{\mathrm{s}}-\mathrm{c} \frac{(\Delta \mathrm{T}) p}{566}, c \text { is a constant. } \\
& { }^{3} e=\mathrm{e}_{\mathrm{s}}-\left[c_{1}\left(1+c_{2} t_{w}\right)\right] p(\Delta T), c_{1} \text { and } c_{2} \text { are constants. }
\end{aligned}
$$


Experimenters at the National Physieal Laboratories [4] have shown that when an Assman hygrometer is used with thermometers aceurate to within $0.1{ }^{\circ} \mathrm{C}$, the optimum expected aceuracy in the determination of the water vapor pressure is $0.2 \mathrm{mbar}$. Errors three times as great can be expected at extreme eonditions. Hence, it would appear that measurement of water vapor limits the aceuracy of the determination of the refractive index to approximately $\pm 1.0 \mathrm{~N}$ units.

Temperature, pressure, and humidity are standard measurements of the world's weather services. Methods of measuring these parameters are fairly standard. Usually the temperature is read from mercurial or alcohol thermometers, the pressure from mereurial barometers, and the humidity from a conversion of wet-and dry-bulb thermometers. The degree of accuracy of these measurements is usually a funetion of the care exercised by the observer. Thermometers protected by radiation shields are usually aceurate to within $\pm 0.1^{\circ} \mathrm{C}$, barometers to within $\pm 1.0 \mathrm{mbar}$. Reading errors can easily be in excess of the instrument error, especially in the determination of humidity where the wet-bulb depression is subjeet to many sourees of error. Contamination of the wiek or water, insufficient wetting, and inadequate aspiration are common sources of error. The wet-bulb determination ean be used below freezing, if proper preeautions are observed.

Automatic-recording systems have been devised for measurement of temperature, pressure, and humidity. The simplest are the hygrothermograph and the microbarograph, in which the sensors are connected by mechanical linkages to pens or ehart recorders. The pressure is recorded by means of an aneroid eapsule, the temperature by means of a bimetal strip or a curved Bourdon tube, and the humidity by means of a hair hygrometer. The accuraey of such devices limits the determination of $N$ to within 2 or $3 \mathrm{~N}$ units.

Sensors producing electrical outputs are used to measure meteorological parameters in a variety of automatic-recording systems, such as stripchart recorders, punched-paper tape, and magnetic-tape recorders. The more common temperature sensors include resistance thermometers, thermocouples, and thermistors. The platinum resistance thermometer, an international standard, is capable of measuring temperature in still air to within $\pm 0.05{ }^{\circ} \mathrm{C}$ if used in a well-compensated bridge eircuit. However, the platinum resistance thermometer is velocity-sensitive in a moving air stream. Thermocouples avoid this problem and yield shortterm accuracies of approximately $\pm 0.1{ }^{\circ} \mathrm{C}$ with time constants measured in milliseconds.

The total atmospheric pressure can be measured by a variety of electrical sensors. The simplest device is the pressure potentioneter where an aneroid capsule is mechanically linked to a potentiometer. One or two millibar's can be considered the limit of aecuracy in a differential 
device operating over a range of $\pm 100 \mathrm{mbar}$ with reference to an average value with time constants of perhaps $5 \mathrm{msec}$. The capacitive microphone is capable of accuracies of $\pm 0.01 \mathrm{mbar}$ and lag constants of several milliseconds over a limited range $( \pm 2 \mathrm{mbar})$. The resolution diminishes to about $\pm 0.1 \mathrm{mbar}$ over the normal range of variations of atmospheric pressure. The capacitive microphone requires correction for significant temperature variations. Strain-gage pressure transducers have approximately the same characteristics as capacitive microphones with some degradation in lag constants. Differential strain gauge transducers operating over a range of 4 to 5 mbar may yield accuracies of several hundredths of a millibar and are relatively free of temperature effects.

Relative humidity requires the greatest care in measurement. The common lithium chloride strip is accurate to within \pm 5 percent in relative humidity. In general, the lag constant is of the order 8 to 10 sec for temperatures in excess of $0{ }^{\circ} \mathrm{C}$ (see sec. 2.5). Other sensors under development show promise. The barium fluoride strip [5] yields accuracies, hysteresis effects, and response times far superior to lithium chloride but has the disadvantage of rapid aging. Phosphorous pentoxide sensors [6] and aluminum oxide sensors [7] appear to be quite promising. The most accurate method that is available for measuring water vapor pressure is the wet-dry bulb technique using electrical thermometers $( \pm 0.02 \mathrm{mbar})$. The lag constant will be relatively large, as it is a function of the wetting properties of the wick, the rate of aspiration, and even the relative humidity, and is thus recommended only for temperatures above $-24^{\circ} \mathrm{C}$.

Measurements up to several thousand feet can be made from towers or with tethered balloons or a wiresonde. Only the sensors need be sent aloft. All auxiliary devices are on the ground, and long cables transmit the information from the sensors to the recording equipment $[8,9]$.

The radiosonde is in almost universal use for high-altitude measurement of the meteorological parameters influencing the refractivity. There are many models of the radiosonde, differing from country to country. The principle of operation for present American radiosondes is illustrated in figure 2.2. An aneroid capsule is used as the active element of a baroswitch. The temperature sensor, humidity sensor, and a reference resistance are alternately switched into the grid circuit of a blocking oscillator as the baroswitch wiper moves under the action of decreasing pressure. The blocking oscillator controls the pulse rate of the rf transmitter; hence, the pulse rate of the transmission is indicative of the value of the sensor being sampled. The number of the contact energized is a measure of the pressure; the switching sequence permits identification of temperature and humidity values. A constant rate of ascent of the balloon is assumed so that the values of the parameter's can be identified with the proper altitude. 


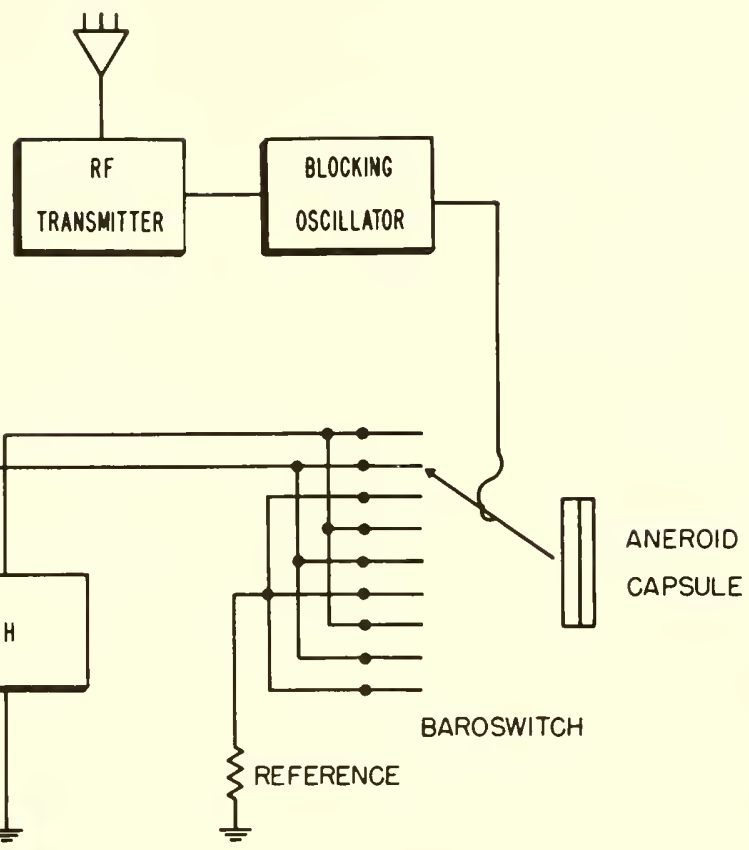

Figure 2.2. Block diagram of the radiosonde.

There is no uniformity of sensors used in the world's radiosondes. Hence, it is difficult to compare measurements from different countries. At one international comparison at Payerne, Switzerland, in 1956, 14 nations participated [10]. The results indicated significant differences. Temperature measurements corresponded to within $\pm 1.5^{\circ} \mathrm{C}$ for night flights but corresponded only to withm $\pm 3.5^{\circ} \mathrm{C}$ for daytme flights. Pressure measurements agreed well at low altitudes but indicated a dispersion of $\pm 1.5 \mathrm{mbar}$ above $9,000 \mathrm{~m}(29,000 \mathrm{ft})$, and $\pm 2.5 \mathrm{mbar}$ above $16,000 \mathrm{~m}(50,000 \mathrm{ft})$. Humidity comparisons were poor, indicating that 15 percent would be the most optimistic estmate of the standard deviation from the mean for all flights. On the average, it is estimated that the standard American temperature sensor indicated a value approximately $1.5^{\circ} \mathrm{C}$ below the mean for all sondes used in the test. The pressure determination was below the mean by 0.5 mbar, whereas the humidity sensor could not be quantitatively evaluated due to the erratic behavior of all sensors.

No attempt was made at these comparison trials to determine the absolute accuracy of any radiosonde. Although the absolute accuracy of the American sonde has not been determined, satistical evaluation of 
the uniformity of American radiosondes has been conducted by both the U. S. Air Force and the U. S. Army Signal Corps. Results indicate a standard deviation for the temperature sensor of $0.8{ }^{\circ} \mathrm{C}$ to $6,000 \mathrm{~m}$ and $1.0^{\circ} \mathrm{C}$ above that altitude. The standard deviation for the pressure determination was 2.2 mbar below $9,000 \mathrm{~m}$ and 1.1 mbar above $9,000 \mathrm{~m}$. Under ideal conditions the humidity sensor (lithium chloride) exhibited a standard deviation of 5 percent. This accuracy is possible only if the element is not subjected to high humidity (95 to 100 percent), or saturation by liquid water, and if the temperature is above $0{ }^{\circ} \mathrm{C}$. The response of the element is especially poor where both temperature and humidity are low. The measurement means little if the relative humidity is below 15 percent at a temperature of $20^{\circ} \mathrm{C}, 20$ percent at a temperature of $0{ }^{\circ} \mathrm{C}$, or 30 percent at a temperature of $-30{ }^{\circ} \mathrm{C}$.

Hence, "under ideal conditions" at sea level, the standard deviation in the determination of the refractivity from radiosonde data is approximately $3 \mathrm{~N}$ units; at $1 \mathrm{~km}$, the standard deviation would be approxi-

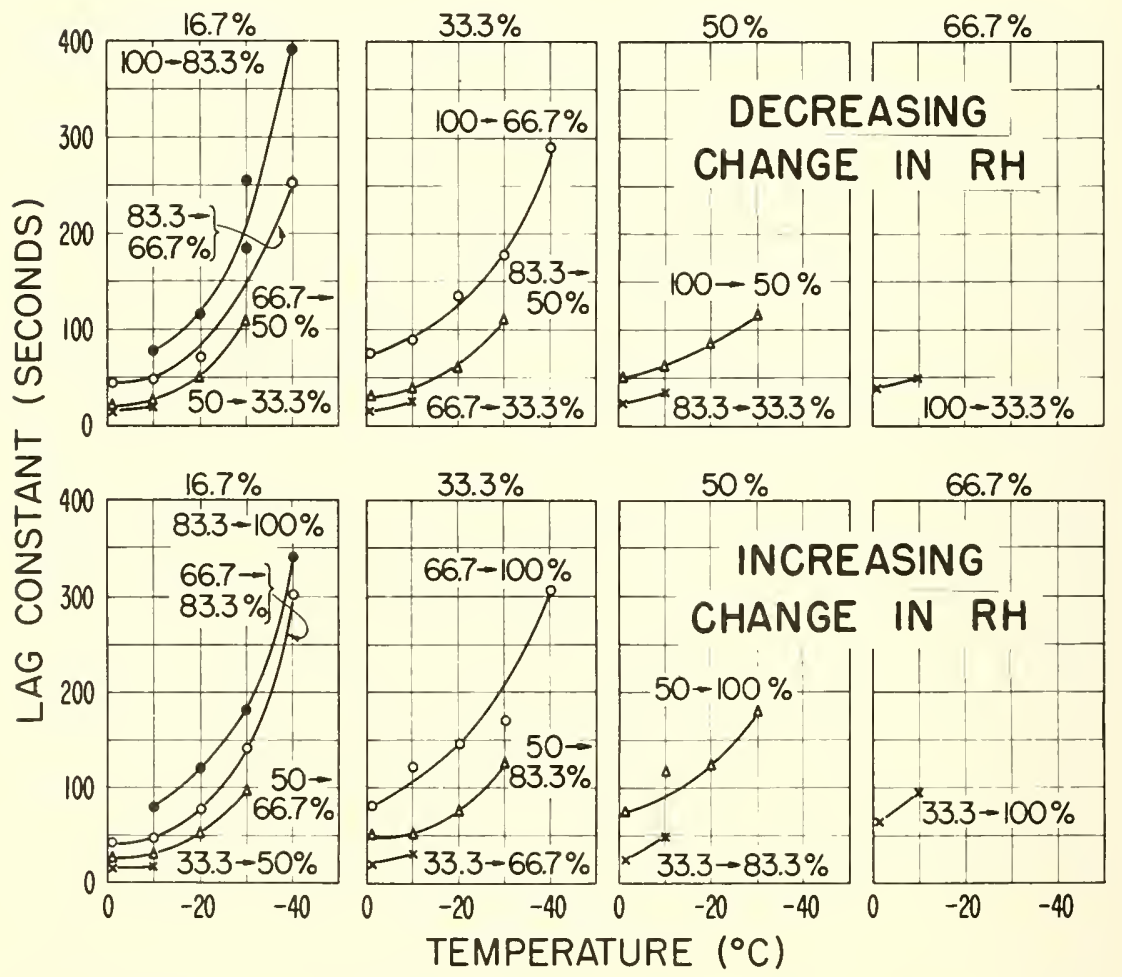

Figure 2.3. Factors affecting the lag constant of the lithium chloride humidity sensor. (After wexler.) 


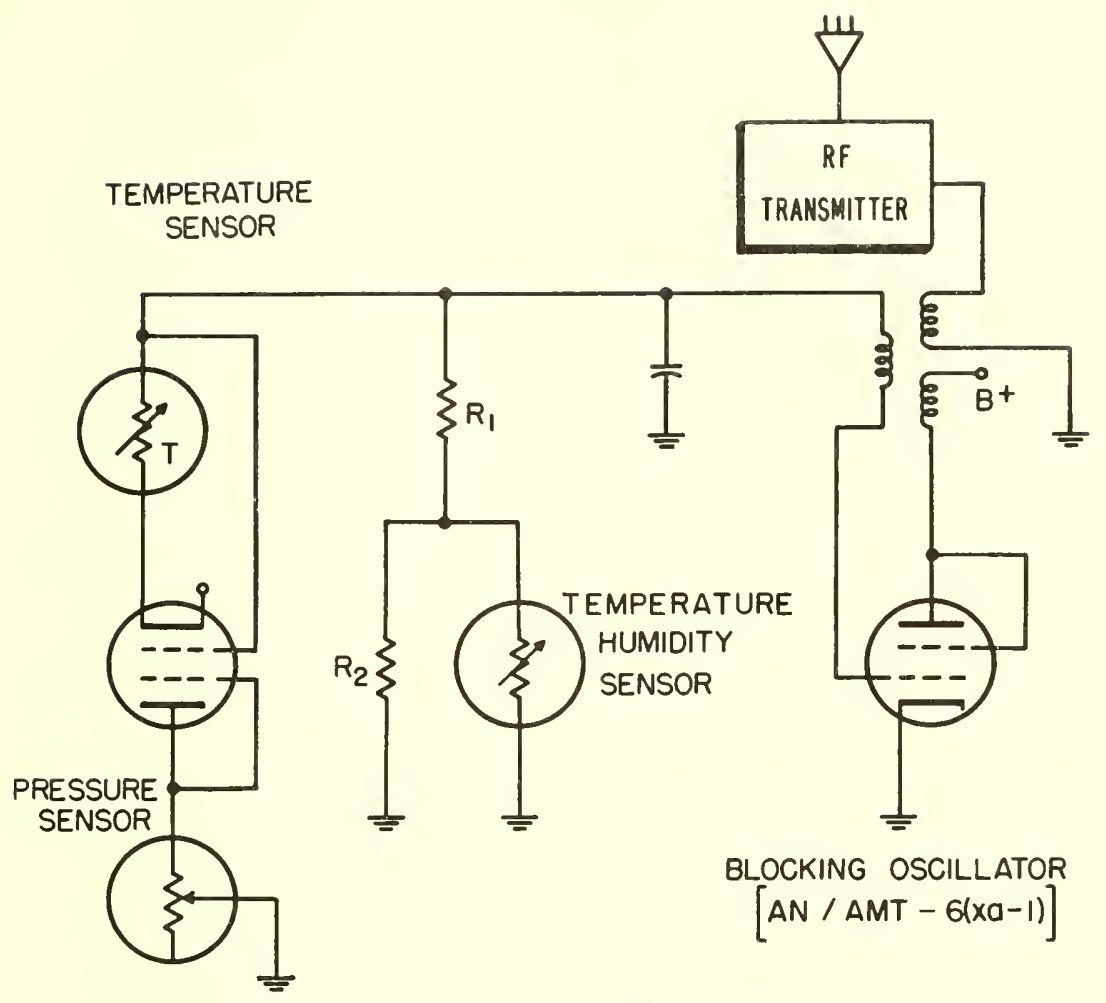

Figure 2.4. Clinger-Straiton radiosonde.

(Transmission in $N$ units.)

mately $2 N$ units. This then would appear to be the ultimate precision with which present conventional radiosonde sensors can yield the refractivity.

The lag constants of the radiosonde sensors are also of importance. Since the sonde is rising at a relatively rapid rate, it passes into regions of changing refractivity before the sensor's are aware of it. The lag coefficient associated with the radiosonde introduces an error in the estimation of the true gradient. Lag constants of sensors have been analyzed by Wexler [11] and by Bean and Dutton [12]. Some correction can be made to the radiosonde data. Wexler (fig. 2.3) shows that the lag coefficient of the lithium chloride strip is a function not only of the temperature, but also of the absolute value of the relative humidity, as well as of the size and direction of the gradient.

The radiosonde samples temperature and humidity in sequence rather than simultaneously. Several experimenter's have devised means to correct this deficiency. Misme [13] decreased the cycling time in one radio- 


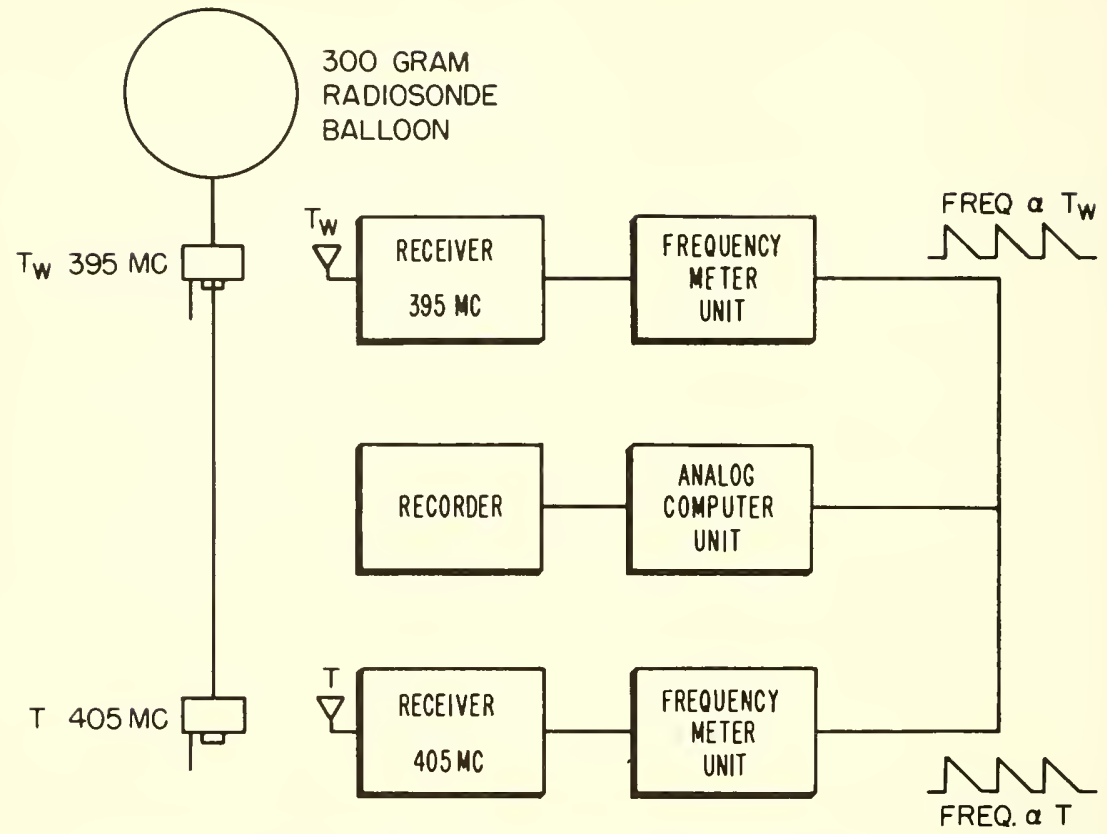

FIgure 2.5. Schematic diagram of the Navy Electronics Laboratory (Thiesen) system.

sonde so that many more samples of each parameter were produced per unit time. Clinger and Straiton [14] developed a radiosonde that combines the parameters so that the output signal is in terms of the refractive index (see fig. 2.4). Since the wet and dry terms are additive, a parallel combination of independent conductances can be used. The dry term sensor has a conductance proportional to temperature and inversely proportional to pressure; the wet term sensor is such that it not only yields a value proportional to the relative humidity, but also adjusts the relative magnitude of the two terms. Thiesen [15] devised a similar radiosonde utilizing two separate sondes on the same balloon (see fig. 2.5). The temperature and humidity information are combined at the ground station by means of an analog computer. For captive balloon application, Hirao and Akita [8] and Crozier [9] developed similar systems using wet and dry bulb thermistors to produce direct output in refractive index.

\subsection{Direct Measurement of the Refractive Index}

The resonant frequency of a microwave cavity is a function of its dimensions and the refractive index of its contents. Hence, if a cavity is open to the atmosphere, the resonant frequency changes as the refractive index of the air passing through it changes. If a sealed reference cavity is 
used for comparison, the difference between resonant frequencies beeomes a convenient measure of the refractive index variations in the sampling cavity. This method of measurement was used in three different instruments: the Crain type, the Birnbaum type, and the Vetter type. The Crain refractometer utilizes the cavities as frequency determining elements in ultra-stable oscillators. The difference in frequency is then the unit of measure. The Birnbaum refractometer utilizes the eavities as passive resonance frequencies of the cavities. The time difference between resonances is the unit of measure. The Vetter instrument, an improvement on Sargent's [16] modification of the Birnbaum's refractometer, utilizes servo techniques to achieve a null system, thus eliminating the necessity for extreme electronic stability.

The resonant cavities are significant components in any of these instruments. Of prime importance is the temperature coefficient of the cavity. Most cavities today are made of invar having a temperature coefficient of approximately one part per million per degree centigrade $[17,18]$. This is equivalent to $1 N$ unit per degree centigrade. Further temperature compensation of the cavity has produced temperature coefficients of 0.2 and $0.1 N$ unit per degree centigrade [19]. Most recently, improved invar eavities, when compensated, yielded temperature coefficients of $0.03 N$ units per degree centigrade [20]. The possibility of improving cavity performance by use of special ceramics $[21,22]$, with temperature coefficients (without compensation) of $0.1 \mathrm{~N}$ units per degree centigrade has been investigated.

The response time of the refractometer is a function of how much of the end plates of the eavity can be opened to the air without appreciable loss of resonant characteristics. It has been found [23, 24], that as mueh as 92 percent of the end plate area could be eliminated without serious resonant degradation. Such end plates do not impede the flow of air, and the response time of the instrument is essentially instantaneous.

In general, the accuracy of the refractometer is at least one order of magnitude smaller than can be achicved by indirect measurement. With proper care, these instruments are capable of discerning changes of the refractive index that are less than a tenth of an $N$ unit. As a relative instrument, i.e., used to measure variations about an undetermined mean, the time constant is such as to easily allow detection of rates of up to $100 \mathrm{c} / \mathrm{s}$.

The development of microwave refractometers led to an immediate increased interest in the fine structure of refractive index variations and its application to radio wave propagation. A summary of this development has been given by Herbstreit [25]. Detailed investigations of the inhomogeneities of refractive index structure using spaced cavities yielded significant results pertinent to the spectrum of turbulence [26, 27]. Refractometers have been widely used in aircraft [18, 28, 29]. The Crain 
refractometer [29, 30], as illustrated in figure 2.6 , utilizes one sealed eavity as a reference eavity and a ventilated cavity as the sampling element. Each of these cavities serves as a frequency-determining element of a Pound [31] oscillator. Very good cavity resonance is required. The Crain refraetometer is so designed that the cavity resonators operating in the $9400-\mathrm{Mc} / \mathrm{s}$ range are slightly different in resonant frequency. The resultant difference frequency is the center frequeney from which departures due to changing refractive index are measured. Recent models utilize a $43 \mathrm{Me} / \mathrm{s}$ center frequency. As the refractive index of the contents of the sampling cavity changes, the difference frequency at the output of the mixer changes according to

$$
\frac{\Delta f}{f}=-\Delta n \text {. }
$$

Then, since

$$
\Delta N=\Delta n 10^{6},
$$

a change of $1 \mathrm{~N}$ unit corresponds to a change in the resonant frequency

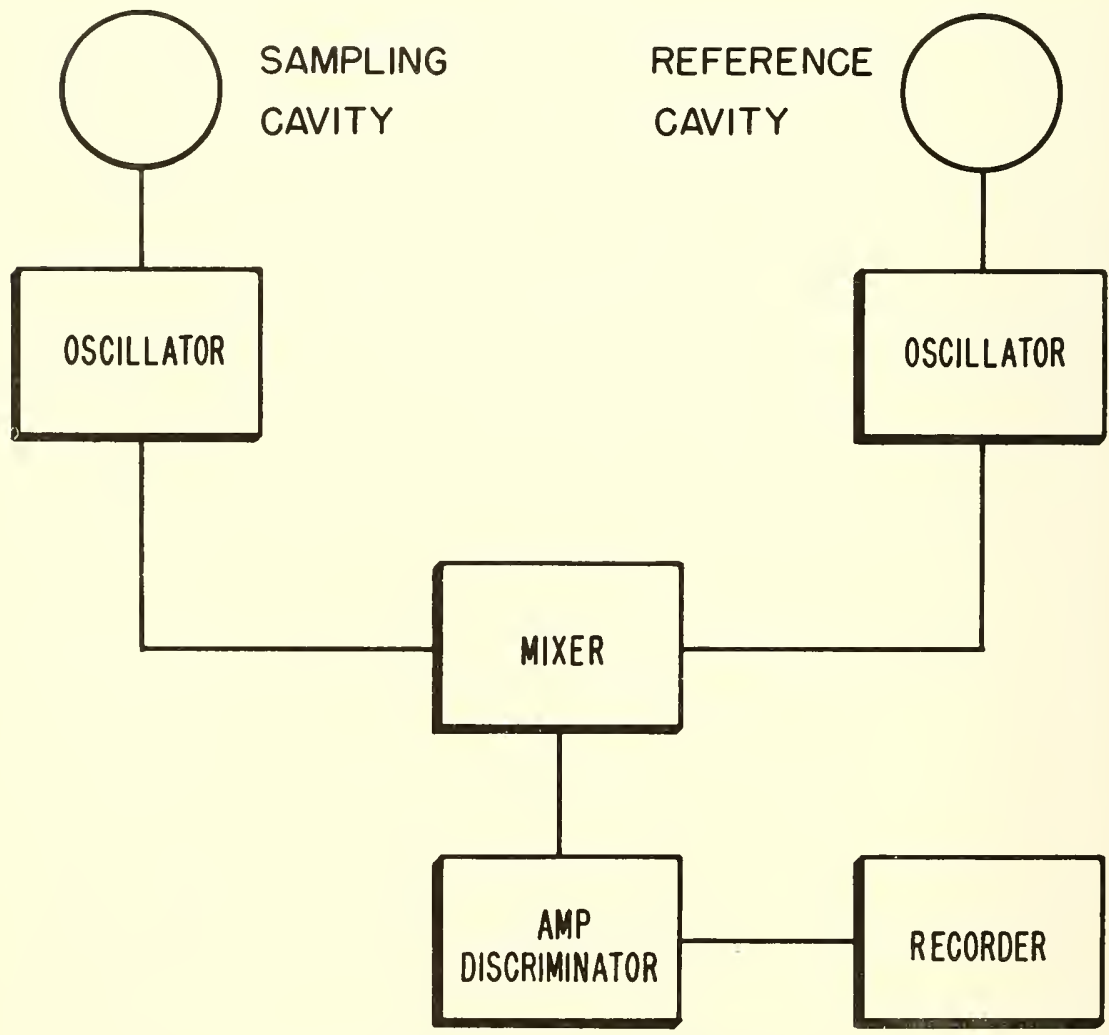

Figure 2.6. The Crain refractometer. 


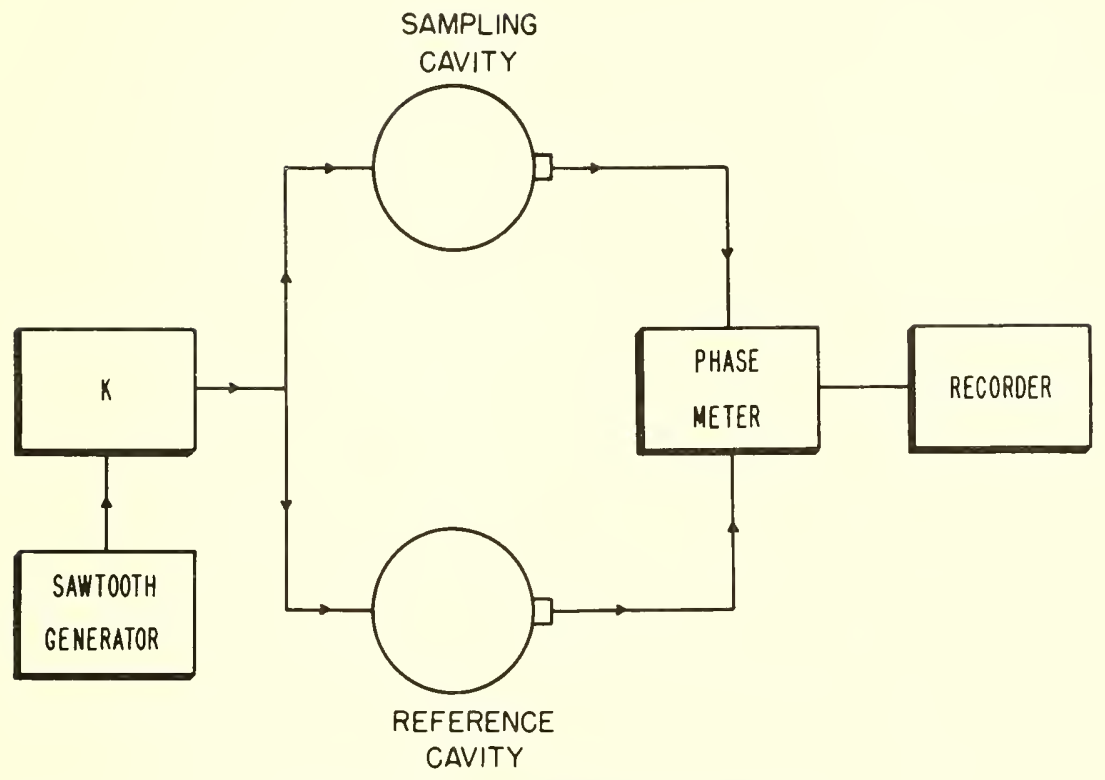

Figure 2.7. The Birnbaum refractometer.

of the sampling cavity of approximately $9.4 \mathrm{kc} / \mathrm{s}$ if the nominal operating frequency is $9400 \mathrm{Mc} / \mathrm{s}$.

For the measurement of small seale variations of refractive index, a modified Crain refractometer utjlizing a $10.7-\mathrm{Me} / \mathrm{s}$ center frequency with a 200-ke/s linear range has been used [29]. Scales of 1 to $20 \mathrm{~N}$ units for full seale deflections of a 1-mA chart recorder have been used.

The Birnbaum refractometer [32], illustrated in figure 2.7, applies the resonance principle in a somewhat different fashion. Both the reference, or sealed, cavity and the sampling, or open, eavity are passive clements. The cavities are slightly different in frequency and are of the transmission type having erystal detectors at the output.

A single klystron, frequency modulated by a sawtooth voltage and having an output frequency linear with time, excites each eavity in sequence. During the frequency excursion of the klystron, the respective cavity resonances are excited, and a pulse formed at each crystal detector. Since the resonant frequencies of the cavities differ, the two output pulses will be displaced in time. If the modulation on the klystron is periodic, then the output pulses will be also displaced in phase, the displacement being a function of the difference in resonant frequencies. Any change in the refractive index of the contents of the sampling eavity will alter the phase difference between the two pulse trains. The relative phase between the two outputs can then be measured by an electronic phase meter. 
The phase meter in use is merely a multivibrator having a constant amplitude output. The pulse from each cavity alternately switches the multivibrator "on" and "off." The width of the constant amplitude output pulse from the multivibrator is a measure of the time difference between pulses. The resulting train of constant amplitude variable width pulses is then applied to appropriate recording circuits. The Birnbaum refractometer is adaptable to multi-cavity operation. A single klystron may be used to sweep simultaneously a number of spaced sampling cavities. A single reference eavity permits simultaneous comparison between the several sampling cavities, and important consideration when the scale and form of refractive index variations are desired ([27].

The accuracy of the Birnbaum instrument is dependent upon maintaining a linear frequency sweep. This problem can be circumvented in the manner of Sargent [16], who modified the Birnbaum refractometer to operate as a microwave hygrometer. A servomechanism is used to tune the sampling eavity to the resonant frequency of the reference cavity. The servomechanism positions a tuning probe in the sampling cavity. The depth of penetration is the measure of the refractive index of the contents of the sampling cavity. This technique minimizes the dependence on the sweep characteristics of the klystron.

The Vetter refractometer [20], figure 2.8, virtually eliminates the dependence of the refractometer on electronic characteristics and shifts the limitations to the cavities themselves. In addition, while previous instruments are primarily relative refractive index indicators, the Vetter refractometer was developed as an absolute refractive index device by using klystron stabilization techniques [33, 34, 35, 36].

Figure 2.8 is a simplified diagram of the Vetter refractometer illustrating the basic prineiple of operation. The reference cavity is excited by a klystron which is modulated by a small sine voltage on the repeller. Any output at the fundamental modulating frequency at the detector of the reference cavity is compared in phase to the original modulating signal, and an error signal is applied to the repeller of the klystron to lock the center frequency of the klystron to the reference cavity. At coincidence, the fundamental disappears in the output of the reference cavity. The same klystron exeites the sampling cavity. Any fundamental appearing at the output after phase comparison to the modulating signal develops another error signal. This error signal is used to drive a mechanical servomechanism which tunes the reference cavity to the resonant frequency of the sampling cavity with concomitant shifting of the klystron center frequency. This then is a double loop system; the reference cavity controls the klystron; the sampling cavity controls the reference cavity.

Tuning of the reference cavity is accomplished by a motor-driven probe that penetrates the reference cavity. The design of the probe is 


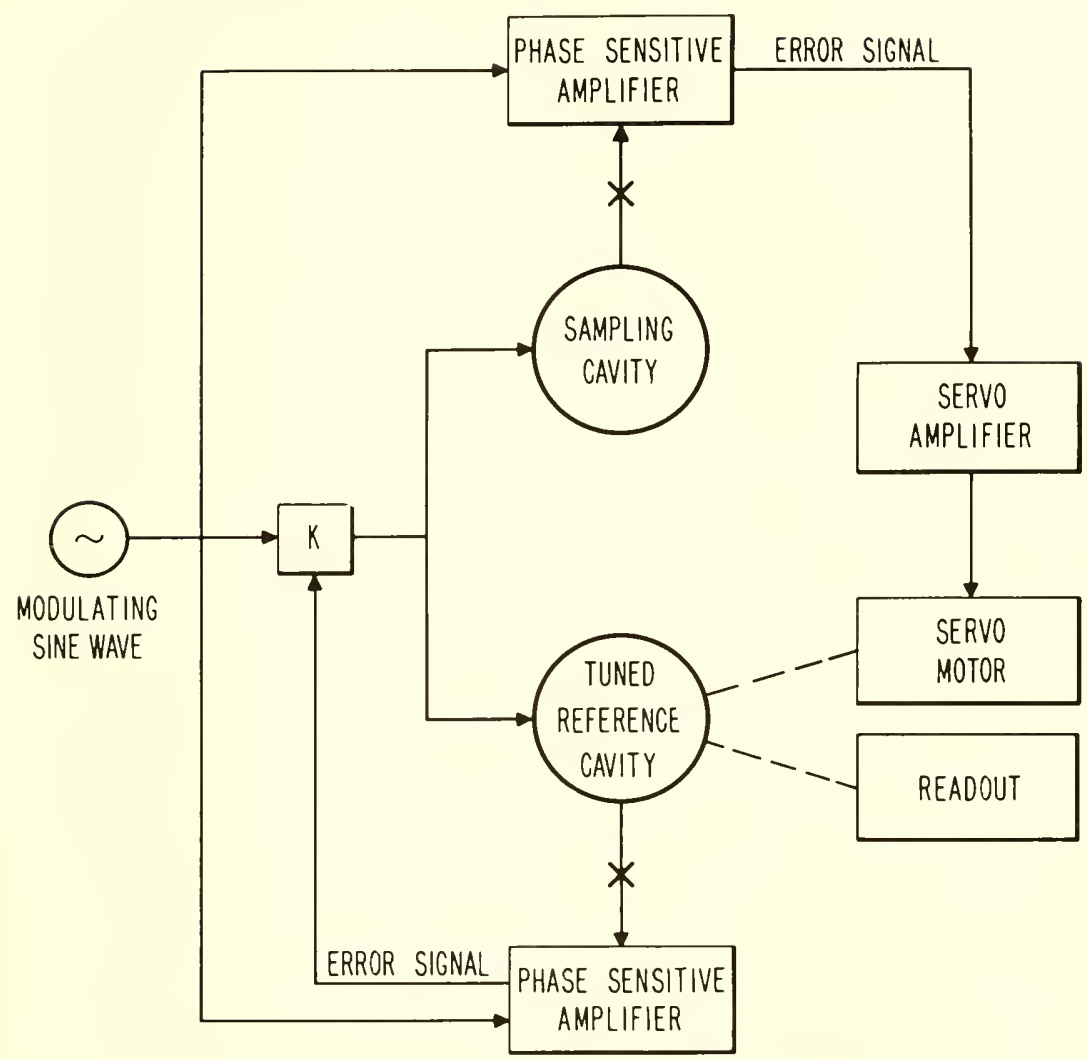

Figure 2.8. Simplified diagram of the Vetter refractometer.

such as to permit very nearly a linear function of $\Delta N$ versus probe penetration over the range of operation. Once the probe is calibrated with the cavity, the relative calibration is static and practically independent of the contents of the cavity. Although the reference cavity is sealed, there is no necessity for a vacuum. Absolute calibration is accomplished by determining the zero point, or the intercept, where $N=0$, by evacuating the sampling cavity.

Since the Vetter refractometer is mechanically tuned by a servosystem having considerable inertia, the high-frequeney response is relatively poor. The upper frequency response is $10 \mathrm{c} / \mathrm{s}$. Accuracy and simplicity of operation are its distinct advantages.

Refractometers have been used to measure both the surface value and the gradient of the refractive index. Although the units so far described were designed originally as ground-based equipment, modifications have 
been made to convert them to airborne use. However, where vertical gradients to high altitude are desired, practical aspects of aircraft operation such as the turning radius and the angle and rate of climb or glide are factors influencing how well the vertical gradients are estimated. Considerable horizontal variation in $N$ is often observed. In addition, the time involved in ascent or descent is relatively long, and the measurements are valid only under static conditions for the total time of measurement. Due to expense and weight, conventional refractometers are not economical or practical for use in balloon ascent, whether frec or tethered. This led to the development of several lightweight refractometers to be used either in wiresonde, radiosonde, or dropsonde applications.

Figure 2.9 is a block diagram of a lightweight $(6 \mathrm{lb})$, expendable refractometer developed by Deam [37] and Deam and Cole [38] for use as a balloon-borne unit or a dropsonde. The device is a Pound oseillator [31], operating at nominal frequency of $403 \mathrm{Mc} / \mathrm{s}$; the instantaneous frequency is determined by coaxial cavity. The refractive index is sampled by the cavity, and is reflected as a capacitance in the tuned circuit of the oscillator.

Operational tests indicate the electronics are sufficiently stable to produce the desired accuracy although the cavity size and mass appear to introduce a sizable time constant. Present accuracy is estimated to be better than $\pm 5 \mathrm{~N}$ units for a complete profile.

The Hay refractometer [39] was developed as a compromise between the microwave refractometer and the conventional radiosonde (see fig. 2.10 ). It lacks the accuracy of the microwave refractometer by an order of magnitude but, weighing only $7 \mathrm{lb}$, it incorporates the lightweight feature of the radiosonde. As a balloon-borne instrument it fills a need for more accurate, faster probing of the refractive index at the higher elevations.

This refractometer is a $10-\mathrm{Mc} / \mathrm{s}$ oscillator whose frequency is determined by an air-scusing capacitor. A change in the refractive index of the air passing through the capacitor is reflected as a frequency change in the oscillator. The scusing capacitor is alternately switched with a reference capacitor of identical design but which is protected from the free atmosphere. The use of a reference capacitor diminishes the problem of temperature compensation since both capacitors will be at very nearly the same tempcrature. To further improve the temperature characteristics, the 12 plates of each capacitor are made of invar and are separated by 0.25 -in. quartz spacers. The output of the $10-\mathrm{MIc} / \mathrm{s}$ oscillator is doubled in frequency and transmitted to the ground station. 


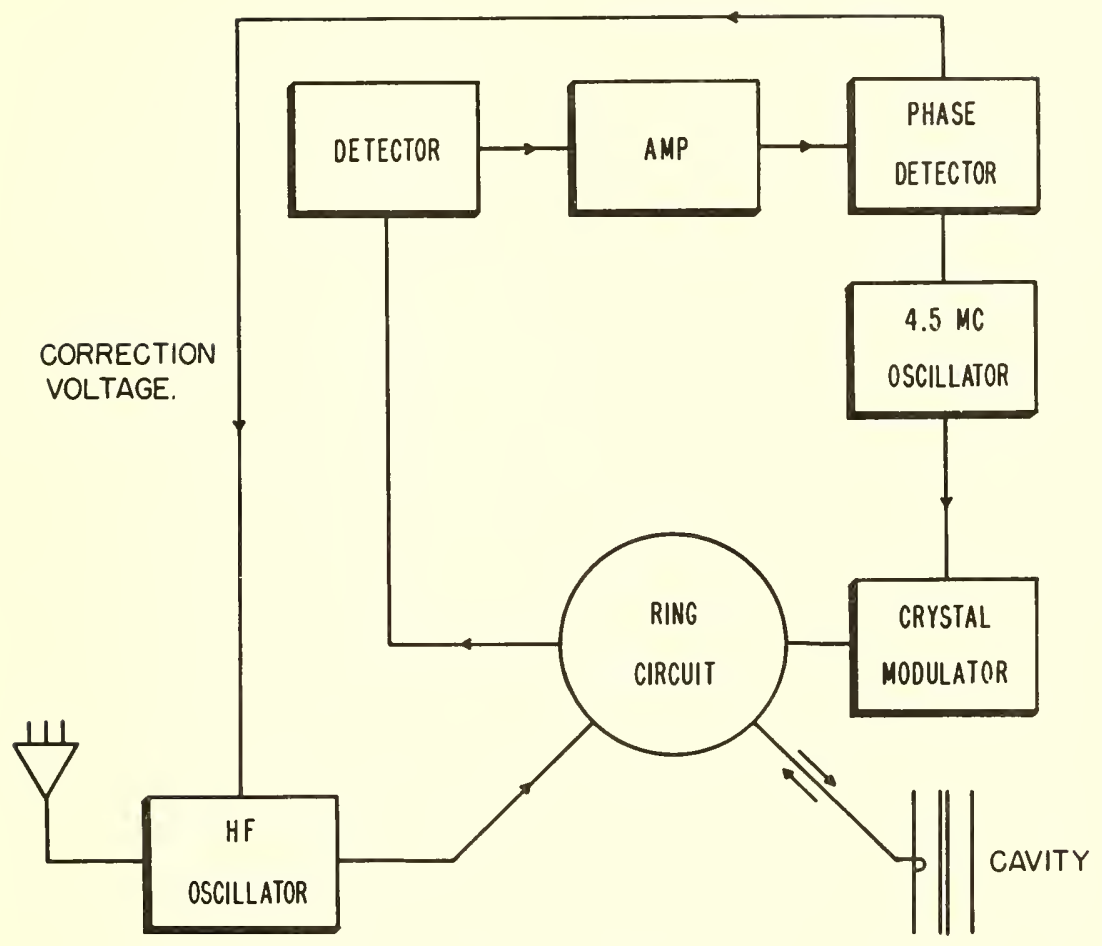

Figure 2.9. The Deam expendable refractometer (modified Pound oscillator).

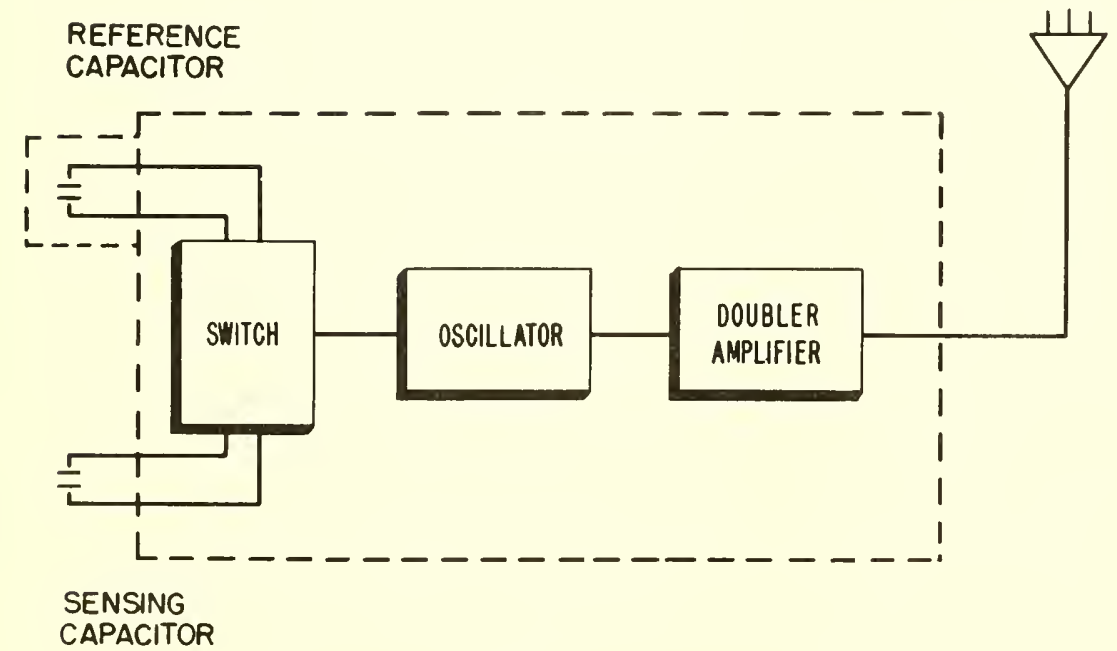

Figure 2.10. The Hay refractometer. 


\subsection{Comparison Between the Direct and Indirect Methods of Measurement}

Absolute accuracy may not be the only consideration in the comparison between the direct and indirect method of measurement. Although refractometers may be capable of superior accuracy, the factors of requirements, economics, and availability of competent technical personnel may outweigh this advantage. Refractometers are relatively expensive, somewhat complex, and require competent technical personnel to maintain, calibrate, and operate them.

In many cases where average values or long-term statistics are adequate, the use of refractometers may not be indicated. The data on refractive index structure derived from weather service data has long been used successfully for the determination of average conditions.

Where extreme accuracy is required, the use of refractometers is indicated. Radar and radio navigation are examples where accurate estimates of both surface values and gradient are necessary to determine the refraction through the atmosphere. The necessity for true vertical gradients would demand the use of a balloon-borne or dropsonde type of refractometer. In many applications the indirect method may be sufficient; however, determination of the fine structure of the refractive index appears to be presently limited to some type of radio-frequency refractometer.

\subsection{Radiosonde Lag Constants}

\subsubsection{Introduction}

The determination of $N$ from radiosonde data is subject to all of the errors inherent in the radiosonde observation. Recently Wagner [40] has analyzed the errors in $N$ arising from time lag of the sensing elements, data transmission techniques, and significant level-selection criteria. Of these soulces of error, Wagner concludes that the time lag of the sensing elements is the most serious source of error. Further, for the southern California coastal inversions, Wagner concludes that only the time lag of the humidity strip need be considered. A similar conclusion has been reached by Clarke [41] for practical applications involving ship-borne radar and over-water air-to-air communications. Although there is a significant correction associated with the time lag of the U.S. radiosonde's lithium chloride humidity-sensing element, there is also a time lag in the temperature element, which, as will be shown, must also be taken into consideration. The correction for the temperature element yields a twofold correction to $N$ due to the actual error in temperature and the ancillary correction in vapor pressure resulting from the more correct estimate of the true saturation vapor pressure. This arises from the fact that 
when the lithium chloride element measures relative humidity it must be used with the saturation vapor pressure. Since the saturation vapor pressure of water is a function of temperature, an error in temperature produces an error in the estimated water vapor pressure.

The lag constant of the lithium chloride ( $\mathrm{LiCl})$ humidity element becomes significantly larger for temperatures below $0{ }^{\circ} \mathrm{C}$ [42]. Wexler [11] has made detailed studies of the lag constants of the $\mathrm{LiCl}$ elements at low temperatures under laboratory conditions. Bunker [43], however, has found quite different lag constants in the free atmosphere. He attributes this discrepancy in lag constants to the laboratory-determined values which were obtained for isothermal conditions; whereas, in rising through the free atmosphere, the radiosonde normally passes from warm to cooler air. Bunker has raised a serious question, namely that the temperature lag of the lithium chloride element is possibly as important as the isothermal humidity lags studied by Wexler. It is quite possible that the interplay of these two lags could produce a total effect either greater or less than the humidity lag alone. We now have a quandary since there is not currently in the literature a complete analysis of the interplay of the temperature and humidity lags of the LiCl humidity element. Although Bunker considers some aspects of this problem, he does not consider the case of decreasing humidity and inereasing temperature, a typical condition giving rise to the superrefraction of radio waves. The differences between Wexler's and Bunker's estimates of the LiCl lag constants are so great as to make one wonder at the validity of applying any correction for this effect. Since neither Wexler's nor Bunker's tabulation of lag constants is complete, the choice of lag constant appears to be arbitrary. Wexler's lag constants will be adopted for the present discussion. Both the temperature and humidity elements are corrected (as much as is possible) for their respective time lags in this chapter for the purpose of preparing refractive index profiles.

In what follows the theory of sensor time lag will be examined. Data from several climatically diverse locations will then be examined to illustrate the relative importance of the various lag constant corrections under conditions of superrefraction of radio waves.

\subsubsection{Theory of Sensor Time Lags}

Middleton and Spilhaus [44] give

$$
\frac{d \theta_{i}}{d t}=-\frac{1}{\lambda}\left(\theta_{i}-\theta_{e}\right)
$$

as the basic differential equation of the time lag of a meteorological sensor, measuring the variable $\theta$, where $t$ is time, $\lambda$ the appropriate lag 
constant, and the subscripts $i$ and $e$ stand for the indicated and environmental values, respectively. The solution to (2.1) depends upon the manner in which the envirommental value of $\theta$ varies. For example, if it is assumed that $\theta_{e}$ varies linearly with time,

$$
\theta_{e}=\theta_{0}+\beta t
$$

one obtains for the solution to $(2.2)$

$$
\theta_{e}-\theta_{i}=+\beta \lambda[1-\exp (-t / \lambda)],
$$

as compared to

$$
\theta_{e}=\frac{[(\beta \lambda-1) \exp (t / \lambda)] \theta_{i}}{\beta \lambda \exp (\beta t)-\exp (t / \lambda)}
$$

under the assumption that $\theta_{e}$ varies exponentially with time,

$$
\theta_{e}=\theta_{0} \exp (-\beta t)
$$

For a column of air, one normally knows the initial reading of the sensing strip, $\theta_{0}$, and for an assumed linear decrease of $\theta$ with height the coefficient $\beta$ becomes

$$
\beta=\frac{\theta_{i}-\theta_{0}}{t-\lambda[1-\exp (-t / \lambda)]},
$$

with the result that $(2.2)$ is written

$$
\theta_{e}=\theta_{0}+\frac{\left(\theta_{i}-\theta_{0}\right) t}{t-\lambda[1-\exp (-t / \lambda)]} .
$$

Once the value of $\lambda$ is determined, estimates of the true properties of the air can be found at all heights up to the point where the gradient changes.

One may proceed by a different course by noting in (2.1) that

$$
\frac{d \theta_{i}}{d t}=\frac{d \theta_{i}}{d h} \cdot \frac{d h}{d t}=R \frac{d \theta_{i}}{d h},
$$

where $R$ is ascension rate of the radiosonde (assumed a constant 300 $\mathrm{m} / \mathrm{min})$.

If it is further assumed that $\theta_{i}$ varies linearly between reported values (an assumption compatible with radiosonde reporting procedure), then

$$
R \frac{d \theta_{i}}{d h}=R \frac{\theta_{i, k+1}-\theta_{i, k}}{h_{k+1}-h_{k}}
$$

where the $k$ th and the $(k+1)$ st layers are the boundaries considered. 
Thus the envirommental value of $\theta$ can be determined from

$$
\theta_{e, k+1}=\theta_{i, k+1}+R \lambda \frac{\theta_{i, k+1}-\theta_{i, k}}{h_{k+1}-h_{k}}
$$

which involves, in a simple fashion, only the indicated or actually meassured values of the parameter $\theta$.

One assumes that $\theta_{i}$ and $\theta_{e}$ are identically the same at $h=0$ and that successive application of $(2.10)$ will yield a more realistic est imate of the distribution of $\theta$ with height. When one applies a correction procedure of the form of (2.10) to temperature, where the temperature lag constant, $\lambda_{T}$, is always 3 sec [45], one obtains immediately the corrected temperature profile. The same is true for humidity (provided the temperature is greater than $0^{\circ} \mathrm{C}$ ), when $\lambda_{f}$ is assumed to be always $10 \mathrm{sec}[40]$. Although there is some indication [46] that, for room temperatures, the humidity lag constant may be nearer 5 sec. For temperatures less than $0{ }^{\circ} \mathrm{C}$, however, $\lambda_{f}$ is a function of true temperature, true value of relative humidity, $f_{e}$, and change of the true value of $f_{e}$ (see fig. 2.3). This means that one must use an iterative solution for $f_{e}$ since $\lambda_{f}$ will change as one's estimate of the true value of $f_{e}$ and $\Delta f_{e}$ changes. Since our knowledge of $\lambda_{f}$ is essentially empirical, the correction procedure is limited to the temperatures and values of $f_{e}$ and $\Delta f_{e}$ reported by Wexler [11].

In applying the above equations one generally assumes that the time lags are always known and that the envirommental and indicated values are identical at the base of each layer [47]. These two conditions are approximately satisfied only for the ground layer since the total lag constant of the humidity strip is not known. It is not clear that any correction for sensor lag may be made above the initial layer since, for subsequent layers, the initial indicated and environmental values are not identical and, further, lag ronstants have not been determined for this case.

\subsubsection{Radiosonde Profile Analysis}

The utility of the above lag constant corrections is illustrated by analyzing past radiosonde data for the oceurrence of ground-based radio ducts. A ground-based radio duct is one in which the gradient of $N$ is sufficient to refract a radio ray to the same curvature as that of the earth. Thus, for ducting, since ray curvature is given by the gradient of the refractive index,

$$
\frac{\Delta N}{\Delta h} \leq \frac{-1}{r_{0}}=-157 \mathrm{Nunits} / \mathrm{km}
$$

where $r_{0}$ is the earth's radius. The data analyzed were from the months of expected maximum duct occurrence at Fairbanks, Alaska (Feb.), 
Washington, D.C. (May), and Swan Island, W.I. (Aug.). All data were for the year 1953 .

As an example of past work, Wagner [40] has assumed that the refractivity lagged its environmental value according to (2.3) with the result that

$$
N_{e}-N_{i}=\beta_{N} \lambda_{N}\left[1-\exp \left(-t / \lambda_{N}\right)\right] .
$$

After an analysis of the meteorological conditions of his area of application, he set $\lambda_{N} \equiv \lambda_{f} \equiv 10$ sec; i.e., the time lag of $N$ derived from pressure, temperature, and humidity was identically that of the humidity sensor for temperature $<0{ }^{\circ} \mathrm{C}$. Comparing this method of $N$-lag correction with the uncorrected data (first and last columns of table 2.1), one notes an increase in both intensity and incidence of ducts in all climates. This is particularly marked for Fairbanks, where a sixfold incidence increase is obtained. However, when one makes individual time-lag corrections for both temperature and humidity by means of (2.10) a quite different picture of duct statistics is obtained. For example, the Fairbanks data indicate a twofold increase rather than a sixfold increase. On the other hand, Washington shows an incidence of nine rather than six ducts with a marked increase in $N$ gradient when one corrects for both temperature and humidity.

The change of ducting statistics at all three locations obtained by the two methods of time-lag correction yields paradoxical results. The near contradiction of the two correction procedures is easily explained and serves as an illustration of the necessity of correcting both the temperature and humidity elements for general application to ducting statistics.

Consider typical temperature and humidity conditions associated with ground-based ducts within each climate. Such cases are shown in figure 2.11. It is sufficient to note that the temperate ducts arise from typical radiation inversion conditions of increasing temperature and decreasing relative humidity with height; the aretic ducts are associated with the intense arctic radiation inversion with ground temperature near $-25^{\circ} \mathrm{C}$ and nearly constant relative humidity with respect to height; the tropical ducts, however, appear to be due to slight decreases of both temperature and humidity with height at temperatures near $+25^{\circ} \mathrm{C}$. The effect of sensor lag upon these different gradients is always to make the indicated gradient appear less than the true or environmental gradient. Thus correcting for sensor lags makes the temperature and humidity gradients more intense. This, in turn, effects the resultant $N$ gradient. One may write

$$
d N=\frac{\partial N}{\partial T} d T+\frac{\partial N}{\partial e} d e+\frac{\partial N}{\partial P} d P
$$


TABLE 2.1. Comparison of ground-based ducting statistics derived from various sensor time lag corrections

\begin{tabular}{|c|c|c|c|c|c|c|c|}
\hline \multirow{2}{*}{ Station } & \multirow{2}{*}{$\begin{array}{l}\text { No. } \\
\text { profiles }\end{array}$} & \multicolumn{2}{|c|}{ Reported data } & \multicolumn{2}{|c|}{$\begin{array}{c}\text { Corrected for } T \text { and } f \\
\text { sensor lag }\end{array}$} & \multicolumn{2}{|c|}{$\begin{array}{c}\text { Assuming } \lambda_{N}=\lambda_{f}=10 \\
\text { sec and eq }(12)\end{array}$} \\
\hline & & $\begin{array}{l}\text { No. } \\
\text { duets }\end{array}$ & $\begin{array}{l}\text { A verage } \\
\text { gradient }\end{array}$ & $\begin{array}{l}\text { No. } \\
\text { duets }\end{array}$ & $\begin{array}{l}\text { Average } \\
\text { gradient }\end{array}$ & $\begin{array}{l}\text { No. } \\
\text { duets }\end{array}$ & $\begin{array}{l}\text { A verage } \\
\text { gradient }\end{array}$ \\
\hline $\begin{array}{l}\text { Washington, D.C., } \\
\text { (May), temperate } \\
\text { climate. }\end{array}$ & \multirow{3}{*}{$\begin{array}{r}123 \\
62 \\
51\end{array}$} & 1 & $\begin{array}{r}\text { Nunits } / \mathrm{km} \\
-163\end{array}$ & 9 & $\begin{array}{r}N \text { units } / k m \\
-202\end{array}$ & 6 & \multirow{3}{*}{$\begin{array}{l}-181 \\
-238 \\
-250\end{array}$} \\
\hline $\begin{array}{l}\text { Swan Island, (Aug.), } \\
\text { tropical climate. }\end{array}$ & & 15 & -186 & 20 & -206 & 23 & \\
\hline $\begin{array}{l}\text { Fairbanks, Alaska, } \\
\text { (Feb.), arctic climate }\end{array}$ & & 2 & -211 & 4 & -212 & 12 & \\
\hline
\end{tabular}

which, for normal conditions of $15^{\circ} \mathrm{C}, 1013 \mathrm{mbar}$, and 60 percent $\mathrm{RH}$, is approximated by

$$
\Delta N \cong-1.27 \Delta T+4.50 \Delta e+0.27 \Delta P .
$$

Since, for ducting, a large negative gradient of $N$ is required, the effect of temperature sensor lag correction is to make temperate and aretic temperature increases with height more pronounced with a resultant larger contribution to a negative $N$ gradient. Humidity lag corrections under all conditions lead to a larger decrease of $\mathrm{RH}$ with height than indicated and concomitant more rapid decrease of $N$ with height. Such an explanation does not seem so evident for tropical conditions, however, where both temperature and relative humidity decrease with height. This apparent paradox is explained by the relatively great change in saturation vapor pressure of water associated with a small change of temperature near $25{ }^{\circ} \mathrm{C}$ which then produces the required large decrease of $e$ and $N$ with height. It is seen, then, that the temperature sensor lag correction produces an added humidity correction. Thus one must correct both sensing elements for the purpose of preparing $N$ profiles. Even in the case of the aretic inversion the interplay of the temperature and humidity lag corrections is very important, as has been noted by Yerg [48].

Thus, the correction for humidity sensor lag alone tends to overestimate ducting incidence since the corrected relative humidity decrease, coupled with the indicated temperature increase, produces a greater decrease in water vapor pressure than is actually present.

\subsubsection{Conclusion}

It appears from the present study that if studies of refractive index profile characteristics are to include sensor lag correction, then allowance should be made for both temperature humidity sensor time lags, regardless of climate. Any systematic application of these conclusions to large 
bodies of data, however, must await the appearance in the literature of effective lag coefficients that combine the effects of both temperature and humidity lags upon the $\mathrm{LiCl}$ element.

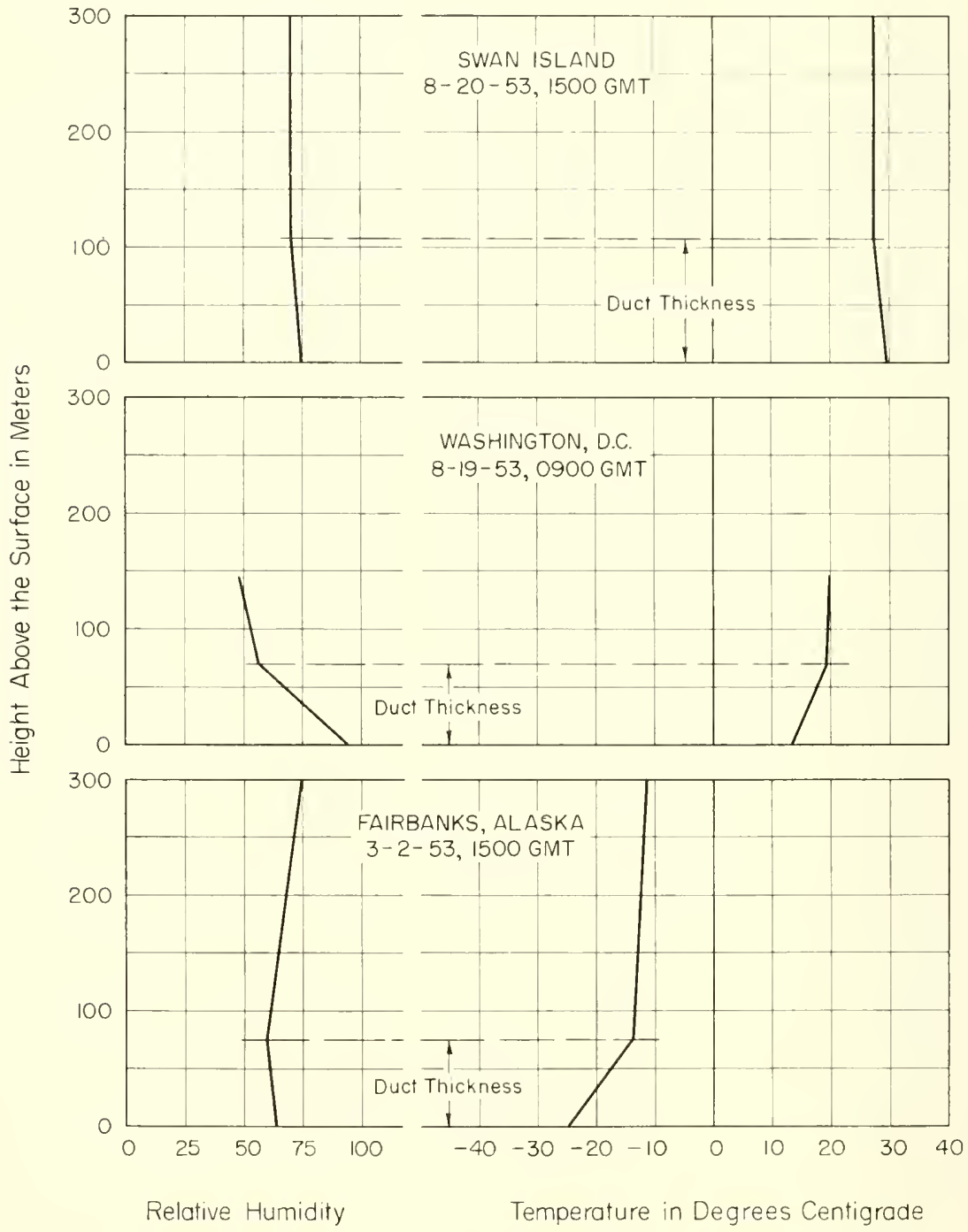

Figure 2.11. Temperature and humidity profiles. 


\subsection{References}

[1] Johnson, W. E. (Dec. 1953), An analogue computer for the solution of the radio refractive index equation, J. Res. NBS 51, No. 6, 335-342.

[2] Sprung, A. (1888), Bestimm. d. Luftfeuchtigk. nit Hülfe d. Assmann'schen Asperationspsychrometers, Das Wetter 5, 105-109.

[3] List, R. J. (1958), Smithsonian Meteorological Tables, Washington, D.C. (Published by the Snithsonian Institution).

[4] National Physical Laboratory, Dept. Science Research (1960), The refractive index of air for radio waves and microwaves (Teddington, Middlesex, England).

[5] Jones, F. E., and A. Wexler (July 1960), A barium fluoride filn hygrometer element, J. Geophys. Res. 65, No. 7, 2087-2095.

[6] Macready, P. B., Jr. (1960), Field applications of MRI Model 901 water vapor meter, Internal Memo Report, Meteorol. Research Inc. of Altadena, Calif.

[7] Stover, C. M. (Apr. 1961), Preliminary report on a new aluminum humidity element, Internal Tech. Memo, Sandia Corporation, Albuquerque, N. Mex.

[8] Hirao, K., and K. I. Akita (Oct. 1957), A new type refractive index variometer, J. Radio Res. Lab. 4, No. 18, 423-437.

[9] Crozier, A. L. (Apr. 1958), Captive balloon refractovarioneter, Rev. Sci. Instr. 29, No. 4, 276-279.

[10] Cline, D. E. (1957), International radiosonde comparison tests, Tech. Memo NRM-1907, U.S. Army Signal Eng. Lab. Task NR 3-36-11-402.

[11] Wexler, A. (July 1949), Low temperature performance of radiosonde electric hygrometer elements, J. Res. NBS 43, 49-56.

[12] Bean, B. R., and E. J. I)utton (Nov. 1961), Concerning radiosondes, lag constants, and refractive index profiles, J. Geophys. Res. 66, No. 11, 3717-3722.

[13] Misme, P. (Jan. 1956), Methode de mesure thermodynamique de l'indice de refraction de l'air- description de la radiosonde MDI, Ann. Telecommun. 11, No. 1, 81-84.

[14] Clinger, A. H., and A. W. Straiton (May 1960), Adaptation of the radiosonde for direct measurement of radio refractive index, Bull. Am. Meteorol. Soc. 41, No. 5, 250-252.

[15] Thiesen, J. F. (Apr. 1961), Direct measurement of the refractive index by radiosonde, Bull. Am. Meteorol. Soe. 42, No. 4, 282.

[16] Sargent, J. A. (May 1959), Recording microwave hygromoter, Rev. Sci. Instr. 30, 345-355.

[17] Lement, B. S., C. S. Roberts, and B. L. Averbach (Mar. 1951), Determination of small thermal expansion coefficients by a micrometric dilatometer method, Rev. Sci. Instr. 22, No. 3, 194-196.

[18] Bussey, H. E., and G. Birnbaum (Sept.-Oct. 1953), Measurement of variation in atmospheric refractive index with an airborne microwave refractometer, J. Res. NBS 51, No. 4, 171-178.

[19] Crain, C. M., and C. E. Williams (Aug. 1957), Method of obtaining pressure and temperature in sensitive microwave cavity resonators, Rev. Sci. Instr. 28, No. 8, 620-623.

[20] Vetter, M. J., and M. C. Thompson, Jr. (June 1962), An absolute microwave refractometer, Rev. Sci. Instr. 33, 656-660.

[21] Thompson, M. C., Jr., F. E. Freethey, and 1). M. Waters (Oct. 1958), Fabrication techniques for ceramic $X$-band cavity resonators, Rev. Sci. Instr. 29, No. 10, $865-868$.

[22] Fraser, 1). W., and E. G. Holmes (1953), Precision frequency control techniques, Final Report Proj., 229-298 (Georgia I.st. Tech., State Eng. Exp. Sta.). 
[23] Adey, A. W. (1957), Microwave refractometer cavity design, Can. J. Tech. 34, $519-521$.

[24] Thompson, M. C., Jr., F. E. Freethey, and D. M. Waters (July 1959), End plate modification of $X$-band $T E$ cavity resonators, IRE Trans. Microwave Theory Tech. MTT-7, No. 3, 388-389.

[25] Herbstreit, J. W. (July 1960), Radio refractometry, NBS Tech. Note 66.

[26] Birnbaum, G. (Apr. 1951), Fluctuations in the refractive index of the atmosphere at microwave frequencies, Phys. Rev. 82, 110-111.

[27] Birnbaum, G., and H. E. Bussey (Oct. 1955), Amplitude, scale, and spectrum of refractive index inhomogeneities in the first 125 meters of the atmosphere, Proc. IRE 43, 1412-1418.

[28] Crain, C. M., and A. P. Deam (Aug. 1951), Measurement of Tropospheric Index of Refraction Profiles with an Airplane-Carried Direct Reading Refractometer, Electrical Engineering Research Laboratory, University of Texas, Austin, Tex.

[29] Crain, C. M. (Oct. 1955), Survey of airborne microwave refractometer measurements, Proc. IRE 43, No. 10, 1405-1411.

[30] Crain, C. M. (May 1950), Apparatus for recording fluctuations in the refractive index of the atmosphere at 3.2 centimeter wavelength, Rev. Sci. Instr. 21, No. 5, 456-457.

[31] Pound, R. V. (Nov. 1946), Electronic frequency stabilization of microwave oscillators, Rev. Sci. Instr. 17, No. 11, 490-505.

[32] Birnbaum, G. (Feb. 1950), A recording microwave refractometer, Rev. Sci. Instr. 21, No. 2, 164-176.

[33] Murray, G., and T. B. Watkins (Apr. 1957), Automatic frequency control, U.S. Patent 2,788,445. Filed Feb. 5, 1952, granted Apr. 9, 1957.

[34] Silsbee, R. H. (Jạn. 1956), A high sensitivity paramagnetic resonance spectrometer, Cruft Lab. Tech. Report 221, Harvard Univ., Cambridge, Mass.

[35] Jung, P. (Oct. 1960), Transistorized frequency stabilization for reflex klystrons used in magnetic resonance, J. Sci. Instr. 37, 372-374.

[36] Smith, M. J. A. (Oct. 1960), Frequency stabilization of klystrons, J. Sci. Instr. 37, 398-399.

[37] Deam, A. P. (May 1959), An expendable atmospheric radio refractometer, EERL Report 108, Univ. of Texas, Austin, Tex.

[38] Deam, A. P., and C. F. Cole, Jr. (Jan. 1960), Development of a lightweight expendable microwave refractometer, EERL Report 5-46, Univ. of Texas, Austin, Tex.

[39] Hay, D. R., H. C. Martin, and H. E. Turner (June 1961), Lightweight refractometer, Rev. Sci. Instr. 32, No. 11, 693-697.

[40] Wagner, N. K. (July 1960), An analysis of radiosonde effects on measured frequency of occurrence of ducting layers, J. Geophys. Res. 65, 2077-2085.

[41] Clarke, L. C. (1960), Theory of atmospheric refraction, Part II of Meteorological Aspects of Radio-Radar Propagation, pp. 31-82, NWRF31-0660-035 (U.S. Navy Weather Research Facility, Norfolk, Va.).

[42] Dunmore, F. W. (1938), An electric hygrometer and its application to radio meteorography, J. Res. NBS 20, 723-744.

[43] Bunker, A. F. (1953), On the determination of moisture gradients from radiosonde records, Bull. Am. Meteorol. Soc. 34, 406-409.

[44] Middleton, W. E. K., and A. F. Spilhaus (1953), Book, Meteorological Instruments, p. 63 (Univ. of Toronto Press, Toronto, Canada).

[45] Sion, E. E. (Jan. 1955), Time constants of radiosonde thermistors, Bull. Am. Meteorol. Soc. 36, 16-21.

[46] Wexler, A., S. Garfinkel, F. Jones, s. Hasegawa, and A. Krinsky (Aug. 1955), A fast responding electric hygrometer, J. Res. NBS 55, 71-78. 
[47] Wagner, N. K. (May 1961), The effect of time constant of radiosonde sensors on the measurement of temperature and humidity discontinuities in the atmosphere, Bull. Am. Meteorol. Soc. 42, 317-321.

[48] Yerg, D. G. (May 1950), The importance of water vapor in microwave propagation at temperatures below freezing, Bull. Am. Meteorol. Soc. 31, 175-177. 



\section{Chapter 3. Tropospheric Refraction}

\subsection{Introduction}

If a radio ray is propagated in free space, where there is no atmosphere, the path followed by the ray is a straight line. However, a ray that is propagated through the earth's atmosphere encounters variations in atmospheric refractive index along its trajectory that cause the ray path to become curved. The geometry of this situation is shown in figure 3.1, which defines the variables of interest. The total angular refraction of the ray path between two points is designated by the Greek letter $\tau$, and is commonly called the "bending" of the ray. The atmospheric radio refractive index, $n$, always has values slightly greater than unity near the earth's surface (e.g., 1.0003), and approaches unity with increasing height. Thus ray paths usually have a curvature that is concave downward, as shown in figure 3.1. For this reason, downward bending is usually defined as being positive.

If it is assumed that the refractive index is a function only of height above the surface of a smooth, spherical earth (i.e., it is assumed that the refractive index structure is horizontally homogeneous), then the path of a radio ray will obey Snell's law for polar coordinates:

$$
n_{2} r_{2} \cos \theta_{2}=n_{1} r_{1} \cos \theta_{1}
$$

the geometry and variables used with this equation are shown in figures 3.1 and 3.19. With this assumption $\tau$ may be obtained from the following integral,

$$
\tau_{1,2}=-\int_{n_{1}, \theta_{1}}^{n_{2}, \theta_{2}} \cot \theta \frac{d n}{n}
$$

which can be derived as shown later in the chapter or as derived by Smart $[1] .{ }^{1}$

The elevation angle error, $\epsilon$, is an important quantity to the radar engineer since it is a measure of the difference between the apparent

${ }^{1}$ Figures in brackets indicate the literature references on p. 87 . 


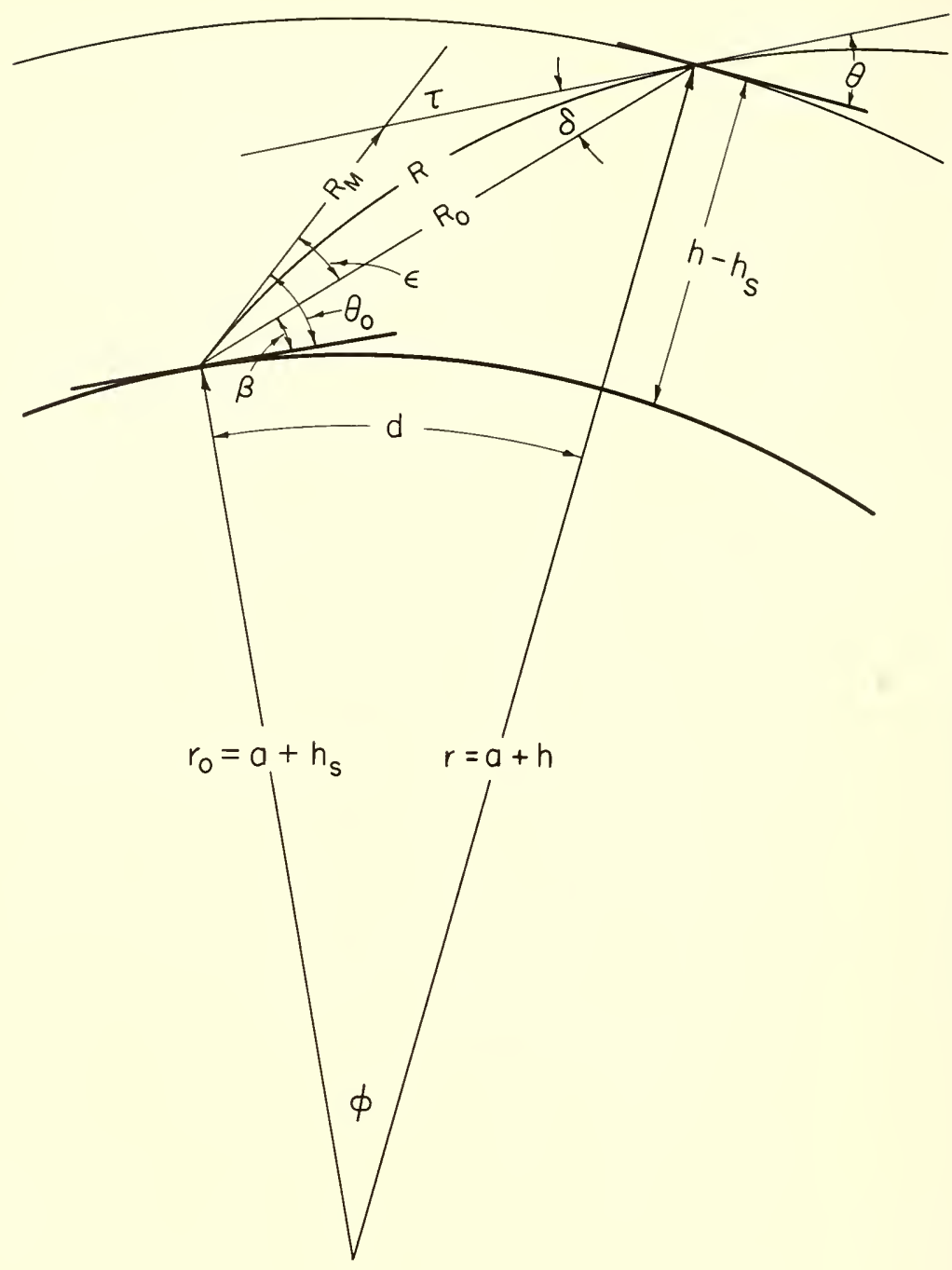

FIGURE 3.1. Geometry of the refraction of radio waves.

elevation angle, $\theta_{0}$, to a target, as indicated by radar, and the true elevation angle. Under the same assumptions made previously, $\epsilon$ is given as a function of $\tau, n$, and $\theta$ by

$$
\epsilon=\operatorname{Arctan}\left\{\frac{\cos \tau-\sin \tau(\tan \theta)-\frac{n}{n_{s}}}{\frac{n}{n_{s}} \tan \theta_{0}-\sin \tau-\cos \tau \tan \theta}\right\} .
$$




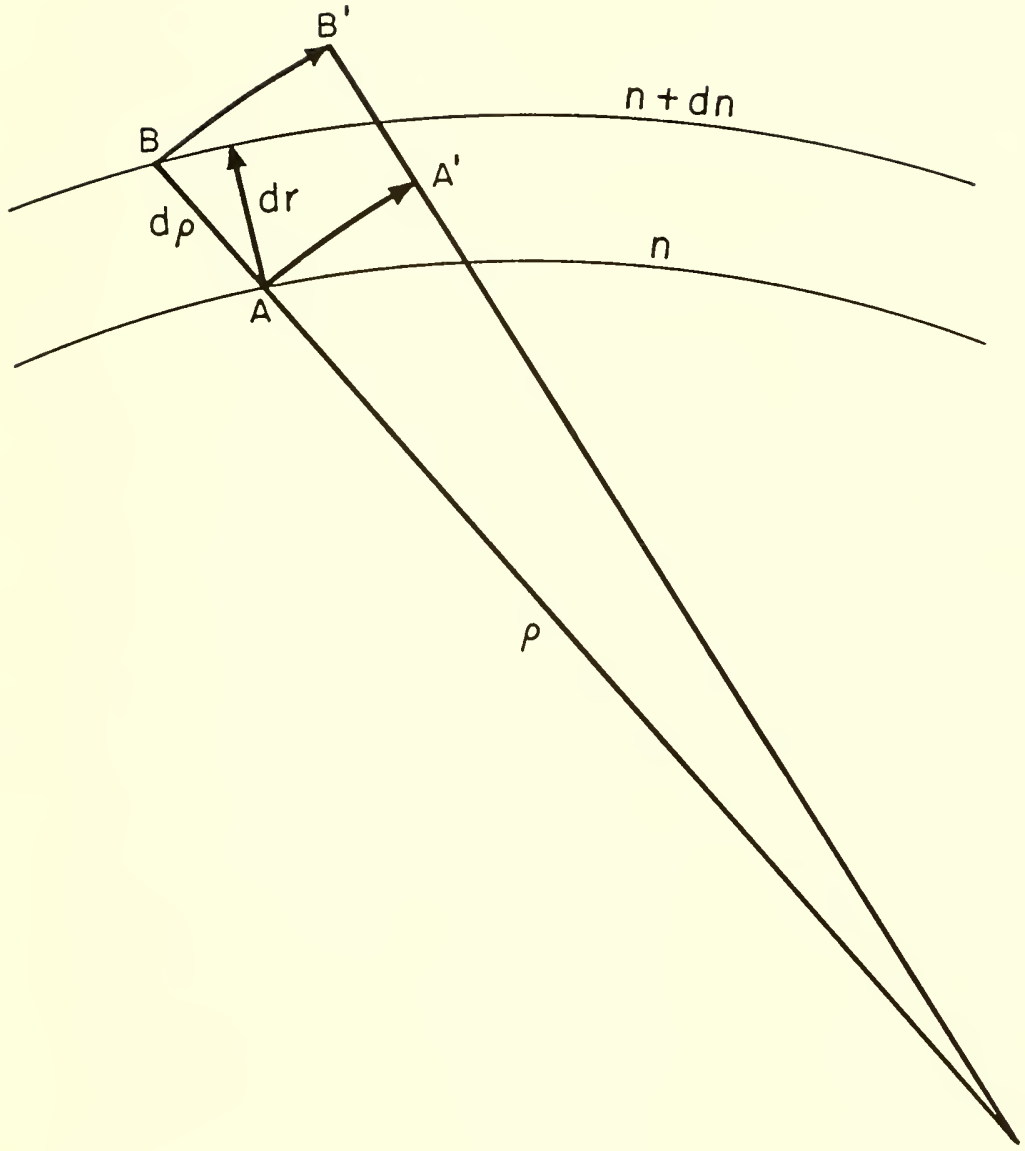

FIGURE 3.2. Differential geometry used in the derivation of the effective-earth's-radiusmodel atmosphere.

The apparent range to a target, $R_{e}$, as indicated by a radar, is defined as an integrated function of $n$ along the ray path,

$$
R_{e}=\int_{0}^{R} n d R=\int_{0}^{h} \frac{n d h}{\sin \theta} .
$$

However, the maximum range error $\left(R_{e}\right.$ minus the true range) likely to be encountered is only about $200 \mathrm{~m}$, hence the evaluation of (3.4) is not of great importance unless one is dealing with an interferometer or phasemeasuring system. 
The integral for $\tau,(3.2)$, eannot be evaluated directly without a knowledge of the behavior of $n$ as a function of height. Consequently, the approach of the many workers in this field has been along two distinet lines: the use of numerical integration techniques and approximation methods to evaluate $\tau$ without full knowledge of $n$ as a function of height, and the construetion of model $n$-atmospheres in order to evaluate average atmospherie refraction. The following sections are devoted to a diseussion of these methods.

\subsection{Limitations to Radio Ray Tracing}

The user should keep in mind that the equations given in the preceding section are subjeet to the following restrietions of ray tracing:

(1) The refractive index should not ehange appreciably in a wavelength.

(2) The fractional change in the spacing between neighboring rays (init ially parallel) must be small in a wavelength.

Condition (1) will be violated if there is a diseontinuity in the refractive index (which will not oceur in nature), or if the gradient of refractive index, $d n / d r$, is very large, in which ease condition (2) will also be violated. Condition (1) should be satisfied if

$$
\frac{(d n / d h) \text { per } \mathrm{km}}{N}<0.002 f_{k c},
$$

where refractivity, $N$, is defined as $N=(n-1) \times 10^{6}$ and $f_{\mathrm{kc}}$ is the carrier frequency in kilocyeles per second [2]. Condition (2) is a basie requirement resulting from Fermat's prineiple for geometrical opties. An atmospheric condition for which both conditions (1) and (2) are violated is known as "trapping" of a ray, and it can oceur whenever a layer exists with a vertical decrease of $N$ greater than $157 N$-units per kilometer. A layer of this type is ealled a "duct," and the mode of propagation through such a layer is similar to that of a waveguide [3]. Taking into account refractive index gradients, a eutoff frequency may be derived for waveguidelike propagation through a dueting layer [4].

In addition to the above limitations, it should be remembered that the postulate of horizontal homogeneity, made in order to use (3.1), is not realized under actual atmospheric conditions; some degree of horizontal inhomogeneity is always present (see chapter \$). 


\subsection{An Approximation for High Initial Elevation Angles}

A method may be derived for determining ray bending from a knowledge only of $n$ at the end points of the ray path, if it is assumed that the initjal elevation angle is large. Equation (3.2) in terms of refractivity, $N$, is equal to

$$
\tau_{1,2}=-\int_{N_{1}, \theta_{2}}^{N_{2}, \theta_{2}} \cot \theta d N \cdot 10^{-6}
$$

assuming $n \cong 1$ in the denominator. Integration by parts yields:

$$
\begin{aligned}
\tau_{1,2} & =-\int_{N_{1}, \theta_{1}}^{N_{2}, \theta_{2}} \cot \theta d N \cdot 10^{-6} \\
& =-\left[N \cot \theta \cdot 10^{-6}\right]_{N_{1}, \theta_{2}}^{N_{2}, \theta_{2}}-\int_{\theta_{1}, N_{1}}^{\theta_{2}, N_{2}} \frac{N}{\sin ^{2} \theta} d \theta \cdot 10^{-6} .
\end{aligned}
$$

Note that the ratio, $N / \sin ^{2} \theta$, beeomes smaller with increasing $\theta$ for values of $\theta$ close to $90^{\circ}$. If point 1 is taken at the surface, then $\theta_{1}=\theta_{0}$ and $N_{1}=N_{s}$. Then for $\theta_{0}=10^{\circ}, N_{2}=0$ and $\theta_{2}=\pi / 2$, the last term of (3.6) amounts to only 3.5 percent of the entire equation, and for the same values of $N_{2}$ and $\theta_{2}$, but with $\theta_{0}=87 \mathrm{mrad}\left(\sim 5^{\circ}\right)$, the seeond term of $(3.6)$ is still relatively small ( $\sim 10$ pereent). Thus it would seem reasonable to assume that for

$$
\theta_{0} \geq 87 \operatorname{mrad}\left(\sim 5^{\circ}\right)
$$

the bending, $\tau_{1,2}$, between the surface and any point, $r$, is given sufficiently well by

$$
\tau_{1,2}=-\left[N \cot \theta \times 10^{-6}\right]_{N_{s}, \theta_{0}}^{N_{r}, \theta_{r}}
$$

or

$$
\tau_{1,2}=N_{s} \cot \theta_{0} \times 10^{-6}-N_{r} \cot \theta_{r} \times 10^{-6} .
$$

The term $-N_{r} \cot \theta_{r} \times 10^{-6}$ is practically constant and small with respect to the first term, for a given value of $\theta_{0}$ and $r$, in the range $\theta_{0} \geq 87$ mrad. Thus $\tau_{1,2}$ is seen to be essentially a linear function of $N_{s}$ in the range $\theta_{0} \geq 87 \mathrm{mrad}$. For bending through the entire atmosphere (to a point where $N_{r}=0$ ), and for $\theta_{0}<87 \mathrm{mrad}$, (3.8) reduces to

$$
\tau=N_{s} \cot \theta_{0} \times 10^{-6} \text {. }
$$


For initial elevation angles less than about $5^{\circ}$, the errors inherent in this method exceed 10 percent (except near the surface) and rise quite rapidly with decreasing $\theta_{0}$.

\subsection{The Statistical Method}

Another method for determining high-angle bending is the statistical linear regression technique developed by Bean, Cahoon, and Thayer [5]. It has been found that for normal conditions and all heights the righthand integral of (3.6) is approximately a linear function of $N_{s}\left(\theta_{0}, r\right.$ constant) for $\theta_{0}<17 \mathrm{mrad}\left(\sim 1^{\circ}\right)$ and that the second term of (3.8) tends to be constant. Thus (3.6) reduces to a linear equation,

$$
\tau_{1,2}=b N_{s}+a,
$$

where $b$ and $a$ are constants (as in tables 9.1 to 9.9 ) and $N_{s}$ is the surface refractivity.

The form of (3.10) is very attractive, since it implies two things:

(1) The value of $\tau_{1,2}$ may be predicted with some accuracy as a function only of $N_{s}$ (surface height and $\theta_{0}$ constant), a parameter which may be observed from simple surface measurements of the common meteorological elements of temperature, pressure, and humidity.

(2) The simple linear form of the equation indicates that, given a large number of observed $\tau_{1,2}$ versus $N_{s}$ values for many values of $h$ and $\theta_{0}$, the expected (or best estimate) values of $b$ and $a$ can be obtained by the standard method of statistical linear regression.

This is what was done to obtain values listed in tables 9.1 to 9.9 .

Tables 9.1 to 9.9 also show the values of the standard error of estimate, $S E$, to be expected in predicting the bending, and the correlation coefficients, $r$, for the data used in predicting the lines. Linear interpolation can be used between the heights given to obtain a particular height that is not listed in the tables. For more accurate results, plot the values of $\tau$ from the tables (for desired $N_{s}$ ) against height, and then plot the values of the standard error of estimate on the same graph. Then connect these points with a smooth curve. This will permit one to read the $\tau$ value and the $S E$ value directly for a given height.

\subsection{Schulkin's Method}

Schulkin has presented a relatively simple, numerical integration method of calculating bending for $N$-profiles obtained from ordinary significant-level radiosonde (or "RAOB") data [6]. The $N$ profile obtained from the RAOB data consists of a series of values of $N$ for different 
heights; one then assigns to $N(h)$ a linear variation with height in between the tabulated profile points, so that the resulting $N$ versus height profile is that of a series of interconnected linear segments. Under this assumption, (3.2) is integrable over each separate linear $N$-segment of the profile (after dropping the $n$ term in the denominator, which can result in an error of no more than 0.04 percent in the result), yielding the following. result:

$$
\Delta \tau_{1,2}(\mathrm{rad}) \cong-\int_{n_{1}, \theta_{1}}^{n_{2}, \theta_{2}} \cot \theta d n \cong \frac{2\left(n_{1}-n_{2}\right)}{\tan \theta_{1}+\tan \theta_{2}},
$$

or

$$
\Delta \tau_{1,2}(\mathrm{mrad}) \cong \frac{2\left(N_{1}-N_{2}\right) \times 10^{-3}}{\tan \theta_{1}+\tan \theta_{2}}
$$

For the conditions stated above, this result is accurate to within 0.04 percent or better of the true value of $\Delta \tau_{1,2}$, an accuracy that is usually better than necessary. Thus it is possible to simplify (3.11) further by substituting $\theta$ for $\tan \theta$; this introduces an additional error that is less than 1 percent if $\theta$ is under $10^{\circ}(\sim 175 \mathrm{mrad})$. Now (3.11) becomes

$$
\Delta \tau_{1,2}(\mathrm{mrad}) \cong \frac{2\left(N_{1}-N_{2}\right)}{\theta_{1}+\theta_{2}},
$$

where $\theta$ may be determined from (3.58).

The bending for the whole profile can now be obtained by summing up the $\Delta \tau_{1,2}$ for each pair of profile levels:

$$
\tau_{n}(\mathrm{mrad}) \cong \sum_{k=0}^{n} \frac{2\left(N_{k}-N_{k+1}\right)}{\theta_{k}+\theta_{k+1}}
$$

This is Schulkin's result. The degree of approximation of (3.13) is quite high, and thus most recent "improved" methods of ealeulating $\tau$ will reduce to Schulkin's result for the accuracy obtainable from RAOB or other similar data. Thus, provided that the $N$-profile is known, (3.13) is the most useful form for computing bending (for all practical purposes) that should concern the communications or radar engineer. Some other methods have been published which are actually the same as Schulkin's, but have some additional desirable features; e.g., the method of Anderson [7] employs a graphical approach to avoid the extraction of square roots to obtain $\theta_{k}$. 


\subsection{Linear or Effective Earth's Radius Model}

The classical method of accounting for the effects of atmospheric refraction of radio waves is to assume an effective earth's radius, $a_{e}=k a$, where $a$ is the true radius of the earth and $k$ is the effective earth's radius factor. This method, advanced by Schelleng, Burrows, and Ferrell [8], assumes an earth suitably larger than the actual earth so that the curvature of the radio ray may be absorbed in the curvature of the effective earth so that the relative curvature of the two remains the same, thus allowing that radio rays be drawn as straight lines over this earth rather than curved rays over the true earth. This method of accounting for atmospheric refraction permits a tremendous simplification in the many practical problems of radio propagation engineering although the height distribution of refractive index implied by this method is not a very realistic representation of the average refractive index structure of the atmosphere. This section will consider the refractive index structure assumed by the effective carth's radius model and how this differs from the observed refractive index structure of the atmosphere. Further, the limits of applicability of the effective earth's radius approach will be explored and a physically more realistic model, the exponential, will be described for those conditions where the effective earth's radius model is most in error.

It is instructive to give a derivation of the expression relating the curvature of radio rays to the gradient of refractive index. In figure 3.2 a wave front moves from $A B$ to $A^{\prime} B^{\prime}$ along the ray path. If the phase velocity along $A A^{\prime}$ is $v$ and $v+d v$ along $B B^{\prime}$, then, from considering the angular velocity,

$$
\frac{v}{\rho}=\frac{v+d v}{\rho+d \rho}
$$

or

$$
\frac{d v}{v}=\frac{d \rho}{\rho}
$$

where $\rho$ is the radius of curvature of the are $A A^{\prime}$. Now, since the phase velocit $\mathbf{y}, v$, is

$$
v=\frac{c}{n}
$$

where $c$ is the velocity of light in vacuo, one obtains

$$
\frac{d v}{v}=-\frac{d n}{n}
$$


combining (3.15) and (3.17), the familiar equation,

$$
\frac{1}{\rho}=-\frac{1}{n} \frac{d n}{d \rho}
$$

is obtained. If the ray path makes an angle $\theta$ with the surface of constant refractive index

$$
d r=d \rho \cos \theta
$$

and

$$
\frac{1}{\rho}=-\frac{1}{n} \frac{d n}{d r} \cos \theta
$$

If the curvature of the effective earth is defined as

$$
\frac{1}{a_{e}}=\frac{1}{a}-\frac{1}{\rho}
$$

then

$$
a_{e}=k a=\frac{1}{1 / a-1 / \rho}
$$

and

$$
k=\frac{1}{1+\frac{a}{n} \frac{d n}{d h} \cos \theta} .
$$

For the small values of $\theta$ normally used in tropospheric propagation, $\cos \theta$ may be set equal to unity. Further, by setting

$$
\frac{d n}{d h} \equiv-\frac{1}{4 a}
$$

one obtains the familiar value of $k=4 / 3$ for the effective earth's radius factor. By assuming that the gradient of $n$ is constant, a linear model of $N$ versus height has been adopted.

For this model, the bending

$$
\tau_{1,2}=-\int_{n_{1}}^{n_{2}} \cot \theta d n
$$

is written

$$
\tau_{1,2}=\int_{h_{1}}^{h_{2}} \frac{\cot \theta}{4 a} d h
$$


since

$$
N=N_{0}-\frac{h}{4 a} 10^{6}
$$

and

$$
d n=d N \times 10^{-6}=-\frac{d h}{4 a} .
$$

Further, for the case $h_{1}=h_{0}=0$, and

$$
0 \leq \theta_{0} \leq 10^{\circ} \text {, }
$$

where $\theta_{0}$ is the initial elevation angle of a ray, (3.26) may be approximated by

$$
\tau_{0, h}=-\int_{0}^{h} \frac{d h}{4 a \theta} .
$$

The angle $\theta$ may be determined from

$$
\begin{aligned}
\theta_{h} & =\left\{\theta_{0}^{2}+2\left(N-N_{0}\right)+\frac{2}{a}\left(h-h_{0}\right) \cdot 10^{6}\right\}^{1 / 2} \\
& =\left\{\theta_{0}^{2}+\frac{3}{2} \frac{h}{a}\right\}^{1 / 2} .
\end{aligned}
$$

For the case when $\theta_{0}=0,(3.29)$ becomes

$$
\begin{gathered}
\left.\tau_{0, h}=\frac{1}{2 \sqrt{6 a}} \int_{0}^{h} \frac{d h}{\sqrt{h}}=\frac{1}{\sqrt{6}} \sqrt{\frac{h}{a}}\right]_{0}^{h} \\
\tau_{0, h}=\frac{1}{\sqrt{6}} \sqrt{h / a} .
\end{gathered}
$$

Now, from the geometrical relationship,

$$
\tau_{0, h}=\frac{d_{0, h}}{a}+\left(\theta_{0}-\theta_{h}\right),
$$

one finds, for $\theta_{0}=0$,

$$
d_{0, h}=a\left(\tau_{0, h}+\theta_{h}\right),
$$


which upon substitution from (3.33) and (3.31) gives

$$
d_{0, h}=\sqrt{2 h(4 / 3)} \bar{a},
$$

or, more familiarly,

$$
d_{0, h}=\sqrt{2 k a h} .
$$

A very convenient working formula is derived from (3.37) by $k=4 / 3$, $a=3960$ miles and using units of miles for the ground distance to the radio horizon, $d_{0, h}$, and feet for the antenna height, $h$ :

$$
d_{0, h}=\sqrt{2 h} \text { miles }
$$

This is the familiar expression often used in radio propagation engineering for the distance to the radio horizon.

\subsection{Modified Effective Earth's Radius Model}

The effective earth's radius model, although very useful for engineering practice, is not a very good representation of actual atmospheric $N$ structure. For example, the data on figure 3.3 represent the average of individual radiosonde observations over a 5 -yr period at several locations chosen to represent the extremes of refractive index profile conditions within the United States. The Miami, Fla., profile is typical of warm, humid sea-level stations that tend to have maximum refraction effects while the Portland, Me., profile is associated with nearly minimum sealevel refraction conditions. Although Ely, Nev., has a much smaller surface $N$ value than either Miami or Portland, it is significant that when its $N$ profile is plotted in terms of altitude above sea level, it falls within the limits of the maximum and minimum sea level profiles. It is to take advantage of this simplification, that altitude above sea level rather than height above ground is frequently used throughout this monograph. The $N$ distribution for the $4 / 3$ earth atmosphere is also shown on figure 3.3. It is quite evident that the $4 / 3$ earth distribution has about the correct slope in the first kilometer above the earth's surface but decreases much too rapidly above that height. It is also seen, by noting that figure 3.3 is plotted on semi-logarithmic paper, that the observed refractivity distribution is more nearly an exponential function of height than a linear function of height as assumed by the $4 / 3$ earth atmosphere. One might expect the refractivity to decrease exponentially with height since the first term of the refractivity equation (1.20) involving $P / T$, comprises at least 70 percent of the total and is proportional to air density, a wellknown exponential function of height. 


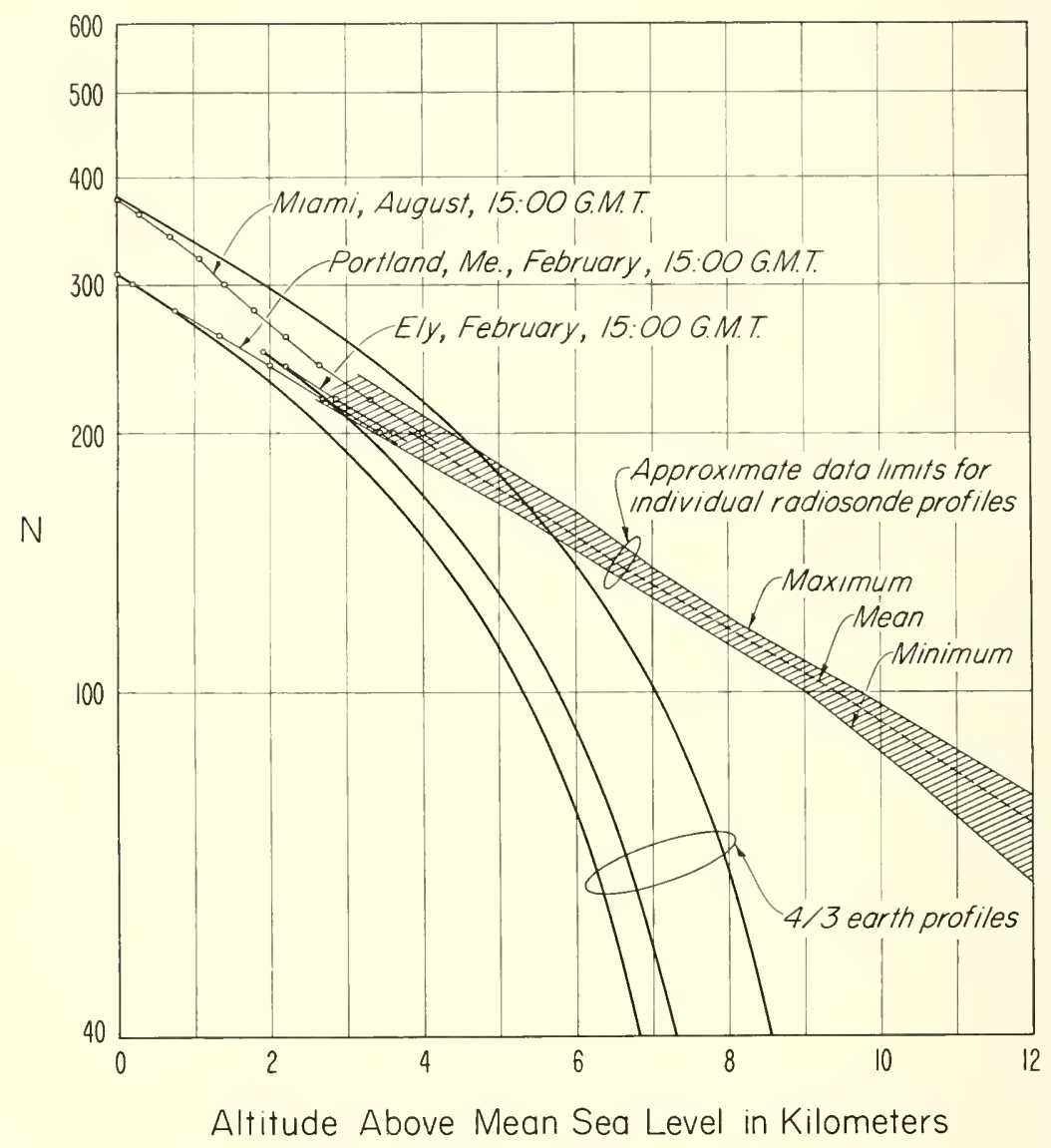

Figure 3.3. Typical $\mathrm{N}$ versus height distributions.

One might wonder, in the light of the data of figure 3.3, why the effective earth's radius approach has served so well for so many years. It appears that this success is due to the $4 / 3$ earth model being in essential agreement with the average $N$ structure near the earth's surface which largely controls the refraction of radio rays at the small values of $\theta_{0}$ common in tropospherıc communications systems.

It would seem that the deficiency of the effective earth's radius approach could be lessened by modifying that theory in the light of the average $N$ structure of the atmosphere. An indication of the average $N$ structure was obtained by examining a variety of $N$ profiles which were carefully selected from 39 station-years of individual radiosonde observations to represent the range of $N$ profile conditions during summer and winter at 13 climatically diverse locations. The results of this examination are given in table 3.1 . 
TABLE 3.1. Refractivity statistics as a function of altitude above sea level as derived from individual radiosonde observations

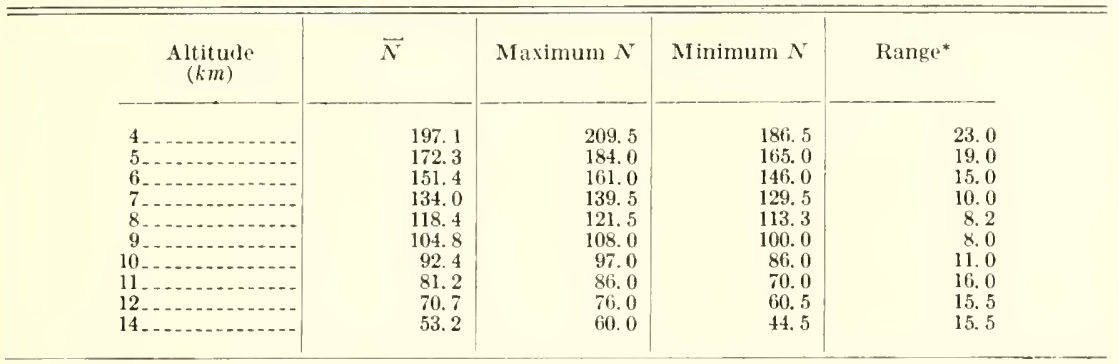

${ }^{*}$ Range $=\operatorname{maximum} N-\operatorname{minimum} N$.

It is interesting to note that the range of $N$ values has a minimum at 8 to $9 \mathrm{~km}$ above sea level but is systematically greater above and below that altitude. The average value of 104.8 at $9 \mathrm{~km}$ corresponds to a similar value reported by Stickland [9] as typical of the United Kingdom. Further the altitude of $8 \mathrm{~km}$ corresponds to the altitude reported by Humphreys [10] where the atmospheric density is nearly constant regardless of season or geographical location. Since the first term in the expression for refractivity is proportional to air density, and the water-vapor term is negligible at an altitude of $9 \mathrm{~km}$, the refractivity also tends to be constant at this altitude. It seems quite reasonable, then, to adopt a constant value of $N=105$ for $9 \mathrm{~km}$, thus further facilitating the specification of model atmospheres. Further, as also noted in chapter 1, when the values of table 3.1 are plotted such as on figure 3.3 , it is seen that the data strongly suggest that $N$ may be represented by an exponential function of height of the form:

$$
N(h)=N_{0} \exp \{-b h\}
$$

in the altitude range of 1 to $9 \mathrm{~km}$ above sea level.

The following recommendation is made when dealing with problems involving ground-to-ground communications systems or other types of low-altitude radio propagation problems where the ray paths involved do not exceed 1 , or at most $2, \mathrm{~km}$ above the earth's surface: use the effective earth's radius method to solve the associated refraction problems. The user should refer to the tables in chapter 9, where effective earth's radius factors are tabulated along with other refractivity variables. Table 9.27 may be entered with $N_{8}$ and table 9.28 may be entered with $\Delta N\left(N_{s}\right.$ subtracted from the $N$ value at $1 \mathrm{~km}$ above the surface). In both these tables linear interpolation will suffice for any practical problem. The variables listed in these tables are for the exponent ial model of $N(h)$ that is covered in the following subsection. 
When the effective earth's radius treatment is used, height is calculated as a function of distance, for a ray with $\theta_{0}=0$, with the equation $h=d^{2} / 2 k a$, where $d$ is the distance, $k$ is the effective earth's radius factor, and $a$ is the true radius of the earth $(\sim 6373 \mathrm{~km})$. The errors likely to be incurred when using this equation, assuming as a true atmosphere an exponential $N(h)$ profile as given in the following subsection will not exceed 5 percent for heights of $1 \mathrm{~km}$ or less.

The preceding background discussion has presented the material necessary for the consideration of the suitability of various models of refractivity to describe atmospheric refraction of radio waves. As a guide to what follows, let us ask what a logical sequence of models (or assumptions) would be to describe the effects of atmospheric refraction.

One such sequence might be:

(1) Assume an invariant model that is near to the actual average conditions and facilitates the calculation of radio field strengths. This has been done by the $4 / 3$ earth model.

(2) Assume a variable effective earth's radius factor for the calculation of radio field strengths in various climatic regions. This approach has been followed by Norton, Rice, and Vogler [11]. When it has become apparent that the effective earth's radius approach is inadequate, one might proceed by :

(3) Correctıng the effective earth's radius model by assuming a more realistic $N$ structure in the region where that model is most in error. This "modified effective earth's radius" model would then maintain, for some applications, the advantages of the original model.

(4) Assume an entirely new model of $N$ structure guided by the average $N$ structure of the atmosphere.

It is assumed that models (3) and (4) would allow for seasonal and climatic changes of the average refractive index structure of the atmosphere.

In the following sections, models (3) and (4) will be developed and tested by their relative agreement with the ray bendings obtained from actual long-term average $N$ profiles.

The first model of atmospheric refractivity that will be considered is based upon the effective earth's radius concept in the first kilometer. In this atmosphere $N$ is assumed to decay linearly with height from the surface $h_{s}$ to $1 \mathrm{~km}$ above the surface $h_{s}+1$. This linear decay is given by

$$
N(h)=N_{s}+\left(h-h_{s}\right) \Delta N, h_{s} \leq h \leq h_{s}+1,
$$

where

$$
-\Delta N=7.32 \exp \left(0.005577 N_{s}\right)
$$


This last relationship comes from the observed relationship between 6 to 8 year averages of daily observations of $N_{s}$ and $\Delta N$, the difference between $N_{s}$ and the value of $N$ at $1 \mathrm{~km}$ above the earth's surface:

$$
-\Delta N=N_{s}-N(1 \mathrm{~km}) .
$$

It is evident from figure 3.4 that for average conditions, a relationship exists between $\Delta N$ and $N_{s}$. The least squares determination given by (3.40) was based upon 888 sets of monthly mean values of $\bar{\Delta} \bar{N}$ and $\bar{N}_{s}$ from 45 United States weather stations representing many diverse climates. This relationship between $\bar{\Delta} \bar{N}$ and $\bar{N}_{s}$ is expected to represent the best estimate of a majority of individual profiles and certainly will closely agree with average conditions for the United States with one notable exception, southern California in the summer. These data, although shown on figure 3.4, were excluded from the least squares determination due to their singular large range of $\Delta N$ compared to their small range of $N_{s}$ which resulted in a marked "finger" of points rising from the main body of the data. This obvious departure of data points (24 points out of a total of 912) plus the well-known unique nature of the southern California summer climate were taken as sufficient justification for ignoring these points, although, as shall be seen, the ray bendings based upon

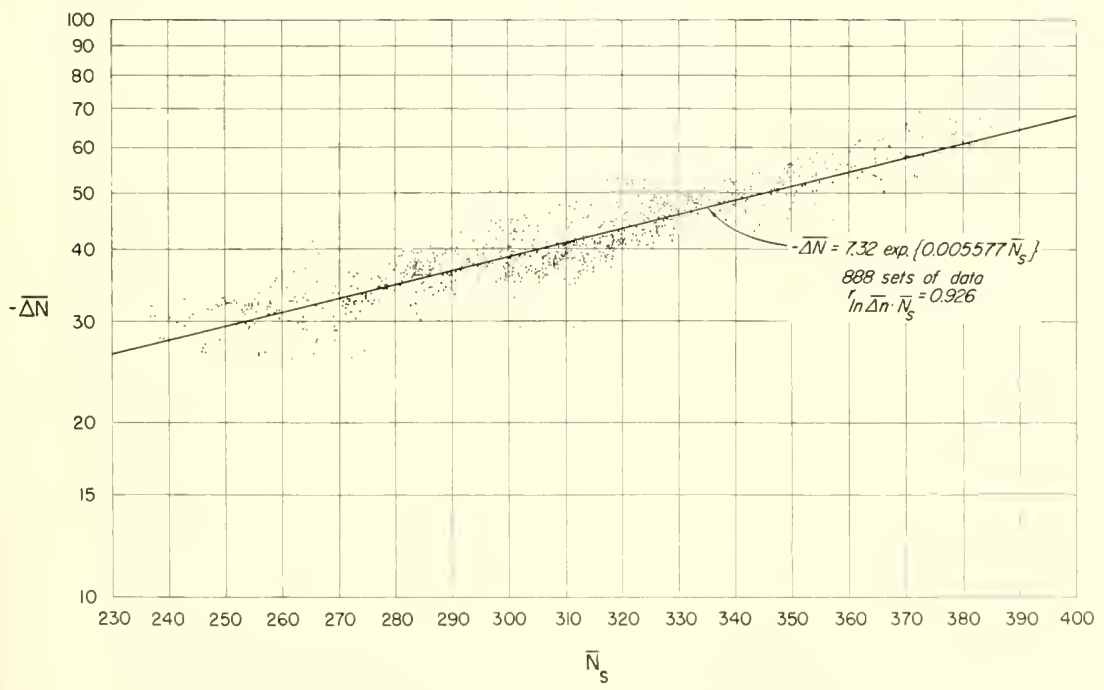

Figure 3.4. Eight-year average $\mathrm{N}_{\mathrm{B}}$ versus 6-year average $\Delta \mathrm{N}$ at 0800 and 1500 GMT' 
the $\Delta N$ obtained from (3.40) are in rather good agreement with the values calculated from actual $N$ profiles, even those observed in southern California.

Equation 3.40 offers a convenient method of specifying various models of the refractivity structure of the atmosphere, since it allows an estimation of the value of $N$ at $1 \mathrm{~km}$ in addition to the two values already known; i.e., $N_{s}$ and $N=0$ at $h=\infty$.

It may be further assumed that $N$ decreases exponentially from $h_{s}+1$ to a constant value of 105 at $9 \mathrm{~km}$ above sea level. In this altitude range $N$ is defined by:

$$
N=N_{1} \exp \left\{-c\left(h-h_{s}-1\right)\right\}, h_{s}+1 \leq h \leq 9 \mathrm{~km},
$$

where

$$
c=\frac{1}{8-h_{s}} \ln \frac{N_{1}}{105},
$$

and $N_{1}$ is the value of $N$ at $1 \mathrm{~km}$ above the surface.

Above the altitude of $9 \mathrm{~km}$, where less than 10 percent of the total bending occurs, a single exponential decrease of $N$ may be assumed. The coefficients in the exponential expression:

$$
N=105 \exp \{-0.1424(h-9)\}, h \geq 9 \mathrm{~km},
$$

were determined by a least squares analysis of The Rocket Panel data [12]. This expression is also in agreement with the ARDC Model Atmosphere 1956 [13] and Dubin's [14] conclusion that a standard densitydistribution may be used to determine the refractivity distribution at altitudes in excess of $20,000 \mathrm{ft}$.

The three-part model of the atmosphere expressed by (3.39)-(3.42) has the advantage of the effective earth's radius approach, particularly for such applications as point-to-point radio relaying over distances up to, say, $100 \mathrm{mi}$, where the radio energy is generally confined to the first kilometer, plus being in reasonably good agreement with the average $N$ structure of the atmosphere. The reader is cautioned, however, that application of this model to mode-theory calculations would be misleading, since the resultant diffraction region fields would be enhanced by the addition of strong reflections from the $n$-gradient discontinuities at $h_{s}+1$ and at $9 \mathrm{~km}$. The specific combinations of $N_{s}, h_{s}$, and $\Delta N$, that define the CRPL Reference Atmosphere-1958 are given in table 3.2.

The station elevations corresponding to given combinations of $N_{s}$ and $\Delta N$ were chosen to correspond with an average decay of $N$ with station elevation. Although the error in neglecting this height dependence has 
TABLE 3.2. Constants for the CRPL Reference Atmosphere-1958

\begin{tabular}{|c|c|c|c|c|c|c|}
\hline$N_{s}$ & $h$ s & $a^{\prime}$ & $-\Delta N$ & $k$ & $a_{e}$ & $c$ \\
\hline 0 & ${ }^{f t}{ }_{0}$ & $\begin{array}{c}m i \\
3960.0000\end{array}$ & 0 & 1.00000 & $\begin{array}{c}m i \\
3960.00\end{array}$ & $0_{0}^{\text {per } \mathrm{km}}$ \\
\hline $200 \ldots$ & 10,000 & 3961.8939 & 22.3318 & 1. 16599 & 4619. 53 & 0.106211 \\
\hline $250 \ldots$ & 5,000 & 3960.9470 & 29.5124 & 1. 23165 & 4878.50 & 0.114559 \\
\hline 301 & 1,000 & 3960.1894 & 39.2320 & 1. 33327 & 5280.00 & 0.118710 \\
\hline $313_{-}$ & 700 & 3960.1324 & 41.9388 & 1.36479 & 5403.88 & 0. 121796 \\
\hline $350 \ldots$ & 0 & 3960.0000 & 51.5530 & 1.48905 & 5896.66 & 0. 130579 \\
\hline 400 & 0 & 3960.0000 & 68.1295 & 1.76684 & 6996.67 & 0.143848 \\
\hline $450 \ldots$ & 0 & 3960.0000 & 90.0406 & 2. 34506 & 9286.44 & 0.154004 \\
\hline
\end{tabular}

Note: $a_{e}$ is the effective earth's radius and is equal to $a^{\prime} k, a^{\prime}=a+h_{s}$, where $h_{s}$ is the altitude of the earth's surface above sea level, $a=3960$ miles and $c=1 / 8-h_{8} \ln N_{1} / 105$.

been estimated to be no more than a few pereent, it could be important in such high precision applications as radar tracking of earth satellites. It should be remembered in subsequent applieations that a unique feature of these reference atmospheres is the dependence of $N_{s}$ on the altitude of surface above sea level. This feature was built in so that the referenee atmospheres would be completely specified by the single parameter $N_{s}$.

\subsection{The Exponential Model}

The next model of the atmosphere to be considered may be specified by assuming a single exponential distribution of $N$ :

$$
N=N_{s} \exp \left\{-c_{e}\left(h-h_{s}\right)\right\},
$$

where

$$
c_{\epsilon}=\ln \frac{N_{s}}{N(1 \mathrm{~km})}=\ln \frac{N_{s}}{N_{s}+\Delta N}
$$

and these equations are used to determine $N$ at all heights. This model of atmospherie refractivity is a elose representation of the average refractivity structure within the first $3 \mathrm{~km}$. Further, the single exponential model has the advantage of being an entire function, and therefore is easily used in theoretical studies. This model of the atmosphere has been adopted for use within the National Bureau of Standards with speeific values of the constants in (3.43) and (3.44). These constants are given in table 3.3 and speeify the CRPL Exponential Reference Atmosphere1958.

Figure 3.5 compares the $N$ structure of the above two models plus the $4 / 3$ earth model. It ean be seen that the assumption agrees with the reference atmosphere in the first kilometer, which is to be expeeted since $N_{s}=301$ is the value required to yield the $4 / 3$ gradient from figure 3.5 . Figure 3.5 illustrates the essential agreement of the reference atmosphere 

TABLE 3.3. Table of the constant $\mathrm{c}_{\text {e for the }}$ fRPL exponential radio refractivity
atmospheres

$N=N_{s} \exp \left[-c_{e}\left(h-h_{s}\right)\right]$

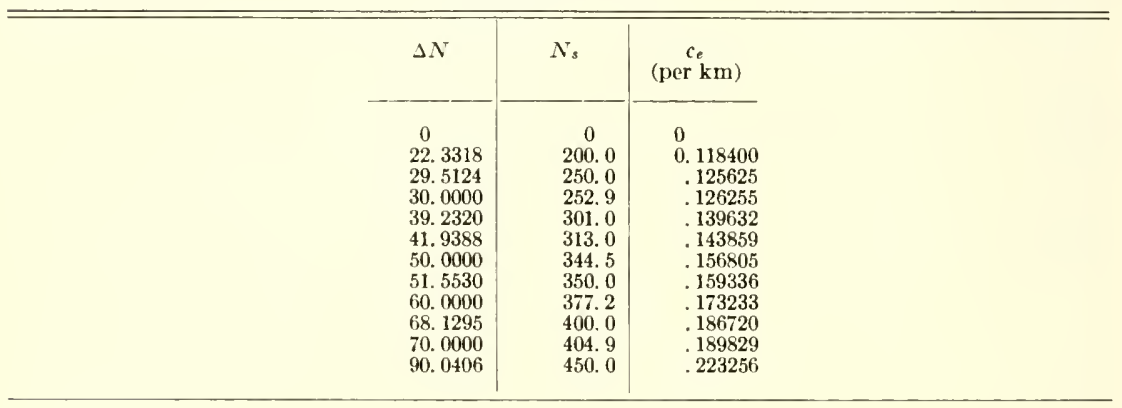

with the Rocket Panel and ARDC data. The exponential reference atmosphere is also shown on figure 3.5 for $N_{s}=313$, the average value of the United States. The exponential reference atmosphere appears to be a reasonable single line representation of $N$ throughout the height interval shown. The differences between the various models becomes more apparent by examining their agreement with observed $N$ profiles over the first $10 \mathrm{~km}$ as in figure 3.6 .

The reference and exponential reference atmospheres are given for the $N$ profiles corresponding to near-maximum $N_{s}$ (Lake Charles, La.) and near minimum-at-sea-level $N_{s}$ conditions (Caribou, Me.). The two reference atmospheres were determined solely from the $N_{s}$ values of each profile. Several observations can be made of these data. First, the $4 / 3$ earth model closely represents the slope of the minimal $N_{s}$ profile over the first kilometer, but then decreases too rapidly with height. Note, however, that the $4 / 3$ earth model with its constant decay of $39.2 \mathrm{~N}$ units per kilometer would be a very poor representation of the maximum profile which decreases over $66 \mathrm{~N}$ units in the first kilometer. The exponential reference atmosphere is in good agreement with the initial $N$ distribution but tends to give values systematically low above approximately $3 \mathrm{~km}$. At first glance, the exponential reference atmosphere does not appear to be as good a representation of the two observed profiles as the reference atmosphere, particularly above approximately $5 \mathrm{~km}$. Subsequent analysis of the refraction obtained from the two model atmospheres will show that this systematic disagreement of the exponential reference atmosphere in the 5 - to 20 - $\mathrm{km}$ interval is a minor defect of the model compared to its closer agreement with observed $N$ distributions over the first 1 to $3 \mathrm{~km}$. This is particularly true for the higher values of $N_{s}$ such as that for Lake Charles.

The above models are more in agreement with long-term mean $N$ profiles than is the $4 / 3$ earth model. The application at hand would aid in deciding which of the reference atmospheres would be most useful. To 


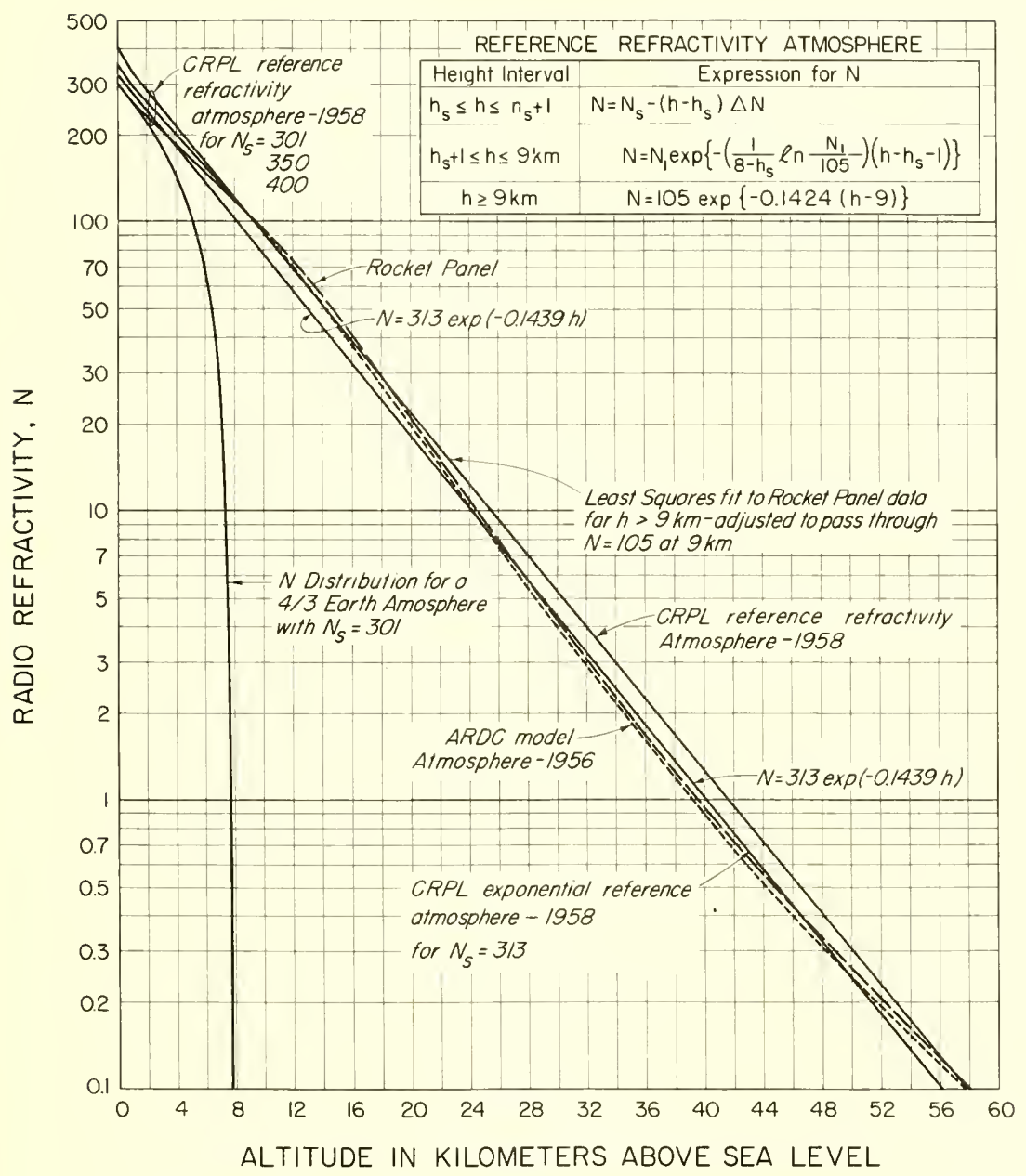

FIGURE 3.5. CRPL reference refractivity atmospheres-1958.

aid in distinguishing between the various models, the following sections will be concerned with eomparing the ray bending between the models.

A comparison of the ray paths in the reference atmospheres in the $4 / 3$ earth model will illustrate the systematic differences between these models. Such a eomparison is given in figure 3.7 for a distance of $200 \mathrm{mi}$ and a height of $14,000 \mathrm{ft}$ and in figure 3.8 for a distance of $800 \mathrm{mi}$ and a height of $240,000 \mathrm{ft}$. The particular graphical presentation used in figures 3.7 and 3.8 shows the $4 / 3$ earth rays as straight lines. It is noted that the $4 / 3$ earth ray at $\theta_{0}=0$ is in relatively good agreement, with the values from the reference atmosphere for distances out to $200 \mathrm{mi}$ and heights up to $14,000 \mathrm{ft}$, but systematically departs from the reference 


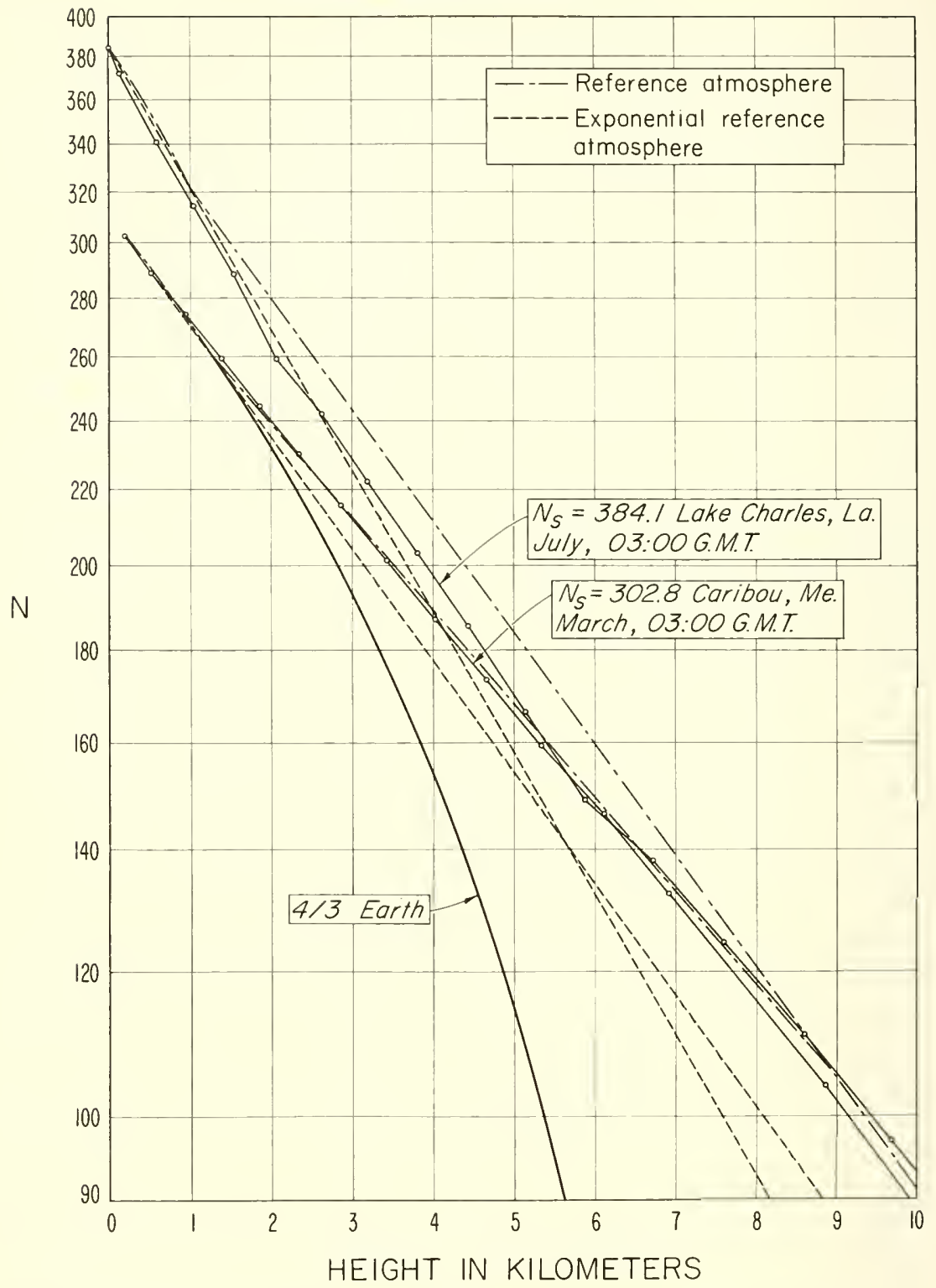

FIGURE 3.6. Comparison of reference atmospheres with observed $\mathrm{N}$ profiles. 
atmosphere for greater distances and heights. For a range of $600 \mathrm{mi}$, where the ray reaches heights of about $200,000 \mathrm{ft}$, the $4 / 3$ earth ray is some $9000 \mathrm{ft}$ lower than the $N_{s}=400$ reference atmosphere and $36,000 \mathrm{ft}$ lower than the $N_{s}=250$ reference atmosphere. This height discrepancy is due to the $4 / 3$ earth model's unrealistically large $N$ gradient at great heights with resultant increased bending.

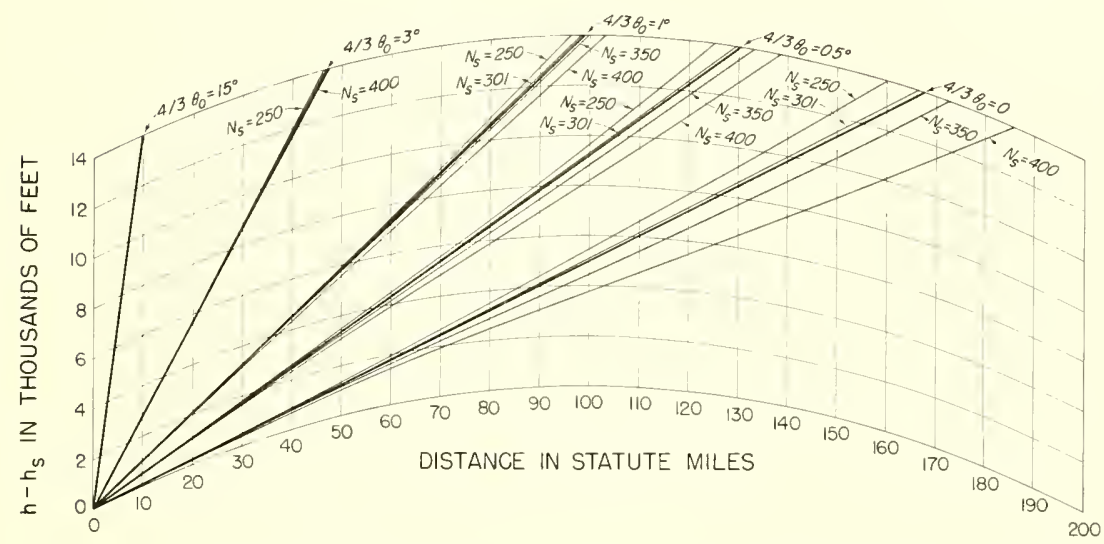

Figure 3.7. Comparison of rays in the CRPL reference refractivity atmospheres-1958 and the 4/3 earth atmosphere.

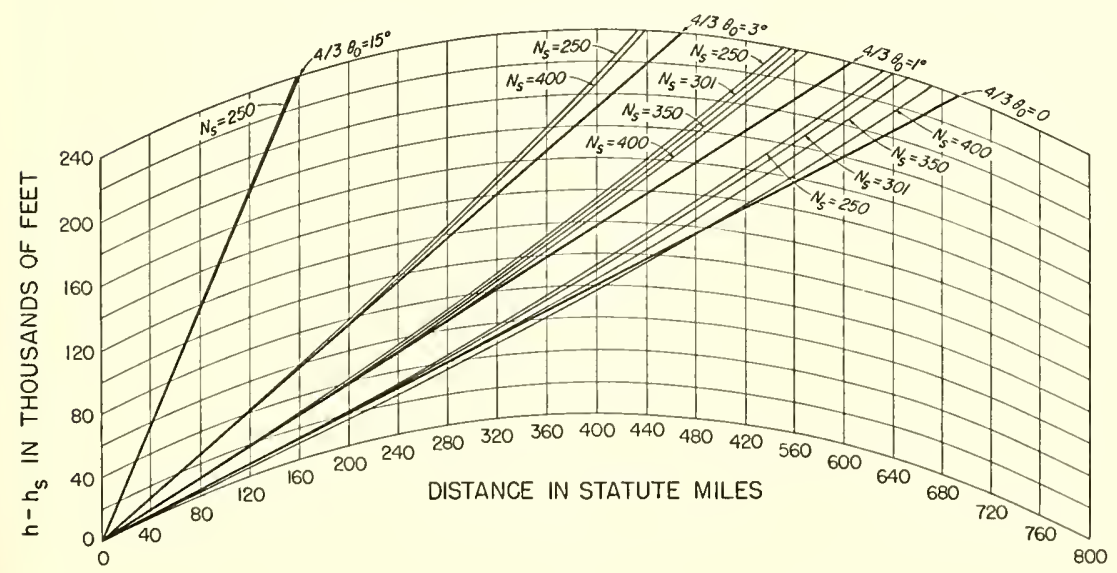

FIGURE 3.8. Comparison of rays in the CRPL reference refractivity atmospheres-1958 and the $4 / 3$ earth atmosphere. 


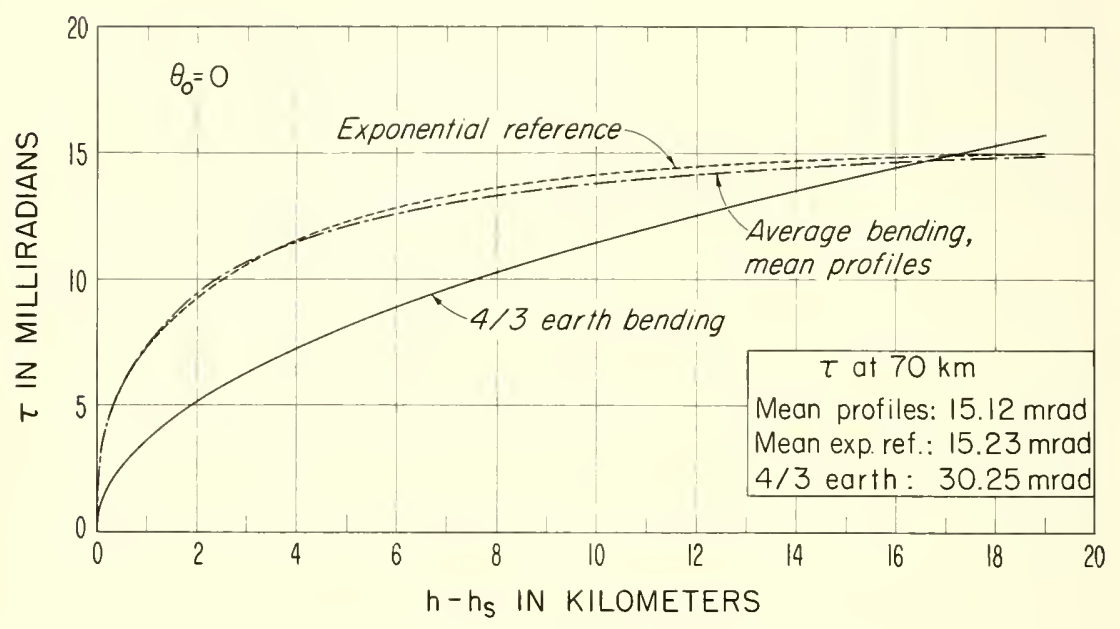

Figure 3.9. Bending versus height.

Further, the bending in the $4 / 3$ earth atmosphere is compared with that in the exponential reference atmosphere in figure 3.9. The bending in an "average" atmosphere is also given. This average atmosphere is a composite of the 5-year mean profiles for both summer and winter at the 11 U.S. radiosonde stations enumerated in the following paragraph, and was used as a readily available measure of average conditions. The important point made by figure 3.9 is that the $4 / 3$ earth model is systematically in disagreement with average bending; at low heights it gives too little bending, while at high altitudes it gives too much bending. The exponential reference atmosphere does not appear to be systematically biased, and deviates less than 5 percent from the average atmosphere. It is significant that the exponential reference and the average atmosphere are in essential agreement as to the shape of the $\tau$-height curve.

It would now be instructive to compare the bendings obtained from the various models with values obtained from each of the 5 -year mean $N$ profiles from different climatic regions. The 5 -year mean $N$ profiles were obtained for both summer and winter for a variety of climates as represented by the states of Florida, Texas, Maine, Illinois, Nevada, California, North Dakota, Washington, Nebraska, Wyoming, and by the District of Columbia.

Comparisons of the bending obtained from the $4 / 3$ earth model and the bendings obtained from the 5 -year mean $N$ profiles with the reference atmospheres are shown in figures 3.10 and 3.11. These figures were selected to illustrate the range of agreement between the models and the expected long-term average bendings. Figure 3.10 gives a comparison 


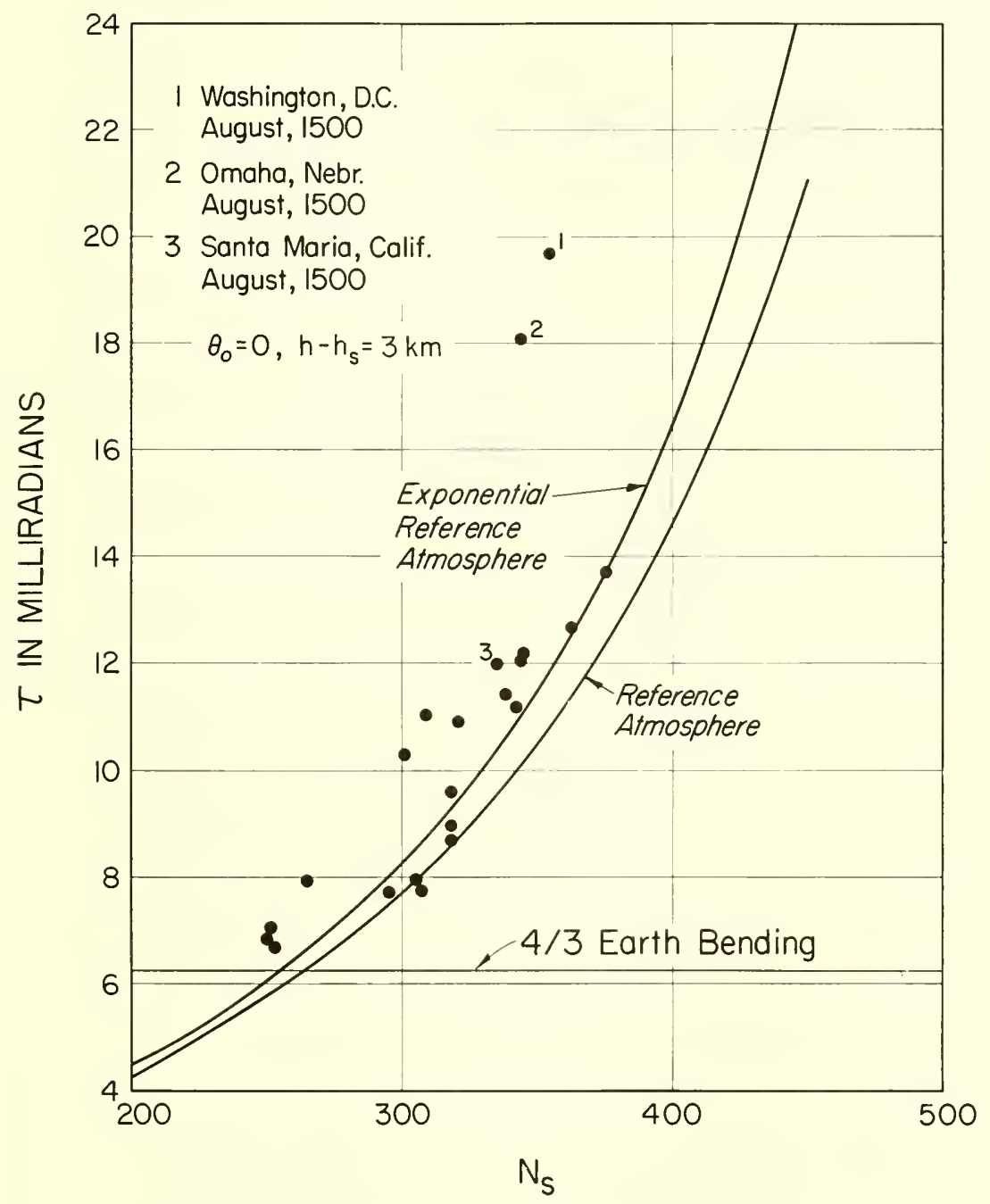

Figure 3.10. Comparison of $\tau$ versus $\mathrm{N}_{\mathrm{s}}$ as obtained from CRPL reference atmospheres-1958 and 5-year mean radiosonde data for $\theta_{0}=0, \mathrm{~h}-\mathrm{h}_{\mathrm{s}}=3 \mathrm{~km}$.

for a small initial elevation angle, $\theta_{0}=0$, and a small height inerement, $h-h_{s}=3 \mathrm{~km}$, and shows that both reference atmospheres tend to set a lower limit to the bendings. In this case, the exponential reference atmosphere appears to be in better agreement with the expected long-term mean bendings than does the reference at mosphere. The numbered data points for Washington, D.C., Omaha, Nebr., and Santa Maria, Calif., are of special interest. Washington and Omaha have the only long-term 


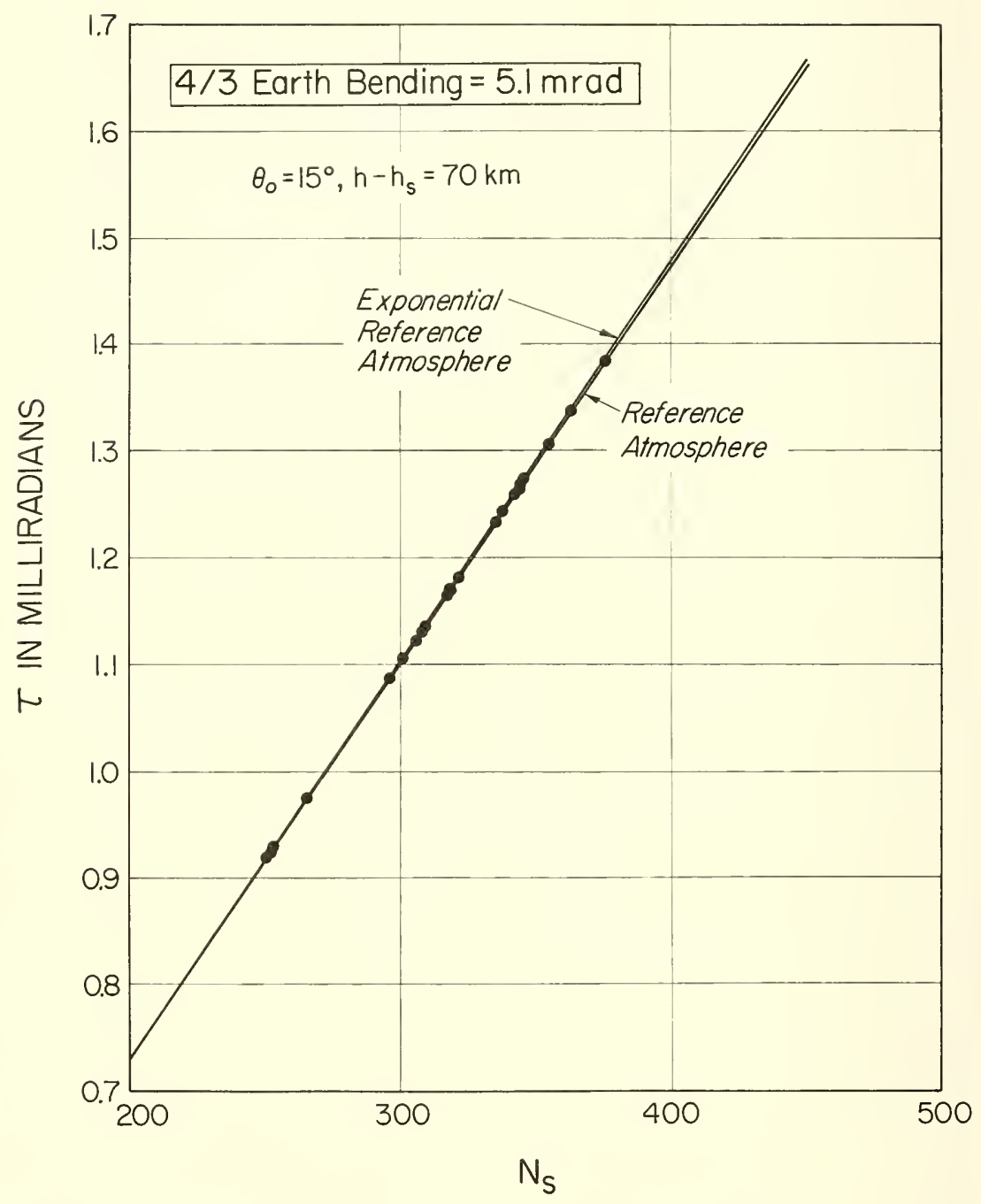

Figure 3.11. Comparison of $\tau$ versus $\mathrm{N}_{\mathrm{B}}$ as obtained from CRPL reference atmospheres-1958 and 5-year mean radiosonde data for $\theta_{\mathrm{o}}=15^{\circ}, \mathrm{h}-\mathrm{h}_{\mathrm{s}}=70 \mathrm{~km}$.

mean $N$ profiles with initial $N$ gradients (i.e., $-112 / \mathrm{km}$ and $-106 / \mathrm{km}$, respectively) that are significantly greater than would be expected from the $\Delta N$ versus $N_{s}$ relationship. Both of these stations have an unusually large humidity decrease near the ground. The third point, Santa Maria, Calif., is of interest since it is in relatively good agreement with the reference atmospheres, even though it represents the southern California summer climate which was excluded from the original $\Delta N$ versus $N_{s}$ 
relationship. This agreement is attributed to the fact that the reference atmosphere is a good representation of the $N$ distribution below the California elevated inversion and to the fact that a majority of the bending is accomplished below the elevated inversion height of about $500 \mathrm{~m}$. Further, it can be easily shown that the bending integral is increasingly insensitive to strong $N$ discontinuities as the height increases.

Figure 3.11 shows a similar comparison for a high initial elevation angle, $\theta_{0}=15^{\circ}$ and a large height increment, $h-h_{s}=70 \mathrm{~km}$. This comparison shows that both of the reference atmospheres are in closer agreement with the long-term mean bendings than are the $4 / 3$ earth bendings. Note that, whether $\tau$ is predicted from $N_{s}$ or $\Delta N$, the $4 / 3$ earth model gives but a single value of bending that is outside the limits of the values of $\tau$ obtained from the long-term mean profiles.

In considering the comparisons of figures 3.10 and 3.11 , one might ask if they reflected the form of the basic equation for bending; namely, at low angles is $\tau$ determined by the $N$ gradient throughout the $N$ profile, and at high angles is $\tau$ essentially a function of the value of $N$ at both ends of the $N$ profile (i.e., the limits of integration). Thus one might expect the deviations to be smaller if the comparisons were made on the basis of a function of the $N$ gradient such as $\Delta N$, particularly for small values of $\theta_{0}$. Such a comparison is given by figures 3.12 and 3.13 for the same initial elevation angles and height increment as before. It is seen that the $\Delta N$-specified reference atmospheres improve the agreement for the low-angle case, but decidedly decrease the agreement for the high-angle case.

A numerical evaluation of the root mean square ( $\mathrm{rms}$ ) deviation of the long-term mean bendings from both the reference atmospheres determined as a function of both $\Delta N$ and $N_{s}$ was made for a variety of initial elevation angles for the height increments 3 and $70 \mathrm{~km}$. Root mean square deviations were not calculated for the $4 / 3$ earth model since it was felt that this model was obviously in marked disagreement with the longterm mean bendings under these conditions. Figure 3.14 summarizes the rms deviations for the $h-h_{s}=3-\mathrm{km}$ case. It is seen that for $\theta_{0}<10 \mathrm{mrad}$ (about $0.5^{\circ}$ ), the $\Delta N$-specified reference atmospheres have the smaller rms deviatiors. Also, the exponential reference atmospheres, whether specified by $\Delta N$ or $N_{s}$, have smaller rms deviations than the reference atmosphere.

It is seen for the 70-km case, figure 3.15, that the $N_{s}$-specified reference atmospheres have a significantly smaller rms deviation than the $\Delta N$ specified atmospheres for $\theta_{0}>5 \mathrm{mrad}$. Again it is seen that the exponential reference atmosphere generally has the smaller rms deviation for values of $\theta_{0}$ less than $10 \mathrm{mrad}$. However, the slightly smaller rms deviations associated with the reference atmosphere for $\theta_{0}>10 \mathrm{mrad}$ reflert that model's closer agreement with the actual $N$ structure of the atmosphere at high heights. 


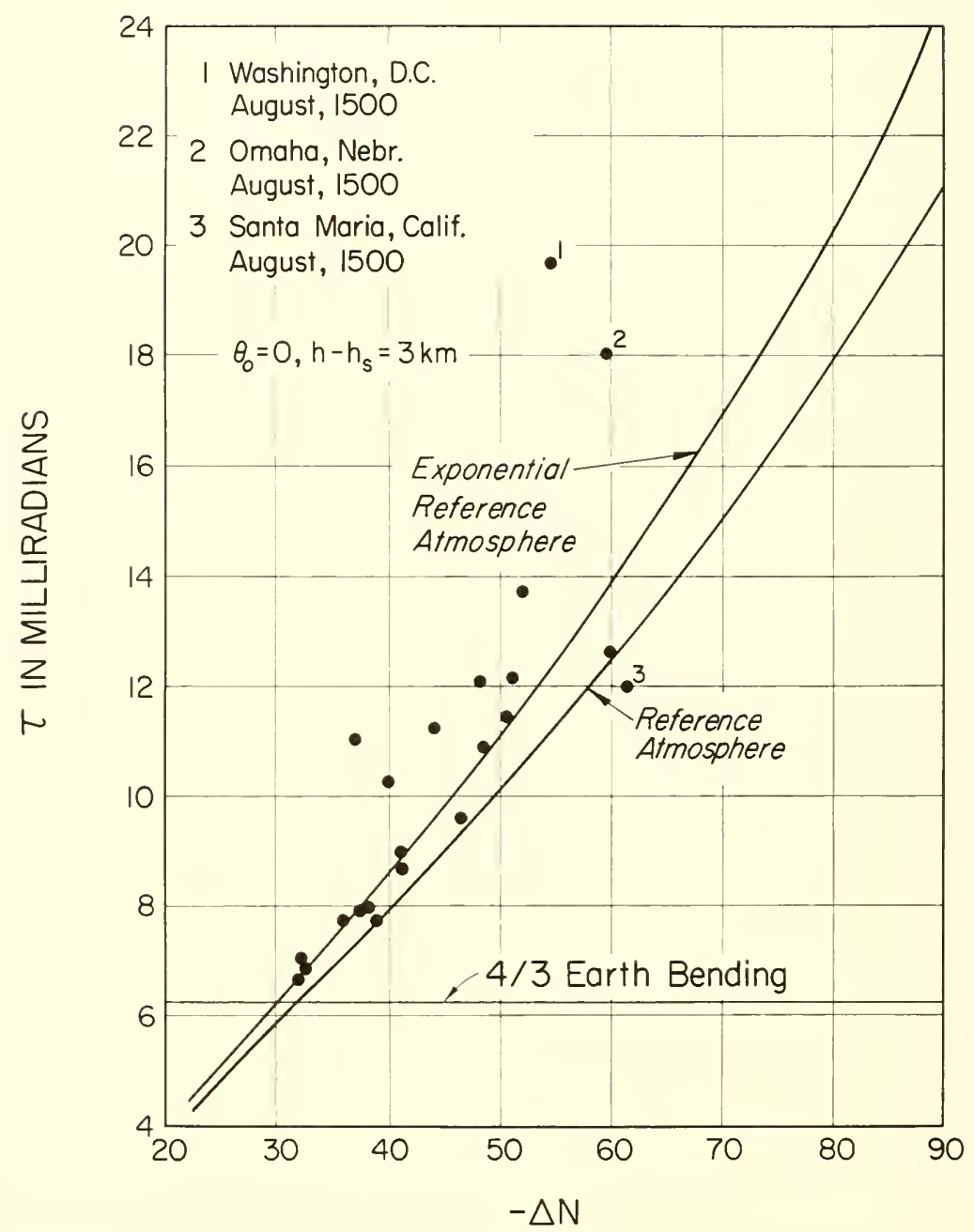

FIgURE 3.12. Comparison of $\tau$ versus $\triangle \mathrm{N}$ as obtained from CRPL reference atmospheres-1958 and 5-year mean radiosonde data for $\theta_{0}=0, \mathrm{~h}-\mathrm{h}_{\mathrm{B}}=3 \mathrm{~km}$. 


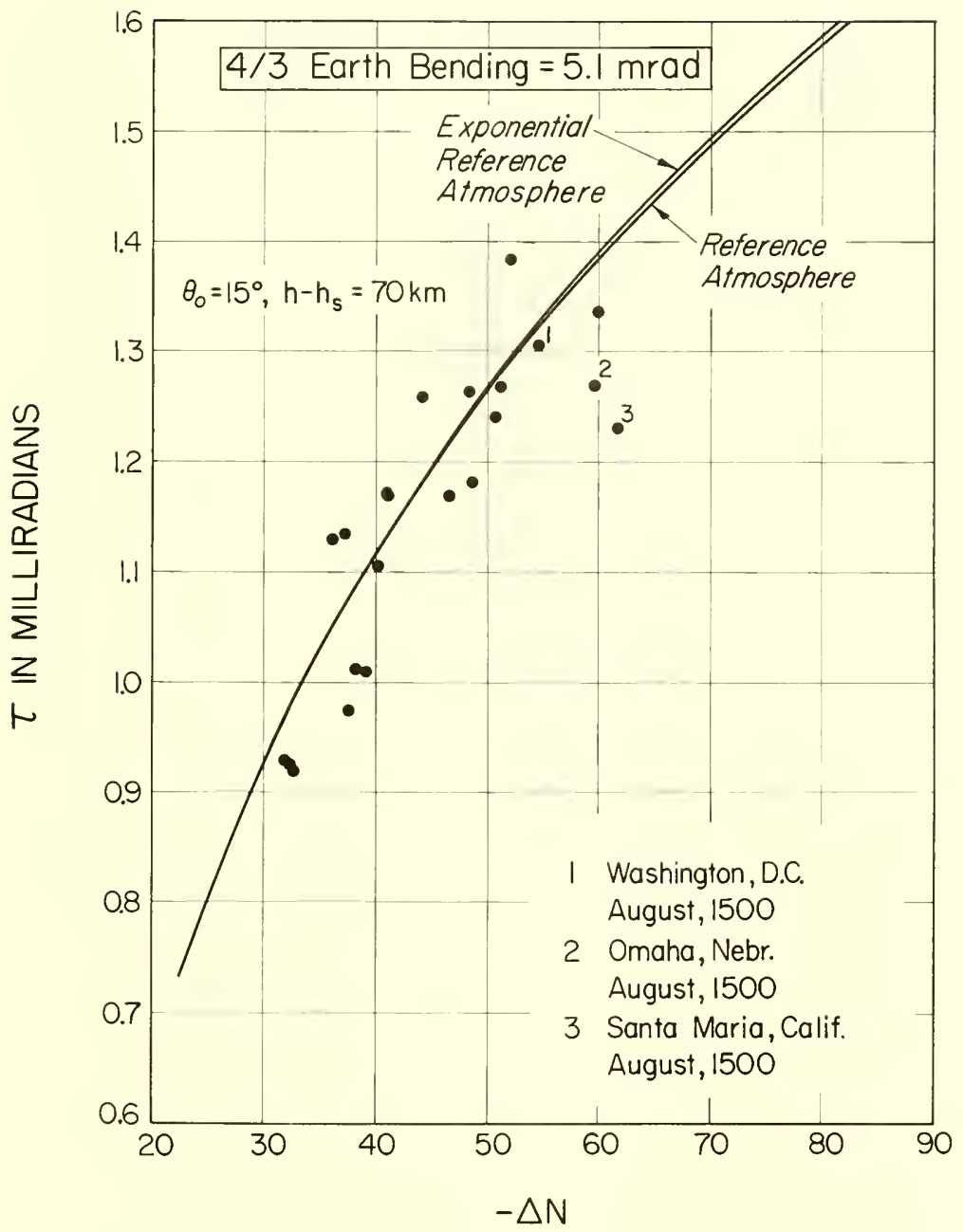

FIgure 3.13. Comparison of $\tau$ versus $\triangle \mathrm{N}$ as obtained from CRPL reference atmospheres-1958 and 5-year mean radiosonde data for $\theta_{0}=15^{\circ} \mathrm{h}-\mathrm{h}_{\mathrm{s}}=70 \mathrm{~km}$. 


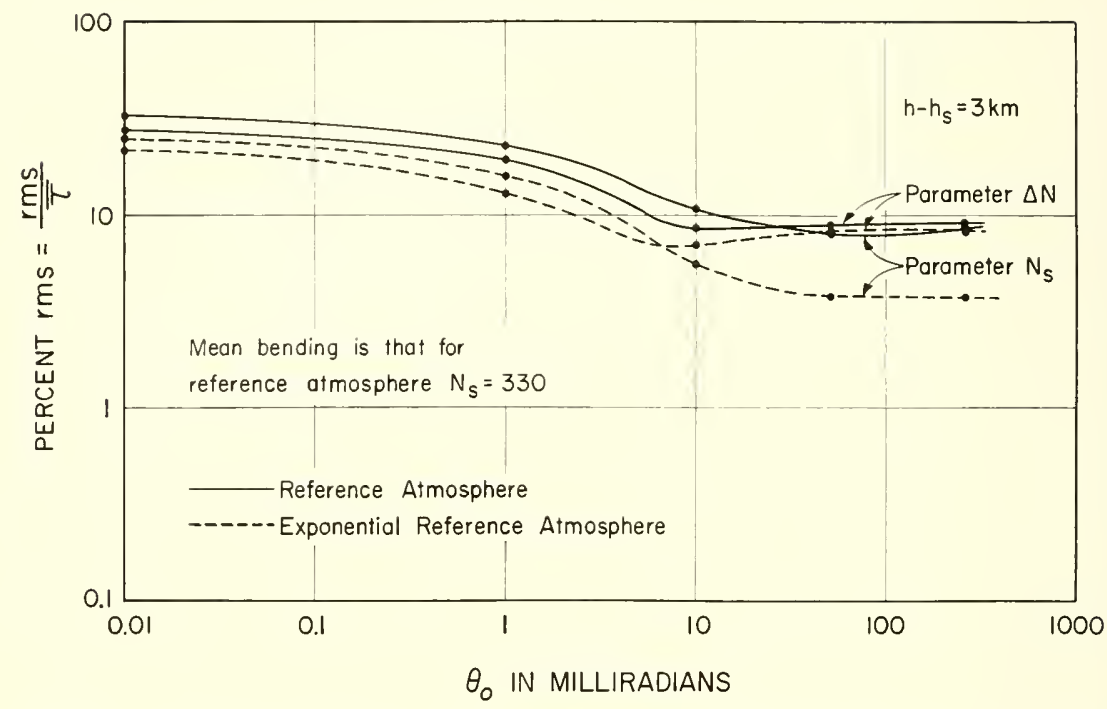

FIgure 3.14. Comparison of percent RMS deviations of 5-year mean profile bendings about CRPL reference atmospheres using two parameters, $\mathrm{N}_{\mathrm{s}}$ and $\Delta \mathrm{N}$ for

$$
\mathrm{h}-\mathrm{h}_{\mathrm{s}}=3 \mathrm{~km} \text {. }
$$

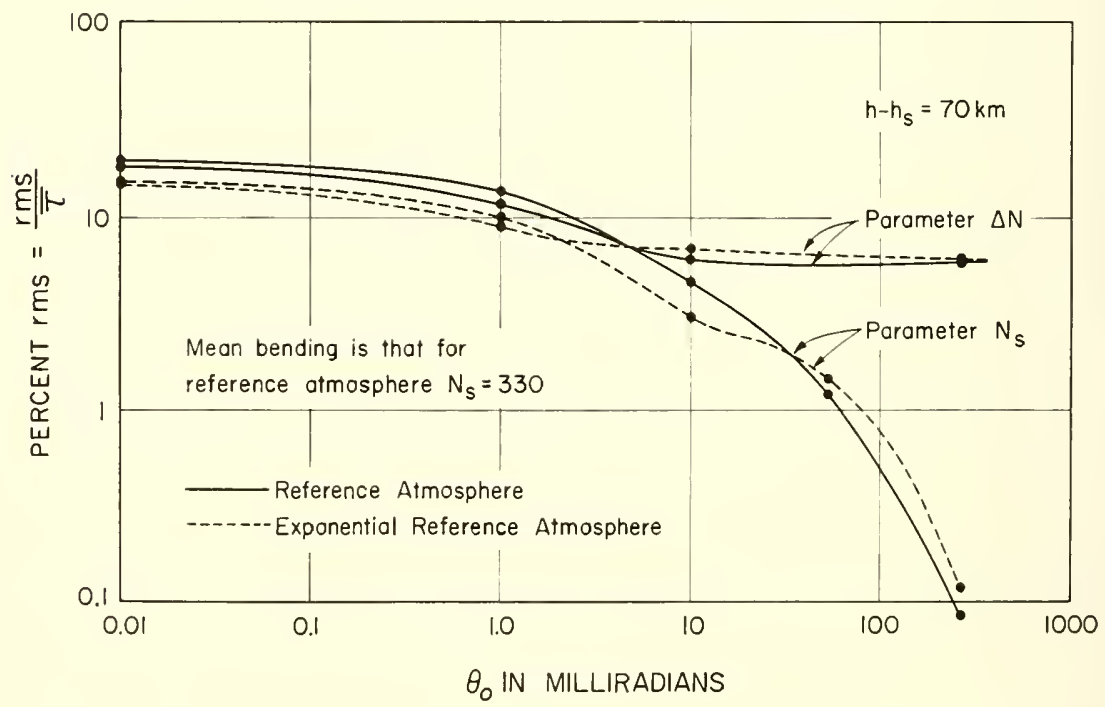

Figure 3.15. Comparison of percent RMS deviations of 5-year mean profile bendings about CRPL reference atmospheres using two parameters, $\mathrm{N}_{\mathrm{s}}$ and $\triangle \mathrm{N}$ for $\mathrm{h}-\mathrm{h}_{\mathrm{s}}=70 \mathrm{~km}$. 


\subsection{The Initial Gradient Correction Method}

The importance of the initial gradient in radio propagation, where the initial elevation angle of a ray path is near zero, has long been recognized. For example if $d n / d h=-1 / a$ (the reciproeal of the earth's radius), then the equation for $\tau$ is indeterminate, an expression of the fact that the ray path remains at a constant height above the earth's surface. This is called ducting, or trapping of the radio ray. The effect of anomalous initial $N$-gradients on ray propagation at elevation angles near zero, and for gradients less than dueting $(|d N / d h|<157 N$ units $/ \mathrm{km}$, or $d N / d h>$ $-157 N$ units $/ \mathrm{km}$ ) may also be quite large. A method has been developed for correcting the predicted refraction (from the exponential reference atmosphere) to account for anomalous initial $N$ gradients, assuming that the actual value of the initial gradient is known [2].

The result is

$$
\tau_{h}=\tau_{h}\left(N_{s}, \theta_{0}\right)+\left[\tau_{100}\left(N_{s}^{*}, \theta_{0}\right)-\tau_{100}\left(N_{s}, \theta_{0}\right)\right],
$$

where $\tau_{h}\left(N_{s}, \theta_{0}\right)=\tau$ at height $h$, for the exponential reference atmosphere corresponding to $N_{s}$, and $N_{s}{ }^{*}$ is the $N_{s}$ for the exponential reference atmosphere that has the same initial gradient as the observed intiial gradient; $\tau_{100}$ is $\tau$ at a height of $100 \mathrm{~m}$.

This procedure has the effect of correcting the predicted bending by assuming that the observed initial gradient exists throughout a surface layer $100 \mathrm{~m}$ thick, calculating the bending at the top of the $100-\mathrm{m}$-thick layer, then assuming that the atmosphere behaves according to the exponential reference profile corresponding to the observed value of $N_{s}$ for all heights above $100 \mathrm{~m}$. This approach has proved quite successful in predicting $\tau$ for initial elevation angles under $10 \mathrm{mrad}$, and will, of course, predict trapping when it occurs.

\subsection{The Departures-From-Normal Method}

A method of ealculating bending by the use of the exponential model of $N(h)$ together with an observed $N(h)$ profile ean sometimes be advantageously employed [15]. This method is primarily intended to point out the difference between actual ray bending and the average bending that is predieted by the exponential $N(h)$ profile and is a powerful method of identifying air mass refraction effects.

The exponential model deseribed in seetion 3.8 can be expeeted to represent average refractivity profile characteristies at any given location, but it cannot be expected to depict aceurately any single refractivity profile selected at random, even though it may oecasionally do so. In order 
to study the differences between individual observed $N(h)$ profiles and the mean profiles predicted by the exponential model, a variable called the $A$ unit has been developed; it is defined simply as the sum of the observed $N$ at any height, $h$, and the refractivity drop from the surface to the height, $h$, which is predicted by the exponential profile for a given value of $N_{s}$.

Thus

$$
A\left(N_{s}, h\right)=N(h)+N_{s}\left(1-\exp \left\{-c_{e} h\right\}\right) .
$$

Thus (3.46) adds to $N(h)$ the average decrease of $N$ with height, so that if a particular profile should happen, by coineidence, to be the same as the corresponding exponential profile, the value of $A\left(N_{s}, h\right)$ for this profile would be equal to $N_{s}$ for all heights. The above analysis shows that the difference between $A\left(N_{s}, h\right)$ from $N_{s}, \delta A\left(N_{s}, h\right)$, is a measure of the departure of $N(h)$ from the normal, exponential profile:

$$
\delta A\left(N_{s}, h\right)=A\left(N_{s}, h\right)-N_{s}=N(h)-N_{s} \exp \left\{-c_{e} h\right\} .
$$

It seems logical that the application of the $A$ unit to bending would indicate the departures of bending from normal, in some way, just as it indieates departures of refractivity, $N$, from normal. This is indeed the case, as can be seen in figure 3.16, where for an $N_{s}=313.0$ exponential atmosphere, $A(313.0, h)$ is plotted on one set of graphs for various typical air masses, and the eorresponding bending departures from normal are shown in the second set of graphs corresponding to the same air masses. Obviously, the bending departures between layers are highly analogous to the $A$ unit variation. It can be seen from figure 3.16 that the similarity exists, although it is less, for higher initial elevation angles. The similarity also decreases with increasing height, owing to the fact that the bending departures from normal are an integrated effect, and at low initial elevation angles are more sensitive to $N$ variations at the lower heights. This causes an apparent damping of the bending departures from normal at greater heights. However, the $A$-unit variation is not similarly influenced; hence, a loss of similarity arises at large heights above the earth's surface.

If (3.46) is differentiated and substituted into (3.2), the following equation results:

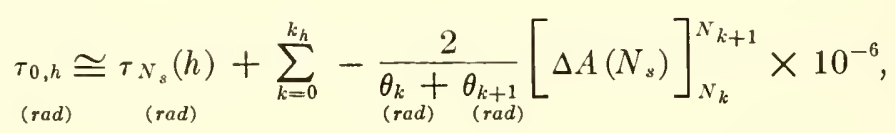




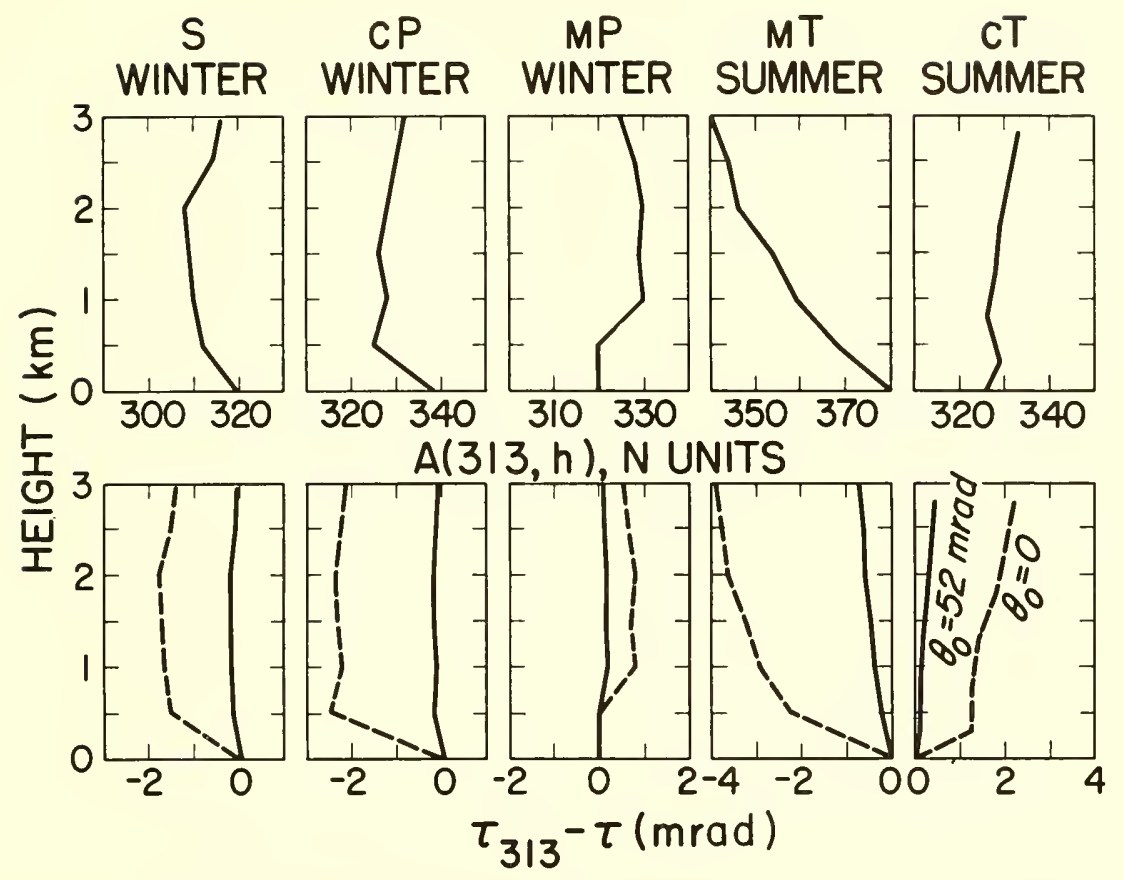

FigURE 3.16. A-unit profiles for typical air masses and refraction deviation from normal.

where

$$
\begin{aligned}
\Delta A\left(N_{s}\right) & =\Delta N(h)+\Delta\left[N_{s}\left\{1-\exp \left(-c_{e} h\right)\right\}\right] \\
& =\Delta N(h)+N_{s} c_{e} \exp \left(-c_{e} h\right) \Delta h
\end{aligned}
$$

and $\tau_{N s}(h)$ is the value of $\tau$ tabulated for various atmospheres in tables 9.10 to $9.17, \theta_{k}$ and $\theta_{k+1}$ are in milliradians and must be from the $N_{s}$ exponential atmosphere used. The value of $\Delta A\left(N_{s}\right)$ is obtained from subtraction of the $A$ value at layer level, $k$, from the value of $A$ at layer, $k+1$. The $A$ value may be obtained by adding any given $N(h)$ value, obtained from RAOB or other similar data, to a value of $N_{s}[1-\exp \{-\operatorname{ch}\}]$ for the same height which may be obtained from figure 3.17. Since $\tau_{N s}(h)$ has been calculated only for a few of the exponential atmospheres, these being the $N_{s}=200.0,252.9,289.0,313.0,344.5,377.2,404.9$, and 450.0 atmospheres, one of these atmospheres must be used in the ealeulation of bending by the departures method. The selection of the particular atmosphere to be used is based on the value of the gradient of $N$, $d N / d h$, between the surface of the earth and the first layer considered. In table 3.2 are shown the ranges of the gradient for the choice of a particular exponential atmosphere. 


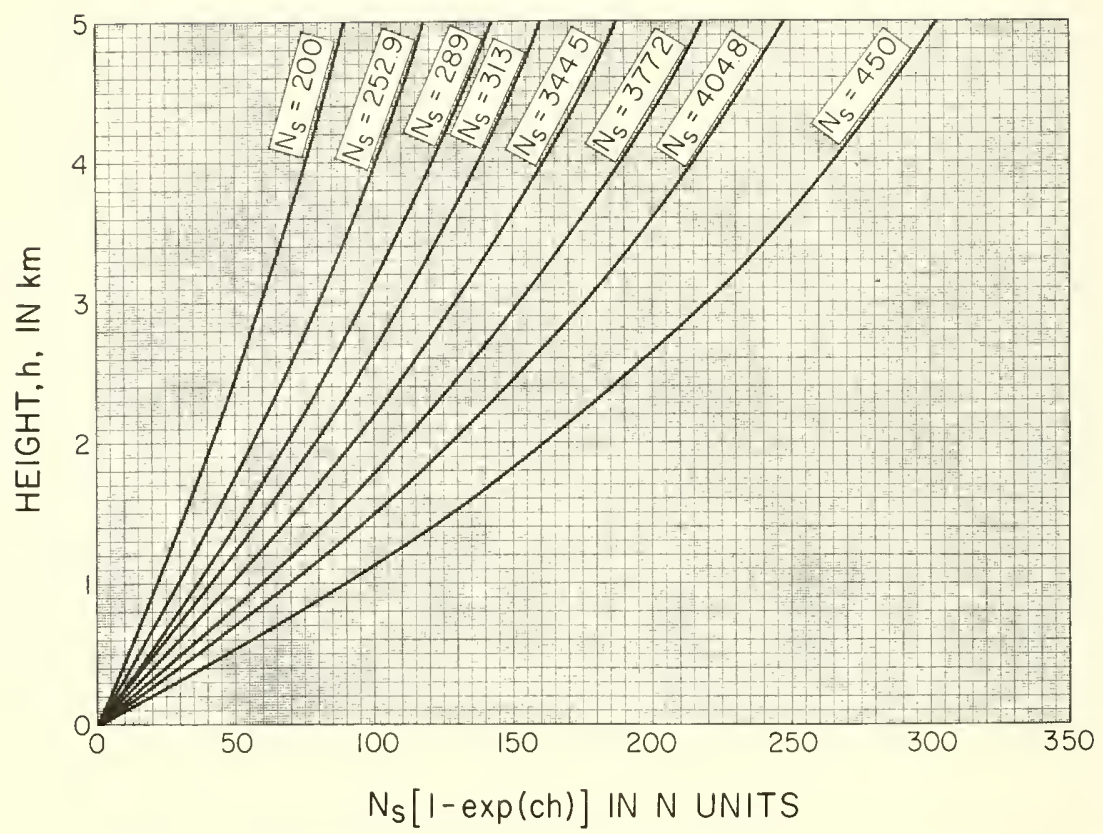

FIgURE 3.17. Graphic representation of $\mathrm{N}_{s}[1-\exp (-\mathrm{ch})]$ in $\mathrm{N}$ units versus height.

\subsection{A Graphical Method}

Weisbrod and Anderson [16] present a handy graphical method for computing refraction in the troposphere. Rewriting and enlarging (3.11), one obtains

$$
\tau(\operatorname{mrad})=\sum_{k=0}^{n} \frac{N_{k}-N_{k+1}}{500\left(\tan \theta_{k}+\tan \theta_{k+1}\right)},
$$

where $\tau$ will be the total bending through $n$ layers. Terms for the denominator can be determined from figure 3.18. Equation 3.49 is essentially Schulkin's result with only the approximation, $\tan \theta_{k} \cong \theta_{k}$, for small angles, omitted.

The procedure for using figure 3.18 follows. Enter on the left margin at the appropriate $N_{s}-N(h)$. Proceed horizontally to the proper height, $h$, interpolating between curves if necessary. Use the solid height curves when $N_{s}-N(h)$ is positive and the dashed curves when $N_{s}-$ $N(h)$ is negative. Then proceed vertically to the assumed $\theta_{0}$ and read $500 \tan \theta$ along the right margin. 


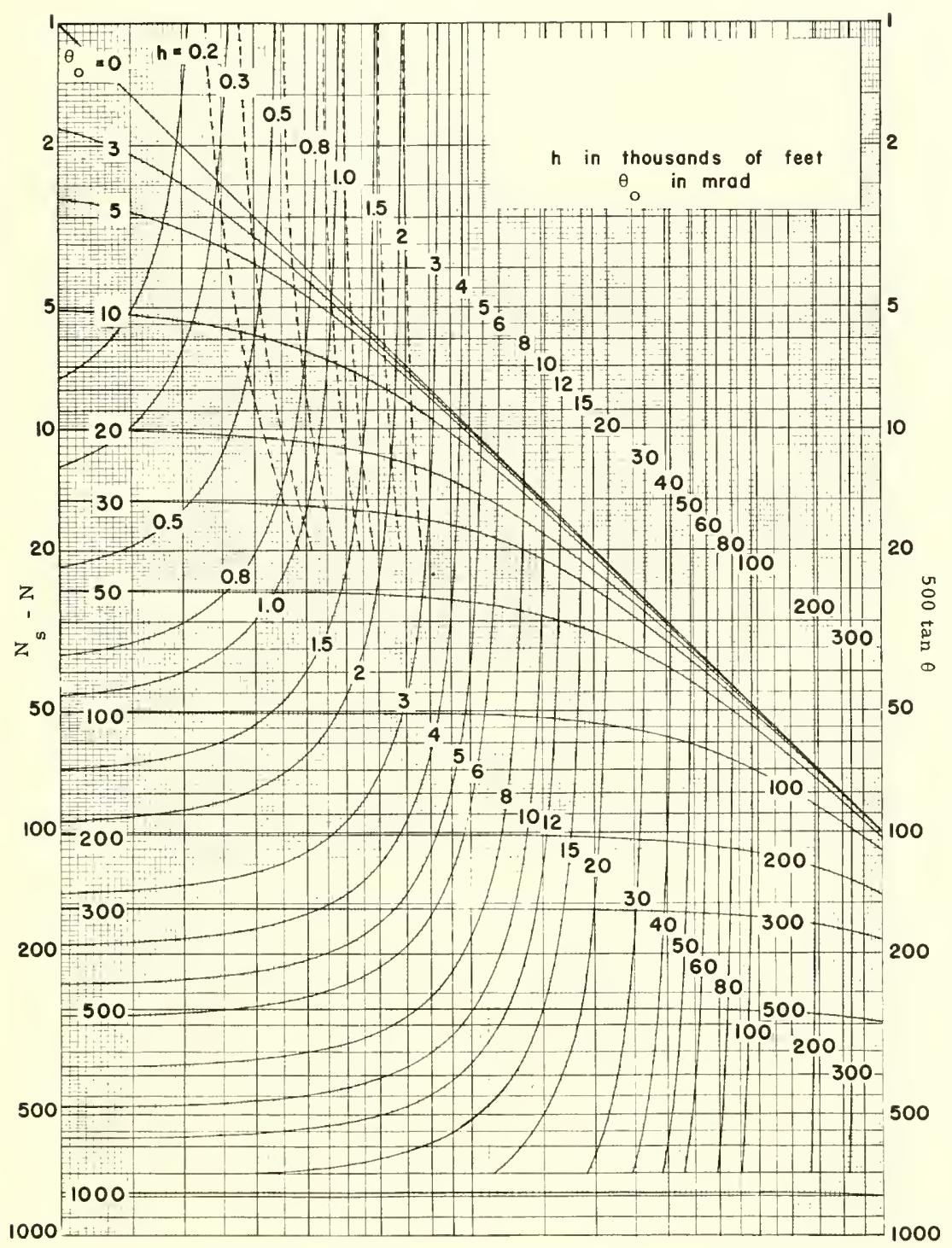

FigURE 3.18. Graphic representation of Snell's Law for finding $500 \tan \theta$. 


\subsection{Derivations}

The approximate relation between $\theta_{1}$ and $\theta_{2}$ is derived here. This relation holds for small increments of height and small $\theta$ 's. The relationship was used in making all sample computations in preceding sections. Since for small $\theta$ 's

$$
\cos \theta_{1} \cong 1-\frac{\theta_{1}^{2}}{2} \text { and } \cos \theta_{2} \cong 1-\frac{\theta_{2}^{2}}{2},
$$

and knowing

$$
r_{2}=r_{1}+\Delta h(\text { fig. 3.19) }
$$

then substituting in (3.1) yields

$$
n_{2}\left(r_{1}+\Delta h\right)\left(1-\frac{\theta_{2}^{2}}{2}\right) \cong n_{1} r_{1}\left(1-\frac{\theta_{1}^{2}}{2}\right)
$$

or

$$
n_{2} r_{1}+n_{2} \Delta h-n_{2} r_{1} \frac{\theta_{2}^{2}}{2}-n_{2} \Delta h \frac{\theta_{2}^{2}}{2} \cong n_{1} r_{1}-n_{1} r_{1} \frac{\theta_{1}^{2}}{2} .
$$

Dividing by $r_{1}$

$$
n_{2}+\frac{n_{2} \Delta h}{r_{1}}-\frac{n_{2} \theta_{2}^{2}}{2}-n_{2} \frac{\Delta h}{r_{1}} \frac{\theta_{2}^{2}}{2} \cong n_{1}-n_{1} \frac{\theta_{1}^{2}}{2}
$$

Since the term $-n_{2}\left(\Delta h / r_{1}\right)\left(\theta_{2}^{2} / 2\right)$ is small with respect to the other terms of (3.53) it may be neglected, and thus:

$$
n_{2}+\frac{n_{2} \Delta h}{r_{1}}-n_{2} \frac{\theta_{2}^{2}}{2} \cong n_{1}-n_{1} \frac{\theta_{1}^{2}}{2}
$$

or

$$
-n_{2} \frac{\theta_{2}^{2}}{2} \cong-n_{1} \frac{\theta_{1}^{2}}{2}-\frac{n_{2} \Delta h}{r_{1}}+\left(n_{1}-n_{2}\right) .
$$

If one now divides both sides of (3.55) by $n_{2}$ and assumes $\left(n_{1}-n_{2}\right) / n_{2} \cong n_{1}-n_{2}$ and $n_{1} / n_{2} \cong 1$, (3.55) may be arranged to yield

$$
\theta_{2} \cong \sqrt{\theta_{1}^{2}+\frac{2 \Delta h}{r_{1}}-2\left(n_{1}-n_{2}\right)} .
$$


Writing (3.56) in terms of $N$ units,

$$
\theta_{2}(\mathrm{mrad}) \cong \sqrt{\theta_{1}^{2}+\frac{2 \Delta h}{r_{1}} \times 10^{6}-2\left(N_{1}-N_{2}\right)}
$$

if $\theta_{1}$ is in milliradians.

Generalizing (3.57) for the $k$ th and the $(k+1)$ st layers,

$\theta_{k+1}(\mathrm{mrad}) \cong \sqrt{\theta_{k}^{2}(\mathrm{mrad})+\frac{2\left(r_{k+1}-r_{k}\right)}{r_{k}} \times 10^{6}-2\left(N_{k}-N_{k+1}\right)}$.

Also from the geometry shown in figure 3.19, a useful relationship for $\tau_{1,2}$ can be obtained. Tangent lines drawn at $A$ and $B$ will be respectively perpendicular to $r_{1}$ and $r_{2}$, since $r_{1}$ and $r_{2}$ describe spheres of refractive indices $n_{1}$ and $n_{2}$ concentric with $O$. Therefore,

$$
\text { angle } A E C=\text { angle } A O B=\phi
$$

also, in triangle $A E C$

angle $A C E=180^{\circ}-$ angle $C A E-$ angle $A E C$

$$
=180^{\circ}-\theta_{1}-\phi .
$$

But from triangle $D C B$

$$
\text { angle } A C E=\text { angle } D C B=180^{\circ}-\tau_{1,2}-\theta_{2} \text {. }
$$

Thus

$$
180^{\circ}-\tau_{1,2}-\theta_{2}=180^{\circ}-\theta_{1}-\phi
$$

Or

$$
\tau_{1,2}=\phi+\left(\theta_{1}-\theta_{2}\right)
$$

Now since $\phi$ in radians $=d / a$, where $d$ is distance along the earth's surface:

$$
\tau_{1,2}=\frac{d}{a}+\left(\theta_{1}-\theta_{2}\right)
$$

or the bending of a ray between any two layers is given in terms of the distance, $d$, along the earth's surface from the transmitter (or receiver), the earth's radius, $a$, and the elevation angles $\theta_{1}$ and $\theta_{2}$ (in radians) at the beginning and end of the layer. 


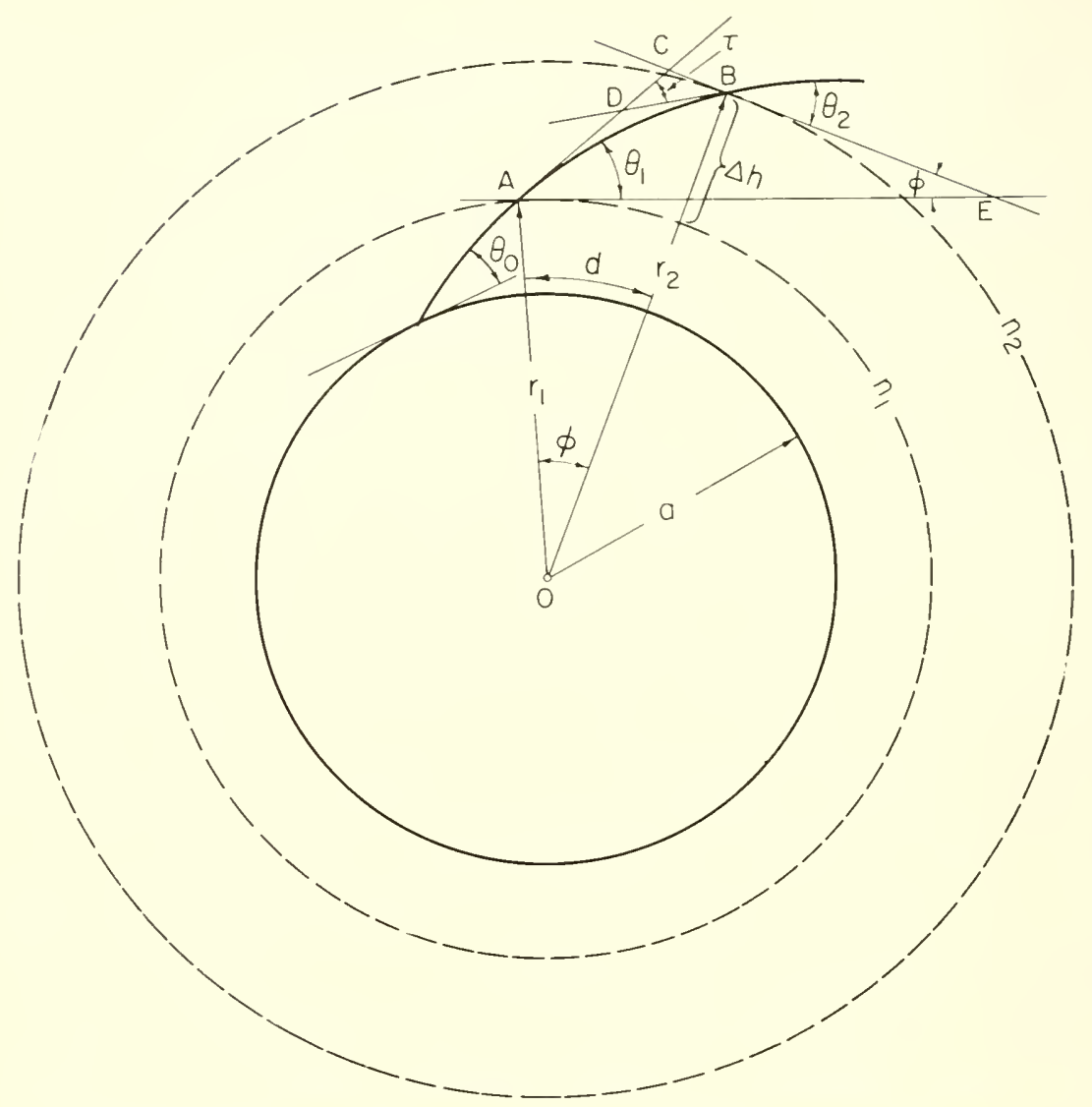

Figure 3.19. Bending geometry on a spherical earth with concentric layers.

If one considers figure 3.20, Snell's law in polar coordinates (3.1) and the refraction formula (3.2) may be obtained from the more familiar form of Snell's law.

Assume that the earth is spherical and that the atmosphere is arranged in spherical layers. In figure 3.20 let $C$ be the center of the earth, $O$ the observer and $C O Z$ the direction of his zenith. Let $n$ and $n+d n$ be the indices of refraction in two adjacent thin layers $M$ and $M^{\prime}$. Let $L P$ be the section of a ray in $M^{\prime}$ which finally reaches the observer at $O$. At $P$ it is refracted along $P Q$. Similarly, it is refracted at the surfaces between successive layers and the final infinitesimal element of its path is TO.

Draw the radii $C P$ and $C Q$. Let angle $P Q F=\theta$, angle $L P S=\theta+d \theta$, and angle $Q P F=\psi$. Then, since the radius $C P$ is perpendicular at $P$ to 


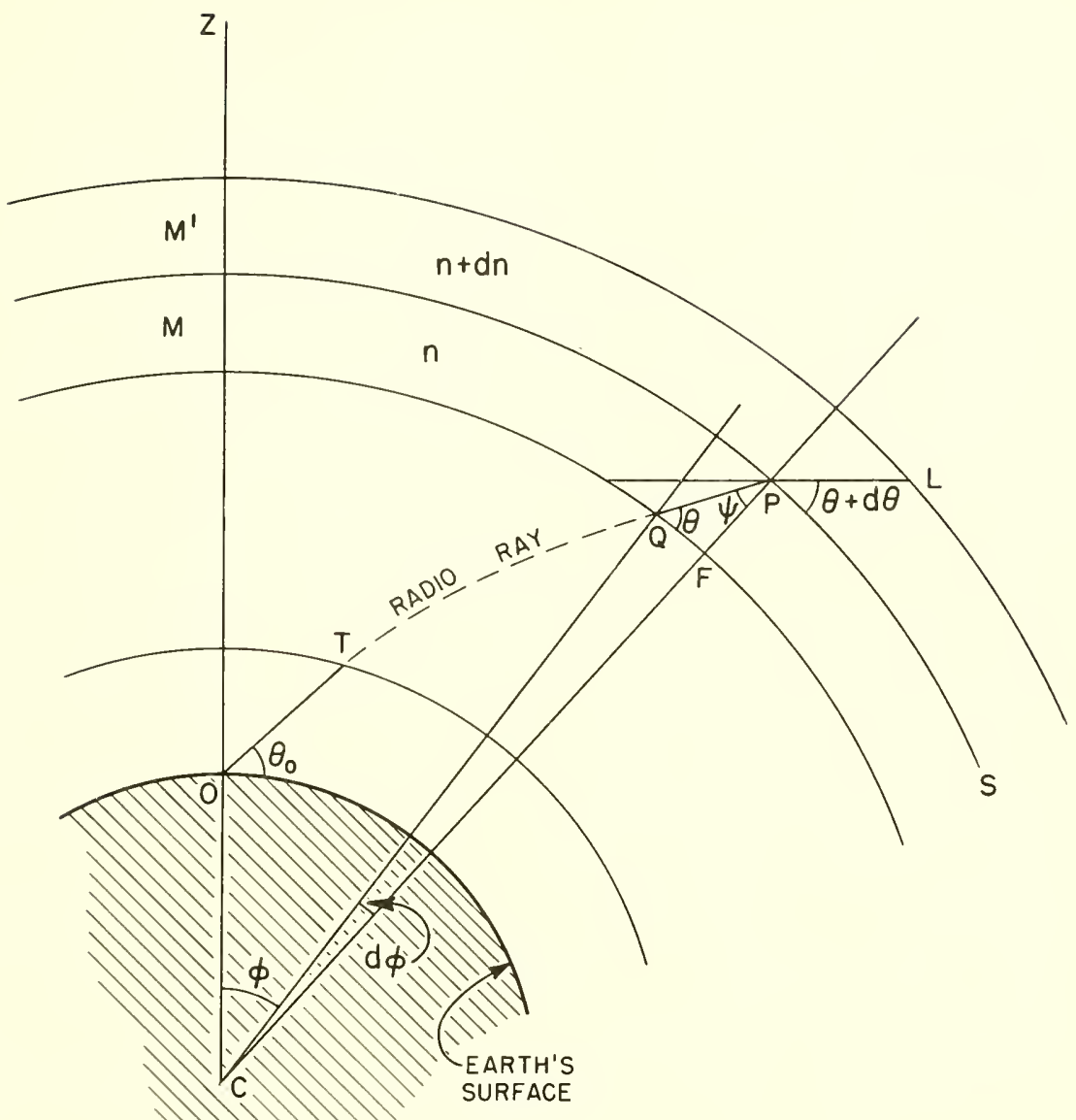

Figure 3.20. Geometry for the derivation of Snell's Law in spherical coordinates.

the bounding surface between layers $M$ and $M^{\prime}$, by Snell's law we have

$$
(n+d n) \sin \left[90^{\circ}-(\theta+d \theta)\right]=n \sin \psi .
$$

Now from the triangle $C Q P^{2}$, in which $C Q=r$ and $C P=r+d r$, and

'The assumption involved in this triangle is that the path of the ray in $\mathbf{M}^{\prime}$ is a straight line, which, of course, can only be true in an isotropie medium. Hence, it can only be true for an infinitesimal layer in the troposphere. Thus only a differential form of Snell's law, (3.65), in polar coordinates, can be obtained by the use of the geometry of figure 3.20 ; not the finite form, (3.68), which has the same appearance. 
angle $C Q P=90^{\circ}+\theta$, we have, from the law of sines,

$$
r \sin \left(90^{\circ}+\theta\right)=r \sin \left(90^{\circ}-\theta\right)=(r+d r) \sin \psi .
$$

Eliminating $\sin \psi$ from (3.63) and (3.64) we then have

$$
(n+d n)(r+d r) \sin \left[90^{\circ}-(\theta+d \theta)\right]=n r \sin \left(90^{\circ}-\theta\right),
$$

or

$$
(n+d n)(r+d r) \cos (\theta+d \theta)=n r \cos \theta .
$$

Multiplication of the $(n+d n)$ and $(r+d r)$ terms, ignoring differential products, yields

$$
(n r+n d r+r d n) \cos (\theta+d \theta)=n r \cos \theta
$$

or

$$
\begin{array}{r}
(n r+n d r+r d n)[\cos \theta \cos (d \theta)-\sin \theta \sin (d \theta)] \\
=n r \cos \theta .
\end{array}
$$

Since $\cos (d \theta) \cong 1$, and $\sin (d \theta) \cong d \theta$, another multiplication, again ignoring products of differentials, yields:

$$
n d r \cos \theta+r d n \cos \theta-n r \sin \theta d \theta=0,
$$

or, dividing all terms by $n r \cos \theta$,

$$
\frac{d r}{r}+\frac{d n}{n}-\tan \theta d \theta=0
$$

Now if (3.67) is integrated between any two thin layers of refractive indices $n_{1}$ and $n_{2}$, whose radial distances from the earth's center are $r_{1}$ and $r_{2}$, and the initial elevation angles of a radio ray entering the layers are $\theta_{1}$ and $\theta_{2}$ :

$$
\int_{r_{1}}^{r_{2}} \frac{d r}{r}+\int_{n_{1}}^{n_{2}} \frac{d n}{n}-\int_{\theta_{1}}^{\theta_{2}} \tan \theta d \theta=\ln \frac{r_{2}}{r_{1}}+\ln \frac{n_{2}}{n_{1}}+\ln \frac{\cos \theta_{2}}{\cos \theta_{1}}=0
$$

or, taking antilogs of both sides,

$$
\frac{r_{2} n_{2} \cos \theta_{2}}{r_{1} n_{1} \cos \theta_{1}}=1,
$$


whence

$$
n_{1} r_{1} \cos \theta_{1}=n_{2} r_{2} \cos \theta_{2},
$$

which is Snell's law for polar coordinates, (3.1).

In figure 3.20 , it can be seen that

$$
\tan \theta \cong \frac{P F}{Q F}=\frac{d r}{r d \phi},
$$

where $\phi$ is the angle at the earth's center between $r$ and $C O Z$. Substituting (3.69) in (3.67)

$$
\tan \theta d \phi+\frac{d n}{n}-\tan \theta d \theta=0
$$

or

$$
(d \phi-d \theta) \tan \theta=-\frac{d n}{n} .
$$

Since, by considering (3.61) for infinitesimal angles,

or, in $(3.70)$

$$
d \tau=d \phi-d \theta
$$

$$
d \tau \tan \theta=-\frac{d n}{n}
$$

or

$$
d \tau=-\cot \theta \frac{d n}{n}
$$

Integration of (3.71) yields (3.2).

\subsection{References}

[1] Smart, W. M. (1931), Book, Spherical Astronomy, Ch. 3 (Cambridge Univ. Press, London, England).

[2] Bean, B. R., and G. D. Thayer (May 1959), On models of the atmospheric refractive index, Proc. IRE 47. No. 5, 740-755.

[3] Booker, H. G., and W. Walkinshaw (1947), The mode theory of tropospheric refraction and its relation to wave guides and diffraction, Book, Meteorological Factors in Radio-Wave Propagation, pp. 80-127 (The Physical Society, London, England).

[4] Freehafer, John E. (1951), Tropospheric refraction, Book, Propagation of Short Radio Waves, pp. 9-22 (McGraw-Hill Book Co., Inc. New York, N.Y.).

[5] Bean, B. R., and B. A. Cahoon (Nov. 1957), The use of surface weather observations to predict the total atmospheric bending of radio waves at small elevation angles, Proc. IRE, 45, 1545-1546. 
[6] Schulkin, M. (May 1952), Average radio-ray refraction in the lower atmosphere, Proc. IRE 40, 554-561.

[7] Anderson, L. J. (Apr. 1958), Tropospheric bending of radio waves, Trans. Am. Geophy's. Union 39, 208-212.

[8] Schelleng, J. C., C. R. Burrows, and E. B. Ferrell (Mar. 1933), Ultra-short-wave propagation, Proc. IRE 21, 427-463.

[9] Stickland, A. C. (1947), Refraction in the lower atmosphere and its application to the propagation of radio waves, Book, Meteorological Factors in Radio Wave Propagation, pp. 253-267 (The Physical Society, London, England).

[10] Humphreys, W. J. (1940), Book, Physics of the Air, p. 82 (McGraw-Hill Book Co., Inc., New York, N.Y.).

[11] Norton, K. A., P. L. Rice, and L. E. Vogler (Oct. 1955), Use of angular distance in estimating transmission loss and fading range for propagation through a turbulent atmosphere over irregular terrain, Proc. IRE 43, 1488-1526.

[12] The Rocket Panel (1952), Pressures, densities, and temperatures in the upper atmosphere, Phys. Rev. 88, 1027-1032.

[13] Handbook of Geophysics for Air Force Designers (1957), Geophysics Research Directorate (Air Force Cambridge Research Center, ARDC, USAF).

[14] Dubin, M. (Sept. 1954), Index of refraction above 20,000 feet, J. Geophys. Res. 59. 339-344.

[15] Bean, B. R., and E. J. Dutton (May-June 1960), On the calculation of departures of radio wave bending from normal, J. Res. NBS 64D (Radio Prop.), No. 3, 259-263.

[16] Weisbrod, s., and L. J. Anderson (Oct. 1959), Simple methods for computing tropospheric and ionospheric refractive effects on radio waves, Proc. IRE 47, $1770-1777$. 


\section{Chapter 4. $N$ Climatology}

\subsection{Introduction}

The contents of this chapter include a study of the surface variation of the radio refractive index on a worldwide scale in terms of a reduced-tosea-level form of the index that gives a significantly more accurate description of refractive index variations than the nonreduced form.

The mean vertical structure of the refractive index parameter in the troposphere over central North America is presented, again in terms of a reduced-to-sea-level form of $N$.

A climatological treatment of the phenomenon of the atmospheric duct, or waveguide, and associated fading regions is also presented. The chapter is concluded with a discussion of refraction of radio waves in various air masses. It is demonstrated that refraction differences within air masses arise from departures of refractive index structure from normal.

\subsection{Radio-Refractive-Index Glimate Near the Ground}

\subsubsection{Introduction}

The radio refractive index of air, $n$, is a function of atmospheric pressure, temperature, and humidity, thus combining in one parameter three of the normal meteorological elements used to specify climate. In the following sections we will examine the variability of $n$ during different seasons of the year and in differing climatic regions. The systematic dependence of $n$ upon station elevation will make it necessary to consider a method of expressing $n$ in terms of an equivalent sea-level value in order to see more clearly the actual climatic differences of the various parts of the world. After a consideration of the $n$ climate of the world, the application of this information to such practical problems as the prediction of radio field strength and the refraction of radio waves will be discussed.

\subsubsection{Presentation of Basic Data}

Near the surface of the carth, for VHF and UHF frequencies, $n$ is a number of the order of 1.000:3. Since, for air, $n$ never exceeds unity by 
more than a few parts in $10^{4}$, it is convenient to consider the climatic variation of $n$ in terms of

$$
N=(n-1) 10^{6}
$$

as defined in chapter 1. The notation $N_{s}$ is used to indicate that (4.1) has been evaluated from standard surface weather observation.

To obtain long-term average values of $N$, one should properly average individual observations over many years. 'This is difficult to do since, in general, only summaries of weather observations are readily available. However, long-term average values of temperature, pressure, and humidity are available and may be converted into an "average" value of $N$. This "average" $N$ differs from the true average since the intercorrelation of pressure, temperature, and humidity is neglected. This difference was examined by an analysis of 2 years of weather records of the months of February and August at an aretic location (Fairbanks, Alaska), a temperate zone location (Washington, D.C.), and a tropical location (Swan Island, W.I.). These data, given in table 4.1, indicate that the difference between the two methods was never more than $1.5 \mathrm{~N}$ units and that the average difference was less than $1 \mathrm{~N}$ unit, which is small compared to commonly observed seasonal and geographic variations of 20 to $100 N$ units.

TABLE 4.1. Two-year average value of $\mathrm{N}_{\mathrm{B}}$ versus the value of $\mathrm{N}_{\mathrm{B}}$ calculated from average temperature, pressure, and humidity

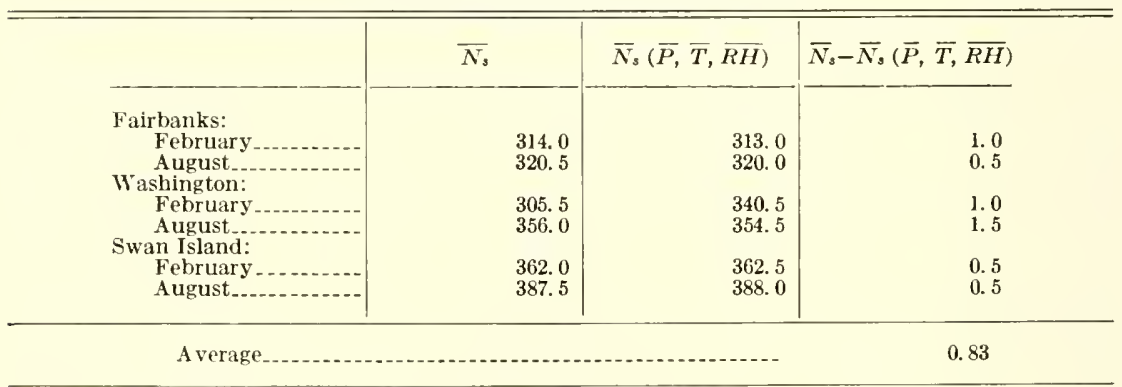

On this basis it was decided to use the long-term means given in the United Nations' monthly publication, Climatic Data for the World. 'This publication is particularly advantageous for our present study since it reports the fictitious value of the relative humidity needed to obtain the actual average vapor pressure from the saturated vapor pressure of the reported mean temperature [1]. ${ }^{1}$

${ }^{1}$ Figures in brackets indicate the literature references on p. 170 . 
Data from 306 weather stations were obtained in order to give reasonable geographical coverage. In general, 5 years of records were obtained for each station from the period 1949 to 1958 , preference being given to the years 1954 through 1958. A noticeable exception, however, was Russia, for which only 1 year of data (IGY) is reported in Climatic Data for the World; thus all charts are drawn with dashed contours for Russia. There are vast expanses of ocean for which there are no meteorologicai observing stations. Climatic atlases were utilized in order to present estimates of world climate in these locales. A reasonable coverage of the sparse data areas of the world was made by estimating temperature from sea surface isotherms [2] and humidity from charts of seasonal average depression of the wet bulb temperature [3]. Pressure was est imated for these locations from average winter and summer pressure charts.

When these data were converted to $N$ [4] and charts prepared, a pronounced altitude dependence could be seen, as in figure 4.1. Figure 4.1 and the following charts of $N$ variations across the United States are from an extensive $N$ climatology now being prepared at the Central Radio Propragation Laboratory. Although the present study is primarily aimed toward worldwide variations, it is felt that the U.S. data better illustrate the height dependence of $N_{s}$ and the subsequent reduction process employed. It is noted that the coastal areas display high values of $N_{s}$, while the inland areas have lower values. There are low values of $N_{s}$ corresponding to the Appalachian and Adirondack Mountains and a decrease with increasing elevation of the Great Plains until the lowest values are observed in the Rocky Mountain region and the high plateau area of Nevada. A corresponding gradient is observed from the west coast eastward. Crosshatching encloses areas where the terrain changes so rapidly that it was felt the data were inadequate to obtain realistic contours of $N_{s}$.

The altitude dependence of $N$ can be studied in terms of the "dr'y" and "wet" components of $N$. These components are those of the two-term expression in (1.17). The dry term, $D$,

$$
D=\frac{77.6 P}{T}
$$

is proportional to air density and nommally constitutes at least 60 pereent of $N$.

The average variation of density with altitude in the atmosphere may be expressed in the first approximation as

$$
\rho=\rho_{0} \exp \{-z / H\}
$$




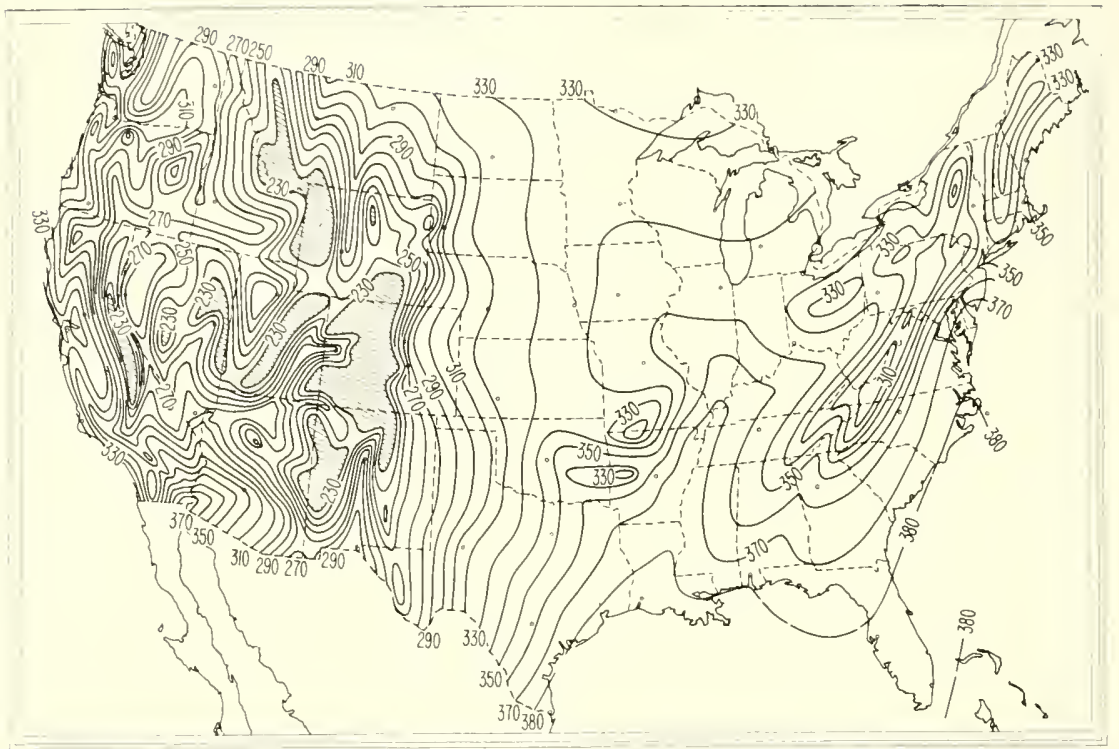

Figure 4.1. Mean $\mathrm{N}_{\mathrm{B}}$, August 0200 local time.

where $z$ is the altitude, $\rho_{0}$ the average sea level density of moist air and $H$ the average scale height between zero and $z$. It is useful to introduce the concept of an effective scale height, $H^{*}$, for the average variation of refractive index in the atmosphere. Many studies have shown that the average refractive index variation with height is quite well represented, to a first approximation, by a formula similar to $(4.3)[5,6,7]$. It is possible to calculate a theoretical value of this effective scale height using a distribution of water vapor. This is, however, quite a complex procedure. Furthermore, the value obtained depends upon the model of the water vapor distribution, and no definite conclusion can be justified considering the extreme variation of water vapor concentration with season, geographic location, and height above the earth's surface. A convenient and simple alternative is to adopt a value for $H^{*}$ from the average $(n-1)$ variation with height in the free atmosphere. Several such values of $H^{*}$ were determined by reference to the NACA standard atmosphere [8] and recent climatological studies of atmospheric refractive index structure [9].

It is seen from table 1.8 , chapter 1 , that $H^{*}$ varies from 6.56 to 7.63 $\mathrm{km}$ in the NACA standard atmosphere, depending on the value of relative humidity assumed. The value of $H^{*}=6.95 \mathrm{~km}$ obtained from climatological studies of $(n-1)$ variations over the first kilometer above 
the earth's surface from nearly 2 million radiosonde observations from many diverse climates.

At the time of analysis of the map series presented here, a value of effective scale height, $H^{*}=9.46 \mathrm{~km}$, was in use. This form was

$$
D_{0}=D_{s} \exp \left\{\frac{z}{H^{*}}\right\}
$$

in which the value $H^{*}=9.46 \mathrm{~km}$ was determined from the NACA dry standard atmosphere. A $D_{0}$ chart is shown on figure 4.2 , presenting a gradient that is remarkably free of detail as compared to the $N_{s}$ chart of figure 4.1, and is easily drawn for all areas of the country.

An investigation of the elevation dependence of the surface wet term

$$
W_{s}=\frac{3.73 \times 10^{5} e}{T^{2}}
$$

revealed low correlations of $\log W_{s}$ and height, indicating that $W_{s}$ is not a marked exponential function of elevation. Contours of $W_{s}$ for all sections of the country are shown on figure 4.3.

The maps that follow are completed in terms of a single reduced form

$$
N_{0} \cong\left(D_{s}+W_{s}\right) \exp \left\{\frac{z}{H^{*}}\right\}=N_{s} \exp \left\{\frac{z}{H^{*}}\right\}
$$

where $N_{s}$ is reduced by the dry term effective scale height, $H^{*}=9.46 \mathrm{~km}$. Figure 4.4 gives the $N_{0}$ contours for the same time as the previous maps of $D_{0}$ and $W_{s}$. The $N_{0}$ maps are no more difficult to prepare than the $W_{s}$ maps and have effectively removed the station height dependence of $N_{s}$. One might wonder at the advisability of arbitrarily reducing the wet term by the dry term correction. For the coastal areas of the country, where the exponential height correction factor is nearly unity, this amounts simply to adding the $D_{0}$ and $W_{s}$ maps for the mountain areas. Where the height correction factor is large, the $W_{s}$ values are small with the result that the gradient of the $N$ isopleths obtained from the $D_{0}$ and $W_{s}$ maps is essentially maintained on the $N_{0}$ maps. As an example, for the series of maps under discussion, the $\left(D_{0}+W_{s}\right)$ difference between Reno, Nev. $(1,340 \mathrm{~m}$ elevation), and Oakland, Calif. (5.5 m elevation), is $21 \mathrm{~N}$ units, while the $N_{0}$ difference is $19 \mathrm{~N}$ units.

The effects of this correction on the worldwide values can be seen from figure 4.5, where $N_{s}$ is plotted versus station elevation in kilometers. A sample line illustrates the decay of $N_{s}$ with height for $N_{0}=348$. The value of $N_{s}$ for any other value of $N_{0}$ would be obt ained from a line parallel to the $N_{0}=348$ line but having a zero intercept equal to the new value of 


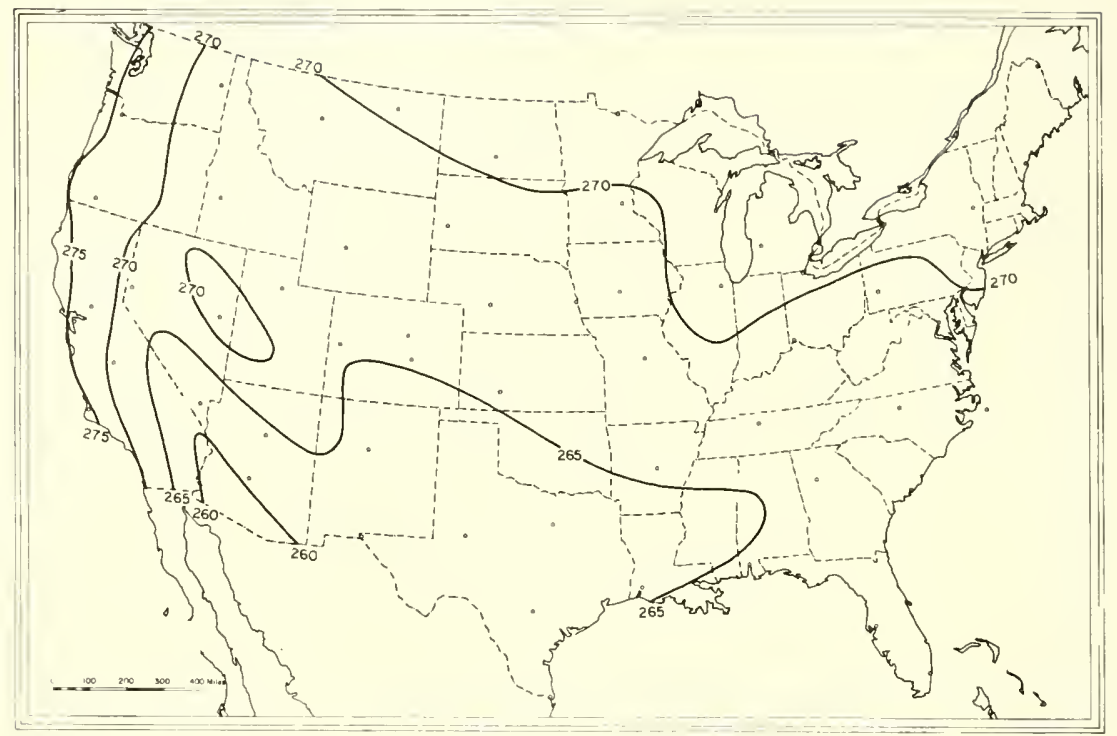

Figure 4.2. Mean $\mathrm{D}_{0}$, August 0200 local time.

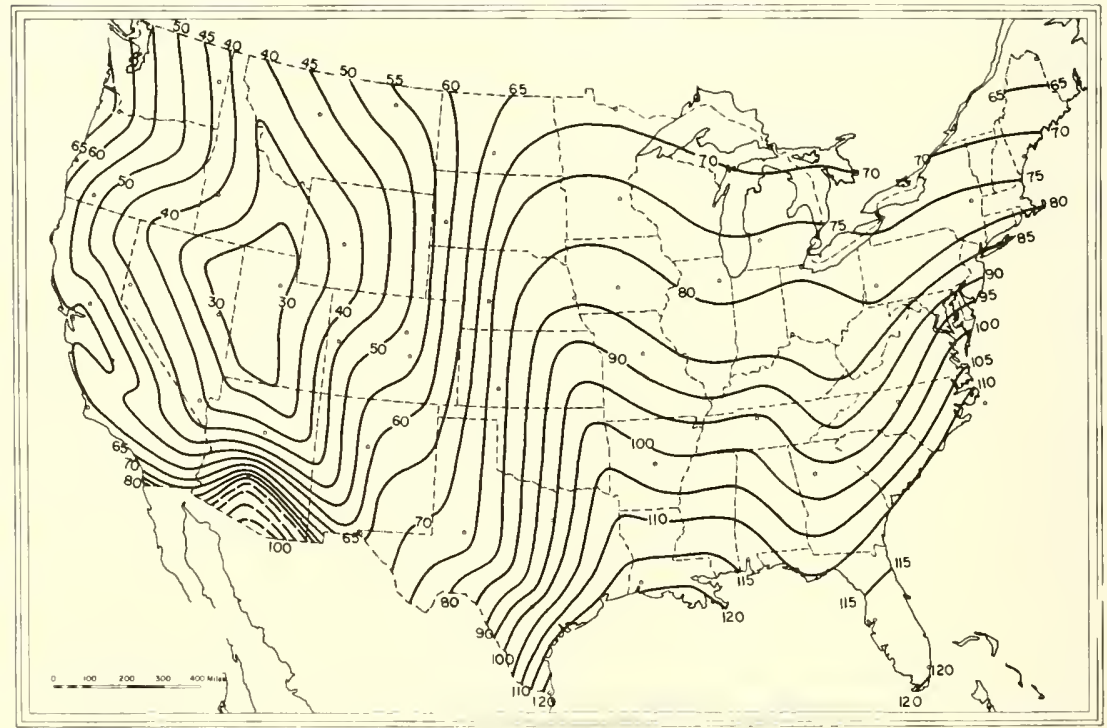

Figure 4.3. Mean $\mathrm{W}_{8}$, August 0200 local time. 


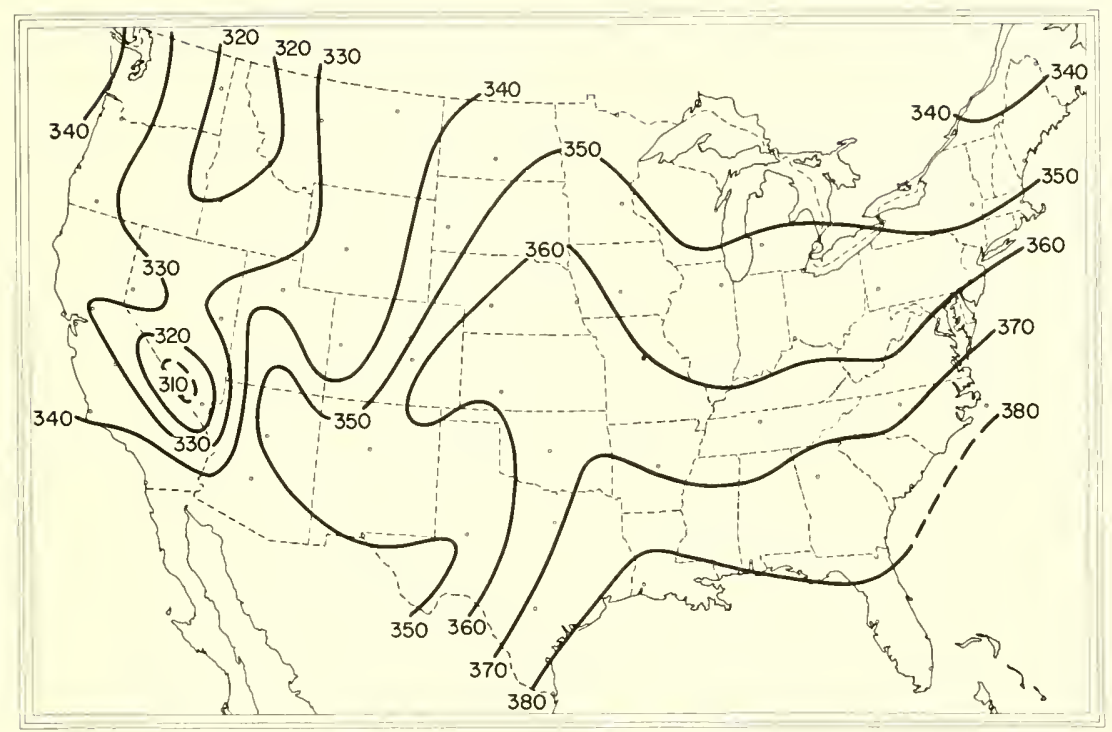

Figure 4.4. Mean $\mathrm{N}_{0}$, August 0200 local time.

$N_{0}$. The advantage of adopting $N_{0}$ is illustrated by the reduction in range from $190 \mathrm{~N}$ units for $N_{s}$ to $115 \mathrm{~N}$ units for $N_{0}$, thus diminishing the number of contours of the resulting maps.

It would appear that by removing the influence of station elevation it would be more efficient to estimate $N_{s}$ from $N_{0}$ charts rather than from charts of $N_{s} . \quad$ As a test of this hypothesis, $N_{s}$ and $N_{0}$ contour charts were prepared for both summer and winter from only 42 of the 62 U.S. Weather Bureau stations for which 8-year means of $N_{s}$ are available. The remaining 20 stations, distributed at random about the country, were used as a test sample by estimating their 8-year mean value of $N_{s}$ from the $N_{0}$ and $N_{s}$ contours. Summertime examples of these charts are given by figures 4.6 and 4.7. Note that due to the reduced range of $N$, the $N_{0}$ charts are drawn every $5 \mathrm{~N}$ units as compared to the 10-N-unit contours of the $N_{s}$ charts. The individual deviations of the values obtained from the contour maps with the actual 8-year means are listed in table 4.2. By comparing the root mean square ( $\mathrm{rms}$ ) deviations of $10.7 \mathrm{~N}$ units in winter and $13.0 \mathrm{~N}$ units in summer obtained by estimating $N_{s}$ from the $N_{s}$ contours with the $2.7-N$-unit rms of estimating $N_{s}$ from $N_{0}$ contours, one concludes that it is at least 4 times more accurate to estimate $N_{s}$ from $N_{0}$ contours than from those of $N_{s}$. An inspection of the individual deviations in table 4.2 indicates that the $N_{0}$ contour method is particularly efficient at elevations in excess of $1,200 \mathrm{~m}$ or where the terrain is changing 


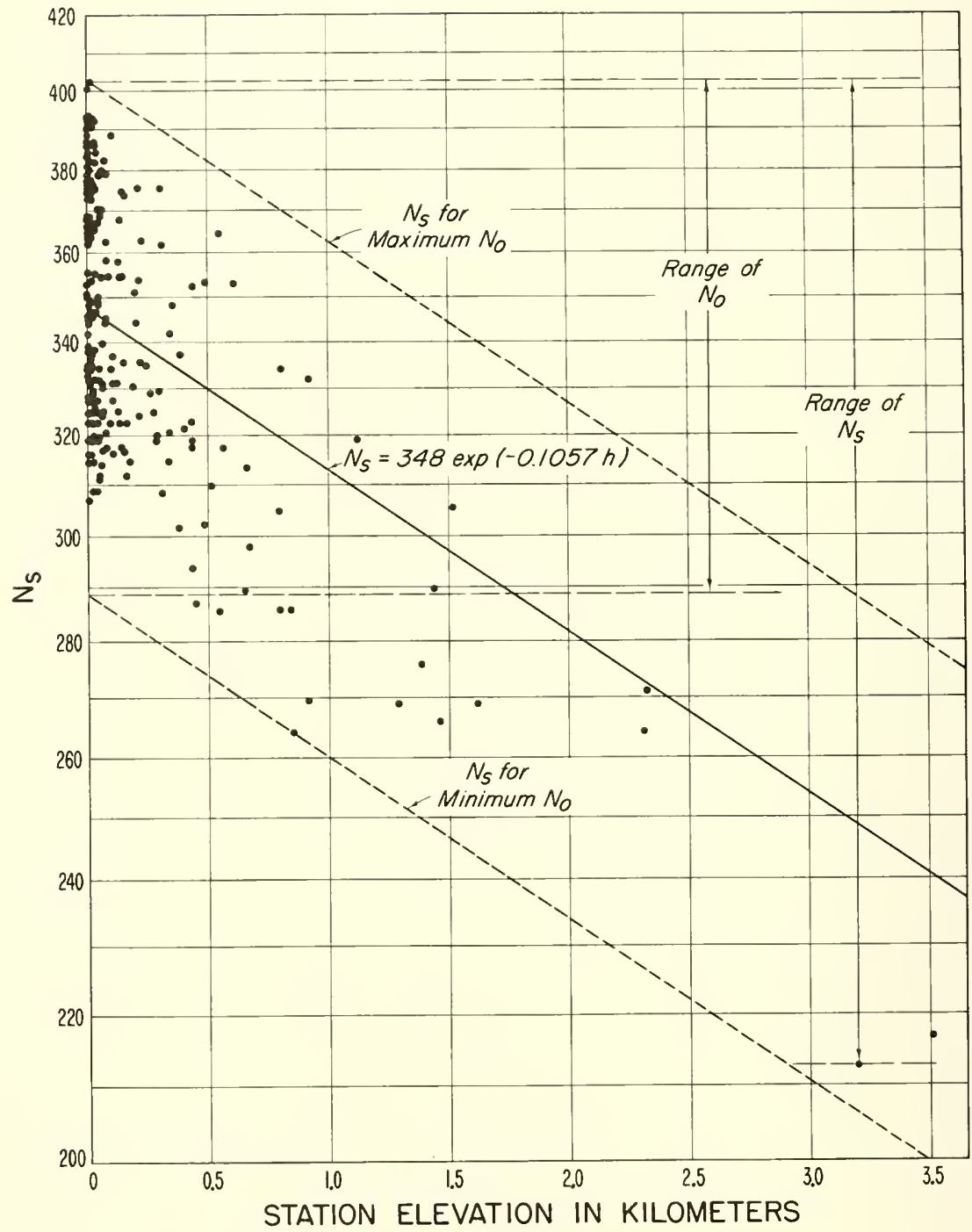

Figure 4.5. Worldwide values of $\mathrm{N}_{\mathrm{s}}$ versus height for August. 


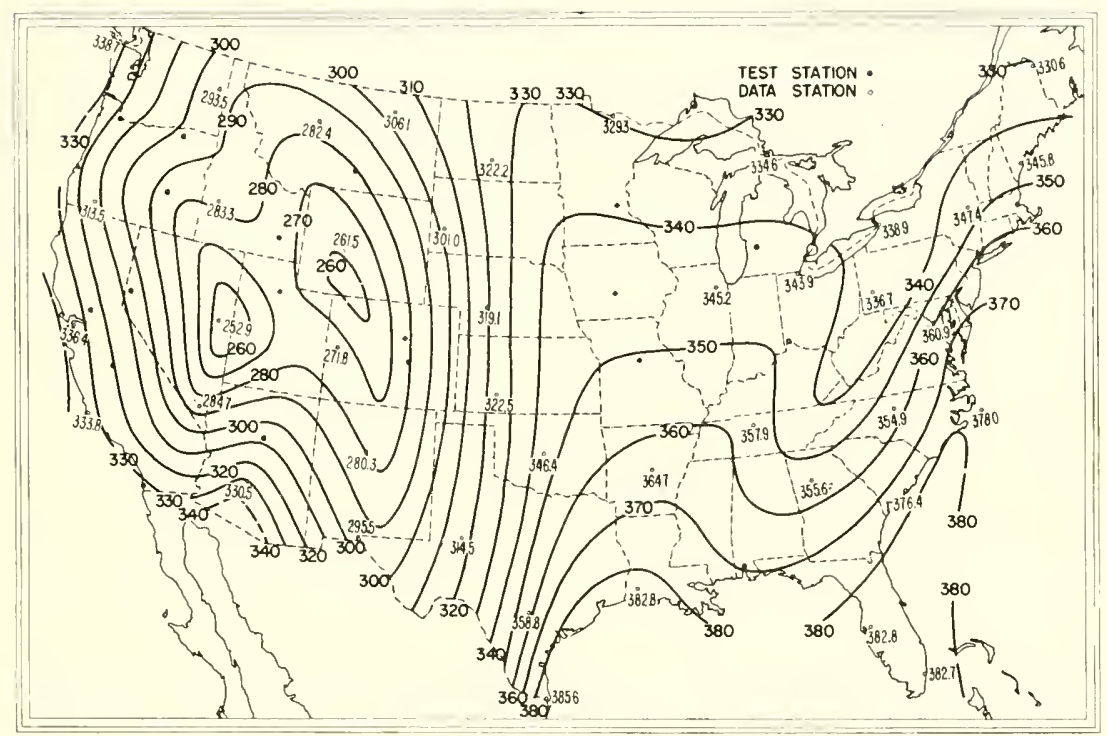

Figure 4.6. Test chart of mean $\mathrm{N}_{3}$ August 0200 local time.

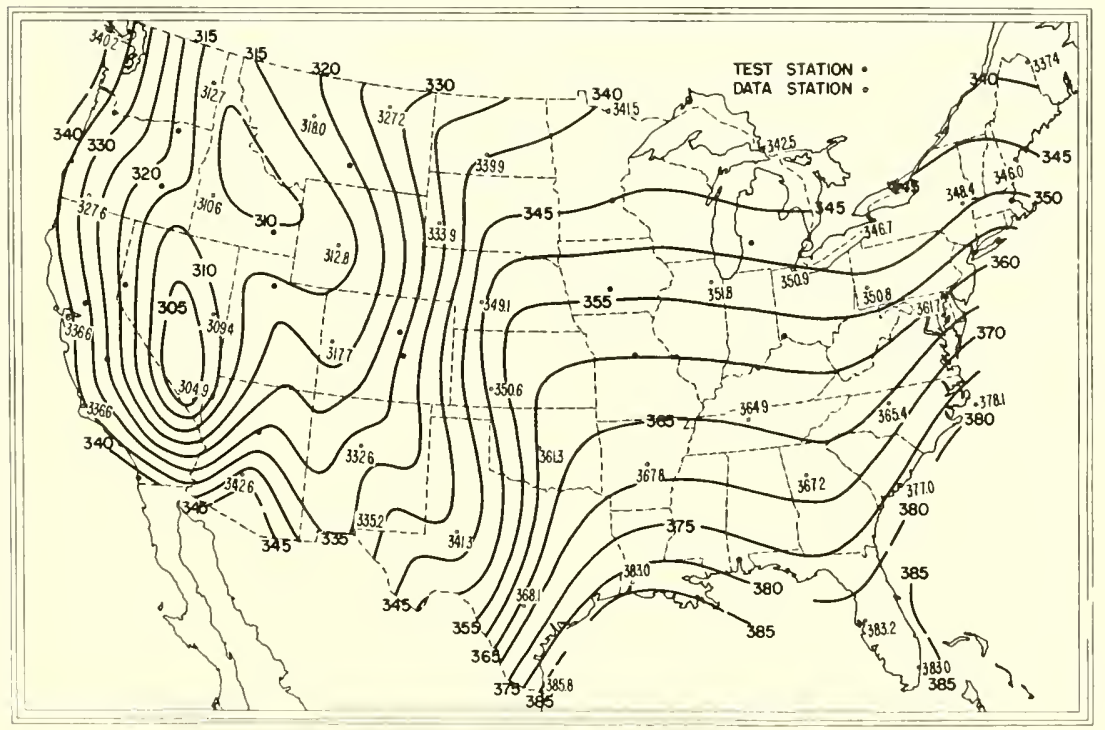

Figure 4.7. Test charl of mean $\mathrm{N}_{0}$ August 0200 local time. 
rapidly with respect to horizontal distance. As a further practical consequence, one notes the remarkable similarity between the $N_{0}$ contours of figures 4.4 and 4.7 , even though the latter contours were derived from only two-thirds of the original data. This indicates that any desired level of accuracy may be maintained with fewer stations (and less expense) by the use of $N_{0}$.

TABLE 4.2. Deviations of estimated 8-year means of $\mathrm{N}_{s}$ (calculated from contour charts) from actual 8-year means selected at random from 20 Weather Bureau stations

\begin{tabular}{|c|c|c|c|c|c|c|c|}
\hline \multirow{3}{*}{ Test station } & \multirow{3}{*}{ Height } & \multicolumn{3}{|c|}{ February 1400} & \multicolumn{3}{|c|}{ August 0200} \\
\hline & & \multirow{2}{*}{$\begin{array}{l}\text { Actual } \\
8-y r \\
\text { mean } \\
\text { value } \\
\text { of } N_{s}\end{array}$} & \multicolumn{2}{|c|}{ Deviation* } & \multirow{2}{*}{$\begin{array}{l}\text { Aetual } \\
8-y r \\
\text { mean } \\
\text { value } \\
\text { of } N_{s}\end{array}$} & \multicolumn{2}{|c|}{ Deviation } \\
\hline & & & $\begin{array}{c}N_{s} \\
\operatorname{map}\end{array}$ & $\begin{array}{c}N_{0} \\
\text { map }\end{array}$ & & ${ }_{\operatorname{map}}^{N_{s}}$ & $\begin{array}{c}N_{0} \\
\operatorname{map}\end{array}$ \\
\hline $\begin{array}{l}\text { Sacramento, Calif. } \\
\text { Portland, Oreg. } \\
\text { San Diego, Calif } \\
\text { Mobile, Ala } \\
\text { Fresno, Calif. }\end{array}$ & $\begin{array}{r}\text { meters } \\
7 \\
8 \\
11 \\
66 \\
86\end{array}$ & $\begin{array}{r}N \text { units } \\
315.6 \\
316.2 \\
314.2 \\
326.6 \\
310.6\end{array}$ & $\begin{array}{r}N \text { units } \\
7.6 \\
4.2 \\
-5.8 \\
6.6 \\
9.6\end{array}$ & $\begin{array}{r}N \text { units } \\
0.8 \\
-.5 \\
-2.4 \\
4.8 \\
3.4\end{array}$ & $\begin{array}{r}N \text { units } \\
329.6 \\
337.7 \\
348.1 \\
376.0 \\
326.2\end{array}$ & $\begin{array}{r}N \text { units } \\
1.0 \\
19.7 \\
16.1 \\
6.0 \\
5.2\end{array}$ & $\begin{array}{r}N u n i t s \\
1.8 \\
6.0 \\
3.5 \\
0.6 \\
4.2\end{array}$ \\
\hline $\begin{array}{l}\text { Boston, Mass, } \\
\text { Grand Rapids, Mieh } \\
\text { Columbia, Mo } \\
\text { Minneapolis, Minn } \\
\text { Cineinnati, Ohio }\end{array}$ & $\begin{array}{r}89 \\
210 \\
239 \\
255 \\
271\end{array}$ & $\begin{array}{l}308.6 \\
304.4 \\
300.8 \\
301.1 \\
302.5\end{array}$ & $\begin{array}{r}-6.4 \\
0.4 \\
-.2 \\
-.1 \\
-.5\end{array}$ & $\begin{array}{r}-0.5 \\
-5.9 \\
2.4 \\
0.6 \\
.7\end{array}$ & $\begin{array}{l}347.5 \\
340.5 \\
348.7 \\
338.5 \\
344.1\end{array}$ & $\begin{array}{l}-7.5 \\
-1.5 \\
-2.3 \\
-0.5 \\
-2.9\end{array}$ & $\begin{array}{r}-0.4 \\
-2.1 \\
2.7 \\
-2.8\end{array}$ \\
\hline $\begin{array}{l}\text { Des Moines, Iowa... } \\
\text { Pendleton, Oreg } \\
\text { Billings, Mont } \\
\text { Burns, Oreg, } \\
\text { Salt Lake City, Utah...... }\end{array}$ & $\begin{array}{r}294 \\
455 \\
1,088 \\
1,262 \\
1,288\end{array}$ & $\begin{array}{l}300.9 \\
295.9 \\
269.3 \\
268.1 \\
266.3\end{array}$ & $\begin{array}{r}3.9 \\
1.9 \\
-2.3 \\
-23.9 \\
1.3\end{array}$ & $\begin{array}{r}2.3 \\
0.4 \\
-3.2 \\
-0.8\end{array}$ & $\begin{array}{l}343.1 \\
300.9 \\
285.6 \\
271.3 \\
279.5\end{array}$ & $\begin{array}{r}-1.9 \\
2.9 \\
5.6 \\
-15.7 \\
8.5\end{array}$ & $\begin{array}{r}-0.1 \\
-3.1 \\
1.2 \\
-4.4 \\
4.4\end{array}$ \\
\hline $\begin{array}{l}\text { Reno, Nev } \\
\text { Poeatello, Idaho } \\
\text { Denver, Colo } \\
\text { Colorado Springs, Colo..... } \\
\text { Flagstaff, Ariz... }\end{array}$ & $\begin{array}{l}1,340 \\
1,355 \\
1,625 \\
1,882 \\
2,131\end{array}$ & $\begin{array}{l}259.6 \\
264.7 \\
244.9 \\
237.1 \\
237.8\end{array}$ & $\begin{array}{r}-20.4 \\
-2.3 \\
-8.1 \\
-15.9 \\
-26.2\end{array}$ & $\begin{array}{r}-6.8 \\
0.4 \\
.7 \\
-.6 \\
2.3\end{array}$ & $\begin{array}{l}277.6 \\
269.7 \\
276.6 \\
272.4 \\
261.4\end{array}$ & $\begin{array}{r}-29.4 \\
-3.3 \\
-1.4 \\
-6.6 \\
-36.6\end{array}$ & $\begin{array}{r}1.6 \\
0.0 \\
.3 \\
1.1 \\
-2.2\end{array}$ \\
\hline Root mean square deviation... &.- & 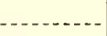 & 10.7 & 2.7 & -.... & 13.0 & 2.7 \\
\hline
\end{tabular}

${ }^{*}$ Deviation equals the aetual long-term mean minus the value obtained from map eontours.

\subsubsection{Worldwide Values of $N_{\mathrm{o}}$}

Mean values of $N_{0}$ were calculated at each of the 306 selected stations and charts were prepared for each month of the year. The charts for February and August are given on figures 4.8 and 4.9. It is seen that the values of $N_{0}$ for sea-level stations vary from 390 in the maritime tropical areas to $290 \mathrm{in}$ the deserts and high plateaus. The interiors of continents and mountain chains in middle latitudes are reflected by low values as compared to those of coastal areas. Further, such pronounced climatic details as the Indian monsoon and the effects of coastal mountain ranges blocking prevailing winds and producing rain shadows are indicated by these $N_{0}$ contours. Regional climatological data of $N_{0}$ for the United States are given in chapter 9. 


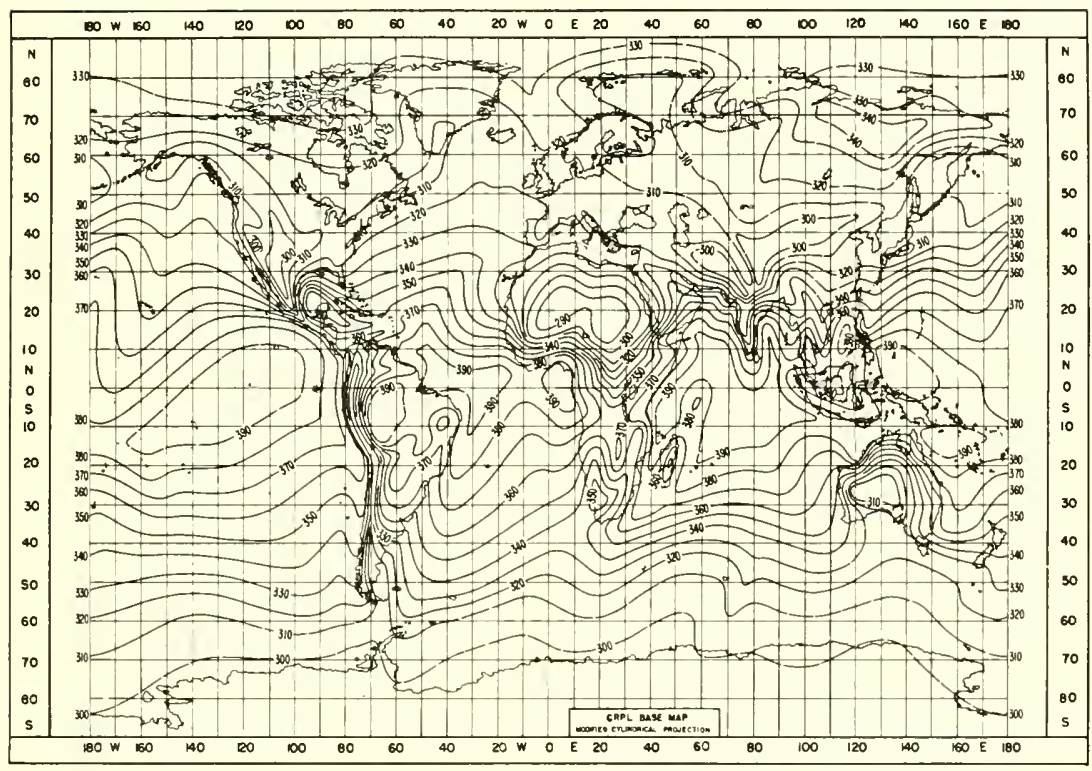

Figure 4.8. Worldwide values of mean $\mathrm{N}_{0}$ for February.

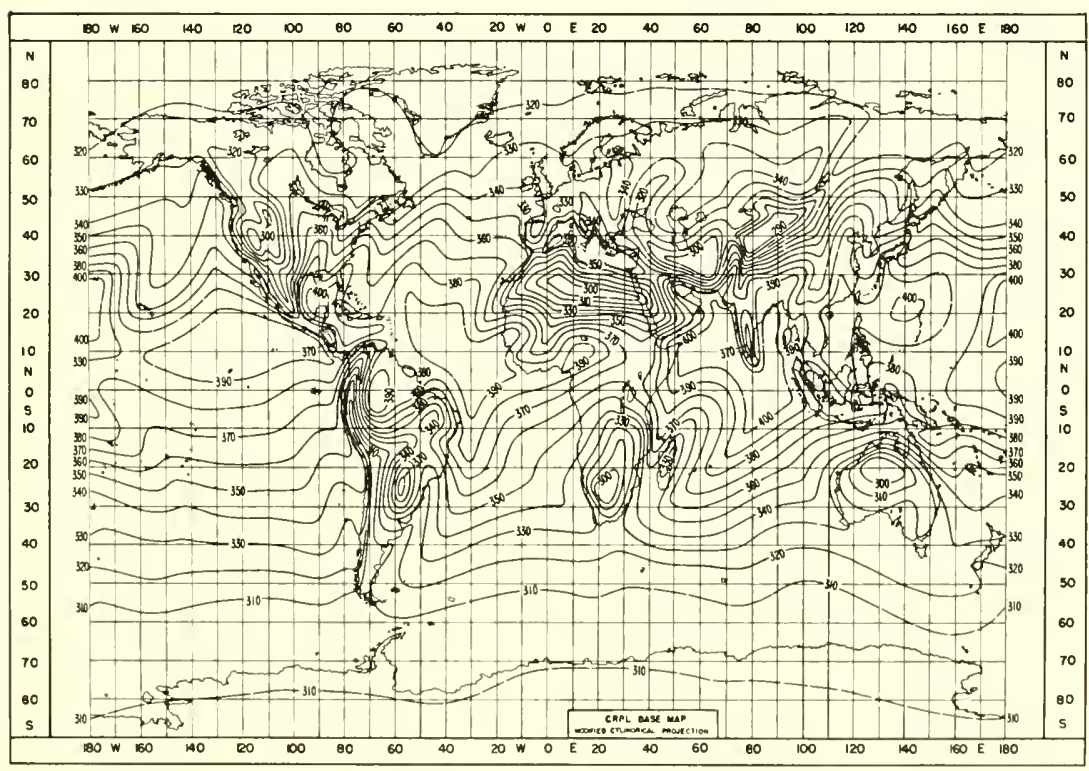

Figure 4.9. Worldwide values of mean $\mathrm{N}_{0}$ for August. 


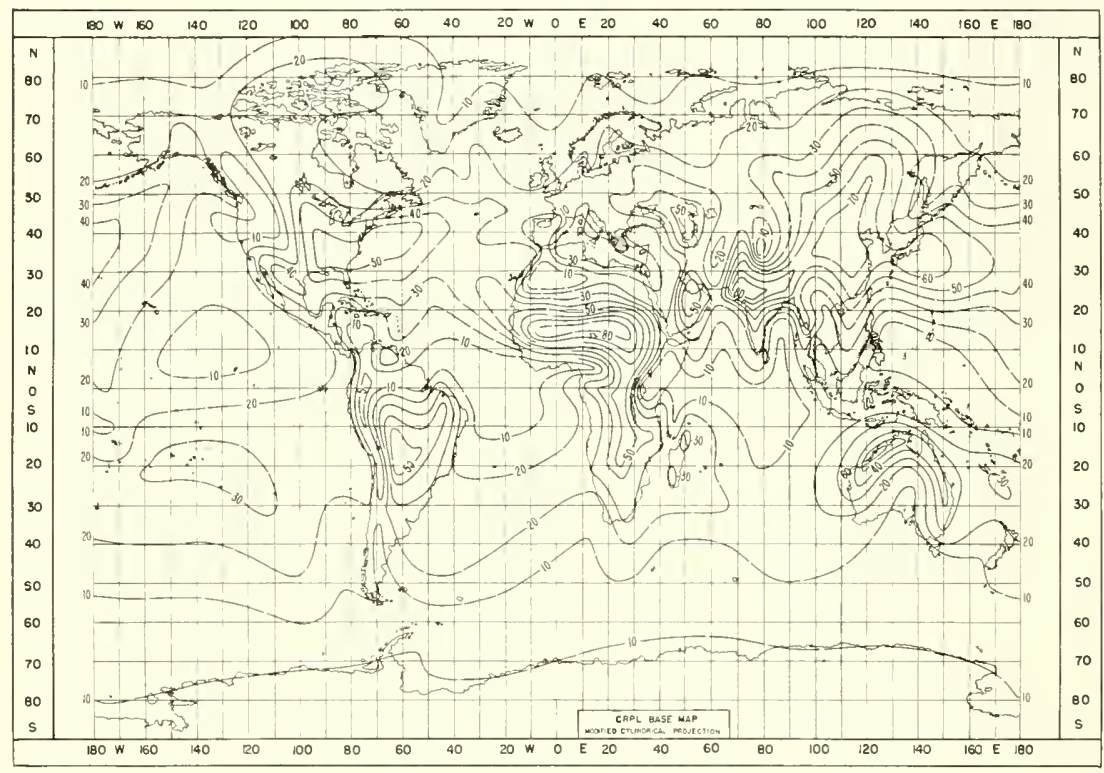

Figure 4.10. Annual range of monthly mean $\mathrm{N}_{\mathrm{B}}$.

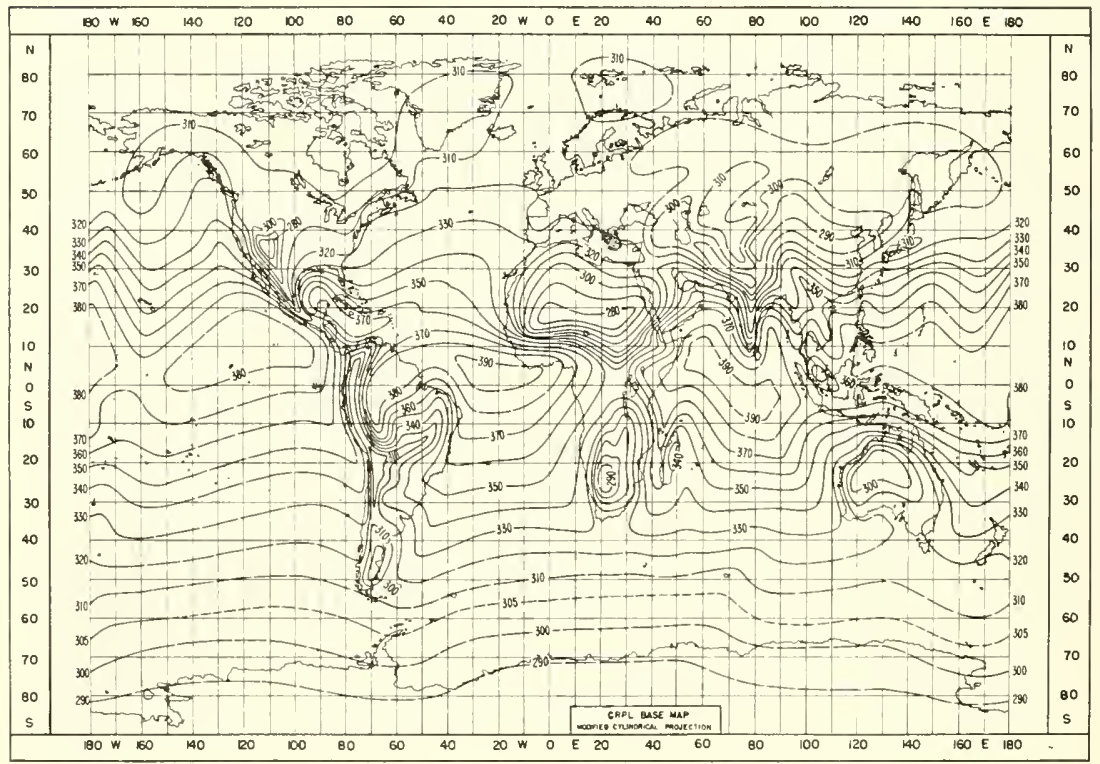

Figure 4.11. Minimum monthly mean value of $\mathrm{N}_{0}$. 


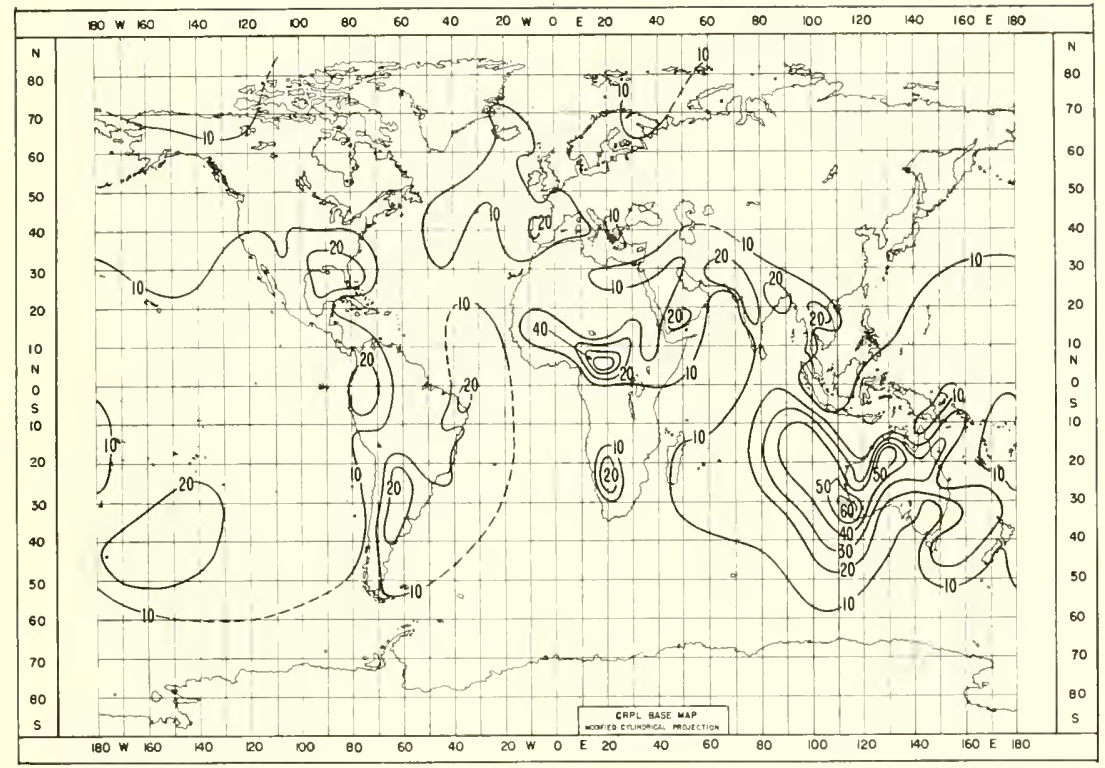

Figure 4.12. Year-to-year range of monthly mean $\mathrm{N}_{\mathrm{a}}$ for February.

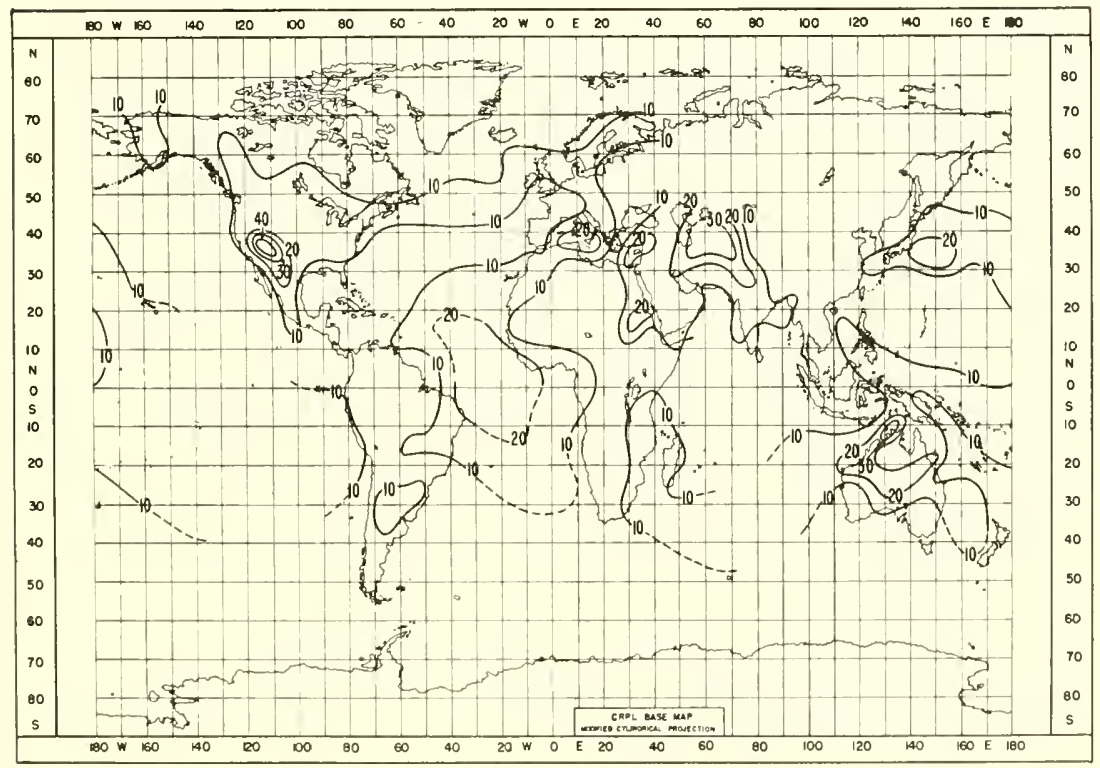

Figure 4.13. Year-to-year range of monthly mean $\mathrm{N}_{\mathrm{B}}$ for August. 
The annual variation of $N_{s}$ is indicated on figure 4.10 by contours of the difference between the maximum and minimum monthly means observed throughout the year. It is quite remarkable how clearly climatic differences are evidenced by the yearly range of $N_{s}$. The prevailing transport of moist maritime air inland over the west coasts of North America and Europe is indicated by relatively small annual ranges (20 to $30 \mathrm{~N}$ units), while, for example, the east coast of the United States with a range of 40 to $50 \mathrm{~N}$ units or more reflects the invasion of that area from time to time by such diverse air masses as arctic continental and tropical maritime. The largest annual ranges of $N_{s}(90 \mathrm{~N}$ units) are observed in the Sudan of Africa and in connection with the Indian monsoon.

An additional $N_{0}$ map (fig. 4.11) was prepared from the minimum monthly mean value of $N_{s}$ observed throughout the year to supplement the range map in order that an estimation might be made of both the minimum and maximum monthly mean $N_{s}$ expected during the year.

A measure of the variability of the February and August mean values of $N_{s}$ is given by monthly range maps (fig. 4.12 and 4.13) determined from monthly averages from 5 years of data. Ranges are given by the maximum difference of the five individual monthly mean values. In contouring the two variability maps only those terrestrial regions having reasonable data coverage are included. Dashed contours are shown for areas of sparse or unreliable data. The general picture of the worldwide distribution of $N_{s}$ variability is that of a number of continentally located cells of moderate range accompanied by somewhat random small-scale variation over ocean areas. Regions of large range, from 40 to as much as $70 \mathrm{~N}$ units, are present, however, in Australia and on islands of the adjoining oceans, on the African equatorial plateau near the Cameroons, and in the Great Basin of the southwestern United States. Common to all these areas of large year-to-year variability, at least during the summer season, are high mean temperatures ranging from about 25 to $30^{\circ} \mathrm{C}$, the variability being due to relatively small variations of humidity. It is felt that when a more dense network of stations is available for a longer period of record, say 10 years, areas of high monthly variability are likely to be more extensive in tropical and desert areas than indicated on our present maps.

\subsubsection{Climatic Classification by $N_{\mathrm{s}}$}

The annual cycle of $N_{s}$ at each station was examined for the purpose of deriving similarities of climatic pattern. As one form of climatic classification, the annual mean value of $N_{s}$ at each station was plotted versus the annual range at the station. When this was done, several distinct groupings of data seemed evident. These groupings, described 
in table 4.3, are intended to give a general idea of the geographic and elimatic character of the majority of the stations found within given values of range and yearly mean of $N_{s}$.

TABLE 4.3. Characteristics of climatic types

\begin{tabular}{|c|c|c|c|c|}
\hline Type & Location & $\begin{array}{l}\text { Annual } \\
\text { mean } N\end{array}$ & $\begin{array}{l}\text { Annual } \\
\text { range of } N_{s}\end{array}$ & Characteristics \\
\hline $\begin{array}{l}\text { I. Midlatitude- } \\
\text { coastal. }\end{array}$ & $\begin{array}{l}\text { Near the sea or in lowlands on } \\
\text { lakes and rivers, in latitude } \\
\text { belts between } 20^{\circ} \text { and } 50^{\circ} \text {. }\end{array}$ & $\begin{array}{l}N \text { units } \\
300 \text { to } 350\end{array}$ & $\begin{array}{l}N \text { units } \\
30 \text { to } 60\end{array}$ & $\begin{array}{l}\text { Generally subtropical } \\
\text { with marine or modi- } \\
\text { fied marine climate. }\end{array}$ \\
\hline $\begin{array}{c}\text { II. Subtropical- } \\
\text { Savanna. }\end{array}$ & $\begin{array}{l}\text { Lowland stations between } 30^{\circ} \mathrm{N} \\
\text { and } 25^{\circ} \mathrm{S} \text {, rarely far from the } \\
\text { ocean. }\end{array}$ & 350 to 400 & 30 to 60 & $\begin{array}{l}\text { Definite rainy and dry } \\
\text { seasons, typical of } \\
\text { Savanna climate. }\end{array}$ \\
\hline $\begin{array}{l}\text { III. Monsoon- } \\
\text { Sudan. }\end{array}$ & $\begin{array}{l}\text { Monsoon-generally between } \\
20^{\circ} \text { and } 40^{\circ} \mathrm{N} \text {, Sudan- across } \\
\text { central Africa from } 10^{\circ} \text { to } 20^{\circ} \mathrm{N} \text {. }\end{array}$ & 280 to 400 & 60 to 100 & $\begin{array}{l}\text { Seasonal extremes of } \\
\text { rainfall and tem. } \\
\text { perature. }\end{array}$ \\
\hline $\begin{array}{l}\text { IV. Semiarid- } \\
\text { mountain. }\end{array}$ & $\begin{array}{l}\text { In desert and high steppe regions } \\
\text { as well as mountainous re- } \\
\text { gions above } 3,000 \mathrm{ft} \text {. }\end{array}$ & 240 to 300 & 0 to 60 & Year-round dry climate \\
\hline $\begin{array}{l}\text { V. Continental- } \\
\text { Polar. }\end{array}$ & $\begin{array}{l}\text { In middle latitudes and polar } \\
\text { regions. (Mediterranean cli- } \\
\text { mates are included because of } \\
\text { the low range resulting from } \\
\text { characteristic dry summers.) }\end{array}$ & 300 to 340 & 0 to 30 & $\begin{array}{l}\text { Moderate or low annual } \\
\text { mean temperatures. }\end{array}$ \\
\hline $\begin{array}{l}\text { VI. Isothermal- } \\
\text { equatorial. }\end{array}$ & $\begin{array}{l}\text { Tropical stations at low eleva- } \\
\text { tions between } 20^{\circ} \mathrm{N} \text { and } 20^{\circ} \mathrm{S} \text {, } \\
\text { almost exclusively along sea- } \\
\text { coasts or on islands. }\end{array}$ & 340 to 400 & 0 to 30 & $\begin{array}{l}\text { Monotonous rainy } \\
\text { climates. }\end{array}$ \\
\hline
\end{tabular}

For a given classification of refractive-index climate, diverse meteorological climates and geographical regions may be represented. Note, for example, that type $\mathrm{V}$ of table 4.3 includes stations from Mediterranean and marine as well as polar climates. Mediterranean stations in this eategory fail to attain a high range because of the characteristic dryness of the subtropical high-pressure pattern that is generally found in this area during the summer montlis. Polar and marine climates in this group maintain a low rauge due to suppressed humidity effects as a result of low to moderate year-around average temperatures.

Annual trends of $N_{s}$ for stations typical of each climatic division are shown by figure 4.14 .

Yet another facet of the climate is the year-to-year variation of the monthly mean value of $N_{s}$. Five consecutive years of monthly means were prepared for each of the six typical stations whose annual cycles are shown in figure 4.14. Then, for each month, the absolute value of the difference between consecutive years was obtained. These values were then averaged for all months and are listed in the second column of table 4.4 . 
TYPE I

MID-LATITUDE COASTAL

WASHINGTON, D.C.

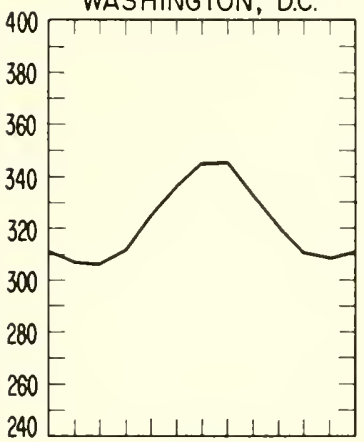

TYPE IV

SEMI-ARID MOUNTAIN

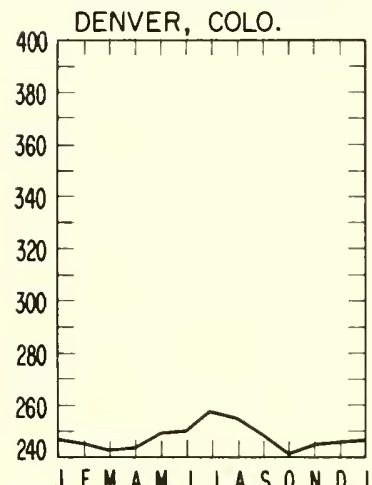

TYPE II SUBTROPICAL - SAVANNA

MIAMI, FLA.

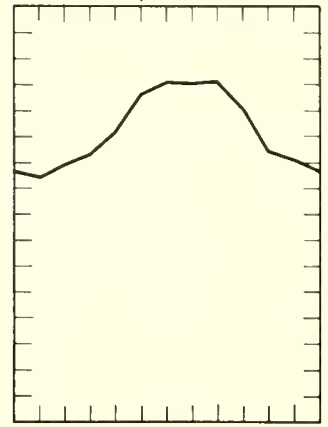

TYPE $\mathbb{Z}$

CONTINENTAL - POLAR OSLO, NORWAY

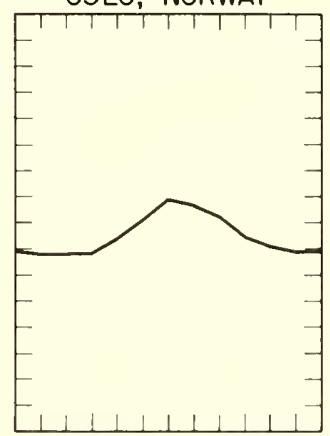

JFMAMJJASONOJ
TYPE III

MONSOON-SUDAN

JODPHUR, INDIA

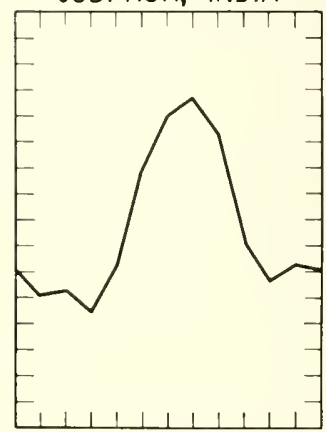

TYPE ZII

ISOTHERMAL EQUATORIAL CANTON ISL., S. PACIFIC O.

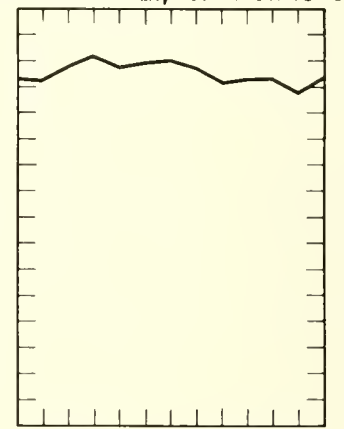

JFMAMJJASONOJ

Figure 4.14. Representative annual cycles of $\mathrm{N}_{\mathrm{s}}$ for the major climatic types.

Another measure of the variation of monthly mean values of $N_{s}$ is obtained by differencing the maximum and minimum values occurring for a given month during the 5-year period. These differences are also given in table 4.9 for the months of February, May, August, and November.

TABLE 4.4. Year-to-year differences of monthly mean $\mathrm{N}_{8}$

\begin{tabular}{|c|c|c|c|c|c|}
\hline \multirow{2}{*}{$\underset{\text { type }}{\text { Climatic }^{*}}$} & \multirow{2}{*}{$\begin{array}{l}\text { Differences between monthly means } \\
\text { in successive years for the same } \\
\text { month, averaged for all seasons over } \\
\text { a } 5 \text {-yr period }\end{array}$} & \multicolumn{4}{|c|}{$\begin{array}{l}\text { Maximum differences between monthly } \\
\text { means over a } 5 \text {-yr period }\end{array}$} \\
\hline & & Feb. & May & Aug. & Nov. \\
\hline $\begin{array}{l}\text { II } \\
\text { IIII } \\
\text { IV } \\
\text { V } \\
\text { VII } \\
\text { VI }\end{array}$ & $\begin{array}{l}5.7 \\
5.4 \\
8.9 \\
5.4 \\
4.7 \\
7.1\end{array}$ & $\begin{array}{r}6.0 \\
8.5 \\
16.5 \\
10.5 \\
5.5 \\
9.5\end{array}$ & $\begin{array}{r}16.5 \\
6.5 \\
14.5 \\
11.5 \\
11.5 \\
25.5\end{array}$ & $\begin{array}{r}17.0 \\
8.0 \\
20.0 \\
13.5 \\
10.0 \\
8.5\end{array}$ & $\begin{array}{r}7.0 \\
11.0 \\
6.5 \\
6.5 \\
5.0 \\
8.5\end{array}$ \\
\hline
\end{tabular}

*Climatic types are the same as those in table 4.3 . 


\subsubsection{Applications}

The communications engineer usually has available a small amount of measured field-strength data from limited tests of a particular system. He must then estimate the expected signal level or practical range of that system, or other systems, for other times of the year, other years, and in other areas. The variation of signal level from month to month and climate to climate can be explained, in part, by its observed correlation with $N_{s}$.

Pickard and Stetson $[10,11]$ were among the first to note the correlation of $N_{s}$ and received field strengths. The correlation of $N_{s}$ and field strength over a particular path has been studied quantitatively $[12,13]$ and found to be highest (correlation coefficients of 0.8 to 0.95 ) when the variables are averaged over periods of a week to a month. This latter study has shown that the regression coefficient (decibel change in field strength per unit change in $N_{s}$ ) varies diurnally from $0.14 \mathrm{~dB}$ in the afternoon hours to $0.24 \mathrm{~dB}$ per unit change of $N_{s}$ in the early morning hours. This correlation is so sufficiently consistent that Gray [14] and Norton [15] have utilized it in their recent prediction methods of transmission loss in a band from 100 to $50,000 \mathrm{Mc} / \mathrm{s}$. In addition, the coefficient $0.2 \mathrm{~dB}$ per unit change in $N_{s}$ has been tentatively adopted by CCIR Study Group $\mathrm{V}$ in their revision of the $30-$ to $300-\mathrm{Mc} / \mathrm{s}$ tropospheric-wave propagation curves to account for the geographic and seasonal variations of field strengths. The estimates of field strength variations attributed to $N_{s}$ given in table 4.5 are based upon the CCIR coefficient.

If one assumes, for comparison only, that the worldwide average value of $N_{s}$ is 330 and that one is able to estimate the field strength level of a particular communications system at a given distance and for $N_{s}=330$, then the above correlations would indicate that the climatic variations of fields given in table 4.5 might be expected.

TABLE 4.5. Climatic variation of hypothetical communications system relative to predicted value for $\mathrm{N}_{\mathrm{s}}=330$, assuming a $0.2-d B$ variation per unit change in $\mathrm{N}_{\mathrm{s}}$

\begin{tabular}{|c|c|c|}
\hline Climatie type* & $\begin{array}{c}\text { Expeeted yearly } \\
\text { mean field strength } \\
\text { level relative to } \\
N_{s}=330\end{array}$ & $\begin{array}{l}\text { Expeeted annual } \\
\text { range on the above } \\
\text { assumption }\end{array}$ \\
\hline 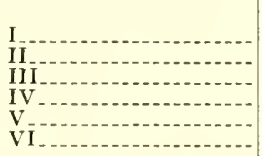 & $\begin{array}{l}d B \\
-6 \text { to }+4 \\
+4 \text { to }+14 \\
-10 \text { to }+14 \\
-18 \text { to }-6 \\
-6 \text { to }+2 \\
+2 \text { to }+14\end{array}$ & $\begin{array}{l}d B \\
6 \text { to } 12 \\
6 \text { to } 12 \\
12 \text { to } 20 \\
0 \text { to } 12 \\
0 \text { to } 6 \\
0 \text { to } 6\end{array}$ \\
\hline
\end{tabular}


The data of table 4.5 indicate, for example, that identically equipped tropospheric communications systems could display as much as a $32-\mathrm{dB}$ difference in mean signal-strength level due to the climatic difference of say, Denver, Colo., and the tropics. Further, one might expect the monthly mean field strength of this hypothetical system to vary throughout the year from less than $12 \mathrm{~dB}$ in the high plains near Denver to as much as $20 \mathrm{~dB}$ in the African Sudan.

Under this same assumption, figures 4.10 and 4.11 allow the communications engineer to estimate the expected maximum and minimum monthly mean field strength expected throughout the year.

The year-to-year variations of the monthly mean $N_{s}$ listed in table 4.4 indicate that the monthly mean of field strength for a particular month may differ in successive years by as little as $1.0 \mathrm{~dB}$ for clmatic category $\mathrm{V}$ in November or as much as $5.1 \mathrm{~dB}$ for category VI in May.

Another application of these worldwide charts is to aid in estimating the refraction of radio waves. The most convenient method of accounting for the effects of atmospheric refraction is by means of the effectiveearth's-radius concept (see chapter 3) of Schelleng, Burrows, and Ferrell [16]. The effective earth's radius, $a_{e}$, is determined from

$$
a_{e}=\left(\frac{1}{1+\frac{a}{n} \frac{d n}{d h}}\right) a,
$$

where $a$ is the true radius of the earth, $n$ is the refractive index, and $d n / d h$ is the initial $n$ gradient with respect to height. A great simplification of propagation calculations is accomplished by assuming that $d n / d h$ is a constant, thus allowing radio rays to be drawn as straight rays over a fictitious earth of radius $a_{e}$ rather than curved rays over the true earth of radius $a$. This simplification allows, for example, the distance to the radio horizon, $d$, of a radio ray leaving an antenna of height, $h$, to be calculated from $d=\sqrt{2 a_{e} h}$.

One notes, however, that the determination of $a_{e}$ involves $d n / d h$ as well as $n$ and that our $N_{0}$ charts allow only an estimation of $n$. This disparity may be resolved by utilizing the observation that $N_{s}$ is highly correlated with the value of $N$ at $1 \mathrm{~km}$ above the surface. The difference between $N_{s}$ and $N$ at $1 \mathrm{~km}$ is denoted $\Delta N$. It has been noted [9] that the correlation coefficient between $\ln |\Delta N|$ and $N_{s}$ is 0.926 for 888 sets of data from 45 U.S. weather stations representing many diverse climates.

The regression equation

$$
-\bar{\Delta} \bar{N}=7.32 \exp \left\{0.005577 \bar{N}_{s}\right\}
$$

results when both variables are averaged over 6 to 8 years of record. 
Approximating $d n / d h$ in (4.7) by $\Delta N$, we may determine that the radio horizon distance of an antenna located $150 \mathrm{~m}$ above the earth would vary from $48 \mathrm{~km}$ when $N_{s}=200$ to $59 \mathrm{~km}$ when $N_{s}=400$. Yet another application of the $N_{s}$ charts is to the exponential models of the decrease of refractive index with height which have been proposed to date $[9,17]$. These models are completely specified by $N_{s}$ and may be used to account for seasonal and geographic variations of such refraction effects as radar range and elevation angle errors.

\subsubsection{Critical Appraisal of Results}

The world maps presented above were based upon data from 306 weather stations. This number of stations appears to be consistent with the scale of map used. The map scale is so small, however, that only large climatic differences can be expeeted to be discerned. For the elimate of any given area one should refer to detailed studies of $N$ such as those currently in preparation for the United States at the Central Radio Propagation Laboratory.

The accuracy of the present charts may be assessed from the charts of maximum range, $R$, of monthly means as given by figures 4.12 and 4.13. The standard deviation of the individual monthly means may be estimated from [18] $0.43 R$, where the coefficient 0.43 is appropriate for five individual observations. Since, in general, $R \leq 20 \mathrm{~N}$ units, then $0.43 R \leq 9 N$ units, although this standard deviation may be as large as 26 $N$ units for the month of February in Australia and $17 N$ units in the southwest of the United States during August, or in the African Sudan during February.

Further, the standard error of estimating a 5-year mean from five individual monthly values is determined from

$$
\frac{0.43 R}{\sqrt{n}}
$$

where $n$ for our case is 5 and thus the error of the 5-year mean would be $0.192 R$. Remembering that $R \leq 20 \mathrm{~N}$ units and assuming perfect skill in drawing the contours, one would expect the standard error of estimate to be less than $4 \mathrm{~N}$ units. This standard error can be as large as $12 \mathrm{~N}$ units in Australia where $R=60 \mathrm{~N}$ units.

The value of $N_{s}$ at each of the 20 test stations of table 4.2 was estimated from the $N_{0}$ contours with an rms error of $5 \mathrm{~N}$ units which is consistent with the standard error of estimate obtained from the range charts. In the large areas of sparse data, such as the oceans and Russia, this uncertainty rises to about $10 \mathrm{~N}$ units and thus the contours in these regions are dashed. 
At the time the present study was initiated it was felt that $N_{s}$ should be reduced to sea level by at least the dry term correction factor as in (4.6). The absence of published work on models of $N$ structure in the free atmosphere encouraged the decision to rest on prudence and adopt this dry term correction factor. Since that time several effective exponential models of the free atmosphere have been demonstrated $[17,9]$. In future work a smaller value of $H^{*}$ on the order of 7.0 , which corresponds to the $N$ decay in the free atmosphere, will be adopted. The adoption of any value of $H^{*}$ between 6.5 and $7.5 \mathrm{~km}$ would have reduced the range of $N_{0}$ values on figure 4.4 by no more than $2 N$ units. Since this reduction in range is more than an order of magnitude less than the reduction of (4.6) used to obtain $N_{0}$, it appears that the basic advantage of the method has been realized.

A map of $N_{0}$, such as that of figure 4.4 , which represents a large continental area, may easily be compared and merged with maps for ocean areas. This would be more difficult with $N_{s}$, since, for example, the strong gradient over California mainly represents the rapid altitude variations of the Sierra Nevada Mountains. It has also been demonstrated that $N_{0}$ is a better indicator of tropospheric storms and air masses than $N_{s}$ when considered on a synoptic or "daily weather map" basis [19].

Also (4.6) aids in comparing air properties as a function of altitude at the same position. The variations of $N_{0}$ will represent the local departures between this quantity and the value in a standard atmosphere and will show the perturbations in the structure of the atmosphere produced by fronts, air masses, and other synoptic features. Although any value of $H^{*}$ between 6.5 and $7.5 \mathrm{~km}$ will remove the gross altitude dependence of the refractive index, the choice of value of $H^{*}$ within this range could depend on the application. The synoptic application, which is discussed in detail in chapter 5 , would be best served by a scale height near $8.0 \mathrm{~km}$, whereas the objectives of the climatic chart usage would best be met by a scale height near $6.5 \mathrm{~km}$. This seeming paradox is easily understood in terms of the physical interpretation of the various scale heights. For example, $H^{*}=8.0 \mathrm{~km}$ is typical of a perfectly dry atmosphere and its use results in emphasis of humidity differences between air masses, whereas $H^{*}=6.5 \mathrm{~km}$ corresponds to a saturated atmosphere and minimizes moisture differences. Thus it would appear that eventually one might use a value of $H^{*}$ as indicated by the application at hand. As a practical matter, however, $H^{*}=7.0 \mathrm{~km}$ appears to reach a desirable compromise between the objectives of the two preceding examples.

\subsubsection{Conclusions}

With the above critical appraisal in mind, the salient conclusions of the present study are: 
(a) The radio refractive index varies in a systematic fashion with climate and different climates may be identified by the range and mean values of the refractive index.

(b) It is 4 or 5 times more accurate to estimate the station value of the index from charts of the reduced-to-sea-level index than from charts of the station value. This improved accuracy results from using a method that allows height dependence to be accurately taken into account.

(c) Identically equipped tropospherie communications systems might be expected to vary as much as $30 \mathrm{~dB}$ in monthly mean signal level in different climatic regions, and the annual range of monthly mean field strength could be as high as $20 \mathrm{~dB}$ in the Sudan of Africa and as low as 0 to $6 \mathrm{~dB}$ in the high plains of the western United States.

\subsection{On the Average Atmospheric Radio Refractive Index Structure Over North America}

\subsubsection{Introduction}

As has been already noted, the radio refractive index of the atmosphere combines three of the meteorological elements normally used to specify the state of the atmosphere on either a synoptic or a climatological basis. This fact has led to its being used as a synoptic tool $[19,20,21,22,23]$ and as a measure of elimatic eharacteristics [7, 24, 25, 26, 27].

The present treatment is concerned with the degree to which the average $N$ structure in the vertical direction reflects the gross differences in climate over the North American continent. Diurnal and seasonal range graphs of $N$ at the earth's surface also shed light upon elimatic characteristics.

\subsubsection{Meteorological Data and Reduction Techniques}

The basic data used in this study are the significant level data of the radiosonde observations from the 18 weather stations shown on figure 4.15 for the 5-year period 1952-57. These observations were converted by means of (1.20) to radio refractivity, $N$.

The significant level data were collected for the values of $N$ at the earth's surface and within height inerements centered upon $0.25,0.5,0.75$, $1.0,1.5,2.0,2.5,3.0$, and $3.5 \mathrm{~km}$. Each value of $N$ was referenced to the center of its height increment by use of the average atmosphere,

$$
N(h)=N_{0} \exp \{-h / 7.0\},
$$

which has been shown to be a reasonable model for the decrease of the refractive index with height for the United States. By use of this exponential model, the gross height dependence of $N$ within each height 


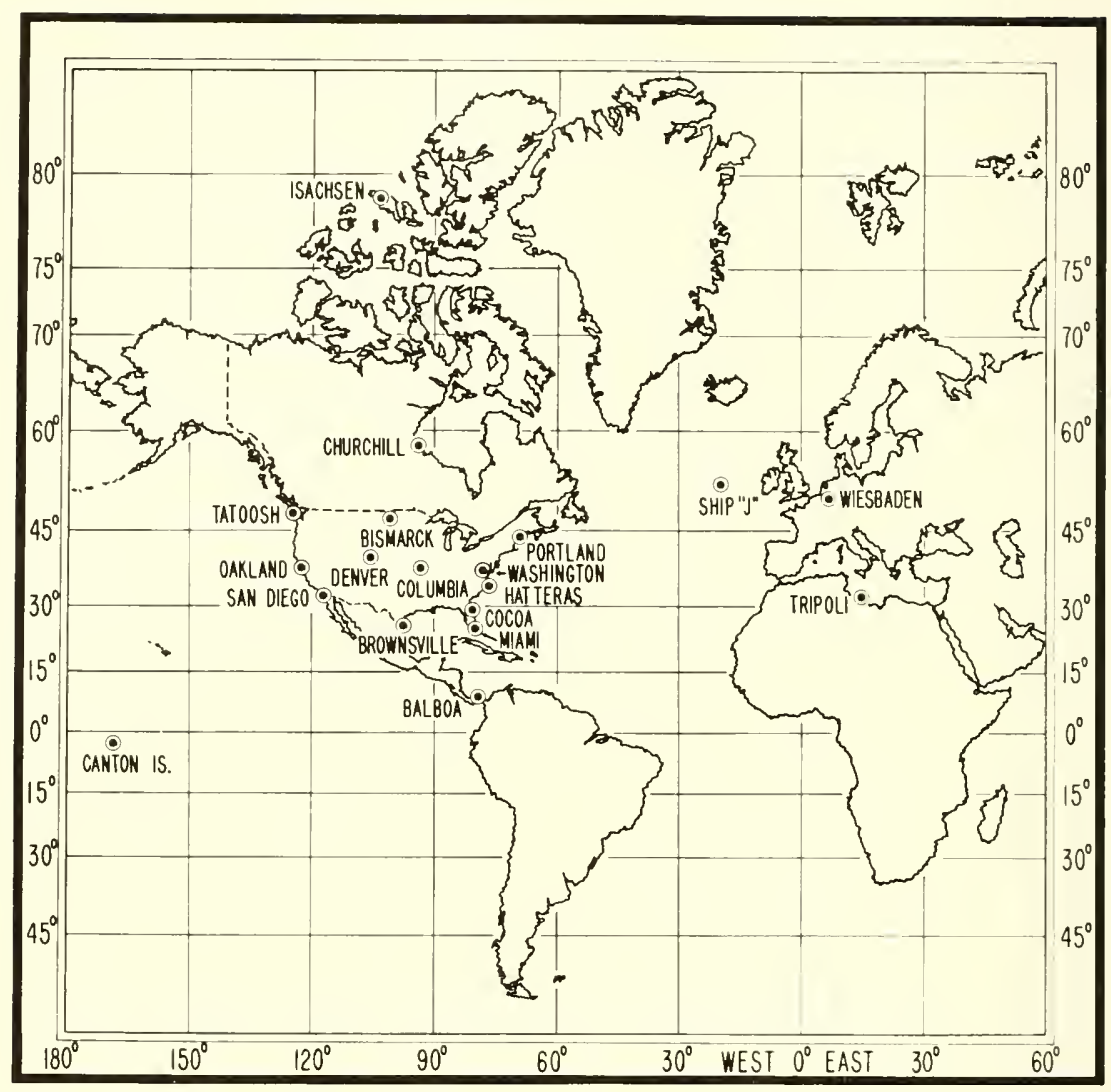

FIgure 4.15. Radiosonde stations used in this study.

increment was effectively removed and there remains a more reliable estimate of the mean $N$ at the center of the height increment. The final value of $\bar{N}_{0}$, for example, at $1 \mathrm{~km}$ over Washington, D.C., would be 300 with a standard deviation of $15.7 \mathrm{~N}$ units for 152 observations. The error in the mean is then determined to be 1.3 from

$$
s(\bar{N})=\frac{s(N)}{\sqrt{k}},
$$

with $k=152$. An examination of similar data for all stations and levels used shows that $s(\bar{N})$ is generally less than $1.0 \mathrm{~N}$ unit.

In addition, when considering atmospheric cross sections, the mean values of $N$ were referred to sea-level by means of (4.9) thus further emphasizing climatic differences [28]. 


\subsubsection{Average $N_{\mathrm{o}}$ Structure}

The first series of charts presented in this analysis are those showing the time variation of $\bar{N}_{0}$ both vertically above each station as well as at the standard ground-observing level. A slight dichotomy in data sample exists since the vertical data are monthly averages of radiosonde observations taken twice a day, corresponding in time to the hours 0300 and 1500 GMT, while the surface data are 8-year means for the even hours of the day local time [29]. This climatic variation at each station is represented by a two-part chart prepared for each station, the first part depicting seasonal changes in the mean value of $N_{0}$ throughout the first $3.5 \mathrm{~km}$ above the station and the second showing the seasonal and diurnal changes of $N_{0}$ at station elevation. These two presentations give a more complete climatic picture of a location than do the usual unidimensional annual cycle graphs.

Tatoosh Island (TTI), figure 4.16, off the coast of Washington State, illustrates typical features of a marine west coast climate. The seasonal profile changes indicate moderate gradients the year around with a small summer maximum in the $N_{0}$ gradients in keeping with the cool temperature regime and small diurnal temperature range of this maritimedominated climate. This consistency of weather conditions is further emphasized by the almost complete lack of diurnal pattern in the surface data throughout the year and small seasonal change in $N_{0}$ for any hour of the day.

The seasonal profile chart of Oakland (OAK), figure 4.17, indicates less maritime effect than at Tatoosh Island and shows clearly the influence of the summer subsidence inversion on the northeastern edge of the Pacific high-pressure area by both the low values of $N_{0}$ and increased gradients at about $1.0 \mathrm{~km}$. The surface data show the moderate seasonal and diural cycles for all months of the year expected in a Mediterranean climate.

The effects of the Pacific High are more pronounced further south along the California coast. The vertical profile data for San Diego (SAN), figure 4.18 , show clearly that during the winter months the strongest gradients of $N_{0}$ are near the surface as one would expect in a maritime dominated climate while, by contrast, in the summer the well-known Southern California elevated inversion produces strong gradients aloft. The subsiding air above the inversion is reflected by a characteristic increase of $N_{0}$ with height. The maritime effect is also evident at the surface in the low diurnal range of about $10 \mathrm{~N}$ units. It is significant that the presence of the elevated layer produces a smaller diurnal range in the summer than in the winter.

The semitropical nature of the humid periphery of the Gulf of Mexico is reflected by the data for Brownsville, Tex. (BRO), figure 4.19, which show a strong seasonal cycle and pronounced diurnal range in the summer 


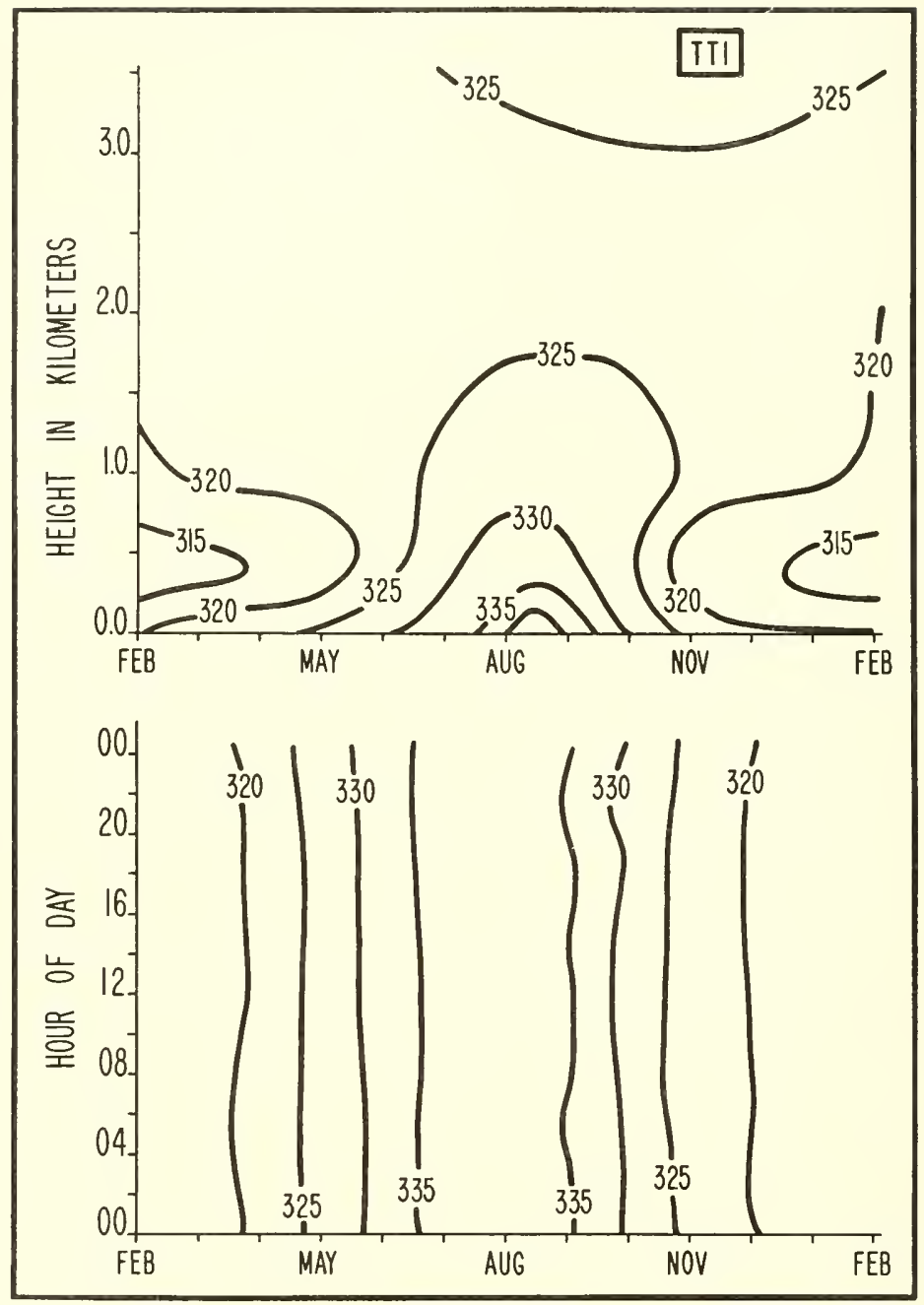

Figure 4.16. Diurnal, seasonal, and vertical variation of $\mathrm{N}_{0}$ for Tatoosh Island, Wash. (TTI).

due in large measure to the very high summer temperatures in this locale with resultant high water-vapor capacity. It is also quite striking that the general level of $N_{0}$ is 25 at $30 \mathrm{~N}$ units above that of the west coast.

The high $N_{0}$ values of the Gulf regions are also found at Miami (MIA), figure 4.20, although the moderating effect of continual onshore winds produces less pronounced vertical gradients and smaller diurnal ranges than Brownsville, particularly during the summer months. 


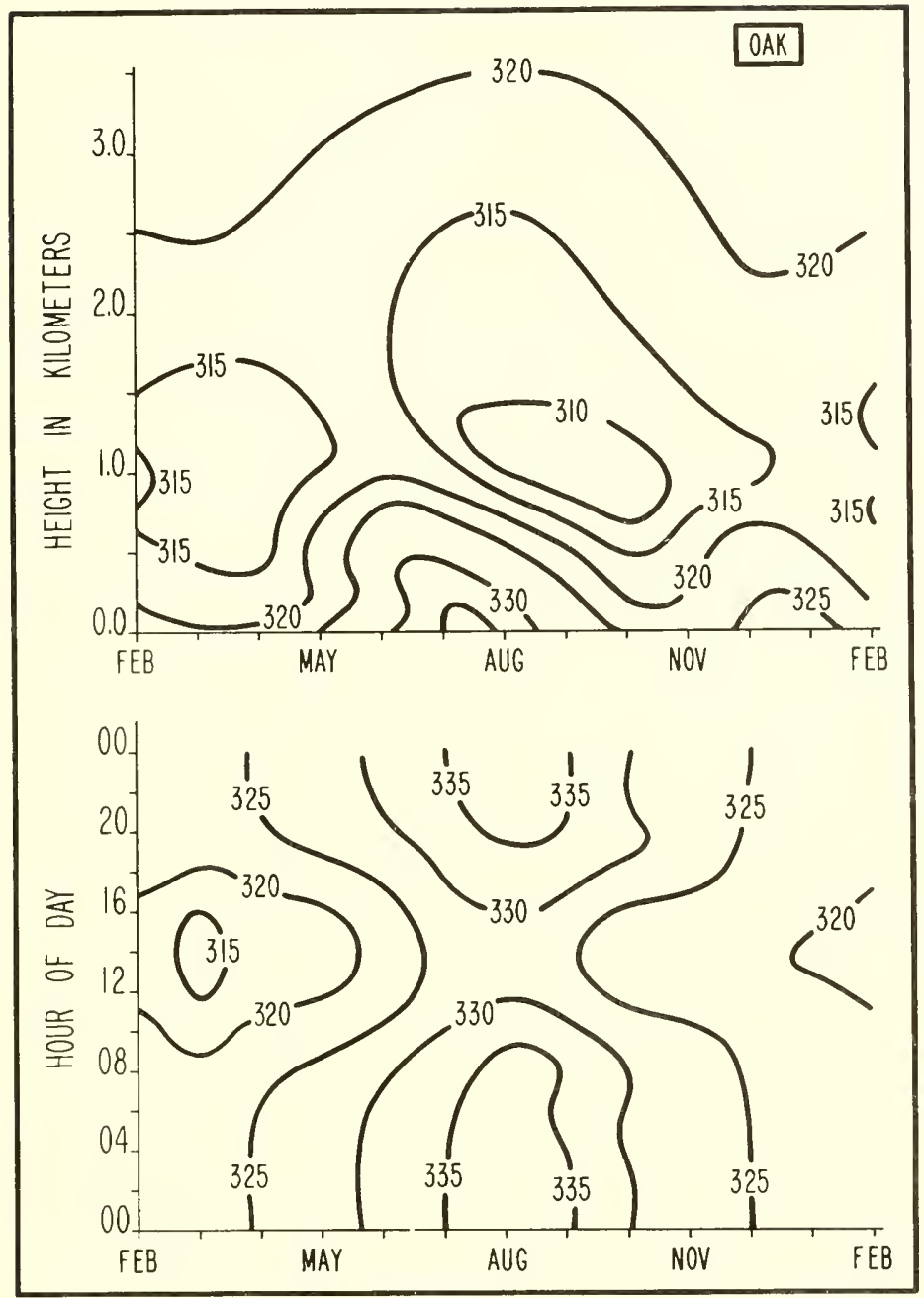

FIgURE 4.17. Diurnal, seasonal, and vertical variation of $\mathrm{N}_{0}$ for Oakland, Calif. $(O A K)$.

The interplay of polar and maritime air masses along the middle east coast of the United States is reflected in the strong seasonal range of $50 \mathrm{~N}$ units at Washington, D.C. (DCA), figure 4.21. The summertime diurnal range of $15 \mathrm{~N}$ units reflects the moderating influence of maritime air so common along the central east coast in the summer.

The latitudinally controlled lower mean temperatures of the northeast coastal regions are reflected in the generally lower values of $N_{0}$ plus 


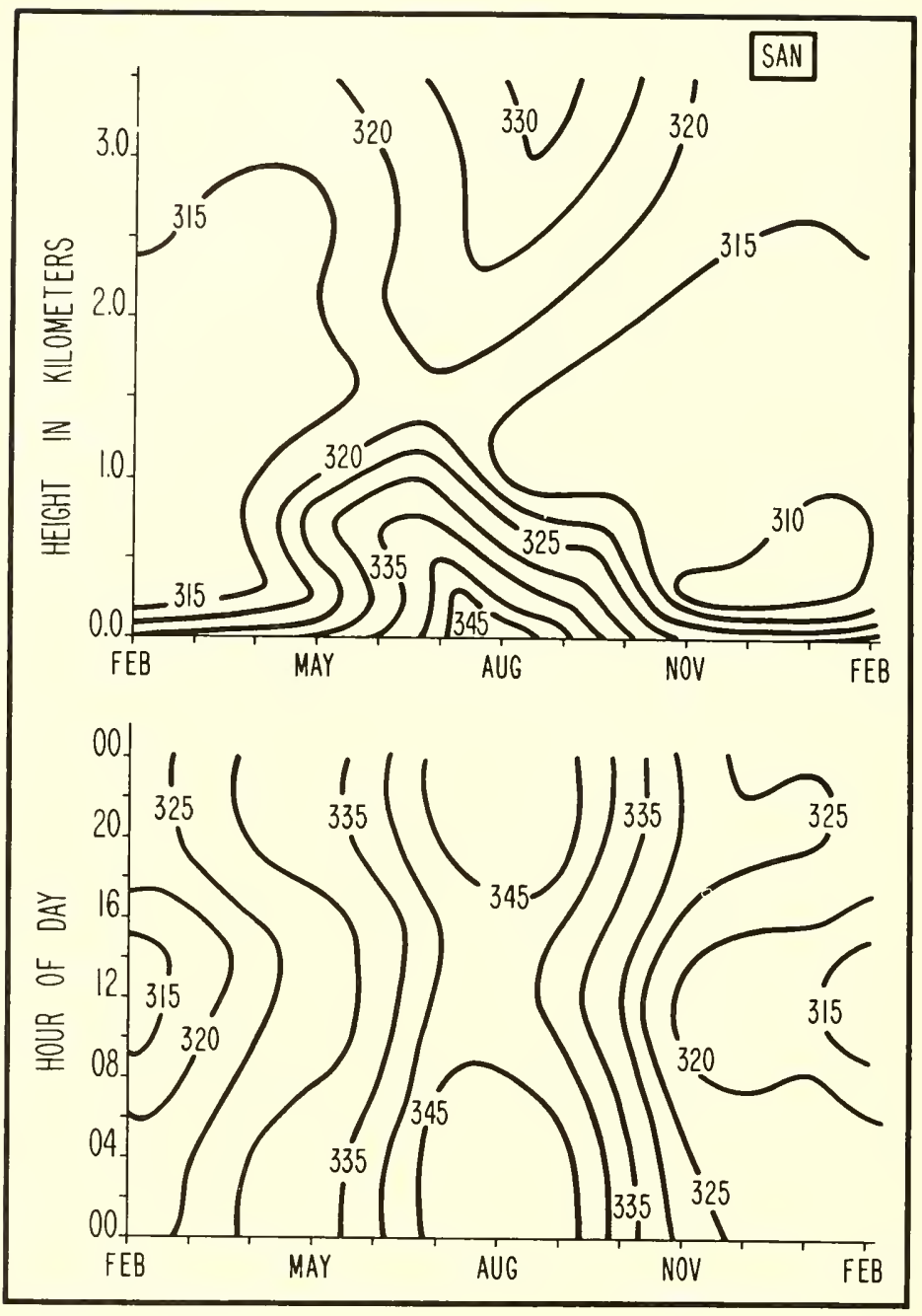

Figure 4.18. Diurnal, seasonal, and vertical variation of $\mathrm{N}_{0}$ for San Diego, Calif. (SAN).

smaller seasonal, diurnal, and vertical ranges as illustrated by Portland, Me. (PWM), figure 4.22. The curious low in late autumn appears to be the result of advection of air from the continental interior and is perhaps indicative of the Indian summer of New England. The long New England winter appears in the surface data as a large area of nearly constant $N_{0}$.

The above data reflect the south-to-north change from humid subtropical to marine-modified continental climates typical of the lee coasts 


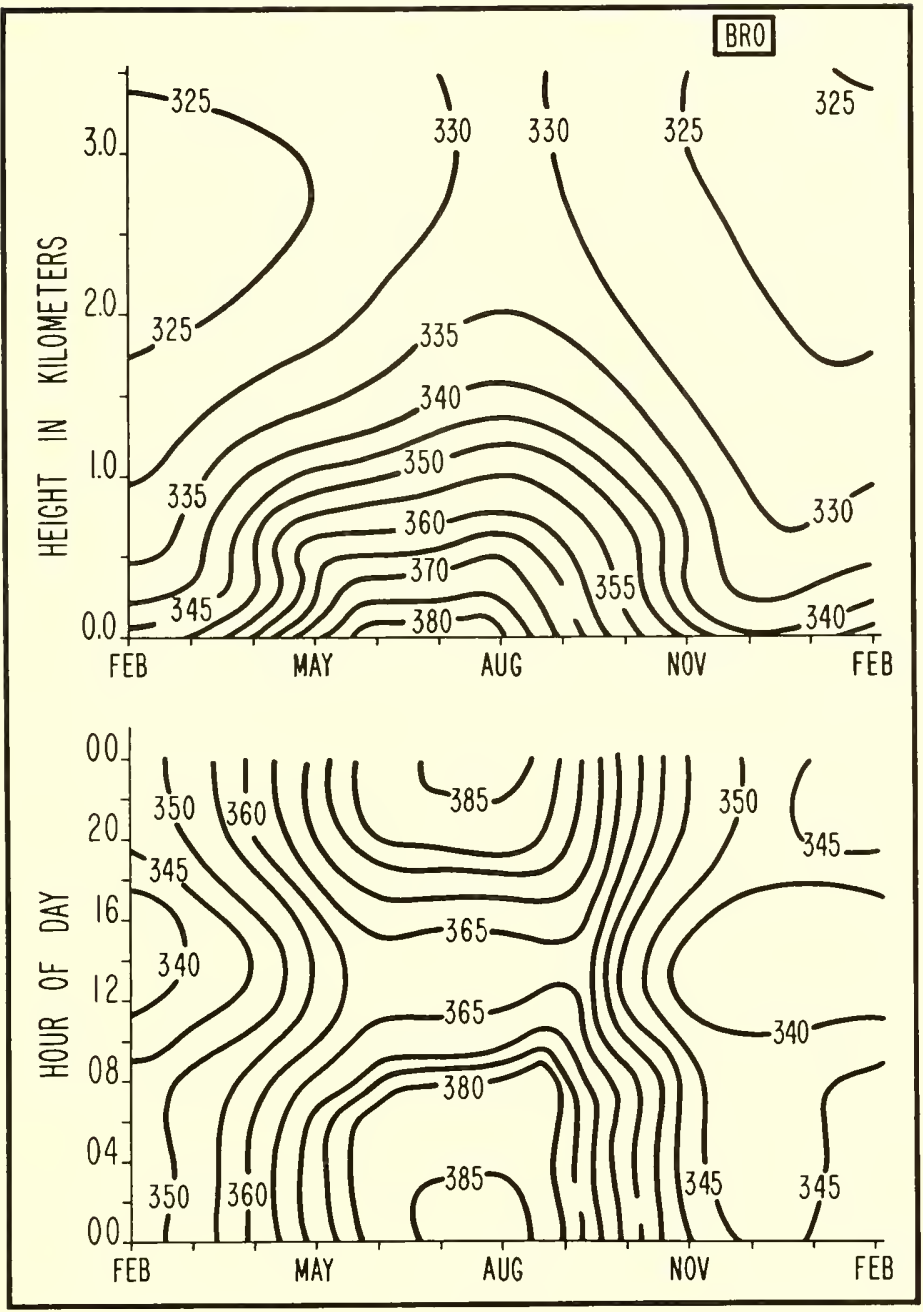

Figure 4.19. Diurnal, seasonal, and vertical variation of $\mathrm{N}_{0}$ for Brownsville, Tex. (BRO).

of continents. By contrast, the island-like station of Cape Hatteras (HAT), figure 4.23, reflects both the characteristic strong seasonal range of a lee coast station that is dominated by dry, cold continental air in the winter and warm, moist maritime air in the summer and the small diurnal ranges for all months of the year of a maritime modified climate.

The data for Denver, Colo. (DEN), figure 4.24, strongly reflect the influence of the climatic controls of altitude and continentality. 'The level of $N_{0}$ is intermediate to that of Oakland and Washington. The vertical 


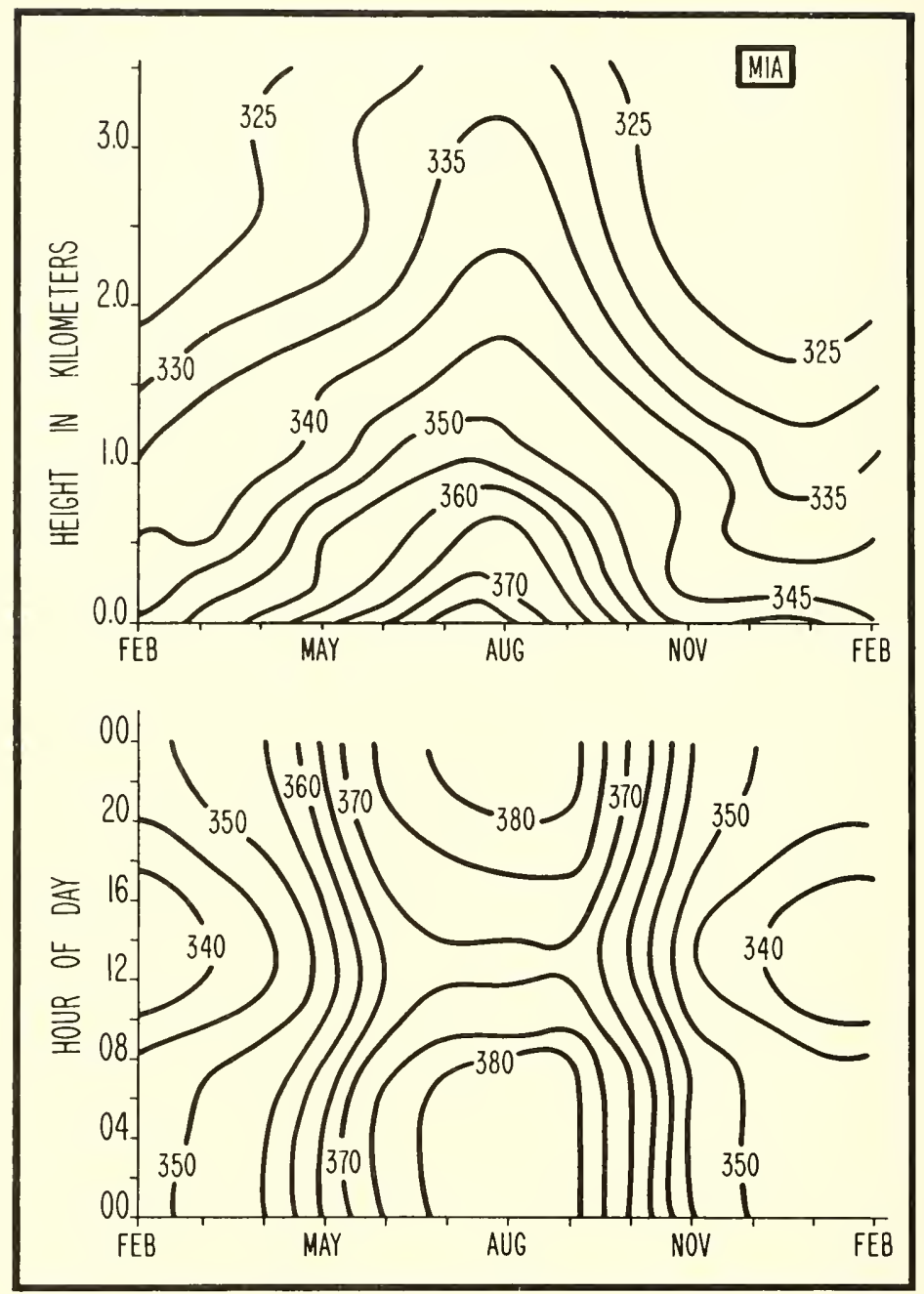

Figure 4.20. Diurnal, seasonal, and vertical variation of $\mathrm{N}_{0}$ for Miami, Fla. (MIA).

profile chart for this station indicates the mild influx of tropical air in the summer at the surface and the convective mixing of this air to great heights by the strong thunderstorm activity common to the area. The high $N_{0}$ values between 2 and $3 \mathrm{~km}$ are perhaps due to superior air subsiding on the lee slopes of the Rocky Mountains. Relatively intense diurnal ranges are apparent in both summer and winter, as the day to night variations of this high plains climate are controlled to a large extent by radiational heating and cooling through the thin atmospheric blanket at high elevations. 


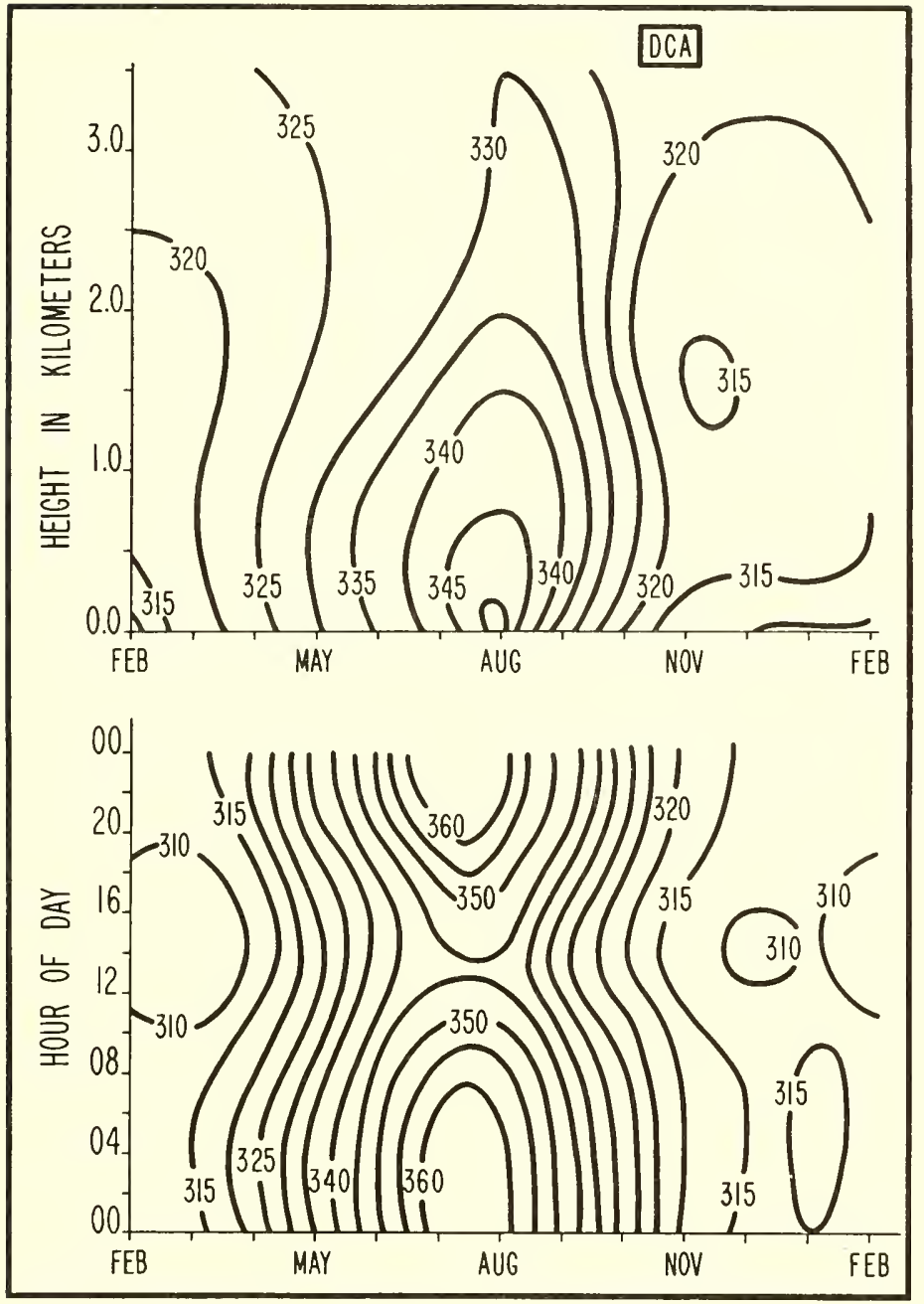

Figure 4.21. Diurnal, seasonal, and vertical variation of $\mathrm{N}_{0}$ for Washington, D.C. (DCA).

The strong warm-season influx of tropical maritime air from the Gulf of Mexico up the Mississippi Valley is spectacularly in evidence at Columbia, Mo. (CBI), figure 4.25. The most significant feature of these charts is the weak year-round diurnal range of $10 \mathrm{~N}$ units or less accompanied by a strong seasonal range of nearly $50 \mathrm{~N}$ units.

In summary, it is seen that the west-coast stations display a rather uniform low average value of $N_{0}$ accompanied by small seasonal and diurnal ranges. This is due to the continual onshore advection of cool 


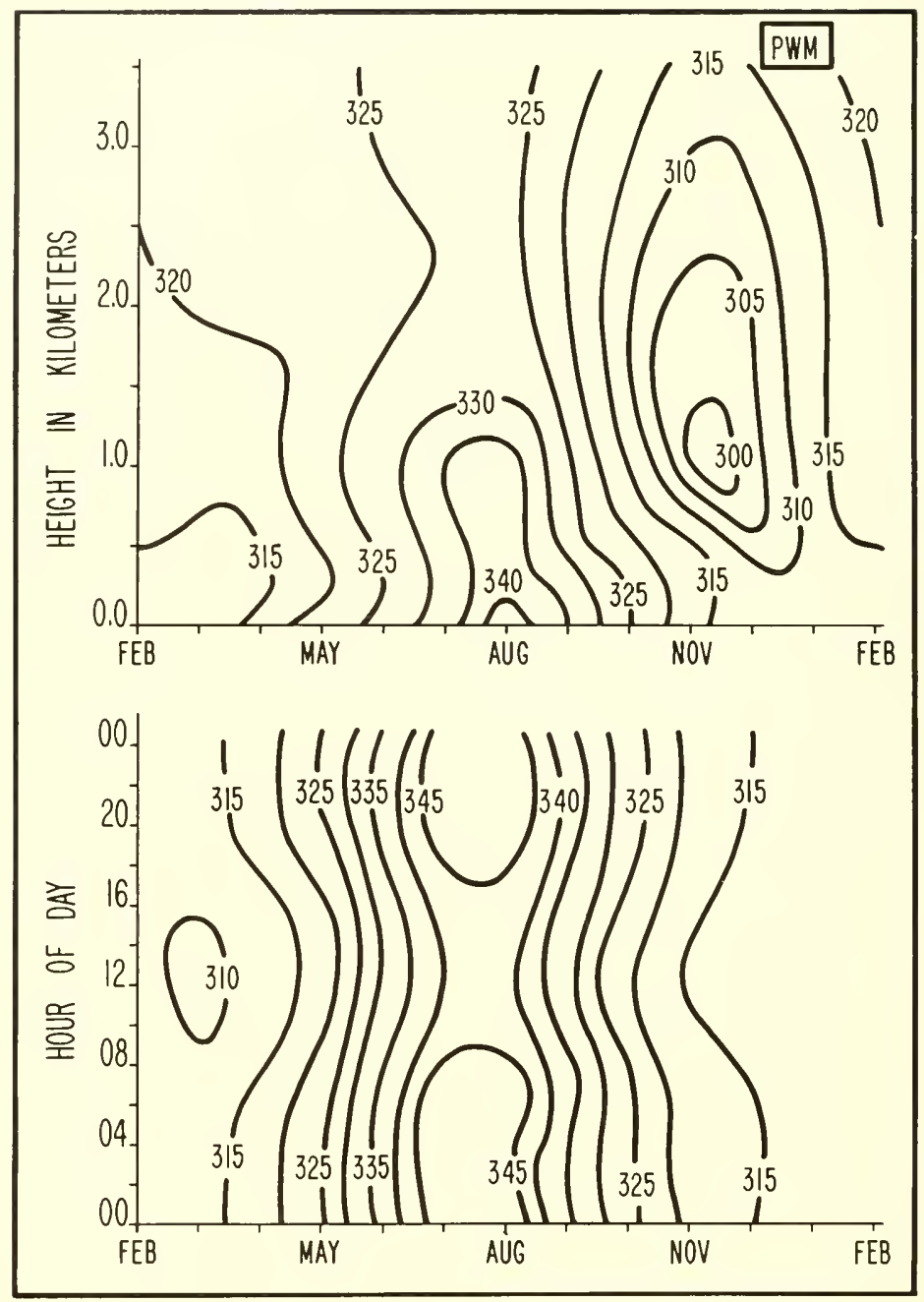

Figure 4.22. Diurnal, seasonal, and vertical variation of $\mathrm{N}_{0}$ for Portland, $M e .(P W M)$.

maritime air that keeps the mean temperatures both low and uniform. By comparison, the continental stations show relatively large diurnal and seasonal ranges controlled in large measure by the radiative heating and cooling, summer to winter and day to night. The east-coast stations display a general increase of $N_{0}$ from north to south arising from the 


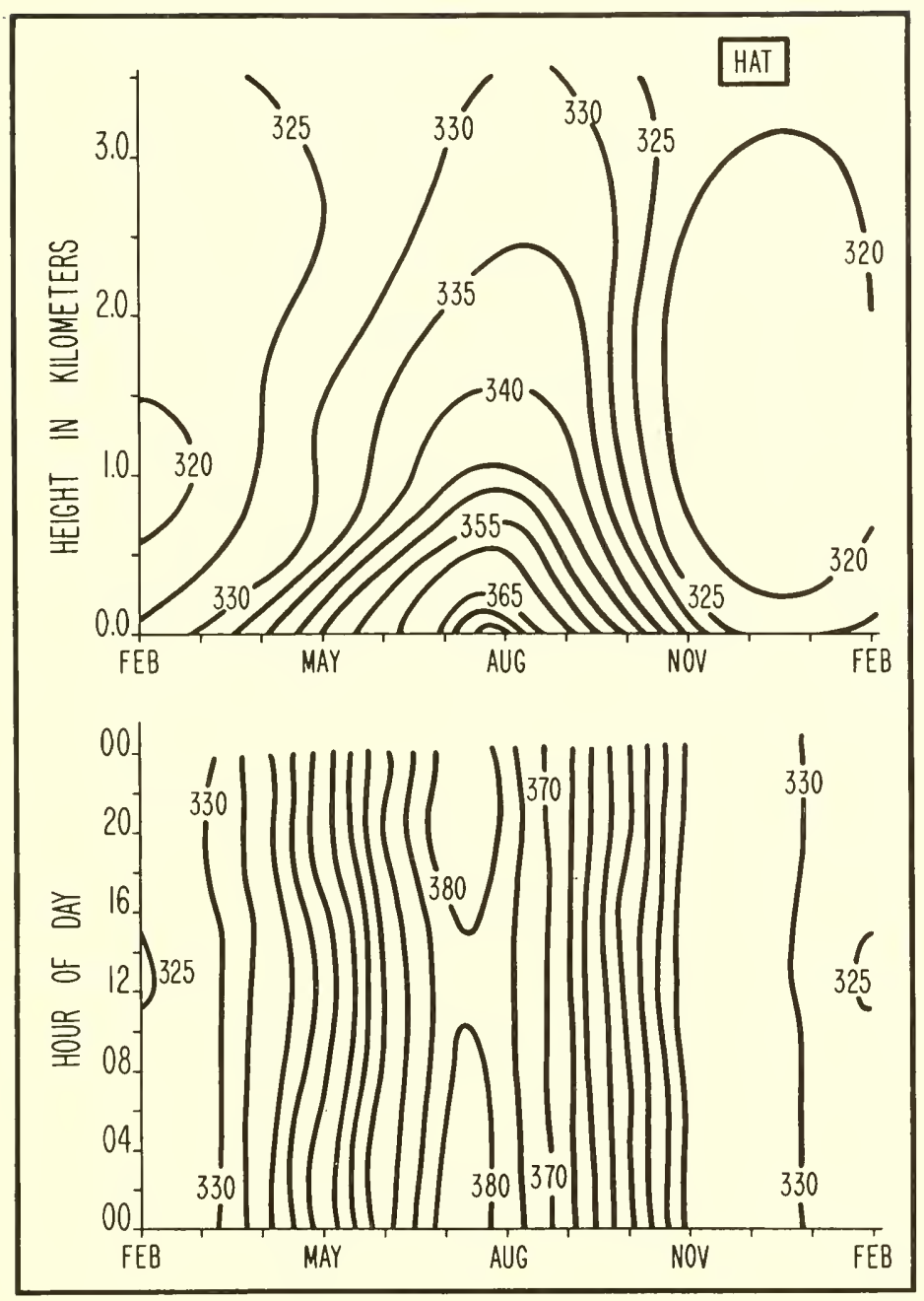

Figure 4.23. Diurnal, seasonal, and vertical variation of $\mathrm{N}_{0}$ for Cape Hatteras, N.C. (HAT).

general increase of mean temperature with resultant increase in watervapor eapacity. These same stations have a marked seasonal range due to the interplay of continental and maritime effects. The relatively small summertime diurnal ranges along the east coast reflect the strong influence of maritime air upon this region during that season. 


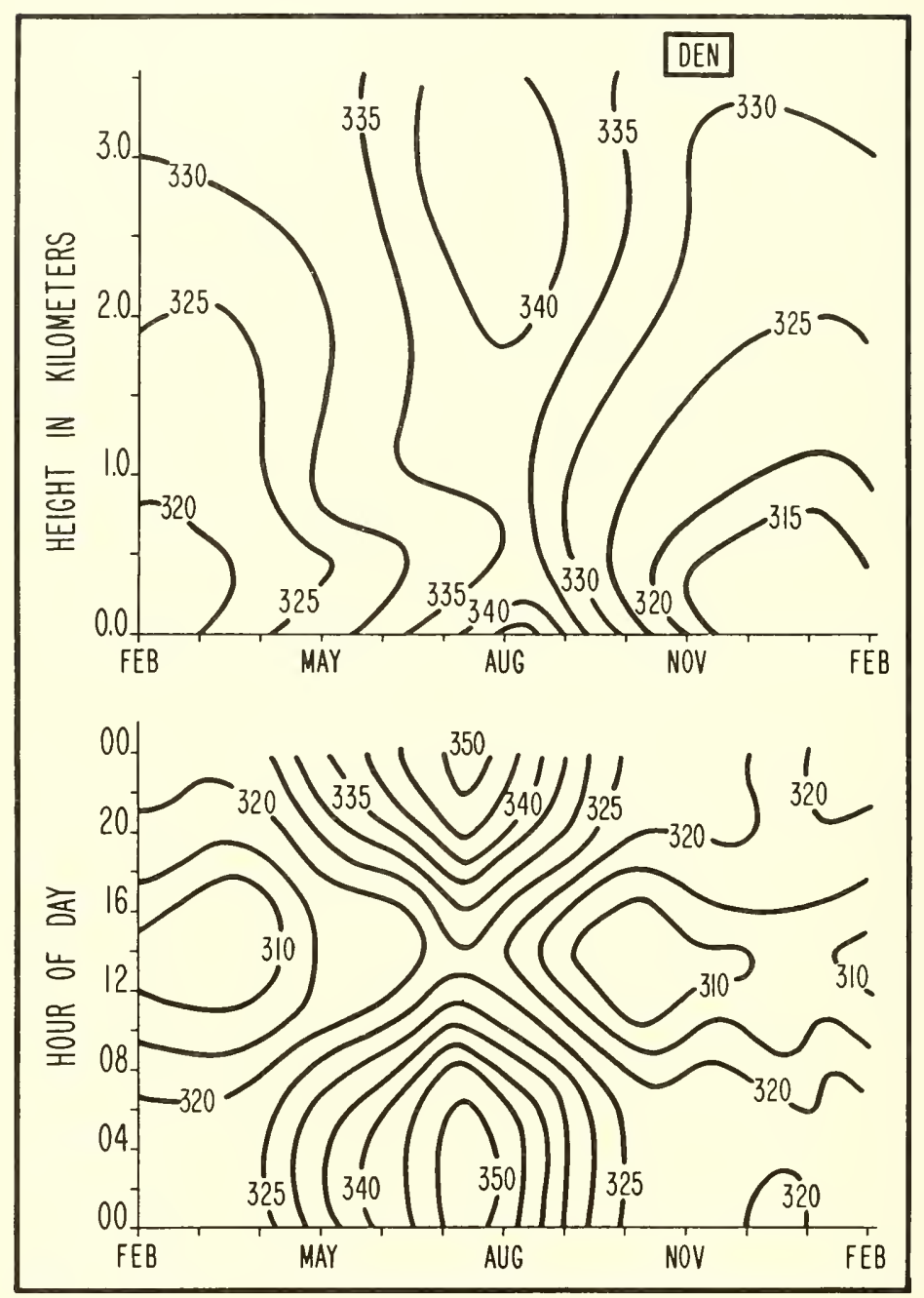

Figure 4.24. Diurnal, seasonal, and vertical variation of $\mathrm{N}_{0}$ for Denver, Colo. $(D E N)$. 


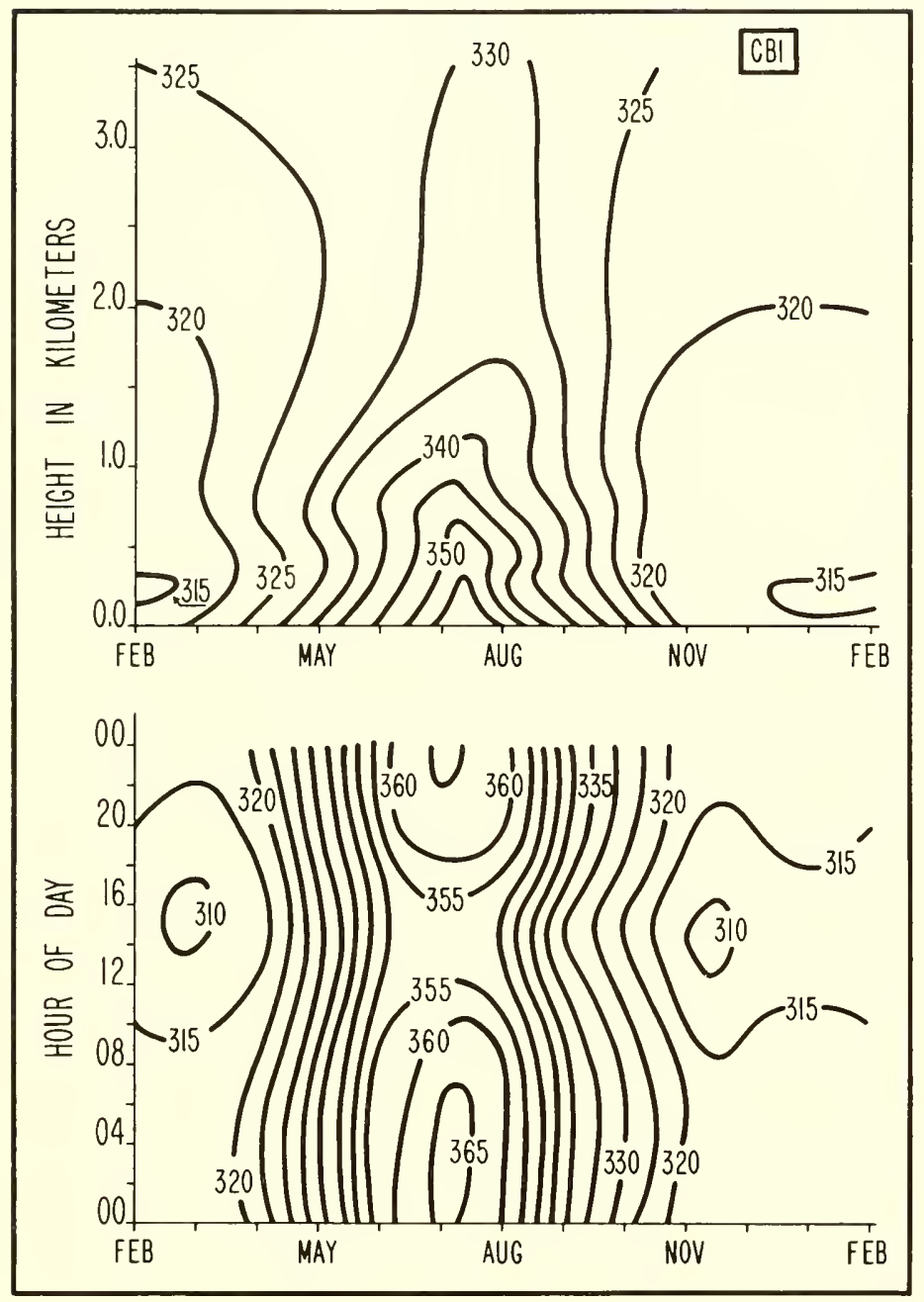

Figure 4.25. Diurnal, seasonal, and vertical variation of $\mathrm{N}_{0}$ for Columbia, Mo. (CBI). 


\subsubsection{Continental Cross Sections}

The vertical profile data obtained for the stations of figure 4.15 provide a means to construct $N_{0}$ cross sections traversing varied climatic zones and geographic regions.

The first cross section of this series, figure 4.26, is taken along the Pacific coast of central North America from Canada to Mexico. Intrusion of polar air at the northern end of the wintertime cross section is responsible for the relatively flat $N$ gradient over Tatoosh. The 320 $N_{0}$-isopleth covering most of the coast southward from Tatoosh shows the uniform modifying influence of the ocean which, at this tıme of year, is considerably warmer than the continent. Over southern California the low at $500 \mathrm{~m}$ results from the drying-out effects of the Pacific high inversion. On the summer cross section moderate $N_{0}$-values typify the coast. The minimum at Oakland stems perhaps from the upwelling of relatively cool ocean waters off the California coast. Striking evidence of the Pacific inversion is apparent from the low at $1 \mathrm{~km}$ between Oakland and San Diego that results from significant decreases in the vapor pressure term contribution to $N$ within the dry subsiding air aloft.

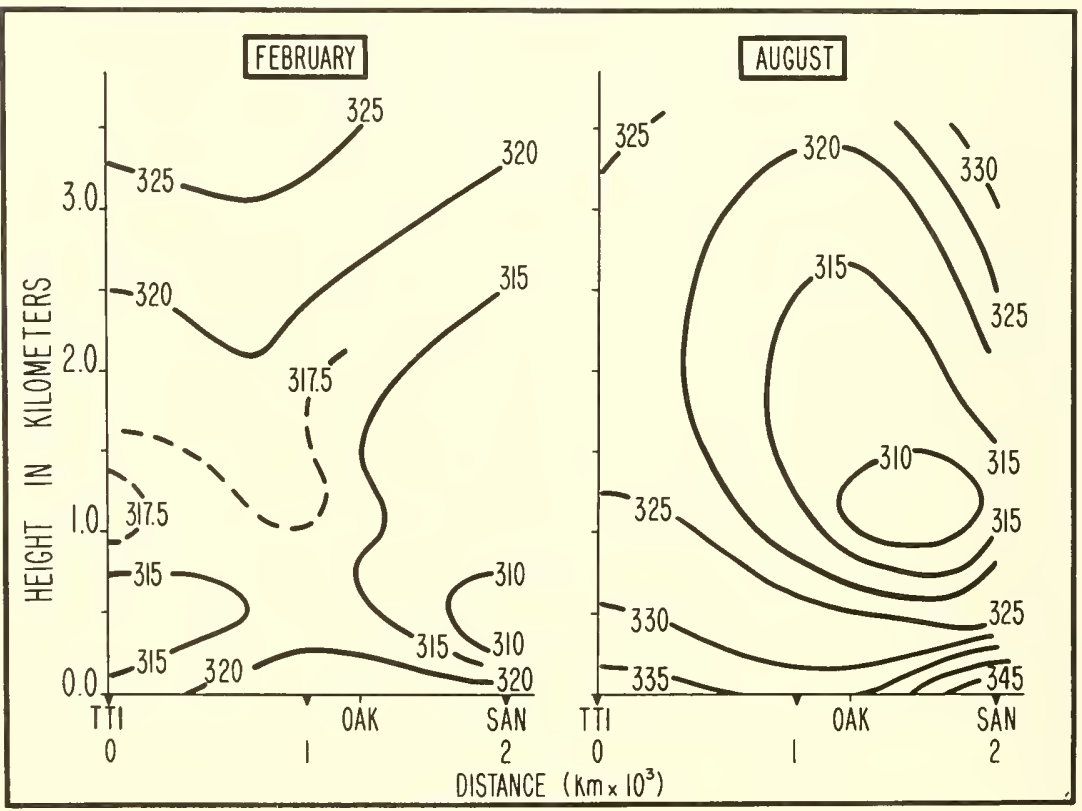

FIgure 4.26. North-south $\mathrm{N}_{0}$ cross section along the western coast of the United States. 


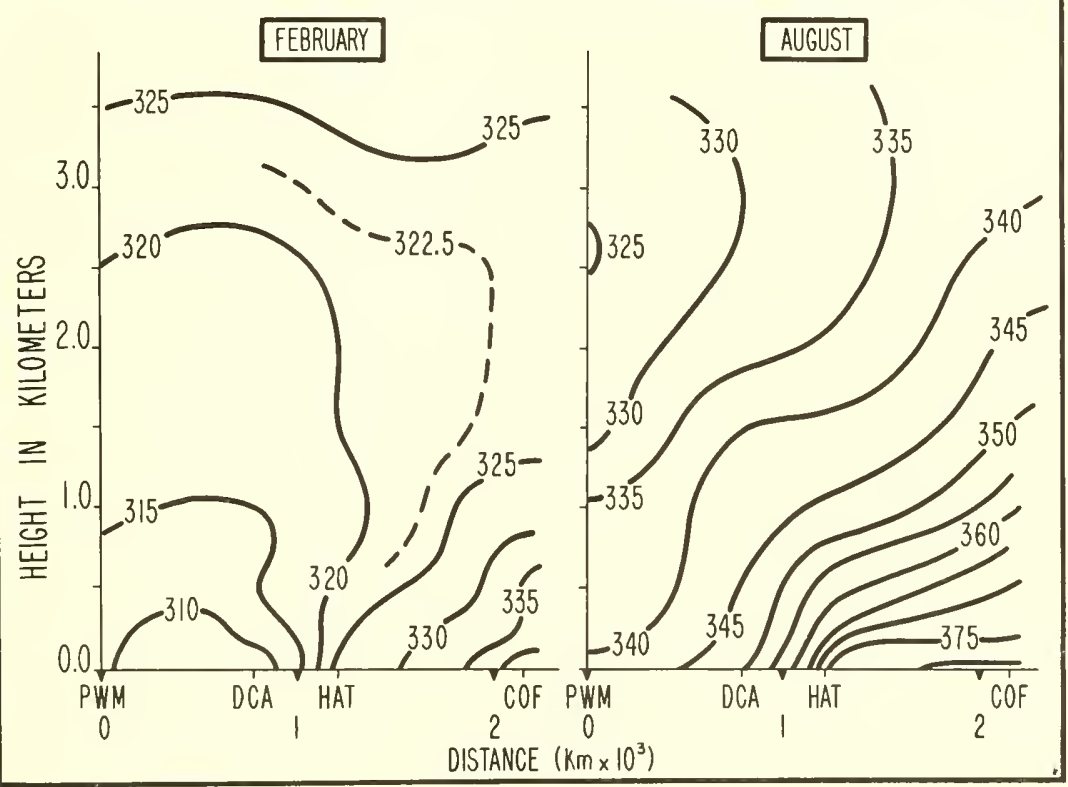

FIgURE 4.27. North-south $\mathrm{N}_{0}$ cross section along the eastern coast of the United States.

The cross section for the eastern seaboard of the United States, figure 4.27 , presents a considerably different picture of refractive index climate than the Pacific coast. On the winter eross section the area between Portland and Washington falls within a low produced by frequent oceurrence of polar air masses during this season of the year. Southward toward Cocoa, Fla. (COF) there is a signifieant $N$ inerease that corresponds to the considerable latitudinal temperature gradient that exists on the east coast of the continent during the winter months. On the summertime cross section the temperature gradient difference between the two coasts is even more pronounced and is reflected in the strong east-coast $N_{0}$ difference of about $40 \mathrm{~N}$ units as compared to the Tatoosh-San Diego difference of about $10 \mathrm{~N}$ units. The vertical gradients are also seen to increase in a systematic fashion from north to south, reflecting the increased vapor pressure gradients in the warmer southern regions.

The extensive continental cross section from Isachsen (IC) to Balboa (BLB), figure 4.28, discloses several interesting features. On the wintertime chart the relatively high $N_{0}$ values between Isachsen and Churchill (YQ) indicate the presence of very cold, dense, dry air. To the south over Bismarck, N.Dak. (BIS), and the Great Plains, relatively warm, dry air of 


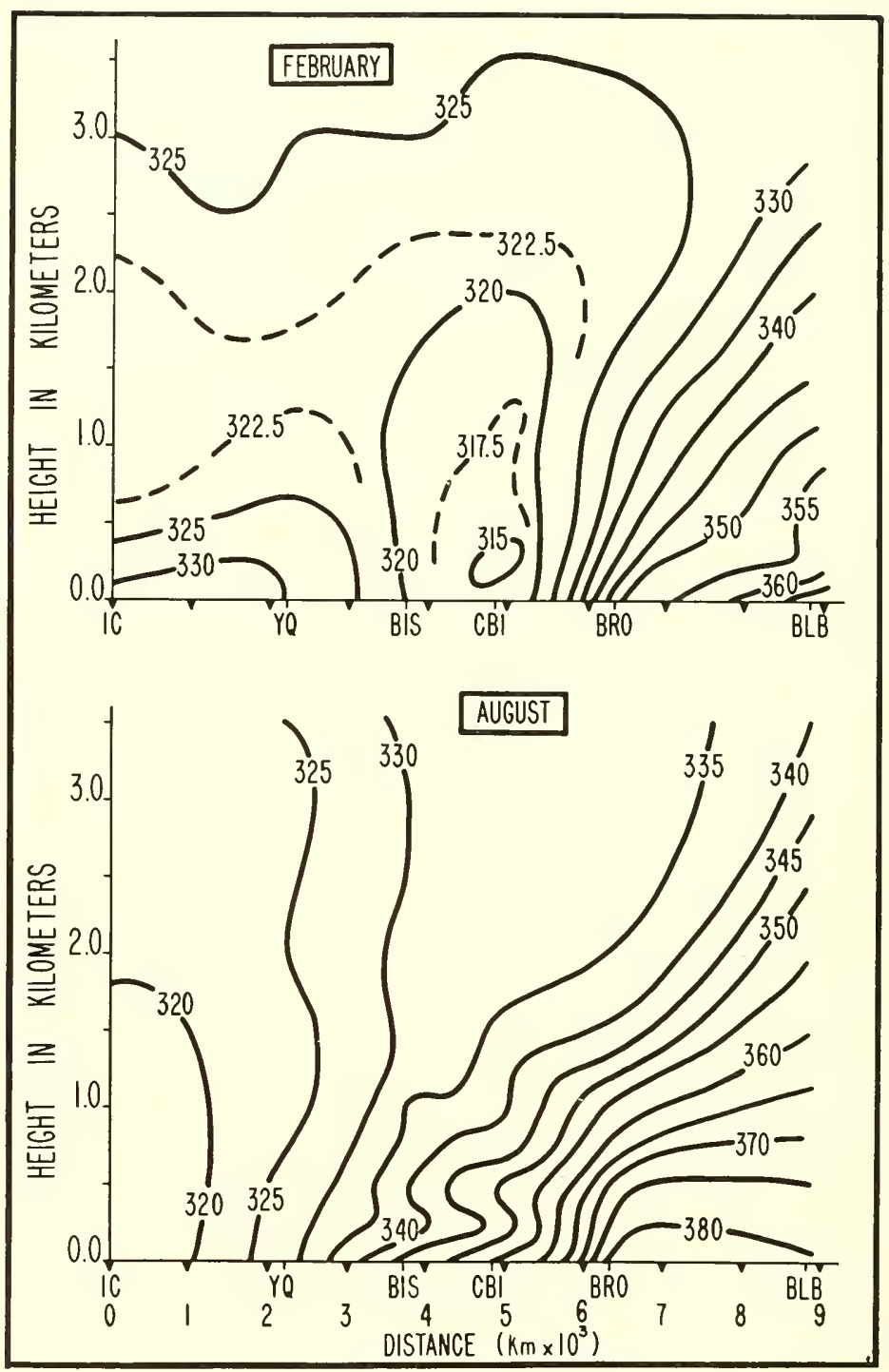

Figure 4.28. North-south $\mathrm{N}_{0}$ cross section from Isachsen, North West Territories (IC). through Churchill (YQ) to Balboa, Canal Zone (BLB). 


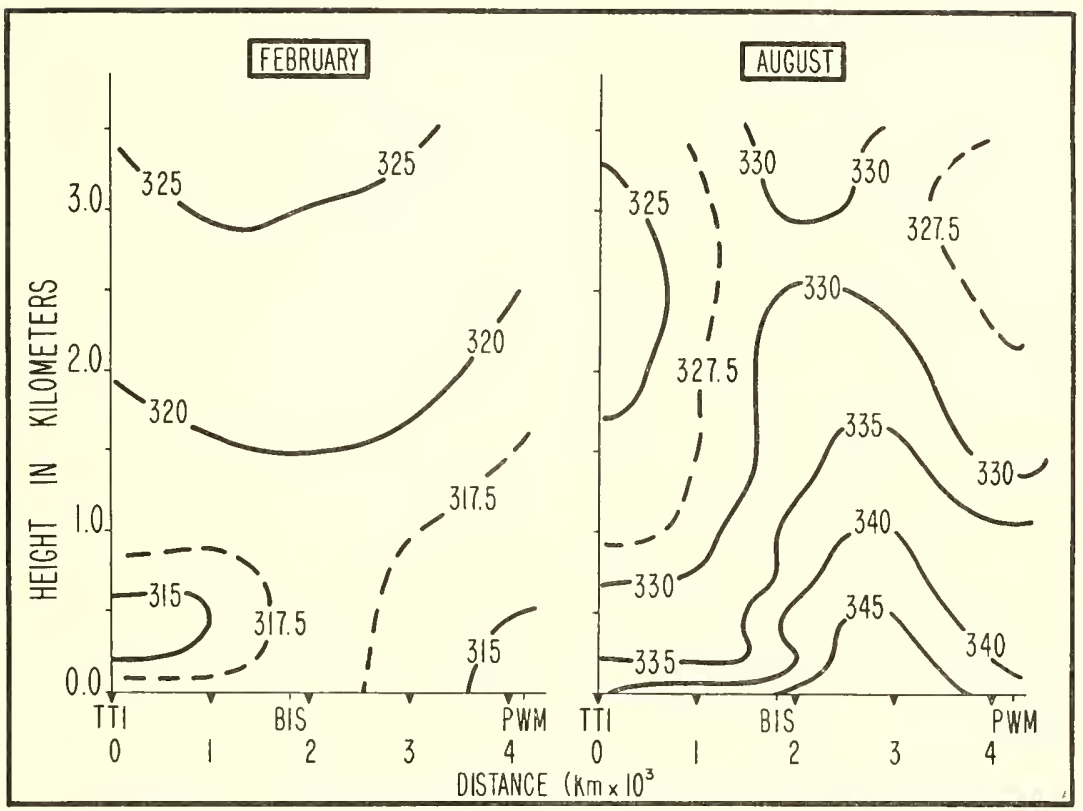

Figure 4.29. West-east $\mathrm{N}_{0}$ cross section for the northern United States.

lower density creates a refractive index low. Southward from the Great Plains a considerable $N_{0}$ gradient is encountered as the Gulf source region for tropical maritime air is approached. Latitudinal temperature gradients produce a continued increase in $N_{0}$ to the southern extreme of the chart at Balboa in the tropics. The $N_{0}$ gradients of the summer cross section are largely thermally controlled, ranging from cool temperatures and low $N_{0}$ in the polar regions to a maximum between Brownsville and Balboa near the warm-season heat equator.

Three zonal cross sections have been prepared extending across the northern, central, and southerm portions of the United States. The February chart for the northern cross section, Tatoosh to Portland, figure 4.29 , exhibits lows at both coasts, with slightly higher values of $N_{0}$ in the cold interior of the continent. The summertime chart clearly shows the intrusion of tropical maritime air from the Gulf of Mexico pushing northward over the Great Plains. The central U.S. cross section, figure 4.30, shows, on the wintertime chart, slightly lower values of $N_{0}$ on the west coast at Oakland than at Hatteras. East of the Rockies, the chinook winds of the prevailing westerlies assist in the formation of a relatively warm, dry air mass of characteristically low $N_{0}$. On the summer map, 


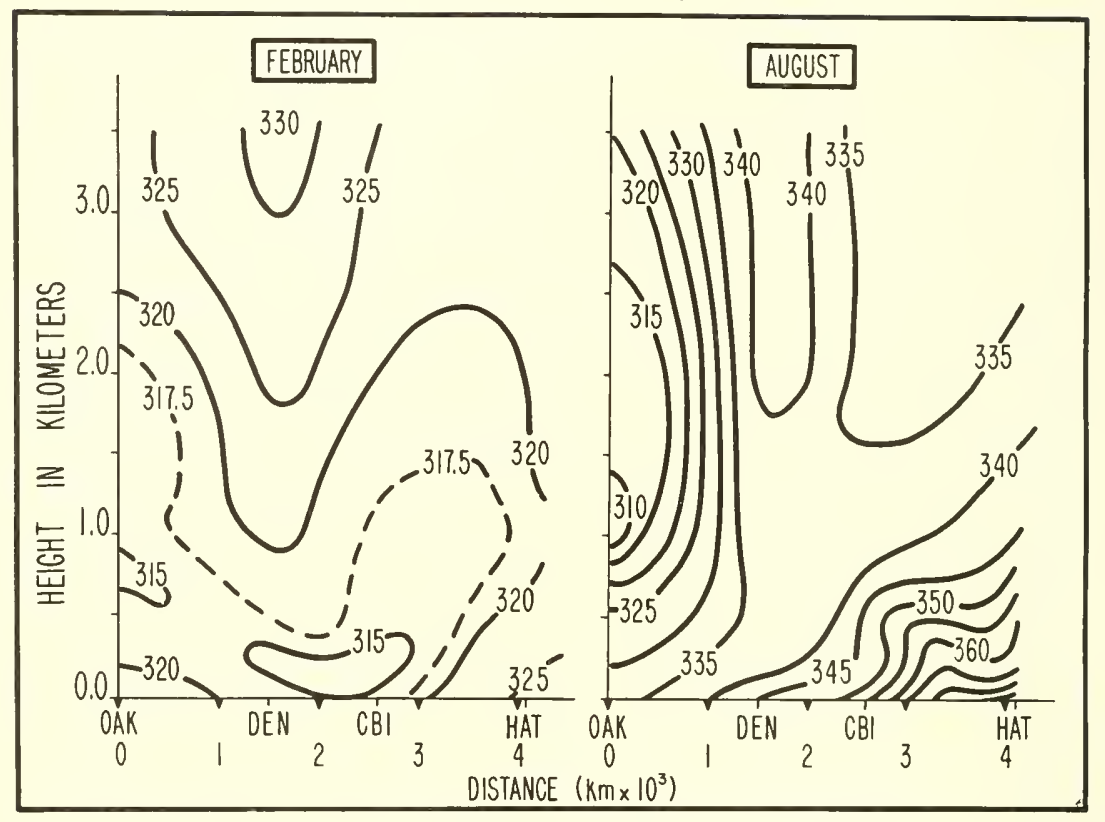

Figure 4.30. West-east $\mathrm{N}_{0}$ cross section for the central United States.

the influence of the Pacific high is in evidence over Oakland, while in the interior of the continent the influence of tropical maritime air is noticeable up the Mississippi Valley. The zonal cross section across the southern United States, figure 4.31, shows a transition from the Mediterranean climate of the Pacific coast to the humid subtropical type from Brownsville eastward. Here again the east-coast $N_{0}$ values, from a higher temperature regime, are larger than those in the west. The summertime chart is similar to the winter one with the $N$-gradient intensified all along the route.

Figure 4.32 is for the longest cross section, extending approximately $22,000 \mathrm{~km}$ from Canton Island (CAN) to Wiesbaden (WSB) in northern Europe. The winter chart shows a gradual decrease in $N$ from Canton Island to Brownsville and Cocoa, with very uniform $N$ structure over the Atlantic to Ship "J," and onwards to Wiesbaden. The summer chart shows a double maximum, one at Canton and the second over the warm North American continent between Brownsville and Cocoa. The gradual decrease in mean temperature northward across the Atlantic Ocean is evidenced by the rather uniform decrease of $N_{0}$ between Cocoa and Wiesbaden. 


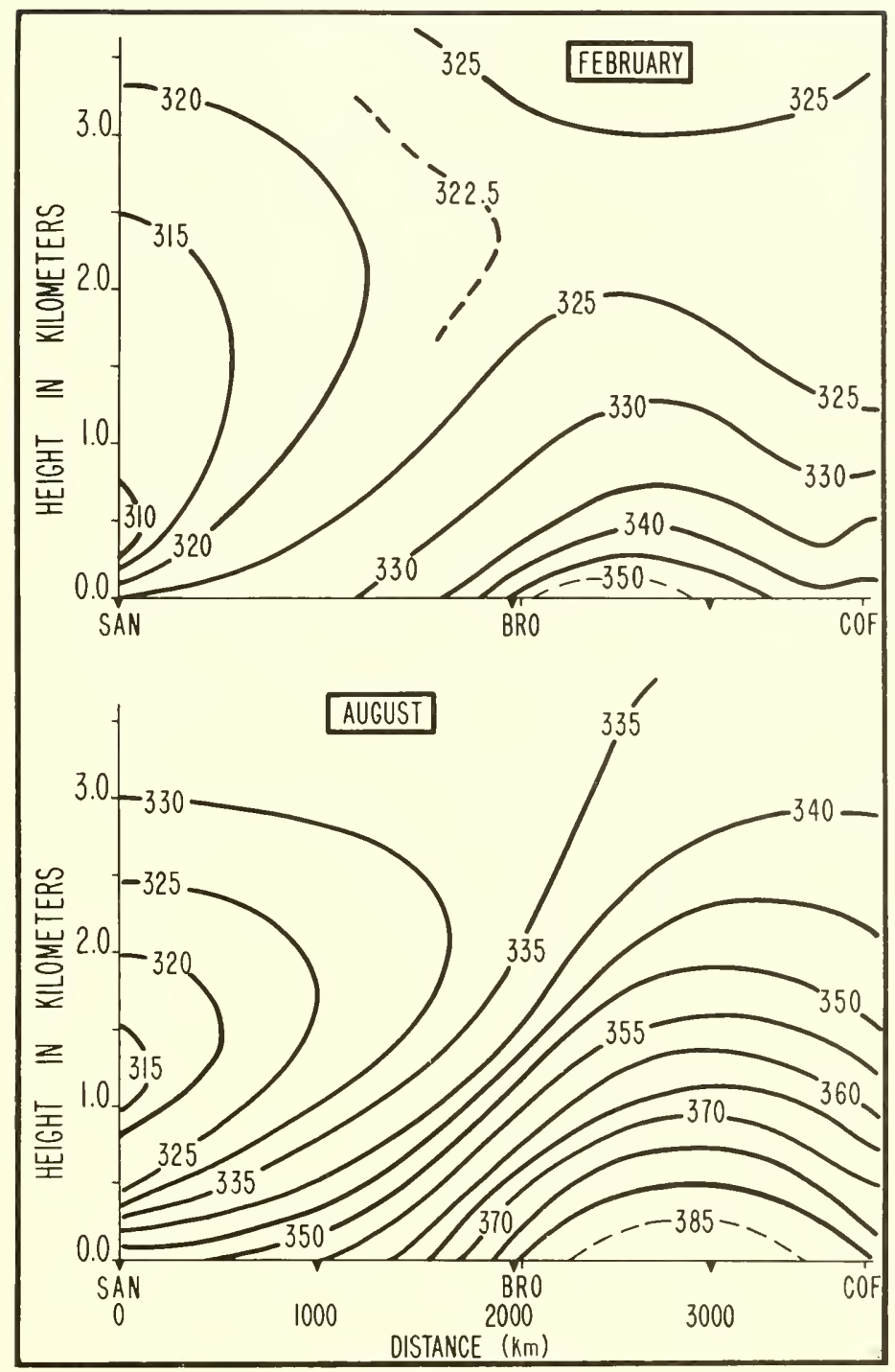

Figure 4.31. West-east $\mathrm{N}_{0}$ cross section for the southern United States. 


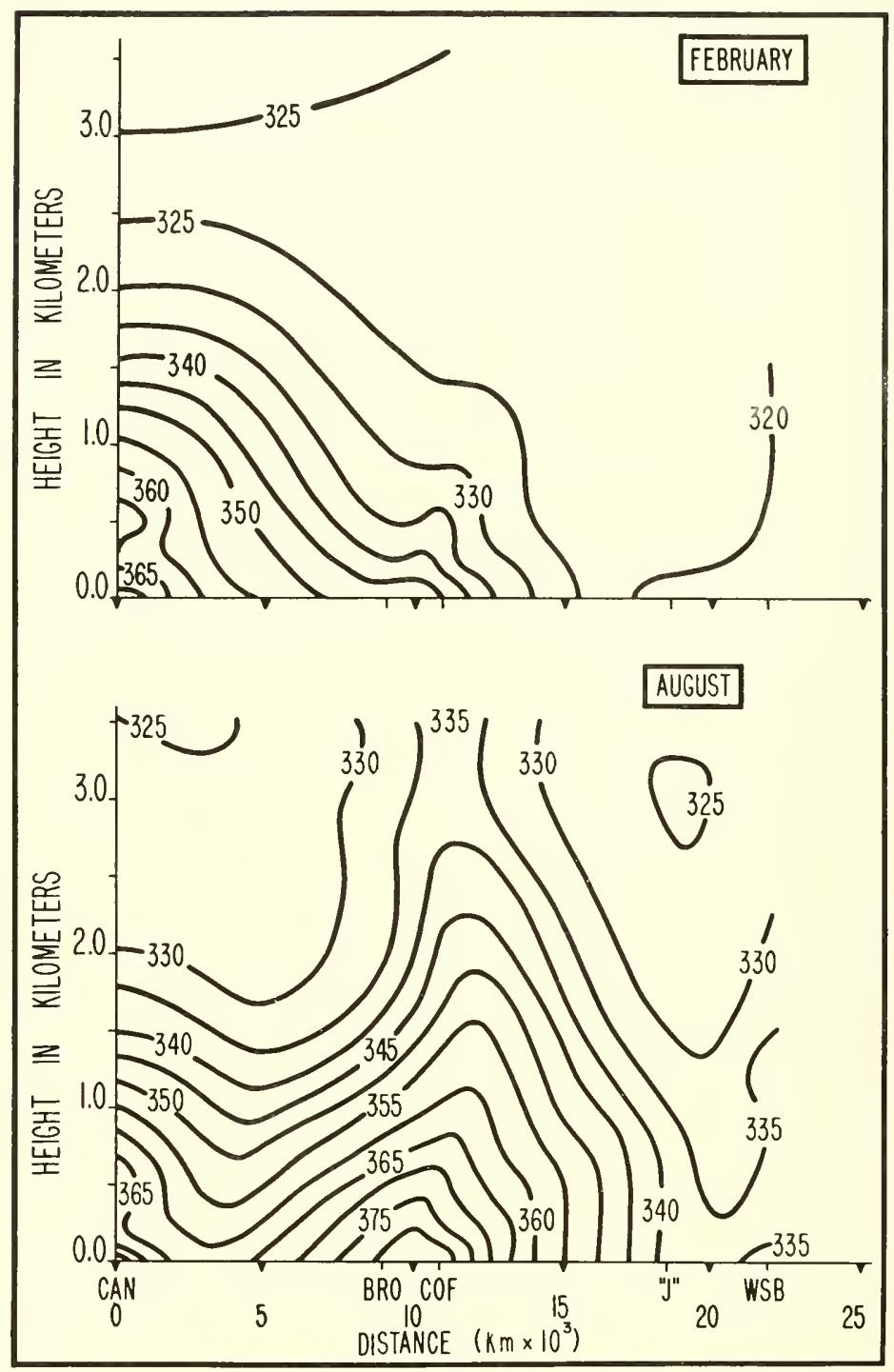

Figure 4.32. West-east $\mathrm{N}_{0}$ cross section from Canton Island, South Pacific (CTN) through Wiesbaden, Germany (WSB). 


\subsubsection{Delineation of Climatic Characteristics}

Since elimate is really a synthesis of weather elements taken over a period of time, it is apparent that no fixed elimatie boundaries exist in nature. These boundaries shift from year to year with ehanging weather and with the addition of new data into the climatie averages. Climatie borders, then, represent transition zones between so-ealled "core elimates" of one type or another. A "core elimate" presumably maintains its elimatic personality consistently over a period of time. The coniferous forest regions of the far north of Canada, for example, maintain a continuously frigid elimate under the aretie inversion during the heart of the winter season. Since a simple system is required for effective elassification, the congeries of minute climatic areas arising from extensive climatic division are, for practical purposes, eoordinated into areas having broad similarity of climatic character. Thus we shall only attempt here to look for general patterns in our limited data sample.

The $N_{0}$ data for the station elevation of figures 4.16 to 4.25 contain information about the radio refractive index climate of the respeetive stations that may be converted to a pair of indices, one seasonal, one diurnal, useful in elassifying climate. The diurnal index is the ratio obtained by dividing the differenee between the highest and lowest hourly means for August by a similar difference for February. This ratio is then plotted versus a ratio of the maximum difference for the 12 mean $N_{0}$ values at 0200 divided by the maximum differenees for the 1400 means.

The results of this analysis ean be seen on figure 4.33 , where the largest pair of ratios are observed for Denver and Bismarck, a consequence of the strong continentality effects at these inland stations. Denver, it is to be noted, displays a large seasonal ratio whereas Bismarek represents the extreme in diurnal ratios. Brownsville, Washington, and Columbia show sizable seasonal ratios coupled with slightly greater than normal diurnal ratios. Summer-to-winter elimate changes are considerable at these three locations.

The remainder of the stations display the near-unity value of both ratios that would be expeeted of maritime-dominated elimates.

A comprehensive, though simple, index of elimatic variation of the vertieal distribution of $N$ is particularly diffieult to envisage. One such index is the ratio, $R(\Delta N)$, of the summer to winter value of $\Delta N$ versus the mean value of $\Delta N$ (the absolute difference in $N$ at the earth's surface and at $1 \mathrm{~km}$ above the earth's surface). The gradient, $\Delta N$, has received wide enginecring application and is currently being mapped on a worldwide basis by the International Consultative Committee for Radio [30]. When this ratio is plotted versus the yearly mean value of $\Delta N$, as on figure 4.34, one again obtains about the same climatic demarcation as above. For example, the maritime-dominated climates generally have values of $R(\Delta N)$ of 1.2 or less. 


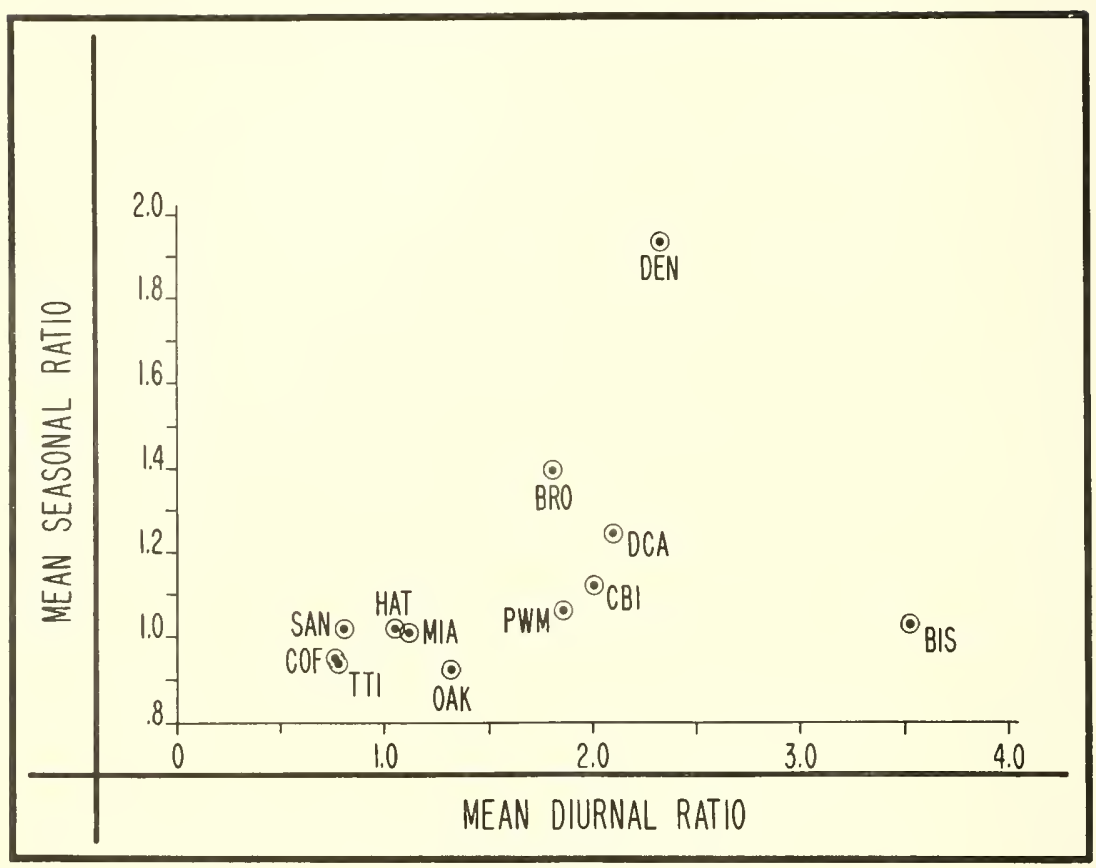

FIgURE 4.33. Comparison of mean seasonal to mean diurnal ratio of $\mathrm{N}_{0}$ at the earth's surface.

The more southerly and humid stations have relatively high mean $\Delta N$ values in excess of $55 \mathrm{~N}$ units $/ \mathrm{km}$. The single European station, Wiesbaden, shows both low average value of $\Delta N$ arising from the low average temperatures and small seasonal variation associated with the continuous onshore advection of cool, moist air from the Atlantic Ocean. The values $R(\Delta N)<1.0$ for the Canadian stations of Churchill and Isachsen reflect the steep gradients associated with the intense stratification during the long winter night. The high, dry climate of Denver produces low mean values of $\Delta N$ but a high seasonal range. The interplay of continental and maritime effects along the east coast produces relatively high values of $R(\Delta N)$ while the presence of the Pacific high inversion layer below $1 \mathrm{~km}$ accounts for similar values along the west coast. The extremely high value of $R(\Delta N)$ of 1.8 for Tripoli (TPI) arises principally from the intense summertime $N$ gradients that exist near the surface in 


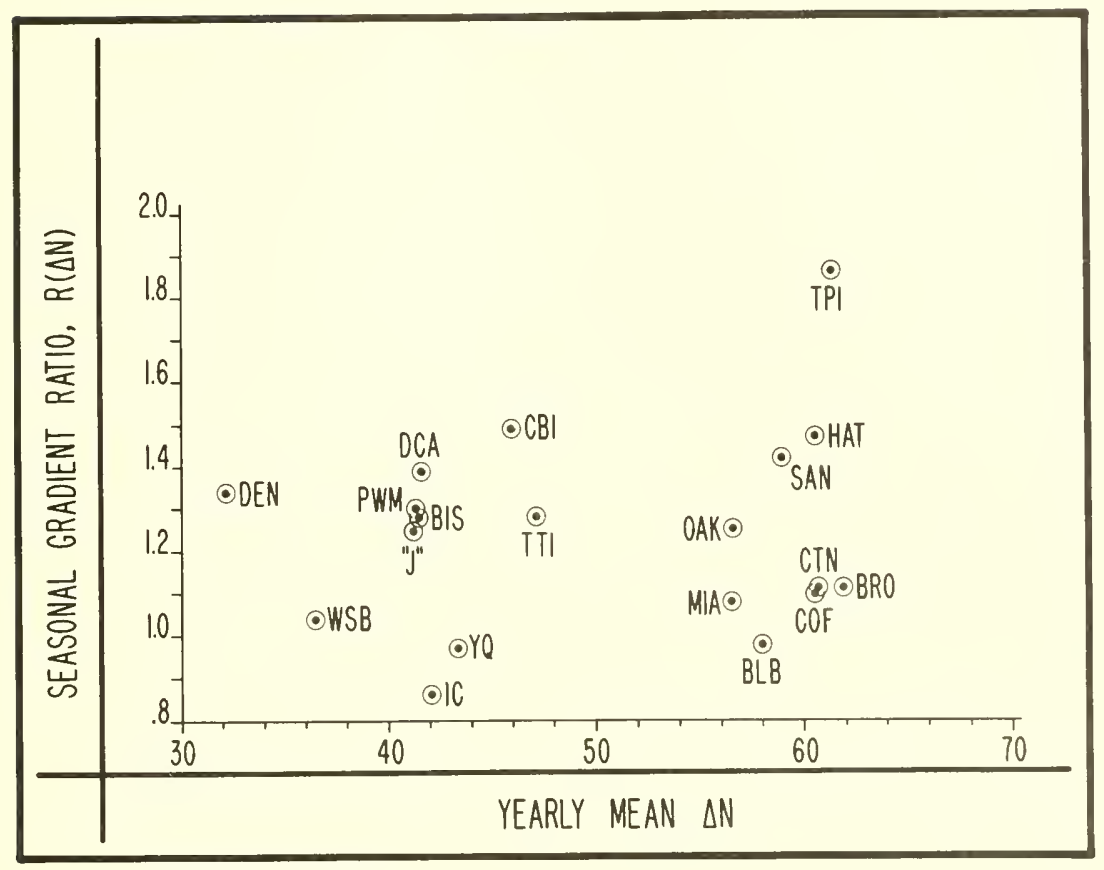

Figure 4.34. Yearly mean and range ratio of $\Delta \mathrm{N}$.

this locale. In the lowest layers of the atmosphere warm, moist air from the Mediterranean Sea is advected inland underneath superheated air from the Sahara Desert. Along the zone of contact between these air masses of widely differing moisture content, spectacular drops in vapor pressure and, consequently, in the wet term component of $N$ occur. During the winter, moderate temperatures and prevailing offshore flow combine to produce near-normal gradients of $N$. Consequently the summer-to-winter $\Delta N$ ratio turns out to be quite high for this borderland station.

The anàlysis above represents a rough and crude first attempt to understand the climatic variations of $N$. It is expected that such classifications will improve with the addition of data from more diverse locations and, in particular, as our knowledge of the role of water vapor in climatie variability increases. 


\subsection{The Climatology of Ground-Based Radio Ducts}

\subsubsection{Introduction}

The occurrence of atmospheric ducts places limitations upon ray tracing of VHF-UHF radio waves. Ducting is defined as occurring when a radio ray originating at the earth's surface is sufficiently refracted so that it either is bent back towards the earth's surface or travels in a path parallel to the earth's surface. Although the rigorous treatment of ducting involves consideration of the full wave equation solution [31] rather than a simple ray treatment, the present study will be based upon a geometrical optics definition of the limiting case in which ray tracing techniques may be used. This simple criterion is then applied to several years of radiosonde observations from stations typical of aretic, temperate, and tropical climates to derive estimates of the variation of the occurrence of radio ducts with elimatic conditions.

\subsubsection{Meteorological Conditions Associated With Radio Refractive Index Profiles}

The path followed by a radio ray in the atmosphere is dependent upon the gradient of the refractive index along that path. Of the vertical and horizontal gradient components that compose the path gradient, the horizontal gradient is normally negligibly small. Thus, the atmosphere is considered horizontally homogeneous and only the vertical gradient of the refractive index is utilized. The numerical value of the vertical gradient of the index of refraction depends on the vertical distribution of atmospheric temperature, humidity, and pressure.

Normally, temperature and humidity decrease with height in the atmosphere, since turbulence prevents any great changes in struoture. However, there are periods of time in which the air becomes fairly calm, whereupon temperature inversions and humidity lapses can be built up and maintained. Temperature inversions have a twofold importance in that (a) they can be widespread in area and persist over a relatively long period of time, and (b) they exercise a stabilizing influence on air motion such that turbulence is suppressed and strong humidity gradients may develop. Layers in which there is intense superrefraction to the point of duct formation may be formed as a result of these gradients and trapping of radio waves may follow. The inversions may start at ground level or at some greater height. The thickness of the layer can show great variability. Three processes that form temperature inversions are:

Advection: Advection is the horizontal flow of air having different heat properties. Such a process is of importance in microwave propagation since it may lead to a different rate of exchange of heat and moisture between the air and the underlying ground or ocean surface, thus affecting 
the physical structure of the lowest layers of the atmosphere. This process results in air with different refractive index characteristics being brought into the area. The most common and important case of advection is that of dry air above a warm land surface flowing out over a cold sea. This type of advection frequently appears in the English Channel during the summer when the weather has been fine for several days.

Advective duct formation depends on two quantities: (a) the excess of the ummodified air temperature above that of the water surface, and (b) the humidity deficit (the difference between the water vapor pressures of the modified and unmodified air). If these quantities are large, especially the humidity deficit, an intensive duct may form.

Important advective processes can also occur over land, but the conditions required for duct formation occur less frequently. However, a duct may be formed when dry, warm air flows over cold, wet ground with resultant temperature and humidity structure previously discussed.

Radiation: Differences in daytime and nighttime radiation are the causes of diurnal variation in refractive conditions. A subrefractive layer may be present during the day, especially at the time of maximum surface heating. Clear skies and light surface winds at night result in considerable cooling of the earth, thus causing the formation of temperature inversions. The surface heat loss produced by nocturnal radiation is a prime factor in the formation of temperature inversions. Atmospheric stratification formed by such a combination of meteorological parameters may produce a trapping layer. A temperature inversion is seldom strong enough to produce a duct in the middle and low latitudes, but it is of major importance in the formation of ducts in the northern latitudes. Low stratus clouds or extreme amounts of moisture (as in the tropies) tend to prevent loss by radiation which lessens the possibility of duct formation.

If the ground temperature in a nocturnal inversion falls below the dewpoint temperature, the water vapor in the lowest layers of the air condenses and the heat of condensation is released directly to the air. Under conditions of radiative fog formation, the humidity lapse tends to counteract any temperature inversion present and may cause substandard refraction if the humidity "inversion" is sufficiently strong. However, the temperature inversion may be strong enough to keep the layer standard or superrefractive.

Subsidence: Subsidence is the slow settling of air from a high-pressure system. The air is heated by adiabatic compression as it descends and spreads out in a layer well above the earth's surface. This process produces stable layers and inversions of temperature with an accompanying decrease in relative humidity. Since the air has come from a high level in the atmosphere, it is dry and may overlay a cooler, moist air mass. 
This type of inversion may cause the formation of an elevated superrefractive layer where the air temperature usually decreases immediately above the ground, rises through the inversion layer and decreases above the layer. This is a common occurrence which may be observed at any time. Subsidence has a tendency to destroy subrefractive layers and to intensify superrefractive layers. Although the effects of subsidence are generally observed at high levels, they are occasionally observed at lower levels, especially in the subtropics. Since subsidence frequently occurs in the lee of mountains and in the southeastern regions of northern hemisphere highs, elevated ducts may also be observed.

Conditions inimical to ducting are those which induce mixing of the lower atmosphere. Small scale atmospheric motions (turbulence) and consequent mixing and mass exchange result from differential surface heating and surface roughness effects. Both of these processes work to destroy stratification. They ultimately result in uniform vertical distribution of moisture through considerable depths of the lower atmosphere and the establishment of neutral temperature lapse rates. Accordingly, where the process of mechanical and convectively induced mixing are at work, the probability of the occurrence of ducting is vanishingly small. Thus, few, if any, ducts are observed over snow-free, low albedo land areas from midmorning to late afternoon when the skies are clear, or in areas of moderate to great surface roughness when the surface winds are more than a few meters per second, irrespective of cloud conditions or the time of the day.

\subsubsection{Refractive Conditions Due to Local Meteorological Phenomena}

Land and sea breezes may produce ducts along the coastal regions since the winds are of thermal origin, resulting from temperature differences between land and sea surfaces. During the day, when the land gets warmer than the sea, the air above the land rises and is replaced by air from the sea, thus creating a circulation from the sea to the land, called a sea breeze. During the night, the land becomes colder than the sea and a circulation, called a land breeze, is set up in the opposite direction. This type of circulation is generally shallow and does not extend higher than a few hundred meters above the land or sea surface.

A land or sea breeze may modify the refractive conditions in different ways depending upon the distribution of moisture in the lower layers. Since these breezes are of a local nature and generally extend only a few miles, only coastal locations are usually affected. The very nature of sea and land breezes results in a marked refractive index pattern. With a 
sea breeze a duct may be formed over the water due to subsidence. The land breeze is accompanjed by subsiding air over the land with resultant duct formation.

The formation of fog results in a decrease of superrefractive or ducting possibilities. When a fog forms by nocturnal radiation, the water content of the air remains practically the same; however, part of the water changes from the gaseous to the liquid phase thus reducing the vapor pressure. The resulting humidity lapse rate tends to counteract the temperature inversion and cause above standard refraction. However, the temperature inversion may be strong enough to keep the layer standard or superrefractive. This process may also occur with advection fogs.

The nighttime temperature profile is a result of the interaction between nocturnal radiation, turbulence, and heat conduction. The associated refractive index profiles are such that a radar duct begins to form about the time of sunset, developing quickly during the early evening, more slowly after midnight, and dissipating rapidly after sunrise. This is mainly an inland effect resulting from large diurnal temperature variations observed in the interiors of large continents. However, a shallow body of water may have an appreciable diurnal temperature variation, as compared to the open ocean so that superrefraction may occur over such a location from time to time.

It is generally recognized that radiosonde observations (RAOB's) do not have a sufflciently high degree of accuracy to be completely acceptable for use in observing changes in the degree of stratification of the very lowest layers of the atmosphere; however, until more accurate methods such as meteorological towers and refractometer measurements are more commonly used, the RAOB will continue to be used as a basis for forecasting the occurrence of superrefractive conditions.

\subsubsection{Background}

The property of the atmosphere basic to radio ray tracing is the radio refractive index of the atmosphere, $n$, which for VHF-UHF frequencies at standard conditions near the surface, is a number of the order of 1.0003 . Although the refractive index is used in ray tracing theory, it is more convenient when evaluating refraction effects from common meteorological observations to use the refractivity $N$, which, for the frequency range 0 to $30,000 \mathrm{Mc} / \mathrm{s}$, is given by $(1.20)$.

When evaluating the meteorological conditions that give rise to refractive phenomena, it is frequently instructive to examine separately the behavior of the dry component, $D$, and wet component, $W$, of $N$. 
The gradient of the refractivity, $\Delta N$, with respect to height may then be expressed:

$$
\Delta N=\Delta D+\Delta W
$$

Average values of $\Delta N, \Delta D$, and $\Delta W$ are given in table 4.6 for two increments between the earth's surface and $1 \mathrm{~km}$ above sea level for Fairbanks, Alaska; Washington, D.C.; and Swan Island, W.I.

TABLE 4.6. Gradient of N, D, and $\mathrm{W}$ in $\mathrm{N}$ units per kilometer

\begin{tabular}{|c|c|c|c|c|c|c|c|}
\hline \multirow{2}{*}{ Station } & \multirow{2}{*}{$\begin{array}{c}\text { Height } \\
\text { increment }\end{array}$} & \multicolumn{3}{|c|}{ February } & \multicolumn{3}{|c|}{ August } \\
\hline & & $-\Delta N$ & $-\Delta D$ & $-\Delta W$ & $-\Delta N$ & $-\Delta D$ & $-\Delta W$ \\
\hline Fairbanks, Alaska.... & $\begin{array}{l}\text { surface-0.5 km } \\
0.5 \mathrm{~km}-1.0 \mathrm{~km}\end{array}$ & $\begin{array}{l}37 \\
35\end{array}$ & $\begin{array}{l}41 \\
35\end{array}$ & $\begin{array}{r}-4 \\
0\end{array}$ & $\begin{array}{l}31 \\
36\end{array}$ & $\begin{array}{l}27 \\
24\end{array}$ & $\begin{array}{r}4 \\
12\end{array}$ \\
\hline Washington, D.C.. & $\begin{array}{l}\text { surface }-0.5 \mathrm{~km} \\
0.5 \mathrm{~km}-1.0 \mathrm{~km}\end{array}$ & $\begin{array}{l}41 \\
30\end{array}$ & $\begin{array}{l}34 \\
26\end{array}$ & $\begin{array}{l}7 \\
4\end{array}$ & $\begin{array}{l}60 \\
46\end{array}$ & $\begin{array}{l}28 \\
24\end{array}$ & $\begin{array}{l}32 \\
22\end{array}$ \\
\hline Swan 1sland, W.I..... & $\begin{array}{l}\text { surface }-0.5 \mathrm{~km} \\
0.5 \mathrm{~km}-1.0 \mathrm{~km}\end{array}$ & $\begin{array}{l}39 \\
58\end{array}$ & $\begin{array}{l}24 \\
24\end{array}$ & $\begin{array}{l}15 \\
34\end{array}$ & $\begin{array}{l}47 \\
66\end{array}$ & $\begin{array}{l}26 \\
24\end{array}$ & $\begin{array}{l}21 \\
42\end{array}$ \\
\hline
\end{tabular}

Several general observations may be made of the data of table 4.6: The gradient of the dry term is relatively less variable than that of the wet term when considered as a function of season or height; the increase of $\Delta N$ from winter to summer at a particular location or from aretic to tropical climate at a given time is most strongly reflected in $\Delta W$ rather than in $\Delta D$. The marked increase of gradient with height for Swan Island reflects the drop of refractivity across the interface of the trade wind inversion where dry subsiding air overlies the moist oceanic surface layer.

A fundamental equation used in radio ray tracing is Snell's law, which, for polar coordinates, is given in chapter 3 as

$$
n r \cos \theta=n_{0} r_{0} \cos \theta_{0}
$$

where $n$ is the radio refractive index of the atmosphere, $r$ is the radial distance from the center of the earth to the point under consideration, and $\theta$ is the elevation angle made by the ray at the point under consideration with the tangent to the circle of radius $r$ passing through that point. The radius to any point, $r$, is equal to $(a+h)$, where $a$ is the radius of the earth and $h$ is the height of the point above sea level. The zero subseript refers to the value of $n, r$, or $\theta$ at the earth's surface.

For the present study, geometrical opties techniques, similar to those considered by Bremmer [32], are used to indicate when refraction is 
sufficiently great to direct a ray either back to earth or in a eircular path at a constant height above the earth; i.e.:

$$
\frac{n_{0} r_{0} \cos \theta_{0}}{n r} \geq 1
$$

This condition then allows one to obtain the value of $\theta_{0}$ which divides the rays into two groups: those that penetrate the duct and those that are trapped within the duct. This particular value of $\theta_{0}$, called the angle of penetration and designated $\theta_{p}$, is obtained by noting those instances where

$$
\frac{n_{0} r_{0}}{n r} \geq 1
$$

and solving for the value of $\theta_{0}$ such that (4.13) is equal to unity.

It is instructive to consider the order of magnitude of refractive index gradient needed for trapping for several commonly observed refractive index profiles. If we rewrite Snell's law,

$$
n_{t} r_{t} \cos \theta_{t}=n_{A} r_{A} \cos \theta_{d}
$$

where the subscripts $t$ and $A$ refer to the values of the variables at the transmitter height and the top of the trapping layer, then trapping occurs when

$$
\frac{n_{t} r_{t}}{n_{A} r_{A}} \geq 1
$$

and the angle of penetration at the transmitter, $\theta_{p}$, is given by setting

$$
\frac{n_{t} r_{t}}{n_{A} r_{A}} \cos \theta_{p}=1
$$

The maximum permissible $n$ gradient for a given value of $\theta_{p}$ is then given by:

$$
\frac{\Delta n}{\Delta r}=\frac{n_{t}-n_{A}}{r_{A}-r_{t}}
$$

where $n_{A}$ must satisfy $(4.17)$; 1.e.:

$$
n_{A}=\frac{n_{t} r_{t}}{r_{A}} \cos \theta_{p}
$$


By designating $r_{A}=r_{t}+h_{A}$, (4.18) becomes

$$
\frac{\Delta n}{\Delta r}=-\frac{n_{t}}{h_{A}}\left[1-\frac{r_{t}}{r_{t}+h_{A}} \cos \theta_{p}\right]
$$

By rewriting (4.20)

$$
\frac{\Delta n}{\Delta r}=-\frac{n_{t}}{h_{A}}\left[1-\frac{1}{1+\frac{h_{A}}{r_{t}}} \cos \theta_{p}\right],
$$

and expanding $\left(1+h_{A} / r_{t}\right)^{-1}$ and $\cos \theta_{p}$, one obtains the expression:

$$
\frac{\Delta n}{\Delta r}=-n_{t}\left[\frac{1}{r_{t}}+\frac{\theta_{p}^{2}}{2 h_{A}}\right]
$$

by neglecting terms $\theta_{p}^{4} / 4$ ! and $\left(h_{A} / r_{t}\right)^{2}$, and terms beyond these. For the case of $\theta_{p}=0,(4.22)$ reduces to:

$$
-\frac{\Delta n}{\Delta r}=\frac{n}{a} \sim \frac{1}{a} \sim 157 \mathrm{~N} \text { units } / \mathrm{km}
$$

It is seen from (4.22) that the $n$ gradient necessary to trap a radio ray at a given value of $\theta_{p}$ is practically independent of transmitting antenna height above the earth. For example, a $\theta_{p}=0$ ray will be trapped by an $n$ gradient of $-157.0 \mathrm{~N}$ units $/ \mathrm{km}$ at sea level, where $n_{t} \cong 1.0003$, while the necessary $n$ gradient at $3 \mathrm{~km}$ above sea level will be -156.9 $N$ units $/ \mathrm{km}$ for an $n_{t} \cong 1.0002$. This indicates, for all practical applications, that the necessary $n$ gradient for trapping is independent of altitude. Further, by considering the temperature and humidity gradients encountered in the troposphere, one is led to the conclusion that ducting gradients would not be expected to occur at altitudes greater than $3 \mathrm{~km}$. In fact, Cowan's [33] investigation indicates that trapping gradients are nearly always confined to the first kilometer above the surface.

A consideration of (4.22) indicates that the magnitude of the negative gradient necessary for ducting is $1 / a$ for $\theta_{p}=0$ but increases in proportion to $\theta_{p}{ }^{2} / 2 h$ as $\theta_{p}$ increases. The gradients necessary for atmospheric ducts as a function of $\theta_{0}$ are given for several different profiles in figure 4.35 An analysis of radiosonde data indicates that gradients in excess of $0.5 \mathrm{~N}$ units per meter are seldom exceeded within atmospheric layers. It is interesting to note how rapidly the necessary gradients increase to 


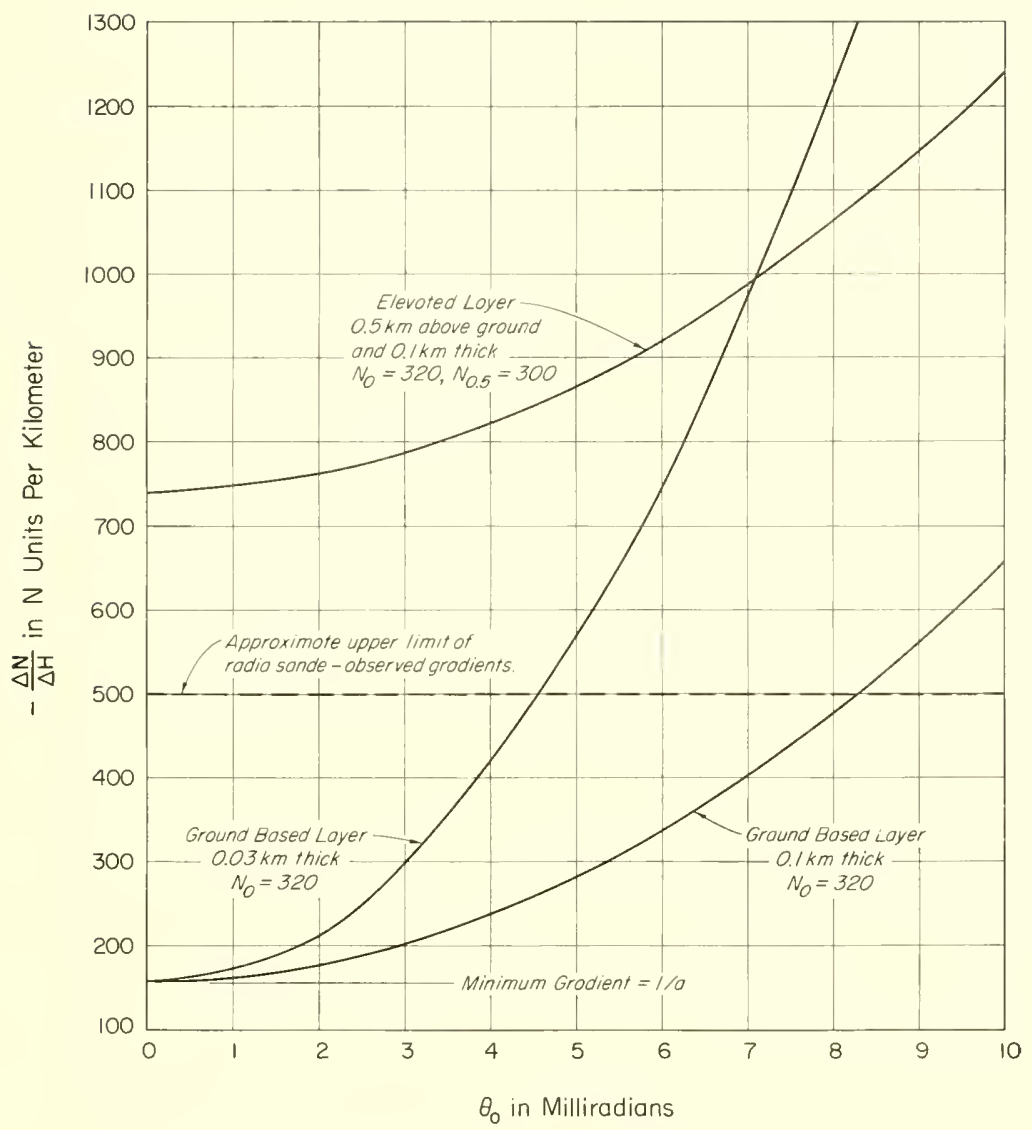

FIgURE 4.35. Refractivity gradients needed for radio ducts.

the approximate upper limit of radiosonde-observed gradients: a groundbased layer $100 \mathrm{~m}$ thick attains this gradient at $8.3 \mathrm{mrad}$ while the maximum observed gradient is intercepted by the $30-\mathrm{m}$ layer curve at $\theta_{0}=4.5 \mathrm{mrad}$. A third example was calculated for an elevated layer 0.5 $\mathrm{km}$ above the ground and $100 \mathrm{~m}$ thick by assuming normal refraction between the ground and the base of the layer and solving for the necessary ducting gradient within the layer. The large values of $n$ gradient necessary for this case explain why elevated ducts were not observed. Although the preceding examples were calculated for a ground transmitter, the combinations of $\theta_{p}, \Delta n / \Delta r$, and $\Delta r$ are very nearly the same as would be obtained for any other transmitter height within the first $3 \mathrm{~km}$ above the surface. 


\subsubsection{Description of Observed Ground-Based Atmospheric Ducts}

Approximately three years of radiosonde data typical of an arctic climate (Fairbanks, Alaska), a temperate climate (Washington, D.C.), and a tropical maritime climate (Swan Island, W.I.) were examined by means of a digital computer for the occurrence of ducts during the months of February, May, August, and November. The percentage occurrence of ducts is shown on figure 4.36. The maximum occurrences of 13.8 percent for August at Swan Island and 9.2 percent for Fairbanks in February are significantly greater than the values observed at other times of the year. The Washington data display a summertime maximum of 4.6 percent. These data indicate that the temperate zone maximum incidence is about one-half the wintertime maximum incidence in the aretic, and about one-third of the summertime tropical maximum.

The range of observed values of $\theta_{p}$ is shown in figure 4.37. The mean value calculated for each month as well as the maximum and minimum values of $\theta_{p}$ observed for the limiting cases are given for each month and location. The mean value of the angle of penetration under these conditions is between 2 and $3 \mathrm{mrad}$ and appears to be independent of climate. The maximum value of $\theta_{p}$ observed during ducting is $5.8 \mathrm{mrad}$.

The refractivity gradients observed during ducting are given on figure 4.38. The maximum gradient of $420 \mathrm{~N}$ units per kilometer was observed during February at Fairbanks, Alaska. The mean values of $N$ gradient appear to follow a slight climatic trend from a high value of $230 \mathrm{~N}$ units per kilometer at Fairbanks to a value of $190 \mathrm{~N}$ units per kilometer at Swan Island.

Another property of radio ducts is their thickness, which is given in figure 4.39. Again there is observed a slight climatic trend as the median thickness increases from $66 \mathrm{~m}$ at Fairbanks to $106 \mathrm{~m}$ at Swan Island. These values of thickness correspond to the gradients given in figure 4.38. One can then obtain, by linear extrapolation, the thickness at which the gradient is equal to $-1 / a$; i.e., the height corresponding to the gradient just sufficient to trap the ray at $\theta_{0}=0$. These values, shown on figure 4.40 , display an increase in the median thickness of about 25 percent for Swan Island, 100 percent for Washington, and 200 percent for Fairbanks, which results in a reversal of the climatic trend of the observed thickness between Fairbanks and Swan Island. This increase in height emphasized the preceding conclusion. Fairbanks is characterized by shallow layers with relatively intense gradients.

These maximum duct widths may be used to estimate the maximum radio wavelengths trapped. Kerr [34] gives the maximum wavelength, $\lambda$, trapped by given thickness, $d$ :

$$
\lambda_{\max }=c \gamma^{1 / 2} d^{3 / 2}
$$




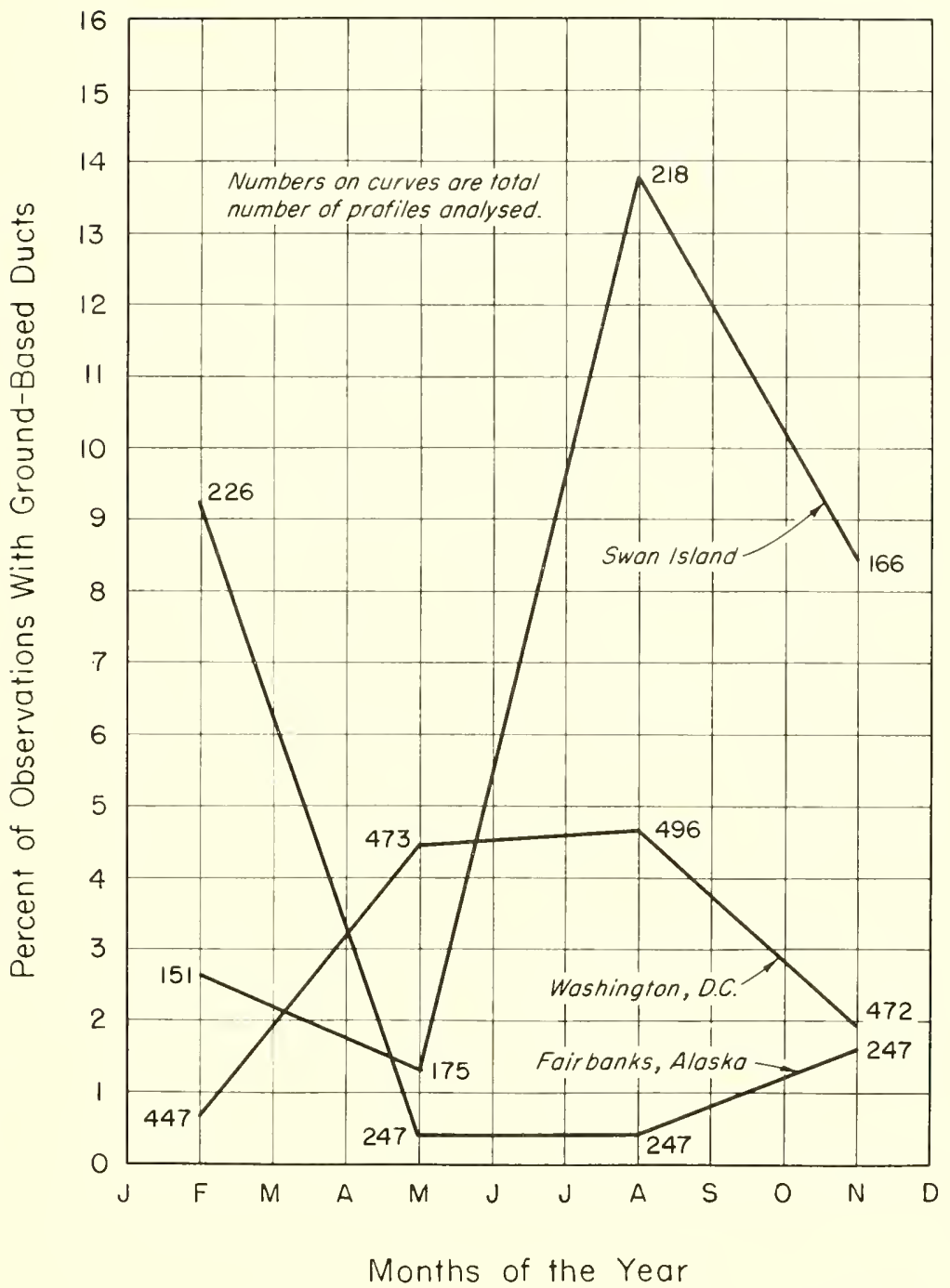

Figure 4.36. Frequency of occurrence of ground-based ducts.

by assuming a linear decrease of $n$ within the duct. The coefficient $c$ is a constant and $\gamma$ is a function of the $n$ gradient excess over the minimum value of $\Delta n / \Delta h=1 / a$. Expressing $\lambda_{\max }$ in centimeters, and $d$ in meters, then

$$
\gamma=\left(\frac{N_{t}-N_{d}}{d}-0.157\right) 10^{-6} .
$$


and

$$
c=2.514 \times 10^{2} .
$$

The maximum wavelengths trapped during ducting conditions were estimated by (4.24) for the maximum duct thicknesses of figure 4.40. These values, given in table 4.7 , were determined for the month with the maximum occurrence of ducts, thus allowing an estimate of the radio frequencies likely to be affected by ducting conditions. Note, for example, that the data of table 4.8 indicate that $1000 \mathrm{Mc} / \mathrm{s}$ rays $(\lambda=30 \mathrm{~cm})$ will be trapped by 50 percent of the ducts regardless of location.
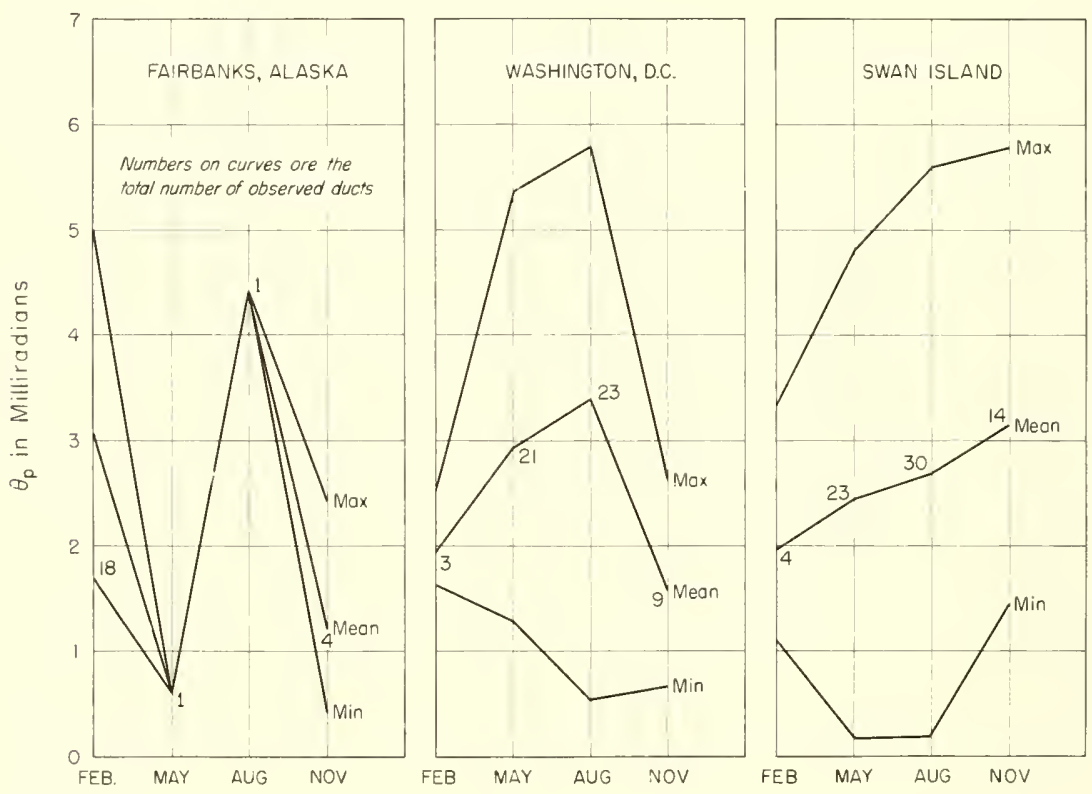

Months of the Year

FIGURE 4.37. Angle of penetration of ground-based ducts. 

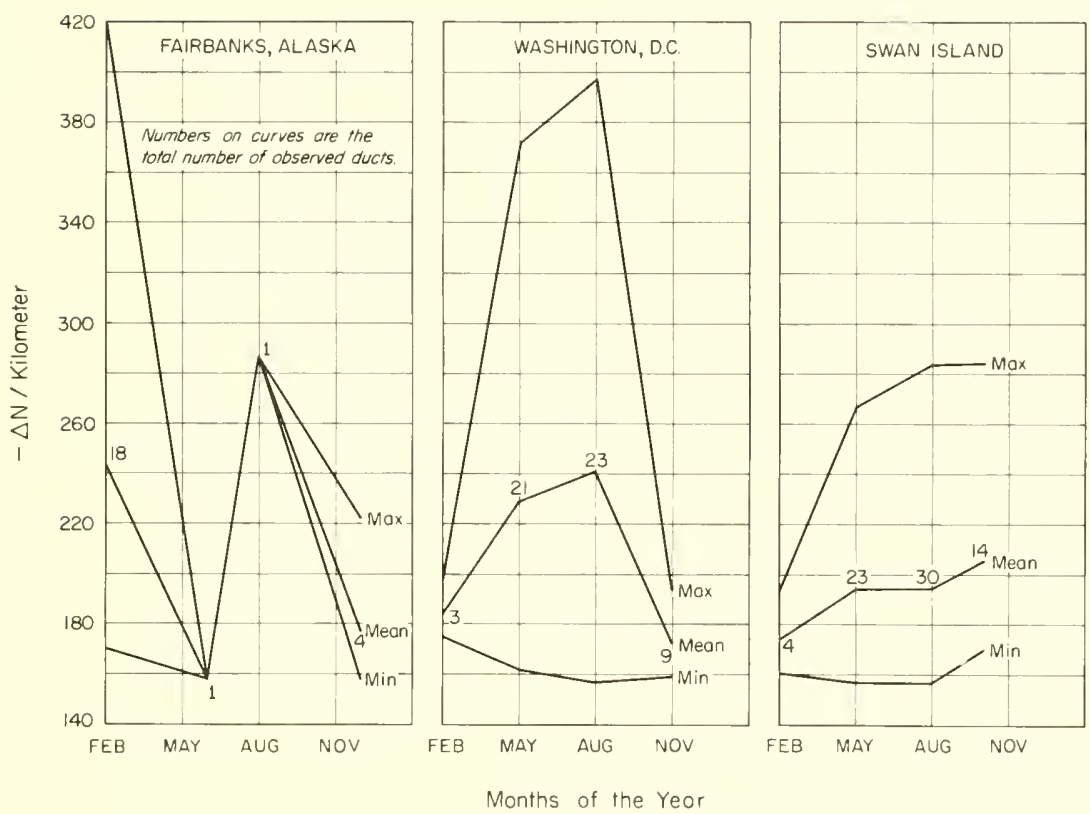

Figure 4.38. Refractivity gradients of ground-based ducts.

TABLE 4.7. Estimated maximum wavelength trapped at $\theta_{0}=0$

\begin{tabular}{|c|c|c|c|c|c|c|c|}
\hline \multirow{2}{*}{ Station } & \multicolumn{7}{|c|}{$\begin{array}{c}\text { Percentage of ducts that trap wavelengths in centimeters equal to } \\
\text { or shorter than tabulated value }\end{array}$} \\
\hline & 95 & 90 & 75 & 50 & 25 & 10 & 5 \\
\hline $\begin{array}{l}\text { Fairbanks, Alaska (February). } \\
\text { Washington, D.C. (August) } \\
\text { Swan Island (August) }\end{array}$ & $\begin{array}{c}20.5 \\
7 \\
12\end{array}$ & $\begin{array}{l}22.6 \\
10 \\
23\end{array}$ & $\begin{array}{l}25.5 \\
27 \\
41.5\end{array}$ & $\begin{array}{l}34.0 \\
50 \\
60\end{array}$ & $\begin{array}{r}43.5 \\
112 \\
82.3\end{array}$ & $\begin{array}{c}61.5 \\
164 \\
92\end{array}$ & $\begin{array}{l}69.0 \\
200 \\
132\end{array}$ \\
\hline
\end{tabular}

TABLE 4.8. Ranges of surface ducting gradients

\begin{tabular}{|c|c|c|c|c|}
\hline $\begin{array}{l}\text { Duct gradients } \\
\text { at Washington, D.C. }\end{array}$ & $\underset{\text { February }}{N \text { units/km }}$ & $\underset{\text { May }}{N \text { units } / \mathrm{km}}$ & $\begin{array}{c}N \text { units } / \mathrm{km} \\
\text { August }\end{array}$ & $\begin{array}{l}\text { Nunits/km } \\
\text { November }\end{array}$ \\
\hline $\begin{array}{l}\text { Max } \\
\text { Mean } \\
\text { Min }-1\end{array}$ & $\begin{array}{l}-200 \\
-185 \\
-175\end{array}$ & $\begin{array}{l}-372 \\
-230 \\
-162\end{array}$ & $\begin{array}{l}-395 \\
-240 \\
-158\end{array}$ & $\begin{array}{l}-195 \\
-170 \\
-159\end{array}$ \\
\hline
\end{tabular}




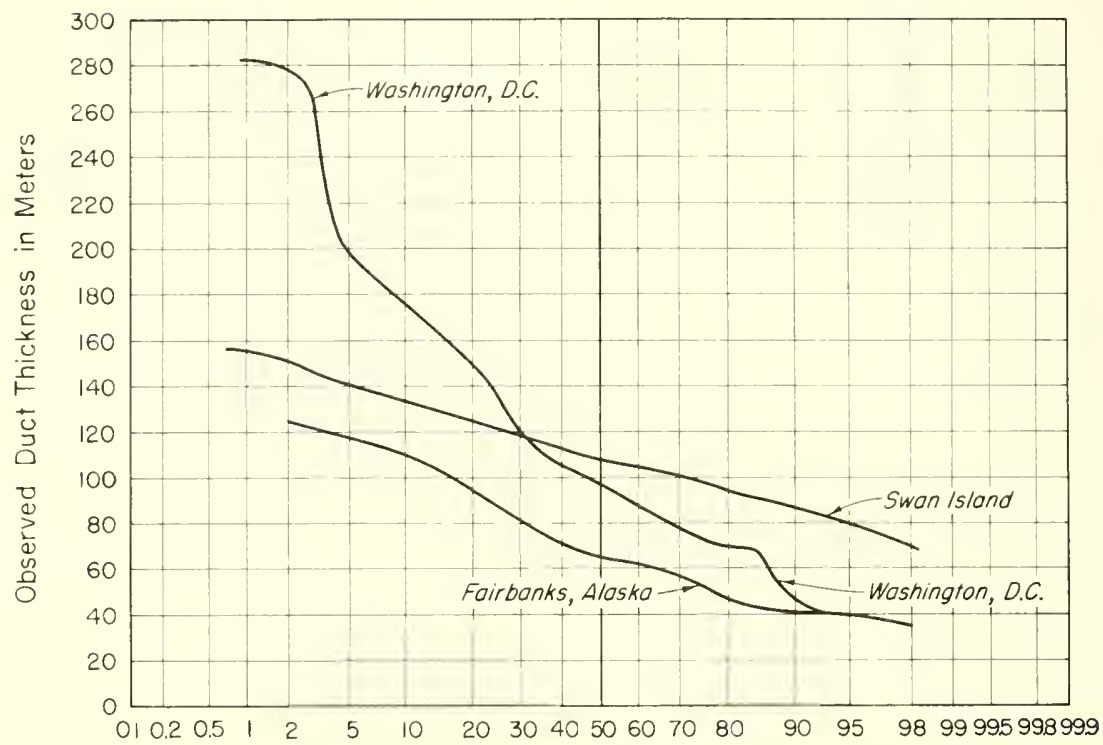

Percentage of Observations That Equal or Exceed the Ordinate Value

FIgURE 4.39. Observed ground-based duct thickness.

The reader is cautioned that an atmospheric duct does not have the sharp boundaries of a metallic waveguide. Thus the maximum wavelengths obtained by (4.24) do not represent cutoff frequencies but, as Kerr is so careful to emphasize, merely suggest lower limits under the assumptions of this rudimentary theory.

The results given above were derived from a consideration of radiosonde data. Although the radiosonde is not an extremely sensitive instrument, it is readily available and is the only source of climatic information involving the temperature and humidity structure of the atmosphere. It is believed that the radiosonde data will at least yield the climatic trend of radio ducts as well as their probable temperature and humidity distributions. Further, it is evident that the choice of stations will definitely affect the percentage of ducts observed. For example, it is almost certain that a greater percentage of ducts would be observed over water in the subtropics than over Swan Island. 
With these reservations in mind, the present study has shown:

(a) Ducts occur no more than 15 percent of the time.

(b) The annual cycle of the incidence of ducts is reversed for the aretic and tropical stations studied. The aretic station has a wintertime maximum and the tropic station a summertime maximum. The temperate station has a summertime maximum incidence of less than 5 percent.

(c) The maximum initial elevation angle is limited to $5.8 \mathrm{mrad}$ with a mean value of about $3 \mathrm{mrad}$.

(d) The steepest gradient of $N$ observed is $-420 \mathrm{~N}$ units per kilometer.

(e) The maximum thickness of observed ducts is such as to trap radio waves of $1000 \mathrm{Mc} / \mathrm{s}$ and above at all locations for at least 5 percent of the observed ducts.

(f) Ducts in the arctic appear to be associated with temperature inversions at ground temperatures of $-25{ }^{\circ} \mathrm{C}$ or less; temperate zone, with the common radiation inversion and accompanying humidity lapse; tropics, with a moderate temperature and humidity lapse for temperatures of about $30^{\circ} \mathrm{C}$.

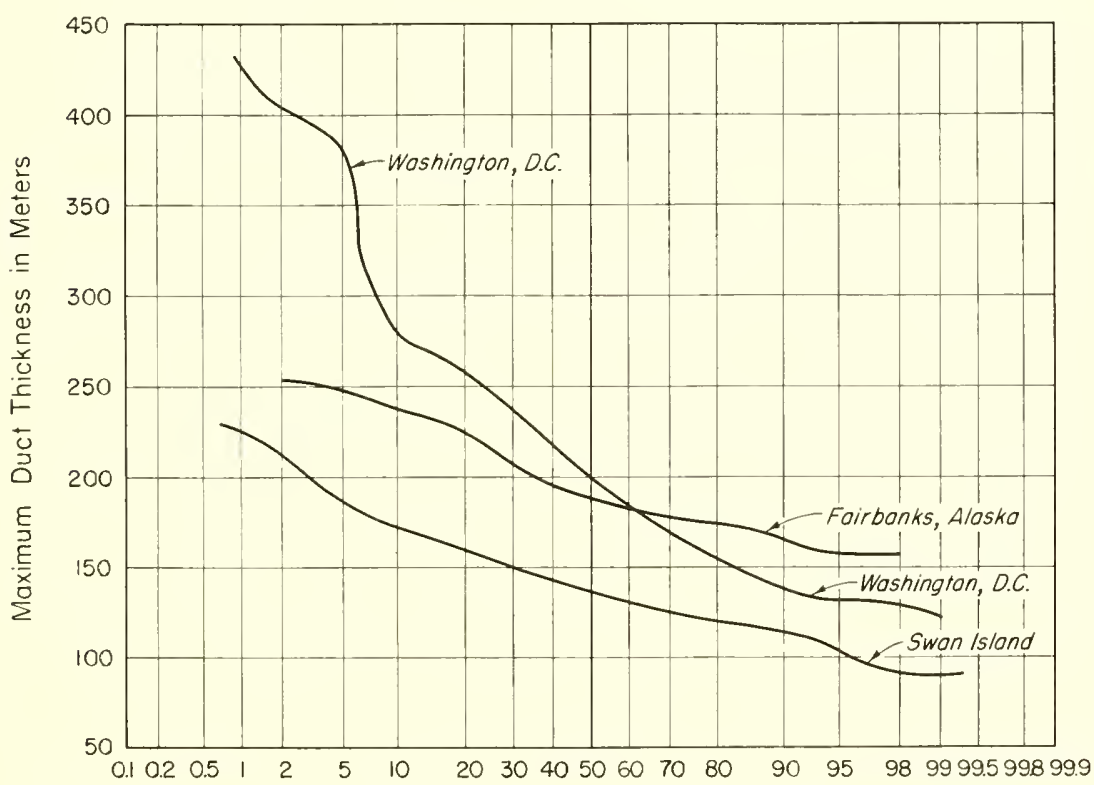

Percentage of Observations That Equal or Exceed the Ordinate Value

FigUre 4.40. Maximum ground-based duct thickness. 


\subsection{A Study of Fading Regions Within the Horizon Caused by a Surface Duct Below a Transmitter}

\subsubsection{Introduction}

Among the factors influencing the choice of an antenna site for a microwave receiver operating on a line-of-sight path is the desirability of locating in a region relatively free from space-wave fadeouts. Although line-of-sight propagation is normally characterized by steady, high, dependable signals, deep, prolonged space-wave fadeouts are observed from time to time. Since serious disruptions occur during such fadeouts, a systematic discussion of fading phenomena is of considerable interest to the radio circuit engineer.

There are [35] results in detail of a year's study of signal fadeouts occurring at certain points within the horizon caused by ground-based superrefractive layérs. The path studied in this case [35] has its transmitter at Cheyenne Mountain near Colorado Springs, Colo., at an elevation of $8800 \mathrm{ft}$. Receivers are at Kendrick, Colo., $49.3 \mathrm{mi}$ from the transmitter, and at an elevation of $5260 \mathrm{ft}$; Karval, Colo., $70.2 \mathrm{mi}$ from the transmitter, and at an elevation of $5260 \mathrm{ft}$; and Haswell, Colo., 96.6 mi from the transmitter, and at an elevation of $4315 \mathrm{ft}$. A study of the profile of this path reveals that Kendrick is well within the radio horizon, Karval is near, but still witnin the radio horizon, and Haswell is beyond the radio horizon.

The Cheyenne Mountain study found that throughout the period of observation, fadeouts (of $5 \mathrm{~dB}$ or more) occurred regularly at Karval on a frequency of $1046 \mathrm{Mc} / \mathrm{s}$, in conjunction with superrefractive $N$-profile conditions, and coincided with enhanced field strengths at Haswell: A particular instance of this, the night of June 21-22, 1952, is shown in figures 4.53 and 4.54. Figure 4.53 shows the field strengths recorded simultaneously in a 12-hr period at Haswell, Karval, and Kendrick. Here it is to be noted that with the sudden intense rise in field strength at Haswell, a progressively deepening fadeout appears in the Karval data relative to the monthly median, while only insignificant changes are noted at Kendrick. Meanwhile, figure 4.54 indicates the shift in the refractivity profile at Haswell (presumably the same for Kendrick and Karval) towards conditions of superrefraction throughout the evening of 21-22 June, 1952.

The great number of fadeouts at Karval as compared to Kendrick strongly suggested a dependence of fading on distance from the transmitter within the horizon. The following sections describe the conditions under which fading, variations in fading, and locations that favor the occurrence of fading within the radio horizon (in the presence of a groundbased duct) occur. 
Fading, or fadeout, of a radio wave may be defined as a drop in power or field strength below a specified level of intensity. For a given site, criteria may be set up by the communications engineer to establish the magnitude of drop that defines the onset of fading conditions. This magnitude usually ranges from about 5 to $30 \mathrm{~dB}$ below the specified level of intensity. The specified level is derived from some ideal propagation condition.

Fading is also a time-dependent occurrence. It may be classified as (a) prolonged fading, which is fading of sufficient interval to cause continued communication disruptions, and (b) short-term Rayleigh fading, which is only instantaneously observed by a receiver.

One of the main causes of decp fading of field strength within the horizon as compared with the free space field strength value is the $N$ structure of the atmosphere. Theoretically, the atmosphere can be considered horizontally homogeneous and in spherical stratifications concentric with the earth, and $N$ can be considered to decrease exponentially with increasing height above the earth's surface. However, in reality, this picture of the atmosphere is rarely, if ever, realized because of the synoptic meteorological conditions that are perpetually present. Stratifications caused by the synpotic meteorological pattern give rise to field strength fading within the horizon by defocusing the lobe pattern of the transmitter along a given path. Whenever the rate of change of refractivity from the surface value with height (called the gradient of $N$ with height) is less than $-157 \mathrm{~N}$ units per kilometer, a "ducting" condition is said to exist at the surface, and, as shown in figure 4.35, certain rays will tend to be "trapped" or guided within the surface duct. It is this atmospheric condition of surface ducting which will be further explored herein with respect to fading within the radio horizon.

It should be realized that not all within-the-horizon fading has been attributed to refractivity gradient discontinuities in the lower atmosphere. Misme [36] shows the influence of frontal effects and frontal passage on signal fading within the horizon, the frontal passage even occurring at a time when one would most expect a fadeout caused by a surface duct.

\subsubsection{Regions and Extent of Fading Within the Horizon in the Presence of Superrefraction}

Serious disruptions in reception from a transmitter above a duct to a receiver within a duct can occur at particular points within the horizon. It is these disruptions, and these locations, which are of interest to the communications engineer establishing a given transmitting-receiving path. Whenever these disruptions occur between a transmitter and receiver within the horizon, a corresponding increase in the field strength characterized by steady, high, dependable signals, is usually expected 
beyond the horizon. The high signal strength is in keeping with the properties of a surface duct. Nevertheless, deep, prolonged fadeouts can occur in regions beyond the horizon as well as within the horizon.

Price [37] has theoretically determined regions of deep radio fading associated with surface ducts, which he has termed "shadow zones." In the case of a transmitter above a surface duct, a representation of what occurs is shown in figure 4.43. It shows the location of a shadow zone above the radio horizon line in the normal interference region. In the interference region, a fadeout of signal strength due to the presence of superrefraction must be compared with the value of the field when only the interference pattern is present. Papers such as those by Norton [38] and Kirby, Herbstreit, and Norton [39] give methods for calculation of the normal interference field. Ikegami [40] gives a more general method for the calculation of received power in the presence of ground based ducts.

Ikegami's procedure is based on a simple geometrical optics ray-tracing technique of determining the power relative to free space transmission that is received at different locations from the transmitter. A more refined, yet more complex, procedure is the field-strength calculations along the lines followed by Doherty [41], in which a strict mode-theory treatment of the problem reveals that geometrical optics is not sufficient in the presence of refraction anomalies. Doherty expands techniques originally considered by Airy [42] to determine relative field strengths in the neighborhood of caustics (apparent ray intersections) that result vicinal to refraction anomalies such as ducts.

In the procedure that follows, a model for the determination of the location and extent of shadow zones, rather than the determinations of the actual received field strengths and powers at any particular point, will be given for conditions typical of the temperate climate to aid the radio circuit engineer in avoiding these troublesome interference areas.

Washington, D.C., is taken to represent an average temperate zone climate. Fairly extensive work has been done in determination of various ducting conditions at this station as well as with the average exponential refractivity above this station. Therefore, Washington, D.C., will be used as a model for all following calculations.

The gradient of $N$ with height determines whether or not a surface duct will exist. Therefore, a surface ducting atmosphere corresponding to conditions at Washington, D.C., consisting of a ducting gradient up to any desired height, and the $N_{s}=313.0$ exponential atmosphere [9] above this height, have been chosen for this study. The ducting gradients chosen are maximum, mean, and minimum values for February, May, August, and November (representing the middle of winter, spring, summer, and fall, respectively) taken from figure 4.38 and given in table 4.8. Also in this particular model the duct is assumed to be of uniform height throughout the region considered in the calculations. 


\subsubsection{Theory and Results}

In figure 4.41, although it is known that ray theory does not explain what happens to the radio ray at the point $A$ when the ray is tangent to the top of the duct, because of the apparent ray intersection, it is sufficient to define the first shadow zone as that zone between the two possible paths of the ray tangent to the duct at a point $A$ (this is the ray emitted from the transmitter with an initial angle of $\theta_{0}$ with the horizontal). This is possible because it can be readily seen that no radiation will be able to enter the shadow zone, and thus a receiver located in the shadow zone will theoretically receive no signal from the transmitter. The portion of the "split" ray that enters the duct at $A$ will be reflected at point $B$ to point $C$, where it "splits" again. The distance, $d$ from point $A$ to $C$ projected along the earth's surface will be designated the "length" of the first shadow zone. If the surface duct is homogeneous throughout, the ray will be symmetrical about point $B$ and, therefore,

$$
d_{1}=d_{2}=d / 2=d_{h}
$$

where $d_{1}$ and $d_{2}$ are the "half-lengths," $d_{h \iota}$, of the shadow zone.

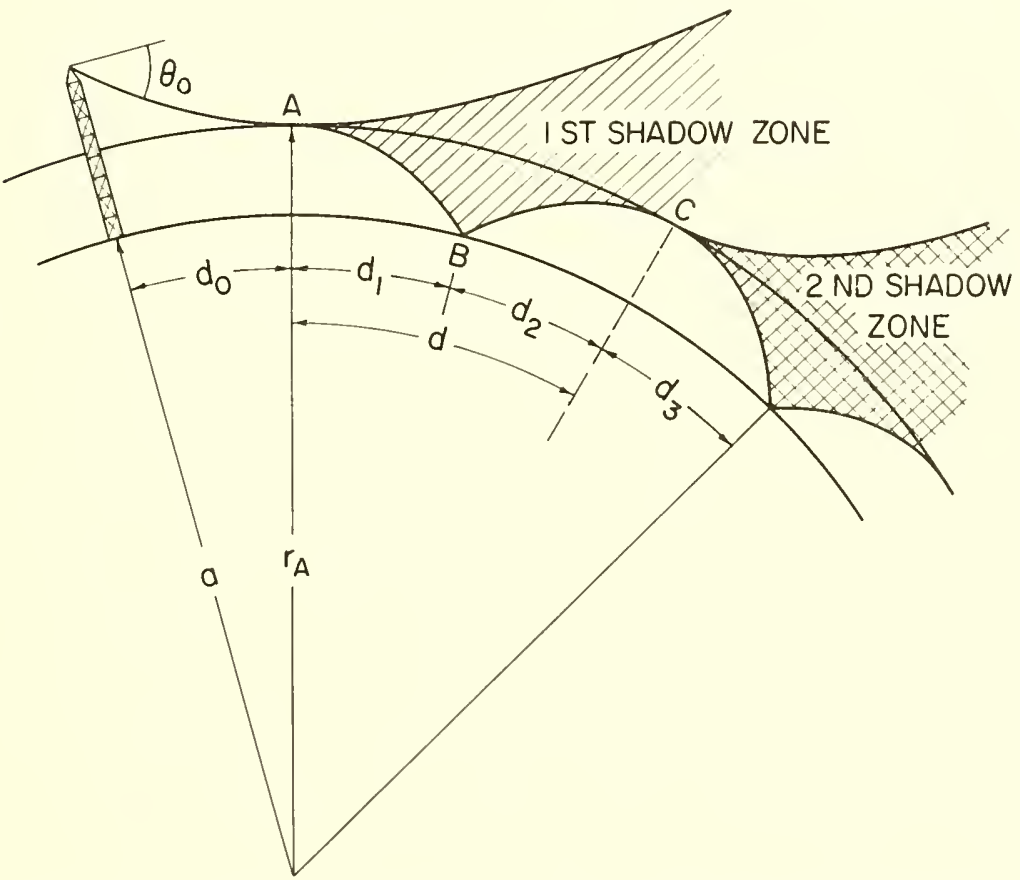

Figure 4.41. Shadow zone occurrence of a curved earth with the transmitler above the duct. 
Chapter 3 shows that the bending, $\tau_{1,2}$, of the radio wave between any two points in the atmosphere is given by

$$
\tau_{1,2}=\frac{d_{1,2}}{a}+\left(\theta_{1}-\theta_{2}\right)
$$

where $d_{1,2}$ is the distance between the two points 1 and 2 (see fig. 4.42) projected along the earth's surface, $a$ is the radius of the earth, and $\theta_{1}$ and $\theta_{2}$ are the angles in radians the ray makes with the horizontal at points 1 and 2 respectively. As described in chapter 3, Schulkin [43] obtained $\tau_{1,2}$ in radians as

$$
\tau_{1,2}=\frac{\Delta n_{1}-\Delta n_{2}}{\theta_{m}} \text { for } 0 \leq \theta_{0} \leq 10^{\circ}
$$

where $\Delta n_{1}=n_{1}-1, \Delta n_{2}=n_{2}-1, \theta_{m}=\left(\theta_{1}+\theta_{2}\right) / 2$. In terms of refractivity, (4.28) becomes

$$
\tau_{1,2}=\frac{\left(N_{1}-N_{2}\right) \times 10^{-6}}{\theta_{m}}=\frac{\Delta N \times 10^{-6}}{\theta_{m}}
$$

Considering now the case of the half-length, $d_{h \iota},(4.27)$ becomes

$$
\tau_{1,2}=\frac{d_{h \ell}}{a}+\theta_{p}
$$

since $\theta_{2}=0$ at the top of the duct, and $\theta_{1}=\theta_{p}$. Now substituting (4.29) in $(4.30)$,

$$
\frac{2 \Delta N \times 10^{-6}}{\theta_{p}}=\frac{d_{h \ell}}{a}+\theta_{p}
$$

since here $\theta_{m}=\theta_{p} / 2$. Rearranging (4.31)

$$
d_{h \ell}=a\left[\frac{2 \Delta N \times 10^{-6}}{\theta_{p}(\mathrm{rad})}+\theta_{p}(\mathrm{rad})\right] .
$$

Since

$$
\begin{aligned}
& \theta_{p}=\cos ^{-1} \frac{n_{A} r_{A}}{n_{0} a} \cong \sqrt{2\left[\Delta N \times 10^{-6}-\frac{\left(r_{A}-a\right)}{a}\right]} \\
& (\mathrm{rad})
\end{aligned}
$$




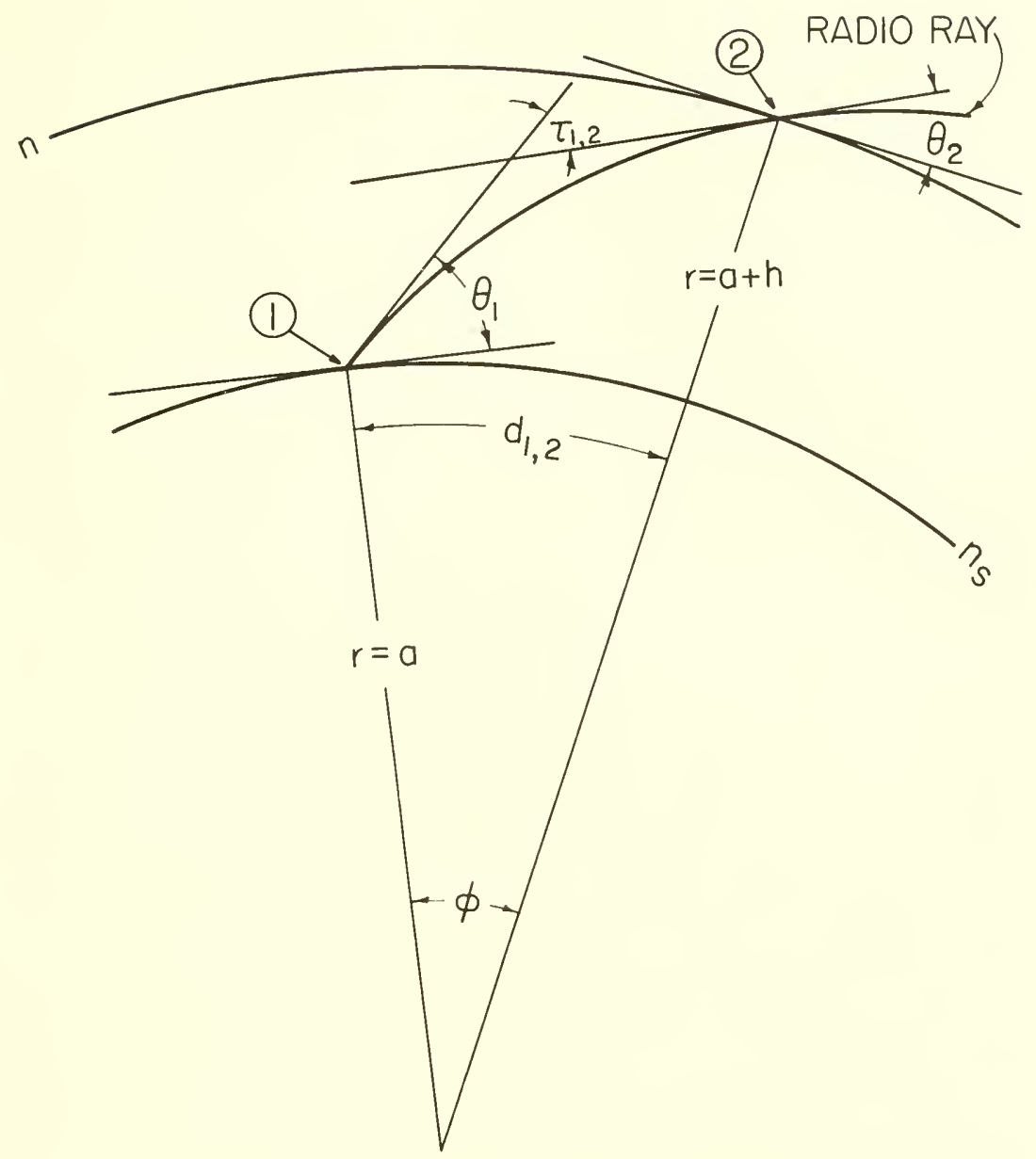

Figure 4.42. Geometry of radio ray refraction.

where $n_{A}$ is the index of refraction at the top of the duct, $r_{A}$ is the distance from the center of the earth to the top of the duct, and $n_{0}$ is the index of refraction at the earth's surface, it is seen that $d_{h \ell}$ is uniquely determined from the surface gradient of $N$ with height inside the duct, provided the height of the duct is known.

If one desires to know the distance, $d_{h}$, for a particular height, $h$, from the reflection point of the ray in the duct (point $B$ in fig. 4.41), where $d_{h}$ is such that 
DIST SHADOW ZONE

DIRECT AND REFLECTED RAY

INTERFERENCE REGION

ZX. ZONE BEYOND THE HORIZON

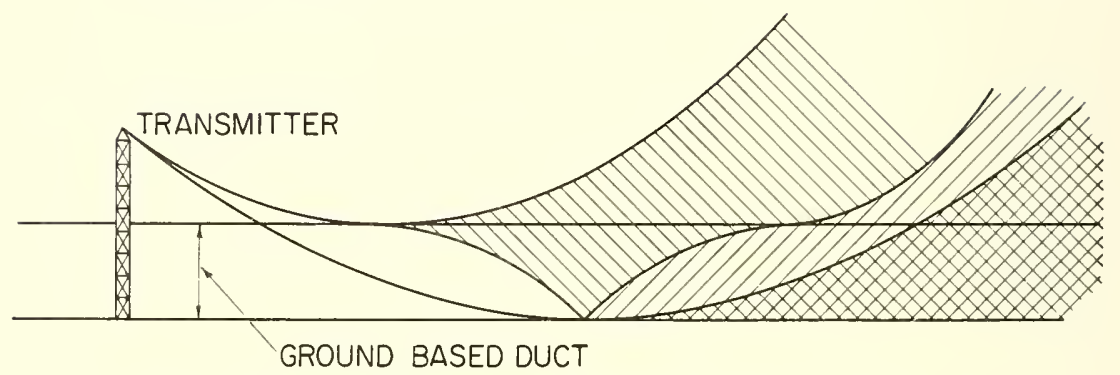

FIGURE 4.43. Regions of fading from different effects of the radio rays.

then (4.37) becomes

$$
d_{h}=a\left[\frac{2 \Delta N^{\prime} \times 10^{-6}}{\theta_{p}+\theta_{2}}+\left(\theta_{p}-\theta_{2}\right)\right]
$$

since $\theta_{2}$ is no longer just equal to zero, but is such that

$$
0 \leq \theta_{2} \leq \theta_{p}
$$

and $\Delta N^{\prime}$ will no longer be the same as the $\Delta N$ for the duct width. The value of $\theta_{2}$ may be determined from the formula:

$$
\theta_{2} \cong \sqrt{\theta_{p}^{2}+\frac{2 h}{a}-2 \Delta N^{\prime}}
$$

In figures 4.44 to 4.46 the shadow zone half-lengths are given as a function of duct height for the various seasons of the year. As shown in figure 4.39, the 50 percent level, or median of observed ground-based duct thickness at Washington, D.C., is about $100 \mathrm{~m}$. Figure 4.40 shows that the percent level of observed maximum ground-based duct thickness is about 200 for Washington, D.C. In table 4.9, the values of $d_{h}$ during the four seasons are shown for the 100- and 200-m surface duct heights.

As previously mentioned, the atmosphere above the assumed duct was taken to coincide with the $N_{s}=313.0$ exponential atmosphere [9] because this value most nearly corresponds to the average annual $N_{s}$ value at 


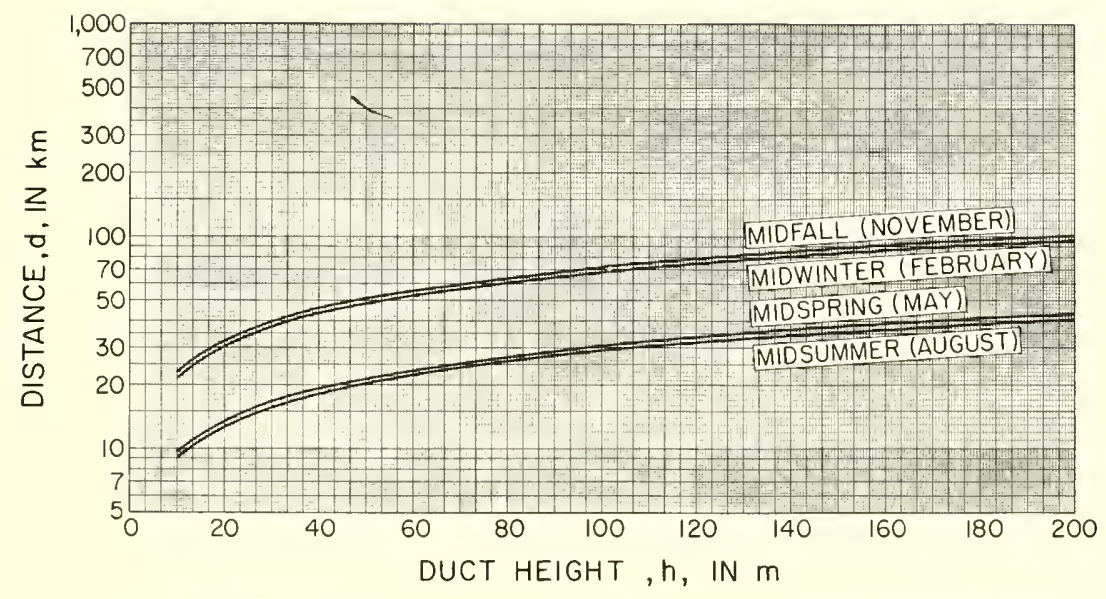

FIGURE 4.44. Shadow zone half-lengths for maximum ducting conditions at Washington, D.C.

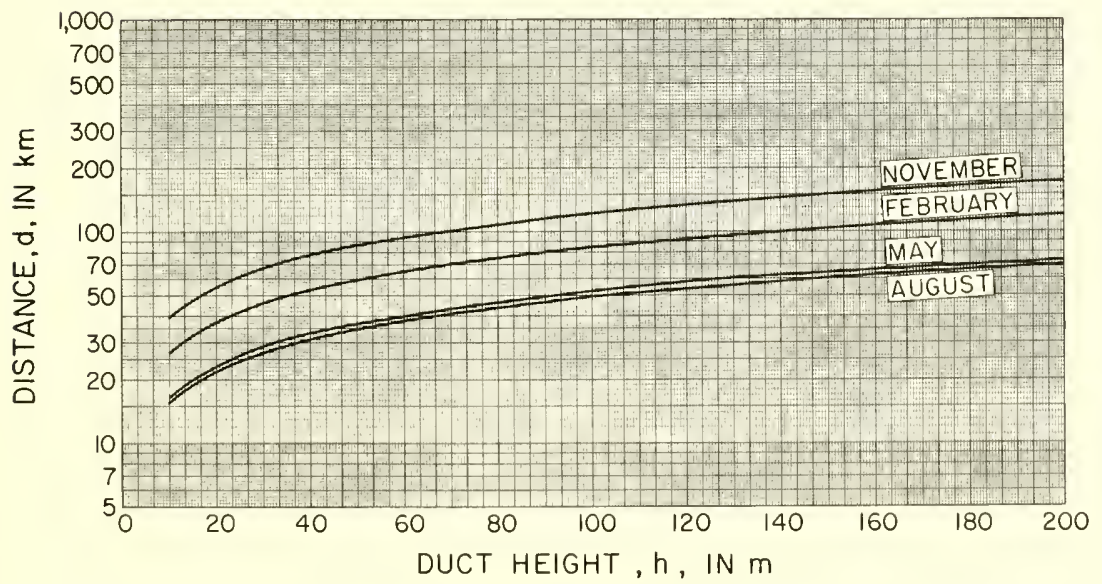

FIgURE 4.45. Shadow zone half-lengths for mean ducting conditions at Washington, D.C. 


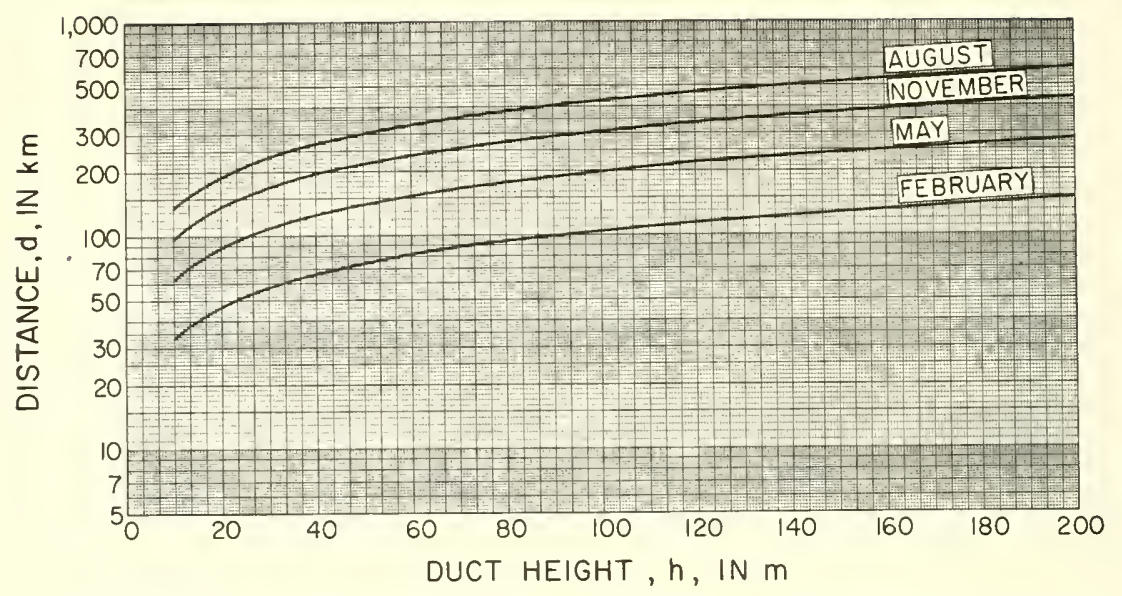

FIGURE 4.46. Shadow zone half-lengths for minimum ducting conditions at Washington, D.C.

TABLE 4.9. Half-lengths at Washington, D.C. for various duct heights and surface gradient conditions

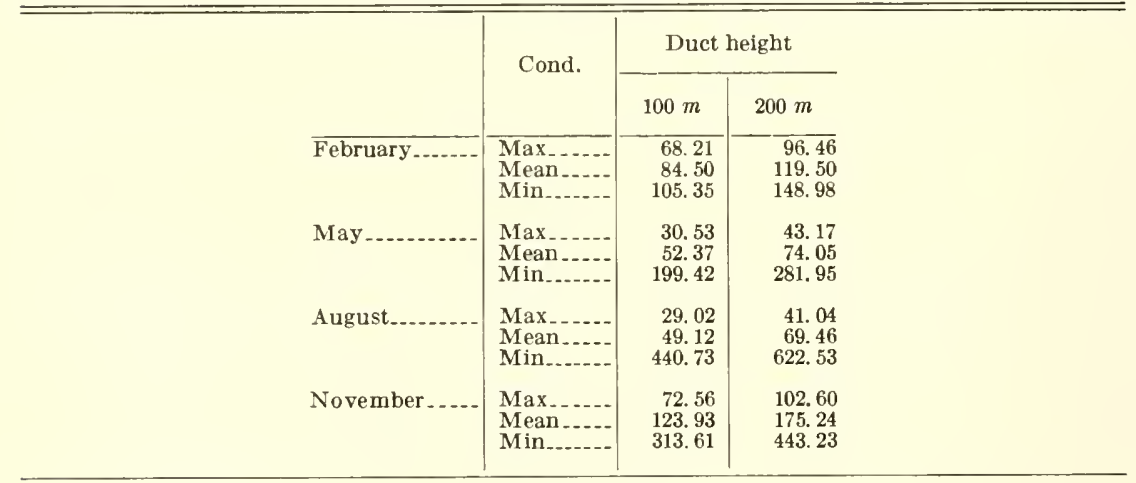

Washington, D.C. Figures 4.47 and 4.48 show the distance measured along the earth's surface that a ray with an initial elevation angle of zero will travel in the $N_{s}=313.0$ exponential atmosphere. The value of zero for an initial elevation angle is used because the ray can be thought of as leaving the position $A$, tangent to the duct in figure 4.41 and "arriving" at the transmitter, even though the reverse process is actually what is occurring. Thus, from figures 4.47 and 4.48 , knowing the height of the transmitter, $h_{t}$, and the height of the ground-based duct, $h_{A}$, the 


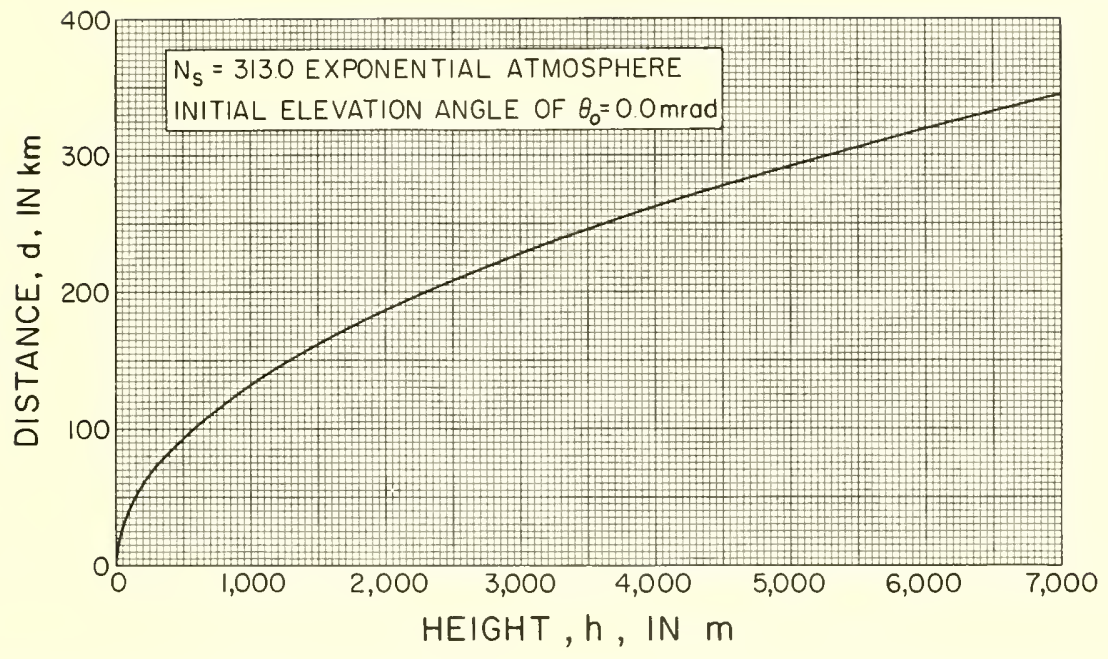

Figure 4.47. Distance versus height above duct representing average Washington, D.C., and Fairbanks, Alaska, atmosphere.

distance, $d_{0}$, that the radio ray travels from the transmitter to the top of the duct is given by

$$
d_{0}=d_{\left(h_{t}-h_{A}\right)}
$$

i.e., the distance obtained by using the difference of $h_{t}$ and $h_{A}$ as the value of height on the abscissa in figures 4.47 and 4.48 gives the distance $d_{0}$.

The distance, $d_{h o}$, to the standard radio horizon from the transmitter is

$$
d_{h_{0}}=\sqrt{2 h_{t}}
$$

if $h_{t}$, the transmitter height, is in feet and $d_{h_{o}}$ is in miles. Using this fact and figures 4.47 and 4.48 , table 4.10 , which shows the ratio of $d_{0}$ to $d_{h o}$,

TABLE 4.10. Ratio of onset point of fading zones from transmitter to distance of radio

\begin{tabular}{|c|c|c|c|c|c|}
\hline \multirow{2}{*}{$\begin{array}{l}\text { Height of } \\
\text { transmitter }\end{array}$} & \multicolumn{5}{|c|}{ Duct height, meters } \\
\hline & 10 & 20 & 50 & 100 & 200 \\
\hline $200 \ldots$ & 0.795 & 0.699 & 0.512 & 0.300 & 0 \\
\hline 300 & .837 & .759 & .605 & .432 & 0.187 \\
\hline 500 & .878 & .818 & 699 & .565 & .375 \\
\hline 800 & .907 & .860 & .765 & .659 & .509 \\
\hline $1000 \ldots$ & .918 & .876 & .792 & .697 & .563 \\
\hline 1500 & .935 & .900 & .831 & .754 & .644 \\
\hline 2000 & .944 & .914 & .854 & .787 & .692 \\
\hline 3000 & .953 & .928 & .880 & .825 & .747 \\
\hline $5000 \ldots$ & .959 & .940 & .902 & .859 & .799 \\
\hline $7000 \ldots \ldots$ & .959 & .943 & .911 & .875 & .825 \\
\hline
\end{tabular}
horizon from the transmitter, $\mathrm{d}_{0} / \mathrm{d}_{\mathrm{ho}}$, for various transmitter heights at Washington, D.C. 


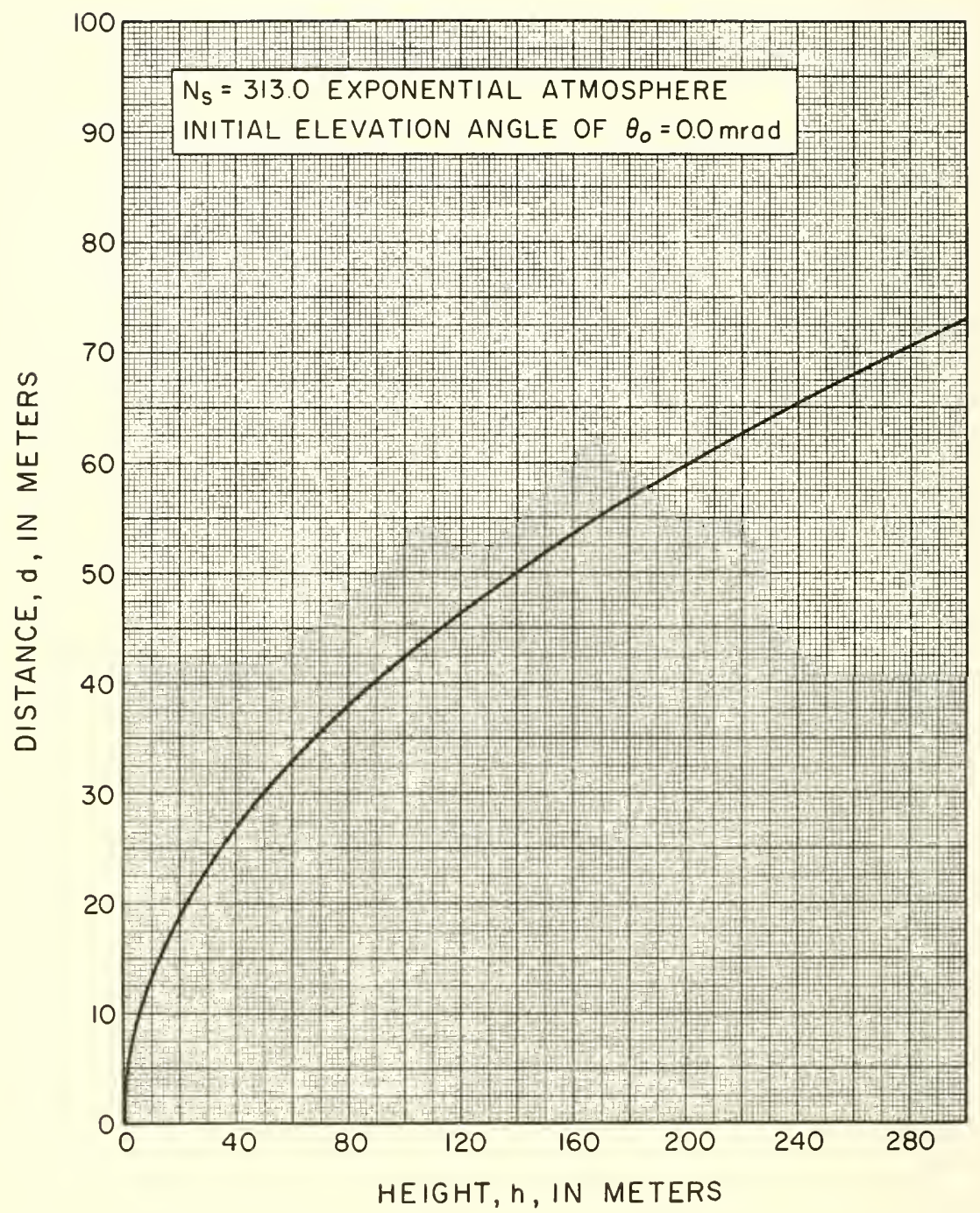

FIGURE 4.48. Distance versus height above duct representing average Washington, D.C., and Fairbanks, Alaska, atmosphere.

is derived for various transmitter heights and various duct heights. This table is useful in determining the portion of the distance (measured from the transmitter) within the horizon where fading due to the presence of superrefraction has no effect on transmission. 


\subsubsection{Sample Computations}

It can be seen from figure 4.52 that

$$
h_{1} \leq \text { loss region } \leq h_{A}+h_{2}
$$

where $h_{1}$ can be determined from figures like 4.49 to 4.51 , which depend upon the station location and the season of the year; $h_{A}$ is the height of the duct; and $h_{2}$ is determined from figures 4.47 and 4.48 . The following example will illustrate the determination of the shadow zone.

As an illustrative problem, assume a 500-m transmitting antenna in the spring of the year at $40^{\circ} \mathrm{N}$ latitude, and that one desires to place the receiving antenna at (a) $40 \mathrm{~km}$ and (b) $90 \mathrm{~km}$ from the transmitter. How high should the receiving antenna be to be free from fading loss caused by a ground-bäsed superrefractive layer?

Since both of the distances required are within the standard horizon distance, $d_{h o}$, which, for a $500-\mathrm{m}$ transmitter is

$$
d_{h o}=92.181 \mathrm{~km},
$$

this is a case of within-the-horizon propagation.

Since the $40^{\circ} \mathrm{N}$ latitude position is within the temperate zone, use will be made of the Washington, D.C., model ducting atmosphere. As previously mentioned, $100 \mathrm{~m}$ is the median mean duct thickness at Washington, D.C., and $200 \mathrm{~m}$ is the median maximum duct thickness observed at Washington. These are the two duct thicknesses to use in this calculation. Also, since it is the spring of the year, figures 4.49 to 4.51 must be used to determine $h_{1}$.

For the 40-km distance, case (a), the subscript of (4.38) becomes

$$
h_{t}-h_{A}=500-100=400 \mathrm{~m},
$$

which is then entered on the abscissa of figure 4.47, where,

$$
d_{0}=d_{400}=83 \mathrm{~km} \text {. }
$$

For a 200-m-thick duct,

$$
h_{t}-h_{A}=300 \mathrm{~m} .
$$




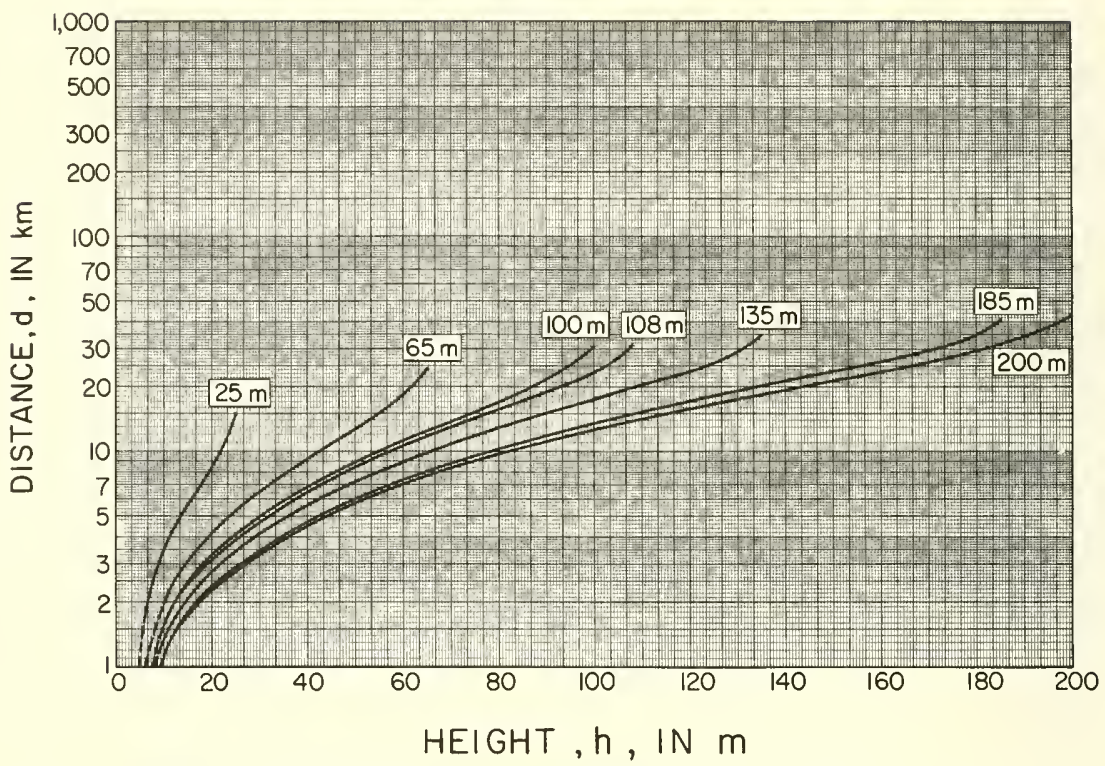

FIgure 4.49. Height versus distance at Washington, D.C., for maximum midspring (May) ducting conditions.

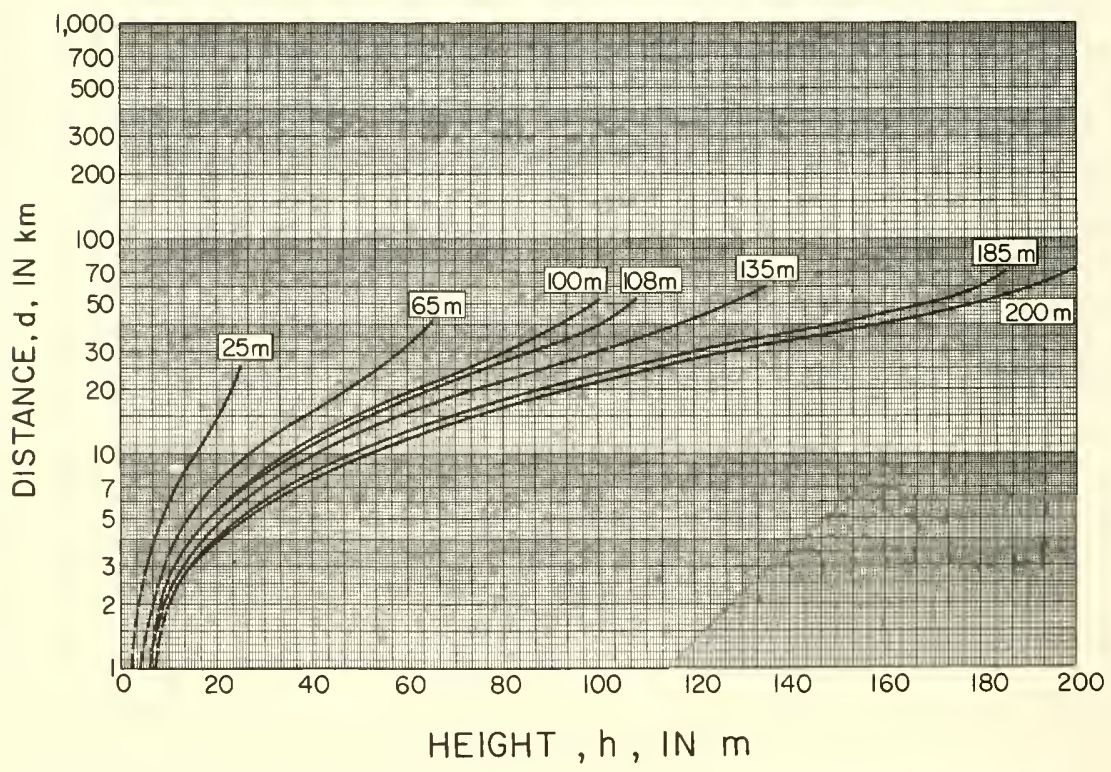

FIgURE 4.50. Height versus distance at Washington, D.C., for mean midspring (May) ducting conditions. 


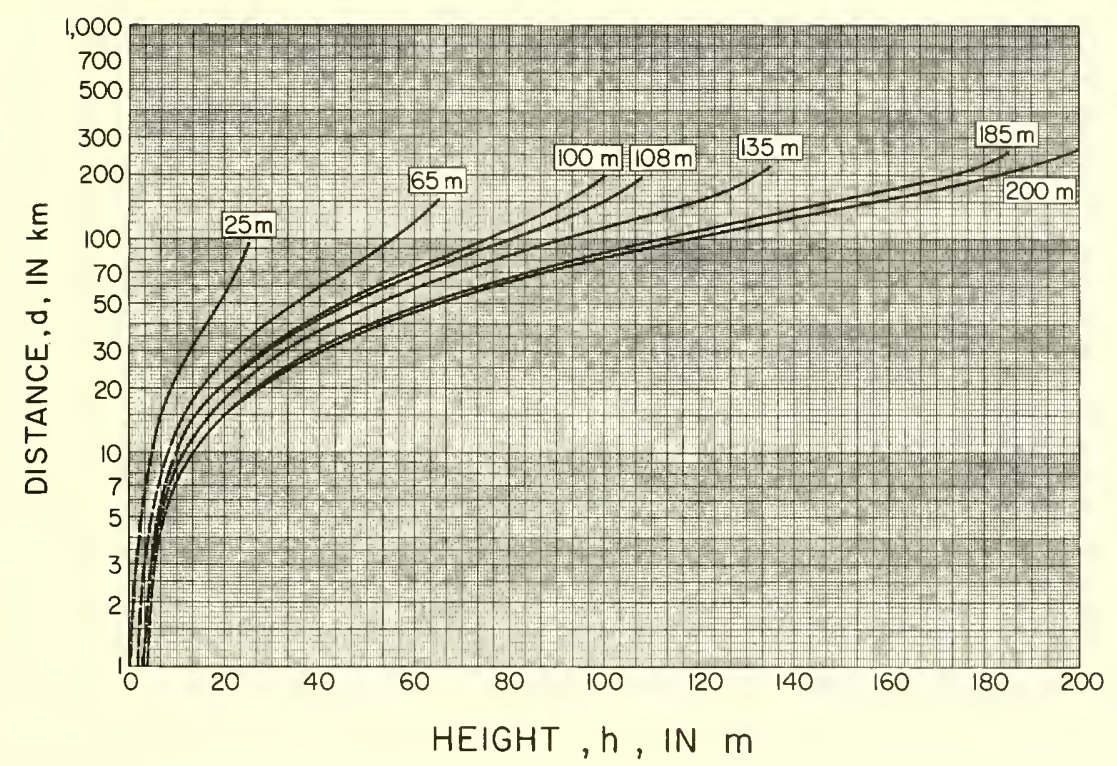

FIGURE 4.51. Height versus distance at Washington, D.C., for minimum midspring (May) ducting conditions.

Therefore, in this case,

$$
d_{0}=d_{300}=72 \mathrm{~km} \text {. }
$$

In either case, as can be seen from figure $4.41, d_{0}$ is greater than $40 \mathrm{~km}$. Therefore, any height of the receiving antenna will be satisfactory at this point, since the first shadow zone has not yet been reached.

For the $90-\mathrm{km}$ distance, case (b), it can be seen that this distance is beyond the onset point of the first shadow zone, and figures 4.49 to 4.51 must be used here.

Figures 4.49 to 4.51 are used differently depending upon which side of the reflection point $B$ of figure 4.52 one desires to locate an antenna. If on the lefthand side of point $B$, one must subtract the desired distance from the distance from $B$ to the top of the duct traversed by the ray and use this new distance to determine height, $h$, in figures 4.49 to 4.51 . This procedure must be followed every time the ray heads downward. If on the righthand side of point $B$ of figure 4.52 (or any other reflection point), one simply uses the distance from the reflection point directly. One must keep in mind that any previous half-lengths must be subtracted from the desired distance from the transmitter, as well as subtracting $d_{o}$, in order to obtain the distance used in figures 4.49 to 4.51 . 


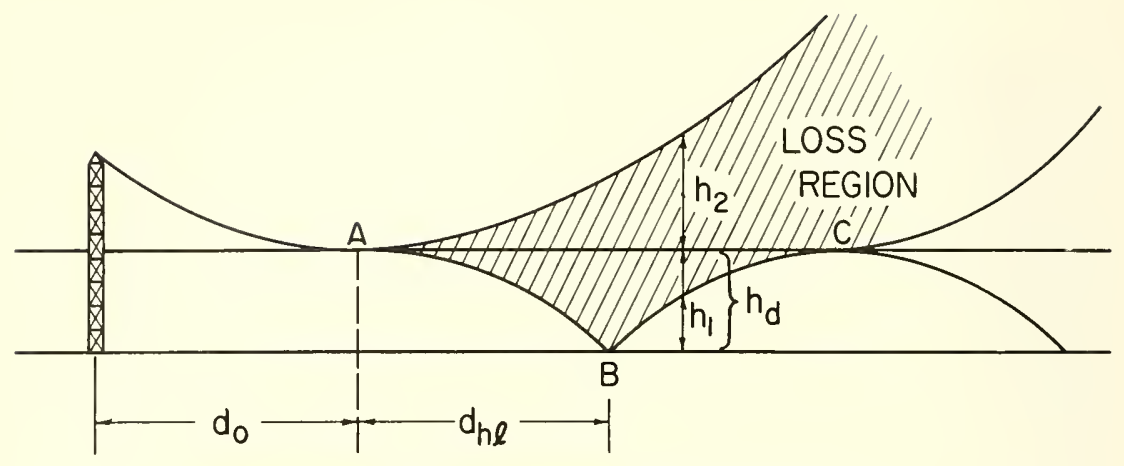

Figure 4.52. Geometry of the shadow zone loss region.

For the maximum midspring ducting conditions (fig. 4.49) and the 100 -m-thick duct, recalling $d_{0}=83 \mathrm{~km}$, the antenna is to be located

$$
90-83=7 \mathrm{~km}
$$

beyond the onset point of the shadow zones. For the $200-\mathrm{m}$ duct, the antenna is located

$$
90-72=18 \mathrm{~km}
$$

beyond point $A$ of figure 4.52. From figure 4.44, the half-length traveled by a ray in a 100-m duct under maximum midspring ducting conditions is $30.5 \mathrm{~km}$. Thus, as described above, the distance used in figure 4.49 to find $h$ is

$$
30.5-7.0=23.5 \mathrm{~km}
$$

because at $25 \mathrm{~km}$ the ray is still sloping downward and hence is on the lefthand side of point $B$ of figure 4.52 . From figure $4.44, d_{h \ell}=43 \mathrm{~km}$ for a $200-\mathrm{m}$ duct; therefore, the distance used in figure 4.49 is

$$
43-18=25 \mathrm{~km} \text {. }
$$

Similarly, utilizing figures 4.45 and 4.46 for mean ducting and minimum ducting conditions for a 100-m and 200-m duct:

Figure 4.50 distance to be used for the $100-\mathrm{m}$ duct $=45 \mathrm{~km}$,

Figure 4.50 distance to be used for the $200-\mathrm{m}$ duct $=56 \mathrm{~km}$, Figure 4.51 distance to be used for the $100-\mathrm{m}$ duct $=191 \mathrm{~km}$, Figure 4.51 distance to be used for the $200-\mathrm{m}$ duct $=262 \mathrm{~km}$, 

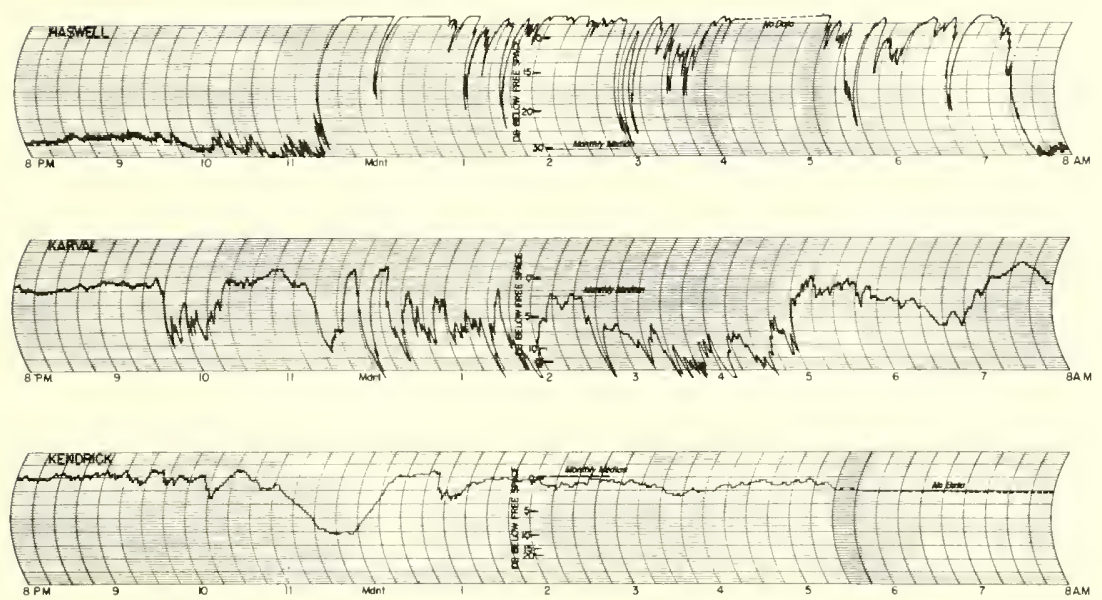

Figure 4.53. 1,046-Mc/s fields for the night of June 21-22, 1952, at Kendrick, Karval, and Haswell, Colo.

and hence

$h_{1}$ for a $100-\mathrm{m}$ duct under maximum spring ducting conditions $=92.3 \mathrm{~m}$, $h_{1}$ for a $200-\mathrm{m}$ duct under maximum spring ducting conditions $=166.0 \mathrm{~m}$, $h_{1}$ for a $100-\mathrm{m}$ duct under mean spring ducting conditions $=96.0 \mathrm{~m}$, $h_{1}$ for a $200-\mathrm{m}$ duct under mean spring ducting conditions $=187.0 \mathrm{~m}$, $h_{1}$ for a $100-\mathrm{m}$ duct under minimum spring ducting conditions $=99.0 \mathrm{~m}$, and

$h_{1}$ for a $200-\mathrm{m}$ duct under minimum spring ducting conditions $=199.0 \mathrm{~m}$.

Figures 4.47 and 4.48 will be used to determine $h_{2}$ from the total distance, $d$. The distance to be used is obtained by subtracting the distance, $d_{0}$, from the desired distance. For the $100-\mathrm{m} \mathrm{duct}, d_{0}=83 \mathrm{~km}$ and

$$
d=90-83=7 \mathrm{~km} \text {. }
$$

Likewise for the $200-\mathrm{m}$ duct, $d_{0}=72 \mathrm{~km}$ and

$$
d=90-72=18 \mathrm{~km} .
$$

Thus it is found that for the model assumed:

(a) $h_{2}$ for the $100-\mathrm{m}$ duct height case will always be $3 \mathrm{~m}$,

(b) $h_{2}$ for the 200-m duct height case will always be $18 \mathrm{~m}$.

Since from $(4.40)$

$$
h_{1} \leq \text { loss region } \leq h_{A}+h_{2}
$$




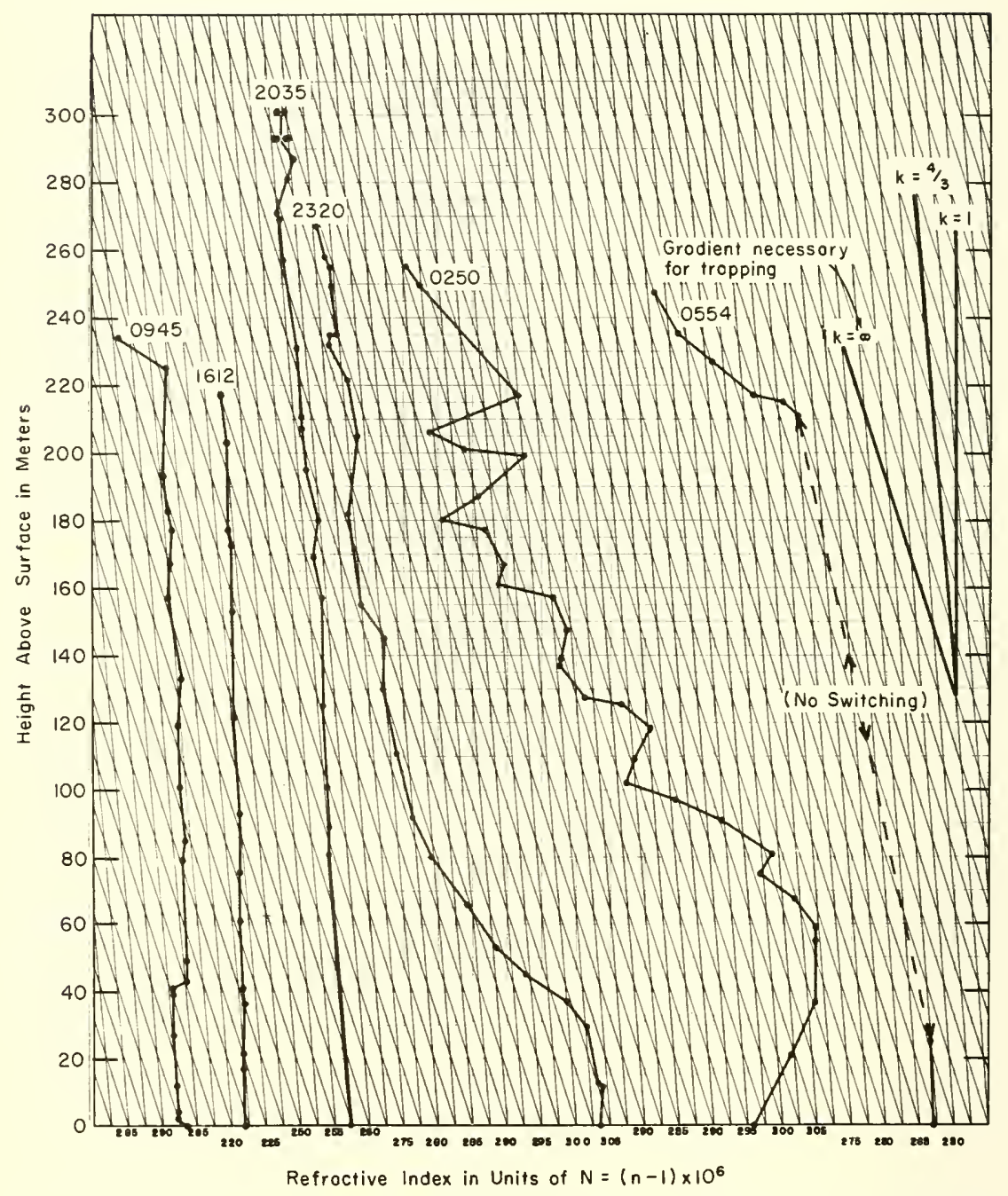

Figure 4.54. Vertical distributions of refractivity at Haswell, Colo., for June 21-2Q,1952

Therefore, in answer to part (b) of the problem, for maximum ducting conditions with a $100-\mathrm{m}$-thick duct:

$92.3 \mathrm{~m} \leq$ loss region $\leq 3+100 \mathrm{~m}$, or

$92.3 \mathrm{~m} \leq$ loss region $\leq 103 \mathrm{~m}$. 
With a $200-$ m-thick duct:

$$
\begin{aligned}
& 166 \mathrm{~m} \leq \text { loss region } \leq 18+200 \mathrm{~m} \text {, or } \\
& 166 \mathrm{~m} \leq \text { loss region } \leq 218 \mathrm{~m} .
\end{aligned}
$$

For mean ducting conditions with a 100 -m-thick duct:

$$
96 \mathrm{~m} \leq \text { loss region } \leq 103 \mathrm{~m} .
$$

With a 200-m-thick duct:

$$
187 \mathrm{~m} \leq \text { loss region } \leq 218 \mathrm{~m} .
$$

For minimum ducting conditions with a 100 -m-thick duct:

$$
99 \mathrm{~m} \leq \text { loss region } \leq 103 \mathrm{~m} \text {, }
$$

with a $200-m-$ thick duct:

$$
199.3 \mathrm{~m} \leq \text { loss region } \leq 218 \mathrm{~m} \text {. }
$$

\subsection{Air Mass Refractive Properties}

\subsubsection{Introduction}

The foregoing material on refractive index climatology concerned the geographical variation of mean $N$ (or $N_{0}$ ) over the surface of the earth and the three-dimensional distribution of mean $N$ over North America. This section develops another aspect of the climatological variation of the refractive index: the mean profile of $N$ in various air masses. The meteorologist defines air masses as bodies of air in the troposphere having approximately homogeneous character at the surface. An air mass is described in terms of its origin, as, for example, polar continental or tropical maritime.

Profiles of $N$ and values of bending of radio rays are given in terms of departures from normal for various air masses.

Recent studies have led to the evaluation of radar elevation angle errors in different climates and air masses $[43,44]$. In general, these studies did not emphasize the relation between air mass refractive index structure and the refraction of radio waves within the air mass. For example, mean angular bendings for radio rays passing completely through 


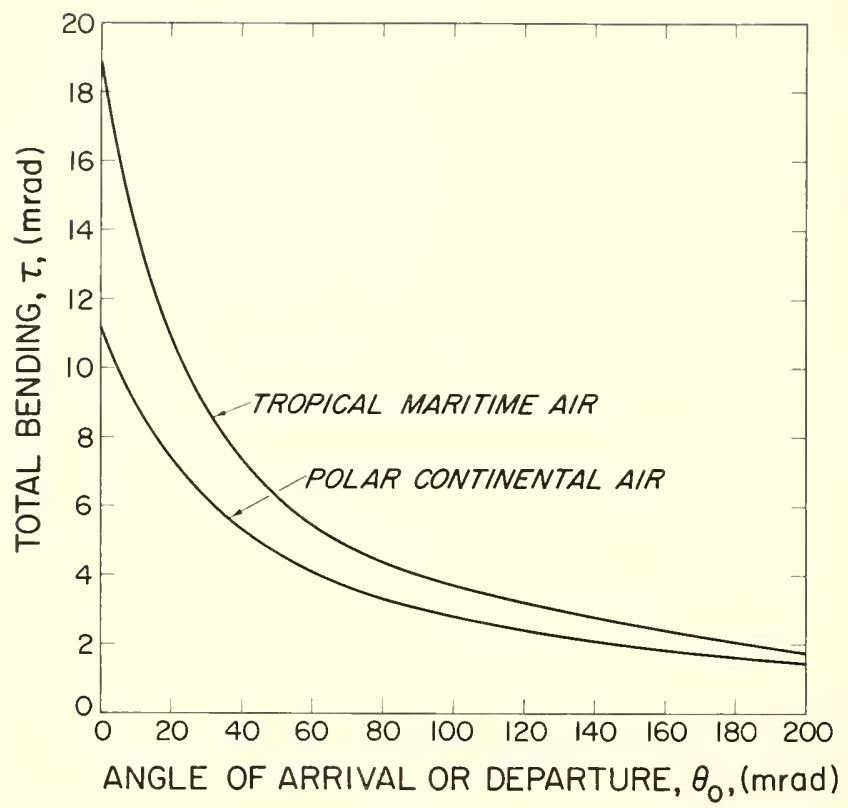

Figure 4.55. Comparison of total angular bending of radio rays within air masses. After Schulkin [1952].

the earth's atmosphere are given for extremes of air mass types and a range of initial elevation angles, $\theta_{0}$, on figure 4.55 [43]. It is seen that maritime tropical air produces 30 percent more bending than continental polar air at initial angles or arrival or departures of about $10^{\circ}(175 \mathrm{mrad})$ and that this difference increases to about 70 percent at zero initial elevation angle. Further, the magnitude of the total bending increases rapidly with decreasing values of $\theta_{0}$.

Figure 4.55 does not make it clear that differences in radio ray refraction arise from differences in the refractive index profiles of the two air masses. It is the purpose of this section to show that various air masses have characteristic refractive index profiles and that the radio ray refraction within each air mass is mirrored by the difference of the actual refractive index structure from a standard atmosphere of exponential form.

\subsubsection{Refraction of Radio Rays}

The angular bending of a radio ray, $\tau_{1,2}$ (see fig. 4.56) between two points in the atmosphere of refractive indices $n_{1}$ and $n_{2}$ is given by the 


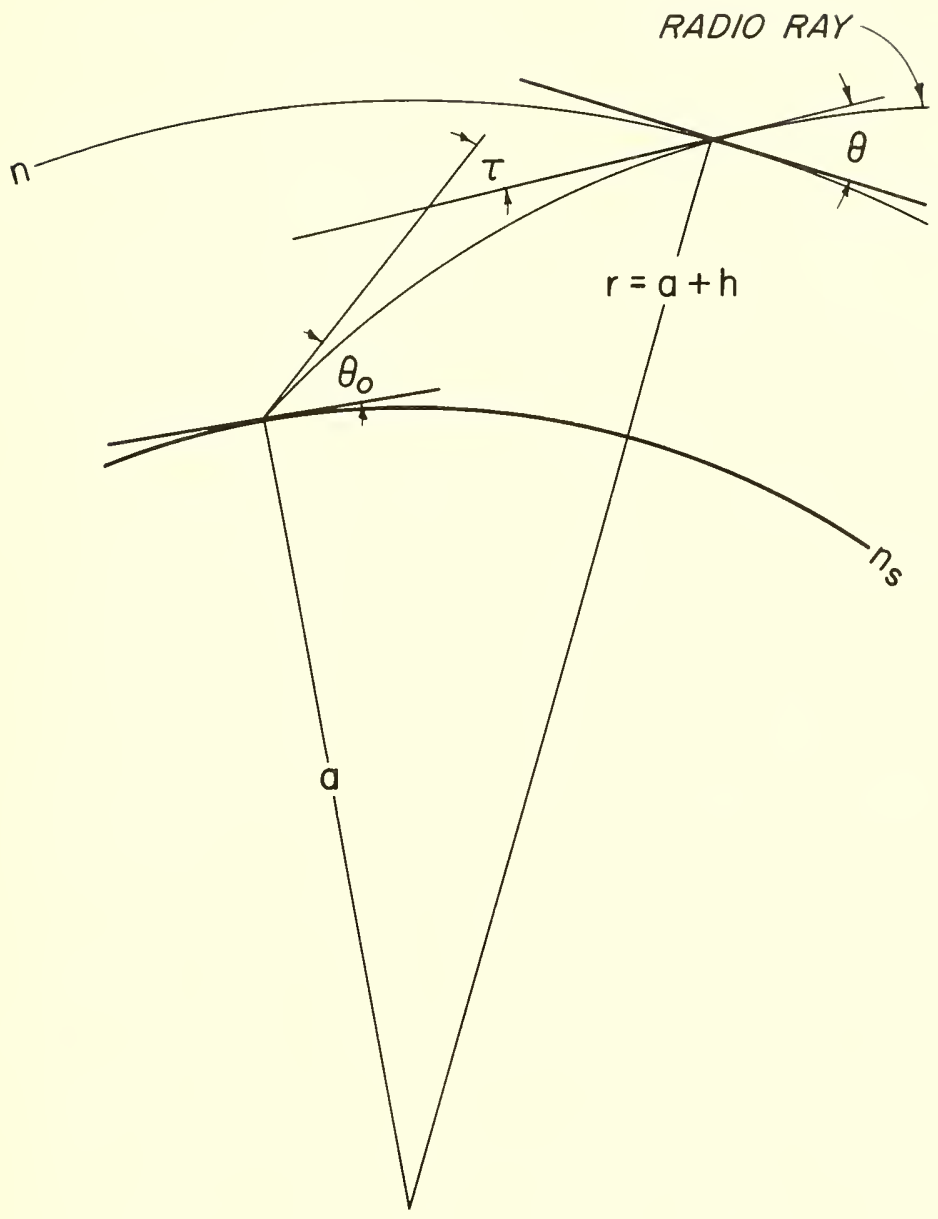

Figure 4.56. Geometry of radio ray refraction.

geometrical optics formula $[45,43]$,

$$
\tau_{1,2} \cong-\int_{n_{1}}^{n_{2}} \cot \theta d n
$$

under the assumption that $d n / n \cong d n$ and $\theta$ is the local elevation angle. It is also assumed that $n$ is spherically stratified and concentric with the earth. The value of $\cot \theta$ is determined from Snell's law, and $\tau_{1,2}$ is completely determined from a knowledge of the variation of $n$ with height. An empirical formula to describe the long-term average variation of $n$ 
with height is

$$
\overline{N(h)}=\overline{[n(h)}-1] 10^{6}=313 \exp \{-h / 6.95\}
$$

where $h$ is the height above the earth's surface in kilometers and 313 is the long-term average value of $(n-1) 10^{6}$ at the earth's surface for the United States. In practice it is convenient to utilize this average $N(h)$ function to refer the observed $N(h)$ data to the common level of $h=0$ by means of

$$
A(h, 313)=N(h)+313[1-\exp \{-h / 6.95\}] .
$$

Chapter 1 shows that $A(h, 313)$ is analogous in concept to potential temperature but utilizes the normal $N$ gradient rather than the adiabatic gradient of the potential refractive modulus of Lukes [46] and Katz [20]. The notation $A(h, 313)$ is used to indicate that $A$ is determined from the refractive index at the height $h$ and the 313 exponential atmosphere. It has been found to be advantageous to use several atmospheres of exponential form in applications involving different climatic regions [47]. The particular form of (4.43) emphasizes departures of $N$ structure from normal as shown by recent studies of synoptic variations of $N(h)$ about frontal systems [21] and, in addition, permits direct calculation of radio ray bending for any observed refractive index structure. When $\boldsymbol{A}(h 313)$ is introduced into (4.41) for ray bending, one obtains (see chapter 3, sec. 3.10$)$.

$$
\tau_{1,2}=-\int_{A_{1}}^{A^{2}} \frac{10^{-6} \cot \theta}{n} d A(h, 313)+\tau(h, 313)
$$

which indicates that the bending can be regarded as the resultant of the bending in the normal atmosphere, $\tau(h, 313)$, and a perturbation component representing departures of refractive index structure from that of the 313 exponential atmosphere. The value of $\tau(h, 313)$ can be obtained from refraction tables [9], and the perturbation term can be evaluated by graphical methods to yield an overall accuracy of a few percent in estimating $\tau_{1,2}[47]$.

Some $A(h, 313)$ profiles were prepared from typical temperature, pressure, and humidity distributions within a range of air masses as published in the literature $[48,49,50]$ and climatic summaries of upper air data [31]. Two of these profiles, one for maritime tropical air and the other representing continental tropical air, are plotted on figures 4.57 and 4.58 to represent the extremes of $A(h, 313)$ profiles. These profiles clearly show that the two air masses have quite different refractive index structures. The difference is most pronounced near the ground. At heights of 20 $\mathrm{km}$, however, $A(h, 313)$ rapidly approaches the asymptotic value of 313 , 


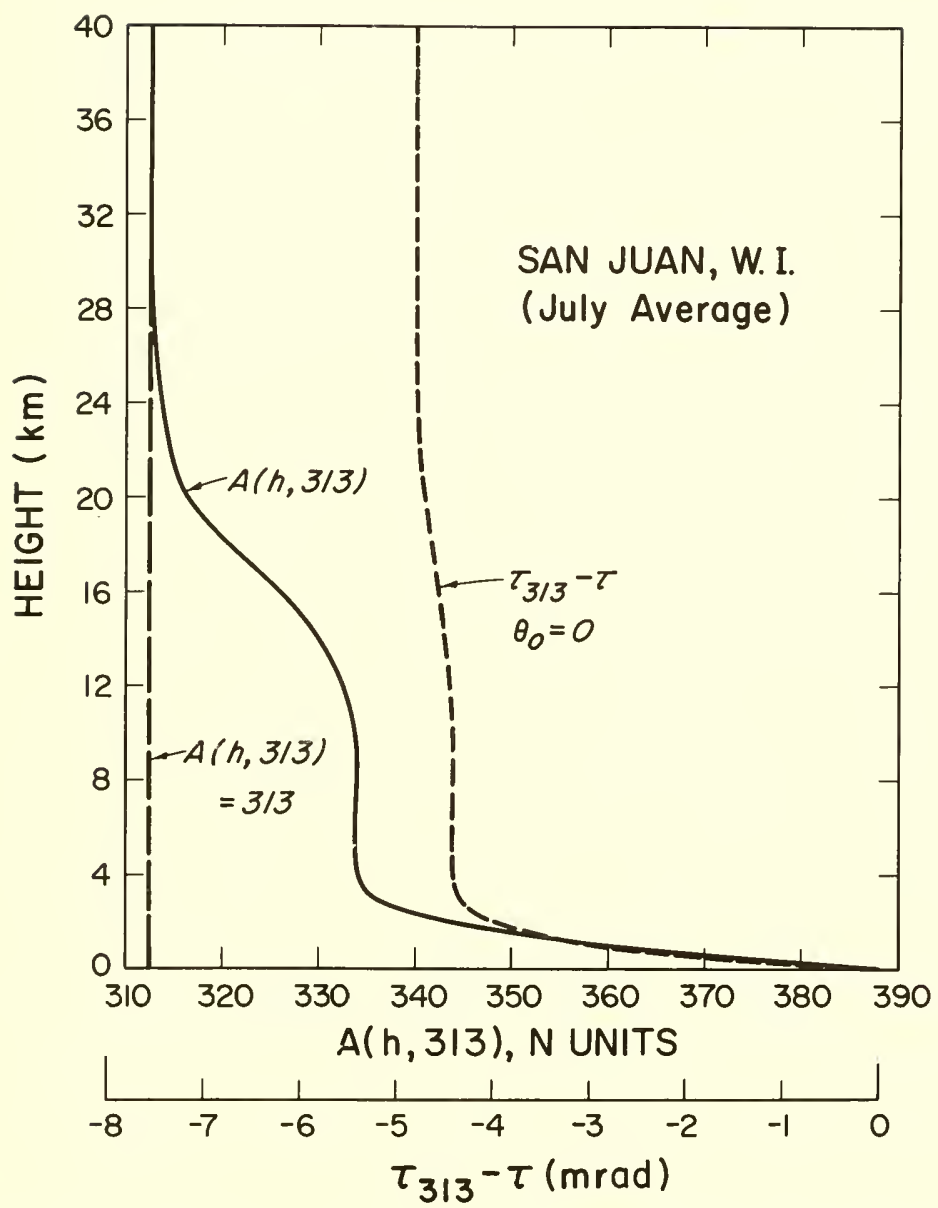

Figure 4.57. Comparison of $\mathrm{A}(\mathrm{h}, 313)$ profiles with departures from normal bending of radio waves for maritime tropical air. After Byers [1944]. 


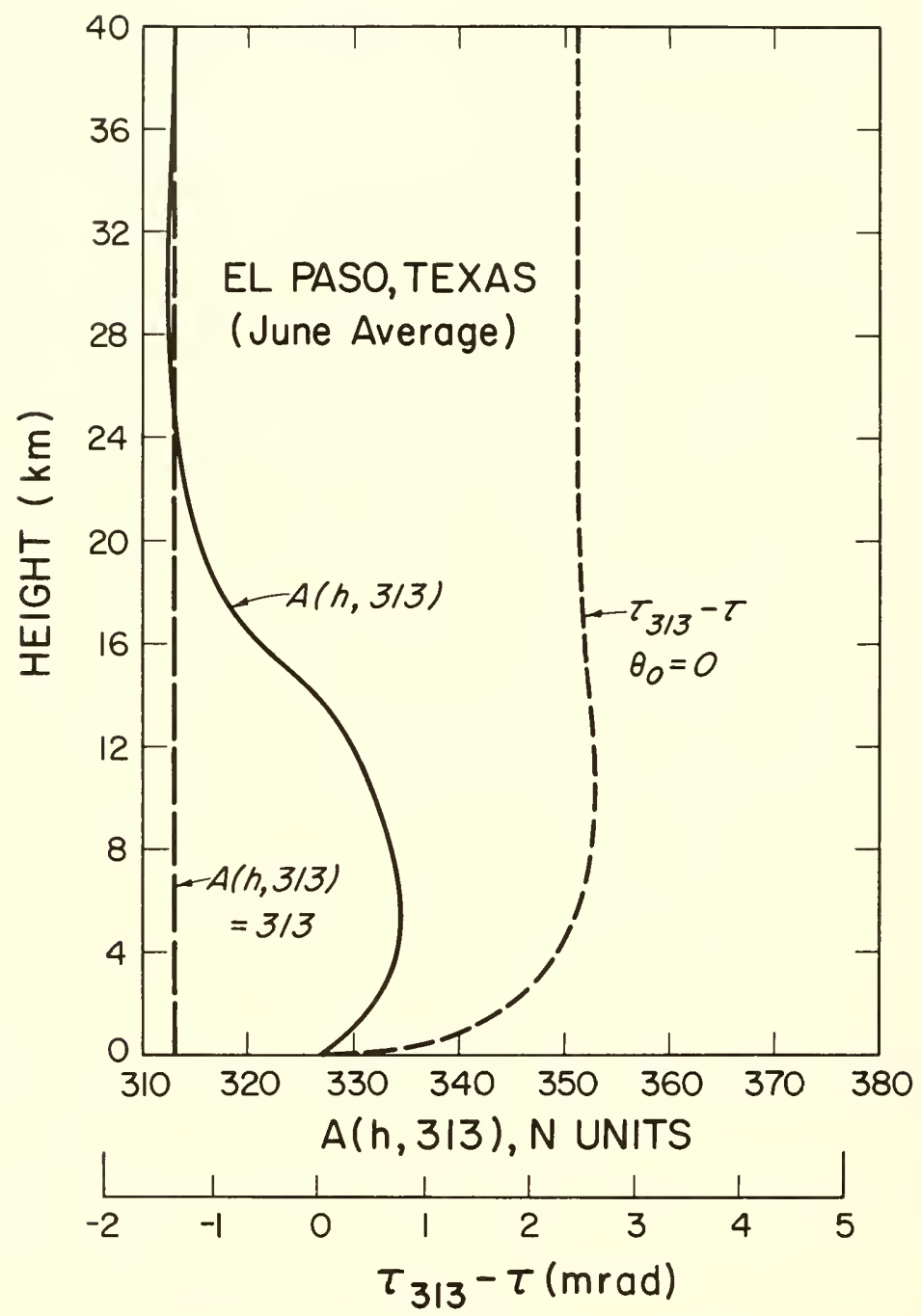

Figure 4.58. Comparison of $\mathrm{A}(\mathrm{h}, 313)$ profiles with departures from normal bending of radio waves for continental tropical air. After Ratner (1945). 
regardless of air mass type. The radio ray bending is also plotted on these figures relative to the value expected in the $N(h)=313 \exp$ $\{-h / 6.95\}$ atmosphere. Near the ground, bending departures are seen to be mirrored by the $A$ unit profiles. That is, a negative gradient of $N$ or $A$ produces a positive increase of bending. Above a few kilometers, however, the bending departures approach a fixed, usually nonzero, value. It is apparent that the asymptotic value of $\tau_{313}-\tau$ at large heights is determined by the bending in the first few kilometers, where 60 to 75 pereent of the low-angle bending normally oecurs. The marked differences in air mass $n$ structure and bending are confined, therefore, to the first few kilometers above the earth, as is illust rated by figure 3.16. The distribution of the meteorological elements within each air mass is reflected in the $A(h, 313)$ profiles. For example, the steep humidity gradient characteristic of maritime tropical air is reflected by the rapid decrease of $A(h, 313)$ with height. Comparatively, the high ground-level temperatures and rapid decrease of temperature with height in continental tropical air are reflected by the increase of $A(h, 313)$ with height within that air mass. These, and $A$-profile characteristics of other air masses, are listed in table 4.11 and are evident from the form of the equation for the refractive index at radio frequencies [see chapter 1, (1.20)].

TABLE 4.11. Refractive characteristics of air masses.

\begin{tabular}{|c|c|c|c|}
\hline Air Mass & $\begin{array}{l}\text { Ref- } \\
\text { erence }\end{array}$ & Meteorological charaeteristies & Refractive characteristies \\
\hline $\begin{array}{l}\text { Superior (S) } \mathrm{S} / \mathrm{mT} \\
\text { typieal of Gulf coast } \\
\text { (Lake Charles, La.). }\end{array}$ & {$[48]$} & $\begin{array}{l}\text { Formed from subsidence of high- } \\
\text { level air with resulting dry adiabatie } \\
\text { temperature lapse rate and low hu- } \\
\text { midity. Often found overlying other, } \\
\text { more humid air masses that show a re- } \\
\text { sultant eharacteristie drying with } \\
\text { height in the lower levels. }\end{array}$ & $\begin{array}{l}\text { Rapid decrease of } N \text { and } A \\
\text { with height in the lowermost } \\
\text { layer produces a superrefrac- } \\
\text { tive* layer. The overlying } \\
\text { superior air is nearly normal in } \\
\text { bending eharaeteristies. }\end{array}$ \\
\hline $\begin{array}{l}\text { Continental Polar (eP) } \\
\text { souree region. }\end{array}$ & {$[49]$} & $\begin{array}{l}\text { Characterized by low temperature } \\
\text { and humidity with a pronounced tem- } \\
\text { perature inversion at the surface } \\
\text { created by progressive nocturnal cool- } \\
\text { ing during the arctie night. }\end{array}$ & $\begin{array}{l}\text { Ground-based superrefrae- } \\
\text { tive layer arising from the tem- } \\
\text { perature inversion. }\end{array}$ \\
\hline $\begin{array}{l}\text { Maritome Polar (mP), } \\
\text { typieal low-level } \\
\text { ground modification } \\
\text { (Seattle, Wash.). }\end{array}$ & {$[48]$} & $\begin{array}{l}\text { Cool air, nearly saturated to a height } \\
\text { of several kilometers. Example shows } \\
\text { typical drying in lower levels when } \\
\text { this air mass moves over land. Over- } \\
\text { lying } m P \text { has inereasing humidity and } \\
\text { deereasing temperature with height. }\end{array}$ & $\begin{array}{l}\text { Surface layer produces near- } \\
\text { normal bending. Inereasing } \\
N \text { in the overlying } m P \text { air pro- } \\
\text { duces an elevated subrefrac- } \\
\text { tive layer. }\end{array}$ \\
\hline $\begin{array}{l}\text { Continental Tropieal } \\
\text { (e'T) souree region } \\
\text { (El Paso, Tex.). }\end{array}$ & {$[51]$} & $\begin{array}{l}\text { Charaeterized by superheated lower } \\
\text { layers, rapid deerease of temperature } \\
\text { with height, and very low humidity. }\end{array}$ & $\begin{array}{l}\text { Strong temperature lapse } \\
\text { produces a subrefractive layer } \\
\text { reaching to several kilometers. }\end{array}$ \\
\hline $\begin{array}{l}\text { Maritime Tropieal } \\
\text { (mT) source region } \\
\text { (Pensacola, Fla.). }\end{array}$ & {$[48]$} & $\begin{array}{l}\text { Relatively warm air, high water- } \\
\text { vapor eontent in lowest layer, whieh } \\
\text { deereases rapidly with height. Mod- } \\
\text { erate ehanges in temperature and hu- } \\
\text { midity strueture protuee large re- } \\
\text { fraetive gradient ehanges. }\end{array}$ & $\begin{array}{l}\text { Strong superrefraetion to } \\
\text { great heights arising ehiefly } \\
\text { from rapid decrease of hu- } \\
\text { midity with height. }\end{array}$ \\
\hline
\end{tabular}

"Normal refraction is taken to mean $r(h, 313)$, suiperrefraction to mean $r(h)>_{r}(h, 313)$, and subrefraetion $\tau(h)<r(h, 313)$. 
The average decrease of pressure, temperature, and humidity with height produces a normal decrease of $N$. If, however, the temperature increases with height, as in a temperature inversion, $N$ decreases more rapidly and radio rays are superrefracted, dependent, of course, upon the vapor pressure. Conversely, an unusually rapid decrease of temperature, or an merease of humidity, with height produces a subnormal decrease of $N$ with height, or subrefraction of radio waves. In any event it is evident from these figures that $A$-profile effects on ray bending are most pronounced at $\theta_{0}=0$, are significantly less pronounced at $\theta_{0}=52 \mathrm{mrad}$ $\left(\sim 3^{\circ}\right)$, and continue to diminish with increasing $\theta_{0}$ until, at $\theta_{0}=\pi / 2$, there is no bending at all and consequently all departures are zero.

\subsubsection{Conclusions}

The work of Schulkin and others has shown that characteristic total bending differences in radio ray refraction exist between various air masses. The present study extends Schulkin's conclusion by identifying abnormal bending of radio rays with departures of refractive index structure from average in the lowermost layers of the air masses. Consideration of departures of both ray bending and refractive index structure from their value in a standard exponential atmosphere results in a suitable method of cataloging air masses in terms of either refractive index structure or bending characteristics.

\subsection{References}

[1] Resolution No. 71 (1948), Conf. of Directors, Internat. Meteorol. Organization (Lausanne, Switzerland).

[2] World Atlas of Sea Surface Temperature Charts (1944), Hydrographic Office Publ. 225, chap. 4, No. 2.

[3] Atlas of climatic charts of the oceans (1938), U.S. Weather Bureau Publ. 1247, Washington, D.C.

[4] Johnson, W. E. (Nov.-Dec. 1953), An analogue computer for the solution of the radio refractive index equation, J. Res. NBS 51, No. 6, 335-342.

[5] Gerson, N. C. (1948), Variations in the index of refraction of the atmosphere, Geofis. Pura Appl. 13, 3-4.

[6] Bean, B. R. (Apr. 1953), The geographical and height distribution of the gradient of refractive index, Proc. IRE 41, No. 4, 549-550.

[7] Misme, P. (Nov.-Dec. 1958), Essai de radioclimatologie d'altitude dans le nord de la France, Anna. Telecommun. 13, No. 11-12, 303-310.

[8] Smithsonian Meteorological Tables (1951), Table 63, Sixth Revised Ed., Book (Washington, D.C.).

[9] Bean, B. R., and G. I. Thayer (1959), CRPL Exponential Reference Atmosphere, NBS Mono. 4.

[10] Pickard, G. W., and H. T. Stetson (1959), Comparison of tropospheric reception, J. Atmos. Terrest. Phys. 1, 32-36.

[11] Pickard, G. W., and H. T. Stetson (1950), Comparison of tropospheric reception at $44.1 \mathrm{Mc}$ with $92.1 \mathrm{Mc}$ over the 167 -mile path of Alpine, New Jersey to Needham, Mass., Proc. IRE 38, No. 12, 1450. 
[12] Bean, B. R. (1956), Some meteorological effects on scattered radio waves, IRE Trans. Commun. Syst. CS4, 32-38.

[13] Onoe, M., M. Hirai, and S. Niwa (Apr. 1958), Results of experiment of longdistance overland propagation of ultra-short waves, J. Radio Res. Labs. 5, 79.

[14] Gray, R. E. (Jan., Feb., Mar. 1959), The refractive index of the atmosphere as a factor in tropospheric propagation far beyond the horizon, IRE Natl. Conv. Record, Pt. 1, 3-11 (1957), Elec. Commun. 36, No. 1, 60.

[15] Norton, K. A. (Mar. 1956), Point-to-point radio relaying via the scatter mode of tropospheric propagation, IRE Trans. Commun. Syst. CS-4, 39-49.

[16] Schelleng, J. C., C. R. Burrows, and E. B. Ferrell (Mar. 1933), Ultra-short-wave propagation, Proc. IRE 21, 427-463.

[17] Anderson, L. J. (1958), Tropospheric bending of radio waves, 'Trans. Am. Geophys. Union 39, 208-212.

[18] Snedecor, G. W. (1946), Statistical Methods, Book, 4th Ed., pp. 97-98 (Iowa State College Press, Ames, Iowa).

[19] Bean, B. R., and L. P. Riggs (July-Aug. 1959), Synoptic variation of the radio refractive index, J. Res. NBS 63D (Radio Prop.), No. 1, 91-97.

[20] Katz, I. (1951), Gradient of refractive modulus in homogeneous air, potential modulus, Propagation of Short Radio Waves by D. E. Kerr, Book, pp. 198-199 (McGraw-Hill Book Co., Inc., New York, N.Y.).

[21] Bean, B. R., L. P. Riggs, and J. D. Horn (Sept.-Oct. 1959), Synoptic study of the vertical distribution of the radio refractive index, J. Res. NBS 63D (Radio Prop.), No. 2, 249-258.

[22] Jehn, K. H. (June 1960), The use of potential refractive index in synoptic-scale radio meteorology, J. Meteorol. 17, 264.

[23] Bean, B. R., and J. D. Horn (Nov.-Dec. 1959), The radio refractive index near the ground, J. Res. NBS 63D (Radio Prop.), No. 3, 259-273.

[24] duCastel and P. Misme (Nov. 1957), Elements de radio climatologie, L'Onde Electrique 37, 1049-1052.

[25] Hay, D. R. (Dec. 1958), Air mass refractivity in central Canada, J. Phys. 36, $1678-1683$.

[26] Tao, K., and K. Hirao (Mar. 1960), Vertical distribution of radio refractive index in the medium height of atmosphere, J. Radio Res. Labs. 7, No. 30, 85-93.

[27] Fehlhaber, L., and J. F. Grosskopf (1959), Beitrage zur Radioklimatologie Westdeutschlands, technische Bericht 5546, FTZ.

[28] Bean, B. R., and R. M. Gallet (Oct. 1959), Applications of the molecular refractivity in radio meteorology, J. Geophys. Res. 64, No. 10, 1439-1444.

[29] Bean, B. R., J. D. Horn, and A. M. Ozanich, Jr. (1960), Climatic charts and data of the radio refractive index for the United States and the world, NBS Mono. 22.

[30] Report 147 (1959), IX Plenary Assembly, International Radio Consultative Committee, pp. 299-337 (Los Angeles, Calif.).

[31] Booker, H. G., and W. Walkinshaw (1947), The mode theory of tropospheric refraction and its relation to wave guides and diffraction, Book, Meteorological Factors in Radio Wave Propagation, pp. 80-127 (The Physical Society and Roy. Meteorol. Soc., London, England).

[32] Bremmer, H. (1949), Terrestrial Radio Waves, Book, pp. 131-138 (Elsevier Publ, Co., New York, N.Y.).

[33] Cowan, L. W. (1953), A radio climatological survey of the U.S., Proc. Conf. Radio Meteorol. (Univ. of Texas, Austin, Tex.).

[34] Kerr, D. E. (1951), Propagation of Short Radio Waves, Book, pp. 9-22 (McGrawHill Book Co., Inc., New York, N.Y.).

[35] Bean, B. R. (May 1954), Prolonged space wave fadeouts at 1046 Me observed in Cheyenne Mountain propagation program, Proc. IRE 42, 848-85i3. 
[36] Misme, P. (Apr. 1956), Methode de mesure thermodynamique de l'indice de refraction de l'air-description de la radiosonde MDI, Ann. Telecommun. 11, No. 4, 81-84.

[37] Price, W. L. (July 1948), Radio shadow effects produced in the atmosphere by inversions, Proc. Phys. Soc. 61, 59-73.

[38] Norton, K. A. (Dec. 1941), The calculation of ground-wave field intensity over a finitely conducting spherical earth, Proc. IRE 29, 623-639.

[39] Kirby, R. S., J. W. Herbstreit, and K. A. Norton (May 1952), Service range for air-to-ground and air-to-air communications at frequencies above $50 \mathrm{Mc}$, Proc. IRE 40, No. 5, 525-536.

[40] Ikegami, F. (Aug. 1959), Influence of an atmospheric duct on microwave fading, IRE Trans. Ant. Prop. AP-7, 252-257.

[41] Doherty, L. H. (1952), Geometrical optics and the field at a caustic with applications to radio wave propagation between aircraft, Res. Rept. EE138 (Cornell Univ., Ithaca, N.Y.).

[42] Airy, G. B. (1938), On the intensity of light in the neighbourhood of a caustic, Cambridge Phil. Trans. 6, 379-402.

[43] Schulkin, M. (May 1952), Average radio-ray refraction in the lower atmosphere, Proc. IRE 40, No. 5, 554-561.

[44] Fannin, B. M., and K. H. Jehn (May 1957), A study of radar elevation angle error due to atmospheric refraction, IRE Trans. Ant. Prop. AP-5, 71-77.

[45] Smart, W. M. (1931), Spherical Astronomy, ch. 3, Book (Cambridge Univ. Press, London, England).

[46] Lukes, G. D. (1944), Radio meteorological forecasting by means of the thermodynamics of the modified refractive index, Third Conf. on Prop., NRDC, pp. 107-113 (Committee on Propagation, Washington, D.C.).

[47] Bean, B. R., and E. J. Dutton (May-June 1960), On the calculation of departures of radio wave bending from normal, J. Res. NBS 64D (Radio Prop.), No. 3, $259-263$.

[48] Byers, H. R. (1944), General Meteorology, Book, pp. 255-277 (McGraw-Hill Book Co., Inc., New York, N.Y.).

[49] Willett, H. C. (1944), Descriptive Meteorology, Book, pp. 190-220 (Academic Press, Inc., New York, N.Y.).

[50] Trewartha, G. T. (1943), An Introduction to Weather and Climate, Book, pp. 206-215 (McGraw-Hill Book Co., Inc., New York, N.Y.).

[51] Ratner, B. (1945), Upper air average values of temperature, pressure, and relative humidity over the United States and Alaska (U.S. Weather Bureau). 


\section{Chapter 5. Synoptic Radio Meteorology}

\subsection{Introduction}

This ehapter treats the variation of refractive index structure in the troposphere with synoptic tropospherie disturbances. Within the seope of the synoptic field are timewise and spacewise variations in the atmosphere from mieroscale fluctuations to broad-seale systems of weather map dimensions.

The microseale fluctuations of the refractive index are those that one would expeet to observe at a particular point along a radio path that reflect detailed terrain and weather conditions in the immediate vieinity of, say, the transmitter or receiver site.

Mesoseale variations, by way of contrast, are those which eover tens of kilometers and thus encompass a substantial portion of a radio path. Examples of this type of variation are land-sea breeze effeets and convection eells of thunderstorm activity.

Large-seale weather systems, affecting vast areas, perhaps even on a continental seale, fall under the elassification of macroseale variation. Examples of this type of activity in the atmosphere are sweeping air mass changes and frontal systems traversing thousands of kilometers on the earth's surface. A detailed analysis of such a system is given as an illustration later in this ehapter.

\subsection{Background}

The problem of determining the vertical and horizontal distribution of the radio refractive index has engaged the attention of radio meteorologists on an international seale for the better part of two deeades $[1,2,3,4,5$, $6] .{ }^{1}$

By analysis of eurrent synoptic conditions from standard weather charts, one may aseertain the air mass type appearing over a given region and, likewise, may predict with reasonable aceuracy the air mass type expeeted over a particular locale in, say, 24 hours. Then, from the air mass profile characteristies table of seetion 4.4, one is able to estimate what the departures of refractive index (and radio ray bending) from normal will be over a certain region. The bending predietions permit an

${ }^{1}$ Figures in brackets indicate the literature references on p. 224. 
estimate of refraction errors and the introduction of appropriate corrections for radio range and elevation angle errors for radio navigational equipment.

VHF-UHF radio field strengths beyond the normal radio horizon will also differ from air mass to air mass. It has been known for many years that the seasonal cycle of VHF radio field strengths received far beyond the normal radio horizon were correlated with the refractive index $[7,8$, $9,10]$ and that significant changes in field strength level are observed from air mass to air mass $[11,12,13$.] Speaking about signal level on a 60 $\mathrm{Me} / \mathrm{s}$ beyond the horizon radio path near Boston, Mass., Hull [11] states that during the winter low signal levels prevail during the presence over the path of fresh polar air. Periods of high signal occur when a cold, dry polar air mass is overrun by warm, moist air of tropical maritime origin. Hull's analysis represents early recognition of refraction and reflection phenomena on a synoptic scale. Later work on seasonal changes of fields and $N$ represents, in a way, a summary of synoptic conditions over a period of time.

Gerson [2] was one of the first to consider the variation of the radio refractive index, $n$, in terms of seasonal and air mass changes. Gerson divided $n$ into two parts, one density-sensitive and the other moisturesensitive. This division is equivalent to the wet- and dry-term separation of $N=(n-1) 10^{6}$ in chapter 4 , section 1 . Gerson was able to measure seasonal thermal changes by variation in the dry term and seasonal moisture changes by observed variation in the wet term. Gerson prepared graphs showing a sinusoidal variation of the dry term with a warm season trough and a cool season crest, indicating density changes in inverse proportion to the temperature. The wet-term component of $n$, on the other hand, was observed to attain its maximum during the warm season when the dry term was at its minimum. In arctic and antarctic locales, the surface variation of the wet term was found to be quite small while in temperate and tropical climates there was a sizable annual variation of the moisture component. As a pertinent aside, Yerg [14] showed that even during the long, cold, arctic night, vertical variations in moisture made significant contributions to the $N$ profile. Apparent ducting gradients, obtained by neglecting the wet term at low temperatures may, in actuality, be only slightly more refractive than standard.

Continuing his investigation, Gerson next turned to the analysis of refractive index changes within various air masses. Using air mass data available in the meteorological literature of the day, he charted mean refractive index profiles for different air mass types. The largest initial values and also the largest vertical gradients of $n$ occurred with tropical maritime air. The air mass with the weakest gradients and, therefore, the poorest refraction properties was found to be the polar continental type. Common to all of Gerson's air mass refractive index graphs is an 
approximately exponential decrease of $n$ with respeet to height. Schelleng, Burrows, and Ferrell [15] attempted to remove this systematic $n$ decrease with height by utilizing a correction factor of linear form, although, as has been seen in chapter 3 , this leads to serious overestimation of refraction effects in the more modern problem of satellite telecommunications.

The work of Hay [6] confirmed and extended the observations Gerson had made concerning air mass profiles. Measuring air mass characteristies at Maniwaki, Quebee, Hay coneluded that each large-seale air mass type in central Canada has a distinetive refractive index profile. Hay determined the height distribution of $N$ for four basic air masses by fitting a second-degree polynominal to each of the four sets of air mass data, indieating that an average $N$ profile eannot be approximated effectively by a linear eurve except over small height inerements. Hay obtained further diserimination by constructing a "dry-term" curve for each air mass. The dry-term eurves display a smaller standard deviation than the total $N$ eurves for all air masses except the continental aretie which is, of course, itself very dry. The largest variations in total $N$ are due to fluctuations in the wet term. The saturation vapor pressure is approximately an exponential function of the temperature, so that during the warm season normal temperature changes cause the saturation vapor pressure and, therefore, the wet term is vary sharply.

Contained also in Hay's paper [6] is a discussion of the use of $N$ profiles to estimate corrections for radio ray refraction by use of a table of mean effective earth's radius factors for each of four air masses within 95 percent probability limits.

Misme $[5,16]$ has had an interest in synoptic radio meteorology in connection with telecommunications networks in France and North Africa. Interests of this French radio engineer include vertical gradients of the refractive index and atmospheric reflection of radio waves.

A reasonable question at this point is, to what extent are model exponential atmospheres applicable to various elimatic zones around the earth? While it is readily apparent that individual profiles vary widely from any sort of exponential norm, there is an increasing backlog of experimental evidence [17] that shows that long-term averages of synoptie refractive index variations do tend toward an exponential form with respect to height. Indeed, the 5-year mean $N$ profiles of figures 5.1 and 5.2 , representing aretic and tropical climates respectively, bear out this contention by showing close agreement with models developed from midlatitudinal data. Indeed, these examples verify the results obtained by Gerson [2] for various air masses.

Randall [4] investigated the relationship of surface meteorological data to surface $N, N_{s}$, and field strength in the FMI frequency band. The results described were drawn from a very limited data sample that covered 


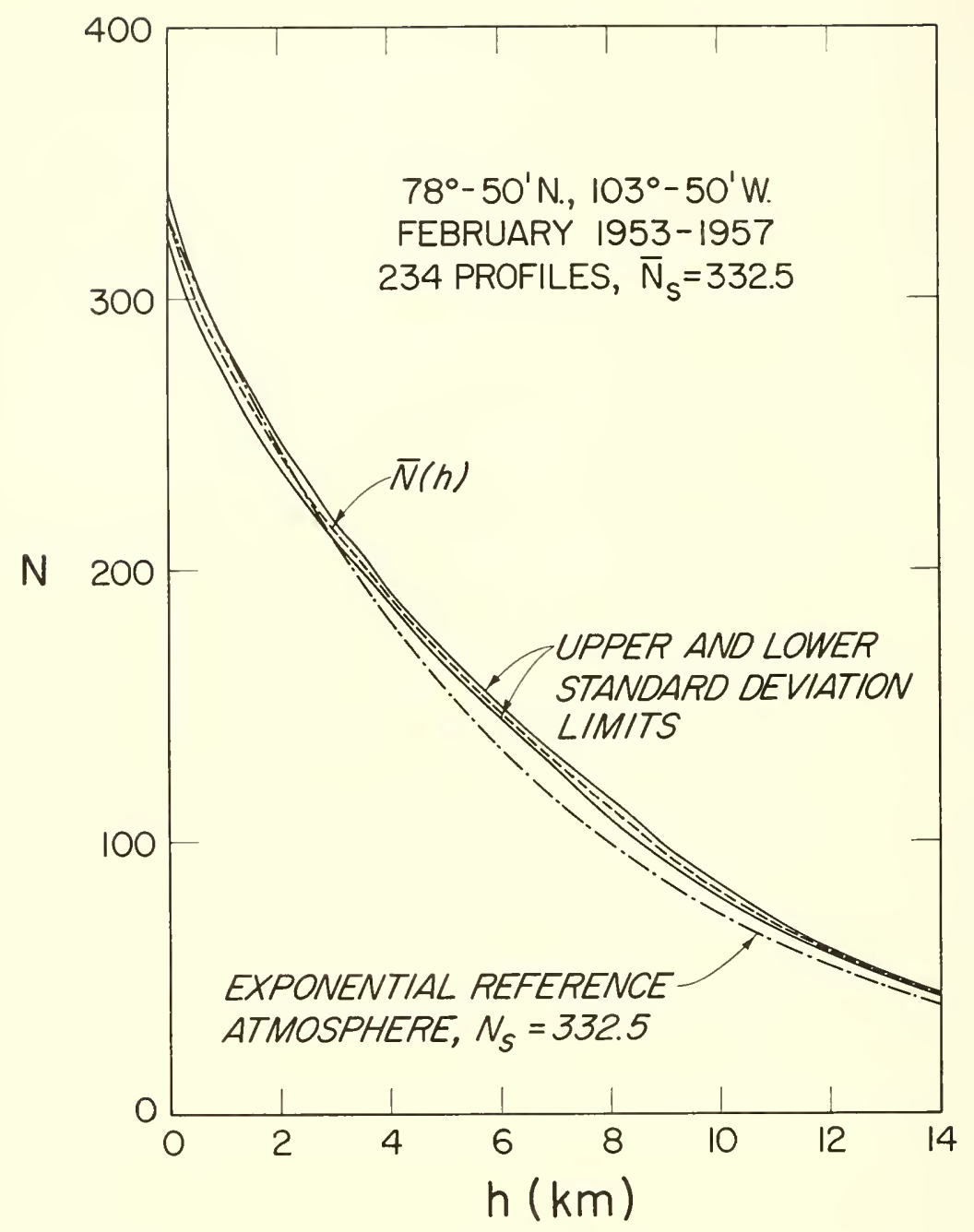

Figure 5.1. Mean N distribution for Isachson, N.W.T., Canada.

less than a month during the summer of 1947. Within the framework of this specialized study, however, Randall found that polar continental air masses were associated with low field strengths and low $N_{s}$ while tropical maritime air masses were associated with high field strengths and high $N_{s}$, as shown on figure 5.3. Randall advanced the hypothesis that the observed field strength changes were due to the existence of characteristic $N$ profiles typical of each air mass type. Randall was curious also as to the behavior of $N$ and radio fields during the passage of fronts and squall lines. Figure 5.4 shows the results of this investigation and indicates that 
definite field strength changes may occur during frontal and squall line passage. Caution should again follow in the interpretation of these results since they represent but a single example of frontal and squall line passage.

Gray [18] made measurements of correlation of $N$ and the gradient on $N$ with path losses at UHF frequencies for observing points representative of various climatic areas around the world and concluded that changes in transhorizon telecommunications are strongly dependent on atmospheric changes.

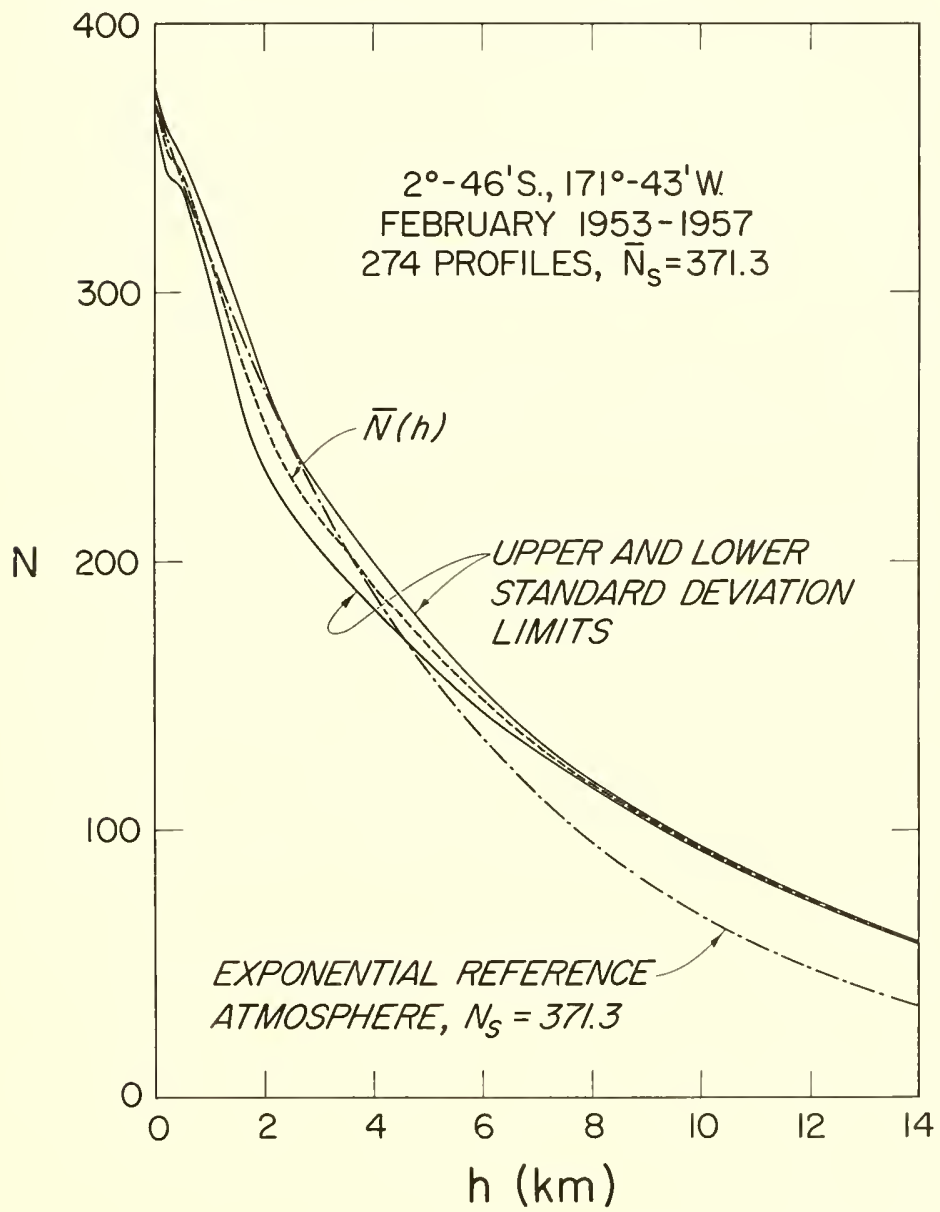

Figure 5.2. Mean N distribution for Canton Island, Pacific Ocean. 


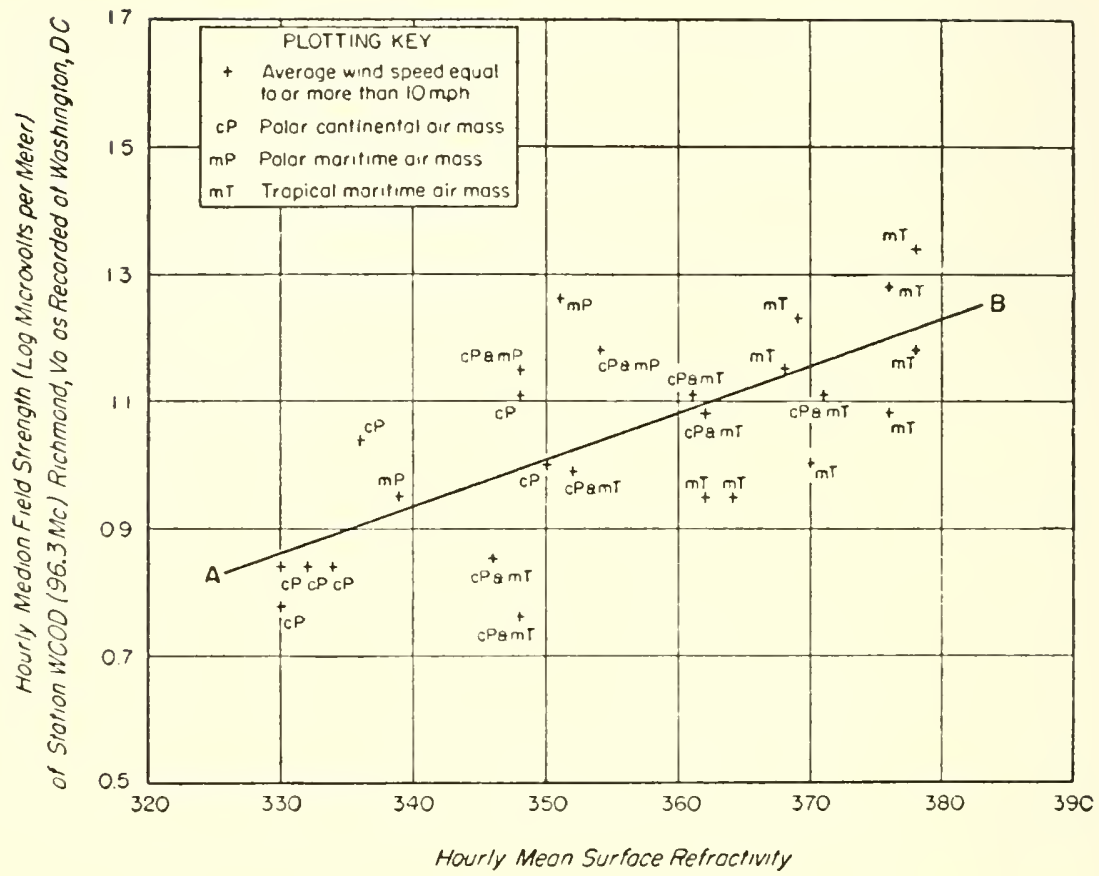

Figure 5.3. Field strength versus refractivity.

Scatter diagram of select hourly median field strength vs. hourly mean refractivity July 17 to Aug. 8, 1947.

In another article Gray [19] considered radio propagation and related meteorological conditions over the Caribbean Sea. Utilizing the effective earth's radius factor as a representative index, Gray presented an empirical curve of annual median scatter loss versus effective propagation distance designed to fit both Caribbean and temperate regions. Effective distance as defined by Gray is the angular distance in radians multiplied by the radius of the earth modified for normal refraction. Gray reports that the refractive gradient in the first $100 \mathrm{~m}$ is in general the determining factor in median scatter loss for transhorizon telecommunications, as one would expect from earlier refraction studies [20].

Other studies on refraction problems during the 1950's have led to systematic computation of refraction effects and to significant applications such as the evaluation of radar elevation angle errors in differing air masses and climates $[21,23]$. Schulkin [21] advanced a practical and very fundamental method for numerical calculation of atmospheric refraction (radio ray bending) from radiosonde data. Figure 4.55 [after Schulkin] gives mean angular bendings for radio rays passing completely through the earth's atmosphere for two extremes of air mass type. Fannin and Jehn concluded that a particular refractive index profile depends on air 
mass type and the climatic controls of season and latitude, as is substantiated by mean $N$ profiles for 34 weather observing sites located in or near four distinct air mass source regions about the world. These data show that a definite difference does exist in the profiles of various air masses. Refraction effects were found to be largest in tropical maritime air, intermediate in polar continental and polar maritime, and least in tropical continental. Fannin and Jehn also published graphs showing day-to-day variations in profiles representing the effects of air mass changes over a given observing station and graphs of diurnal profile variations.

In other studies along these lines, Bean, Horn, and Riggs [23], demonstrated that radio ray refraction within the lower layers of an air mass is mirrored by the difference between the observed refractive index structure and that of a standard atmosphere. Figure 5.5 shows a graph of bending, $\tau$, plotted together with a modified refractive index profile for an example of summertime tropical maritime air. It is apparent that near the ground bending departures reflect refractive index profile departures from standard. Figure 3.16 presents a series of graphs of departures of refractive

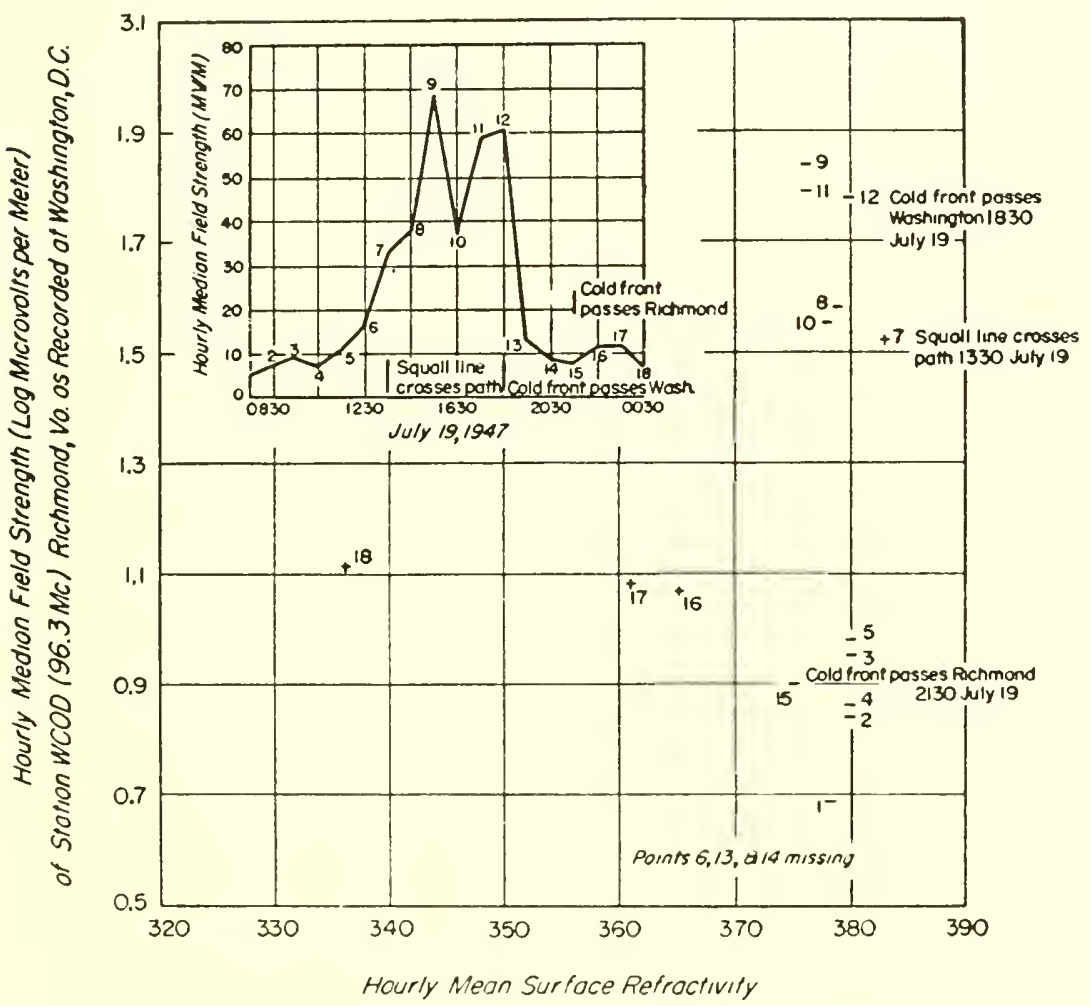

FIGURE 5.4. Field strength and $\mathrm{N}$ changes during frontal passaye. Seatter diagram showing passage of a cold front system over the Washington-Richmond path and graph indicating the fluctuation of hourly median field strength with time. 
index and bending from normal for each air mass and emphasizes the close relation between the two. This affords the synoptic radio meteorologist a set of standard reference profiles for the study of a given air mass or the confluence of contrasting air masses at a frontal zone.

Arvola [24] discussed the changes in refractive index profiles caused by migratory weather systems. Examining a series of synoptic situations in the midwestern portion of the United States during November 1951 that gave rise to greater-than-normal refraction, Arvola found that ridges and accompanying subsidence effects generally gave rise to strong $N$ gradients and enhanced signal strength over a $200-\mathrm{km}$ link broadcasting at 71.75 $\mathrm{Mc} / \mathrm{s}$. Refractive gradients were stronger in the warmer air masses and at times when moist air was present below the inversion created by the subsidence mechanism. Strong gradients which appeared behind a squall line later weakened with the approach of a cold front. After the passage of this front stratification in the cold air again increased the gradient.

Subsequent investigations of polar continental air across central North America make use of reduced-to-sea-level forms of the radio refractive index as synpotic parameters. The reduced forms are sensitive indicators of synoptic changes and afford a clearer picture of storm structure than that obtained using analyses in terms of unreduced $N$ or $B$ units (defined in chapter 1). Later portions of this chapter consider in detail these two synoptic parameters.

Jehn [25], at the University of 'T'exas, used a form of potential refractive modulus, $K$, developed by Lukes [26] and Katz [27] to account for the height dependence feature of the refractive index. Articles by Jehn $[28,29]$ on synoptic climatology use composite analysis techniques to study the synoptic properties of the Texas-Gulf cyclone and the central United States type of cold outbreak.

In another application of the potential refractive modulus, Flavell and Lane [30] have utilized Katz's $K$ unit (see chapter 6 ) to study tropospheric wave propagation. Field strength measurements on VHF-UHF transhorizon radio links over the British Isles are analyzed in terms of cross sections in terms of $K$ and regional charts of $\Delta K$, the difference between $K$ at the surface and $K_{850 \mathrm{mbar}}$. These charts show features similar to those obtained by use of $N_{0}$ or $A$ (see chapter 1).

The authors cite a series of measurements on a $500-\mathrm{km}$ path at 877 $\mathrm{Mc} / \mathrm{s}$ on which the received signal was ordinarily below the noise level. The singular occasions on which the received signal could be measured at the normal times of radiosonde ascent all exhibited a symmetric variation of $\Delta K$ over the transmission path. These results lend credence to the hypothesis that synoptic disturbances play an important role in transhorizon telecommunications.

Moler and Arvola [31] advanced the hypothesis that the vertical gradient of the refractive index is affected by broad-scale vertical motion 


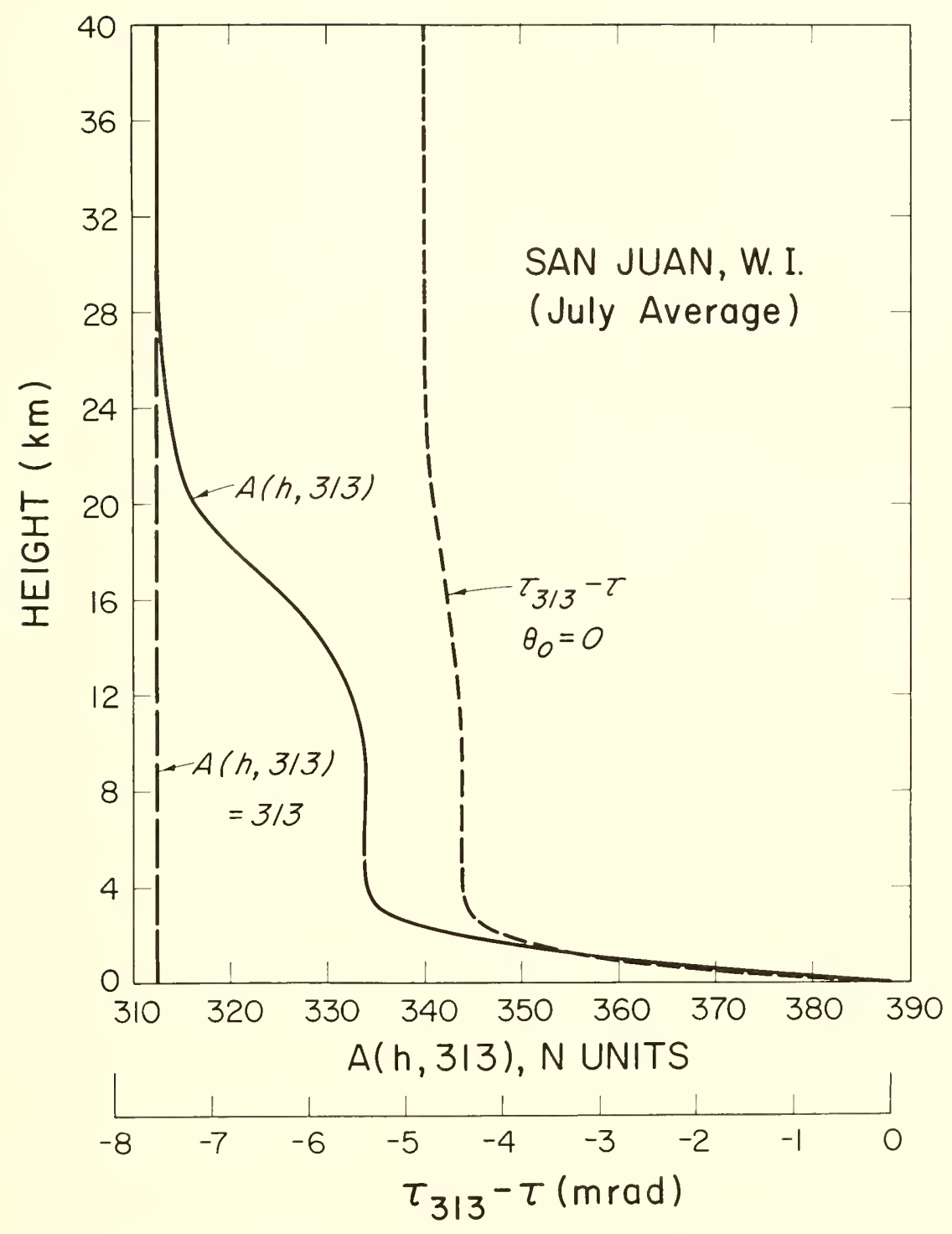

FIGURE 5.5. Departures of $\tau$ and $\mathrm{N}$ from normal for maritime tropical air. 
in the troposphere and suggest that moisture and temperature stratifieation is modified principally by ehanges in vertical velocity. This latter work was extended [32] by a study of mesoscale eenters of horizontal air mass convergence and divergence in the troposphere. Horizontal convergence takes place in a low-pressure area, where the winds around the low have a predominant component toward a local vortex at the center. Upward vertical motion results from the pile-up of air in the vortex region. In a high-pressure area, winds have a predominant component away from the eenter of a high and subsiding air descending from higher levels takes the place of air transported outward from the center of the high. Loeal convergence, then, implies upward vertical motion in the lower levels of the troposphere, while local divergence implies downward vertical motion in the lower levels. It follows that local convergence eenters (smallscale low-pressure eells) produee updrafts in the atmosphere that result in considerable mixing and the destruction of atmospheric layers. Centers of divergenee (small-scale high-pressure cells) ereate strong temperature and humidity inversions by the motion of subsiding air. Such inversions produce large vertieal refractive index gradients that partially refleet microwaves traveling through this meteorological environment [33]. In a well-mixed atmosphere, on the other hand, the primary propagation mechanism is believed to be seattering by turbulent fluctuations of the refractive index [34, 35].

Sea-level measurements showed $N_{s}$ to be essentially invariant during the experiment, yet signal levels ranged over more than $60 \mathrm{~dB}$, a power factor on the order of $10^{6}$. Since scattering theory would aecount for a rise in signal level of only about $13 \mathrm{~dB}$, Moler and Holden [32] conelude that refractive layering and thermal stability over the oceans are prineipally functions of vertical wind velocities. These eonelusions bear out earlier ones of Saxton [36] and later ones of Flavell and Lane [30] stating that high signal levels from a distant transmitter may well be the consequenee of refractive layering and subsequent reflection of radio waves. In the same article Saxton also considered both the scattering of radio energy by turbulent eddies and the effects of superrefraction of radio waves produced by departures from normal of the height variation of the tropospheric refractive index.

A knowledge of the vertieal motion of the atmosphere becomes at this point eentral to the problem of refractive layering. A brief resume of the Moler-Holden method for estimating the relative magnitude and direction of the vertical component of the wind veloeity follows.

Moler and Holden postulate a model atmosphere bound by the following conditions: 
(1) A barotropic level (level of nondivergence) exists at some pressure level greater than 500 mbar.

(2) The horizontal wind velocity divergence changes sign at the level of nondivergence (LND).

(3) The vertical velocity vector in the troposphere is proportional to that at the LND.

(4) The vertical velocity vector (a) vanishes at the surface of the earth and (b) approaches zero at the outer reaches of the atmosphere.

Commencing with the equation of continuity,

$$
\frac{\partial \rho}{\partial t}+\bar{V} \cdot \nabla \rho+\rho \nabla \cdot \bar{V}+\frac{\partial}{\partial z}(\rho W)=0
$$

where $\rho$ is the density of air, $\nabla$ is the horizontal del operator, $\bar{V}$ is the horizontal wind velocity vector, $W$ is the vertical wind speed, and $z$ is the vertical coordinate; and employing horizontal velocity divergence in the natural coordinate system of the form

$$
\nabla \cdot \bar{V}=\frac{\partial v}{\partial s}+v \frac{\partial \theta}{\partial n}
$$

Moler and Holden derive for the magnitude of the vertical vector at the LND, upon employing the vorticity equation,

$$
\begin{aligned}
(W)_{\mathrm{LND}}=-\frac{1}{\rho}\left[\int_{\mathrm{LND}}^{\infty} \frac{\rho}{\zeta} \frac{\partial \zeta}{\partial t} d z+\int_{\mathrm{LND}}^{\infty}\right. & \frac{\rho}{\zeta} V \frac{\partial \zeta}{\partial s} d z \\
& \left.+\int_{\mathrm{LND}}^{\infty} \frac{\rho}{\zeta} W \frac{\partial \zeta}{\partial z} d z\right],
\end{aligned}
$$

where $\zeta$ is the vertical component of the absolute vorticity. Moler and Holden reason that of the labeled integrals (I) decreases in proportion to the height above the LND and is « (II) at 300 mbar. Since $W \ll V$, (III) makes only a small contribution. The expression for $(W)_{\text {LND }}$ becomes

$$
(W)_{\mathrm{LND}}=-\frac{1}{\rho} \int_{\mathrm{LND}}^{\infty} V \frac{\rho}{\zeta} \frac{\partial}{\partial s}\left(\frac{\partial v}{\partial x}-\frac{\partial u}{\partial y}\right) d z
$$



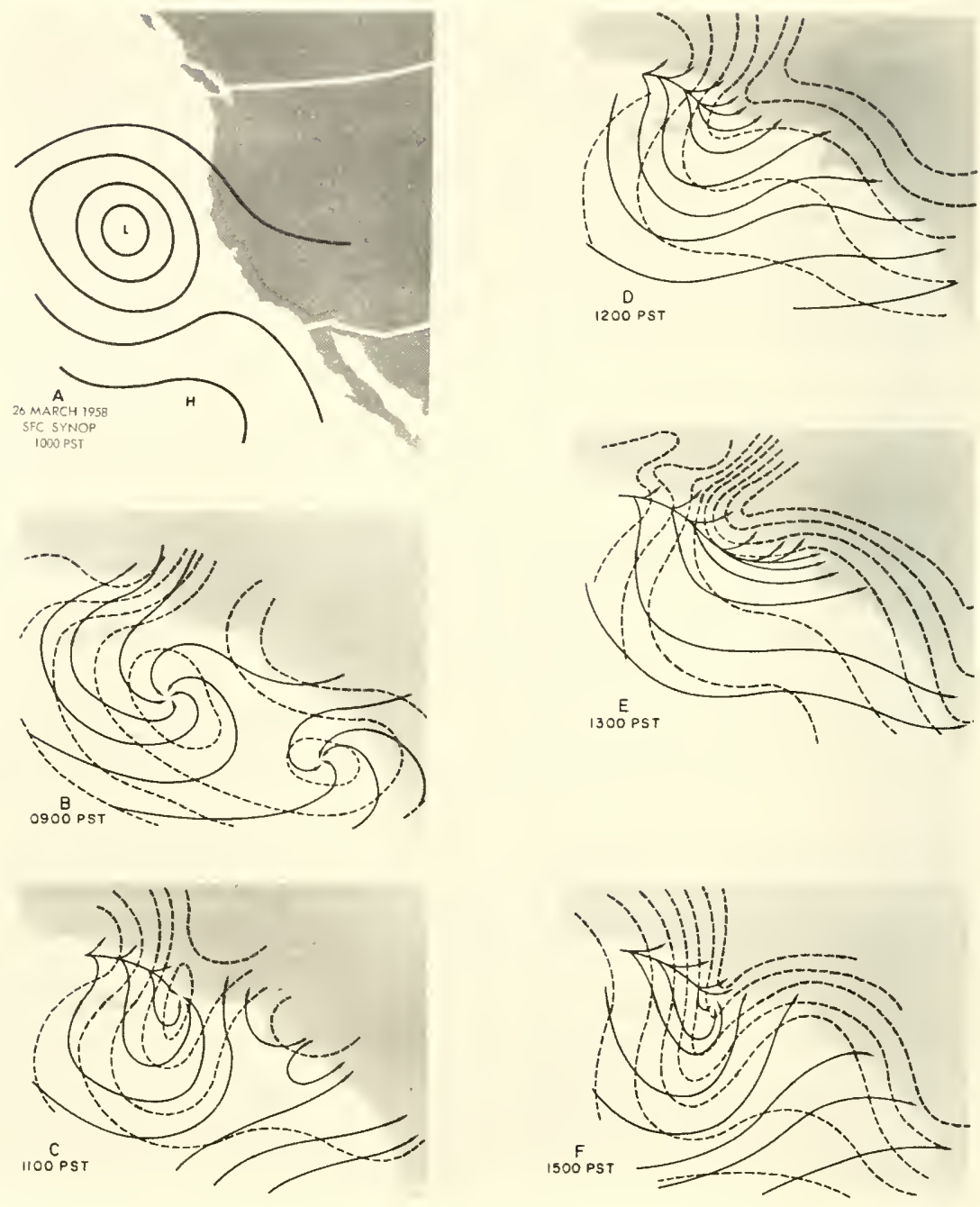

Figure 5.6. Isobaric and streamline maps.

which simplifies to the approximate relation

$$
(W)=\text { relative magnitude of } \frac{V}{\zeta} \frac{\partial}{\partial s}\left(\frac{\partial v}{\partial x}-\frac{\partial u}{\partial y}\right)_{300 \mathrm{mbar}}
$$

for purposes of calculation. 


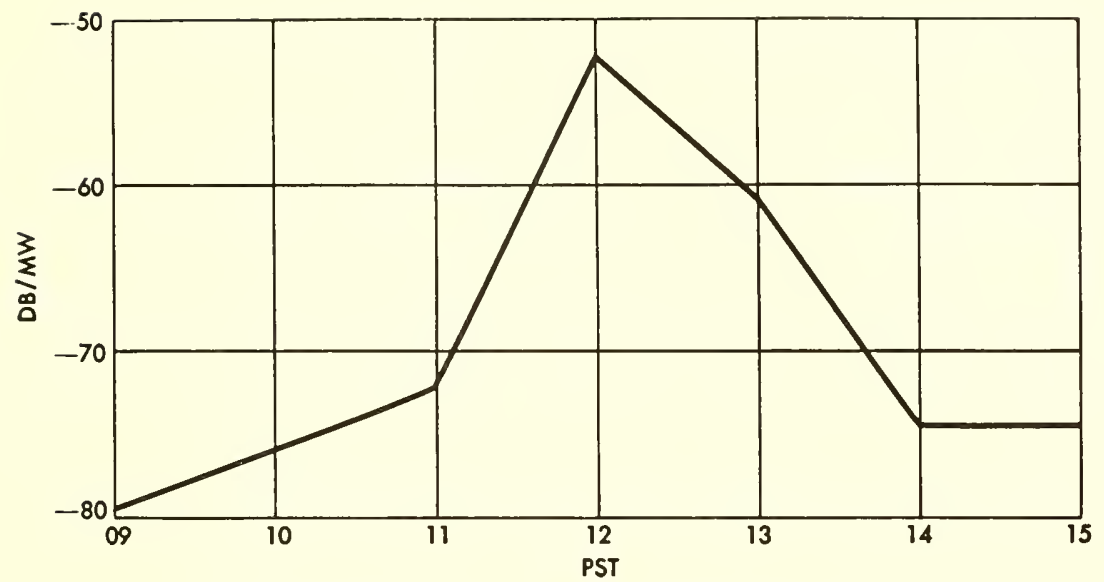

Figure 5.7. X-band signal level versus time. Santa Barbara X-band signal level for 26 Mar. 1958.

Moler and Holden then continue with a description of the large signal enhancement and deep fading that occur as the propagation mechanism varies between partial reflection and scattering on a transhorizon radio path along the California coastline. Reflection occurs when strong refracting layers are present within a kilometer of the surface, typifying meteorological conditions generally associated with the subsidence inversion frequently found along the California coast.

Figure 5.6 shows the sea level pressure chart and a series of streamline analyses by Moler and Holden depicting mesoscale centers of convergence and divergence for a day in March along the southern California coast. Figure 5.7 shows the $X$-band signal level received at Point Loma, San Diego (SD) from Santa Barbara (SB) during the same day. As the centers of convergence along the radio path weaken, refractive layers are formed and the signal level rises sharply during the middle of the day. Later the signal level lowers again with the regeneration of convergence centers and the destruction of stratified layers during the afternoon hours.

\subsection{Refractive Index Parameters}

In later sections, the analysis of a synoptic disturbance in the troposphere will be described in detail. Certain reduced forms of the refractive index that will be useful in the ensuing discussion will be developed here. These forms, already discussed in chapter 1 , are revisited here for the purpose of comparison in synoptic example. 
Figure 4.1 shows contours of the mean value of $N$ at the surface, $N_{s}$, determined from eight years of data for August, 0200 local time. Miniature circles indicate the 62 observing stations used to analyze this chart. It is evident that coastal areas display high values of $N_{s}$ as compared with inland locations. Low values of $N_{s}$ are apparent along the Appalachian mountain chain and in the great mountain systems and inter-mountain plateaus of the western United States. There is a marked similarity between the $N_{8}$ contours on figure 4.1 and the elevation contours of figure 5.8. As a sensitive indicator of changes in atmospheric density, $N_{s}$ displays a strong elevation dependence. To remove this effect the reducedto-sea-level expression, $N_{0}$, was introduced in chapter 4 as:

$$
N_{0}=N_{s} \exp \frac{z}{H^{*}}
$$

where $z$ is height in kilometers and $H^{*}=7.0 \mathrm{~km}$ is the scale height. Scale height is the height at which the mean value of $N$ has decreased to a fraction $1 / e$ of its initial value. A scale height of $7.0 \mathrm{~km}$ is in close agreement with $H^{*}=7.01 \mathrm{~km}$ for the NACA standard atmosphere with 80 percent relative humidity and $H^{*}=6.95 \mathrm{~km}$ obtained from climatic studies utilizing over two million observations of the variation in $N$ over the first kilometer above the surface of the earth [20]. The $N_{0}$ contours

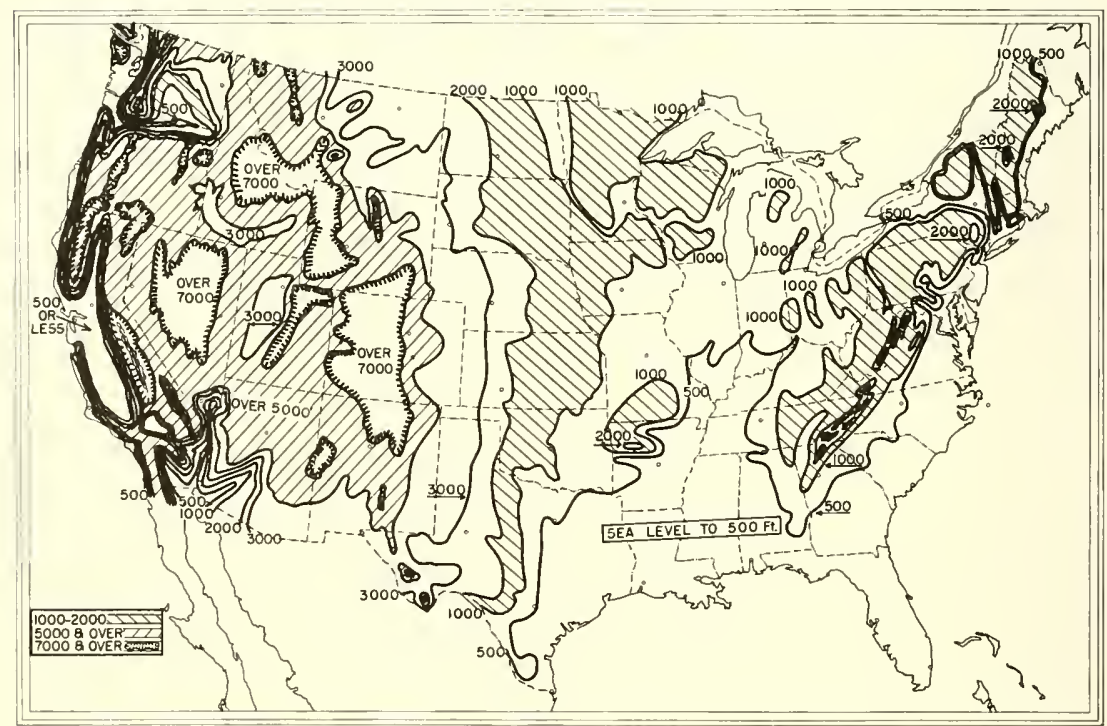

Figure 5.8. Ground elevation above sea level. 


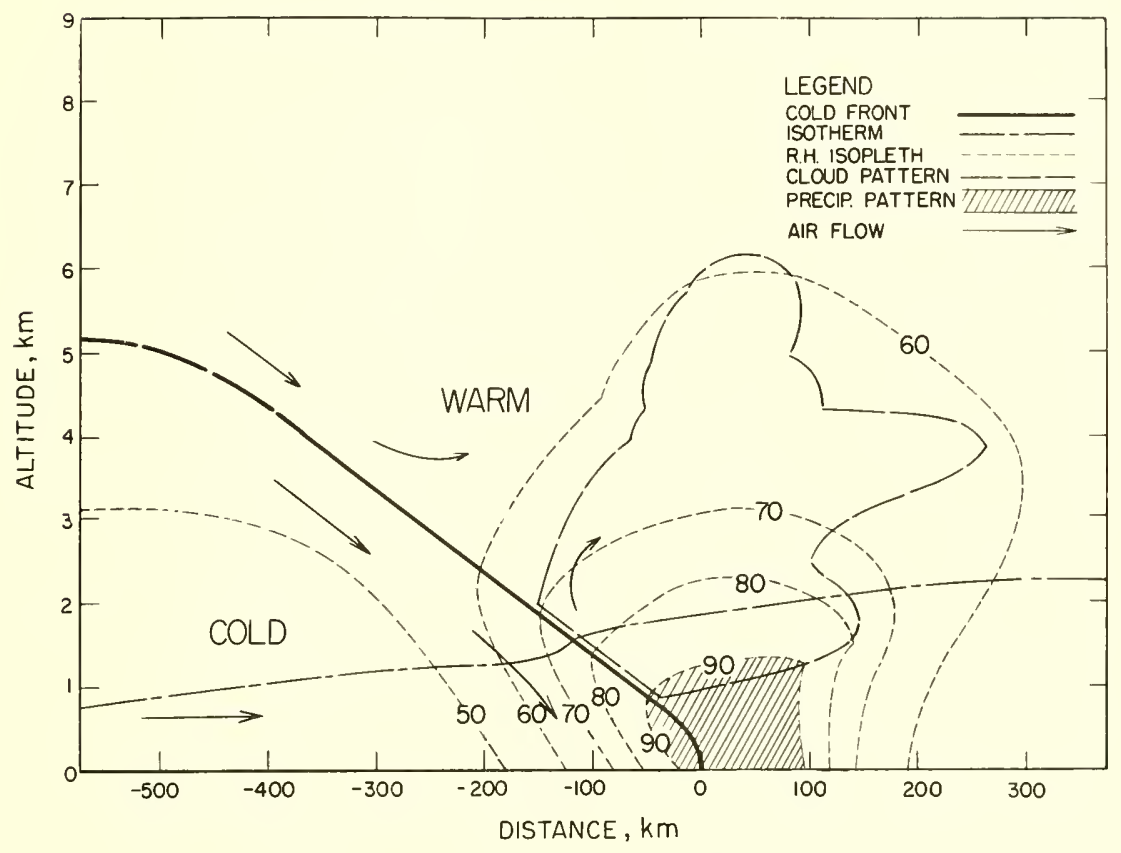

Figure 5.9. Idealized diagram of a fast-moving cold front.

of figure 4.4 utilize the same data as figure 4.1. The use of $N_{0}$ produces a simpler map with a smaller range of variation. Additionally, $N_{s}$ may easily be estimated from the smooth and slowly varying contours of $N_{0}$ providing only that station elevation is known. It was shown in chapter 4 that $N_{s}$ may be more accurately estimated from charts of $N_{0}$ than from charts of $N_{s}$ itself by a factor of 4 or 5 to 1 [37].

The attempt to find a workable method to compensate for the decrease of $N$ with height has brought about the development of various model atmospheres discussed in chapter 3. The paragraphs that follow will outline briefly steps in this development that are relevant to synoptic studies.

Vertical refractive index cross sections are standard working charts for synoptic studies. Such charts constructed from observed values of $N$ suffer from a serious shortcoming in that the natural decrease of $N$ with respect to height effectively masks contrasts between air masses in the lower troposphere. An idealized synoptic example depictng the confluence of contrastıng air masses is presented on figures 5.9 and 5.10. When these idealized systems are analyzed in terms of $N$ as on figures 5.11 and 5.12 the most prominent feature is the laminar structure of the $N$ field. 


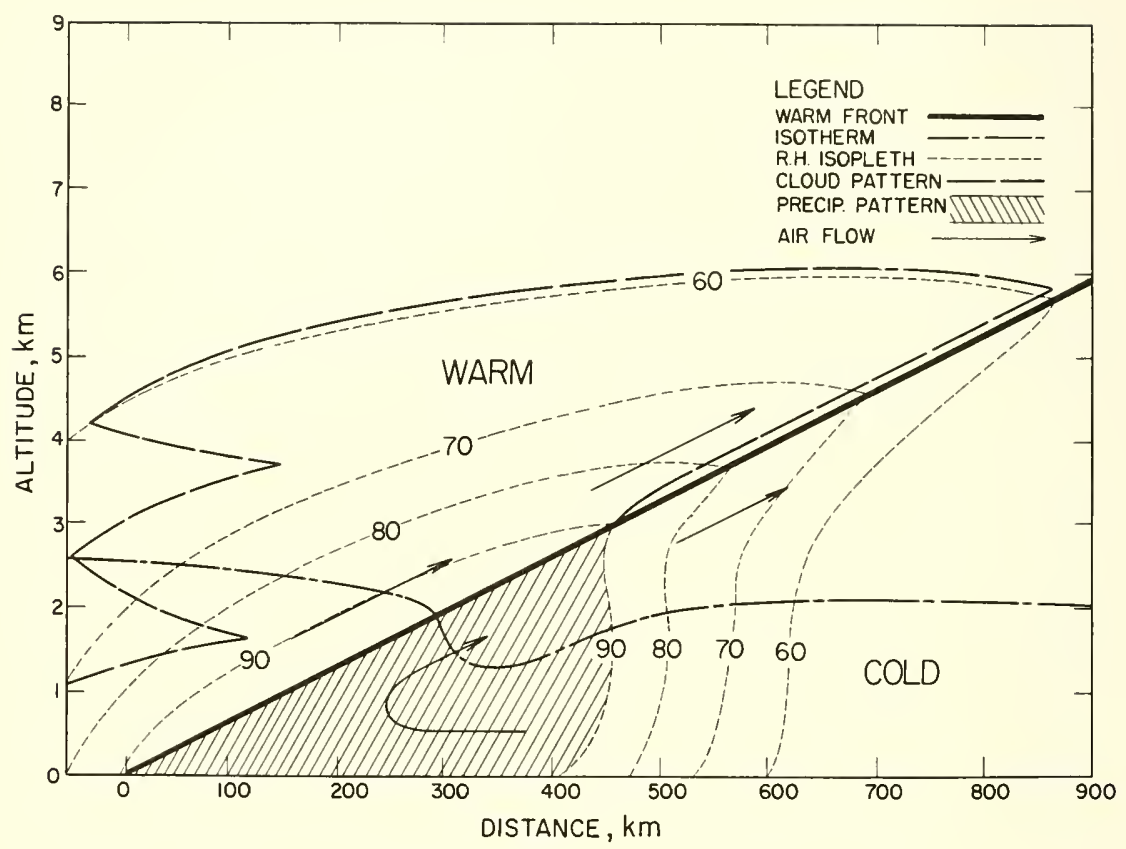

Figure 5.10. Idealized diagram of a warm front.

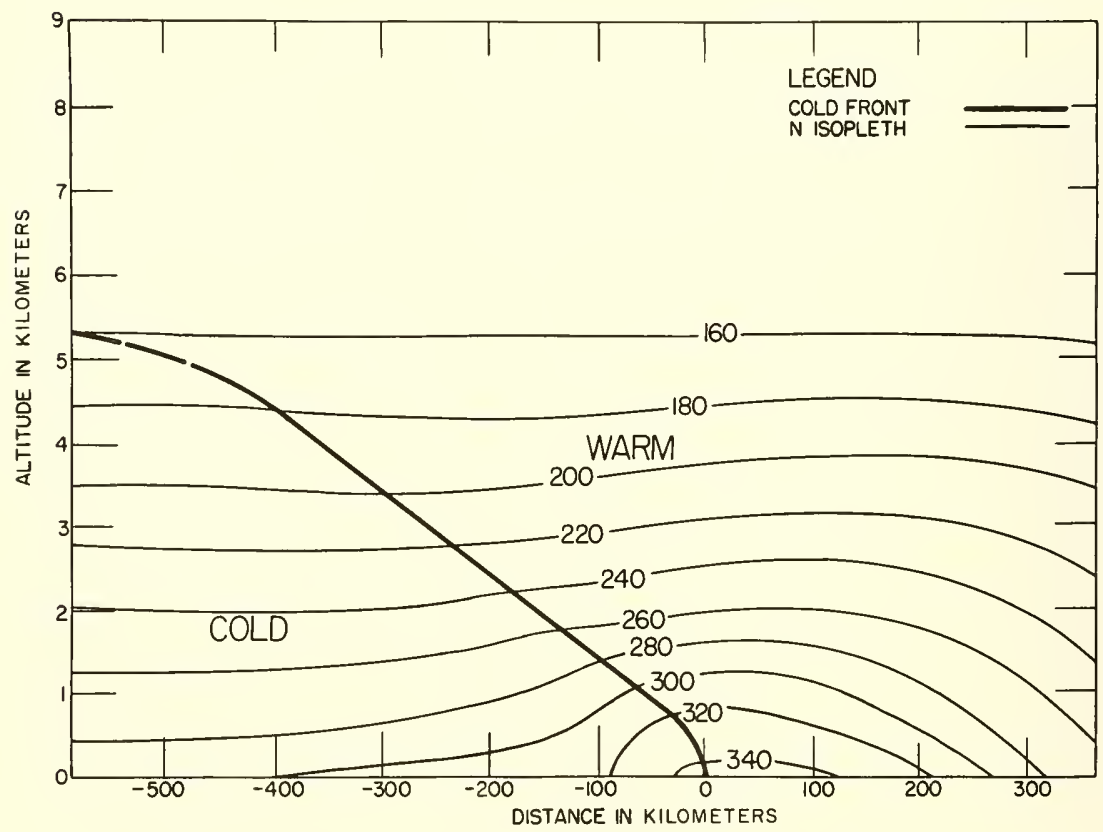

Figure 5.11. Idealized cold front in $\mathrm{N}$ units. 


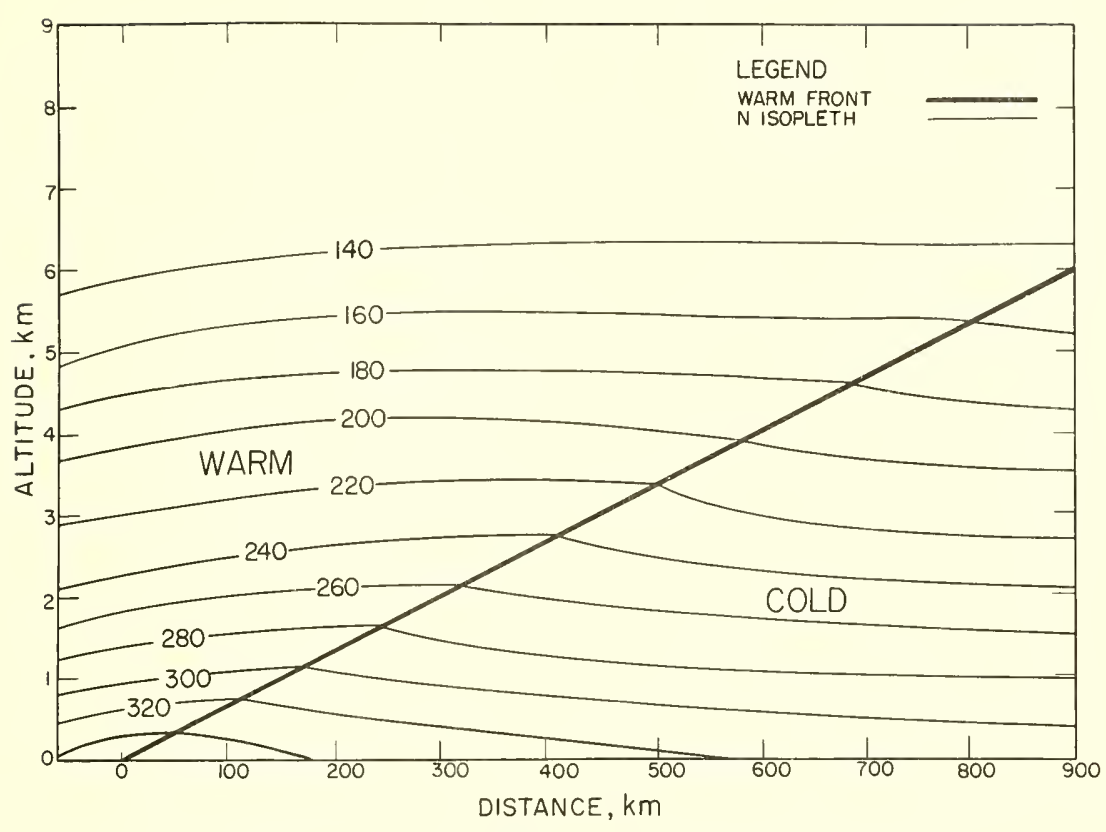

Figure 5.12. Idealized warm front in $\mathrm{N}$ units.

Early attempts to compensate for the decrease of $N$ with height used the constant gradient of the effective earth's radius theory, $1 / 4 a$, where $a$ is the radius of the earth. As an illustration, the strong elevated layer found during the summer in southern California was studied in terms of a form of (1.32) for $B$, given by

$$
B=N(z)+(39.2) z
$$

where $N(z)$ is the value of $N$ at height $z$ in kilometers [38]. Since $N$ tends to be an exponential function of height rather than the linear function assumed by the effective earth's radius theory, the $B$ unit approach overcorrects when $z$ is greater than about $1 \mathrm{~km}$.

This point is illustrated by figures 5.13 and 5.14 where the $N$ data of figures 5.11 and 5.12 are plotted in terms of $B$ units. Note that the overcorrection produces a cross section in which $N$ inereases with height from a value of 310 at the surface to 360 at $5 \mathrm{~km}$. A function of exponential form was designed to account for the systematic decay of density with height that characterizes the terrestrial atmosphere, as given by

$$
A=N(z)+313\left[1-\left\{\exp \left(-\frac{z}{7.0}\right)\right\}\right] \text {. }
$$




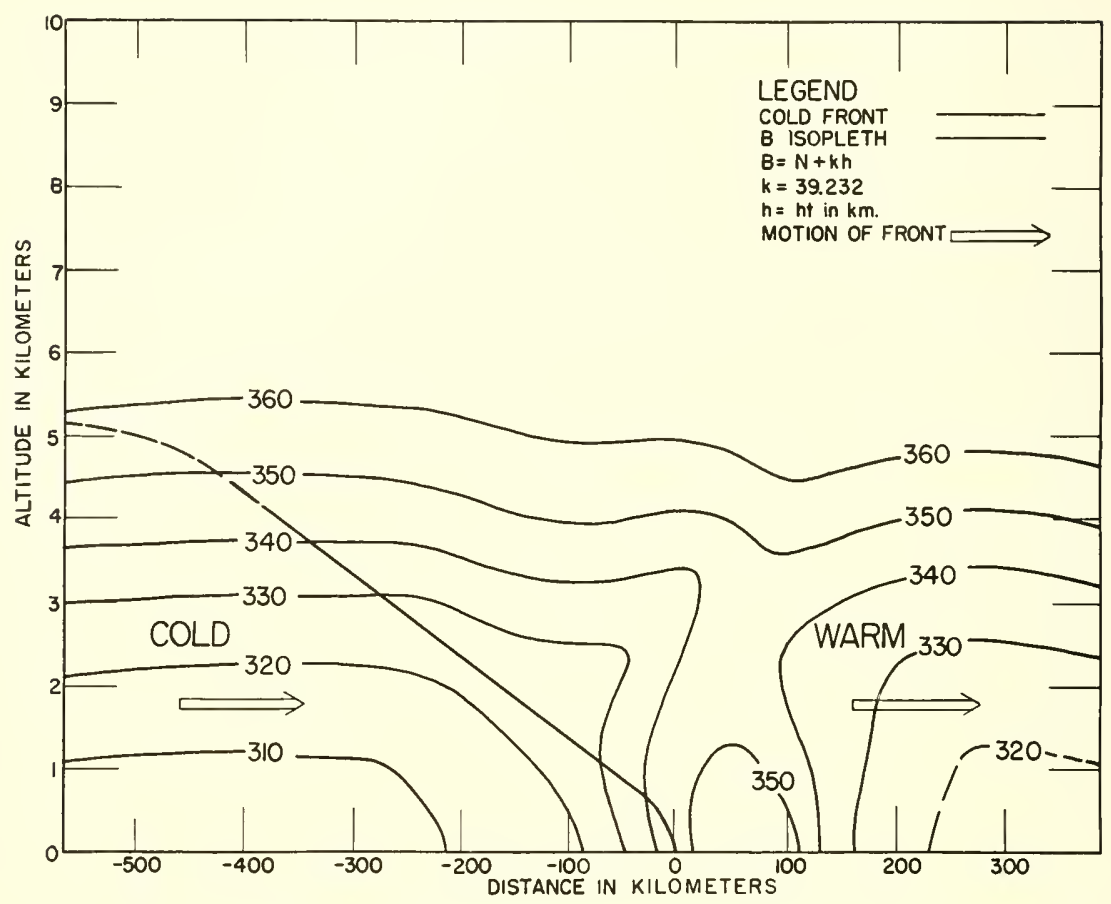

Figure 5.13. Idealized cold front in B units.

The quantity, $A$, enables one to discern departures of $N$ structure from the model atmosphere

$$
N=313 \exp \left\{-\frac{z}{7.0}\right\}
$$

Further, the radio-ray bending,

$$
\tau_{1,2} \doteq-\int_{N_{1}}^{N_{2}} \cot \theta d N \cdot 10^{-6}
$$

where $\theta$ is the local elevation angle of the radio ray to spherically stratified surfaces of constant $N$, may be approximated by

$$
\tau_{1,2} \doteq-\int_{A_{1}}^{A_{2}} \frac{\cot \theta\left(10^{-6}\right)}{n} d A(z, 313)+\tau(z, 313) .
$$

The term $\tau(z, 313)$ is the bending in the average atmosphere given by (5.9), while the integral term represents the departures in bending produced by 


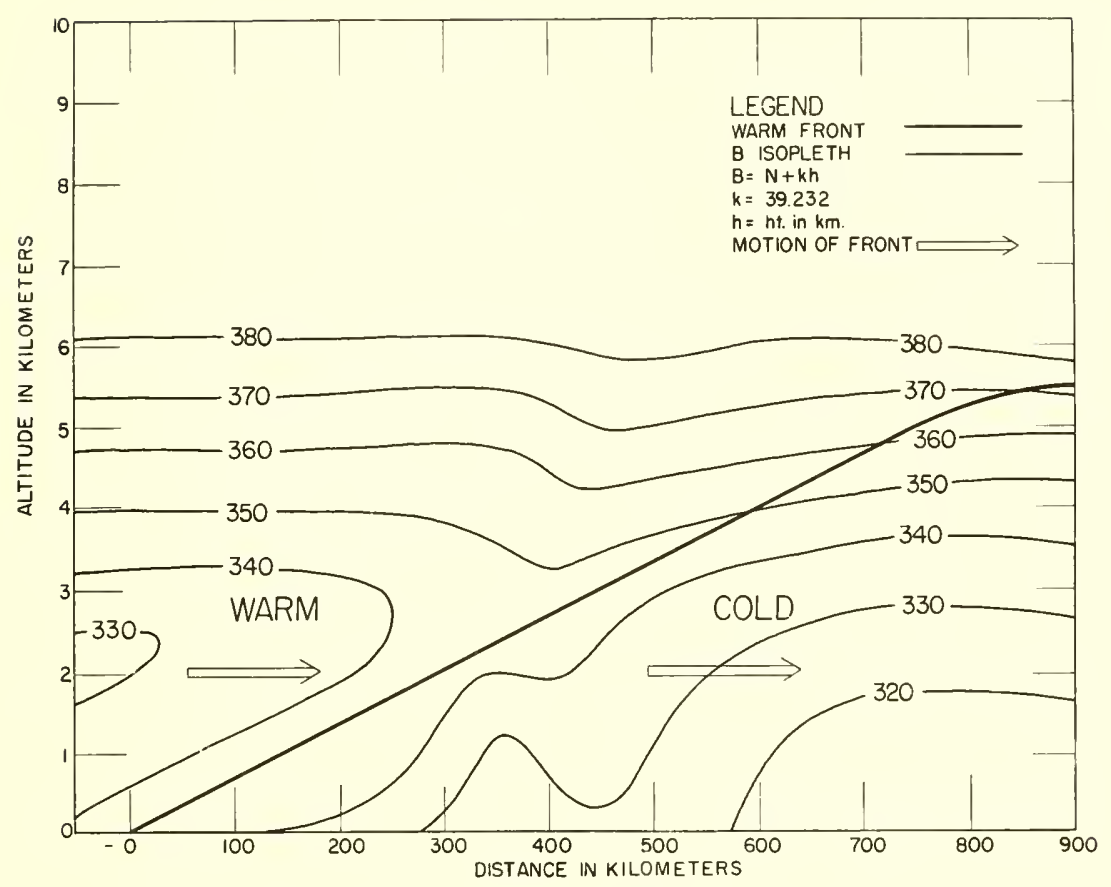

Figure 5.14. Idealized warm front in B units.

various synoptic and air mass effects. The values of bending in the average atmosphere are tabulated [39] and approximate methods of calculating the integral term in (5.11) to within a few percent have been given [40].

The next logical step is to plot the frontal cross sections, previously analyzed in terms of $N$ and $B$, in $A$ units. This has been done on figures 5.15 and 5.16. The range of refractivity values on the new charts is reduced from more than 60 to about 25 units and a pattern emerges that displays sharp contrasts for air mass differences associated with the frontal zone. Note, for the warm front case (fig. 5.16), that the $A$ values increase with height until they reach a maximum associated with the upgliding warm moist air overriding the frontal surface. The region of precipitation in advance of the front is shown as an area of high surface $N$. In the cold-front case (fig. 5.15), the classic push of warm air aloft by the encroaching cold air is evidenced by the "dome" of high $A$ values just before the front. Stratifieation in the cold air due to inversion effects, although impossible to detect in the $N$ charts, is clearly seen by the use of $A$ unit charts. 


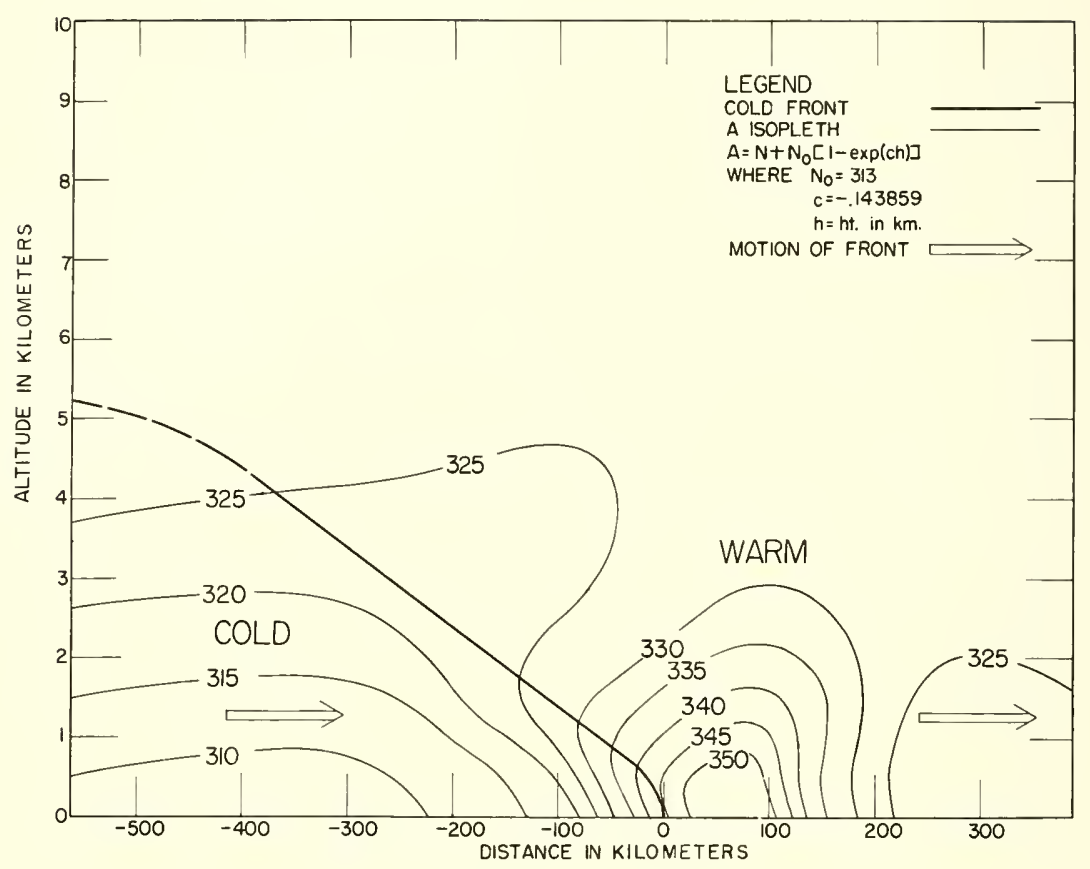

Figure 5.15. Idealized cold front in A units.

$A$ units were used in subsequent cross-section analyses in order to throw frontal discontinuities and air mass differences into sharp relief. The Potential Refractivity Chart of figure 5.17 facilitates the rapid conversion of $N$ to $A$. This simplification eliminates the necessity of using exponential tables for each individual calculation of $A$ and thus lends considerable ease to the preparation of charts of the new parameter.

The reader probably has already observed that the $N_{0}$ and $A$ corrections do substantially the same thing. Their primary distinction is that $A$ is a nonlinear "add-on" correction while $N_{0}$ is a multiplicative one. The disparity between $N_{0}$ and $A$ is tabulated in table 5.1.

These figures are obtained by taking the zero values of $N$ (for example, 300 ), subtracting the add-on correction for, say, $3 \mathrm{~km}(300-109=181)$, and reducing this number, $N=181$, to zero elevation by the $N_{0}$ reduction, $N_{0}=N \exp (3 / 7)$. The 313 exponential atmosphere is adopted for a single reference atmosphere. The large discrepancies of table 5.1 may be avoided for practical applications by choosing a model near to the mean value of $N$ of the site under study.

The $A$ unit has the additional advantage that, while it is a convenient method for height reduction, the ray bending is also readily recoverable 


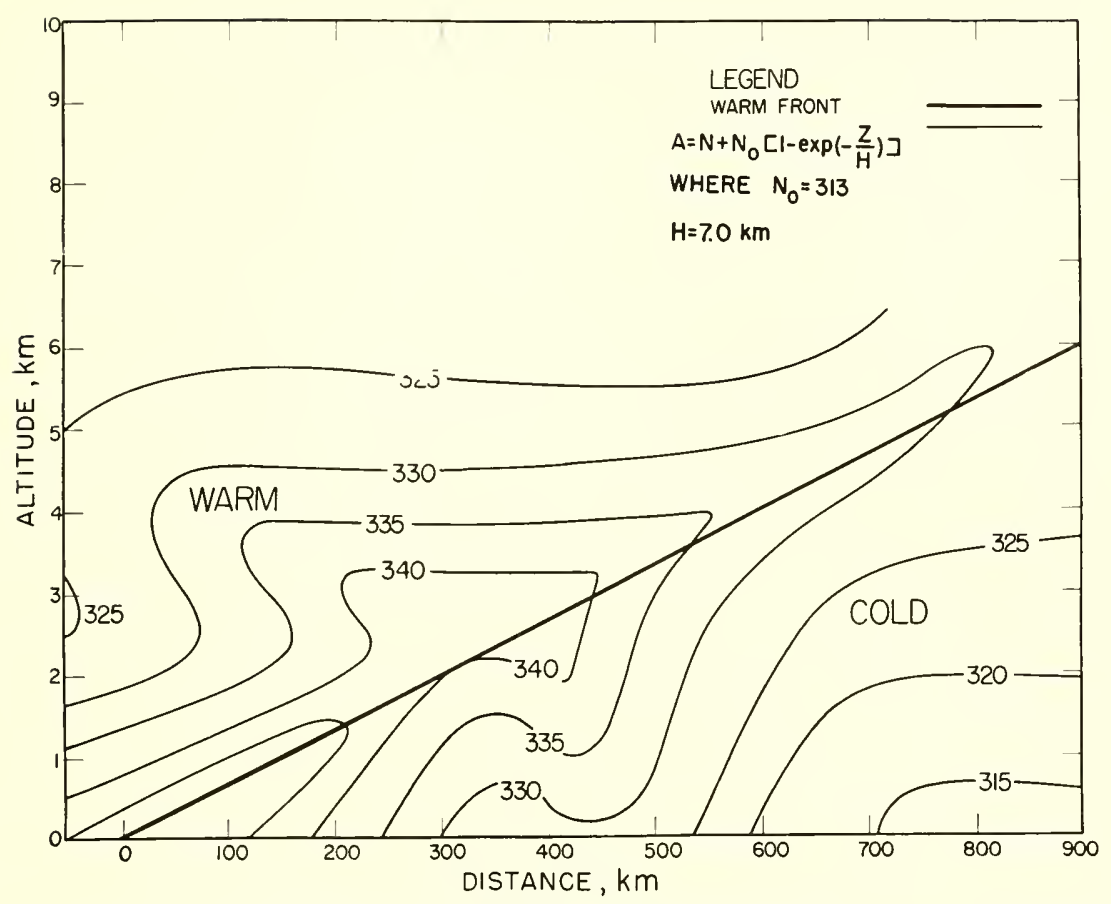

Figure 5.16. Idealized warm front in A units.

from it, requiring only a knowledge of the altitude of the observed refractive index measurement and the local elevation angle of the radio ray.

The potential refractive modulus of I. Katz [27],

$$
\phi=\frac{c}{\theta}\left[P_{0}+b \frac{e_{0}}{\theta}\right]
$$

where $\theta$ is the potential temperature and $e_{0}$ the potential vapor pressure, is also in current use. The constants $b$ and $c$ of $(5.12)$ are given in the development of modification to $N$ data in chapter 1 (1.39). The potential refractive modulus has been employed by Jehn [25] to study polar waves over North America. Refractivity, $N$, cannot aecurately be recovered from $\phi$ for bending ealeulations unless additional information is available; namely, observed temperature and vapor pressure. The eoncept of the potential refractive modulus arose out of the earlier refractive modulus, $M$ (see ch. 1), which may be defined by

$$
M=\left[n-1+\frac{z}{a}\right] \times 10^{6}=N(z)+\left[\frac{z}{a}\right] \times 10^{6},
$$

where $z=$ height above the earth's surface and $a=$ earth's radius. 


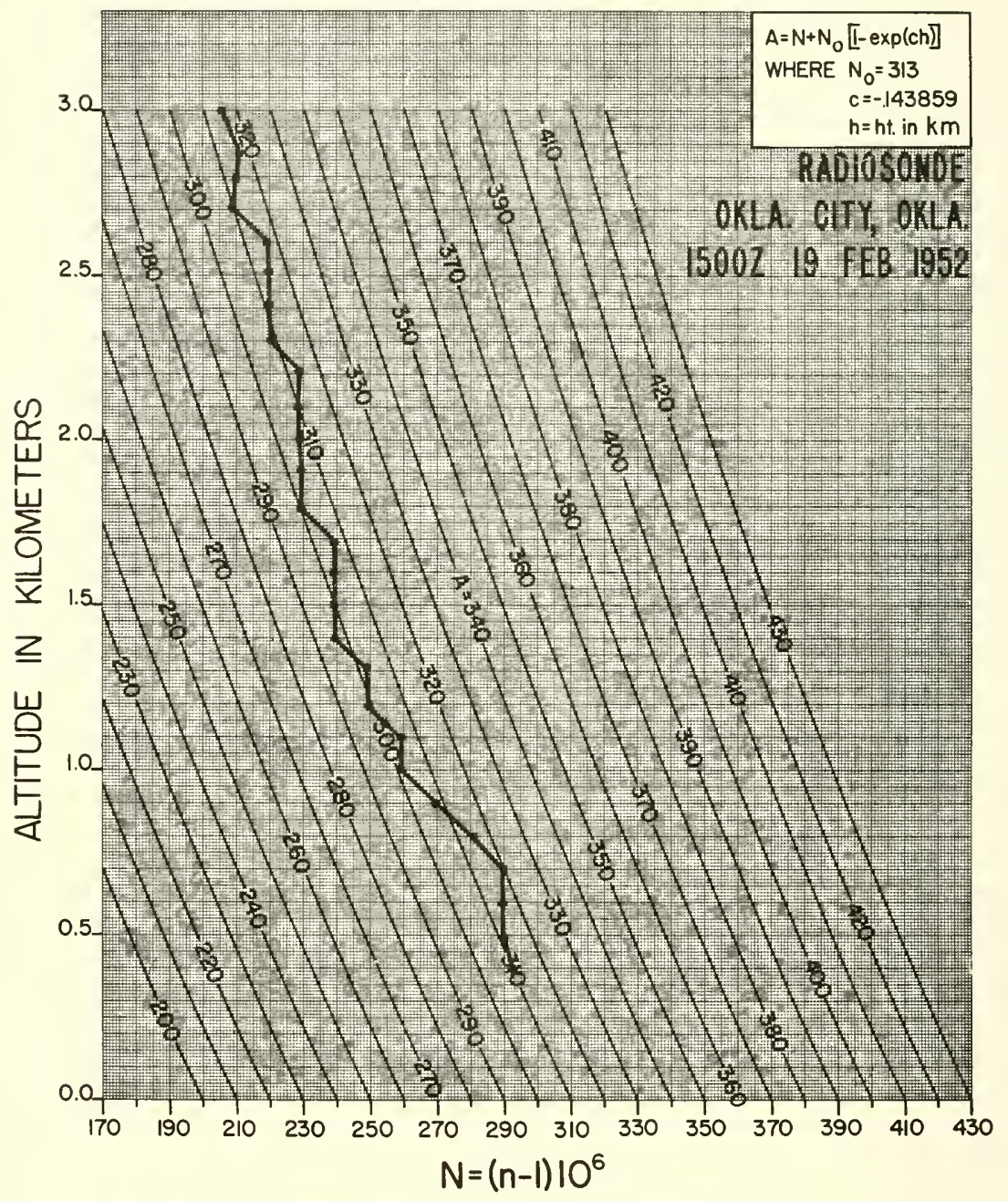

Figure 5.17. Potential refractivity chart.

TABLE 5.1. Differences between $\mathrm{N}_{0}$ and $\mathrm{A}$ at various elevations Value of $N$ at $z=0$

\begin{tabular}{|c|c|c|c|c|c|}
\hline Elevation & 250 & 300 & 313 & 350 & 400 \\
\hline $\begin{array}{c}(\mathrm{km}) \\
1 \ldots \\
2 \\
4\end{array}$ & $\begin{array}{l}-10 \\
-21 \\
-49 \\
-48\end{array}$ & $\begin{array}{r}-2 \\
-5 \\
-22 \\
-29\end{array}$ & $\begin{array}{l}0 \\
0 \\
0 \\
0\end{array}$ & $\begin{array}{r}5 \\
7 \\
5 \\
30\end{array}$ & $\begin{array}{l}13 \\
29 \\
31 \\
68\end{array}$ \\
\hline
\end{tabular}


The $M$ unit came into being out of an approach similar to that which led to the development of the $B$ unit. The condition $d n / d z=-1 / a$ (a radio duet) implies an effective earth of infinite radius (effective earth's radius factor, $k=\infty$, see $c h .1$ ). The $M$ unit is designed so that $d M / d z$ $=0$ when $k=\infty$. $M$ units are employed from time to time in radio meteorological analysis. The Canterbury Project [41], for example, used $M$ unit analyses in the study of ranges of over-water radar signals.

\subsection{A Synoptic Illustration}

The specialized field of synoptic radio meteorology attempts a description of the variations in atmospheric refraction that arise from large scale weather changes such as the passage of a polar front or the movement of an air mass over a particular geographic region. The term air mass is used to describe a portion of the troposphere that has at the surface generally homogeneous properties. Although no air mass is in fact homogeneous, the advantages of the air mass concept as a convenient fiction are evident in the cataloging of meteorological observations for ciimatic or synoptic purposes.

The region of interaction between the cold air of the poles and the warm air of the tropies is referred to as the polar front and is generally located between 30 and $60^{\circ} \mathrm{N}$. From time to time a section of the polar front is displaced northward by a flow of warm tropical air while an adjacent seetion is simultaneously displaced southward by a flow of polar air. The interaction of the flow of polar and tropical air results in the formation of a "wave" that moves along the polar front, often for thousands of kilometers. An example of a fully developed polar front wave is shown on figure 5.18(a), in the same manner that it would appear on a daily weather map. Across the Great Plains and eastern seaboard of the United States the polar front wave normally moves along the line $A B$ in figure 5.18(a). An idealized space cross section along the line $A B$ is shown in figure $5.18(\mathrm{~b})$. The warm tropical air that flows into the warm seetor of the wave overrides the cool air before the wave to form the transition zone denoted as a warm front. The cold front represents the transition between the generally humid air of the warm sector that has been forced upwards and the advancing cold polar air. Squall lines are drawn to represent belts of vigorous vertical convection, intense thunder showers, and sharp wind shifts that frequently precede fast-moving cold fronts. The fronts shown on a daily weather map represent the ground intersection of the transition zones between various air masses. ${ }^{2}$

2 The reader who wishes a critical appraisal of recent meteorological thinking on fronts, air masses, squall lines, etc., is referred to Dynamic Meteorology and Weather Forecasting, by Godske, Bergeron, Bjerknes, and Bundgaard, American Meteorological Society and Carnegie Institute of Washington, D.C., 1957. 


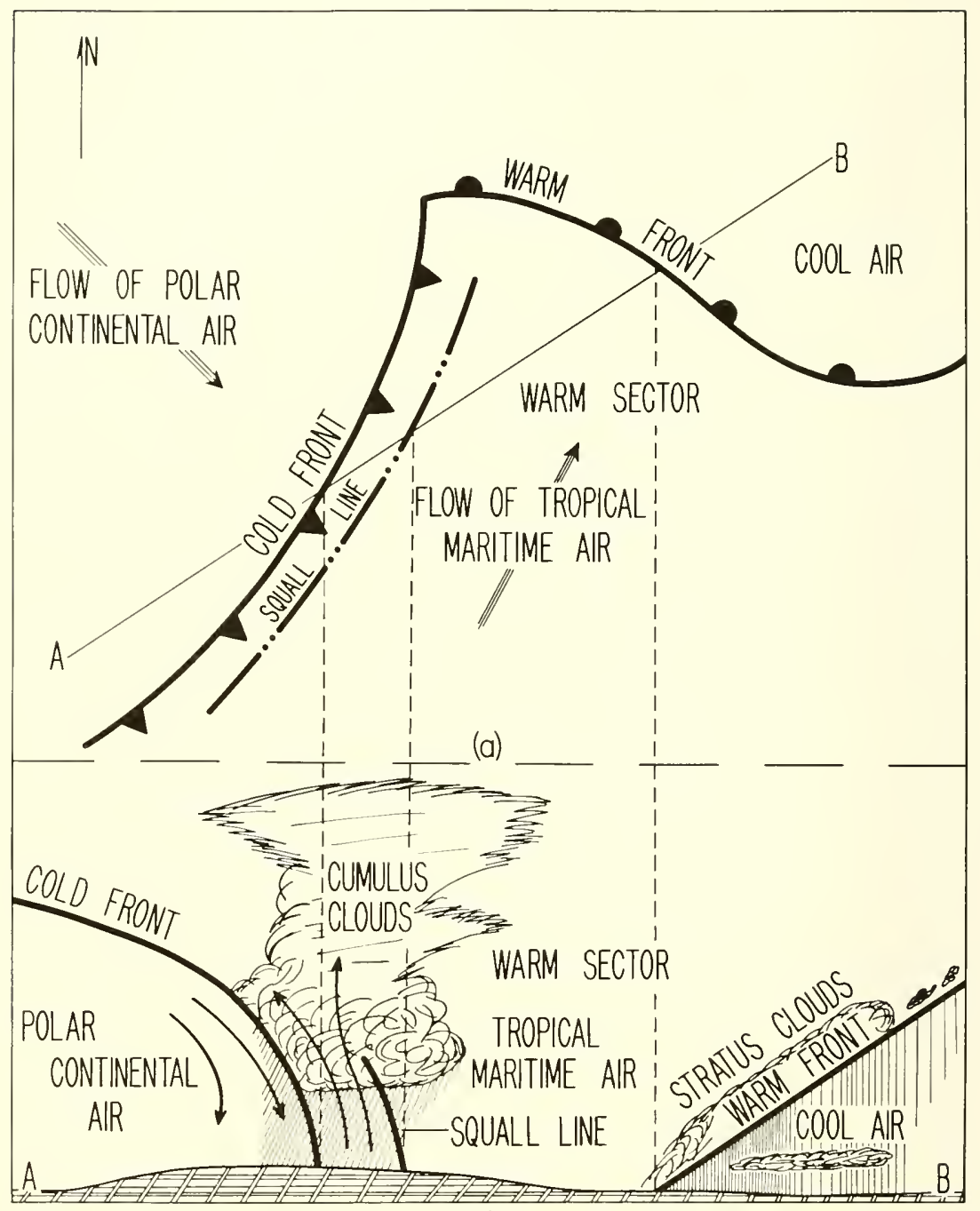

(b)

Figure 5.18. The Polar Front wave. 
As a representative example of the application of these newly developed units to a synoptic situation, a large-scale outbreak of continental polar air which took place over the United States during February 1952 is analyzed in terms of $N_{0}$ and $A[42,43]$.

For this synoptic illustration the reduced expression, $N_{0}$, is used in preparing constant level charts of the storm at the same levels and times as those used in the daily weather map series of the U.S. Weather Bureau. The $A$ unit, on the other hand, is employed to construct vertical crosssections through the frontal system to give a three-dimensional picture of the synoptic changes taking place.

\subsection{Surface Analysis in Terms of $N_{\mathrm{o}}$}

A pronounced cold front developed and moved rapidly across the United States during the period 18 to 21 February 1952.

Prior to February 18, a polar maritime air mass had been moving slowly eastward across the Great Basin and Rocky Mountain regions. This system included a slow-moving cold front extending from northern Utah southwards into Arizona and a quasi-stationary front extending northeastwards into Wyoming. With the outbreak of polar continental air east of the Rocky Mountains, the maritime front became more active and, as it moved ahead of the fast-moving polar continental front sweeping across the Great Plains, was reported as a squall line by the time it crossed the Mississippi River early on the morning of February 20. During the latter stages of the storm system, the polar maritime cold front-squall line was located in the developing warm sector of the polar front wave. The entire ensemble of cold front, polar front wave, and squall line then moved rapidly to the east coast by the morning of the 21 st, thus completing the sequence.

Charts of $N_{0}$ were prepared from Weather Bureau surface observations taken at 12-hour intervals from 0130 EST, February 18 until 0130 EST, February 21, 1952, or, in other words, the period of time that it took the polar front wave to develop and move across the country. The synoptic sequence is seen on figures 5.19 through 5.25 ., where contours of $N_{0}$ are derived for various stages of the storm and compared to the superimposed Weather Bureau frontal analysis. The same procedure of comparing derived contour's with the existing frontal pattern was followed throughout the present example. Observations from 62 weather stations were used in preparing the surface weather maps. Figure 5.19 indicates that the cold front extending from Utah southward displays weak $N_{0}$ changes across the frontal interface. In the early stages of the sequence (figs. 5.19 and 5.20) this lack of air mass contrast is evidenced in another way by the slight change of the position of the $N_{0}=290$ contour encircling west Texas and New Mexico as the frontal system moves through that area. 


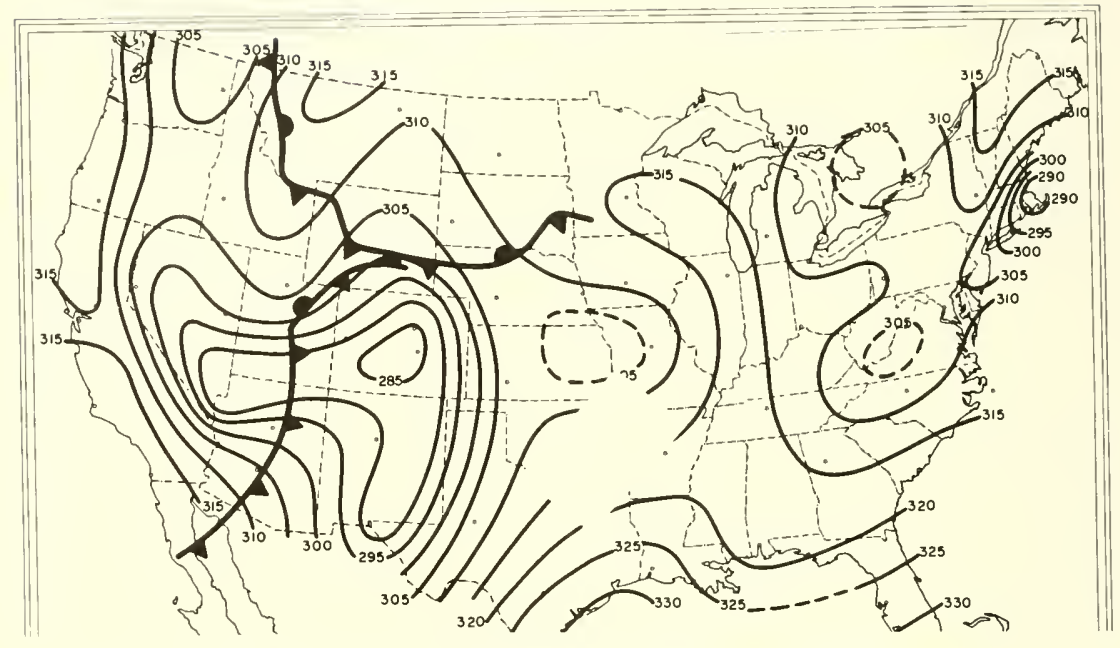

Figure 5.19. $\mathrm{N}_{0}$ chart for storm system 0190E 18 Feb. 1952.

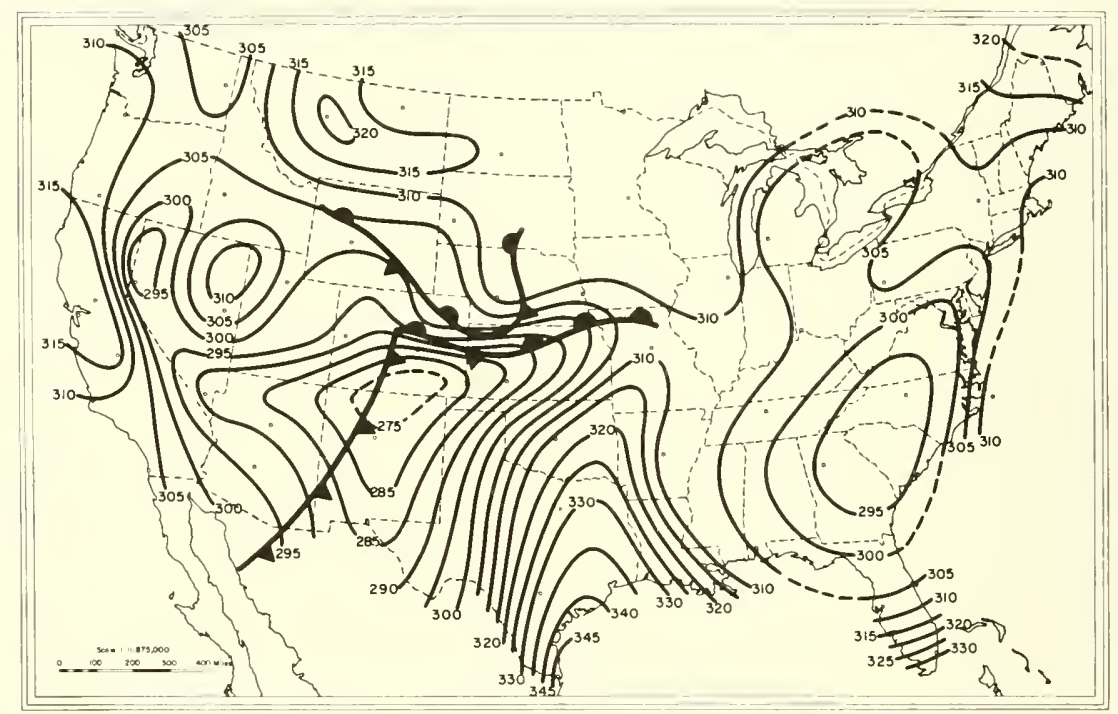

FiguRE 5.20. No chart for storm system 1830E 18 Feb. 1952. 


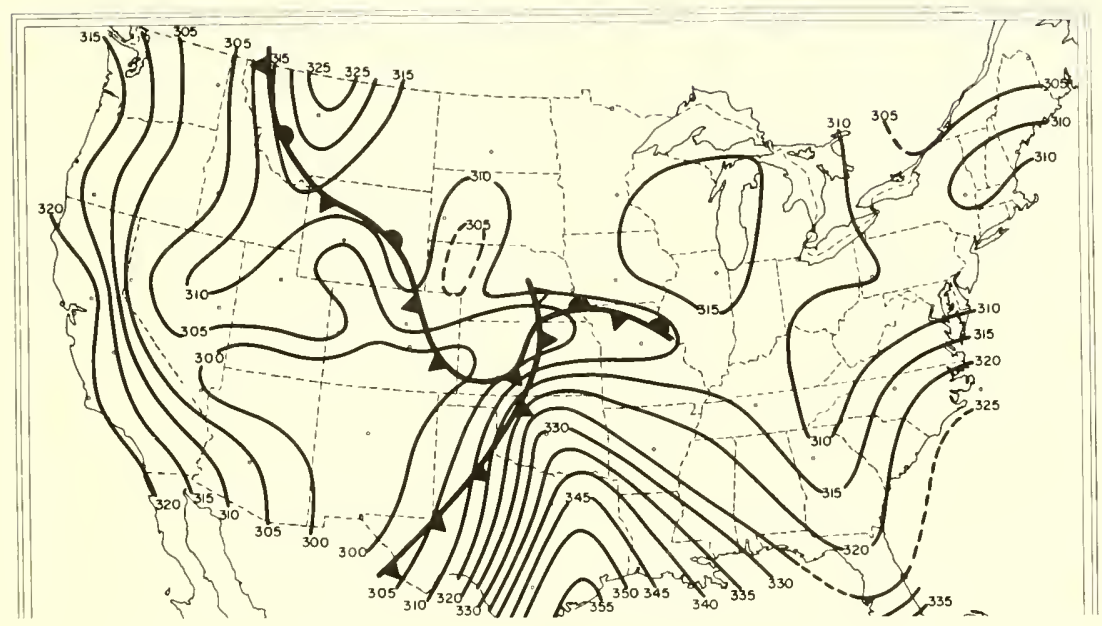

Figure 5.21. $\mathrm{N}_{0}$ chart for storm system 0130E $19 \mathrm{Feb}$. 1952.

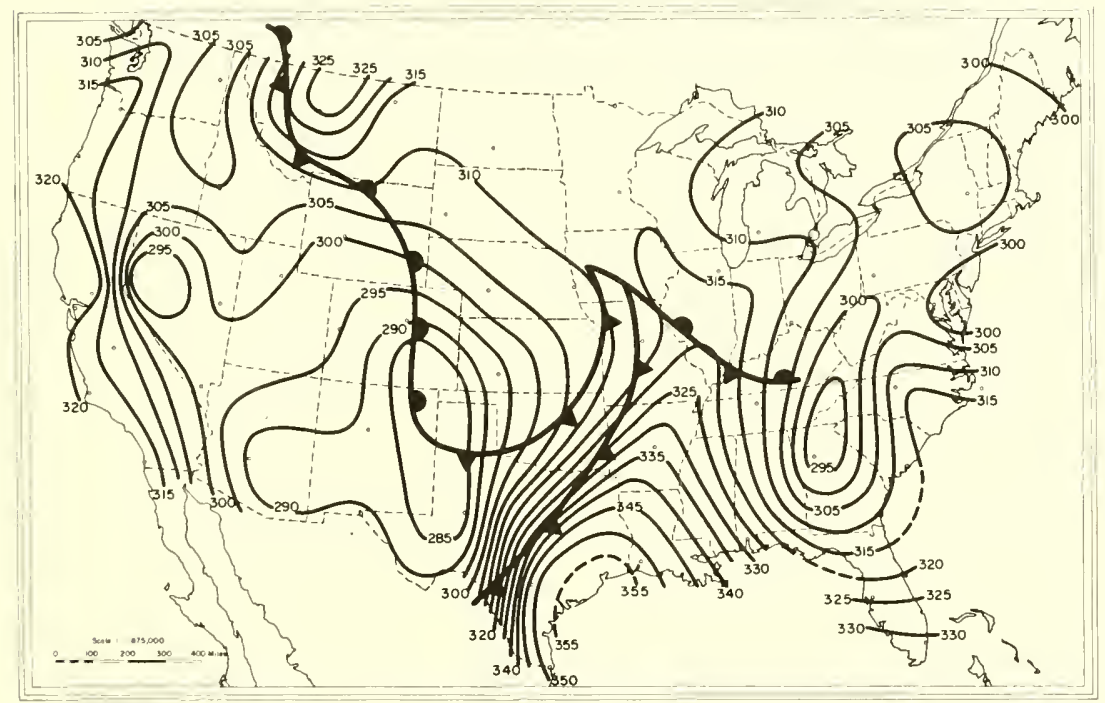

Figure 5.22. $\mathrm{N}_{0}$ chart for storm system 1330E 19 Feb. 1952. 


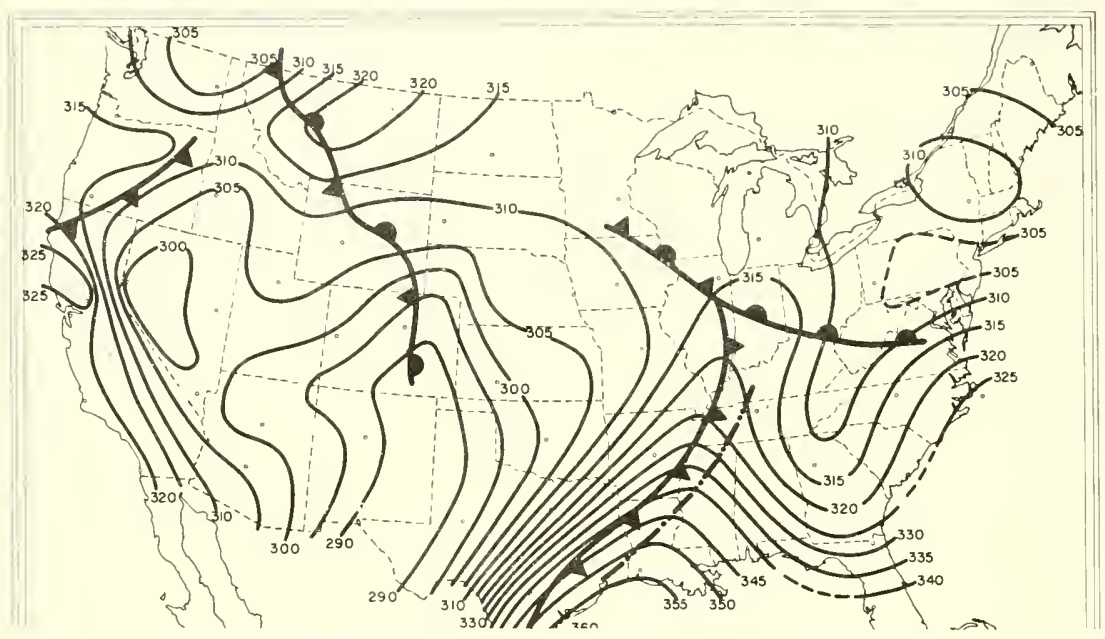

Figure 5.23. No chart for storm system 0190E 20 Feb. 1952.

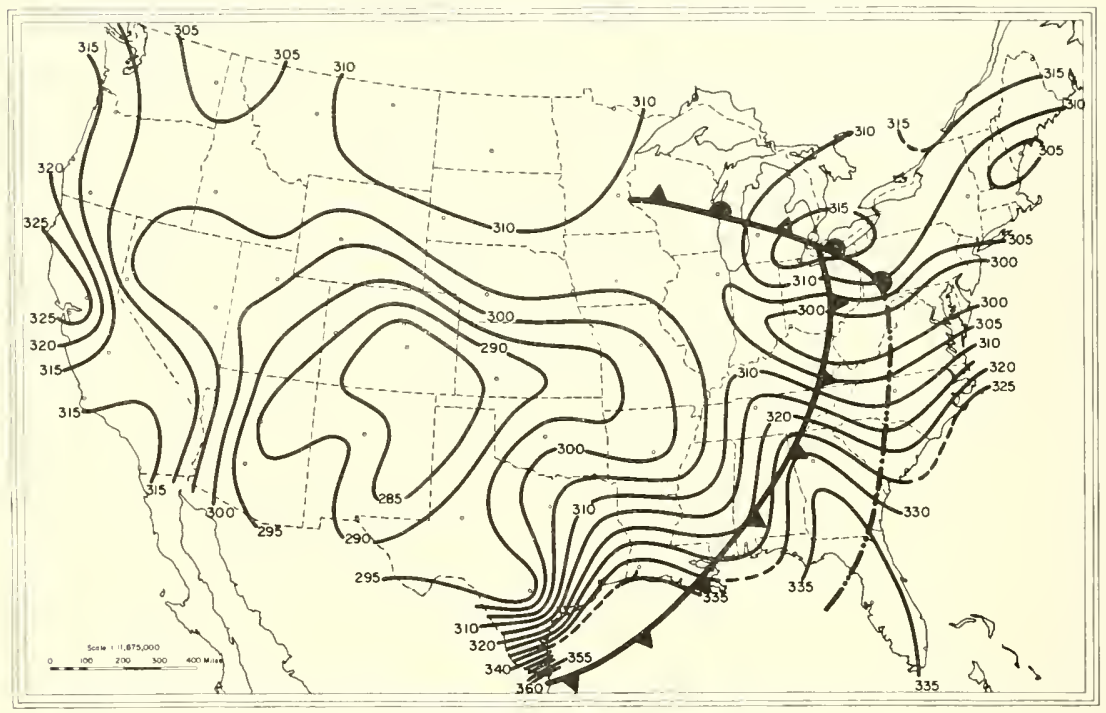

Figure 5.24. No chart for storm system 1330E 20 Feb. 1952. 


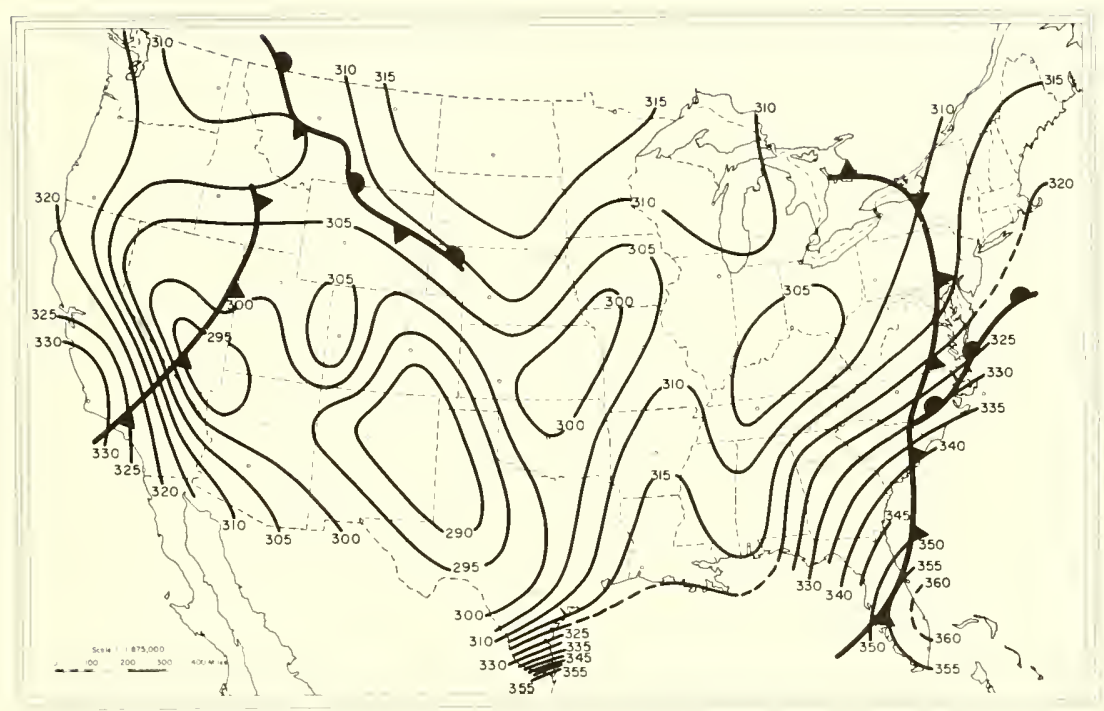

Figure 5.25. $\mathrm{N}_{0}$ chart for storm systems 0130E 21 Feb. 1952.

By comparison, the cold front sweeping down across the Great Plains (figs. 5.20 to 5.22) has a rather marked $N_{0}$ gradient across the front, due in large measure to the northward flow of warm, moist air that forms a definite warm sector by 1330 EST on the 19th. It is perhaps significant that the $N_{0}$ contours indicate that the various frontal systems are transition zones rather than the sharply defined discontinuities of textbook examples, a point that has been enlarged upon by Palmer [44].

Figures 5.22 to 5.25 trace the trajectory of this vigorous push of cold air across the Gulf Coastal Plain and the southeastern states. The most spectacular gradients on this map series are in the eastern half of Texas where the marked contrast of cold, dry polar air of low $N_{0}$ and warm moist Gulf air of very high $N_{0}$ occurs. A prominent feature of meteorological significance on figures 5.20 to 5.23 is the northward advection of tropical maritime air in the warm sector ahead of the cold front. The advection is evidenced by the northward bulge of high $N_{0}$ over the Mississippi Valley on both eharts. Figure 5.25 shows the synoptic situation as the front moves off into the Atlantic Ocean and refractivity gradients across the continent gradually weaken.

The variation of $N_{0}$ due to the passage of the frontal system can be seen on figures 5.26 to 5.30 where the 24 -hour changes of $N_{0}$ have been contoured. The 24-hour change, designated $\Delta N_{0}$, is obtained by subtracting from the current value the value of $N_{0}$ observed 24 hours ago. 


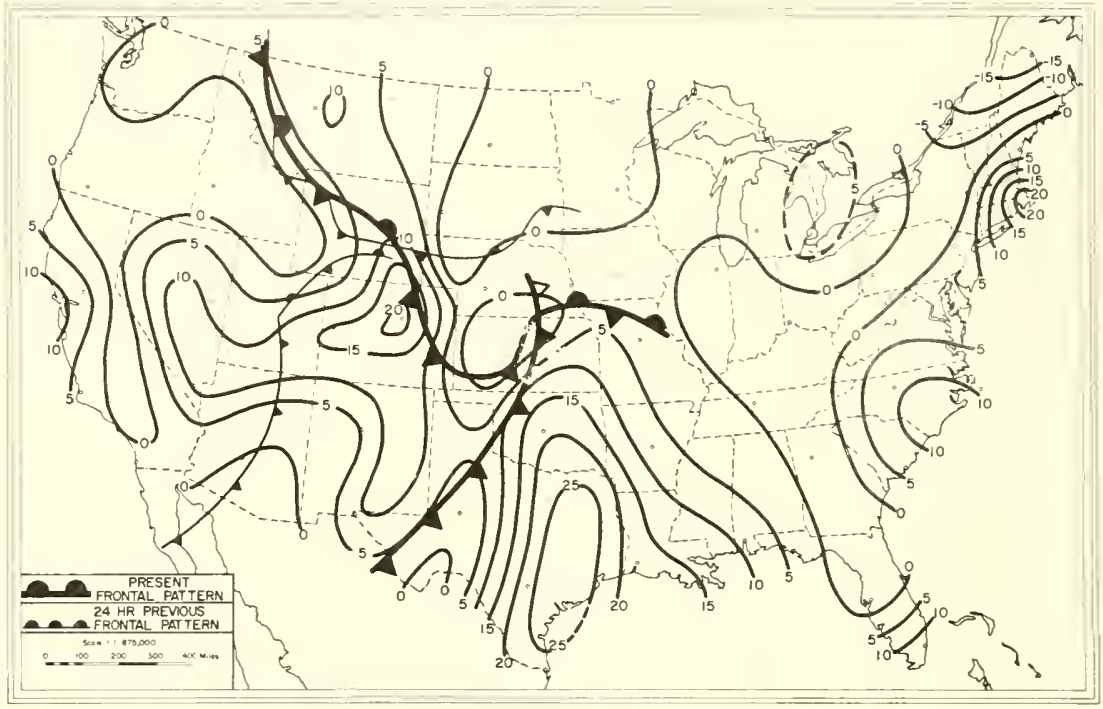

Figure 5.26. 24-hour $\Delta \mathrm{N}_{0}$ chart, O1SOE $19 \mathrm{Feb}$. 1952.

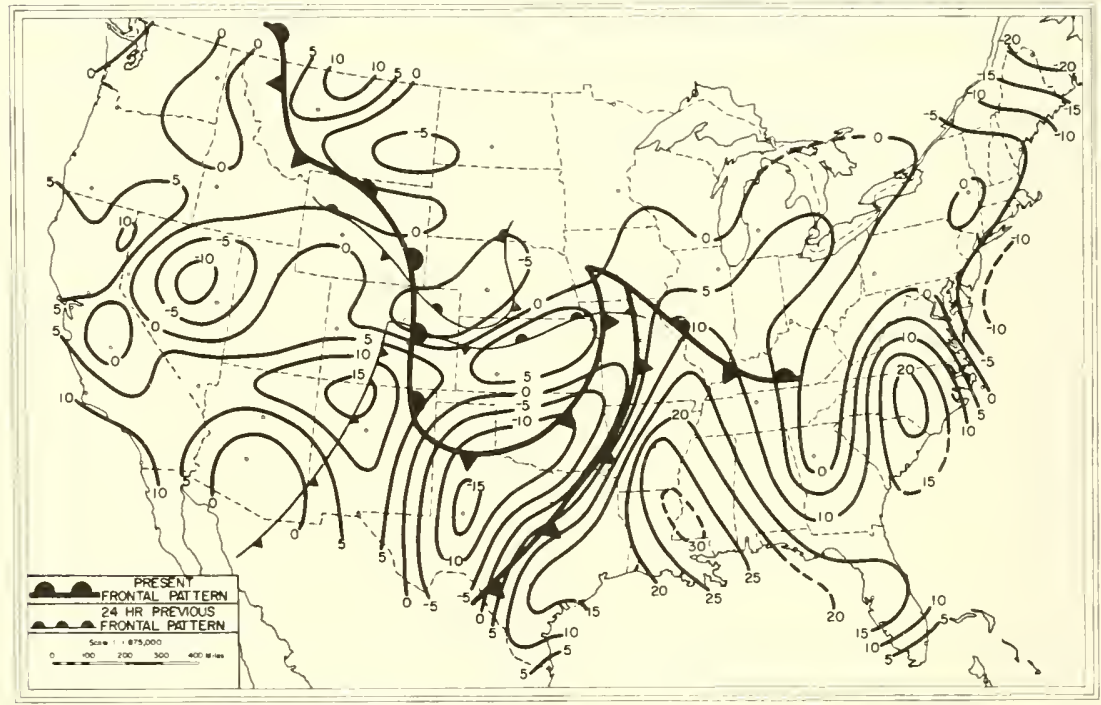

Figure 5.27. 24-hour $\Delta \mathrm{N}_{0}$ chart, 1980E $19 \mathrm{Feb} .1952$. 


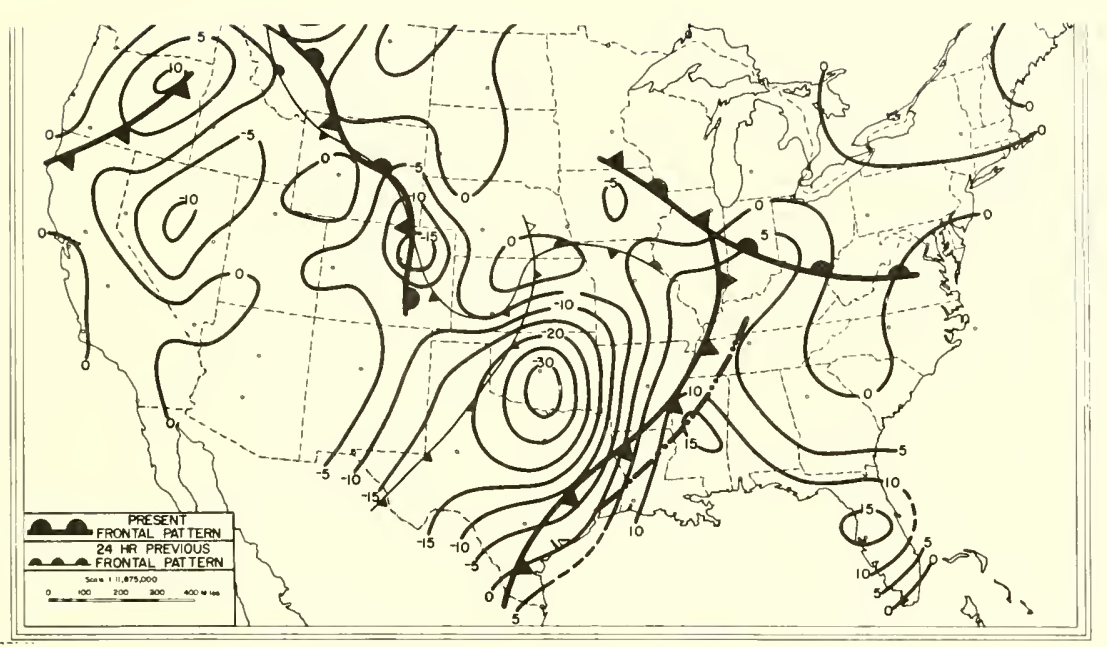

Figure 5.28. 24-hour $\Delta \mathrm{N}_{0}$ chart, O130E $20 \mathrm{Feb} .1952$.

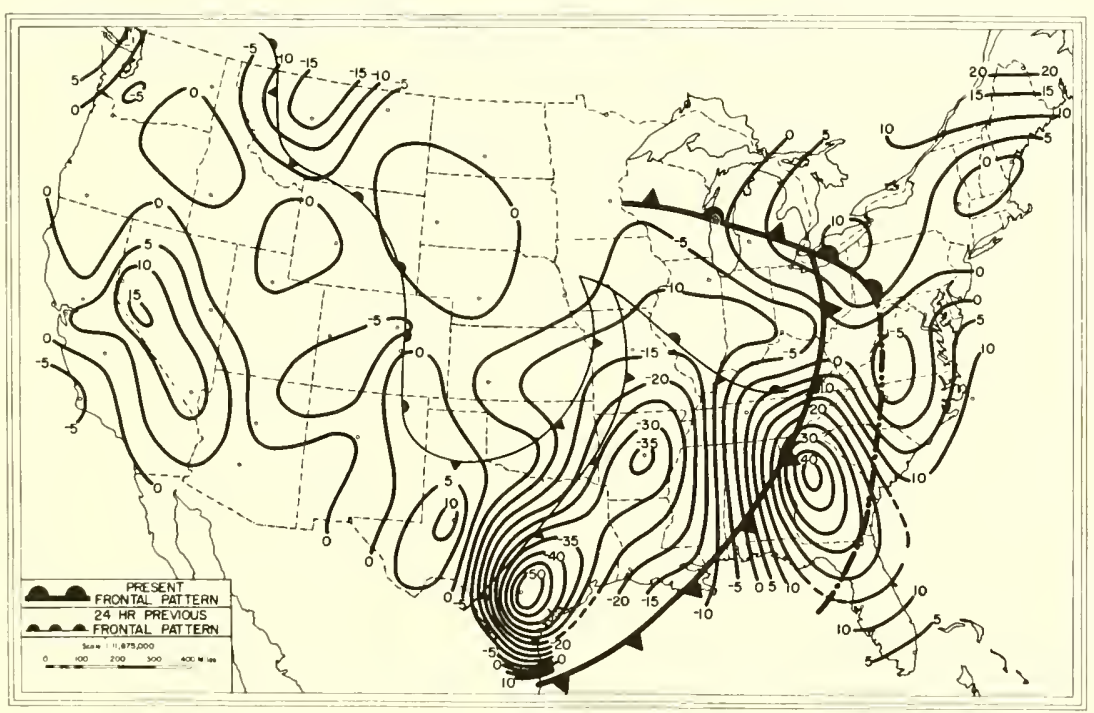

Figure 5.29. 24-hour $\Delta \mathrm{N}_{0}$ chart, 1330E 20 Feb. 1952. 


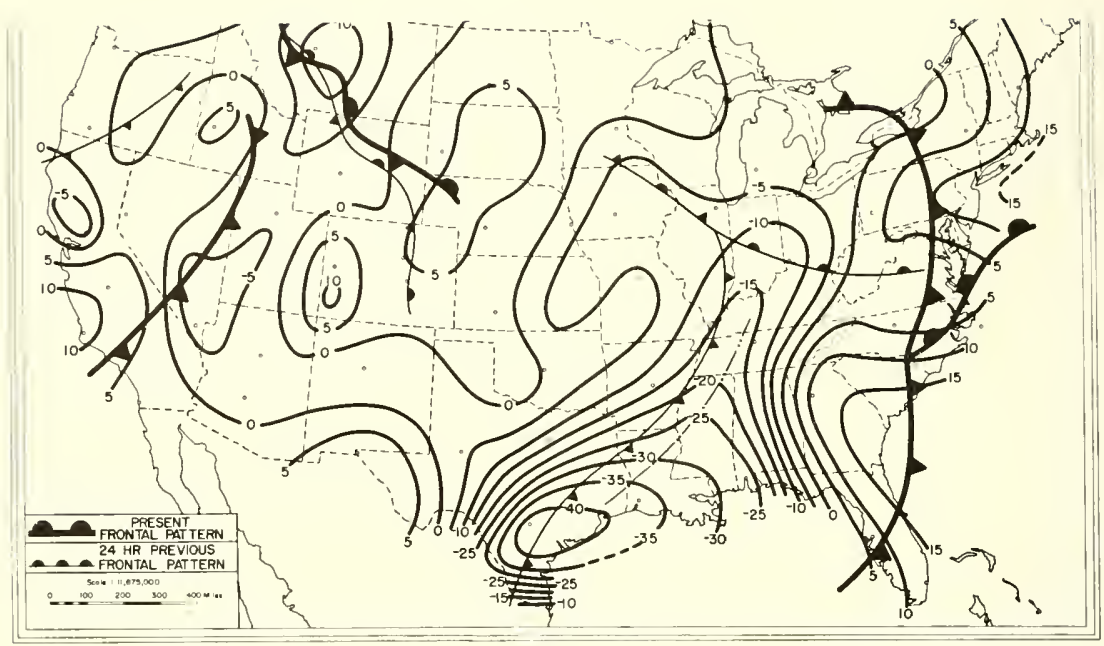

Figure 5.30. 24-hour $\Delta \mathrm{N}_{0}$ chart, O130E 21 Feb. 1952.

The change is determined on a 24 -hour basis in order to remove effects of the diurnal cycle of $N_{0}$. The $\Delta N_{0}$ charts show a general rise of $N_{0}$ in the warm sector and a drop in $N_{0}$ behind the front amounting, in the warm sector, to 35 to $40 \mathrm{~N}$ units by 1330 EST on February 20 (fig. 5.29) accompanied by a 40 to $50 \mathrm{~N}$ drop behind the front.

The relative sensitivity of $N_{0}$ to humidity changes is emphasized by the $\Delta N_{0}$ charts. The $N$ drop behind the cold front occurs in a region of increasing pressure and decreasing temperature-a combination that increases the dry term and depresses the wet term. The decrease in the wet term from rapidly dropping dewpoint more than compensates for the increased dry term. As an example, in the 24-hour period ending 0130 EST on the 19th, the station pressure at Oklahoma City increased 13 mbars. The dry term increased $12 \mathrm{~N}$ units while the wet term dropped $42 \mathrm{~N}$ units, giving a net change of minus $30 \mathrm{~N}$ units. This $N_{0}$ rise in the warm sector and the drop behind the cold front is consistent throughout the development of the polar front wave and appears to be what one would expect for this type of weather system. The present system had about a $35 \mathrm{~N}$ unit rise and a $40 \mathrm{~N}$ unit drop. This general pattern might be expected to occur in all fast-moving cold fronts with varying intensity, depending upon the individual synoptic pattern. In any case, it appears that the $N_{0}$ pattern is a sufficiently stable and conservative property of the atmosphere so that it should be possible to develop forecasting rules for $N_{0}$, but not, of course, without the analysis of many more $N$ patterns. 


\subsection{Constant Pressure Chart Analysis}

The same frontal system as above was analyzed for selected constant pressure levels. The 850 mbar charts (about 5,000 ft above mean sea level) and the $700 \mathrm{mbar}$ charts (about 10,000 ft above mean sea level) were prepared for the times of radiosonde aseent (10 A.MI. and 10 P.MI. EST) throughout the synoptic sequence from the radiosonde reports of 43 U.S. sounding stations. It is not necessary to reduce the $850 \mathrm{mbar}$ or 700 mbar level data, since it is already referenced to the indicated constant pressure level. Contours for the charts aloft are shown on figures 5.31 to 5.36 while their respective 24 -hour changes, $N_{850}$ and $N_{700}$, are given on figures 5.37 to 5.40 .

The $N_{850}$ charts show that the northerly flow of warm humid air within the warm sector that was so prominent on the $N_{0}$ maps is also clearly in evidence at the 850-mbar level. Further, a change patteru similar to that on the $N_{0}$ maps is also observed at the 850 -mbar level, particularly on figure 5.34. That is, a rise in $N_{850}$ values in the warm sector and a decrease behind the cold front is apparent. Surprisingly enough, by the time the frontal system is well-developed, at $1000 \mathrm{ES}$ 'I on the 20 th, the $N_{850}$ values are nearly as large as those on the surface.

The $N_{700}$ charts are more difficult to interpret than those of $N_{0}$ or $N_{850}$. It appears that at this altitude the wet term is usually negligible and $N$ will normally vary inversely as temperature since the pressure is, of course, constant at the $700-$ mbar level. By 1000 EST on the 19 th (fig. 5.34) an intrusion of low $N$ values is observed in the 700 -mbar warm sector due to the advection of warm, low-density air northwards. 'The chart for 24 hours later (fig. 5.42) displays two prominent highs in which $N_{700}=225$. One of these highs lies between the squall line and the cold front and the other just south of the apex of the $700-m b a r$ wave. Interestingly enough, these two highs are due to quite different causes. The high centered over Atlanta appears to have arisen from the unusually high transport of moisture to the 10,000-ft level, since the 700-mbar wet term at Atlanta increases from 4.5 to $25 \mathrm{~N}$ units in the 24-hour period ending with 1000 EST on February 20. The second high, centered over Omaha, appears to be due to an intense dome of cold air, as indicated by the drop of the 700-mbar temperature from $-7.3{ }^{\circ} \mathrm{C}$ to $-21.4{ }^{\circ} \mathrm{C}$ in 12 hours preceding map time. When temperatures are below $0^{\circ} \mathrm{C}$, the wet term contribution to $N$ is quite small and density changes become significant in producing changes in $N$. Falling temperatures produce higher density air and, consequently, a region of high $N$ values around Omaha as depicted on the $N_{700}$ chart of figure 5.31 and $\Delta N_{700}$ chart of figure 5.40 which shows this change more clearly. 


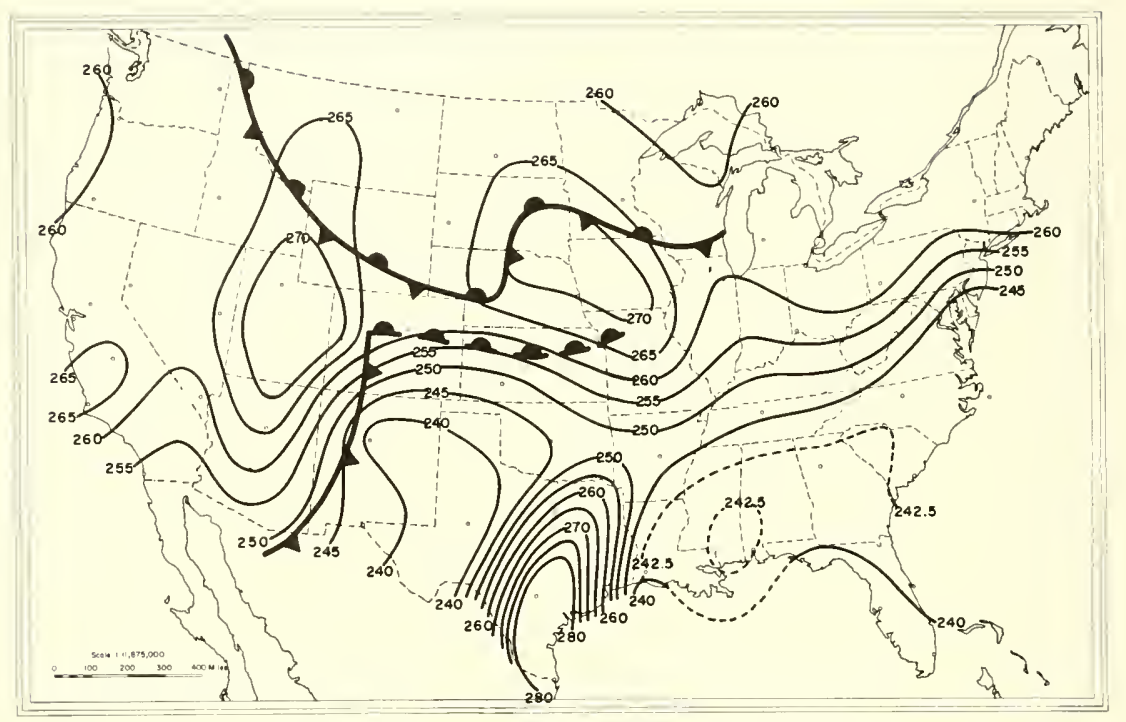

Figure 5.31. N $\mathrm{N}_{850}$ chart, $1000 E 18$ Feb. 1952.

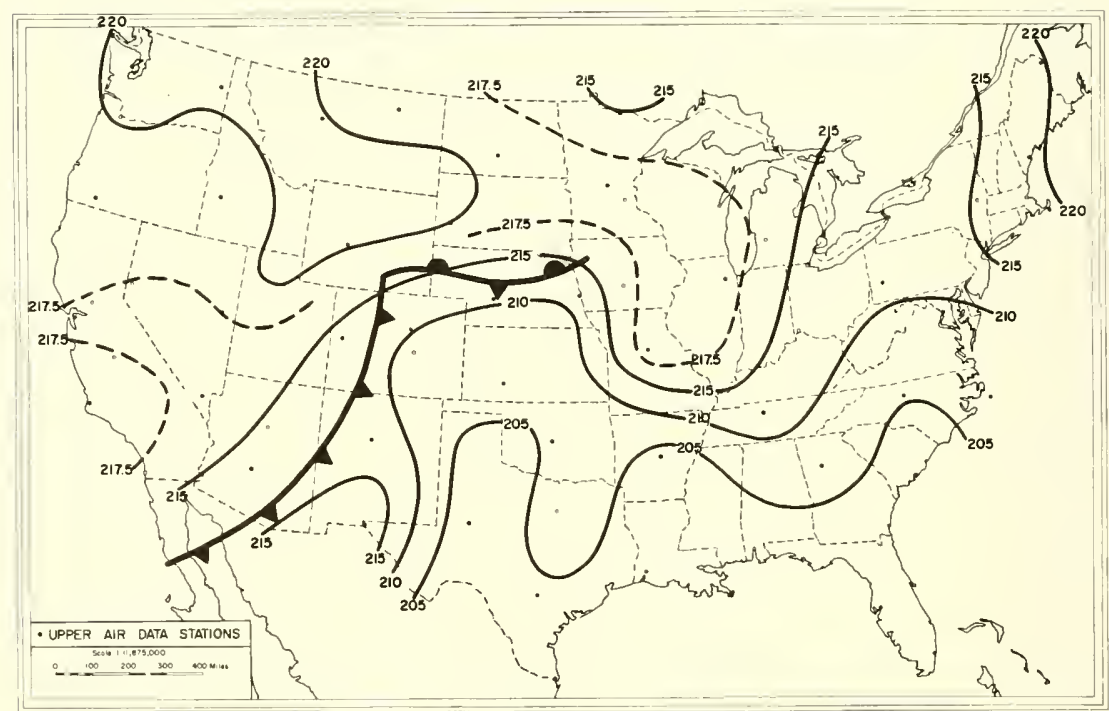

Figure 5.32. $\mathrm{N}_{700}$ chart, 1000E $18 \mathrm{Feb} .1952$. 


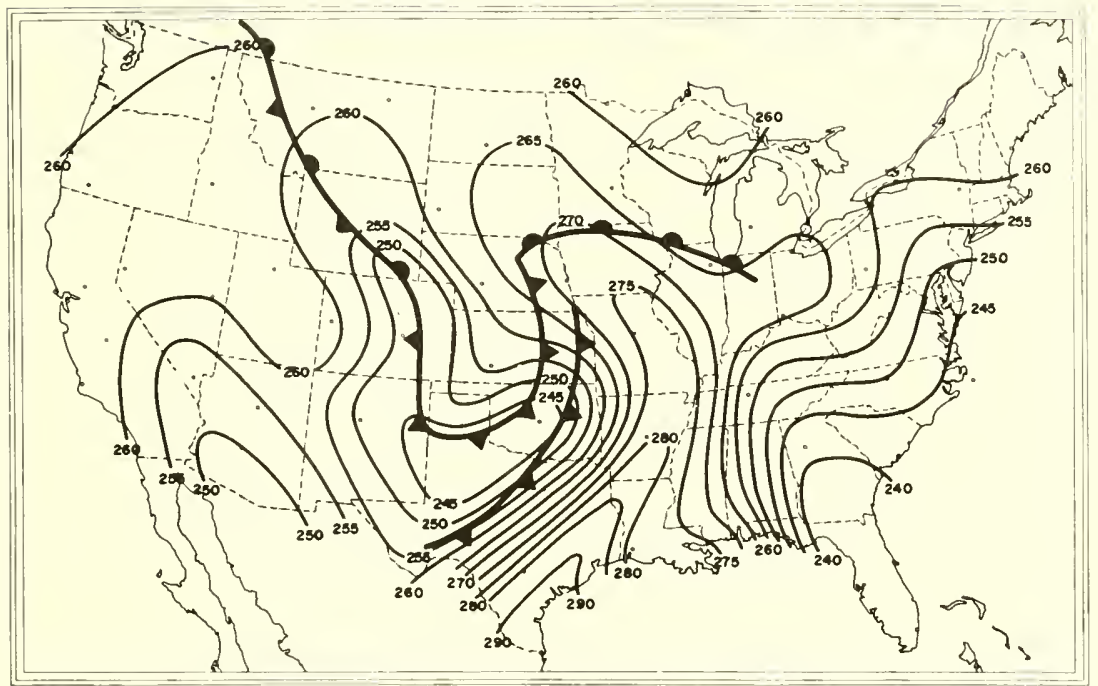

Figure 5.33. $\mathrm{N}_{850}$ chart, 1000E 19 Feb. 1952.

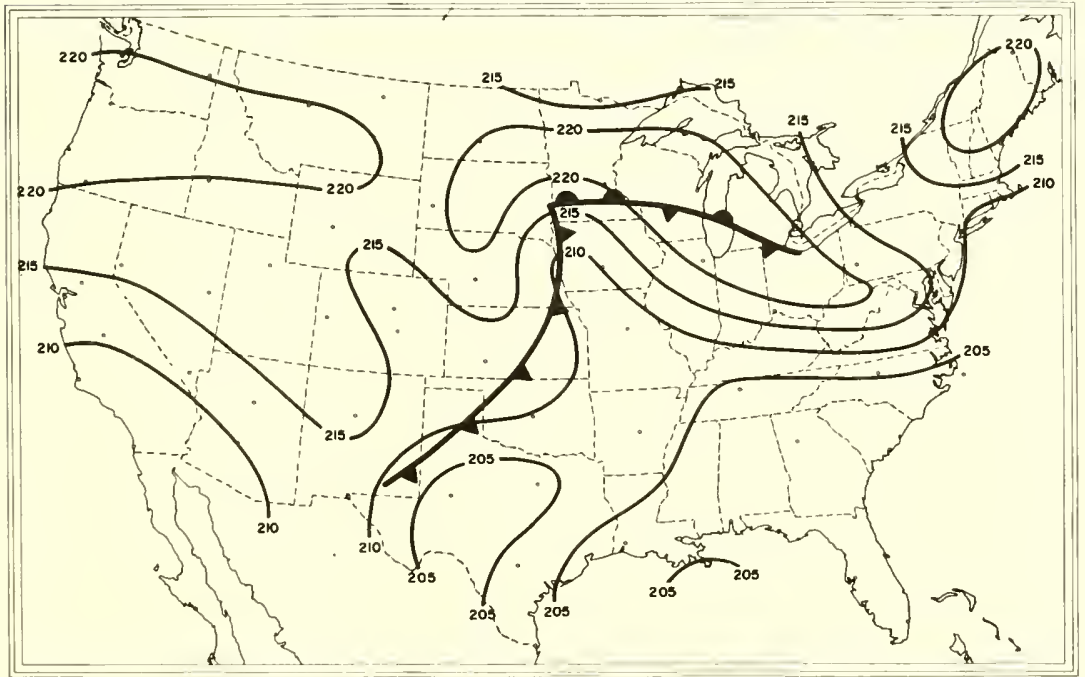

Figure 5.34. N $\mathrm{N}_{700}$ chart, 1000E 19 Feb. 1952. 


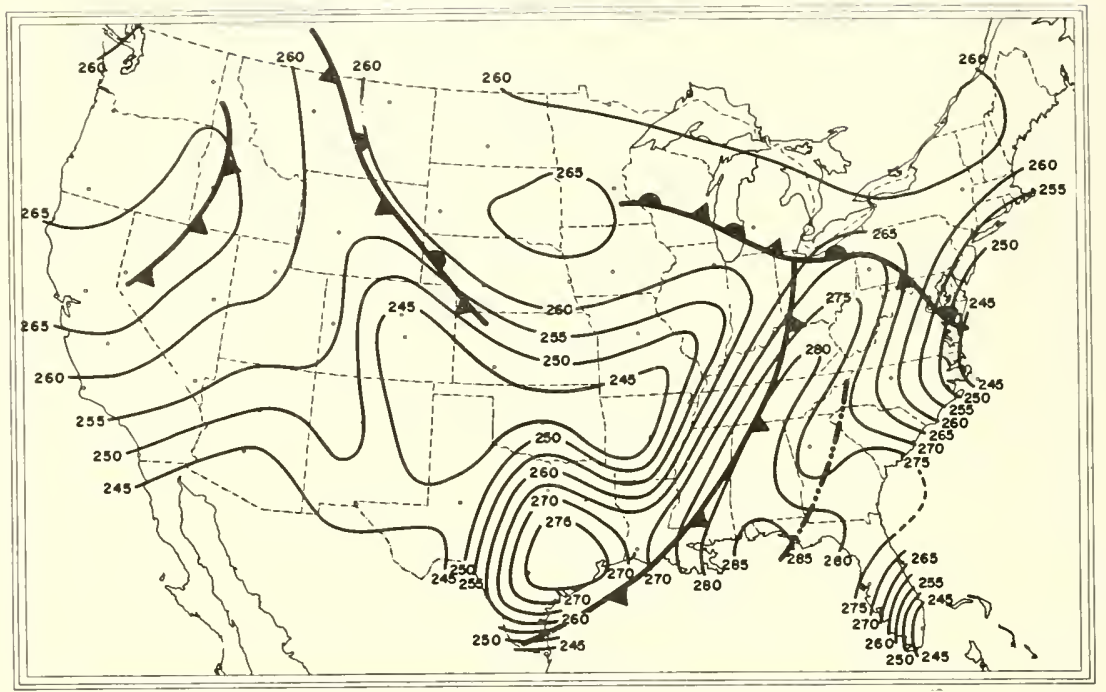

Figure 5.35. $\mathrm{N}_{850}$ chart, 1000E 20 Feb. 1952.

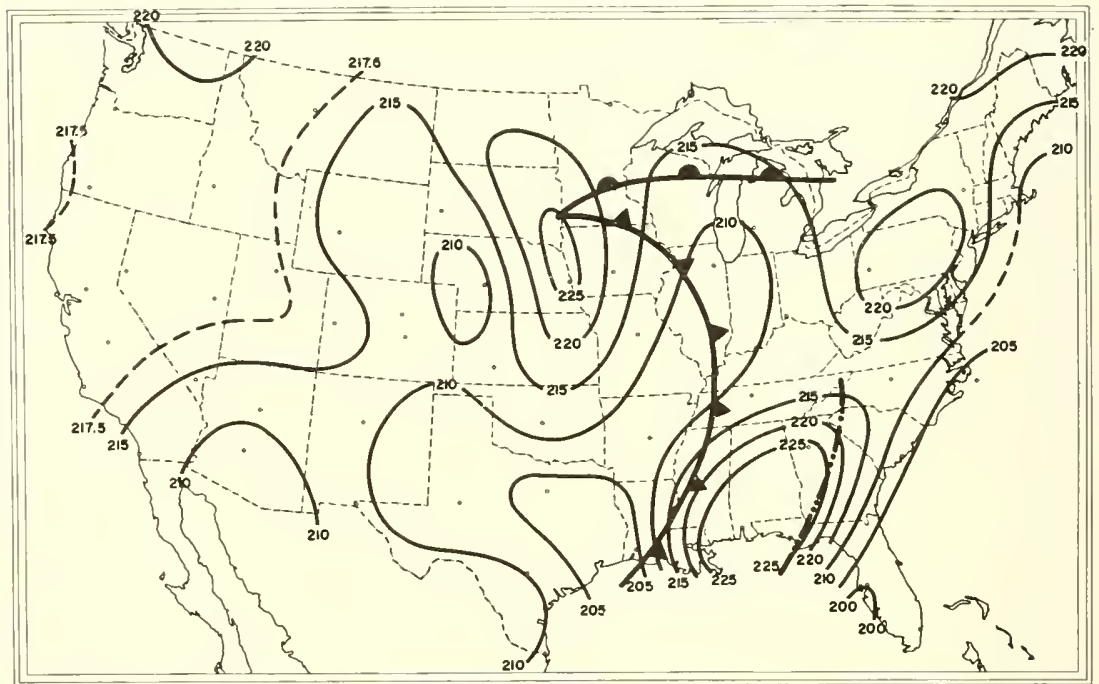

Figure 5.36. N $\mathrm{N}_{700}$ chart, 1000E 20 Feb. 1952. 


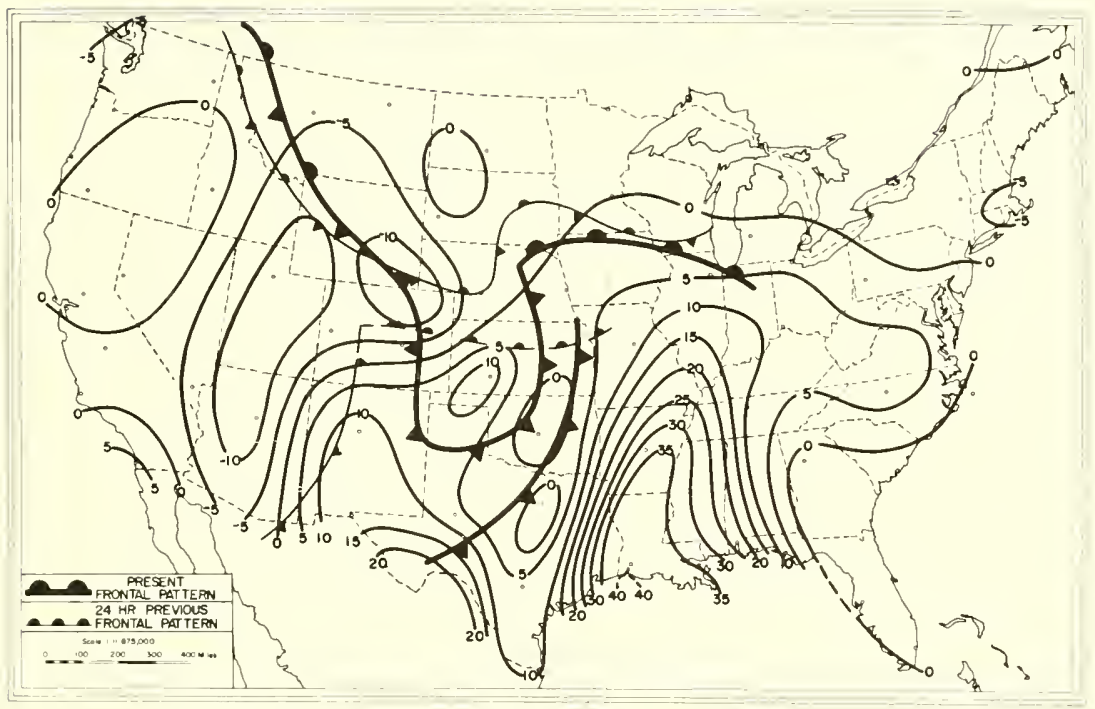

Figure 5.37. 24-hour $\Delta \mathrm{N}_{850}$ chart, 1000E 19 Feb. 1952.

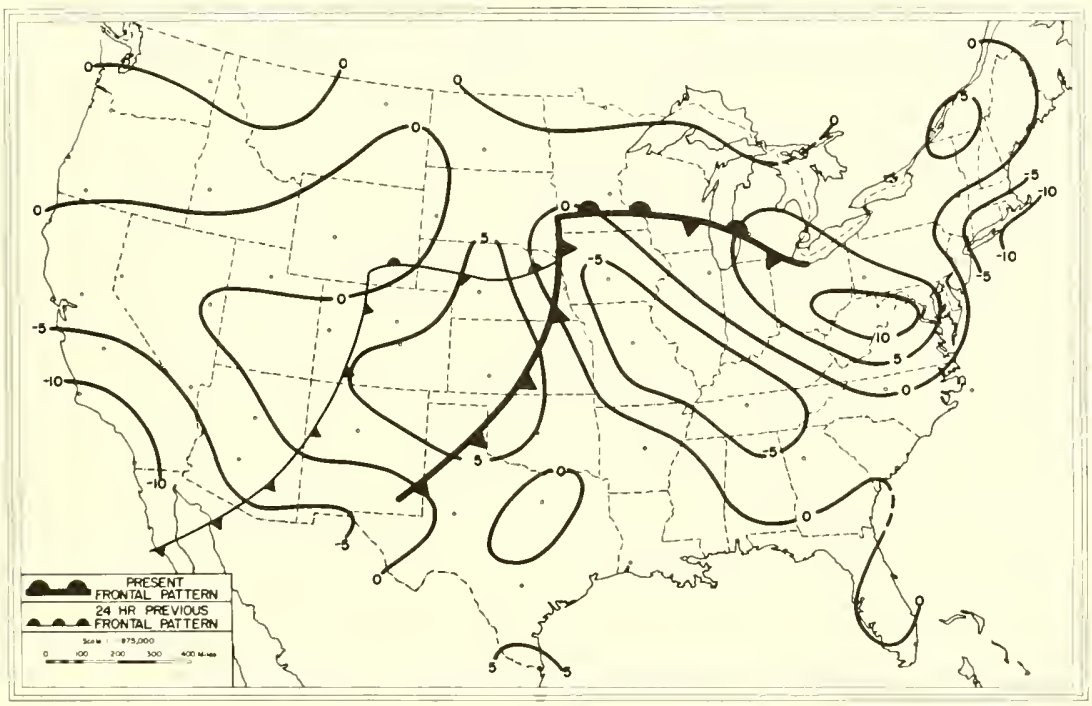

Figure 5.38. 24-hour $\Delta \mathrm{N}_{700}$ chart, 1000E 19 Feb. 1952. 


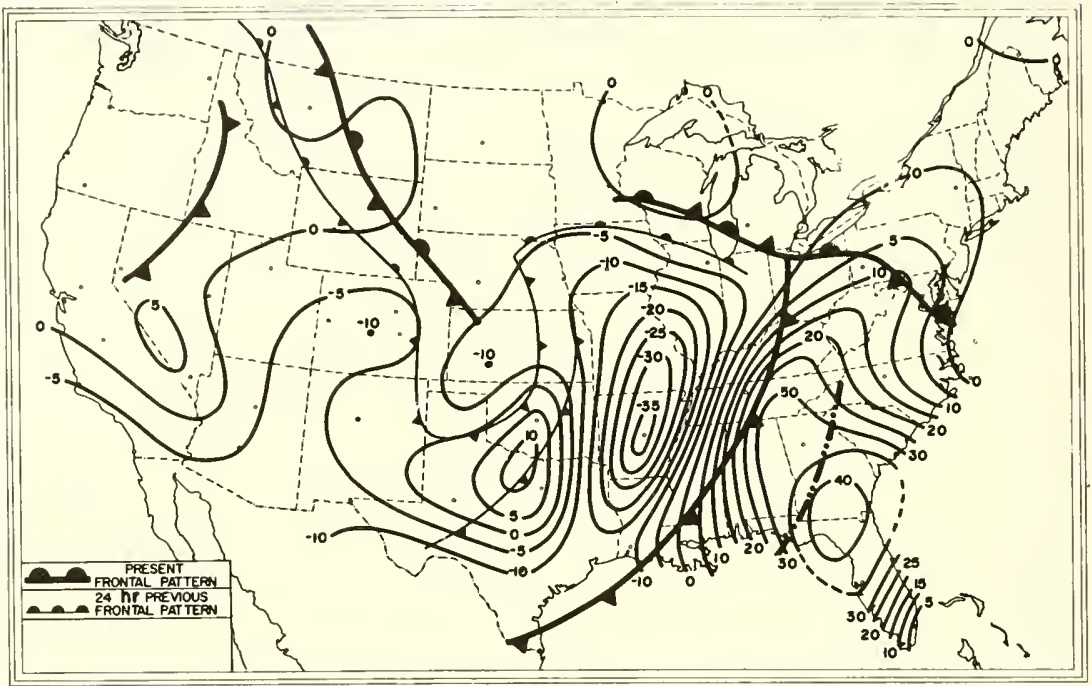

Figure 5.39. 24-hour $\Delta \mathrm{N}_{850}$ chart, 1000E 20 Feb. 1952.

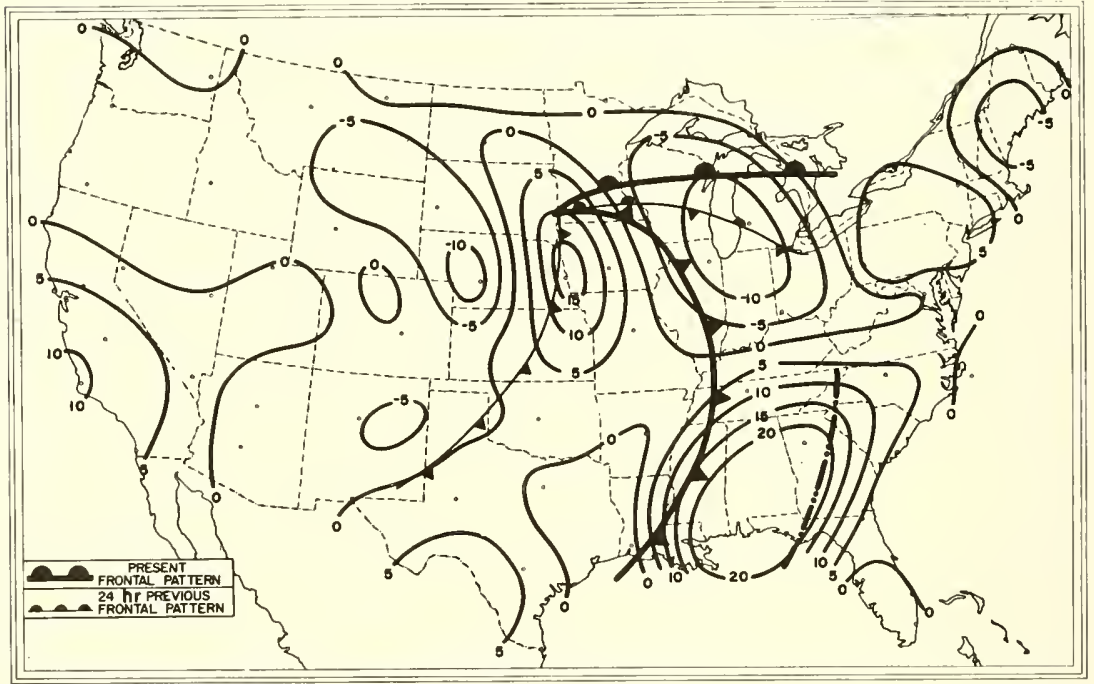

Figure 5.40. 24-hour $\Delta \mathrm{N}_{700}$ chart, 1000E 20 Feb. 1952. 


\subsection{Vertical Distribution of the Refractive Index Using A Units}

The synoptic study of the vertical distribution of the radio refractive index extends the foregoing constant-level analyses by considering the problem of whether the air mass properties associated with this typical wintertime outbreak of polar air are reflected in the vertical refractive index structure. Charts showing the structure of the storm have been prepared using radiosonde measurements from stations located along a line normal to the frontal zone between Glasgow, Mont., and Lake Charles, La. (fig. 5.41). Plots of $N$ versus height along this cross section line were obtained at 12-hour intervals during a 4-day period and converted to $A$ units.

Figure 5.42 is an example cross section along the Glasgow-Lake Charles line analyzed in terms of unmodified $N$ as in the idealized cases of figures 5.11 and 5.12. Compare this figure with the $A$ unit analysis of figure 5.48 for the highlighting of air mass differences refractive-index-wise. Examples of the distribution of $N$ components, temperature and humidity, around the front are charted on figures 5.43 and 5.44. Various stages in the advance of this intense storm system across the continent are represented by figures 5.45 to 5.50 in terms of modified $N$ ( $A$ units). At the outset of the period of observation (fig. 5.45), the polar front was located over the northern Great Plains, between Rapid City, S. Dak.

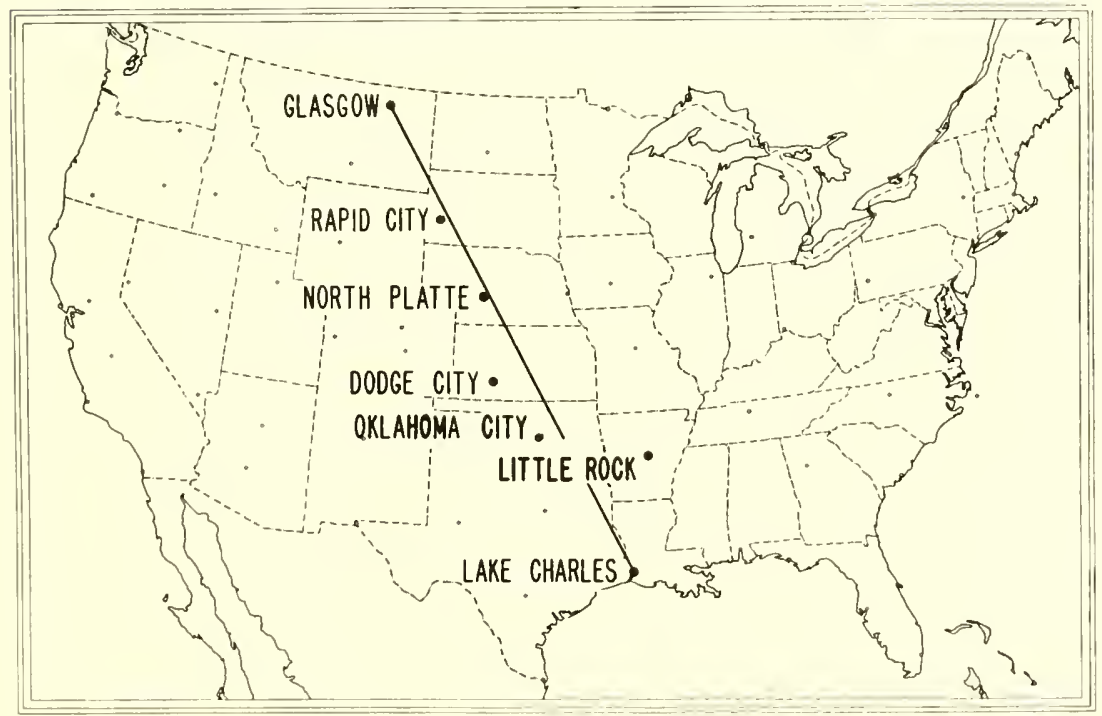

FIGURE 5.41. Station identification chart. 


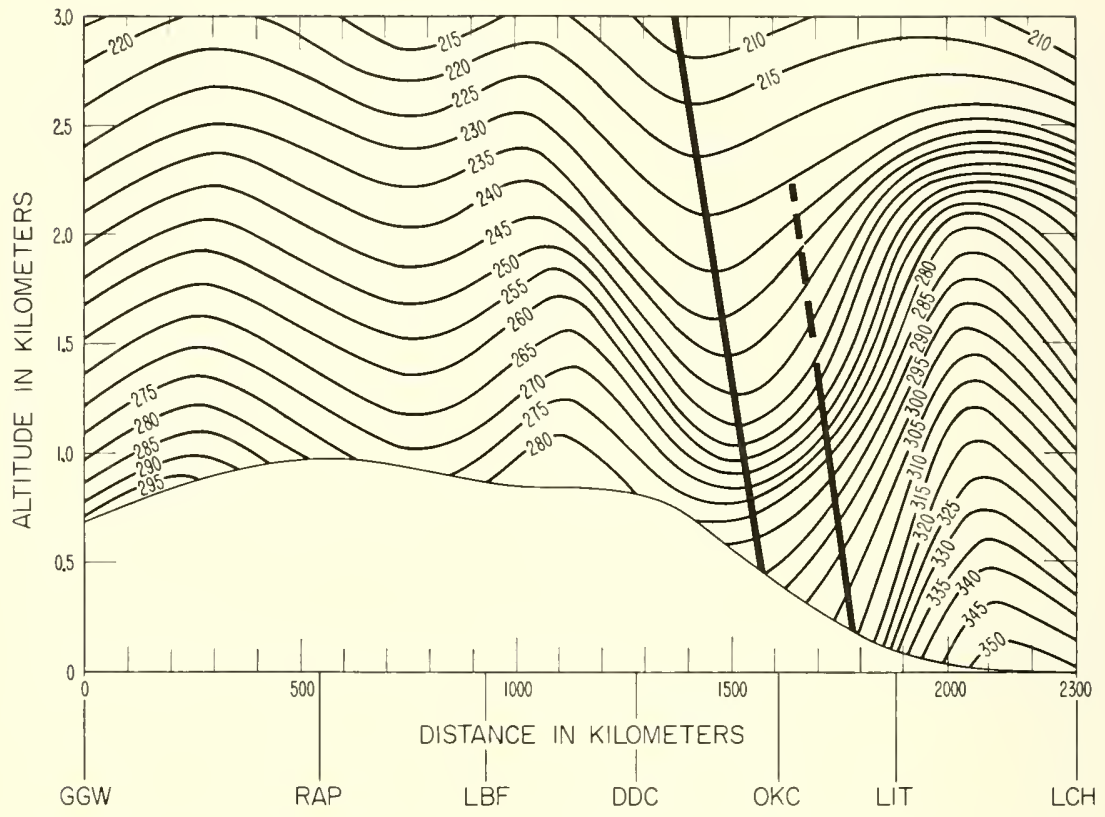

Figure 5.42. Space cross section in N units, 1500Z, 19 Feb. 1952.

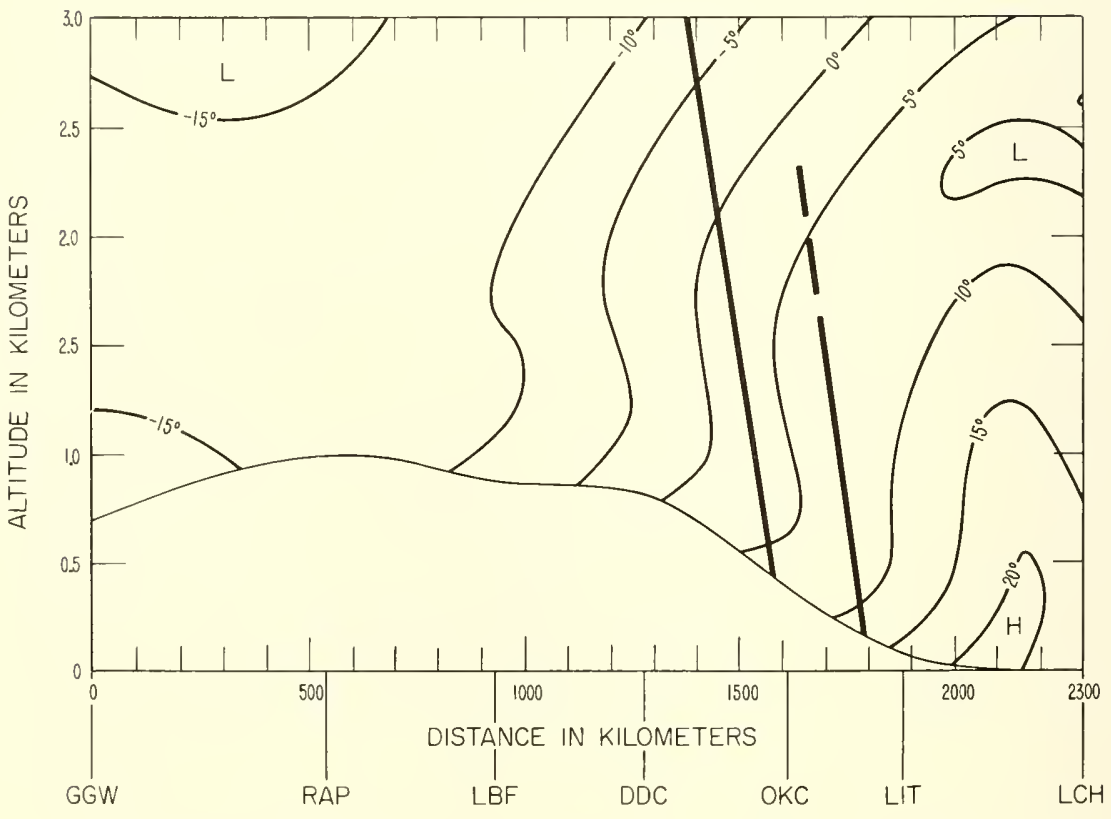

Figure 5.43. Temperature cross section in ${ }^{\circ} \mathrm{C}, 1500 \mathrm{Z}, 19 \mathrm{Feb} .1952$. 
and North Platte, Nebr. At this time the entire cross section is characterized by weak to moderate gradients of refractivity. On figure 5.46 12 hours later, this front had moved some $300 \mathrm{~km}$ southward. The contrast of the southward push of polar air and the northerly advection of tropical maritime air from the Gulf of Mexico into the developing warm sector of the polar front wave is evidenced by the relatively large gradients in the neighborhood of Dodge City. The core of tropical maritime air has evidently not progressed far enough northward to displace the warm but dry air that had been over the Great Plains prior to the outbreak, with the result that a region of low $A$ values is found between the front and the tropical maritime air. On figure 5.47 the effects of the Pacific front are apparent in the buildup of a secondary region of high $A$ some $400 \mathrm{~km}$ ahead of the polar front, located at this time over Dodge City. Twelve hours later (fig. 5.48) the core of tropical maritime air has become more extensive and now reaches to a height of $3 \mathrm{~km}$. The second (Pacific) front is picked up now on the cross section and the area of low $A$ values is confined between the two fronts. By 0300 U'T on the 20th, figure 5.49, the polar front is approaching Lake Charles and the Pacific front is reported on the daily weather map as a squall line. Finally, by the morning of February 20 (fig. 5.50), both fronts have passed to the south of Lake Charles, and the polar air just behind the front is characterized by relatively low $A$ values.

The use of space cross sections does not always yield measurements at the most desirable points along a frontal zone. Another method of arriving at the probable refractive index structure about the frontal interface is to plot radiosonde observations for a single station arranged according to observation times as on figures 5.51 to 5.57 .

The time cross section for Glasgow, Mont. (fig. 5.51), which is in the cold air behind the polar front for the entire period of observation of the storm, displays gradients of $A$ values that are generally weak. An exception occurs on the evening of the 19th (20/0300 UT) apparently as a result of subsidence effects. Rapid City, S. Dak. (fig. 5.52), similar to Glasgow, shows generally weak gradients throughout in the cold air behind the front. North Platte, Nebr. (fig. 5.53), exhibits moderate gradients with increasing $A$ values in the post-frontal, higher-density polar air. This station is too far north to record much in the way of moisture effects at this time of year. Dodge City, Kans. (fig. 5.54), represents dry low ahead of the polar front and increasing $A$ values in the cold air just behind the front. Oklahoma City, Okla. (fig. 5.55), represents a classic synoptic situation in which advection of tropical maritme air from the Gulf of Mexico produces a st rong high ahead of the front and a low within the cool, dry, polar continental air. Again in the region around Lit te Rock, Ark. (fig. 5.56), there is warm, moist air of extremely high refractivity ahead of the front being replaced by polar continental 


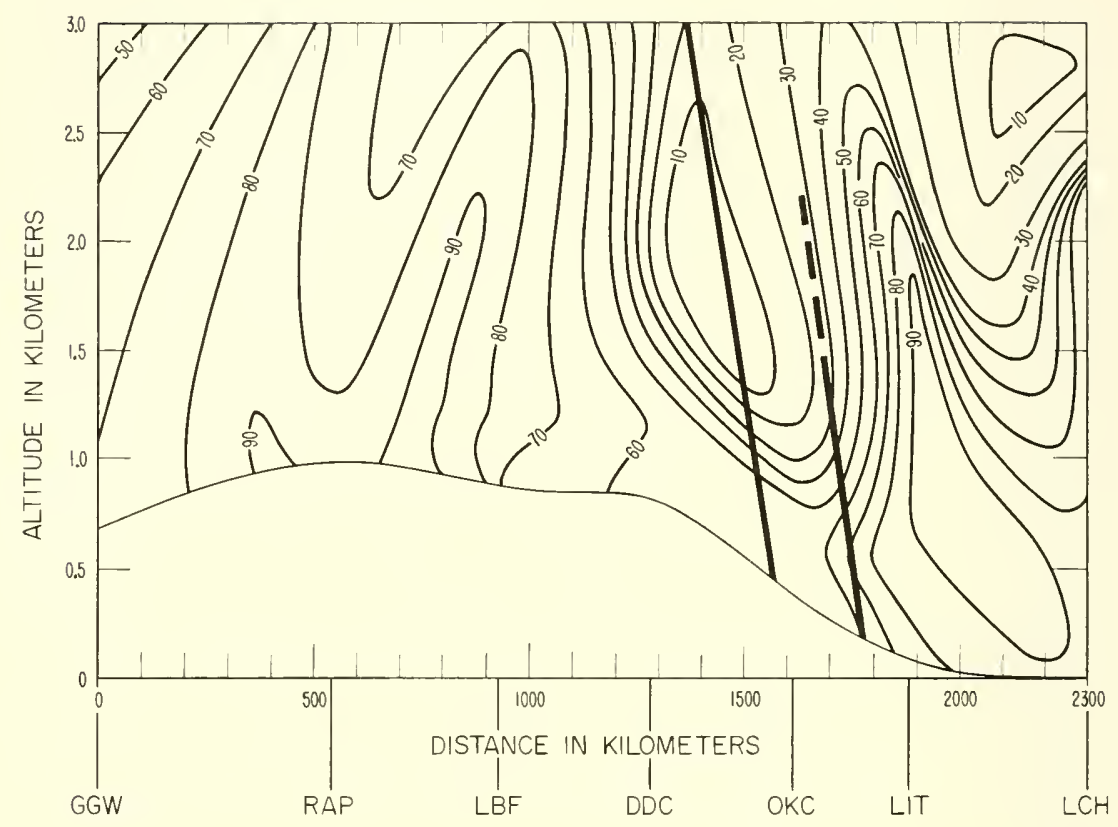

FIgURE 5.44. Relative humidity cross section (percent), 1500Z, 19 Feb. 1952.

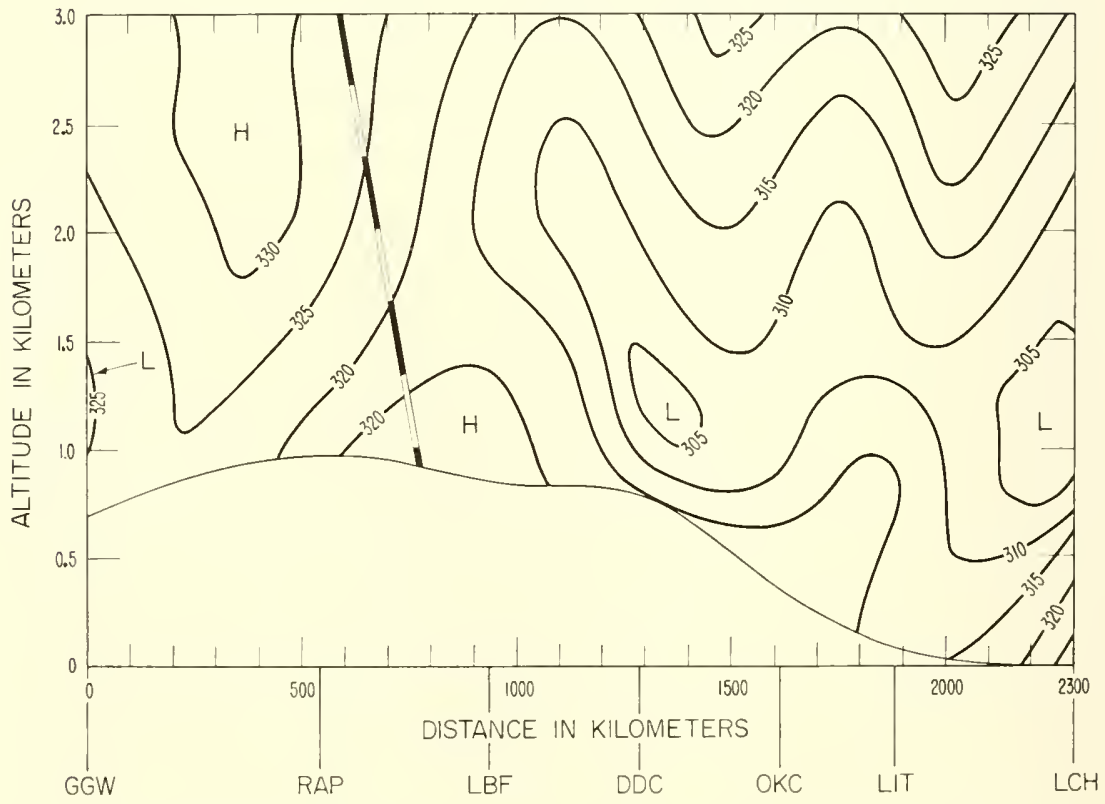

Figure 5.45. Space cross section in A units, 0300Z, 18 Feb. 1952. 


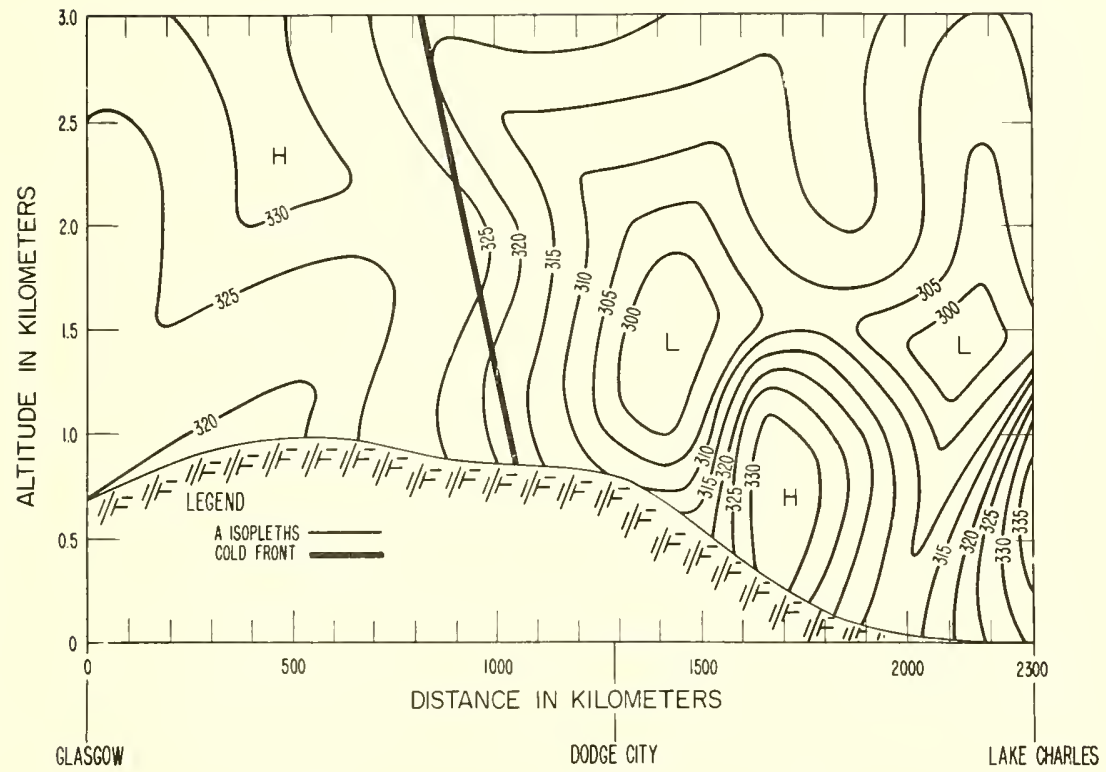

Figure 5.46. Space cross section in A units, 1500Z, 18 Feb. 1952.

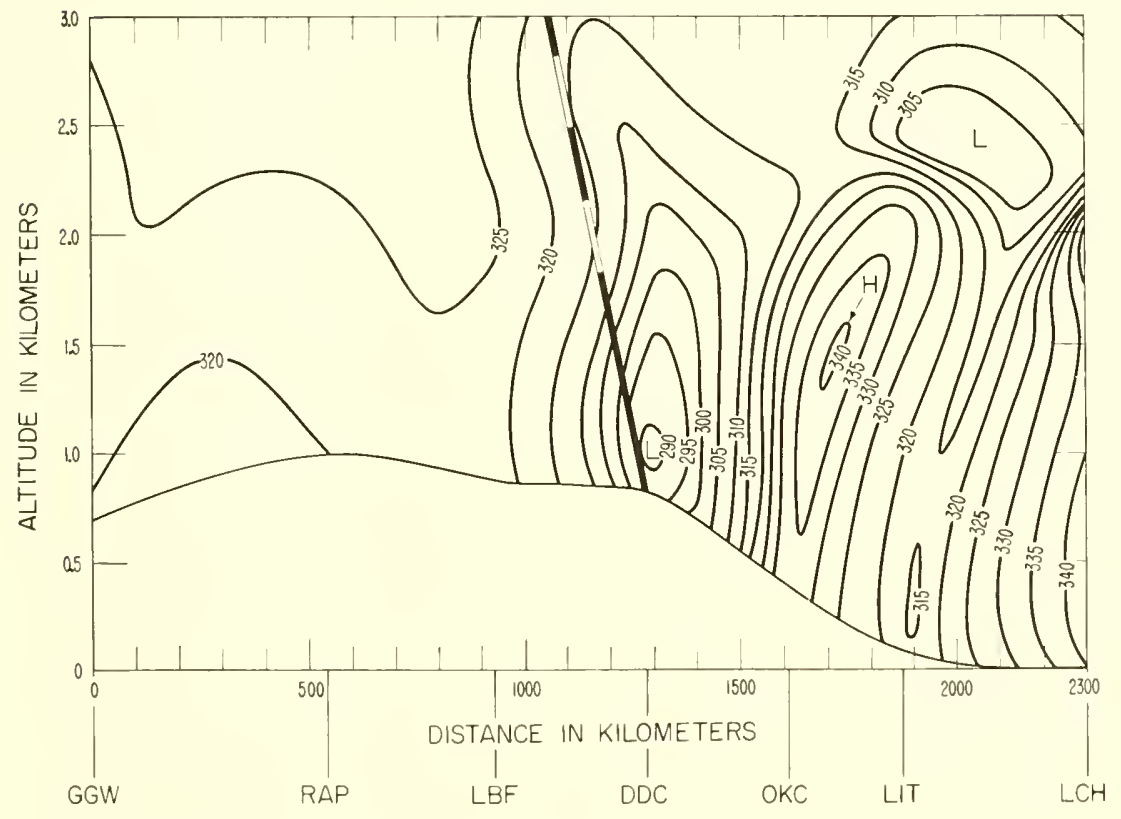

Figure 5.47. Space cross section in A units, 0300Z, 19 Feb. 1952. 


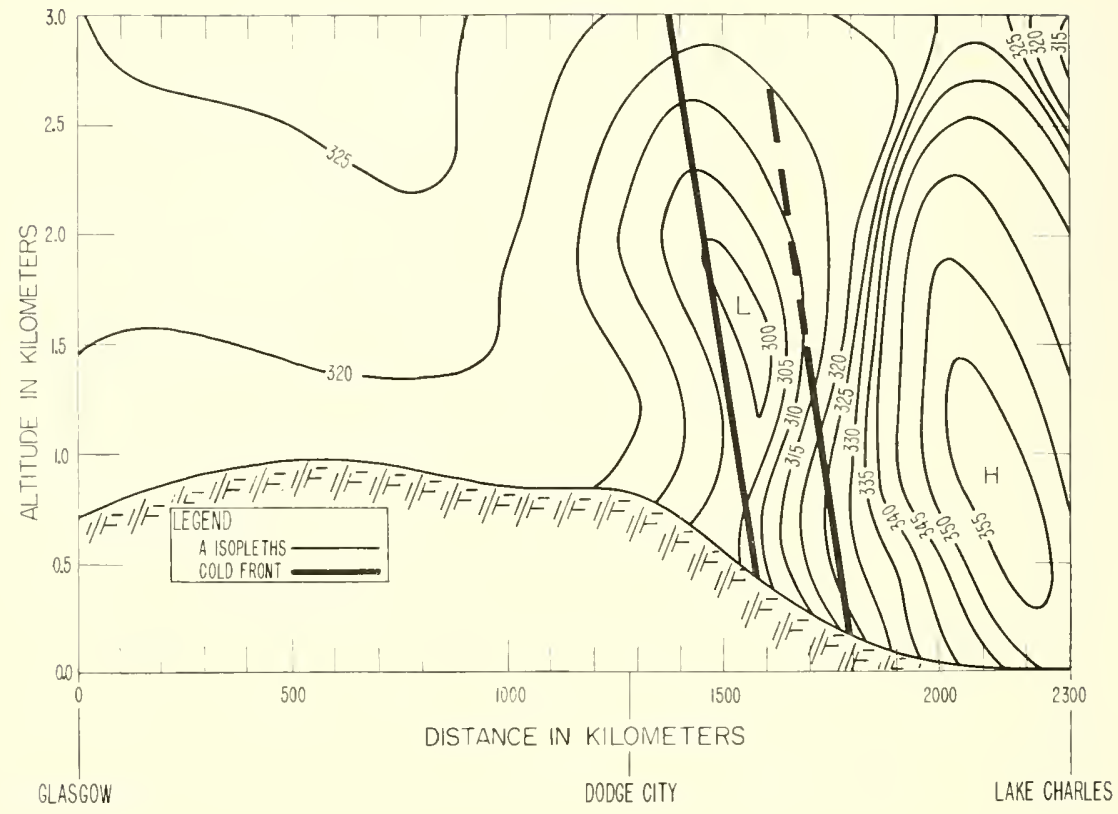

Figure 5.48. Space cross section in A units, 1500Z, 19 Feb. 1952.

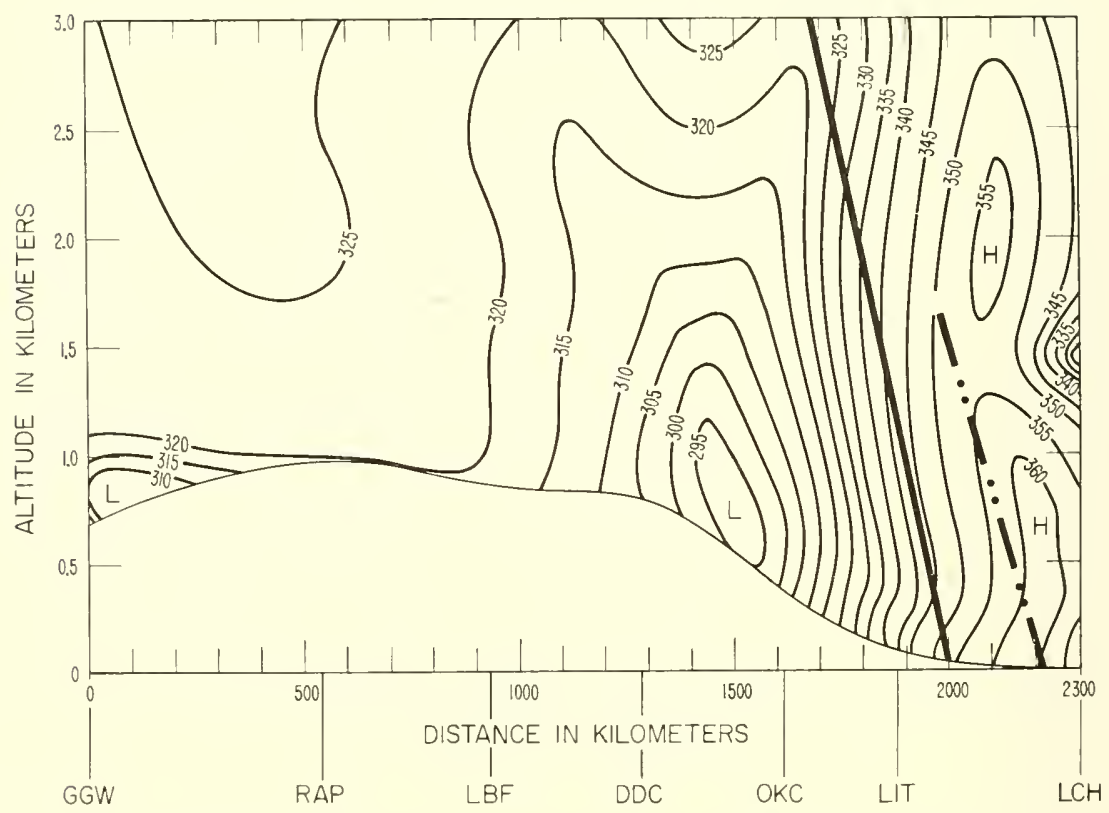

Figure 5.49. Space cross section in A units, 0300Z, 20 Feb. 1952. 


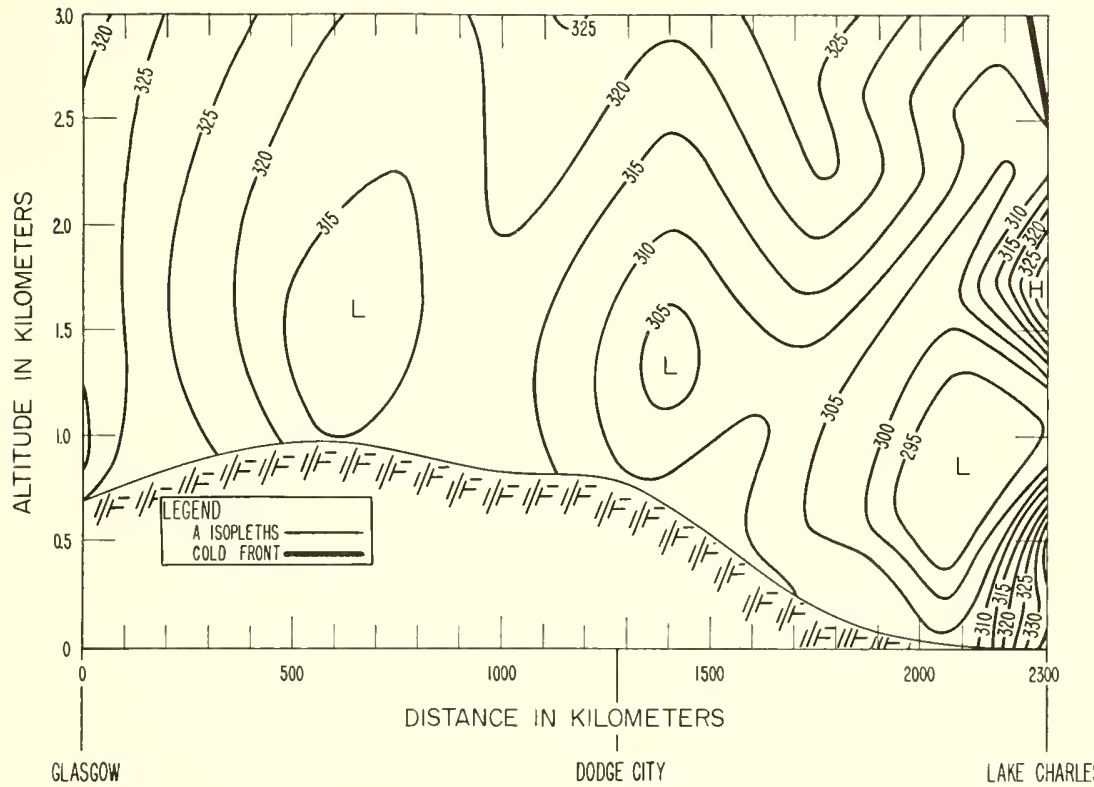

Figure 5.50. Space cross section in A units, 1500Z, 20 Feb. 1952.

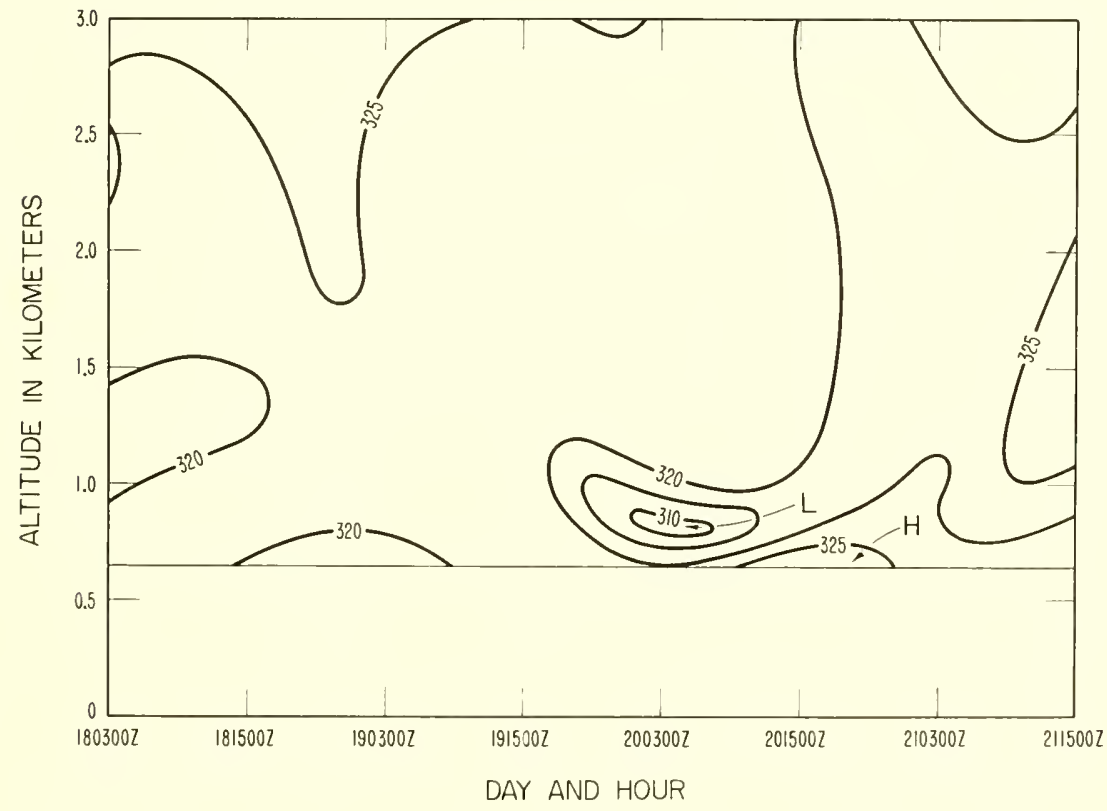

Figure 5.51. Time cross section, Glasgow, Mont., in A units. 


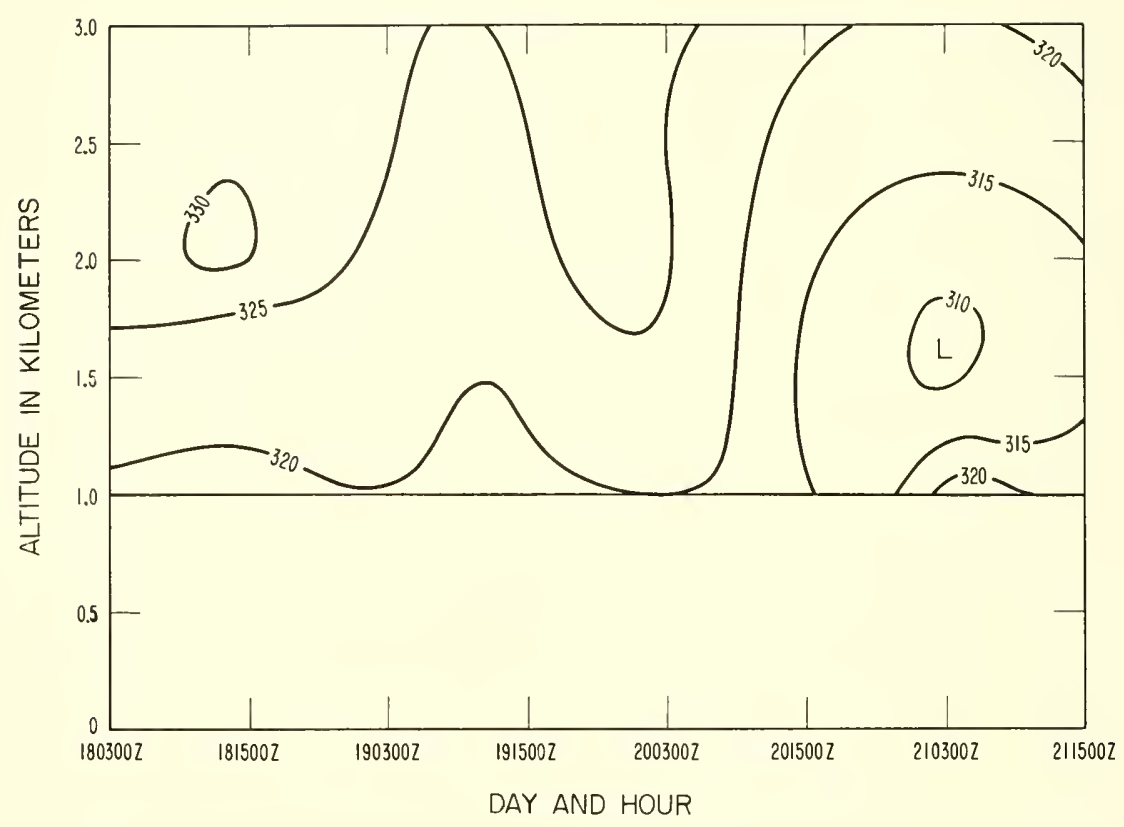

Figure 5.52. Time cross section, Rapid City, S. Dak., in A units.

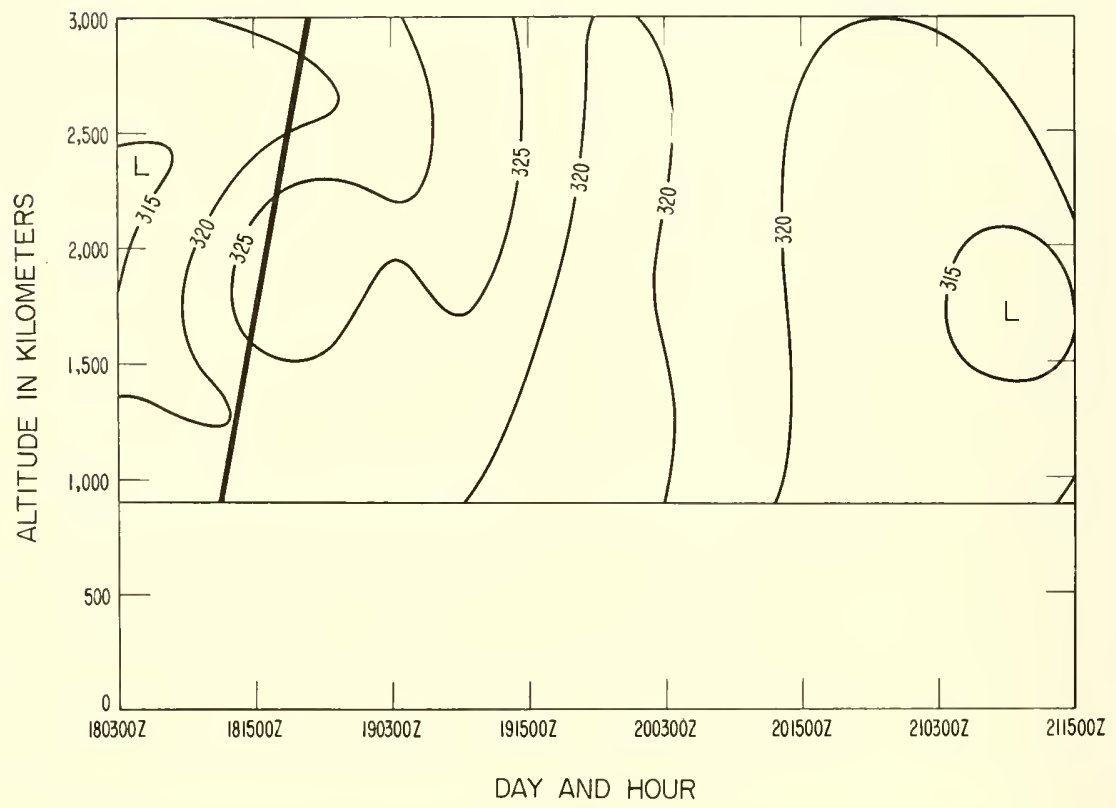

Figure 5.53. Time cross section, North Platte, Nebr., in A units. 


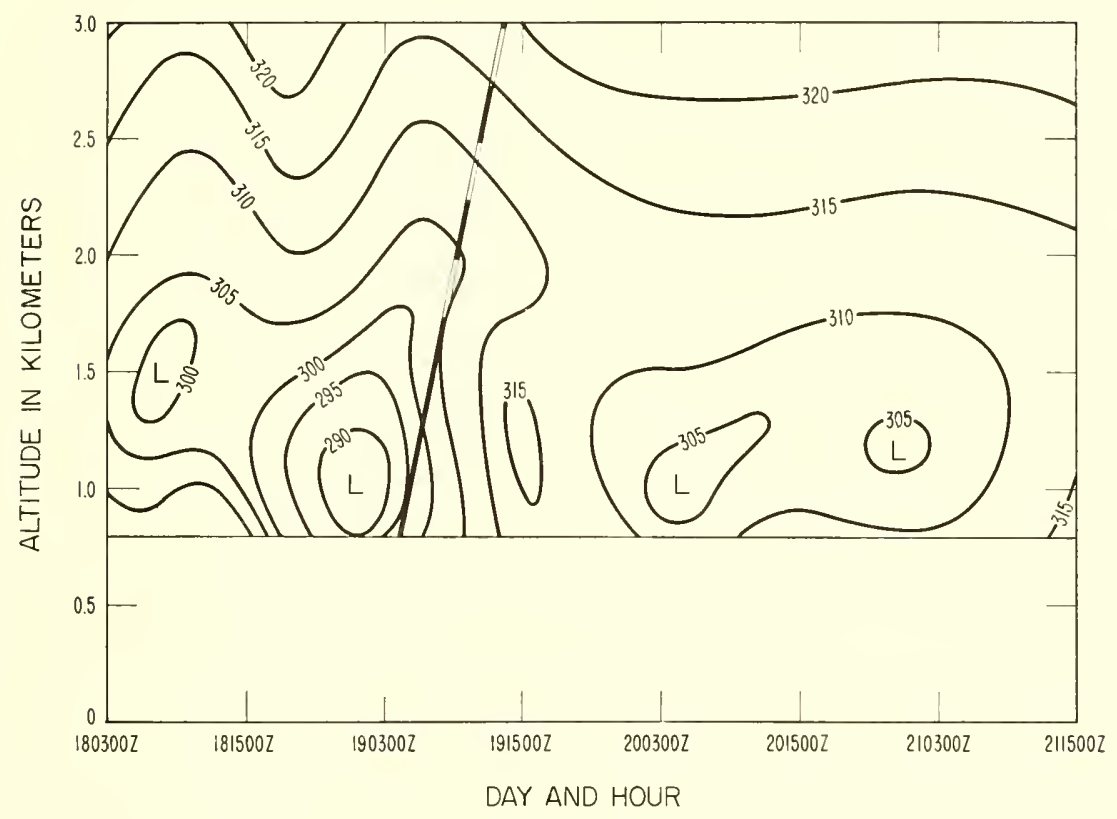

Figure 5.54. Time cross section, Dodge City, Kans., in A units.

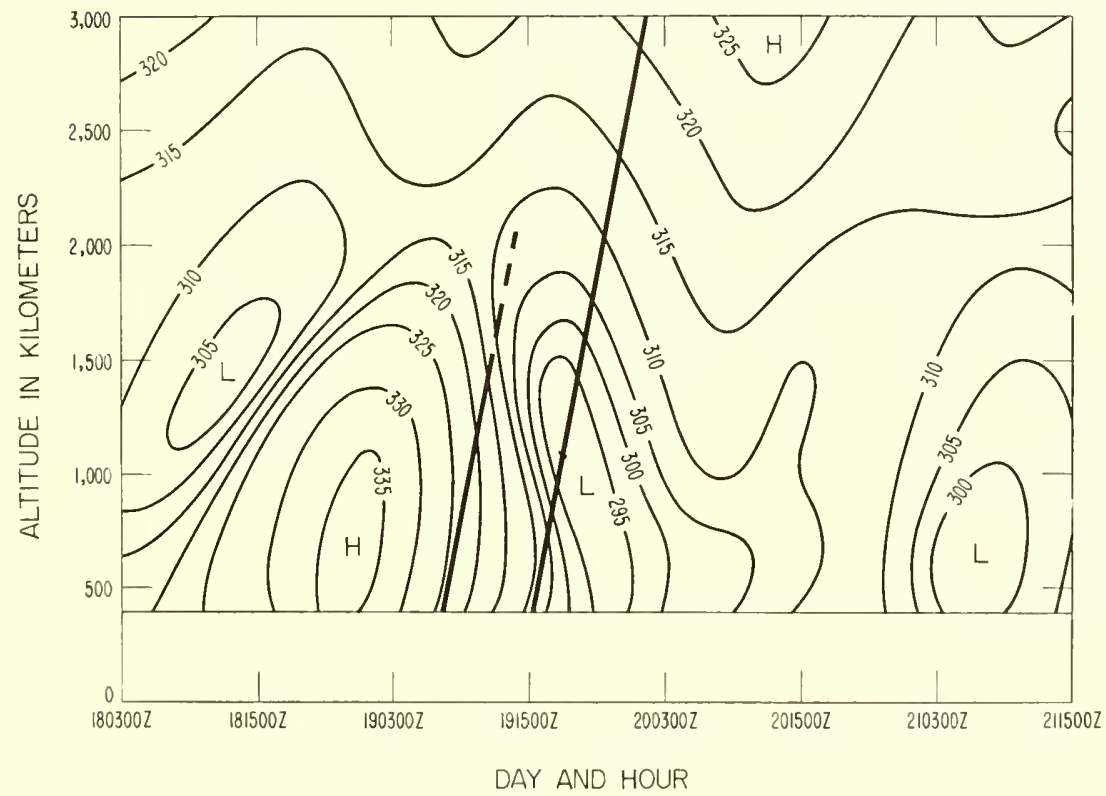

Figure 5.55. Time cross section, Oklahoma City, Okla., in A units. 


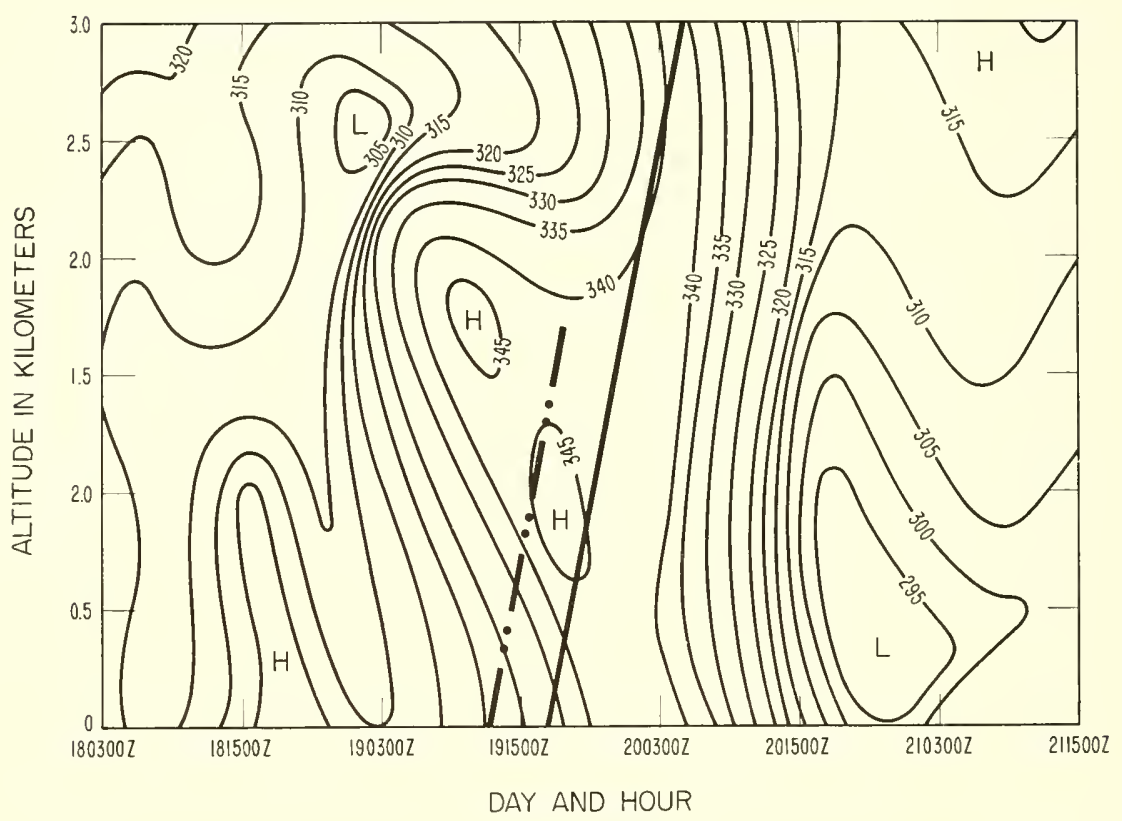

Figure 5.56. Time cross section, Little Rock, Ark., in A units.

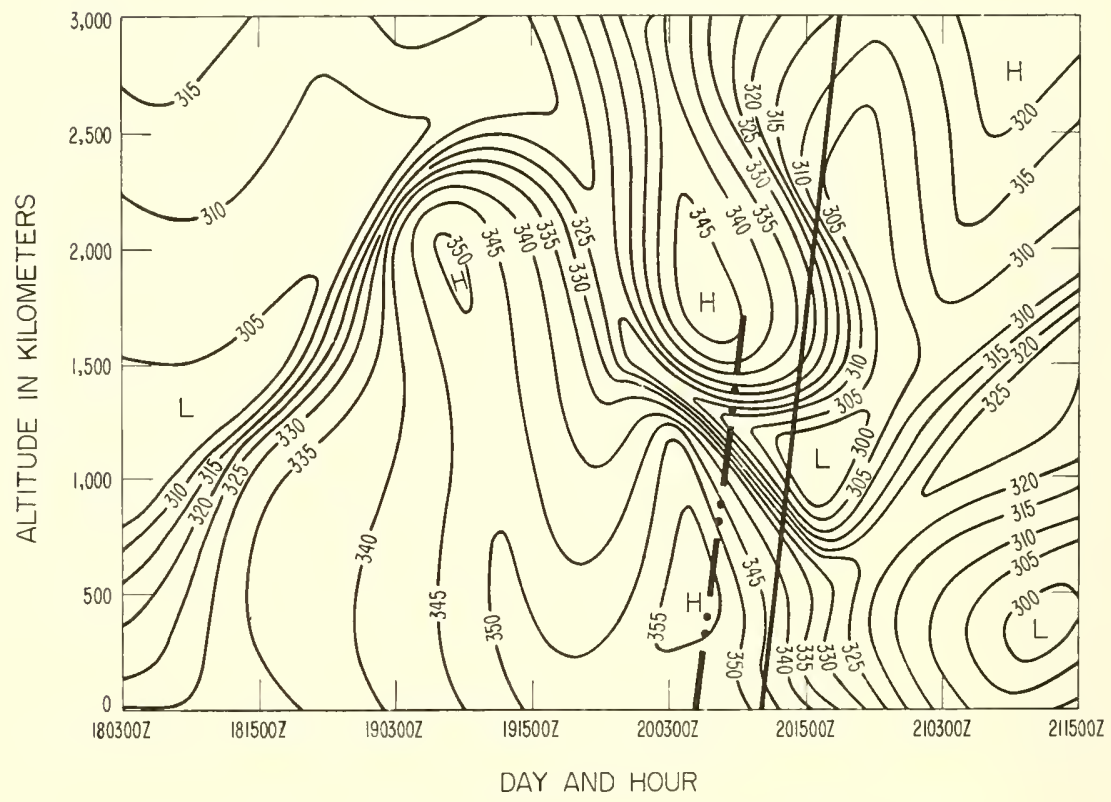

Figure 5.57. Time cross section, Lake Charles, La., in A units. 


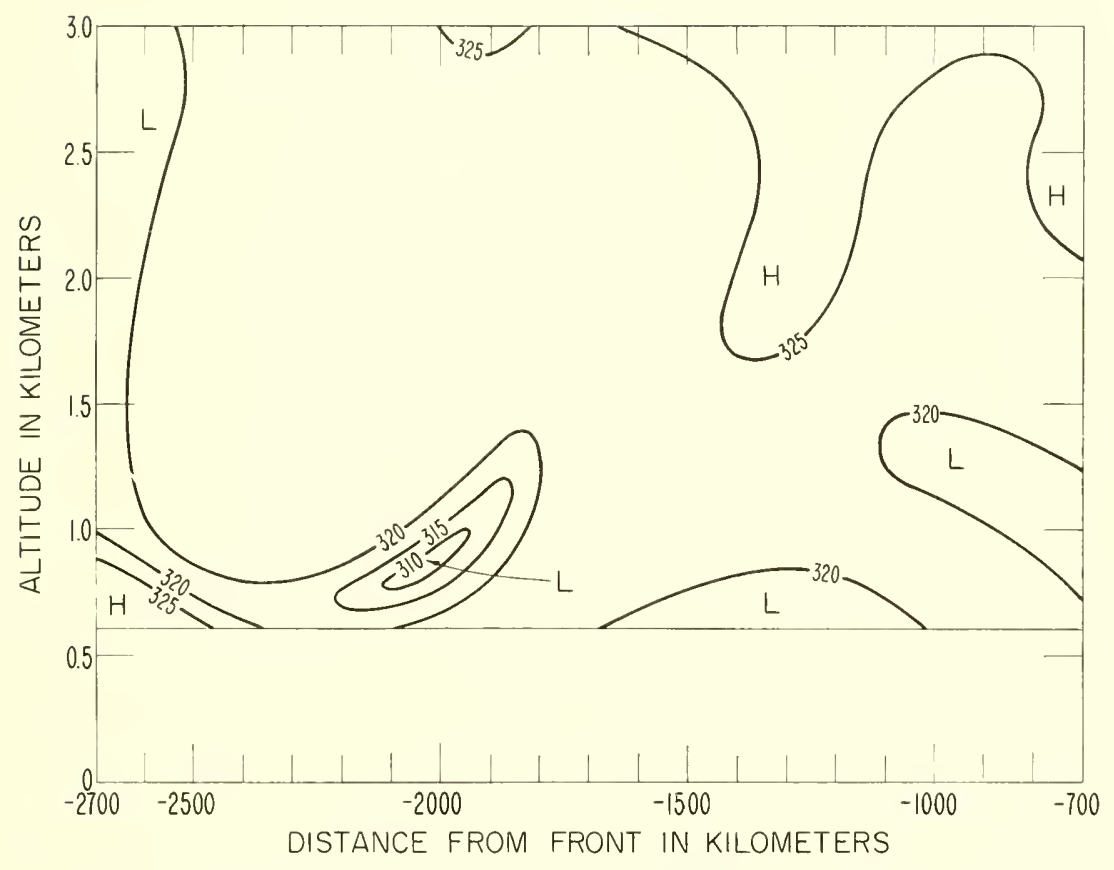

Figure 5.58. Epoch chart, Glasgow, Mont, in A units.

air of characteristically low $A$ value. The time cross section for Lake Charles, La. (fig. 5.57), is complex but represents again the same general features: high $A$ values ahead of the front and low ones behind.

The exponential correction to the refractive index height distribution used in this storm series allows air mass properties to be clearly seen. By use of such an exponential correction, one may construct an idealized refractive index field about a frontal transition zone that shows the temperature and humidity contrasts of the different air masses. Further, when this technique is applied to the analysis of a synoptic tropospheric disturbance, it does indeed highlight air mass differences.

The time cross section presentation is referred to as an epoch chart when the observations are presented as plus or minus time deviations with respect to the frontal passage. Thus, as a frontal system advanees and passes over a station, one obtains yet another perspective of the space cross section. Such a presentation is given on figures 5.58 to 5.64 . Figure 5.59 represents a typical continental station located in the polar 
continental air mass throughout the occurrence of the storm. The essential feature here, as in figure 5.52, is the absence of detail of $A$ structure due to the presence of a uniform air mass over this station. Compare this figure with the epoch chart for Oklahoma City (fig. 5.62), where the structure of the idealized model is clearly reflected by the prefrontal $A$ unit high, strong gradient across the frontal zone, and the $A$ unit low behind the front. This rather fortuitous agreement is felt to be due to the strategic location of Oklahoma City with respect to the motion of contrasting air masses about the polar front. That is, this epoch chart represents a point of confluence of virtually unmodified polar continental and tropical maritime air.

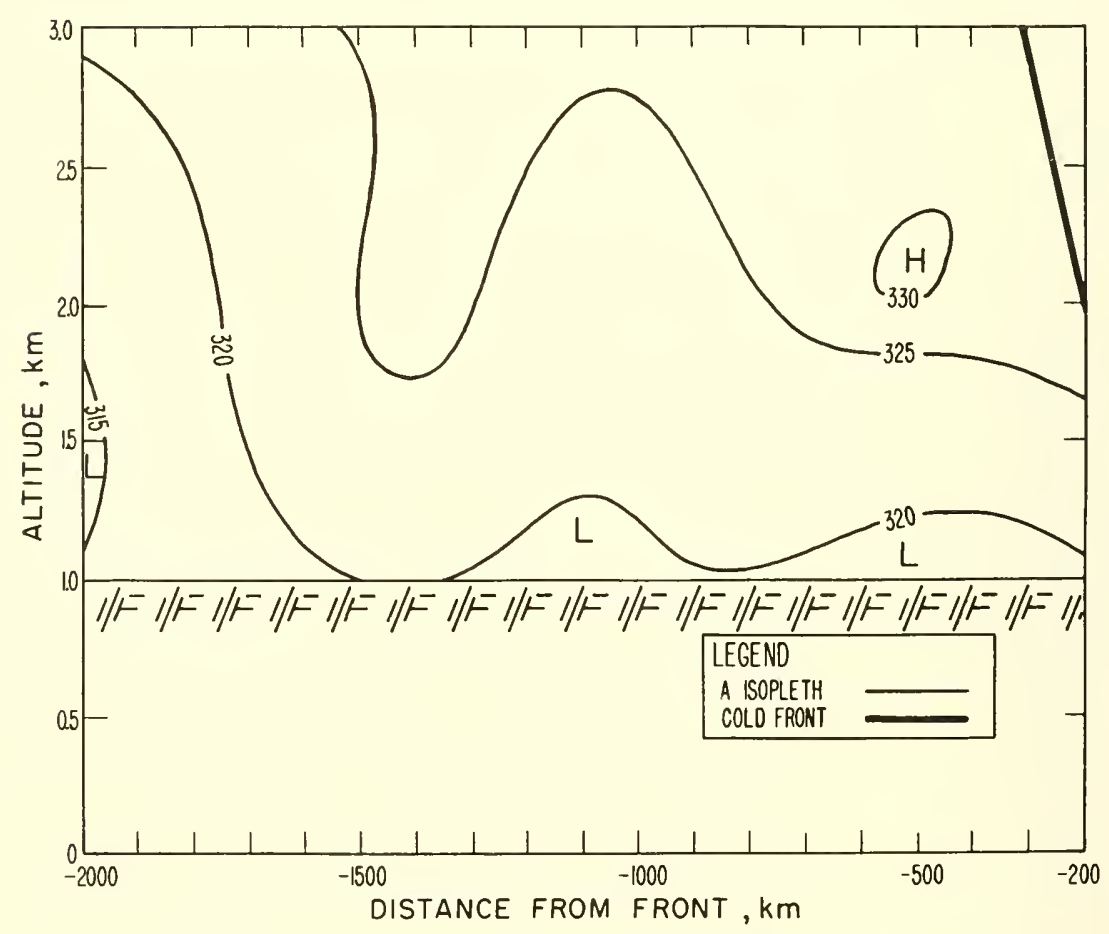

Figure 5.59. Epoch chart, Rapid City, S. Dak., in A units. 


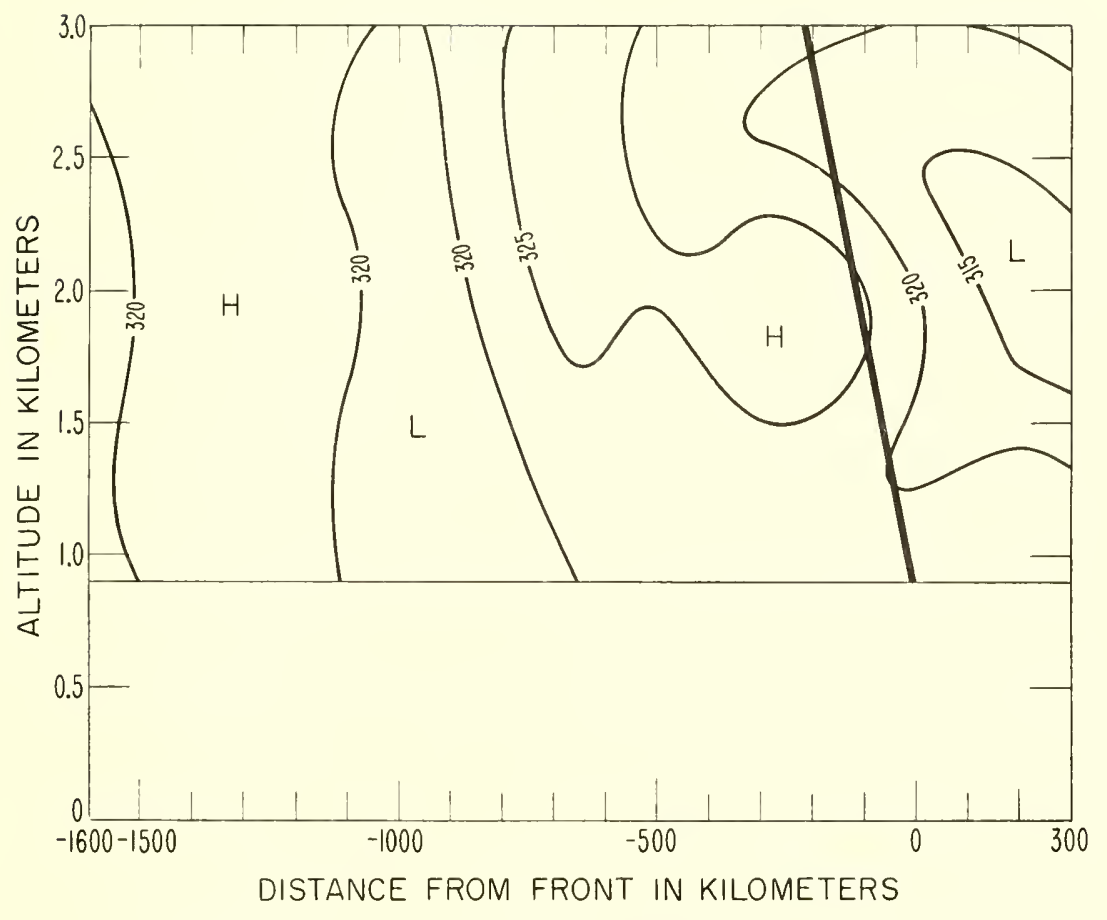

Figure 5.60. Epoch chart, North Platte, Nebr., in A units.

\subsection{Summary}

A survey such as this is designed to indicate the direction of radio meteorological research. Among the brighter prospects is the work of Moler et al., concerning the interrelation of refractive index structure and mesoscale weather changes. Other synoptic features likely to have refractive index significance are the migratory high and low pressure cells of the middle latitudes, since certain surface patterns and vertical profiles occur frequently with a particular type of synoptic system.

A knowledge of the meso-macroscale behavior of $N$ in a synoptic sense enables the propagation engineer to anticipate the occurrence of superrefraction or ducting conditions. Thus, meteorological conditions that give rise to such phenomena as prolonged space-wave fadeouts and interference effects from elevated layers in the troposphere can to some extent be planned for in advance. 


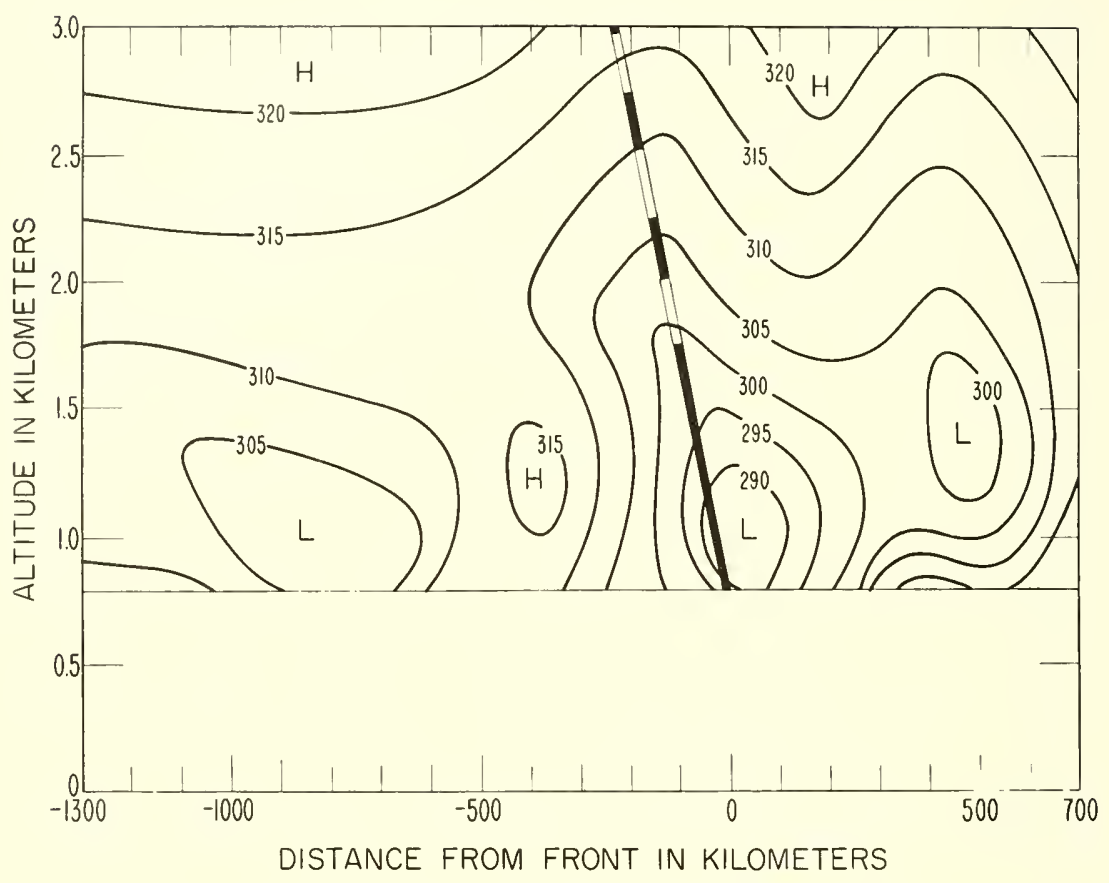

Figure 5.61. Epoch chart, Dodge City, Kans., in A units.

\subsection{References}

[1] Sheppard, P. A. (1947), The structure and refractive index of the atmosphere, Book, Meteorological Factors in Radio Wave Propagation, (Phys. Soc. and Roy. Meteorol. Soc., London, England).

[2] Gerson, N. C. (1948), Variations in the index of refraction of the atmosphere, Geofis. Pura Appl. 13, 88.

[3] Perlat, A. (1948), Meteorology and radioelectricity, L'Onde Elec. 28, 44.

[4] Randall, D. L. (1954), A study of the meteorological effects on radio propagation at 96.3 Mc between Richmond, Va., and Washington, D.C., Bull. Am. Meteorol. Soc. $35,56-59$.

[5] Misme, P. (1957), Influence des discontinuités frontales sur le propagation des ondes decimetriques et centimetriques, Ann. Telecommun. 12, 189-194.

[6] Hay, D. R. (1958), Air-mass refractivity in central Canada, Can. J. Phys. 36, $1678-1683$.

[7] Pickard, G. W., and H. T. Stetson (1950), Comparison of tropospheric reception, J. Atmos. Terrest. Phys. 1, 32.

[8] Pickard, G. W., and H. T. Stetson (1950), Comparison of tropospheric reception at 44.1 Mc with 92.1 Mc over the 167-mile path of Alpine, N.J., to Needham, Mass., Proc. IRE 38, 1450.

[9] Bean, B. R. (1956), Some meteorological effects on scattered radio waves, IRE Trans. Commun. Syst. CS-4, No. 1, 32 . 


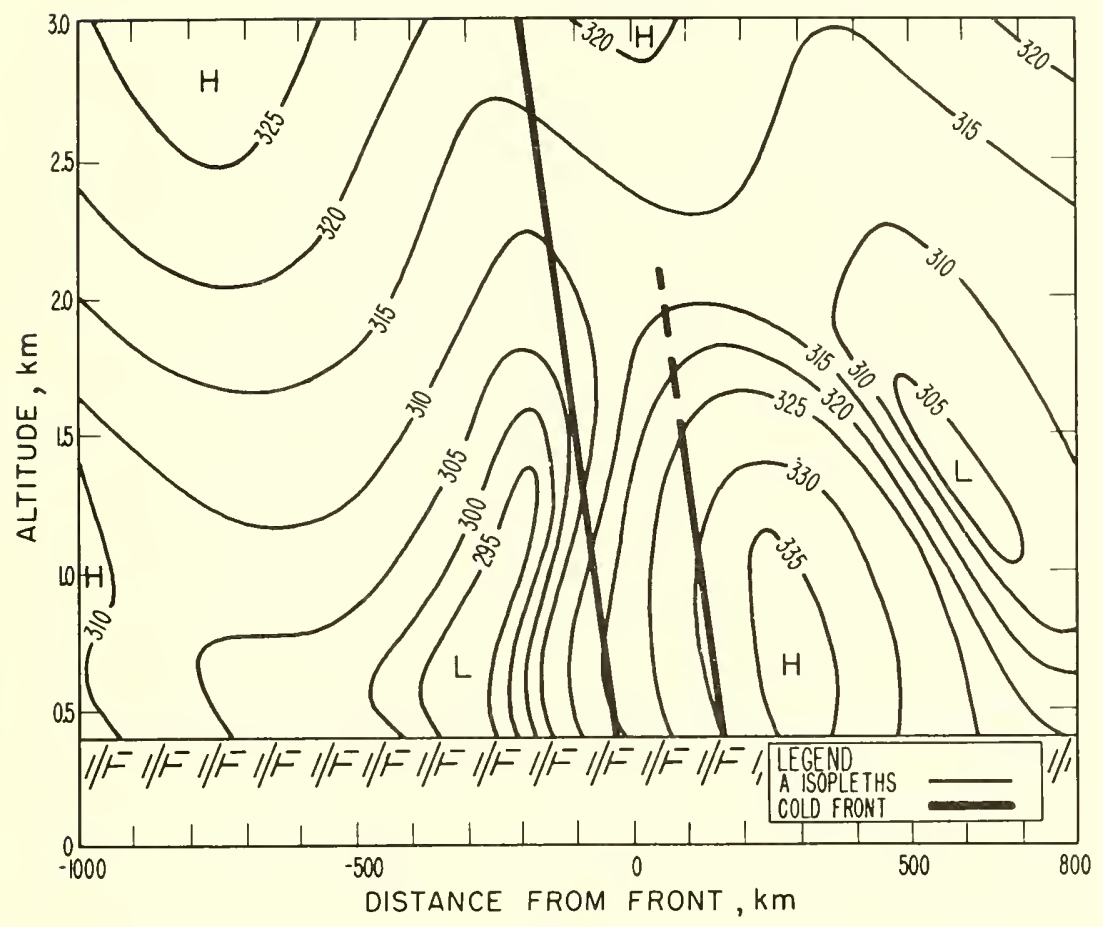

Figure 5.62. Epoch chart, Oklahoma City, Okla., in A units.

[10] Onoe, M., M. Hirai, and S. Niwa (1958), Results of experiment of long-distance overland propagation of ultra-short waves, J. Radio Res. Labs. 5, 79.

[11] Hull, R. A. (1935), Air-mass conditions and the bending of ultra-high-frequency waves, QST 19, 13-18.

[12] Hull, R. A. (1937), Air-wave bending of ultra-high-frequency waves, QST 21, 16-18.

[13] Englund, C. R., A. B. Crawford, and W. W. Mumford (1938), Ultra-short-wave radio transmission through the non-homogeneous troposphere, Bull. Am. Meteorol. Soc. 19, 356-360.

[14] Yerg, D. G. (1950), The importance of water vapor in microwave propagation at temperatures below freezing, Bull. Am. Meteorol. Soc. 31, 175-177.

[15] Schelleng, J. C., C. R. Burrows, and E. B. Ferrell (1933), Ultra-short-wave propagation, Proc. IRE 21, 427-463.

[16] Misme, P. (1960), L'influence du gradient equivalent et de la stabilité atmosphé rique dans les liaisons transhorizon au Sahara et au Congo, Ann. Telecommun. 16, 110.

[17] Misme, P., B. R. Bean, and G. D. Thayer,(1960), Comments on "Models of the atmospheric radio refractive index," Proc. IRE 48, 1498-1501.

[18] Gray, R. E. (1957), The refractive index of the atmosphere as a factor in tropospheric propagation far beyond the horizon, IRE Nat. Convention Record, Pt. 1, 3. 


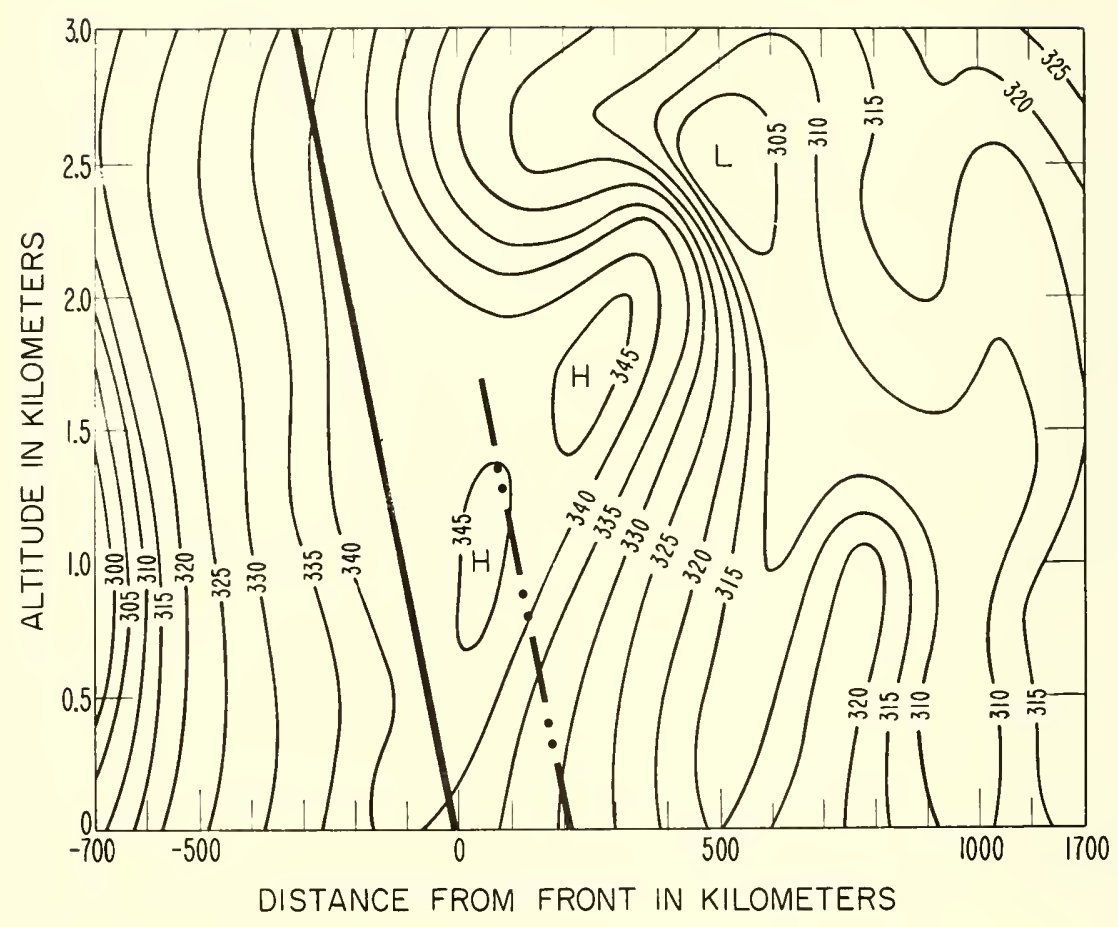

FIgURE 5.63. Epoch chart, Little Rock, Ark, in A units.

[19] Gray, R. E. (1961), Tropospheric scatter propagation and meteorological conditions in the Caribbean, IRE Trans. Ant. Prop. AP-9, No. 5, 492-496.

[20] Bean, B. R., and G. D. Thayer (1959), On models of the atmospheric radio refractive index, Proc. IRE 47, No. 5, 740-755.

[21] Schulkin, M. (1952), Average radio-ray refraction in the lower atmosphere, Proc. IRE 40, No. 5, 554-561.

[22] Fannin, B. M., and K. H. Jehn (1957), A study of radio elevation angle error due to atmospheric refraction, IRE Trans. Ant. Prop. AP-2, No. 1, 71-77.

[23] Bean, B. R., J. D. Horn, and L. P. Riggs (1960), Refraction of radio waves at low angles within various air masses, J. Geophys. Res. 65, 1183.

[24] Arvola, W. A. (1957), Refractive index profiles and associated synoptic patterns, Bull. Am. Meteorol. Soc. 38, No. 4, 212-220.

[25] Jehn, K. H. (1960), The use of potential refractive index in synoptic-scale radio meteorology, J. Meteorol. 17, 264.

[26] Lukes, G. D. (1944), Radio meteorological forecasting by means of the thermodynamics of the modified refractive index, Third Conf. Prop., NDRC, pp. 107-113 (Committee on Propagation, Washington, D.C.).

[27] Katz, I. (1951), Gradient of refractive modulus in homogeneous air, potential modulus, Book, Propagation of Short Radio Waves, pp. 198-199 (McGrawHill Book Co., Inc., New York, N.Y.).

[28] Jehn, K. H. (1960), Microwave refractive index distributions associated with the Texas-Gulf cyclone, Bull. Am. Meteorol. Soc. 41, 304-312. 


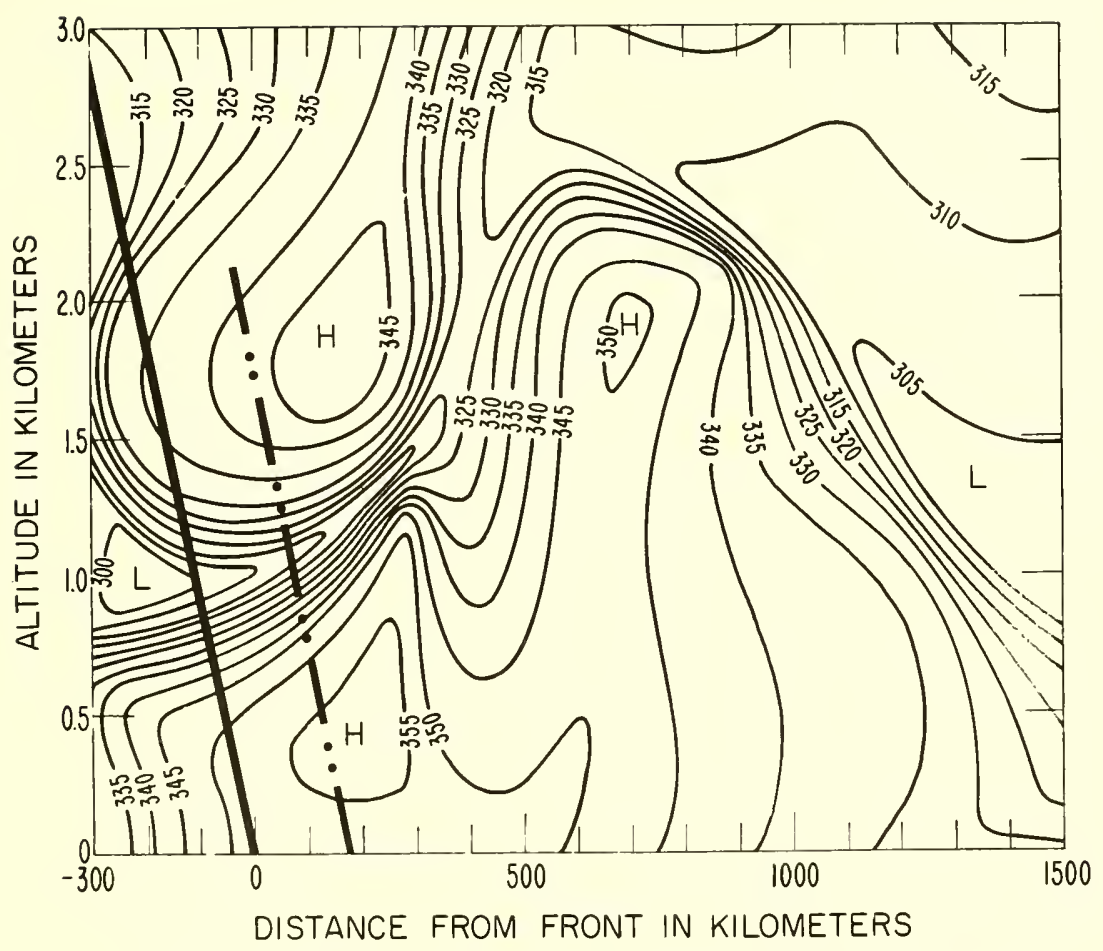

Figure 5.64. Epoch chart, Lake Charles, La., in A units.

[29] Jehn, K. H. (1961), Microwave refractive-index distributions associated with the central United States cold outbreak, Bull. Am. Meteorol. Soc. 42, 77-84.

[30] Flavell, R. G., and J. A. Lane (1962), The application of potential refractive index in tropospheric wave propagation, J. Atmospheric Terrest. Phys. 24, $47-56$.

[31] Moler, W. F., and W. A. Arvola (1956), Vertical motion in the atmosphere and its effects on VHF radio signal strength, Trans. Am. Geophys. Union 37.

[32] Moler, W. F., and D. B. Holden (1960), Tropospheric scatter propagation and atmospheric circulations, J. Res. NBS 64D (Radio Prop.), No. 1, 81-93.

[33] Gossard, E. E., and L. J. Anderson (1956), The effect of super-refractive layers on 50-5,000 Mc nonoptical fields, IRE Trans. Ant. Prop. AP-4, 175-178.

[34] Megaw, E. C. S. (1950), Scattering of electromagnetic waves by atmospheric turbulence, Nature 166, 1100-1104.

[35] Booker, H. G., and W. E. Gordon (1950), A theory of radio scattering in the troposphere, Proc. IRE 38, 401-412.

[36] Saxton, J. A. (1951), Propagation of metre radio waves beyond the normal horizon, Proc. IEE 98, 360-369.

[37] Bean, B. R., J. D. Horn, and A. M. Ozanich, Jr. (1960), Climatic charts and data of the radio refractive index for the United States and the world, NBS Mono. 22.

[38] Smyth, J. B., and L. G. Trolese (1947), Propagation of radio waves in the troposphere, Proc. IRE 35, 1198. 
[39] Bean, B. R., and G. D. Thayer (1959). CRPL exponential reference atmosphere, NBS Mono. 4.

[40] Bean, B. R., and E. J. Dutton (1960), On the calculation of the departures of radio wave bending from normal, J. Res. NBS 64D (Radio Prop.), No. 3, 259-263.

[41] Canterbury Project (1951), Vols. I-III (Department of Scientific and Industrial Research, Wellington, New Zealand).

[42] Bean, B. R., and L. P. Riggs (1959), Synoptic variations of the radio refractive index, J. Res. NBS 63D (Radio Prop.), No. 1, 91-97.

[43] Bean, B. R., L. P. Riggs, and J. D. Horn (1959), Synoptic study of the vertical distribution of the radio refractive index, J. Res. NBS 63D (Radio Prop.), No. 2, 249-258.

[44] Palmer, C. E. (1957), Some kinemsicic aspects of frontal zones, J. Meteorol. 14, No. 5, 403-409. 


\section{Chapter 6. Transhorizon Radio- Meteorological Parameters}

\subsection{Existing Radio-Meteorological Parameters}

\subsubsection{Introduction}

A method of predicting the statistical distribution of field strength on transhorizon paths is an important requirement in tropospheric wave propagation. Consequently, considerable attention has been given in recent years (see figs. 6.1 to 6.6 ) to studies of the correlation between the measured signal level (e.g., the monthly median value) and some quantity derived from surface or upper air meteorological data. Figures 6.1 to 6.6 show relationships between field strength and various meteorological parameters. It can be seen that there are some quite marked similarities (high correlation coefficients) between the two. It thus appears that if a reliable "radio-meteorological parameter" could be developed, then generally available meteorological data would replace expensive radio measurements in deriving the required distribution.

Progress has already been made in this difficult problem, $[1,2]^{1}$. In these investigations, special attention has been given to two parameters: (a) the surface value of refractivity, $N_{s}$, and (b) the difference, $\Delta N$, between $N_{s}$ and $N$ at a height of $1 \mathrm{~km}, N_{1}$. Other groups have studied different parameters $[3,4,5,6,7,8]$, either as possible alternatives to $N_{s}$ and $\Delta N$ in the prediction process or as quantities which elarify the effect of meteorological features, such as anticyelonic subsidence, on signal strength. It is evident from the literature that some differenee of emphasis exists regarding the relative merits of the parameters proposed to date, and particularly on the value of studies of $N_{s}$.

This chapter provides a critical survey of the present position in this field of radio meteorology, and indicates a new approach which incorporates some aspects of all existing treatments. Section 6.1 eontains a study of previous work and attempts to put the various views in proper perspective; section 6.2 diseusses some selected radio data from VHF paths and its classification in terms of refractive index profiles, while section 6.3 introduces a parameter combining the concepts of refraction and atmospheric stability, and compares its properties with those of existing parameters.

${ }^{1}$ Figures in brackets indirate the literature references on p. 266. 


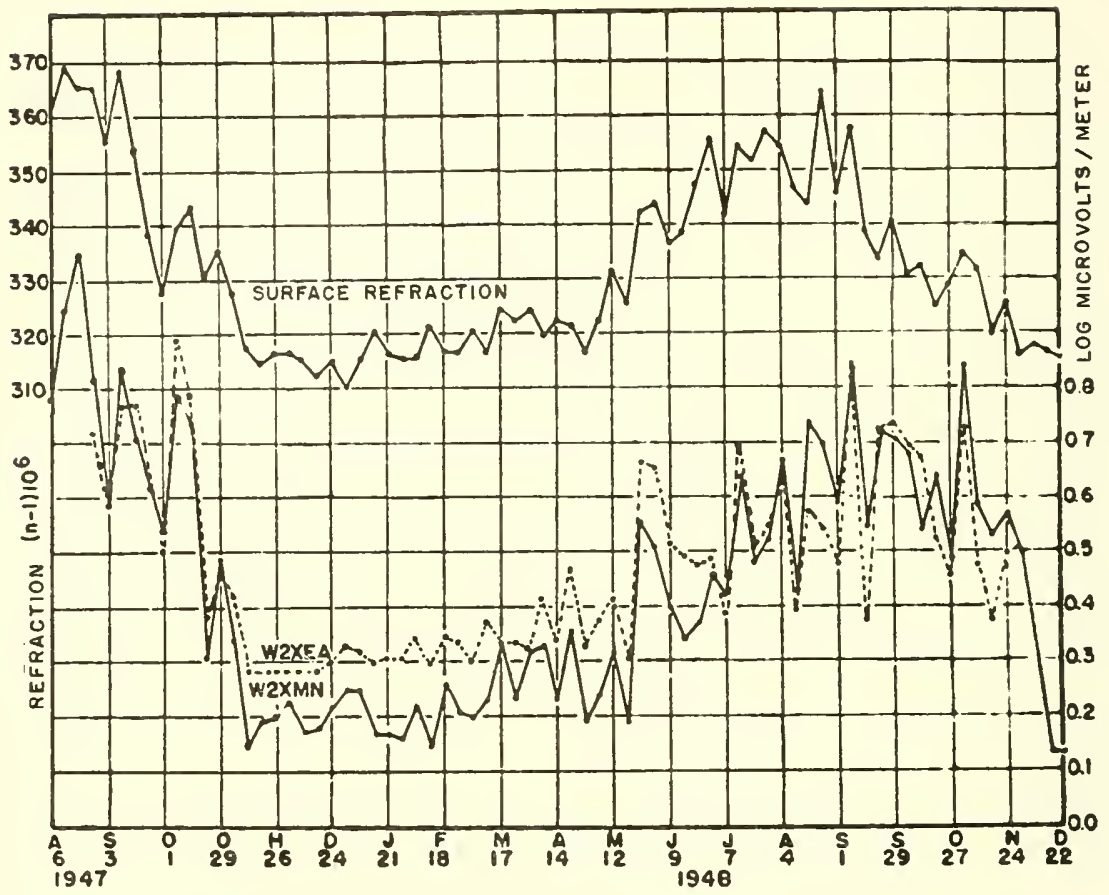

FIGURE 6.1. Weekly means of measured field intensities of W2WMN and W2XEA at Needham, Mass., compared with corresponding atmospheric surface refraction at Boston, Mass.

(After Pickard and Stetson, 1950).

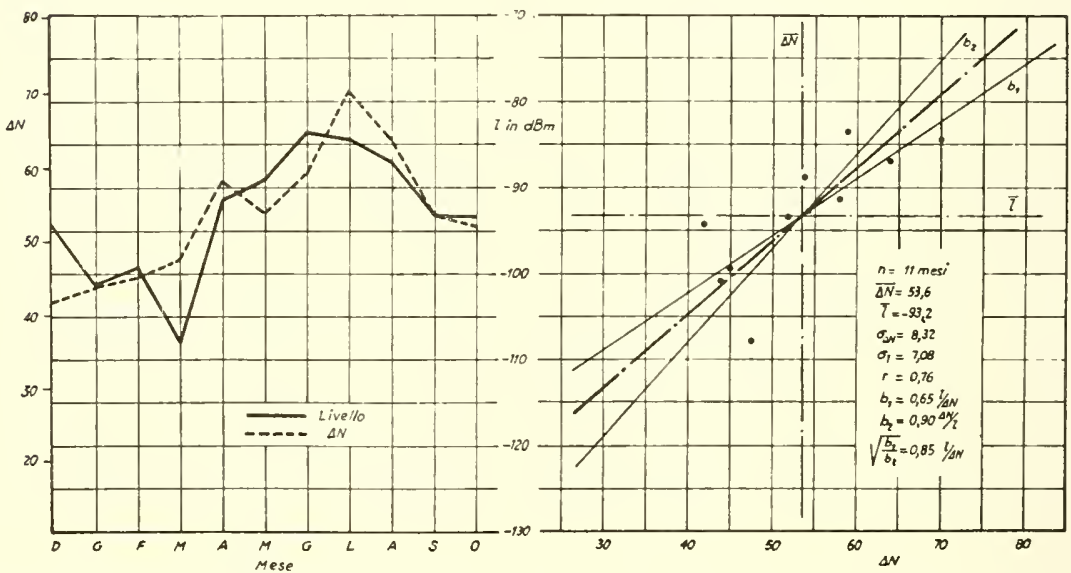

FIGURE 6.2. Correlation of the received monthly mean field strength at Campu Sa Spina and the gradient $\Delta \mathrm{N}$ (between 0 and $1000 \mathrm{~m}$.) of the monthly median index of refraction at Elmas.

(The values of field strength are those obtained after omission of superrefraction). (After Bonavoglia, 1958). 


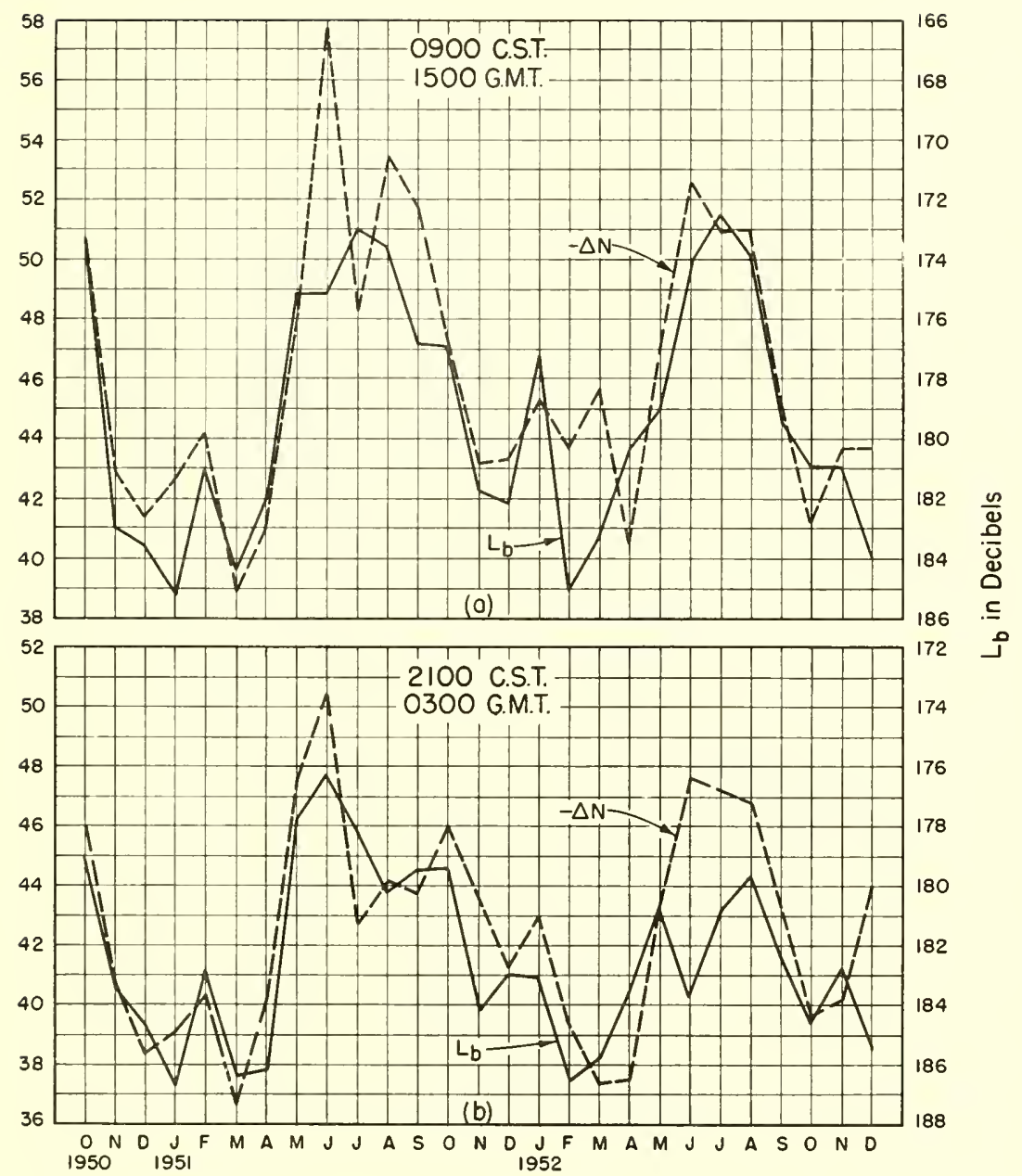

Figure 6.3. Comparison of the monthly median basic transmission loss and refractivity gradient for KIXL-FM, Dallas, Tex., recorded at Austin, Tex.

The development of prediction techniques is especially difficult in the case of:

(a) any path with terminals just within or just beyond the normal horizon, and

(b) signal enhancements which oceur for small percentages of the time. Section 6.2 therefore includes some discussion of propagation characteristies on VHF paths for which considerable radio and meteorological data are already available. The results obtained are particularly relevant to an understanding of the large differences observed between median 


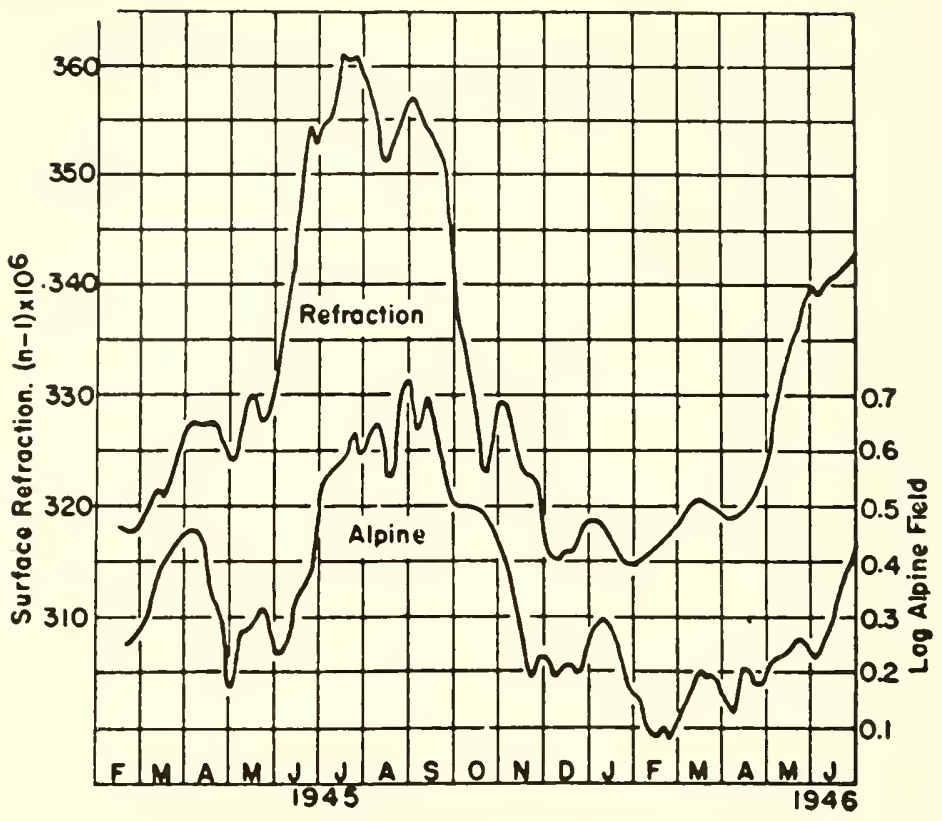

Figure 6.4. Field reception of W2XMN on $42.8 \mathrm{Mc} / \mathrm{s}$ at Needham, Mass., 1945-1946. (After Pickard and Stetson, 1947).

signal levels on VHF paths of comparable length, frequency and angular distance.

The primary purpose of a radio-meteorological parameter is to provide the best estimate of the statistical distribution of field strength (in terms of hourly, daily, weekly, or monthly median values as required on a specific path). The reliability of the parameter must be judged solely in terms of this requirement, and care must be exercised in assessing the value of any given parameter in terms of data obtained over limited intervals of time or from restricted geographical areas. Discussions later in the chapter will consider to what extent it is possible to develop a parameter which, in addition to being statistically reliable, is also characteristic of the physical structure of the atmosphere.

The present practice in applying radio-meteorological parameters consists in determining an average signal level for a given distance and then adjusting this average level for climatic and seasonal differenoes by reference to the changes in some function of the refractive index of the atmosphere. We may express this procedure mathematically, for a linear regression model, thus:

$$
E=b \cdot f(n)+a
$$

where $E$ is the field strength, and $b$ is the regression coefficient expressing 


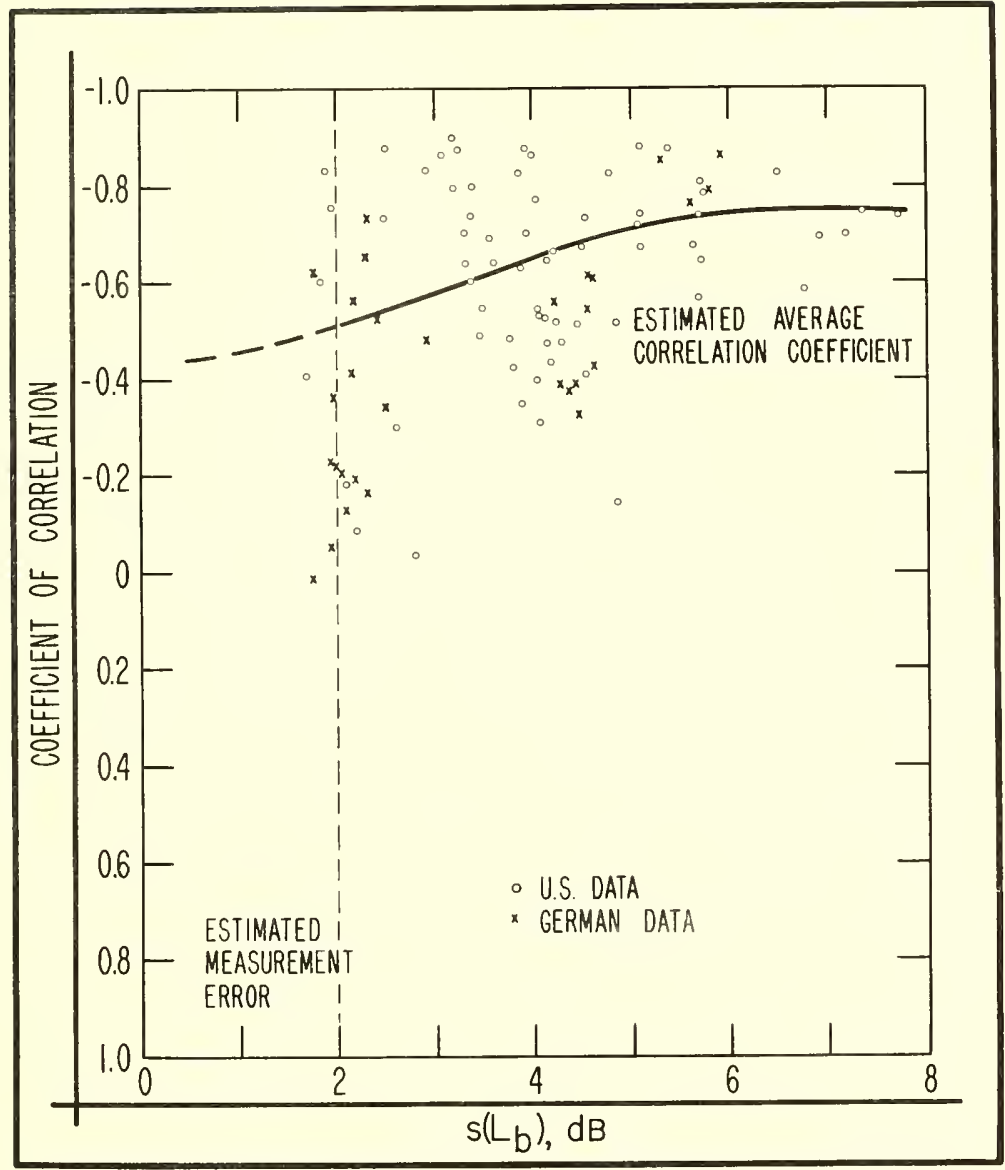

Figure 6.5. Correlation coefficients of seasonal cycles of $\mathrm{N}_{\mathrm{B}}$ and $\mathrm{L}_{\mathrm{b}}$ : night (2000, 2200, 2400) versus the standard deviation of $\mathrm{L}_{\mathrm{b}}$.

the sensitivity of $E$ to a unit change in $f(n)$. The intercept, $a$, is a function of path length, antenna heights, and terrain characteristics, and can be derived from existing prediction procedures [9]. Comments on some results obtained are given in the remainder of this section.

\subsubsection{Parameters Derived From the $N$-Profile}

It has long been recognized that the variations in field strength on transhorizon paths are intimately connected with changes in the vertical gradient of refractive index over the path. Although our knowledge of the detailed fluctuations in refractive index in the troposphere is still inadequate for many requirements, it is nevertheless possible to relate, 


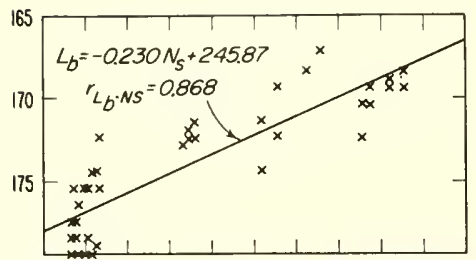

WJAS FM - PATH 12 TRANSMITTER: PITTSBURGH, PA. RECEIVER: STATE COLLEGE, PA. DISTANCE : 188.5 KILOMETERS

$\theta: 30.44$ MILLIRADIANS
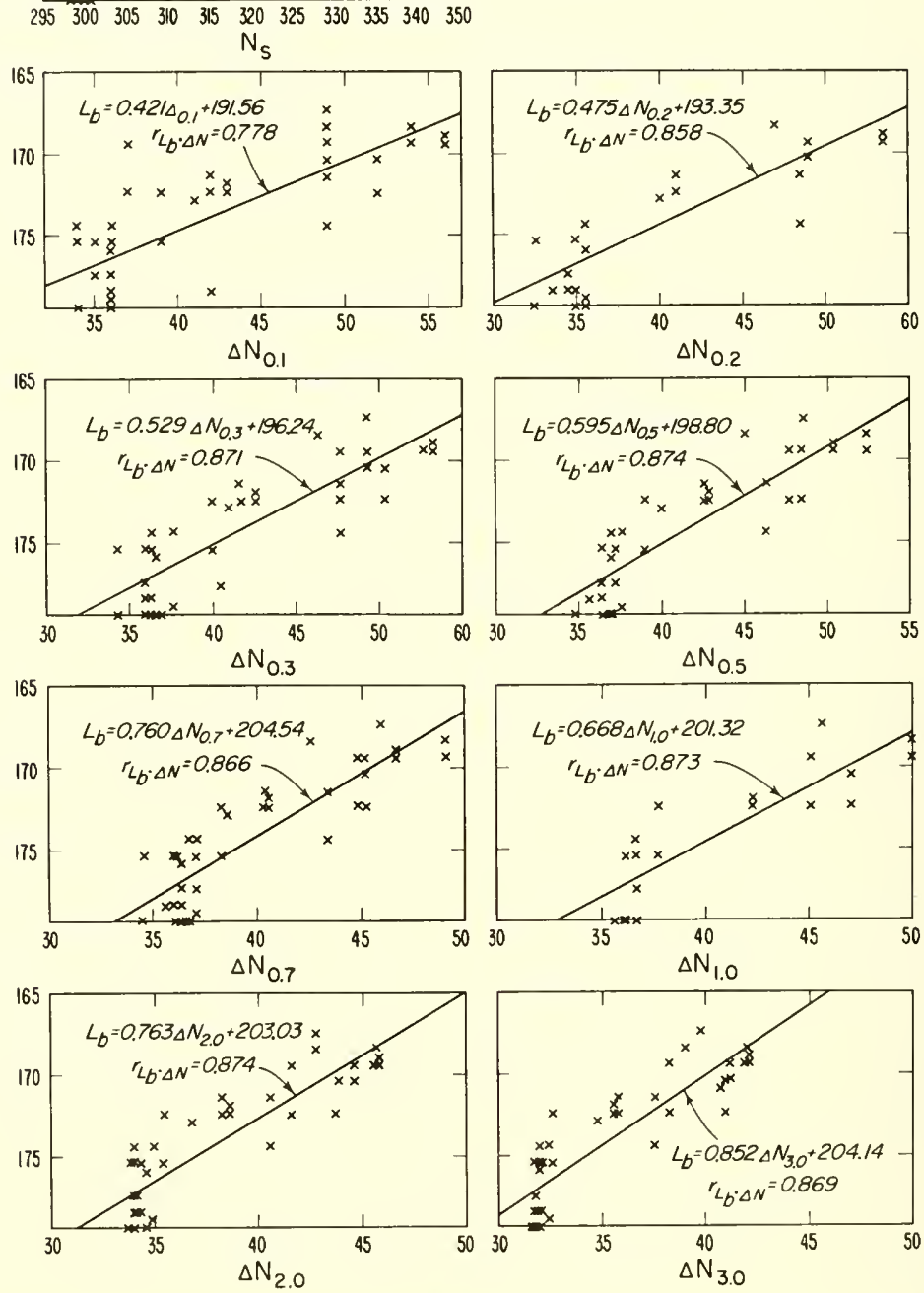

Figure 6.6. Monthly median basic transmission loss versus $\mathrm{N}_{\mathrm{s}}$ or $\Delta \mathrm{N}_{\mathrm{h}}$ for a path from Pittsburgh, Pa., to State College, Pa. 
statistically, changes in signal level and functions of a parameter derived from routine surface and upper-air measurements of pressure, temperature, and humidity.

Following the work of Pickard and Stetson [10], $N_{s}$ and $\Delta N$ have been the subject of detailed studies by several workers $[1,2,11,12,13,14]$. Only the more important conclusions are summarized here. Values of the correlation coefficient, $r$, relating monthly median values of either $N_{s}$ or $\Delta N$ and field strength, derived from a number of paths in diverse climatic conditions [2], range from 0.4 to 0.95 with a median value of about 0.7 . An analysis has also been made of the results obtained by using (a) values of $\Delta N$ obtained from the surface readings and at heights other than $1 \mathrm{~km}$, and (b) values of $\Delta N$ between different levels on the profile up to a height of $3 \mathrm{~km}$. A comparison of measured field strength at frequencies near $100 \mathrm{Me} / \mathrm{s}$ on 20 paths, 130 to $446 \mathrm{~km}$ long, located in various part of the United States, yields the following result: the use of $N_{s}$ gives as good a correlation as any of the $\Delta N$ values, due to the high correlation between the surface value and these differences. The values of $r$ relating monthly median values of $N_{s}$ and $\Delta N$ (the decrease in the first kilometer) have been obtained for the United States [15], France, [16], Germany, [17] and the British Isles [18]. They range from 0.60 to 0.93 . Moreover, the data are consistent with the assumption of a reference atmosphere in which $N$ decreases exponentially with height [15]. These results lead at once to a consideration of the value of $N_{s}$ in predicting the geographical variation of monthly median field strength. This question is considered in more detail later in this section.

It has been shown that, where only past radio or meteorological results are available, one obtains at least as good a prediction of the diurnal and seasonal variations of field strength from long-term meteorological data as from relatively short-term (say 1 year's) radio data. The annual cycle may be represented by a single regression coefficient of $0.18 \mathrm{~dB} / N$-unit for either night or day; however, the regression coefficients for the diurnal cycles lie between 0.2 and $1.1 \mathrm{~dB} / \mathrm{N}$-unit and vary with distance and season, being greatest for paths between 175 and $200 \mathrm{~km}$ long and for the winter months. The possibility of predicting the variation of hourly median values of field strength within any given month by combining the seasonal and diumal correlations has also been discussed [14]. It is recognized that the development of a prediction procedure based on this approach must account for the complex sensitivity of field strength, $E$, to changes in $N_{s}$; this requirement in turn leads to considerations of season, climatic region, distance and frequency. The results for the distance dependence of the $E-N_{s}$ regression coefficient are, of course, intimately connected with the propagation mechanism. In particular, in the case of VHF paths about $200 \mathrm{~km}$ long there will usually be components in the received field due to diffraction around the earth's surface and scattering 
from randomly dispersed eddies; on occasions there will also be a semicoherent field arising from partial reflection at elevated stable layers. Some aspects of this complex situation are discussed in section 6.2.

To sum up other important conclusions reached by the authors mentioned above, the correlation between $E$ and $N_{s}$

(1) increases with increasing variation of $E$ or $N_{s}$,

(2) is greater for seasonal cycles of night-time values of the variables than for the midday values, and

(3) is greater for summer diurnal cycles than for winter ones. Conclusions (1) and (2) are particularly important if we try to assess the utility of $N_{s}$ in prediction work in terms of signal data from areas in which the variation of $E$ is small (e.g., Western Europe), or in terms of data for afternoon periods only.

Another parameter, closely related to $\Delta N$, is the "equivalent gradient," $g_{e}$, proposed by Misme [19]. This is defined as that linear decrease of $n$ with height which produces the same amount of bending as the actual inhomogeneous atmosphere over a given transmission path. The problem is illustrated in figure 6.7 , where the dotted line represents the actual ray path between $T$ and $P$ above an earth of radius $a$. It is required to determine the curvature, $\rho$, of the circular, full-line path which is tangential to the real path at $P$ and which corresponds to a constant value of $d n / d h$ in a fictitious atmosphere. Boithias and Misme [20] have described a graphical method for calculating $g_{e}(=1 / \rho)$, for example, when $P$ is located in the center of the common volume of the antenna beams. Monthly median values of $g_{e}$ are obtained from the corresponding monthly median values of $d n / d h$, and Misme [19] has given tables of $g_{e}$ for various path lengths for different months.

It is evident that $g_{e}$, like $\Delta N$, expresses the amount of refraction produced by the atmosphere and one might expect a high correlation between the two parameters. This is known to be the case in some results quoted by Misme [19] and which are illustrated in figure 6.8. Here the monthly median values of $\Delta N$ and $g_{e}$ (for a $300 \mathrm{~km}$ path) are compared, together with the variation in $N_{s}$, for Leopoldville in the Congo area. The correlation between $g_{e}$ and $\Delta N$ is high, but the maximum values in $N_{s}$ in December and January are accompanied by a local minimum in the values of $g_{e}$ and $\Delta N$. These results, and similar ones from Dakar (W. Africa), have been quoted in support of arguments that $N_{s}$ is of limited value in predicting seasonal and geographical variations in field strength [19]. It is essential, Misme claims, to investigate the nature of the $N_{\mathrm{s}}-\Delta N$ correlation in separate climatic areas; the correlation seems poor for some equatorial climates, probably because of the presence of semipermanent elevated layers. In these conditions, Misme feels that an exponential reference atmosphere and a correlation between $N_{s}$ and $\Delta N$ are not to be expected. 


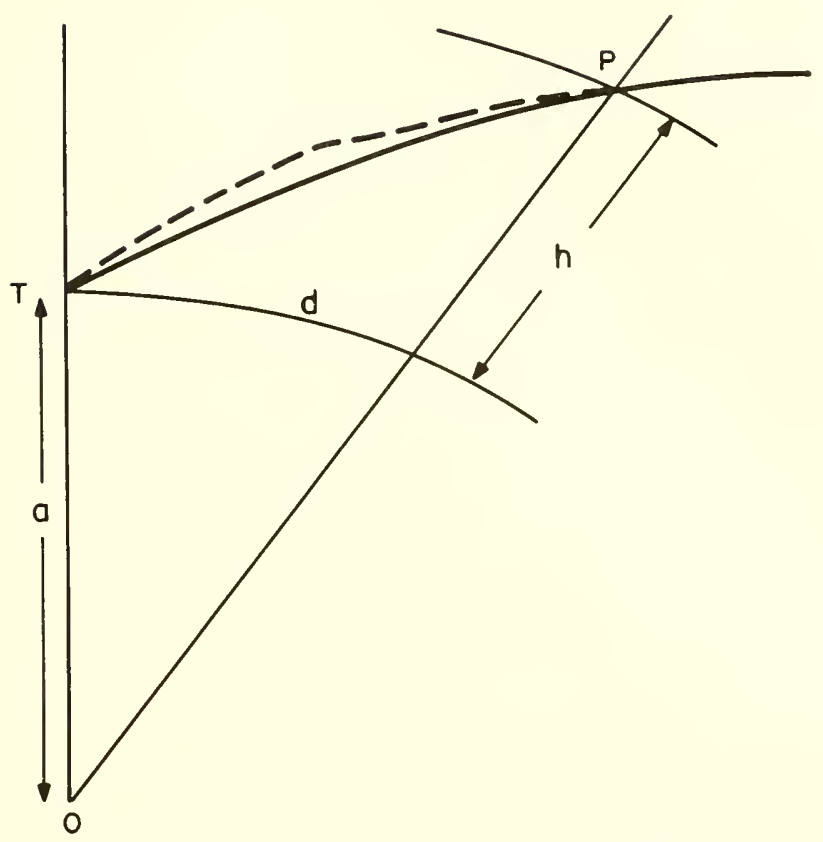

FIgURE 6.7. Geometry of the equivalent gradient.

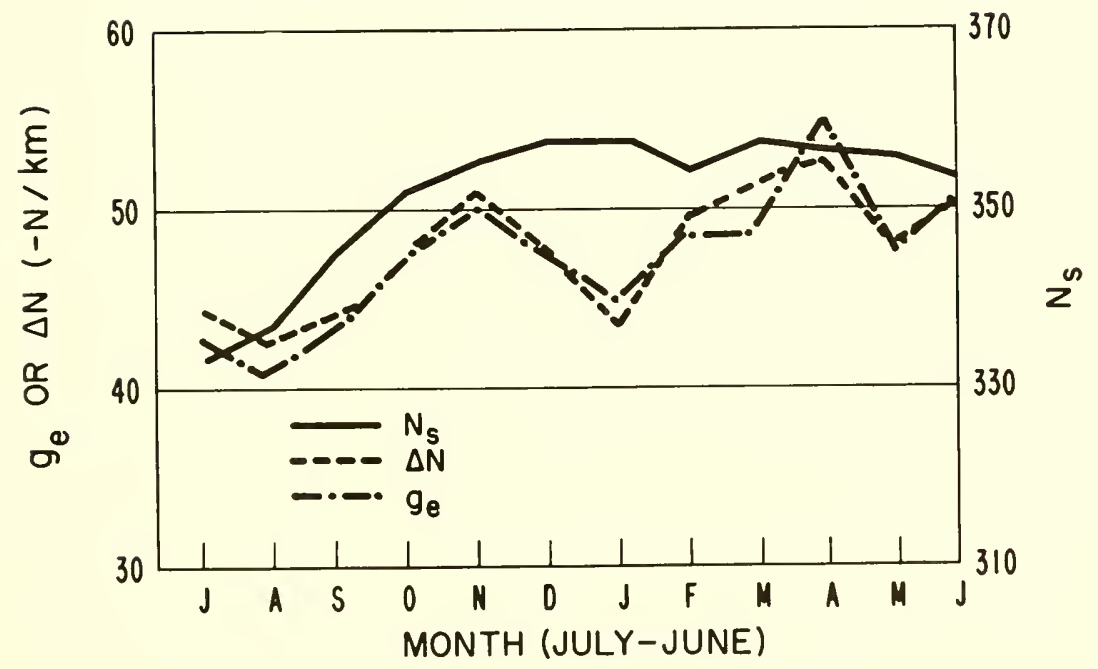

Figure 6.8. Comparison of monthly medians of $\mathrm{g}_{\mathrm{e}}, \Delta \mathrm{N}$, and $\mathrm{N}_{\mathrm{s}}$. (Leopoldville, Congo) 
While admitting the importance of an explanation of the results discussed above, it is difficult at present to accept the argument that $g_{e}$ should be used instead of $\Delta N$. Indeed, it has been shown [19] that for a $471-\mathrm{Mc} / \mathrm{s}$ link, $160 \mathrm{~km}$ long, located in the Sahara region, there is a difference of $20 \mathrm{~dB}$ between the signal levels exceeded for 99 percent of the time during daylight hours in January and June, whereas the January to June variation in $g_{e}$ is less than $2 N / \mathrm{km}$. This result indicates the influence of atmospheric stability on signal strength. We defer, therefore, further consideration of $g_{e}$ until later in the chapter merely noting at this stage that its derivation requires more detailed calculation (i.e., ray tracing) than in the case of $\Delta N$, without any appreciable benefit.

\subsubsection{Parameters Involving Thermal Stability}

The role of stable elevated layers in tropospheric propagation has been discussed by a number of scientists; for example, by Saxton [21], Friis, Crawford, and Hogg [22], and in work done by the French [23] in an important series of papers. These contributions have stimulated interest in the development of radio-meteorological parameters which depend, in part at least, on thermal stability, and this section considers some of the characteristics of these parameters.

\subsubsection{Composite Parameter}

Misme [19] has discussed the Sahara results mentioned above in relation to the theoretical work of Voge [23]. In considering the effect of elevated layers, Voge introduces a parameter, $\eta$, defined by:

$$
\eta=\frac{\text { area of one or several layers at a given height }}{\text { the horizontal area, at the same height, visible from }}
$$

The value $\eta$ is an increasing function of the atmospheric stability measured between two levels in the atmosphere. Stability may be defined as the work, $\Delta W$, required to raise a unit mass of air from one level to another. We have, therefore:

$\eta$ tends to 0 as $\Delta W$ tends to 0.

$\eta$ tends to 1 when $\Delta W$ is large.

The values of $\Delta W$ (in joules per gram) for Aoulef (Sahara) are given by Misme for the January and June months in which measurements were made over the $160 \mathrm{~km}$ path at $471 \mathrm{Mc} / \mathrm{s}$. The relevant data are summarized in table 6.1 . 
TABLE 6.1. Influence of thermal stability on signal level $(\mathrm{d}=160 \mathrm{~km}: \mathrm{f}=471 \mathrm{Mc} / \mathrm{s}$ : North Sahara Region)

\begin{tabular}{ll|c|c}
\hline \hline & Jan. & June \\
\hline$g_{e}(-\mathrm{N} / \mathrm{km}) \ldots \ldots$ & 34.0 & 32.5 \\
\hline$\Delta \mathrm{W}$ for 0.7 to $2 \mathrm{~km} \ldots \ldots$ & 0.17 & 0.0024 \\
\hline Relative signal level, 99 percent value, (d 13) $\ldots$ & 20.0 & 0.0 \\
\hline
\end{tabular}

The dominant propagation mechanisms in January and June are thought to be "diffuse reflection" and "scattering," respectively, and on this basis Misme uses the equations given by Voge to calculate the expected difference in the January and June signal levels. With various assumptions regarding the properties of stable and turbulent layers, a value of $19( \pm 5) \mathrm{dB}$ is obtained for the ratio of predicted field strengths in January and June, in good agreement with the measured value exceeded for 99 percent of the time. This analysis is facilitated by the fact that the two selected months are characteristic of well-defined climatic situations in the Sahara region. Nevertheless, the results indicate that a parameter which combines the concepts of the equivalent gradient and thermal stability may be of general application. Misme [19] has therefore suggested a parameter $M$ of the form:

$$
M=a\left(g_{e}-40+b[\Delta W]^{n}\right)
$$

where $a, b$, and $n$ are constants, $g_{e}$ is the equivalent gradient, and $\Delta W$ is the thermal stability defined above for a $1 \mathrm{~km}$ height interval. With $a=0.5 \mathrm{~dB} / N / \mathrm{km}, M$ provides an estimate of the variation in field strength, $E$, in decibels, caused by changes in equivalent gradient and stability. It remains to define $E$ in a "standard" atmosphere by selecting a mean value of stability and a suitable value for the term $b$.

This composite parameter is thought by Misme to be more representative of the real atmosphere (especially in tropical areas) than $N_{s}$ and $\Delta N$, and it is certainly of great potential value. However, only fragmentary radio data are available for purposes of comparison in the references quoted, and here again the precise value of the parameter in prediction work can only be determined by a more comprehensive study.

\subsubsection{Potential Refractive Index (or Modulus), $K$}

This parameter is defined as the value of $N=(n-1) 10^{6}$ which an air mass would have if brought adiabatically to a standard pressure, say 1000 mbar, assuming a constant humidity mixing ratio. Examples of the use of $K$ in radio metcorology have been given by Katz [24], and by Jehn 
[25]. It has also been applied by Flavell and Lane [6] in studies of the effect of anticyclonic subsidence on tropospheric propagation. Values of $K$ can be derived very rapidly from upper-air data and, since plots of $K$ against height or pressure do not exhibit the large systematic decrease of $N$ with height in the conventional $N(h)$ profile, the structure and motion of meteorological features are clarified considerably. (Below the condensation level, a lapse rate of $-20 N / \mathrm{km} \equiv d K / d h=0$.) In this respect, $K$ is superficially similar to the $A$ unit [26] derived from the exponential reference atmosphere. However, no quantitative results are yet available with this parameter and, furthermore, the method of deriving $K$ assumes a dry adiabatic lapse rate and a constant humidity mixing ratio. The presence of a condensation level in the actual atmosphere is therefore neglected. In addition, accurate values of $N$ can only be derived directly from the $K$ profile in certain restricted conditions. Pending further studies in this direction therefore, $K$ and $d K / d h$ remain more suitable for qualitative synoptic studies than for quantitative predictions of field strength variations. However, it is of interest to note that a close connection exists between $K$ and the composite parameter $M$ discussed above. Misme [27] has shown that the change of $K$ in a given height interval, $\Delta K / \Delta h$, is of the form:

$$
\Delta K / \Delta h=k_{1} \Delta N / \Delta h-k_{2} \Delta W / \Delta h
$$

where $k_{1}$ and $k_{2}$ are constants, and $\Delta W$ is a measure of thermal stability as defined earlier.

\subsubsection{Vertical Motion of the Atmosphere}

The influence of stability has also been discussed by Moler and Arvola [7], and Moler and Holden [8]. These authors assume that the average lapse rate, $\overline{d n / d h}$ and the magnitude of local irregularities on the profile, are primarily determined by changes in vertical velocity. Local centers of convergence (low pressure cells) produce updrafts which result in considerable mixing and the dissipation of any stable layer structure. Horizontal divergence from local high-pressure centers create temperature inversions and associated layer-type discontinuities in the $n$-profile. These latter features are most pronounced in conditions of anticyclonic subsidence.

The direction and relative magnitude of the vertical component of wind velocity can be estimated by techniques outlined by Moler and Holden, and a correlation between hourly median field strength and calculated values of vertical velocity has been noted by these authors. These fundamental studies represent an important attempt to explain signal variations in terms of atmospheric motion, and a survey of available experimental evidence supports the basic assumptions in this approach. It 
is particularly interesting to consider whether local centers of convergence and divergence, ill-defined on Daily Weather Reports, can be distinguished by observations with microbarographs and whether the results are correlated with signal characteristics. Some results of such an investigation, forming part of a more general study of VHF propagation in the United Kingdom, are shown in figure 6.9. The signal records obtained at a frequency of $186 \mathrm{Mc} / \mathrm{s}$ on a $140 \mathrm{~km}$ path are shown for 12 September 1959 together with the pressure variations recorded at transmitting and receiving sites. The steady high signal at 0900 hours begins to fall as the pressure at the transmitter begins to decrease, and at about 1030 hours the median level and fading rate change abruptly as the pressure at the midpoint falls. Between 1200 and 1700 hours the pressure over the whole path is falling, and the signal characteristies remain essentially uniform. However, a significant increase in median level and a reduction in fading rate are evident at 1700 hours as the pressure begins to increase at the transmitter. By about 2300 hours the pressure at the center of the path has reached a steady value and the signal level is again high with negligible fading. The total barometric variation between 0900 and 2200 hours is only 2 mbar.

There is cbviously a fruitful field of radio-meteorological study suggested by the work outlined above, but we have to conclude that the results obtained, while extremely valuable in clarifying a qualitative relationship between signal strength and vertical motion (or stability), are not immediately applicable in the problem of predicting field strength variation.

\subsubsection{Discussion of the Parameters}

The above review emphasizes the need for a critical inspection of representative data, radio and meteorological, with the objective of comparing the merits of the several parameters and explaining, if possible, some of their relative merits and limitations. Some relevant points have been mentioned already, and these issues are developed m more detail in the following discussion.

It is important to bear in mind that in the particular problem of predicting field strength changes we are concerned with a statistical relationship between two quantities, the median signal level and a radiometcorological parameter. It may be advantageous, of course, to investigate the merits of special parameters developed for particular climatic areas; for example, the value of the gradient, $d n / d h$, in a semipermanent elevated layer in the trade winds area. Nevertheless, such a parameter must also be statistically "reliable" if it is to be applied in prediction analysis. 
12 SEPTEMBER 1959
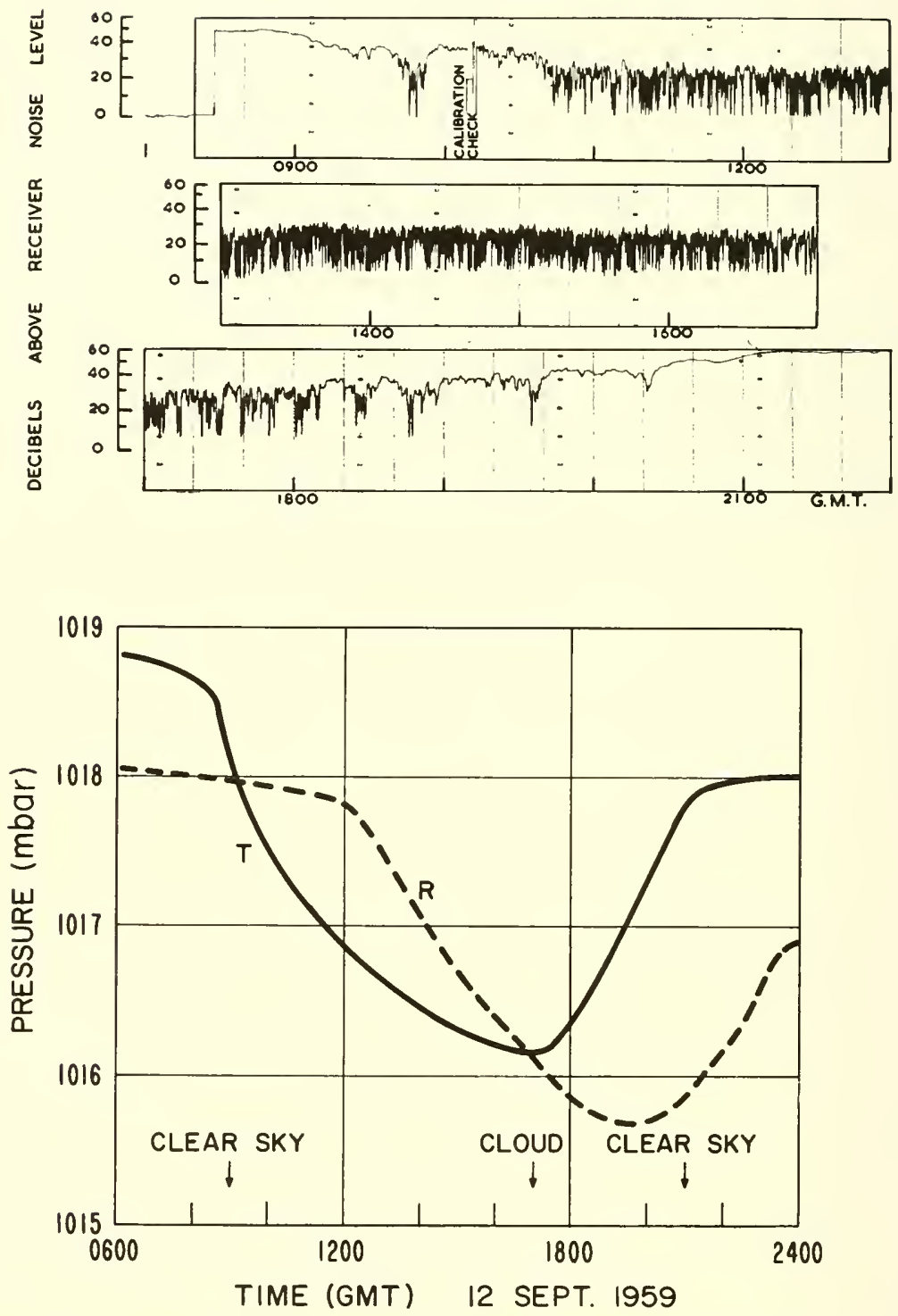

Figure 6.9. Signal records and pressure variations at transmitter, T, and receiver, $\mathrm{R}$, on a $140 \mathrm{~km}$ path; $\mathrm{f}=186 \mathrm{Mc} / \mathrm{s}$. 


\subsubsection{Comparison of Some Parameters}

It is probably fair to say that, at present, the greatest interest is concentrated on the relative merits of $N_{s}, \Delta N$, and $g_{e}$, for these quantities have received more detailed attention than any others. (It might be argued that, since the initial gradient of refractive index with height has a strong influence on refraction, this quantity should be highly correlated with signal level. However, the errors of measuring the initial gradient by radiosonde techniques effectively mask the correlation with the signal.) In a comparison of $N_{s}, \Delta N$, and $g_{e}$, the monthly median values of transmission loss, $L$, were determined for the hours of radiosonde ascents for 20 radio paths in various parts of the United States.

The equivalent gradient, $g_{e}$, was then calculated using standard raytraeing techniques [15] assuming:

(a) the actual antenna heights,

(b) a smooth earth with the sea-level radius,

(c) horizontal stratification of $N$ with a vertical distribution the same as that of the monthly mean.

Standard statistical methods were then employed to determine the correlation between monthly median values of (a) $E$ and $g_{e}$, (b) $E$ and $N_{s}$, and (c) $E$ and $\Delta N$ (surface to $1 \mathrm{~km}$ height). The average values of these correlation coefficients for the 20 paths are given in table 6.2. The paths studied in this comparison were the same as those listed by Bean and Cahoon [2] in their analysis of $N_{s}-\Delta N$ correlation. The data used were representative of climatic conditions ranging from those of New England and the Great Lakes area to Texas and the Pacific coast. This investigation, at least, would seem to justify existing prediction procedures based on $N_{s}$ and $\Delta N$. It is valuable, however, to consider some of the results for specific paths in more detail, since they illustrate some features of interest directly relevant to the arguments eoncerning the value of $N_{8}$ and $\Delta N$.

TABLE 6.2. Average correlations, $\mathrm{r}(\mathrm{z})$, of refraction variables with field strength (20 paths, 130 to $446 \mathrm{~km}$ long, $f=92$ to $106 \mathrm{Mc} / \mathrm{s}$ )

\begin{tabular}{r|r|r|r}
\hline \hline Variable & $g_{e}$ & $N_{s}$ & $\Delta N$ \\
\hline$r(z)_{\ldots} \ldots \ldots$ & 0.59 & 0.70 & 0.71 \\
\hline
\end{tabular}

\subsubsection{Some Exceptions and Anomalies}

In so complex a matter as field-strength prediction, it would be unreasonable to expect any radio-meteorological parameter to be of worldwide application to a uniform degree of reliability, and some conditions have already been quoted in which $N_{s}$ may not be a reliable parameter. The significance of these examples is really the issue on which views 
diverge most at the present time. Unfortunately, adequate radio data are not available for several areas of interest (e.g., equatorial Africa) and in these cases we can only propose tentative explanations based on a critical examination of existing results, for similar but not identical conditions.

An examination of available data, in published and unpublished reports, has shown two particular examples which deserve further study; these are (1) climatic conditions in which stable elevated layers are persistent during certain seasons of the year, and (2) equable climates in which the annual range of $N_{s}, \Delta N$, and field strength is relatively low. Attention has already been drawn to an example in the first category; namely, the path from San Diego to Santa Ana, Calif. [2]. The well-defined coastal inversion in this area occurs at a height of about $0.7 \mathrm{~km}$, and the associated stable layer has a strong influence on radio field strength. Here the correlation between field strength and $N$ gradient for heights up to $0.7 \mathrm{~km}$ above the surface is small and negative (i.e., opposite to the general trend). The correlations with $N_{s}$, however, and with $N$ differences to heights above the base of the inversion, are about 0.8. This result suggests an explanation of some of the results, already mentioned, for Dakar and Leopoldville. Consider the profile of figure 6.10 typical of ascents made through daytime inversions in equatorial and Mediterranean areas. (For comparison purposes, an exponential reference atmosphere is also shown.)

At Dakar, for example, an elevated layer is observed in 40 to 50 percent of the daytime soundings during August, frequently with the base of the inversion above $1 \mathrm{~km}$ height. In these conditions, at least, the high $N_{s}$ values measured in August are not accompanied by high values of $\Delta N$ ( 0 to $1 \mathrm{~km}$ ), for the lapse rate, $d N / d h$, below the inversion is generally less steep than would be expected for the given value of $N_{s}$. It would be valuable, therefore, to examine in more detail the distribution of the height of the layer, particularly in the summer months. If the data given by Misme contain an appreciable fraction of such profiles, then the poor $N_{s}-\Delta N$ correlation he discusses is not surprising. The correlation between $N_{s}$ and signal level, however, could still be significant, as in the San Diego-Santa Ana link [2]. Further radio data are obviously required for areas in which elevated inversions are persistent.

In many temperate regions, a somewhat different situation exists. In western Europe, for example, the annual range of monthly median values of $N_{s}$ is 10 to $20 \mathrm{~N}$ units; similarly, the variation in the monthly median values of field strength is also small and frequently lies within the estimated measurement error. Figure 6.11 shows some results which illustrate these features. Monthly median values of relative field strength are shown for the months January-November, 1959, for a $300-\mathrm{km}$ path at a frequency of $174 \mathrm{Mc} / \mathrm{s}$; the terminals being located at Lille (northern 


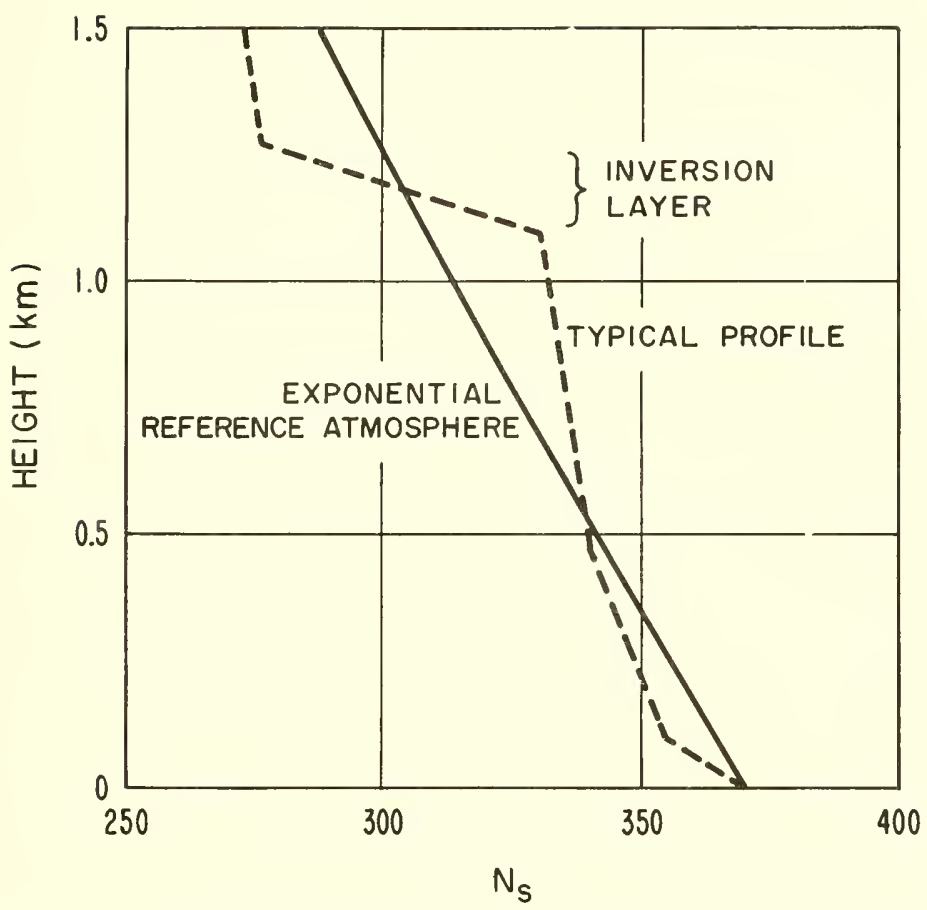

Figure. 6.10. Typical profile through an inversion layer (equatorial and Mediterranean

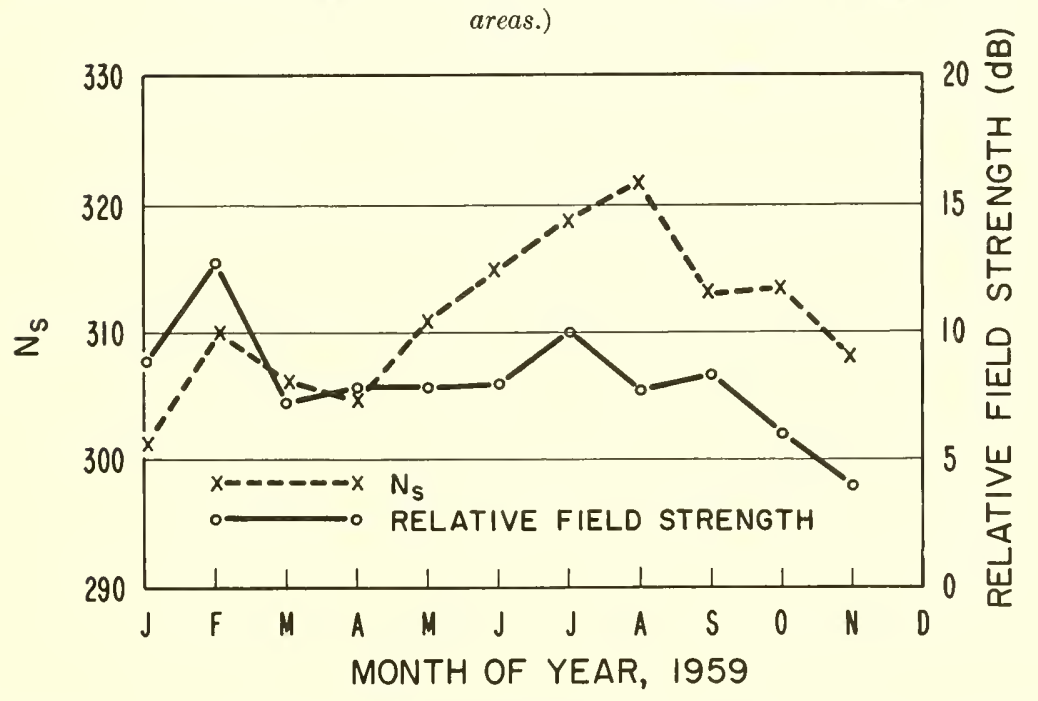

FIgURE 6.11. Variation of monthly median values of $\mathrm{N}_{\mathrm{s}}$ and field strength for a $300 \mathrm{~km}$ path from Lille, France, to Reading, England. 
France) and Reading (England). The variations in $N_{s}$ obtained from the Crawley radiosonde station (close to the midpoint of the path) are also shown. Here $N_{s}$ has a range of $\pm 10 N$ units, and the signal level a range of no more than $\pm 4.5 \mathrm{~dB}$. In fact, from March to September the total variation in observed monthly median field strength is only $\pm 1.5 \mathrm{~dB}$. The measurements of field strength in these experiments were estimated to be subject to possible errors of about $\pm 3 \mathrm{~dB}$. Consequently, one would not be justified in assessing the value of $N_{8}$ as a radio-meteorological parameter on the basis of the correlation coefficient (about 0.25 ) calculated from the two curves in figure 6.11. During the period studied in this work (0900-2300 U'T daily, January-November 1959) the highest signals were observed during anticyclonic winter weather, with elevated inversion layers at a height of about $0.6 \mathrm{~km}$. Extended stable layers at this height were rarely seen on the sonde ascents during the summer months. This result may partly explain the fact that the seasonal variation in $N_{s}$ (highest values in the summer months) is not accompanied on the LilleReading path by a corresponding variation in monthly median field strength.

\subsubsection{Conclusions}

A review of available data shows that no radio-meteorological parameter has yet been proved to be superior to $N_{s}$ or $\Delta N$ for general application in the prediction of field strength distributions. The value of these parameters has been established by studies of many paths in diverse climatic conditions. However, it should be noted that much of the radio data has been obtained at frequencies near $100 \mathrm{Mc} / \mathrm{s}$, and there is a clear need for further analysis of the several parameters in conjunction with field strength measurements at higher frequencies.

Pending more detailed results, it does not seem likely that the equivalent gradient, $g_{e}$, affords any significant advantage over $\Delta N$, especially in view of the many calculations required in its derivation. Some data from selected areas (Congo, Sahara, west Africa) suggest that $N_{s}$ and $\Delta N$ may have limited value in these regions; however, the present lack of adequate radio data precludes any definite conclusions. These examples, and allied work on vertical motion and thermal stability, emphasize the importance of a parameter (such as that suggested by Misme) which takes account of the influence of elevated layers. This approach would probably prove fruitful not only in equatorial areas but also in temperate regions where the annual range of $N_{s}$ and $\Delta N$ is small.

Analysis of some results obtained at a frequency of $100 \mathrm{Mc} / \mathrm{s}$ has shown that it has proved feasible to provide at least as good a prediction of the annual cycle of field strength variations from long-term meteorological data as from relatively short-term radio data. 


\subsection{An Analysis of VHF Field Strength Variations and Refractive Index Profiles}

\subsubsection{Introduction}

It is evident from the discussion in section 6.1 that the further development of radio-meteorological parameters would be assisted by a better understanding of the propagation mechanism on non-optical paths. In particular, the influence of thermal stability on signal level, fading rate and wavelength dependence is an important topic requiring further study.

The effect of varying meteorological conditions on signal characteristics is especially marked in the case of paths of "intermediate" length, with terminals just beyond the radio horizon. As noted in section 6.1 on such a path the residual scattered field in the absence of stable layers or surface ducts will often be comparable with the weak diffracted field. Furthermore, at frequencies up to, say $300 \mathrm{Mc} / \mathrm{s}(\lambda>1 \mathrm{~m})$ relatively strong fields will frequently be observed in conditions favorable to the production of temperature inversions in the first $2 \mathrm{~km}$ or so above the earth's surface. It is the purpose of this section to discuss some aspects of radiometeorology relevant to this situation, especially the field strength distribution observed on a $200 \mathrm{~km}$ path at frequencies between 72 and $180 \mathrm{Mc} / \mathrm{s}$. The signal characteristics are analyzed in terms of a classification of refractive index profiles, with the objective of clarifying the relative importance of different propagation mechanisms and their influence on the measured field strength distribution.

Table 6.3 lists characteristic profile types, the assumed mechanism associated with each type, and typical meteorological conditions. Selected references are given for each eategory, and special mention may be made here of recent work [28] in France which, to a large extent, unifies and extends earlier analyses based on the separate concepts of "reflection" and "scattering."

\subsubsection{Radio and Meteorological Data}

The analysis to follow is limited to paths between Chicago and Urbana, Ill. (fig. 6.12), since several years of radio data were available for four separate wavelengths between 1.67 and $4.18 \mathrm{~m}$. Moreover, two radiosonde stations are located on or near the path, a unique situation in radiometeorologieal investigations. Details of the radio paths are given in table 6.4 (in which $\theta$ is the total angle between the horizon rays from transmitter and receiver on a $4 / 3$-earth profile).

The meteorological data were obtained from the simultaneous radiosonde observations made by the Weather Bureau at Joliet and at Chanute Air Force Base, near Rantoul, Ill. The results used were those from the significant levels reported whenever the temperature or humidity departed by \pm 10 percent from predetermined values. 
TABLE 6.3. Refractive index profile classification, probable propagation mechanisms and meteorological conditions

\begin{tabular}{|c|c|c|c|}
\hline Profile & $\begin{array}{l}\text { Assumed propagation } \\
\text { mechanism }\end{array}$ & $\begin{array}{l}\text { Ref- } \\
\text { erence }\end{array}$ & $\begin{array}{l}\text { Meteorological } \\
\text { conditions }\end{array}$ \\
\hline $\begin{array}{l}\text { Unstratified (U), monotonic decrease } \\
\text { with height, gradient nowhere ex- } \\
\text { ceeds twice normal for that height. }\end{array}$ & Scattering plus diffraction & $\begin{array}{l}{[29]} \\
{[30]} \\
{[31]}\end{array}$ & $\begin{array}{l}\text { Well-mixed atmosphere } \\
\text { due to thermal convec- } \\
\text { tion and, or wind shear }\end{array}$ \\
\hline $\begin{array}{l}\text { Elevated Layer (EL), monotonic de- } \\
\text { crease with height with one or more } \\
\text { distinct layers with gradients at least } \\
\text { twice normal for that height. }\end{array}$ & $\begin{array}{l}\text { Scattering plus diffraction } \\
\text { plus reflection. }\end{array}$ & $\begin{array}{l}{[32]} \\
{[21]} \\
{[28]}\end{array}$ & $\begin{array}{l}\text { Layer formed by subsi- } \\
\text { dence inversion or lift- } \\
\text { ing of radiation inver- } \\
\text { sion. }\end{array}$ \\
\hline $\begin{array}{l}\text { Super-refractive (SR), same as EL but } \\
\text { the layer is ground-based. }\end{array}$ & $\begin{array}{l}\text { Extended radio horizon pro- } \\
\text { ducing enhanced dif- } \\
\text { fracted and scattered } \\
\text { components. }\end{array}$ & [31] & $\begin{array}{l}\text { Radiation inversion } \\
\text { formed during the } \\
\text { night or rapid evapo- } \\
\text { ration from soil after } \\
\text { rain. }\end{array}$ \\
\hline $\begin{array}{l}\text { Ducting (D), same as } \mathrm{SR} \text { but the gra- } \\
\text { dient exceeds the earth's curvature, } \\
\mathrm{I} / a \text {. }\end{array}$ & $\begin{array}{l}\text { Extension of radio horizon } \\
\text { to include the receiver. }\end{array}$ & {$[33]$} & Same as SR. \\
\hline
\end{tabular}

TABLE 6.4. Chicago-to-Urbana radio path characteristics

\begin{tabular}{|c|c|c|c|c|c|}
\hline Station & Distance & $\theta$ & $\lambda$ & $f$ & Period of record \\
\hline $\begin{array}{l}\text { WBKB-TV } \\
\text { WNBQ-TV } \\
\text { WMBI-FM } \\
\text { WENR-TV }\end{array}$ & $\begin{array}{c}k m \\
203.1 \\
202.9 \\
202.7 \\
202.9\end{array}$ & $\begin{array}{c}\operatorname{mrad} \\
16.3 \\
16.7 \\
16.1 \\
16.4\end{array}$ & $\begin{array}{l}c m \\
4.18 \\
3.67 \\
3.15 \\
1.67\end{array}$ & $\begin{array}{r}M c / s \\
71.75 \\
81.75 \\
95.50 \\
179.75\end{array}$ & $\begin{array}{r}5 / 51-5 / 53 \\
10 / 50-10 / 51 \\
7 / 50-6 / 52 \\
7 / 5 \mathrm{l}-6 / 53\end{array}$ \\
\hline
\end{tabular}

\subsubsection{Classification of Radio Field Strengths by Profile Types}

The RAOB significant level data were converted to refractive index by use of (1.20). The gradient of $N$ was then determined between the reported significant levels of each profile and examined as to whether the gradients fell into the category of linear, subrefractive, or superrefractive depending upon the criteria set down in table 6.5, wherein superrefractive is approximately twice normal and subrefractive has a positive gradient. Simultaneous observations of similar profile types at Rantoul and Joliet were necessary for entry as a distinct profile occurrence. If a superrefractive layer occurred above the crossover heights of the radio horizon tangent rays from both transmitter and receiver then it was classified as an elevated layer provided the reported layer heights were within $1 \mathrm{~km}$ of one another at both radiosonde stations.

TABLE 6.5. $\quad \mathrm{N}$ gradient classification of profile types in $\mathrm{N}$-units $/ \mathrm{km}$

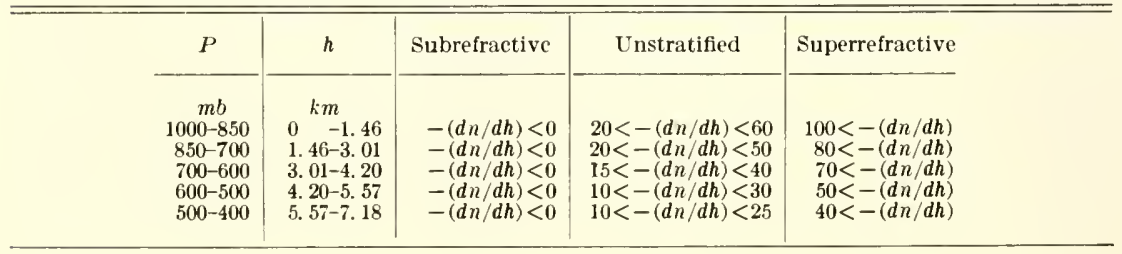


Elevated layers below the crossover height were classified as groundbased superrefractive layers. Elevated layers below the crossover height at one weather station and above that height at the other were classified as tilted elevated layers.

After these characteristic profiles were isolated, the median field strengths for the 3-hourly periods centered upon the radiosonde observation time were arranged into cumulative probability distributions for each profile type. The results are shown in figure 6.13. (There were relatively few examples of subrefractive profiles and no distributions for this category are given.)

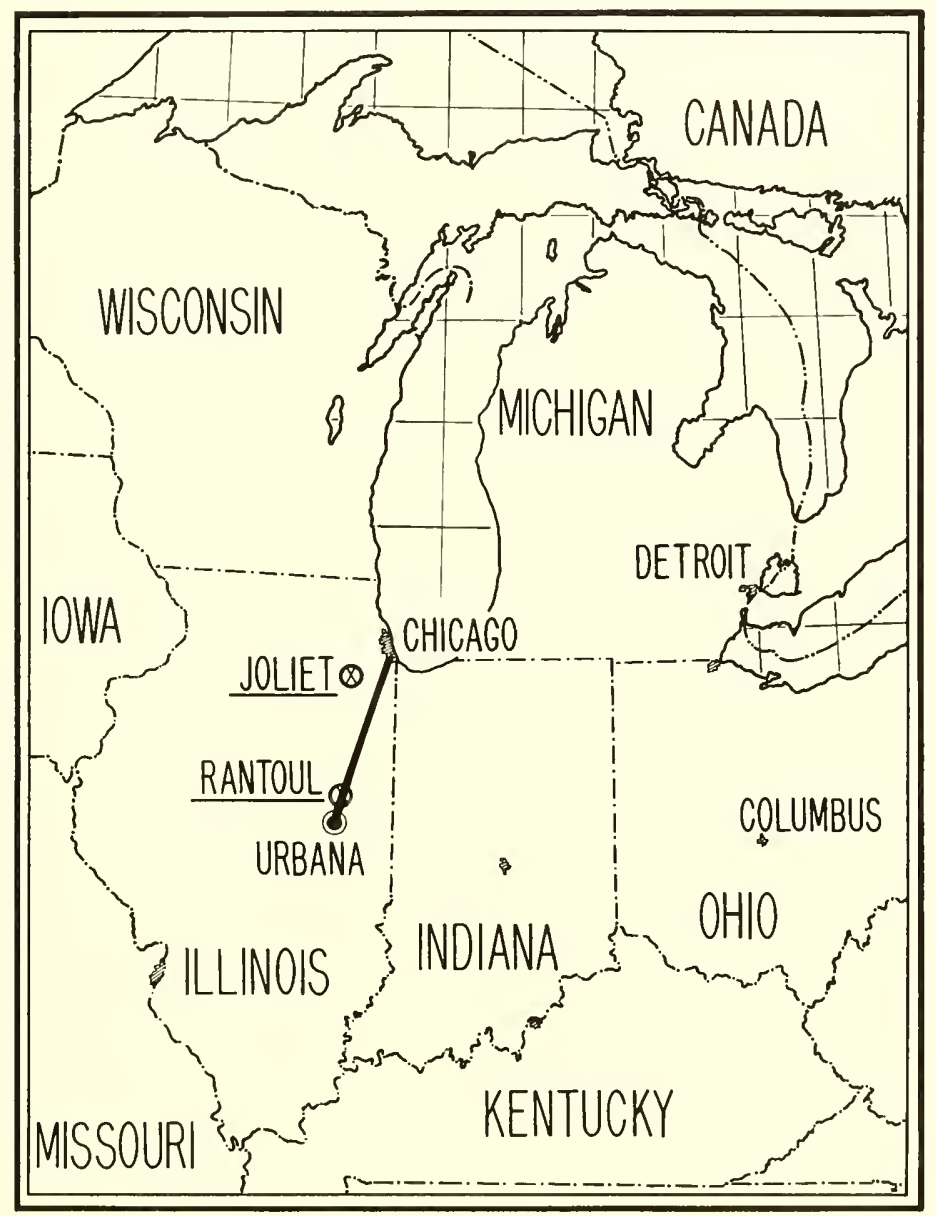

FIGURE 6.12. Location of radio path and radiosonde stations used in this study. 
Generally, the unstratified samples have the lowest overall field strengths throughout the entire distribution range. The presence of any layer (elevated or ground-based) tends to increase the field strength by $10-25 \mathrm{~dB}$ at any percentage level of the distribution. (The exception to this observation, WNBQ-TV, is probably explained by the fact that the observations were limited to six winter months, rather than the 2-year period of the other stations.) Tilted elevated layers appear to produce the greatest enchancement of signal strength, probably as a result of focusing effects due to the layer tilt.

\subsubsection{Prediction of Field Strength for Unstratified Conditions}

The field strengths recorded during the times when the radiosonde ascents at both Joliet and Rantoul indicated nonstratified conditions were compared with the values predicted by Norton, Rice, and others [31] for the case of diffracted plus scattered radio waves. This particular prediction process is adjustable for the average refractive conditions over the path in that it adjusts the effective earth's radius factor to the initial gradient of $N$ for the calculation of diffracted field strengths. One also needs the angular separation of the radio horizon rays at their intersection near midpath. The average initial gradient of $N$ was obtained for each instance of unstratified profile by simply averaging the initial gradients from Rantoul and Joliet, while the angular separation was obtained by determining the amount of radio ray refraction expected over each particular path in atmospheres of exponential decrease with height that closely match the observed $N$ conditions.

Figure 6.14 illustrated a comparison of the predicted and observed field strengths. For WNBQ and WENR there is approximate agreement between the two sets of data. However, the predicted values for WBKB and WMBI are approximately 10 to $12 \mathrm{~dB}$ higher than the observed values.

This tendency to predict field in excess of the measured values suggests that the empirical data, on which the predictions are based may include meteorological conditions with some degree of stratification in the first $2 \mathrm{~km}$ or so, even though most of the empirical data refer to afternoon hours in winter. It will be shown that elevated layers of moderate size (say a few kilometers in horizontal dimensions), that may exist undetected by the radiosonde, could produce field strengths on the Illinois paths comparable with the median values for "unstratified" conditions shown in figure 6.13. Furthermore, the limits placed on the profile gradients specifying unstratified conditions in table 6.5 are such that some layer type profiles may be included in the unstratified category. Consequently, it is important to study in more detail the properties of the elevated layer, 

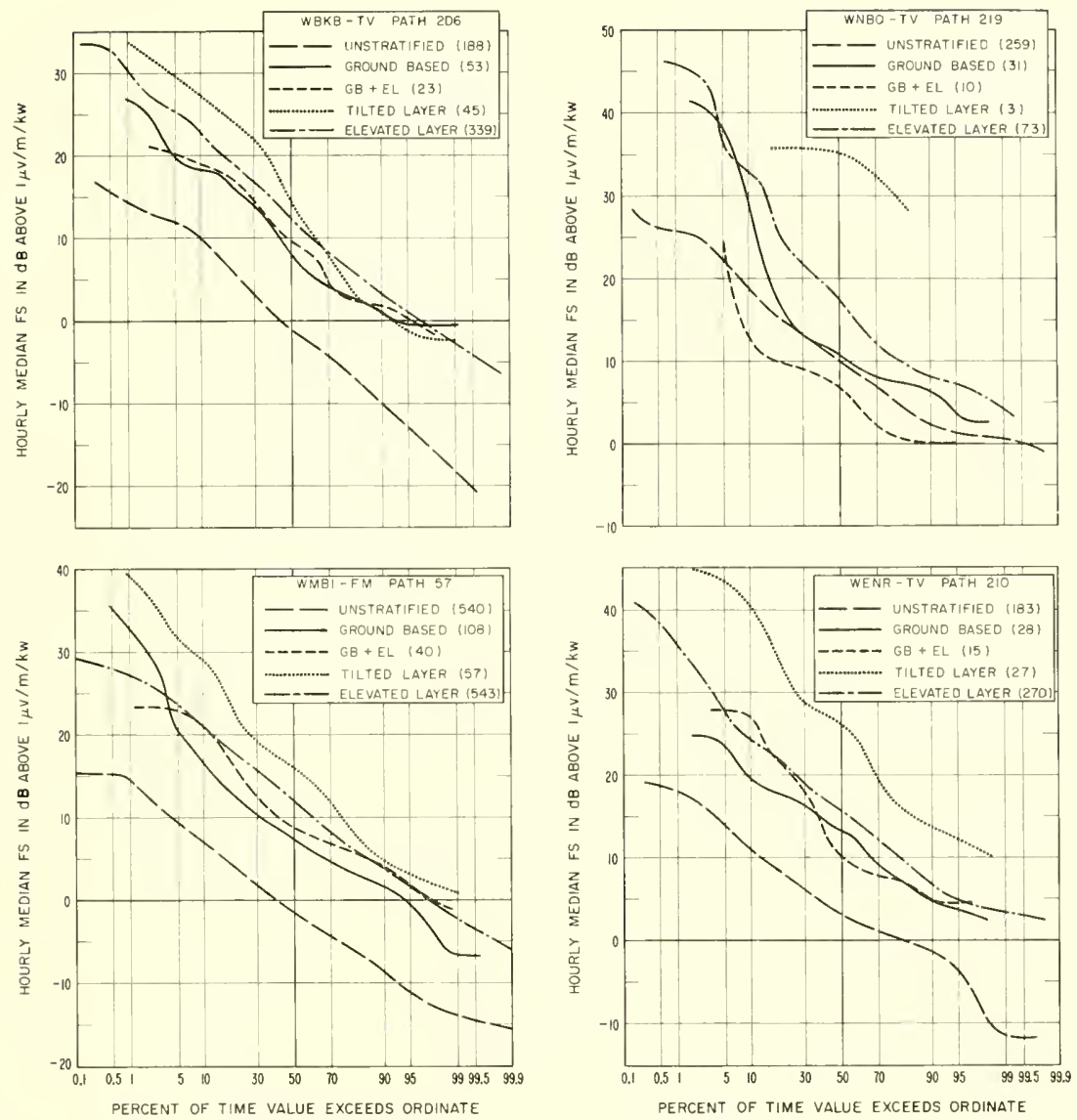

FIGURE 6.13. Distribution of hourly median field strengths with different radiosonde profile conditions.

not only as a feature oceasionally produeing high field strengths, but also as a meehanism which, in less intense form, partly determines the strength of the weaker fields observed for large percentages of the time.

\subsubsection{The Effect of Elevated Layers on the Illinois Path}

The influenee of elevated layers of VHF transmission beyond the horizon has been studied by several workers [21, 22, 28, 34, 35, 36, 37]. However, few investigations have contained any detailed eomparisons of theory and experimental results. The following analysis presents such a comparison, using simple models of the elevated layer for the four Illinois paths. 


\subsubsection{Elevated Layers at Temperature Inversions}

Recent radar and refractometer investigations of tropospheric structure have shown that elevated layers in the refractive index distribution are frequently observed in the stable air of temperature inversions [38].

A typical value of layer thickness is $100 \mathrm{~m}$, with horizontal dimensions of tens of kilometers. On occasions, extended layers no more than $10 \mathrm{~m}$ in thickness have been detected by refractometer soundings. In the present discussion we attempt to evaluate the reflection coefficient of these elevated layers. We may express the modulus of the reflection coefficient $|\rho|$, for a wave incident at a glancing angle $\alpha$ on a layer of thickness $h$, and horizontal extent, $x$, in the form:

$$
|\rho|=\frac{\Delta n}{2 \alpha^{2}} f(\alpha, h, x)
$$

where $f(\alpha, h, x)$ is the ratio of the reflection coefficient of the model to that of the infinitely sharp case (i.e., the Fresnel discontinuity value, $\left.\Delta n / 2 \alpha^{2}\right)$. This function has been evaluated for several layer profiles [28],
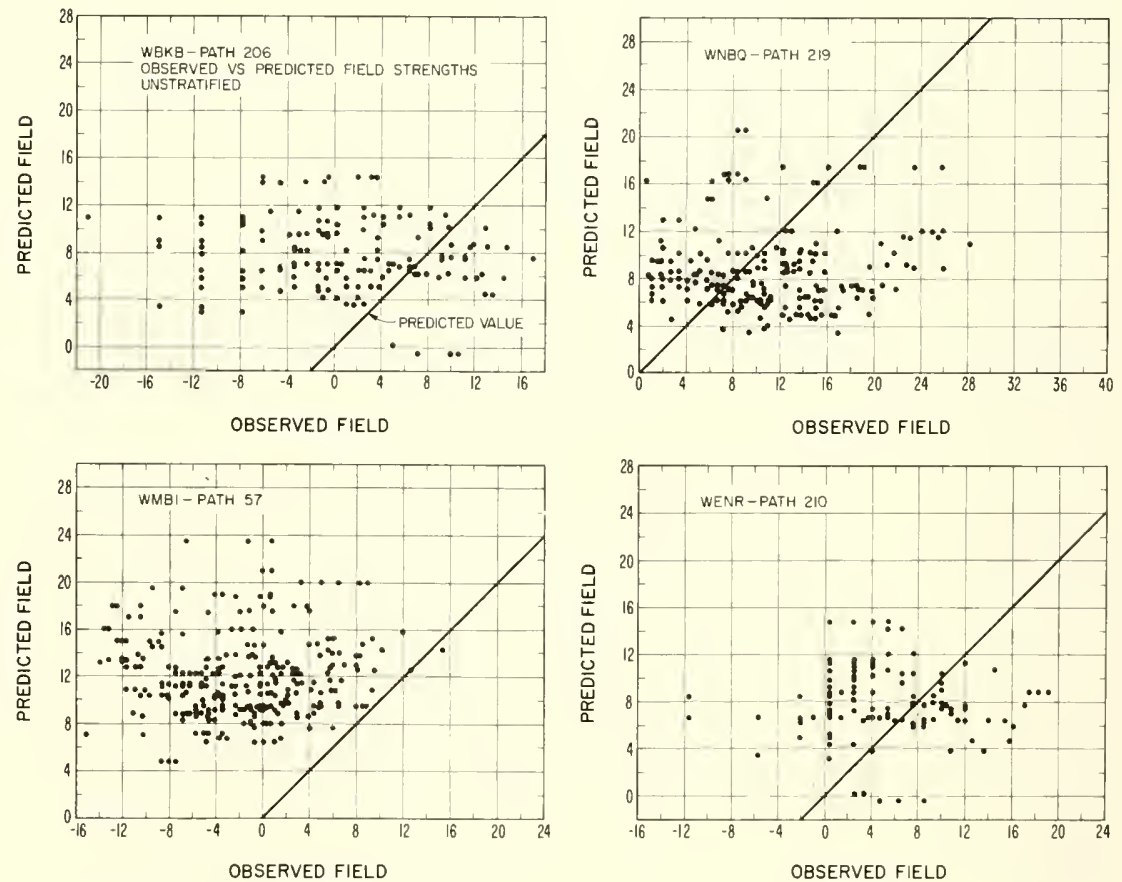

Figure 6.14. Comparison of observed radio field strengths and values predicted for wintertime afternoon hours. 
and preliminary calculations based on this work were made to determine the most suitable model in the present application. It was evident from these calculations that a simple linear profile would yield the best agreement with the measured data, and this model was therefore adopted in the subsequent analysis.

Consider the layer profile shown in figure 6.15, i.e., a linear decrease of $\Delta n$ over a height interval $h$, with transition regions of height $d$. This model and others have been discussed by several authors, but the most detailed treatment is that of Brekhovskikh [39]. His analysis shows that for this linear model:

$$
\begin{aligned}
|\rho| & =\Delta n \cdot \lambda / 8 \pi h \sin ^{3} \alpha \\
& \simeq \Delta n \cdot \lambda / 8 \pi h \alpha^{3} .
\end{aligned}
$$

This equation is valid if:

and

(a) $\Delta n \cdot \lambda \ll \pi h \alpha^{3}$

(b) $4 \alpha d \ll \lambda$.

In the present problem, with values of $\lambda$ of 1.7 to $4.2 \mathrm{~m}, \Delta n \sim 10^{-5}$, $\alpha \sim 0.02$ condition (a) is satisfied for layer thicknesses greater than about $20 \mathrm{~m}$. In addition, condition (b) is fulfilled for the stated conditions if the thickness of the transition region is less than a few meters. These conditions do not seem inconsistent with available refractometer data on elevated layers, but a rigorous justification of the model is impossible at the present time. In any ease, there is almost certainly no unique profile representative of all elevated layers. We assume here, therefore, the linear prafile of figure 6.15 merely as a simple analytical model. It may be noted here that the value of $|\rho|$ given by (6.5) agrees with that quoted by du Castel [40] but is half the value obtained in an earlier analysis [28].

Equation 6.5 was used to calculate reflection coefficient of the layers on each occasion on which these were observed in the sonde ascents. The results, expressed in terms of a reflection loss are compared with the measured values of field strength in figure 6.16. The general agreement is satisfactory for the assumed model. As might be expected, there is a considerable scatter in the data and two considerations are important in assessing the significance of these results. These concern sonde response and layer structure. The work of Wagner [41] on the response of radiosondes shows that, for an elevated inversion layer with $\Delta n=3 \times 10^{-5}$, $h=100 \mathrm{~m}$, a sonde with a 10 sec time constant in the sensing elements, and rising at $5 \mathrm{~m} / \mathrm{sec}$ will give an indicated value for $\Delta n$ of approximately half the true value. The above procedure, using sonde data, therefore underestimates the value of $|\rho|$ for an idealized infinite layer. 


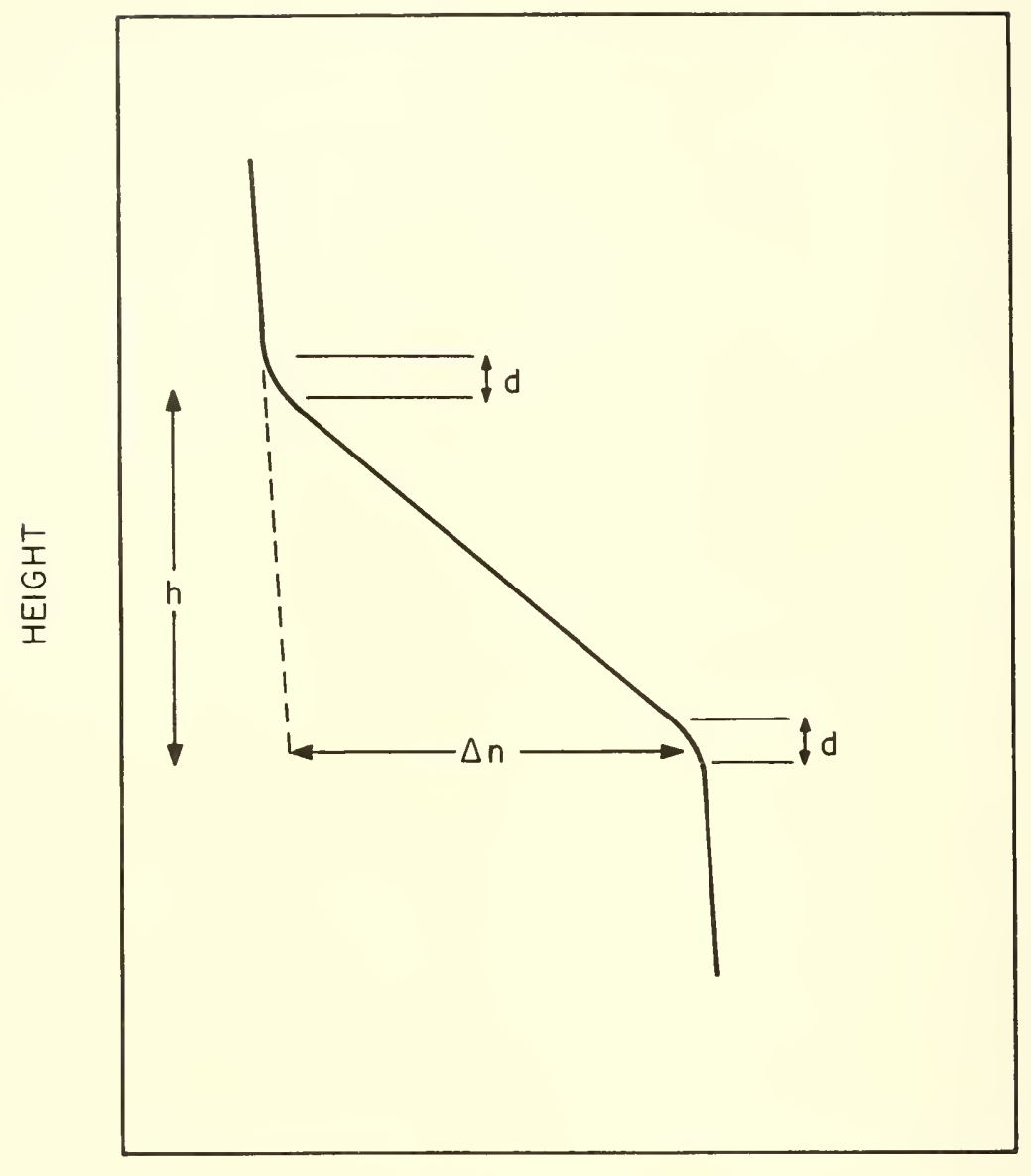

REFRACTIVE INDEX

FIgURE 6.15. Linear profile model of the $\mathrm{n}$ decrease across an elevated layer.

On the other hand, the analysis assumes a smooth layer extending horizontally at least over a distance $x$ equal to the first Fresnel zone. We have:

$$
x=\sqrt{2 a \lambda / \alpha}
$$

where $2 a=$ path length $=203 \mathrm{~km}$ for the Illinois paths. Hence $x$ is of the order of a few tens of kilometers. In addition, we have assumed that the layer is horizontal and smooth over a distance $x$, thus neglecting convergence. If we adopt the Rayleigh criterion, the height of the surface 
irregularities on the layer, $\Delta h$ say, must not exceed $\pm \lambda / 8 \alpha$ for the layer to be considered smooth; i.e.,

$$
\begin{aligned}
& \Delta h< \pm 7 \mathrm{~m} \quad(\lambda=1.67 \mathrm{~m}) \\
& \Delta h< \pm 17 \mathrm{~m}(\lambda=4.18 \mathrm{~m}) .
\end{aligned}
$$

These values apply for $\alpha=0.03 \mathrm{rad}$, corresponding to a layer height of $2.5 \mathrm{~km}$; for lower layers $\Delta h$ will be greater due to the decrease in $\alpha$.

These conditions are not likely to be satisfied in all the examples studied and the analysis therefore overestimates the value of $|\rho|$ in this respect. (Some discussion of this point has been given by Bauer and Meyer [42].) The limitations of sonde soundings, and the effects of layer tilt and surface irregularities therefore provide a partial explanation of the scatter of points in figure 6.16. Further detailed measurements of layer structure are obviously desirable.

\subsubsection{The Influence of Small Layers}

The above discussion has dealt with the particular case of extended elevated layers such as are often associated with temperature inversions. However, it seems quite possible that these layers are merely the more extreme examples of anisotropic irregularities which are thought to be prevalent in the troposphere. There is already some preliminary evidence supporting this concept in the results of refractometer and radar soundings and recent theoretical work $[22,28,40]$ has developed this approach in detail. The relationship of this work to earlier analyses in terms of a "scattering" model is disoussed in the references quoted and need not concern us here. For our purpose it is sufficient to utilize the essential features of the argument as the basis for a simple calculation.

It seems reasonable to assume that even in an atmosphere which sonde data would lead us to classify as "well-mixed" there are often layer-type irregularities. Detailed evidence on the spatial form and stability of this type of layer or "feuillet" is so far lacking, but an inspection of some refractometer results suggests that horizontal dimensions of a few kilometers represent a realistic assumption. Such a layer might exist as a separate entity for, say, several minutes (as compared with a period of several hours for the extended layer in a stable inversion).

For the following analysis, let us consider two layers of horizontal dimensions, $x$, of 2 and $10 \mathrm{~km}$ respectively with the following characteristics:

$$
\begin{aligned}
\Delta n & =10^{-5} \\
h & =100 \mathrm{~m} \\
\alpha & =0.01-0.03 \mathrm{rad} \text { (i.e., layer height of } 0.4 \text { to } 2.5 \mathrm{~km} \text { on the Illinois } \\
& \text { paths). }
\end{aligned}
$$



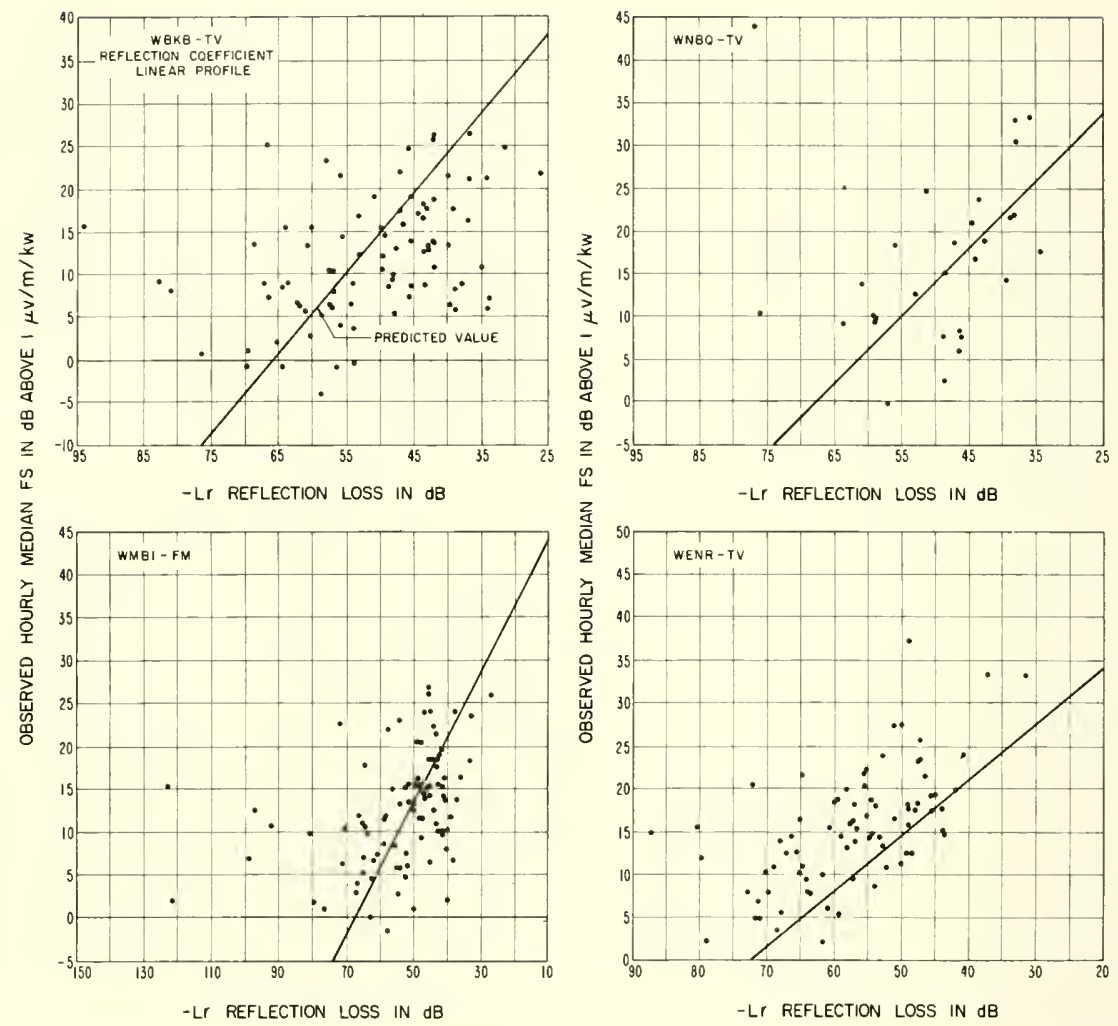

FIGURE 6.16. Observed hourly median field strength versus reflection loss assuming the linear model of figure 6.15.

For these conditions, the layers correspond to those of "intermediate" size in the analysis of Friis, Crawford, and Hogg [22]. They are defined by the inequality:

$$
\sqrt{2 a \lambda} / \alpha>x>\sqrt{2 a \lambda}
$$

where $2 a$ is the path length. In this case, the power received, $P_{R}$, from an antenna of effective aperture, $A_{R}$, with a transmitter radiating a power $P_{T}$ from a antenna of effective aperture $A_{T}$ is given by:

$$
P_{R} / P_{T}=\left(A_{T} A_{R} \lambda^{2} \alpha^{2} \rho^{2}\right) /\left(2 \lambda^{3} a^{3}\right)
$$


We can use this equation to calculate the corresponding field strength, for the Illinois paths, in terms of $\mu \mathrm{V} / \mathrm{m}$ for $1 \mathrm{~kW}$ radiated from a halfwave dipole. We have the following relations:

$$
\begin{aligned}
& A(\lambda / 2 \text { dipole })=0.127 \lambda^{2} \\
& p_{r}(\lambda / 2 \text { dipole })=E^{2} \lambda^{2} / 300 \pi^{2}
\end{aligned}
$$

where $E$ is the field strength in volts/meter if $P_{r}$ is in watts. From (6.8), (6.9), and (6.10), we can calculate $E$ for the two layers specified above. The results obtained are shown in figure 6.17 for various layer heights and the following models of reflection coefficient:

$$
\begin{aligned}
& \text { (a) }|\rho|=\Delta n \cdot \lambda / 8 \pi \alpha^{3} h \\
& \text { (b) }|\rho|=\Delta n / 2 \alpha^{2} .
\end{aligned}
$$

Model (b) is the Fresnel diseontinuity equation which gives the limiting value of $|\rho|$ towards which all models tend as the layer thickness decreases. The curves in figure 6.17 show that the calculated field strength depends considerably on the assumed $n$-profile. If $|\rho|=\Delta n \cdot \lambda / 8 \pi \alpha^{3} h$, values of field strength comparable with the long-term median value may be produced by layers of about $10 \mathrm{~km}$ in lateral dimensions in the height range 0.5 to $1 \mathrm{~km}$. If $|\rho|=\Delta n / 2 \alpha^{2}$, similar field strength may be produced by layers in this height range if the lateral dimension is of the order of $2 \mathrm{~km}$. The effect of the layer deereases with increasing height, but even with layer heights of $3 \mathrm{~km}$, the field strength is still $1 \mu \mathrm{V} / \mathrm{m}$ or greater at both wavelengths for a $10 \mathrm{~km}$ layer with $|\rho|=\Delta n / 2 \alpha^{2}$. However, it should be pointed out that the assumed value of $\Delta n=10^{-5}$ is probably somewhat large for layers as high as $3 \mathrm{~km}$. The results also show that model (b) (i.e., $\rho=\Delta n / 2 \alpha^{2}$ ) gives field strength values which are higher at $\lambda=1.67 \mathrm{~m}(f=179.75 \mathrm{Mc} / \mathrm{s})$ than at $\lambda=4.18 \mathrm{~m}(f=71.75$ $\mathrm{Me} / \mathrm{s})$.

\subsubsection{Conclusions}

Any departure of refractive index structure from a smooth monotonic decrease with height produces an increase in field strength on a $200-\mathrm{km}$ path in the frequency band 70 to $180 \mathrm{Mc} / \mathrm{s}$. In the particular case studied, elevated tilted layers result in signal enhancement of 10 to $25 \mathrm{~dB}$ over the values for unstratified conditions, at all percentage levels. (The importance of the tilted layer is possibly a consequence of the asymmetry of the path, the transmitting antenna being $200 \mathrm{~m}$ above ground and the receiving antenna $30 \mathrm{~m}$. )

The predicted field strengths, for conditions classified as unstratified in terms of sonde data, are in approximate agreement with observed results, although the scatter of the points (plus the tendency to predict 
values in excess of the measured ones) point to the influence of anisotropic layers or eddies of varying size and degrees of stability. This interpretation is consistent with numerical calculations based on the properties of "intermediate" size layers, suggested in the analysis of Friis, Crawford, and Hogg [22].

Calculations of the field strength produced by extended stable layers, using sonde data and a model profile with a linear lapse of $n$ with height, are in reasonable agreement with the experimental results. However, there is probably no unique profile characteristic of elevated layers.

\subsection{A New Turbulence Parameter}

\subsubsection{Introduction}

We shall here attempt to unify past work utilizing the surface value of the refractive index and the concept of atmospheric stability. To do so we shall first review the concepts of atmospheric stability from the viewpoint of temperature structure. In the process we shall show that past efforts have neglected the important role of the conditions at the earth's surface; then we shall extend this work to derive an analogous expression for the radio refractive index.

\subsubsection{The Concept of Thermal Stability}

The stratification of the earth's atmosphere by and large reflects the control of the earth's gravitational field. This average structure is, however, constantly disturbed by the intrusion of thermal plumes of heated air rising from the differentially heated surface of the earth, as well as by the mechanical mixing produced by the passage of air currents. It is customary to assume that parcels of air are forced upwards through the normal stratified atmosphere without mixing with the environmental air mass. Since the parcel is forced to rise, be it by convection or by mechanical mixing, it is also assumed that it expands and cools without exchange of heat, i.e., adiabatically. In such a process it can be shown that the pressure, $P$, is given by

$$
P=P_{0}\left(\frac{T}{T_{0}}\right)^{g / R \alpha}
$$

where $T$ is the temperature $\left({ }^{\circ} \mathrm{K}\right) ; g$, the acceleration of gravity; $R$, the gas constant for air; and, where $\alpha$, the constant lapse of temperature with height is 

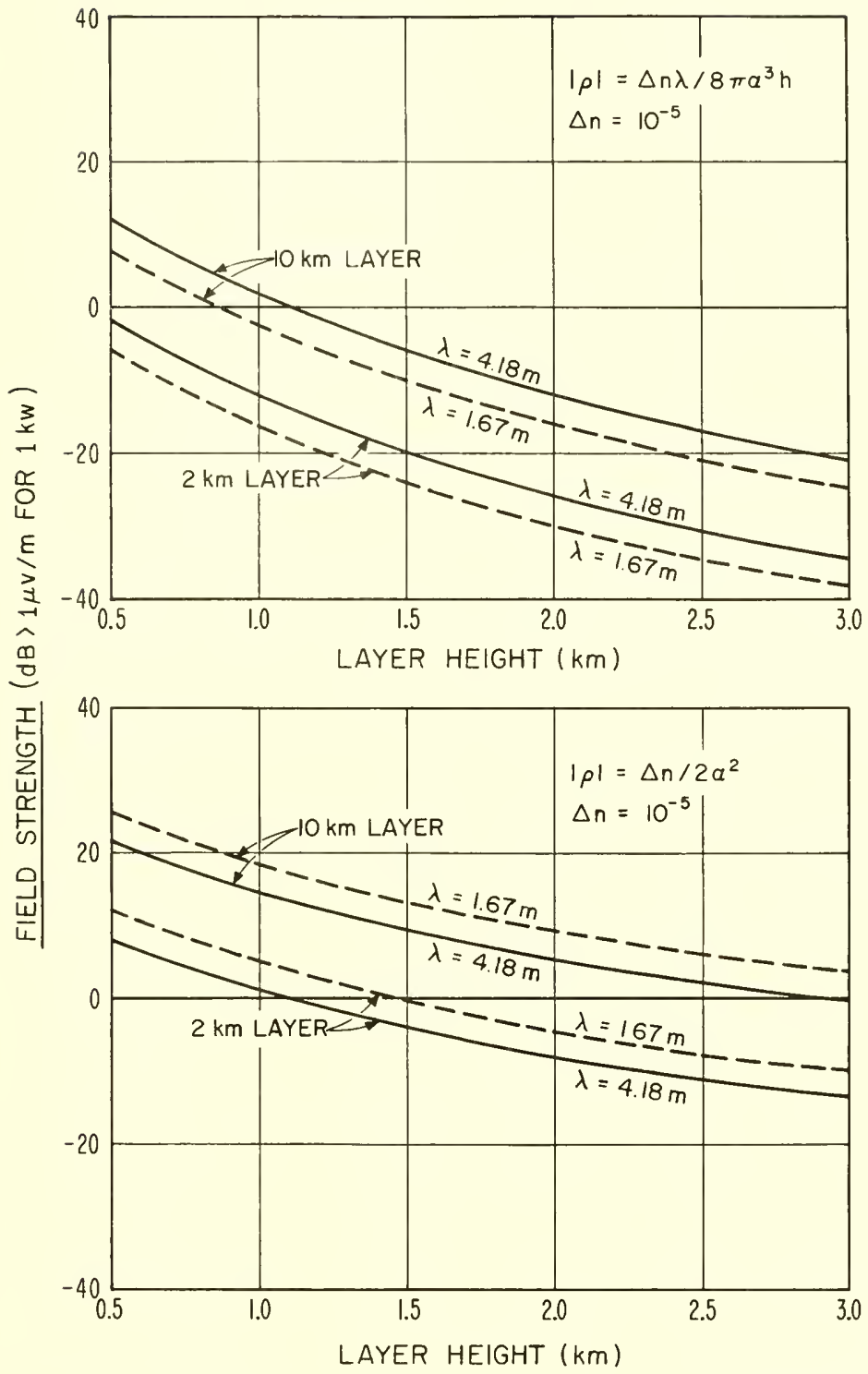

FigURE 6.17. Field strength produced by layers of horizontal dimensions $2 \mathrm{~km}$ and 10 $\mathrm{km}$ on the Chicago-Urbana paths for two model profiles. 
The zero subscripts in (6.11) refer to conditions at the earth's surface. Equations (6.11) and (6.12) are taken to hold as long as saturation does not occur. It is further assumed that the mixing ratio

$$
e \cong e_{0} \frac{P}{P_{0}}
$$

is constant throughout the process. In (6.13) $e$ is the partial pressure of water vapor.

A parcel of air which is caused to rise will cool to the dew point if forced aloft far enough. Further rising will lead to condensation. Conversely, a parcel of air which sinks down will have work done upon it adiabatically and will become warmer than it was in its original elevated position. A parcel of air following a condensation curve has a value of $\alpha$, of $-6^{\circ} \mathrm{C} / \mathrm{km}$ denoted at $\alpha^{*}$. In addition, as water vapor condenses out of the parcel, the vapor pressure will decrease. We here assume that the vapor pressure will follow that of the saturation vapor pressure curve, $e_{s z}$, as given by:

$$
e_{s z}=e_{s \ell} \exp \left[-\beta \alpha^{*}(z-\ell)\right], z \geq \ell
$$

where $\ell$ is the height of the lifting condensation level and $\beta=0.064\left({ }^{\circ} \mathrm{C}\right)^{-1}$. The value of $\ell$ has been found to be given [44] with sufficient accuracy for practical applications by

$$
\ell \cong 0.125\left(T-T_{d}\right)_{0}(\mathrm{~km})
$$

where the zero subscript indicates that the difference between the temperature and the dewpoint, $T_{d}$, need be evaluated only at the earth's surface.

Consider now a parcel rising through some environmental distribution of temperature. The dry adiabatic lapse of temperature with height is greater than that observed on the average in the atmosphere $\left(6{ }^{\circ} \mathrm{C} / \mathrm{km}\right)$ and thus the parcel becomes cooler, and more dense, than the environmental air and will sink to its initial conditions with the removal of the lifting force. If, on the contrary, anywhere in its trajectory it becomes warmer, and thus less dense, it will become unstable and rise of its own buoyancy through the environmental air. Past radio-meteorological studies have taken the area between the adiabatic curves and the environmental temperature distribution on a pressure $(\sim$ height) versus temperature chart as a measure of atmospheric stability. On the general argument that the effects of atmospheric stability, or turbulence, influence radio waves only through the radio refractive index, we shall extend the above concepts to the radio refractive index. 


\subsubsection{The Adiabatic Lapses of $N$}

Introducing the dry adiabatic variations of $P, T$, and $e$ into (1.20), chapter 1 , one obtains the dry adiabatic decrease of $N, N_{d}$ :

$$
N_{d}=N_{0}\left(1-\frac{\alpha z}{T_{0}}\right)^{(\theta / R \alpha)-2}\left\{1-\frac{77.6 P_{0} \alpha z}{T_{0}{ }^{2} N_{0}}\right\}
$$

which is valid for $z \leq \ell$. The distribution of $N_{d}$ with $z$ may be evaluated by taking the logarithms of both sides of $(6.16)$

$$
\begin{aligned}
\ln N_{d}=\ln N_{0}+\left(\frac{g}{R \alpha}-2\right) \ln (1 & \left.-\frac{\alpha z}{T_{0}}\right) \\
& +\ln \left(1-\frac{77.6 P_{0} \alpha z}{T_{0}{ }^{2} N_{0}}\right)
\end{aligned}
$$

and expanding the logarithms on the righthand side of (6.17) with the omission of second-order terms where appropriate for our applications of $z \leq 3 \mathrm{~km}$ :

$$
\ln N_{d} \simeq \ln N_{0}-\Gamma_{d} z
$$

or

where

$$
N_{d} \simeq N_{0} \exp \left(-\Gamma_{d} z\right)
$$

$$
\Gamma_{d}=\frac{g}{R T_{0}}-\frac{2 \alpha}{T_{0}}+\frac{77.6 P_{0} \alpha}{N_{0} T_{0}^{2}}
$$

When evaluated for the standard conditions of $T_{0}=288^{\circ} \mathrm{K}\left(15^{\circ} \mathrm{C}\right)$ and $P_{0}=1013.2 \mathrm{mbar}, \Gamma_{d}$ becomes

$$
\Gamma_{d}=0.08(\mathrm{~km})^{-1}
$$

It is sometimes more convenient to use the dry adiabatic scale height, $H_{d}$ :

$$
H_{d}=\frac{1}{\Gamma_{d}}=12.5 \mathrm{~km}
$$

Above the local condensation level, the expression for $N$ becomes that of the wet adiabatic, $N_{w}$ :

$$
\begin{aligned}
& N_{w}=\left(1+\frac{\alpha^{*} z}{T_{\iota}}\right)^{-2}\left\{D_{\iota}\left(1+\frac{\alpha z}{T_{\iota}}\right)^{\left(g / R \alpha^{*}\right)+1}\right. \\
&\left.+W_{\iota} \exp \left(\beta \alpha^{*} z\right)\right\}
\end{aligned}
$$


where, for convenience,

$$
D_{\iota}=77.6 \frac{P_{\ell}}{T_{\ell}} \text { and } W_{\iota}=\frac{77.6(4810) e_{s \ell}}{T_{\imath}{ }^{2}}
$$

Applying the same approximations as in the derivation of $(6.18)$, one obtains

$$
\ln N_{w}=\ln N_{\ell}-\Gamma_{w} z
$$

where

$$
\Gamma_{w}=\frac{2 \alpha^{*}}{T_{\imath}}+\frac{\left(\frac{g}{R \alpha^{*}}+1\right) \alpha^{*} D_{\iota}}{T_{\imath} N_{\iota}}+\frac{W_{\ell}}{N_{\imath}} \beta \alpha^{*} .
$$

For the standard conditions assumed above and $e_{0}$ corresponding to 60 percent relative humidity, $H_{w} \sim 7.5 \mathrm{~km}$. Again, the form of the wet adiabatic lapse of $N$ is given by

$$
N_{w}=N_{\ell} \exp \left(-\Gamma_{w} z\right), z \geq \ell
$$

We shall now apply these results to the derivation of a refractive index turbulence parameter.

\subsubsection{The Turbulence Parameter, $\Pi$}

Analogously to the concept of thermal stability, we define $I$ as the area between the environmental $N(z)$ curve and the appropriate adiabatic decrease of $N$. That is

$$
\Pi=\int_{0}^{3 \mathrm{~km}}\left(N_{\text {observed }}-N_{\text {adiabatic }}\right) d z
$$

where the integration is arbitrarily taken from the surface to $3 \mathrm{~km}$. This then extends the integration well above the crossover height of the radio horizon rays from transmitter and receiver normally encountered in tropospheric propagation. The integral for II may be written

$$
\begin{aligned}
\Pi=\int_{0}^{3} N d z-\int_{0}^{\ell} N_{0} \exp \left(-\Gamma_{d} z\right) d z \\
-\int_{\ell}^{3} N_{\iota} \exp \left[-\Gamma_{w}(z-\ell)\right] d z .
\end{aligned}
$$


Noting that

$$
N_{\imath}=N_{0} \exp \left(-\Gamma_{d} \ell\right),
$$

(6.28) then becomes, upon integration,

$$
\begin{aligned}
\Pi=\int_{0}^{3} N d z+ & \frac{N_{0}}{\Gamma_{d}}\left[\exp \left(-\Gamma_{d} \ell\right)-1\right] \\
& +\frac{N_{0} \exp \left(-\Gamma_{d} \ell\right)}{\Gamma_{w}}\left\{\exp \left[-\Gamma_{w}(3-\ell)\right]-1\right\} .
\end{aligned}
$$

$\Pi$ is thus dependent upon both $N$ profile eharacteristics and initial conditions. In fact, all the terms on the righthand side of (6.29) save

$$
\int_{0}^{3} N d z
$$

are determined from the conditions at the earth's surface. One might expect, then, that II would constitute a useful radio-meteorological parameter since it includes both the time-proven parameter $N_{0}$, the concept of atmospheric stability, and the integrated $N$-profile characteristics. We shall now apply this new parameter to the radio data presented in detail in section 6.2. The values of $H_{d}$ and $H_{w}$ utilized are those for standard sea-level conditions; i.e., $12.5 \mathrm{~km}$ and $7.5 \mathrm{~km}$, respectively.

\subsubsection{Comparison of Radio-Meteorological Parameters}

The various radio-meteorological parameters discussed in section 6.1 plus II as derived from the Rantoul and Joliet radiosondes were tested for their relative utility by comparison with the radio data of WBKB-TV and WENR-TV (see table 6.4). Correlation coefficients between the radio data (3-hoully medians) of each path and the various radiometeorological parameters were determined for each of the profile categories given in table 6.5 as well as for all available data. It was not possible to fit all of the data into the various categories since these were deliberately made very strict in order to explore the utility of each parameter within each of the propagation mechanisms assumed for the profile categories (table 6.3). Thus it is observed that the sum of the individual categories does not equal all available data. The results of these correlations are given in table 6.6. Before discussing these results it is well to note that the crossover height of the tangent rays from each end of the path were calculated by detailed ray tracing [15] for each radiosonde ascent. The values as determined from the Rantoul and Joliet soundings were averaged. This average crossover height was then used to determine the contribution to the values of II below the crossover 
height $\left(\Pi^{\prime \prime}\right)$, above the crossover height $\left(\Pi^{\prime}\right)$, and $g_{e}$. As listed in table 6.6 , II represents the value of (6.27) for the height increment zero to $3 \mathrm{~km}$.

Before proceeding, the method of ealculating $g_{e}$ will be deseribed. The eoneept of the equivalent gradient is elosely related to that of the effective earth's radius. The curvature of the effeetive earth is given by:

$$
\frac{1}{r_{e}}=\frac{1}{a}+\frac{1}{n} \frac{d n}{d h} \cos \theta
$$

where $r_{e}$ is the effective earth's radius; $a$ is the radius of the earth; and $(1 / n)(d n / d h) \cos \theta$ is the curvature of the radio ray.

The value of $r_{e}$ is determined from the geometrical relationship

$$
h_{c} \cong \frac{d_{c}^{2}}{2 r_{e}}
$$

where $d_{c}$, the ground distance to the crossover height, $h_{c}$, has already been determined by the ray-tracing procedures discussed in chapter 3 . Thus one obtains

$$
\left(\frac{d n}{d h}\right)=\frac{2 h_{c}}{d_{c}{ }^{2}}-\frac{1}{a}
$$

for $\theta=0$ and setting $(\cos \theta) / n \simeq 1$. In order to obtain the equivalent gradient as a positive quantity in the more convenient refraetivity units, the following expression is used:

$$
g_{e}=\left(\frac{1}{a}-\frac{2 h_{c}}{d_{c}^{2}}\right) 10^{6}
$$

It is evident from table 6.6 that the results obtained with $\Pi$ are comparable with those using $\Pi^{\prime}$, due supposedly to the erossover height being on the order of $100 \mathrm{~m}$ for these paths. The value $\Pi^{\prime \prime}$ appears to contribute little to the final value of $\Pi$ and is poorly correlated with the radio data. An interesting exception to this observation is the subrefractive case where II $^{\prime \prime}$ yields a larger correlation coeffieient than either $I$ or $I^{\prime}$. The overall conclusion that one reaches from the data of table 6.6 is that the most promising of the parameters considered is $\Pi$, closely followed by $\Delta N$ and $N_{s}$. In fact, the differenee between the results obtained with $\Pi$ and $\Delta N$ gives one pause to consider if the added complexity of determining $I I$ is worth the relatively small gain in correlation with the radio data. The results obtained with $g_{e}$ and $g_{0}$ (the $N$ gradient of the initial layer as reported by the radiosonde) are disappointingly small and are of no practical signifieance when compared with those of $\Pi, \Delta N$, and even $N_{s}$. 
TABLE 6.6. Summary of correlations

\begin{tabular}{|c|c|c|c|c|c|c|c|c|c|}
\hline & \multirow{2}{*}{$\begin{array}{l}\text { Cate- } \\
\text { gory* }\end{array}$} & \multirow{2}{*}{$\begin{array}{l}\text { Sample } \\
\text { size }\end{array}$} & \multicolumn{7}{|c|}{ Radio-meteorologieal variable } \\
\hline & & & II & $\Pi^{\prime}$ & $\Pi^{\prime \prime}$ & $g_{e}$ & $g_{\circ}$ & $N_{s}$ & $\Delta N$ \\
\hline $\begin{array}{l}\text { W ENR_T V } \\
(179.75 \mathrm{M} \in / \mathrm{s})\end{array}$ & $\begin{array}{l}1 \\
2 \\
3 \\
4 \\
5 \\
6\end{array}$ & $\begin{array}{r}68 \\
21 \\
101 \\
6 \\
196 \\
518\end{array}$ & $\begin{array}{r}-0.3794 \\
-. .2064 \\
-.1748 \\
-.3632 \\
-.5665 \\
-.4624\end{array}$ & $\begin{array}{r}-0.3795 \\
-.2683 \\
-.1754 \\
-.3503 \\
-.5732 \\
-.4602\end{array}$ & $\begin{array}{r}-0.1859 \\
.2003 \\
.0087 \\
-.4315 \\
-.0312 \\
-.0169\end{array}$ & $\begin{array}{r}0.1889 \\
.0035 \\
.0291 \\
.4990 \\
.0555 \\
.0722\end{array}$ & $\begin{array}{r}-0.1884 \\
-.1346 \\
-.0303 \\
-.4149 \\
-.1772 \\
-.1762\end{array}$ & $\begin{array}{r}0.1414 \\
.0531 \\
-.0940 \\
.0905 \\
.3870 \\
.2889\end{array}$ & $\begin{array}{r}-0.3465 \\
-.2653 \\
-.4553 \\
.0873 \\
-.5640 \\
-.4544\end{array}$ \\
\hline $\begin{array}{l}\text { W B K B-TV } \\
(71.75 \mathrm{Me} / \mathrm{s})\end{array}$ & $\begin{array}{l}1 \\
2 \\
3 \\
4 \\
5 \\
6\end{array}$ & $\begin{array}{r}67 \\
27 \\
113 \\
6 \\
213 \\
564\end{array}$ & $\begin{array}{l}-.2952 \\
-.2071 \\
-.2691 \\
-.2445 \\
-.5519 \\
-.4384\end{array}$ & $\begin{array}{l}-.2976 \\
-.1843 \\
-.2709 \\
-.2202 \\
-.5562 \\
-.4398\end{array}$ & $\begin{array}{r}-.2231 \\
-.1378 \\
.0763 \\
-.6849 \\
-.0576 \\
.0087\end{array}$ & $\begin{array}{r}-.0926 \\
-.1037 \\
.1652 \\
.3084 \\
.0624 \\
.0430\end{array}$ & $\begin{array}{r}.0791 \\
.0939 \\
-.0810 \\
-.3686 \\
-.1152 \\
-.1027\end{array}$ & $\begin{array}{l}.0107 \\
.1678 \\
.0703 \\
.2573 \\
.4168 \\
.2128\end{array}$ & $\begin{array}{r}-.1687 \\
-.1608 \\
-.3486 \\
.3485 \\
-.4635 \\
-.3505\end{array}$ \\
\hline
\end{tabular}

* Category

$1=$ Unstratified.

$2=$ Ground-based duet.

$3=$ Elevated layer.

$4=$ Subrefractive layer.

$5=1$ through 4 combined.

$6=$ All data for period of record.

The above data also allow one to evaluate a parameter of the form

$$
H_{1}=a g_{e}+b \Pi^{\prime},
$$

which is analogous to Misme's recently suggested $M$ parameter [19]. The parameter $H_{1}$ given above is physically more realistic for radio purposes since it incorporates a measure of the refractive index turbulence rather than thermal stability. The correlations obtained between field strength and $H_{1}$ are

$$
\begin{aligned}
& \text { WENR-TV : } 0.46 \\
& \text { WBKB-TV : } 0.44
\end{aligned}
$$

which represents, for the significant figures carried, no improvement over the use of $\Pi$ alone. This last analysis suggests that it might be desirable to consider other combinations such as

$$
H_{2}=a_{2} \Delta N+b_{2} \Pi \text {. }
$$

The correlations for this case are:

$$
\begin{aligned}
& \text { WENR-TV : } 0.49 \\
& \text { WBKB-TV : } 0.44
\end{aligned}
$$

which represents a slight improvement over II alone. 
One might well wonder why the correlations of $g_{e}$ and field strengths yield a higher correlation relative to that with $\Delta N$ or $N_{s}$ on a monthly median basis than on a daily basis. This is apparently due to the nonlinear nature of the averaging process of $g_{e}$ which is dependent upon the nonlinear weighting of the $N(h)$ profile inherent in the refraction process. On the other hand, the average $\Delta N$ or $N_{s}$ obtained from the average $N(h)$ curve is the same as that obtained from averaging $\Delta N$ or $N_{s}$. Correlations on $I I$ and field strength on a monthly median basis are now under study.

\subsubsection{Cionclusions}

In conclusion, we reach the opinion that the results of previous experience with the dependence of radio refraction upon $N_{s}$ and the concept of stability are incorporated into $\Pi$. The resultant correlation with radio data on a 3 -hourly median basis is perhaps the most encouraging obtained to date, but still, appears to be a marginal improvement over the time-honored, and much simpler, parameter $\Delta N$. This conclusion could well change with broader experience on other paths and radio frequencies.

\subsection{References}

[1] Bean, B. R., and F. M. Meaney (Oct. 1955), Some applications of the monthly median refractivity gradient in tropospheric propagation, Proc. IRE 43, No. $10,1419-1431$.

[2] Bean, B. R., and B. A. Cahoon (Jan.-Feb. 1961), Correlation of monthly median transmission loss and refractive index profile characteristics, J. Res. NBS 65D (Radio Prop.), No. 1, 67-74.

[3] Misme, P. (Mar.-Apr. 1960), Le gradient equivalent mesure direct et calcul théorique, Ann. Telecommun. 15, No. 3-4, 93.

[4] Misme, P. (Nov.-Dec. 1960), Quelques aspects de la radio-climatologie, Ann. Telecommun. 15, No. 11-12, 266.

[5] Misme, P. (Jan.-Feb. 1961), Essais de radio climatologie dans le bassin du Congo, Ann. Telecommun. 16, No. 1-2, 29.

[6] Flavell, R. G., and J. A. Lane (Jan. 1963), The application of potential refractive index in tropospheric wave propagation, J. Atmos. Terrest. Phys. 24, 47-50.

[7] Moler, W. F., and W. A. Arvola (Aug. 1956), Vertical motion in the atmosphere and its effect on VHF radio signal strength, Trans. Am. Geophys. Union 37, $399-409$.

[8] Moler, W. F., and D. B. Holden (Jan.-Feb. 1960), Tropospheric scatter propagation and atmospheric circulations, J. Res. NBS 64D (Radio Prop.), No. 1, 81-93.

[9] Rice, P. L., A. Longley, and K. A. Norton (1959), Prediction of the cumulative distribution with time of ground wave and tropospheric wave transmission loss, Part I, NBS Tech. Note 15.

[10] Pickard, G. W., and H. T. Stetson (Jan. 1950), Comparison of tropospheric reception, J. Atmos. Terrest. Phys. 1, 32-36.

[11] Bonavoglia, L. (Dec. 1958), Correlazione fra fenomeni meteorologici e propagazione altre l'orizzonte sul Mediterraneo, Alta Frequenza 27, 815. 
[12] Gray, R. E. (Sept. 1961), Tropospheric scatter propagation and meteorological conditions in the Caribbean, IRE Trans. Ant. Prop. AP-9, 492-496.

[13] Onoe, M., M. Hirai, and S. Niwa (Apr. 1958), Results of experiment of longdistance overland propagation of ultra-short waves, J. Radio Res. Labs. 5, 79.

[14] Bean, B. R., L. Fehlhaber, and J. Grosskopf (Jan. 1962), Die Radiometeorologie und ihre Bedeutung für die Ausbreitung der $\mathrm{m}$-, dm-, and $\mathrm{cm}$-Wellen auf grossen Enternungen, Nachrichtentechnische Zeit. 15, 9-16.

[15] Bean, B. R., and G. D. Thayer (May 1959), On models of the atmospheric refractive index, Proc. IRE 47, No. 5, 740-755.

[16] Misme, P. (Nov.-Dec. 1958), Essai de radioclimatologie d'altitude dans le nord de la France, Ann. Telecommun. 13, No. 11-12, 303-310.

[17] Bean, B. R., (Mar. 1962), The radio refractive index of air, Proc. IRE 50, No. 3, $260-273$.

[18] Lane, J. A. (Feb.-Mar. 1961), The radio refractive index gradient over the British Isles, J. Atmos. Terrest. Phys. 21, No. 2-3, 157-166.

[19] Misme, P. (May-June 1961), L'influence du gradient equivalent et de la stabilité atmosphérique dans les liaisons transhorizon au Sahara et au Congo, Ann. Telecommun. 16, No. 5-6, 110.

[20] Boithias, L., and P. Misme (May-June 1962), Le gradient equivalent; nouvelle determination et calculgraphique, Ann. Telecommun. 17, No. 5-6, 133.

[21] Saxton, J. A. (Sept. 1951), Propagation of metre radio waves beyond the normal horizon, Proc. IEE 98, 360-369.

[22] Friis, H. T., A. B. Crawford, and D. C. Hogg (May 1957), A reflection theory for propagation beyond the horizon, Bell Syst. Teeh. J. 36, 627.

[23] duCastel, F., P. Misme, A. Spizzichino, and J. Voge (1958-1960), Resultats experimentaux en propagation troposphérique transhorizon (a series of 10 papers), Ann. Telecommun. 13, 14, and 15.

[24] Craig, R. A., I. Katz, R. B. Montgomery, and P. J. Rubenstein (1951), Meteorology of the refraction problem, Book, Propagation of Short Radio Waves by D. E. Kerr, pp. 198-199 (McGraw-Hill Book Co., Inc., New York, N.Y.).

[25] Jehn, K. H. (June 1960), The use of potential refractive index in synoptic scale radio meteorology, J. Meteorol. 17, 264.

[26] Bean, B. R., L. P. Riggs, and J. D. Horn (Sept.-Oct. 1959), Synoptic study of the vertical distribution of the radio refractive index, J. Res. NBS 63D (Radio Prop.), No. 2, 249-258.

[27] Misme, P. (1962), Private communication.

[28] duCastel, F., P. Misme, and J. Voge (1960), Sur le role des phenomenes de reflexion dans la propagation louitaine des ondes ultracourtes, Electromagnetic Wave Propagation, Book, p. 671 (Academic Press, London and New York).

[29] Booker, H. G., and W. E. Gordon (Apr. 1950), A theory of radio scattering in the troposphere, Proc. IRE 38, No. 4, 401-412.

[30] Villars, F., and V. F. Weisskopf (Oct. 1955), On the scattering of radio waves by turbulent fluctuations in the atmosphere, Proc. IRE 43, No. 10, 1232-1239.

[31] Norton, K. A., P. L. Rice, H. B. Janes, and A. P. Barsis (Oct. 1955), The rate of fading in propagation through a turbulent atmosphere, Proc. IRE 43, No. 10, 1341-1353.

[32] Smyth, J. B., and L. G. Trolese (Nov. 1947), Propagation of radio waves in the lower atmosphere, Proc. IRE 35, No. 11, 1198-1202.

[33] Booker, H. G., and W. Walkinshaw (1947), The mode theory of tropospheric refraction and its relation to wave guides and diffraction, Book, Meteorological Factors in Radio Wave Propagation, pp. 80-127 (The Physical Society, London, England). 
[34] Gossard, E. E., and L. J. Anderson (Apr. 1956), The effect of super refractive layer on 50-5000 Mc nonoptical fields, IRE Trans. Ant. Prop. AP-4, No. 2, 175-178.

[35] Starkey, B. J., W. R. Turner, S. R. Badcoe, and G. F. Kitchen (Jan. 1958), The effects of atmospheric discontinuity layers up to the tropopause height on beyond-the-horizon propagation phenomena, Proc. IEE, Pt. B, 105, Suppl. 8,97 .

[36] Abild, V. B., H. Wensien, E. Arnold, and W. Schilkorski (1952), Über die Ausbreitung ultrakurzer Wellen jenseits des Horizontes unter besonderer Berucksichtigung der meteorologischen Einwiskungen, Tech. Hausmitteilungen des Nordwestdeutschen Rundfunks, p. 85.

[37] Northover, F. H. (Feb. 1952), The anomalous propagation of radio waves in the 1-10 metre band, J. Atmospheric Terrest. Phys. 2, 106-129.

[38] Lane, J. A., and R. W. Meadows (Jan. 1963), Simultaneous radar and refractometer soundings of the troposphere, Nature 197, 35.

[39] Brekhovskikh, L. M. (1960), Waves in layered media, Book (Academic Press, New York and London, England).

[40] duCastel, F. (1961), Propagation troposphèrique et faisceaux hertziens transhorizon, Book, p. 90 (Editions Chiron, Paris).

[41] Wagner, N. K. (1960), An analysis of radiosonde effects on measured frequency of occurrence of ducting layers, J. Geophys. Res. 65, 2077-2085.

[42] Bauer, J. R., and J. H. Meyer (Aug. 1958), Microvariations of water vapor in the lower troposphere with applications to long-range radio communications, Trans. Am. Geophys. Union 39, 624.

[43] Saxton, J. A. (July-Aug. 1961), Quelques reflexions sur la propagation des ondes radioelectrique à travers la troposphere, L'Onde Elect. 40, 505.

[44] Hewson, E. W., and R. W. Longley (1944), Meteorology, Theoretical and Applied, Book, p. 352 (John Wiley \& Sons, New York). 


\section{Chapter 7. Attenuation of Radio Waves}

\subsection{Introduction}

The advent of tropospheric forward scatter techniques has made possible communications over longer distances with higher frequencies than has been heretofore thought practicable. The limitations imposed by gaseous absorption, and by seattering by raindrops, upon the power requirements of a conmunications system for this application become more important with increasing distance and frequency. It has been common in the past to evaluate propagation path attenuation due to absorption by multiplying the ground separation of the terminals by the value of the absorption ealeulated for surface meteorological eonditions $[1]^{1}$ or avoid the problem by restricting the communications system to frequencies that are essentially free of absorption [2]. This is in contrast to another approach [3] which actually used the absorption along the ray path.

The following seetions of this chapter will be devoted to a descriptive treatment of absorption of radio waves by raindrops and gaseous oxygen and water vapor in the atmosphere.

Unless otherwise specified, the following conditions will be assumed in this chapter: (1) All attenuations will be expressed in terms of decibel loss per unit length of the propagation path $(\mathrm{dB} / \mathrm{km})$. The attenuations due to different causes are simply added to give the total attenuation in decibels. (2) In this treatment average conditions of temperature, droplet size, and droplet distribution are assumed for the radio path in order to approximate conditions met in practice.

\subsection{Background}

The attenuation experienced by radio waves is the result of two effects: (1) absorption and (2) scattering. At wavelengths greater than a few centimeters, absorption by atmospherie gases is generally thought to be negligibly small except where very long distances are concerned. However, cloud and rain attenuation have to be considered at wavelengths less than $10 \mathrm{~cm}$, and are particularly pronounced in the vicinity of 1 and $3 \mathrm{~cm}$.

${ }^{1}$ Figures in brackets indicate the literature references on p. 308. 
It is helpful to recall that when an incident electromagnetic wave passes over an object whose dielectric properties differ from those of the surrounding medium, some of the energy from the wave is (a) absorbed by the object and heats the absorbing material (this is called true absorption), and (b) some of the energy is scattered, the scattering being generally smaller and more isotropic in direction the smaller the scatterer is with respect to the wavelength of the incident energy.

In the case of point-to-point radio communications we are interested in the total attenuation of the scattering energy caused by losses resulting from both the true absorption and the scattering.

\subsection{Attenuation by Atmospheric Gases}

The major atmospheric gases that need to be considered as absorbers in the frequency range of 100 to $50,000 \mathrm{Mc} / \mathrm{s}$ are water vapor and oxygen. For these frequencies the gaseous absorption arises principally in the $1.35 \mathrm{~cm}$ line $(22,235 \mathrm{Mc} / \mathrm{s})$ of water vapor and the series of lines centered around $0.5 \mathrm{~cm}(60,000 \mathrm{Mc} / \mathrm{s})$ of oxygen [4]. The variations of these absorptions with pressure, frequency, temperature, and humidity are described by the Van Vleck $[4,5]$ theory of absorption. The frequency dependence of these absorptions is shown in figure 7.1 [4].

In connection with figure 7.1 , the water vapor absorption values have been adjusted to correspond to the mean absolute humidity, $\rho$, (grams of water vapor per cubic meter) for Washington, D.C., $7.75 \mathrm{~g} / \mathrm{m}^{3}$. The reason for this adjustment is that water vapor absorption is directly proportional to the absolute humidity [6] and thus variations in signal intensity due to water vapor absorption may be specified directly in terms of the variations in the absolute humidity of the atmosphere.

It can be seen from figure 7.1 that the water vapor absorption exceeds the oxygen absorption in the frequency range 13,000 to $32,000 \mathrm{Mc} / \mathrm{s}$, indicating that in this frequency range, the total absorption will be the most sensitive to changes in the water vapor content of the air, while outside this frequency range the absorption will be more sensitive to changes in oxygen density. Only around the resonant frequency corresponding to $\lambda=1.35 \mathrm{~cm}$ is the water vapor absorption greater than the oxygen absorption. The absorption equations and the conditions under which they are applicable have been discussed by Van Vleck [4], and the best values to use for this section of the report have been taken from Bean and Abbott [3].

The Van Vleck theory describes these absorptions from $100 \mathrm{Mc} / \mathrm{s}$ to $50,000 \mathrm{MIc} / \mathrm{s}$ in the following manner. The oxygen absorption at 


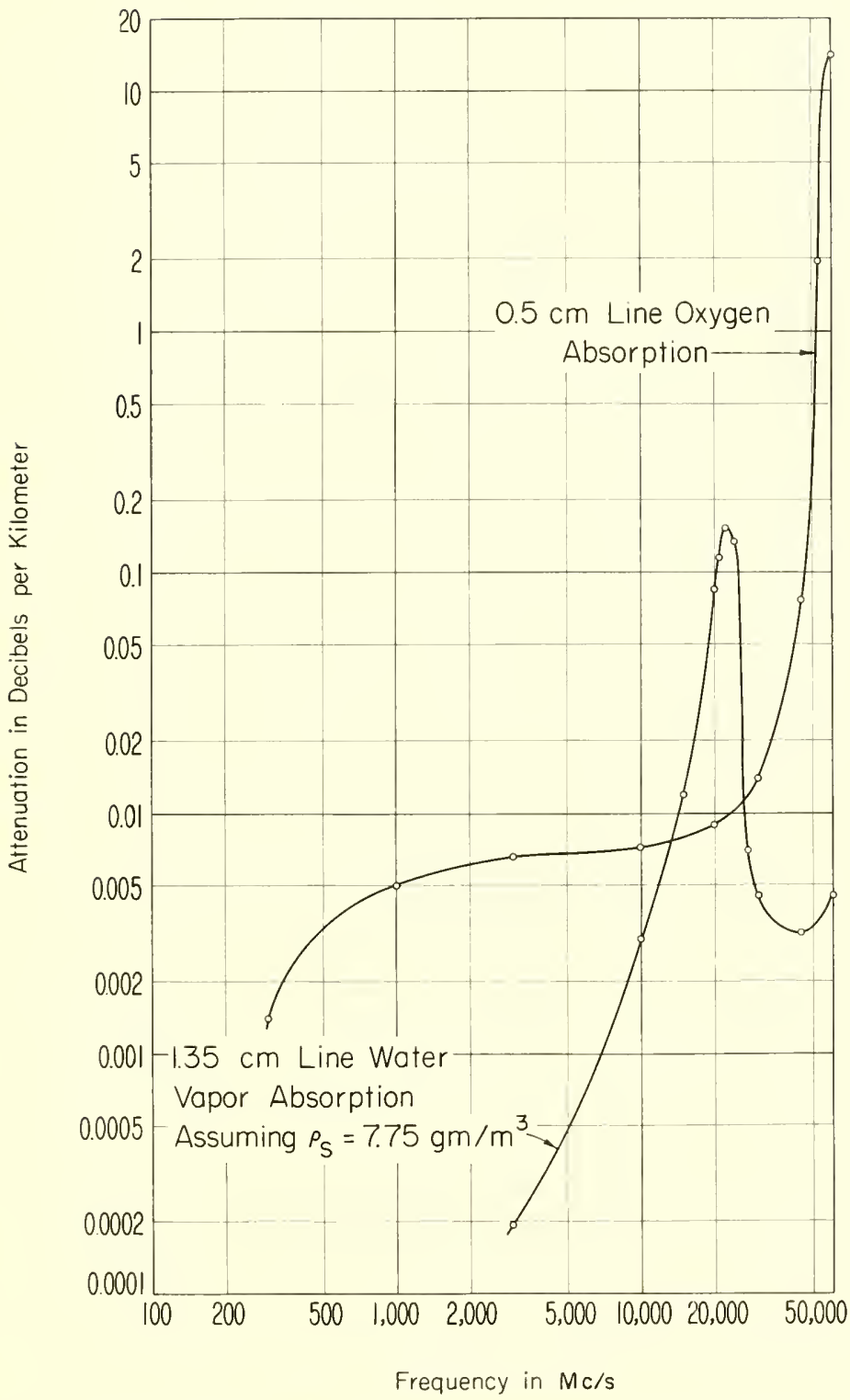

Figure 7.1. Atmospheric absorption by the $1.35 \mathrm{~cm}$ line of water vapor and the $0.5 \mathrm{~cm}$ line of oxygen. 
$T=293^{\circ} \mathrm{K}$ and standard atmospherie pressure in deeibels per kilometer, $\gamma_{1}$, is given by the expression:

$$
\begin{aligned}
\gamma_{1}=\frac{0.34}{\lambda^{2}}\left[\frac{\Delta \nu_{1}}{\frac{1}{\lambda^{2}}+\Delta \nu_{1}^{2}+\frac{\Delta \nu_{2}}{\left(2+\frac{1}{\lambda}\right)^{2}+\Delta \nu_{2}^{2}}}\right. \\
\left.+\frac{\Delta \nu_{2}}{\left(2-\frac{1}{\lambda}\right)^{2}+\Delta \nu_{2}^{2}}\right]
\end{aligned}
$$

where $\lambda$ is the wavelength for which the absorption is to be determined and where $\Delta \nu_{1}$ and $\Delta \nu_{2}$ are line-width factors with dimensions of $\mathrm{cm}^{-1}$. This formula is based on the approximations of collision broadening theory. This theory postulates that, although the electromagnetic energy is freely exchanged between the ineident field and the molecules, some of the electromagnetic energy is converted into thermal energy during molecular collisions and thus a part of the ineident electromagnetic energy is absorbed. The term in (7.1 )involving $\Delta \nu_{1}$ gives the nonresonant absorption arising from the zero frequency line of oxygen molecules while the terms involving $\Delta \nu_{2}$ describe the effeets of the several natural resonant absorptions of the oxygen molecule which are in the vieinity of $0.5 \mathrm{~cm}$ wavelength. The $(2 \pm 1 / \lambda)\left(\mathrm{cm}^{-1}\right)$ terms are the portion of the shape factors that describe the decay of the absorption at frequencies away from the resonant frequeney (the number 2 is the reciprocal of the centroid resonant wavelength $0.5 \mathrm{~cm}$ ).

The water vapor absorption at $293^{\circ} \mathrm{K}$ arising from the $1.35 \mathrm{~cm}$ line, $\gamma_{2}$, is given by:

$$
\begin{aligned}
\frac{\gamma_{2}}{\rho}=\frac{3.5 \times 10^{-3}}{\lambda^{2}}\left[\frac{\Delta \nu_{3}}{\left(\frac{1}{\lambda}-\frac{1}{1.35}\right)^{2}+\Delta \nu_{3}^{2}}\right. & \\
& \left.+\frac{\Delta \nu_{3}}{\left(\frac{1}{\lambda}+\frac{1}{1.35}\right)^{2}+\Delta \nu_{3}^{2}}\right]
\end{aligned}
$$

where $\rho$ is the absolute humidity and $\Delta \nu_{3}$ is the line width factor of the $1.35 \mathrm{~cm}$ water vapor absorption line. The additional absorption arising from absorption bands above the $1.35 \mathrm{~cm}$ line, $\gamma_{3}$, is deseribed by:

$$
\frac{\gamma_{3}}{\rho}=\frac{0.05 \Delta \nu_{4}}{\lambda^{2}}
$$


where $\Delta \nu_{4}$ is the effective line width of the absorption oands above the 1.35-em line. The nonresonant term has been inereased by a factor of 4 over the original Van Vleck formula in order to better satisfy experimental results [7].

Although Van Vleck gives estimates of the various line widths, more recent experimental determinations were used whenever possible. The line-width values used in this ehapter are summarized in table 7.1.

TABLE 7.1. Line width factors used to determine atmospheric absorption

\begin{tabular}{|c|c|c|c|}
\hline$\underset{\text { width }}{\text { Line }}$ & Temperature & Value & Sources \\
\hline $\begin{array}{l}\Delta \nu_{\mathrm{i}} \\
\Delta \nu_{2} \\
\Delta \nu_{3} \\
\Delta \nu_{4}\end{array}$ & $\begin{array}{l}293^{\circ} \mathrm{H} \\
300^{\circ} \mathrm{K} \\
318^{\circ} \mathrm{H} \\
318^{\circ} \mathrm{h}\end{array}$ & $\begin{array}{l}0.018 \mathrm{~cm}^{-1} \mathrm{~atm}^{-1} \\
.049 \mathrm{~cm}^{-1} \mathrm{~atm}^{-1} \\
.087 \mathrm{~cm}^{-1} \mathrm{~atm}^{-1} \\
.087 \mathrm{~cm}^{-1} \mathrm{~atm}^{-1}\end{array}$ & $\begin{array}{l}\text { Birmbaum \& Maryott [8] } \\
\text { Artman \& Gordon [9] } \\
\text { Becker \& Autler [7] } \\
\text { Becker \& Autler [7] }\end{array}$ \\
\hline
\end{tabular}

The preceding expressions for gaseous absorption are given as they appear in the literature and do not reflect the pressure and temperature sensitivity of either the numerical intensity factor or the line widths. This sensitivity must be considered for the present application sinee it is necessary to consider the manner in which the absorption varies with temperature and pressure variations throughout the atmosphere. The dependence of intensity factors upon atmospheric pressure and temperature variations was considered to be that given by the Van Vleck theory. The magnitude and temperature dependence of the line widths is a question not completely resolved. Both theory and experiment indicate the line width to vary as $1 / T^{x}, x<0$. Different measurements on the same line of oxygen have given values of $x$ ranging from 0.71 to 0.90 with differences in the magnitude of $\Delta \nu$ of about 2 pereent $[10,11]$. Experiments have also elearly indicated that the line width changes from line to line, with maximum fluctuations of about 15 percent. In the frequency region considered in this chapter $(10$ to $45 \mathrm{Gc} / \mathrm{s}$ ) the centroid frequency approximation for oxygen is valid and a mean line width can be used with good aceuraey, but in the region of the resonant frequencies of oxygen, the line-to-line line width variations must be taken into account. The expressions used to calculate the absorptions are given in table 7.2. The reference temperatures given are those at which the appropriate experimental determinations were made, and the pressures are to be expressed in millibars. A detailed discussion of the theoretical aspects of the pressure and temperature dependence is given by Artman [12].

Experimental measurements on the absorption of microwaves by the atmosphere (performed after our original work) show different values of the loss than those obtained by theoretical prediction methods. There is reasonably good agreement between the predicted and measured loss for oxygen, but the measured loss of water vapor is considerably greater 
TABLE 7.2. Values used in the calculation of atmospheric absorption

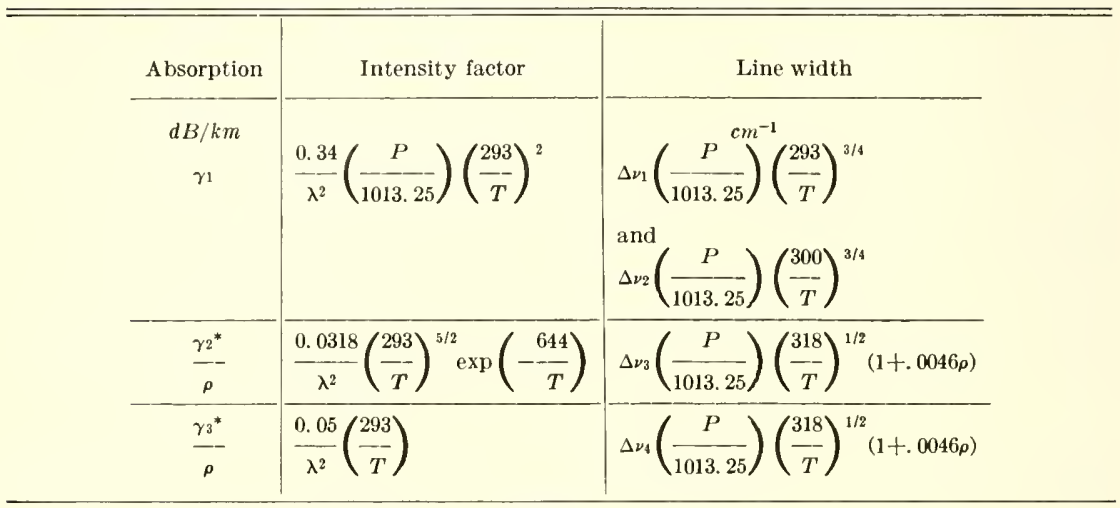

${ }^{*} \rho$ is water vapor density in $\mathrm{g} / \mathrm{m}^{3}$

than that of the predicted amount, particularly above 50,000 Mc/s [13]. These observed discrepancies have little effect upon the present study, which is confined to frequencies less than $50 \mathrm{Gc} / \mathrm{s}$. The results of the present study, for the frequency range 100 to $50,000 \mathrm{Mc} / \mathrm{s}$, agree with those reached by Tolbert and Straiton [14] in their field experiments at Cheyenne Mountain and Pikes Peak, Colo., at altitudes of 14,000 ft.

The above approach represents that presented by Bean and Abbott [3]. The following treatment was given by Gunn and East [15] and based on Van Vleck's two papers [4,5]. This latter presentation is only valid when single line absorption with no appreciable overlap from adjacent lines is considered.

By taking into account the temperature and pressure dependence of the line widths, it is seen that for a given quantity of water vapor, the attenuation is proportional to

$$
P^{-1} \text { and } T^{-2} \exp \left(-\frac{644}{T}\right)
$$

at the resonance line, to

$$
P \text { and } T^{-3} \exp \left(-\frac{644}{T}\right)
$$

at the sides of the curve, and to $P$ and $T^{-3 / 2}$ well away from resonance. In applying the above considerations to absorption approximations it also must be remembered that for a given relative humidity, the density will vary considerably with temperature. Table 7.3 shows attenuation by water vapor at various temperatures and wavelengths. 
The behavior of water vapor attenuation near the resonant line is very remarkable, as ean be seen by inspecting (7.2). Since $\Delta \nu_{3}$ is small compared to $1 / \lambda$, it may be neglected in the denominator of (7.2) for nonresonant wavelengths. The attenuation per unit density is thus direetly proportional to $\Delta \nu_{3}$ and henee to the total pressure for these frequeneies. But at the resonant frequeney, the dominant term in the expression is proportional to $1 / \Delta \nu_{3}$, and thus inversely proportional to the pressure. In the atmosphere, the water vapor density is proportional to the total pressure. Therefore, the attenuation is independent of pressure at the resonant frequeney and now depends only on the fraction of water vapor present. For practical purposes, this means that attenuation can oceur at high altitudes with the same effeetiveness as in the lower, denser layers if the mixing ratio is the same.

On the other hand, oxygen absorption oceurs beeause of a large number of lines around $60 \mathrm{Ge} / \mathrm{s}$. In the region from 3 to $45 \mathrm{Ge} / \mathrm{s}$ the attenuation is proportional to $P^{2}$ and to $T^{-11 / 4}$ [15]. As the temperature deereases the attenuation inereases gradually. At $-40^{\circ} \mathrm{C}$ oxygen attenuation is about 78 pereent higher than at $20^{\circ} \mathrm{C}$ due to inereased density at low temperatures. Table 7.4 shows the pressure and temperature eorreetions for oxygen attenuation at wavelengths between 0.7 and $10 \mathrm{~cm}$.

Figure 7.2 shows the attenuation at a pressure of 1 atmosphere and $20{ }^{\circ} \mathrm{C}$ as a function of wavelength [15]. The solid lines represent values of attenuation measured by Beeker and Autler [7]. The dashed line shows values ealculated from Van Vleck's theory. The water vapor absorption eurve, $c$, corresponds to a water eontent of $1 \mathrm{~g} / \mathrm{m}^{3}$.

Since absorption is so sensitive to the absolute bumidity level, it is helpful to have information on the elimatie variation of absolute humidity throughout the 1 to 99 pereent range of values normally used in radio engineering. Estimates of the values of absolute humidity at the surface expeeted 50 pereent of the time for the United States for February and August are given in figures 7.3 and 7.4 respectively [16]. It is evident that for either month the coastal regions display greater values of absolute humidity than do the inland regions. Note that for any location the August values are eonsistently greater than the February values. Figures 7.5 through 7.8 show the values of absolute humidity expected to be exceeded 1 and 99 pereent of the time throughout the United States in both summer and winter.

In addition to oxygen and water vapor, there are a number of other atmospherie gases which have absorption lines in the microwave region from 10 to $50 \mathrm{Ge} / \mathrm{s}$. These gases normally constitute a negligible portion of the general composition of the atmosphere, but could conceivably contribute to attenuation. Table 7.12 shows the resonant frequeneies, maximum absorption coefficients at $300{ }^{\circ} \mathrm{K}$ (attenuation coefficient if the fraction of molecules present were equal to unity), expected concentration 
TABLE 7.3. Water vapor attenuation (one way) in $\mathrm{dB} / \mathrm{km}$ [After Gunn-East].

$P$, pressure in atmospheres; $W$, water vapor content in $\mathrm{g} / \mathrm{m}^{-3}$

\begin{tabular}{|c|c|c|c|c|c|c|}
\hline$\left(\begin{array}{c}T \\
\left({ }^{\circ} \mathrm{C}\right)\end{array}\right.$ & $\lambda(\mathrm{cm}) 10$ & 5.7 & 3.2 & 1.8 & 1. 24 & 0.9 \\
\hline $\begin{array}{r}20 \\
0 \\
-20 \\
-40\end{array}$ & $\begin{array}{l}0.07 \times 10^{-3} \mathrm{PW} \\
0.08 \times 10^{-3} \mathrm{PW} \\
0.09 \times 10^{-3} \mathrm{PW} \\
0.10 \times 10^{-3} \mathrm{PW}\end{array}$ & $\begin{array}{l}0.24 \times 10^{-3} \mathrm{PW} \\
0.27 \times 10^{-3} \mathrm{PW} \\
0.30 \times 10^{-3} \mathrm{PW} \\
0.34 \times 10^{-3} \mathrm{PW}\end{array}$ & $\begin{array}{l}0.7 \times 10^{-3} \mathrm{PW} \\
0.8 \times 10^{-3} \mathrm{PW} \\
0.9 \times 10^{-3} \mathrm{PW} \\
1.0 \times 10^{-3} \mathrm{PW}\end{array}$ & $\begin{array}{l}\text { 4. } 3 \times 10^{-3} \mathrm{PW}^{*} \\
\text { 4. } 8 \times 10^{-3} \mathrm{PW}^{*} \\
\text { 5. } 0 \times 10^{-3} \mathrm{PW}^{*} \\
\text { 5. } 4 \times 10^{-3} \mathrm{PW}^{*}\end{array}$ & $\begin{array}{l}22.0 \times 10^{-3} \mathrm{PW}^{*} \\
23.3 \times 10^{-3} \mathrm{PW}^{*} \\
24.6 \times 10^{-3} \mathrm{PW}^{*} \\
26.1 \times 10^{-3} \mathrm{PW}^{*}\end{array}$ & $\begin{array}{r}9.5 \times 10^{-3} \mathrm{PW} \\
10.4 \times 10^{-3} \mathrm{PW} \\
11.4 \times 10^{-3} \mathrm{PW} \\
12.6 \times 10^{-3} \mathrm{PW}\end{array}$ \\
\hline
\end{tabular}

* The pressure dependencies shown are only approximate. Near the $1.35 \mathrm{~cm}$ water vapor absorption line (between $1.0 \mathrm{~cm}$ and $2.0 \mathrm{~cm}$ ) no simple power dependency of $\mathrm{P}$ and $\mathrm{W}$ is accurate.

TABLE 7.4. Pressure and temperature correction for oxygen attenuation for wavelengths between 0.7 and $10 \mathrm{~cm}$

[After Gunn-East]

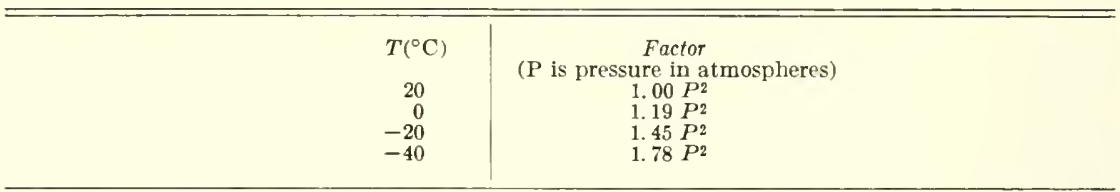

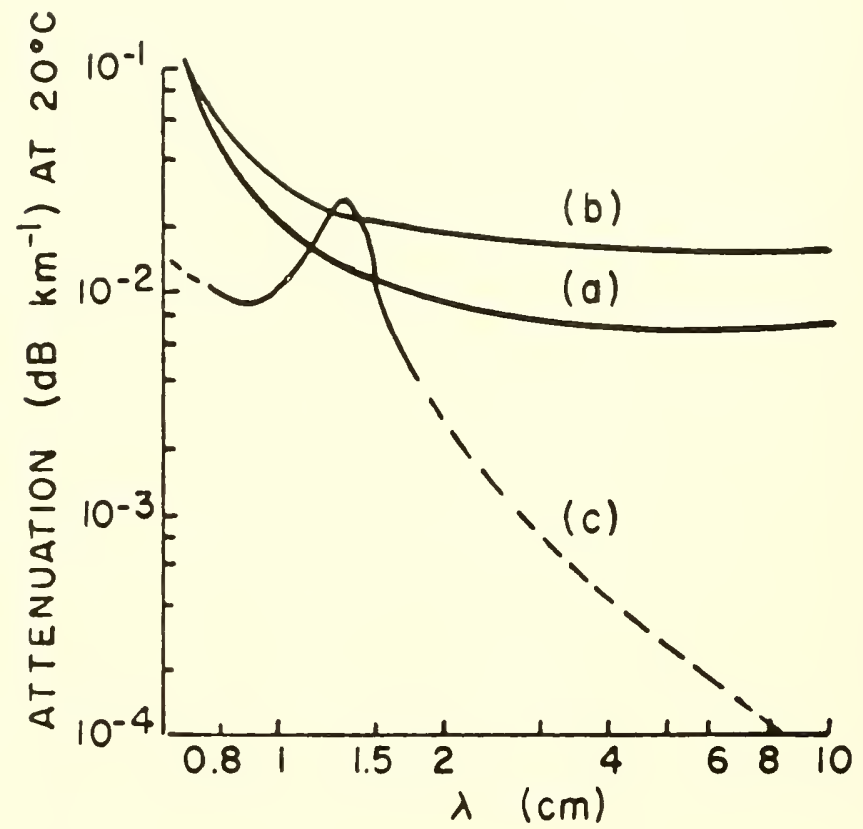

Figure 7.2. Attenuation of microwaves by atmospheric gases. (After Gunn and East, 1954). 


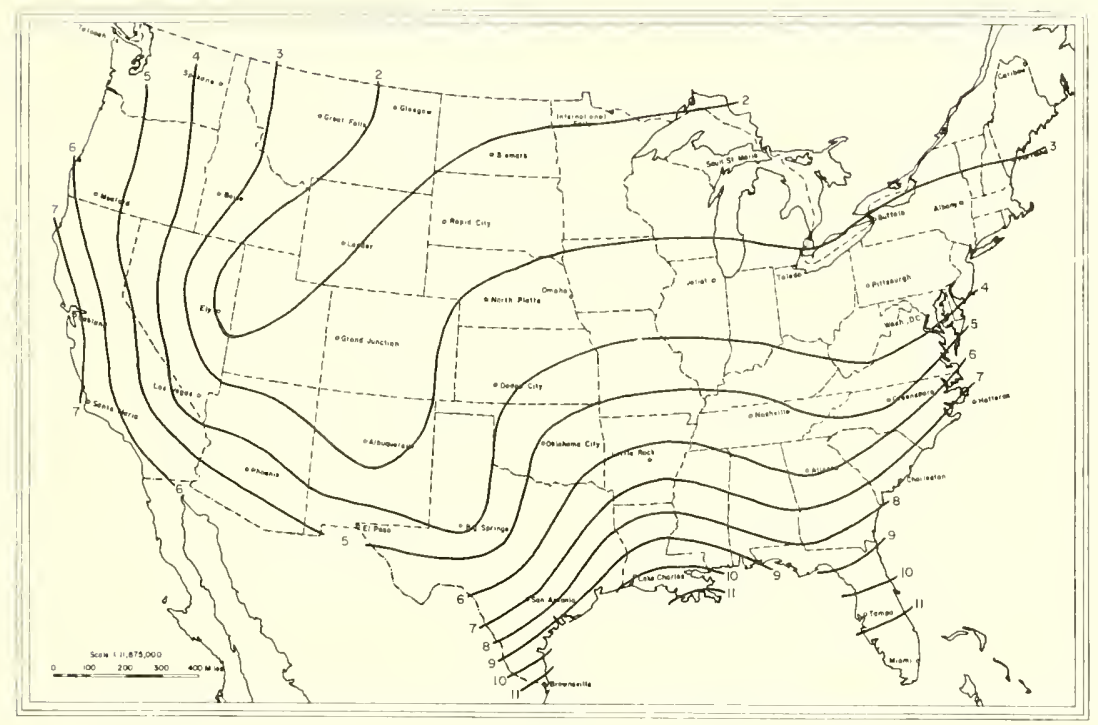

Figure 7.3. Estimate of the value of absolute humidity expected 50 percent of the time for February.

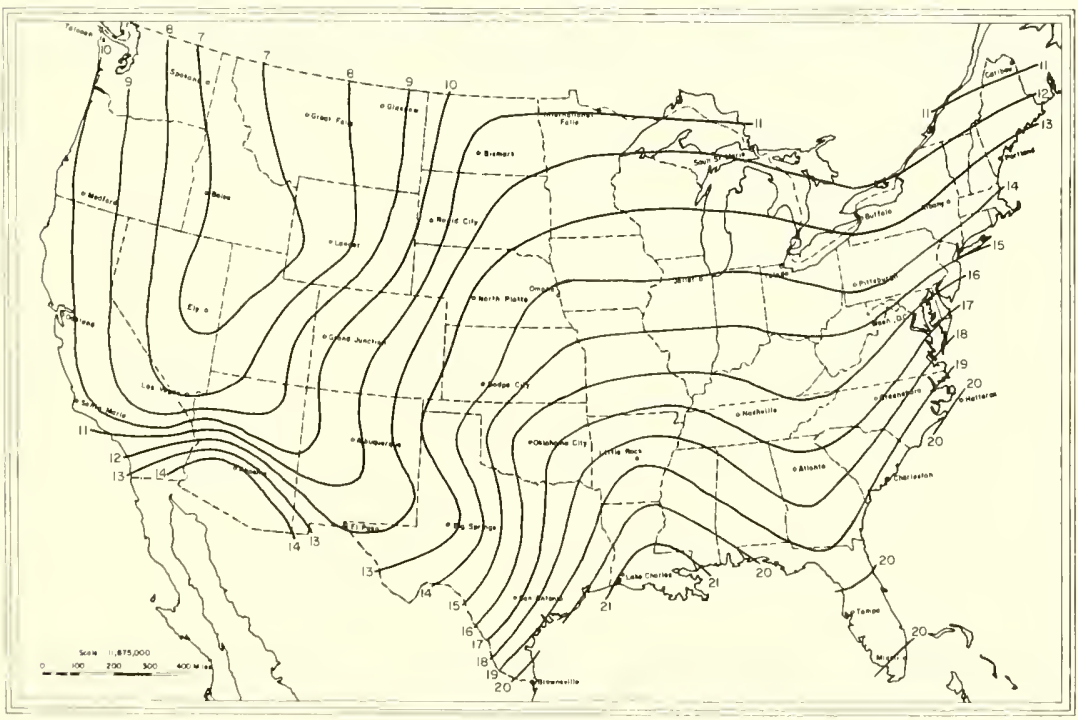

Figure 7.4. Estimate of the value of absolute humidity expected 50 percent of the time for August. 


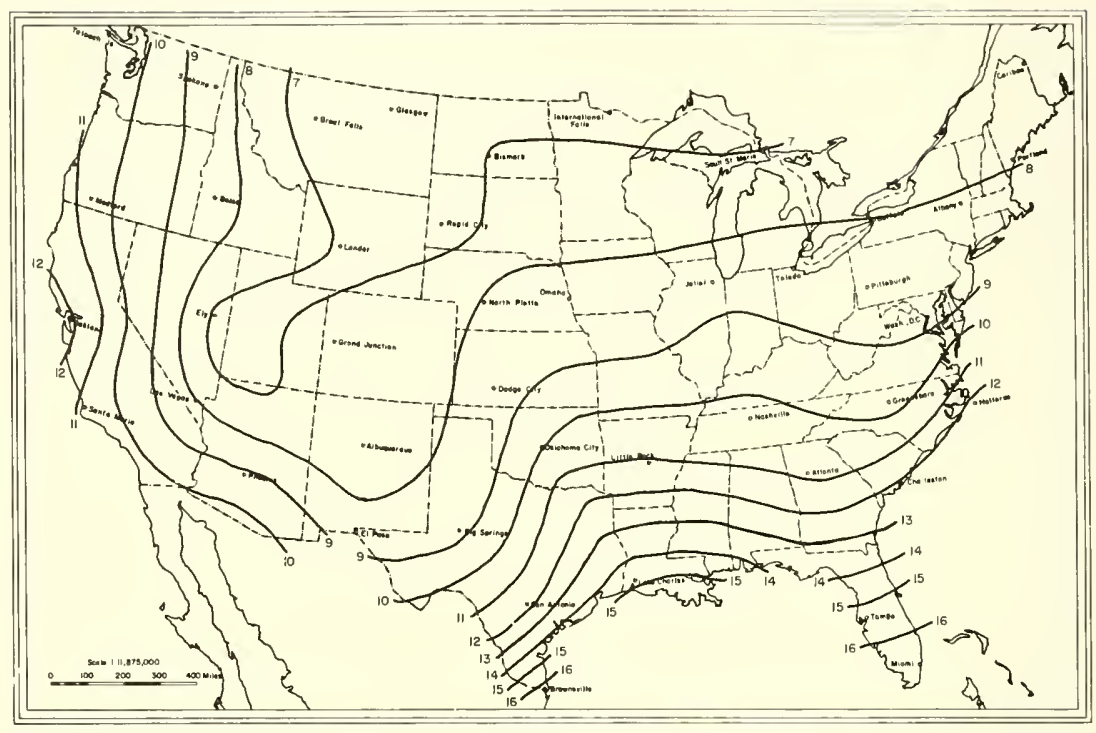

Figure 7.5. Values of absolute humidity expected to be exceeded 1 percent of the time for February.

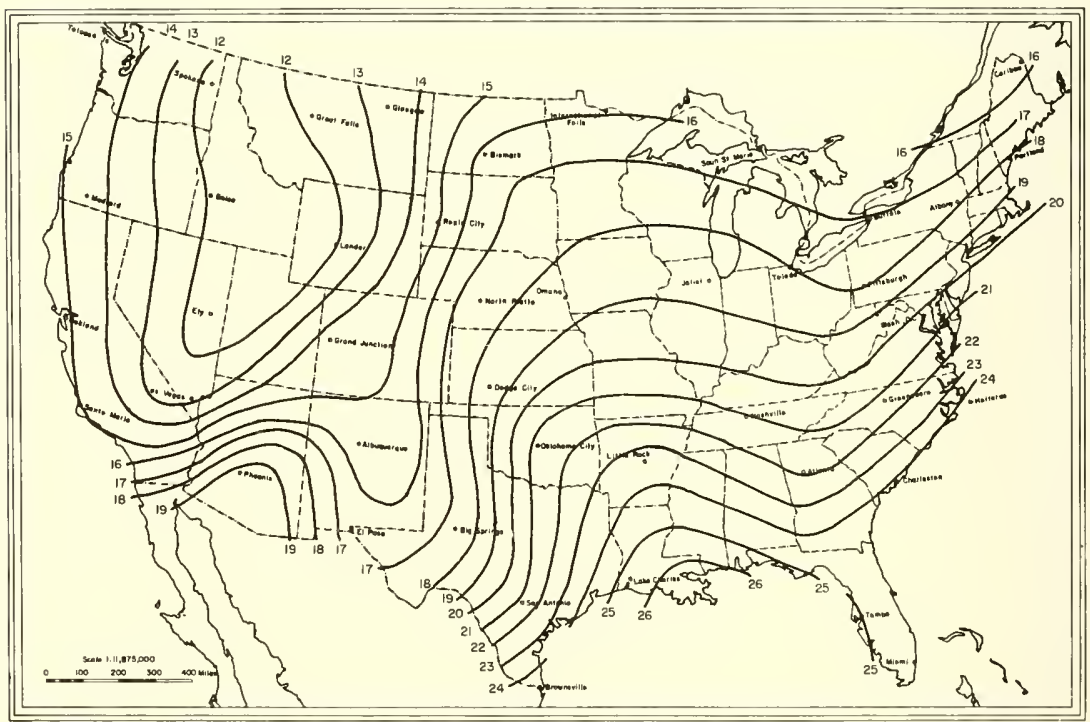

FIGURE 7.6. Values of absolute humidity expected to be exceeded 1 percent of the time for August. 


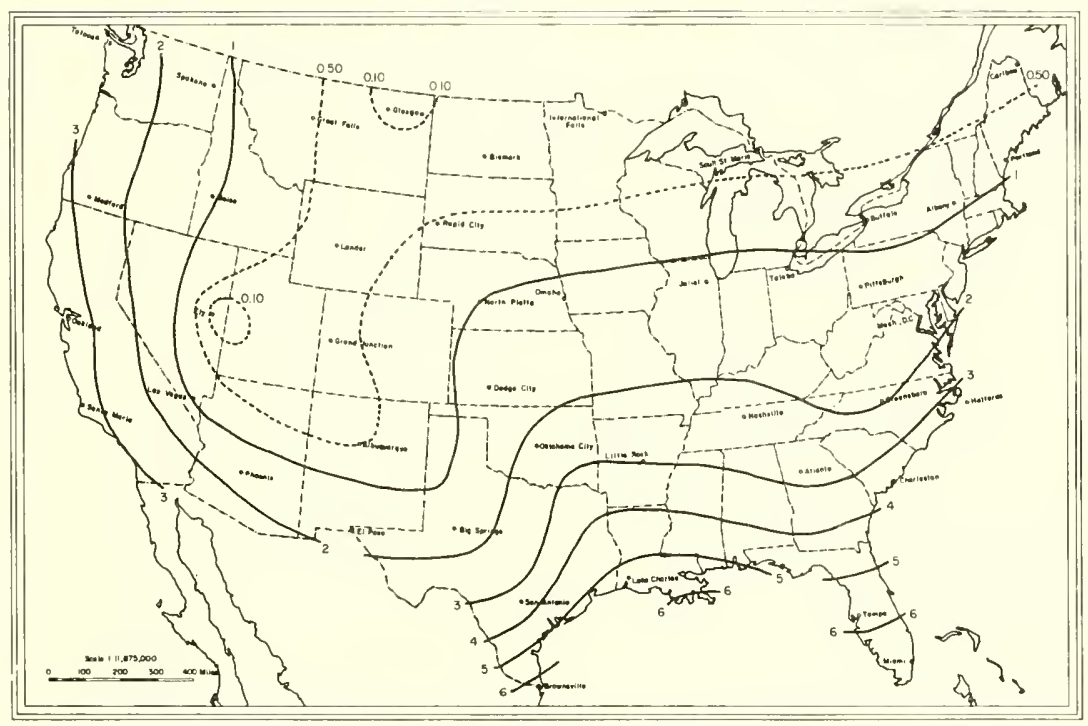

FIGURE 7.7. Values of absolute humidity expected to be exceeded 99 percent of the time for February.

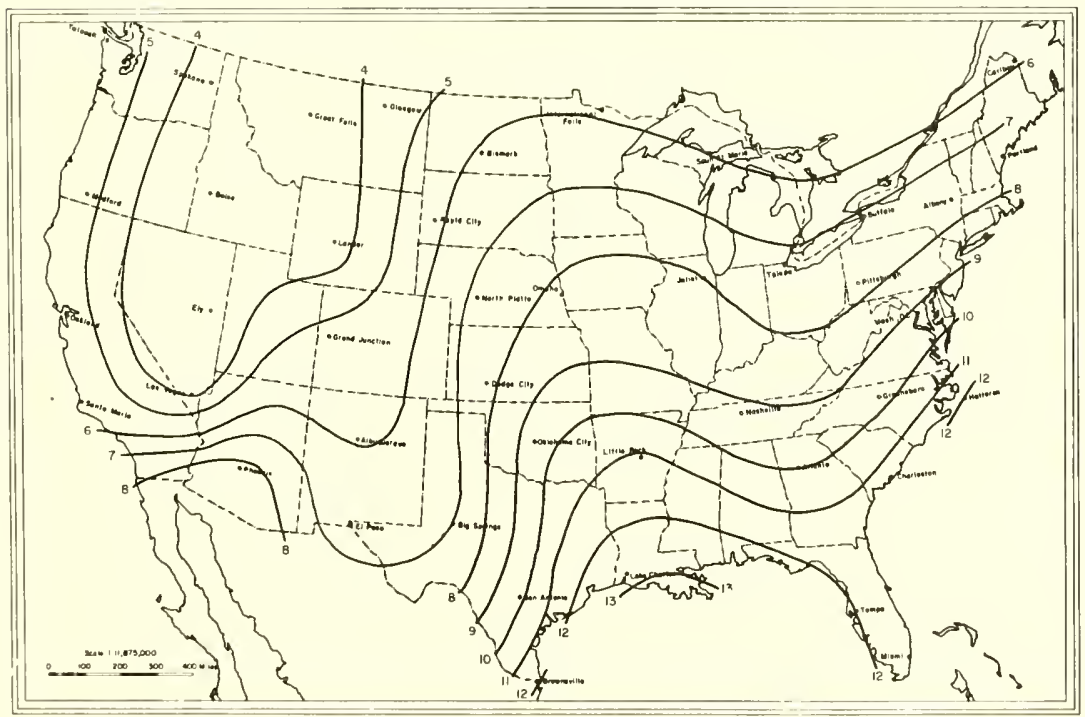

Figure 7.8. Values of absolute humidity expected to be exceeded 99 percent of the time for August. 
in the atmosphere, and expected absorption coefficients due to these trace constituents. The data on molecular absorption coefficients was taken from Ghosh and Edwards [33], that on concentrations from the Glossary of Meteorology [29]. It is readily seen that the attenuation due to these sources is negligible compared to the high absorption due to oxygen and water vapor.

\subsection{Estimates of the Range of Total Gaseous Absorption}

The range in gaseous absorption can be seen by considering the data for the months of February and August at Bismarck, N. Dak., and Washington, D.C., two stations with very different climates. The values of total gaseous absorption (defined as the sum of $\gamma_{1}, \gamma_{2}$, and $\gamma_{3}$, where $\gamma_{1}=$ oxygen absorption in decibels per kilometer, $\gamma_{2}=$ water vapor absorption arising from the $1.35 \mathrm{~cm}$ line and $\gamma_{3}=$ additional absorption arising from absorption lines whose frequencies are considerably higher than that corresponding to the 1.35 line) at each station and elevation up to $75,000 \mathrm{ft}$ are shown in figures 7.9 and 7.10 for each of the four station months for the frequency range of 100 to $50,000 \mathrm{Mc} / \mathrm{s}$. Above $75,000 \mathrm{ft}$ the absorption values for all four station months are identical and are given for each frequency in figure 7.11. The absolute humidity was calculated using the upper air monthly average values of temperature, pressure, and humidity as reported by Ratner [17]. Readings for the relative humidity are not generally given in this report for altitudes greater than about $15 \mathrm{~km}$ due to the inability of the radiosonde to measure the small amount of humidity present at these altitudes. It is believed that the climates represented by these station months encompass the range of those of the majority of the continental United States radio propagation paths.

An interesting property of the annual range of absorption as a function of the frequency may be seen in figures 7.9 and 7.10. For the first 5,000 $\mathrm{ft}$ above the surface, it is noted that in the frequency range of 10 to 32.5 $\mathrm{Gc} / \mathrm{s}$, the summer values are greater than the winter values due to increased humidity of the summer months. Outside of this frequency range, however, the winter values of absorption are greater due to the increased oxygen density.

In the frequency range 6 to $45 \mathrm{Gc} / \mathrm{s}$, atmospheric absorption, $\gamma_{\nu}$, at a frequency $\nu$, arises primarily from oxygen absorption, $\gamma_{d \nu}$, and water vapor absorption, $\gamma_{w \nu}$; i.e.,

$$
\gamma_{\nu}=\gamma_{d \nu}+\gamma_{w \nu}
$$




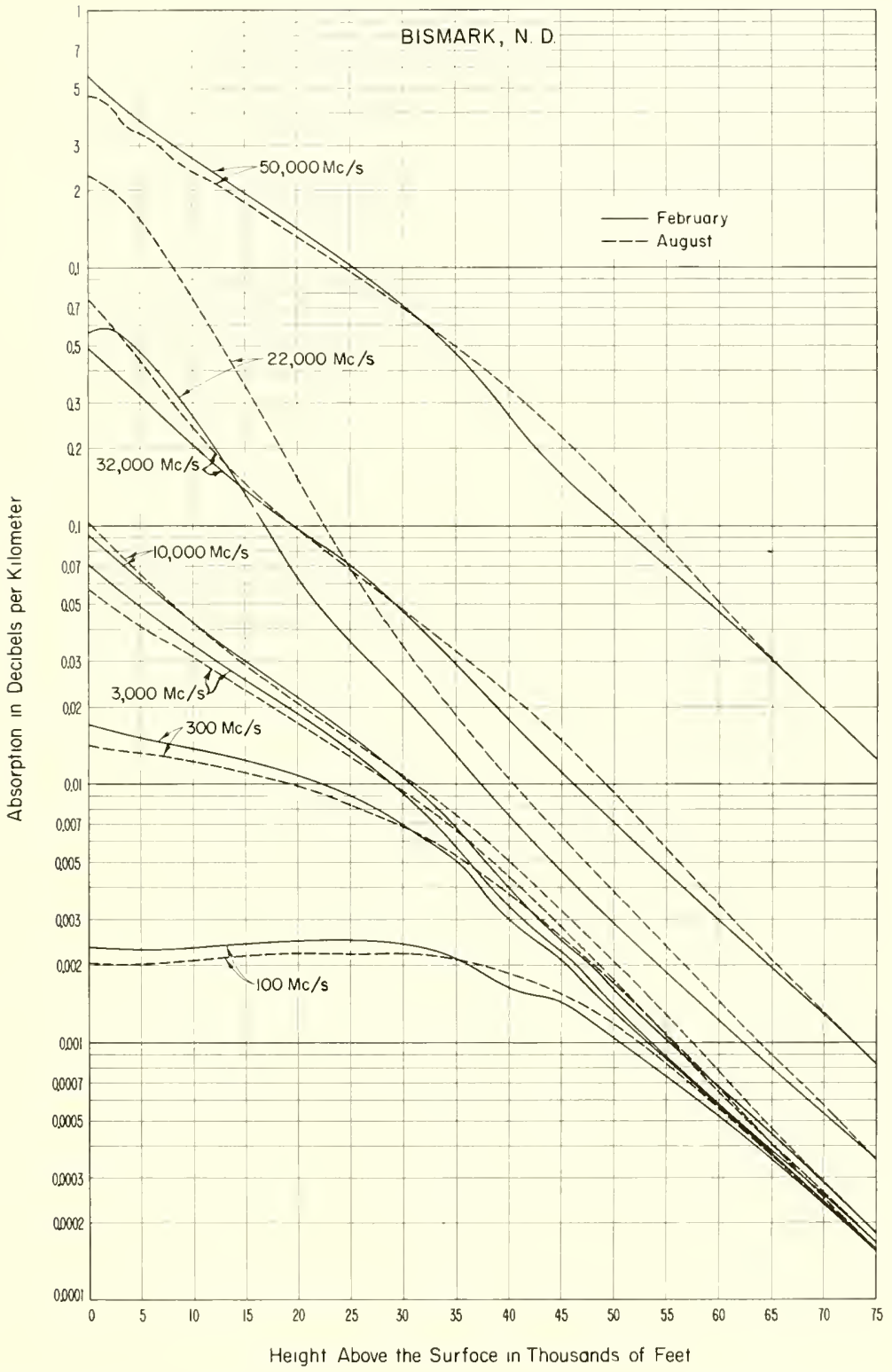

Figure 7.9. Total gaseous atmospheric absorption from the surface to 75,000 feet: Bismarck, N. Dak. 


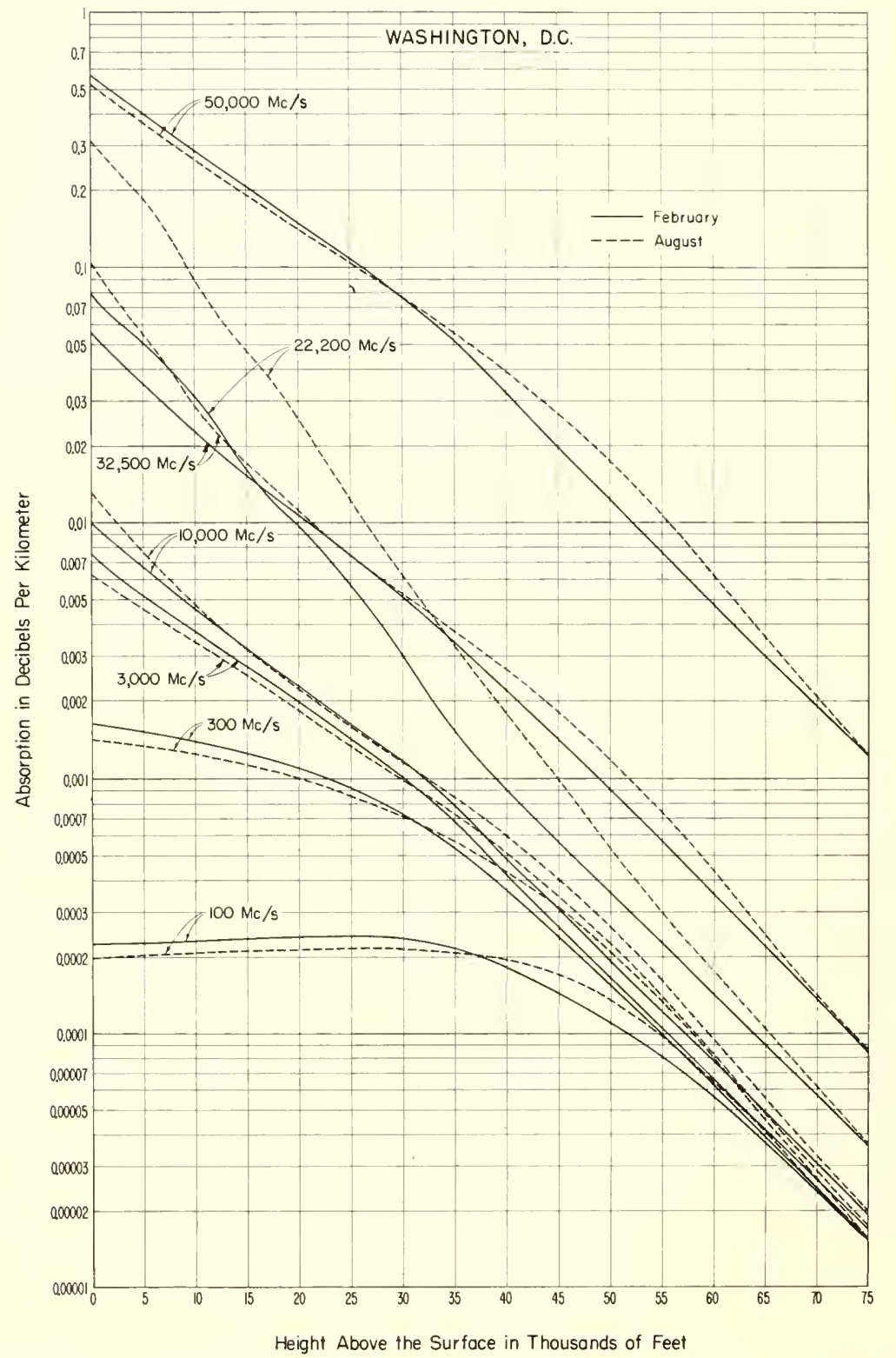

Figure 7.10. Total gaseous atmospheric absorption from the surface to 75,000 feet: Washington, D.C. 
Zhevankin and Troitskii [31] have indicated that $\gamma_{d \nu}$ and $\gamma_{w \nu}$ can be represented as exponential functions of height, $Z$, above the earth's surface,

$$
\gamma_{d \nu}=\gamma_{d \nu 0} \exp \left(\frac{-Z}{H_{d \nu}}\right), \gamma_{w \nu}=\gamma_{w \nu 0} \exp \left(\frac{-Z}{H_{w \nu}}\right)
$$

where $\gamma_{d \nu 0}$ and $\gamma_{w \nu 0}$ are the values of $\gamma_{d \nu}$ and $\gamma_{w \nu}$, respectively, at the earth's surface, and $H_{d \nu}$ and $H_{w \nu}$ are called the "scale heights" of $\gamma_{d \nu}$ and $\gamma_{w \nu}$. This model is known as the "bi-exponential" model of absorption, and $\gamma_{d \nu}$ and $\gamma_{w \nu}$ are often called the "dry" and "wet" terms of $\gamma_{\nu}$. The seale height for the dry term in the frequency range 6 to $45 \mathrm{Ge} / \mathrm{s}$ ean be written as [32]

$$
H_{d \nu}(\text { in } \mathrm{km}) \cong \frac{T_{0}}{c+b \alpha}
$$

where $T_{0}$ is the surface temperature in ${ }^{\circ} \mathrm{K}, \alpha$ is the temperature lapse rate with height in ${ }^{\circ} \mathrm{K} / \mathrm{km}$, and $b, c$, are constants determined from thermodynamic considerations. Because of the hump in the $H_{w_{\nu}}$ eurves as opposed to the flat $H_{d \nu}$ eurve in figure 7.28 , such a handy expression as (7.6) for $H_{w \nu}$ is not possible in the 6 to $45 \mathrm{Ge} / \mathrm{s}$ frequency range (fig. 7.28 was determined from actual radiosonde data at Verkhoyansk, U.S.S.R.).

\subsection{Total Radio Path Absorption}

The total path absorption is determined by ealculating the various absorption coefficients as functions of the heights along the ray path and then numerically integrating the values along the entire path using standard ray traeing teehniques outlined in chapter 3 . The values of total path integration over a $100-\mathrm{km}$ path thus obtained are presented in figure 7.12 for Bismarek, N. Dak., and Washington, D.C. The difference between the two elimates is evident principally at the higher frequencies, where the Washington absorptions are consistently above the Bismarck values. This is apparently due to a combination of generally greater humidities and greater refractive effects. These two effeets are related. The increased humidity at Washington enhances the water vapor absorption and inereases the refraction causing the radio ray to travel consistently through lower levels of the atmosphere with consequent increase in total path absorption. 


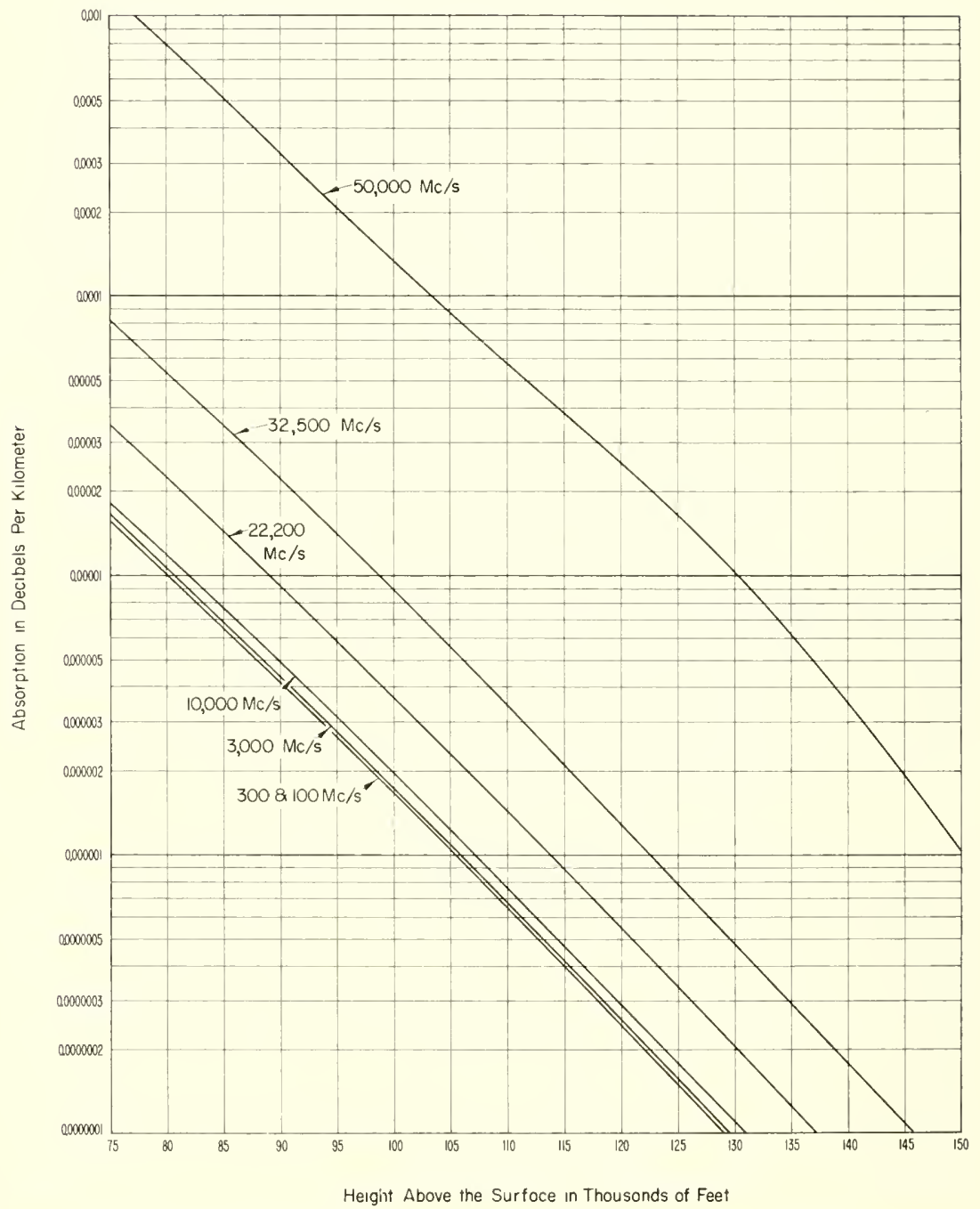

Figure 7.11. Common values of total gaseous atmospheric absorption for elevations greater than 75,000 feet. 


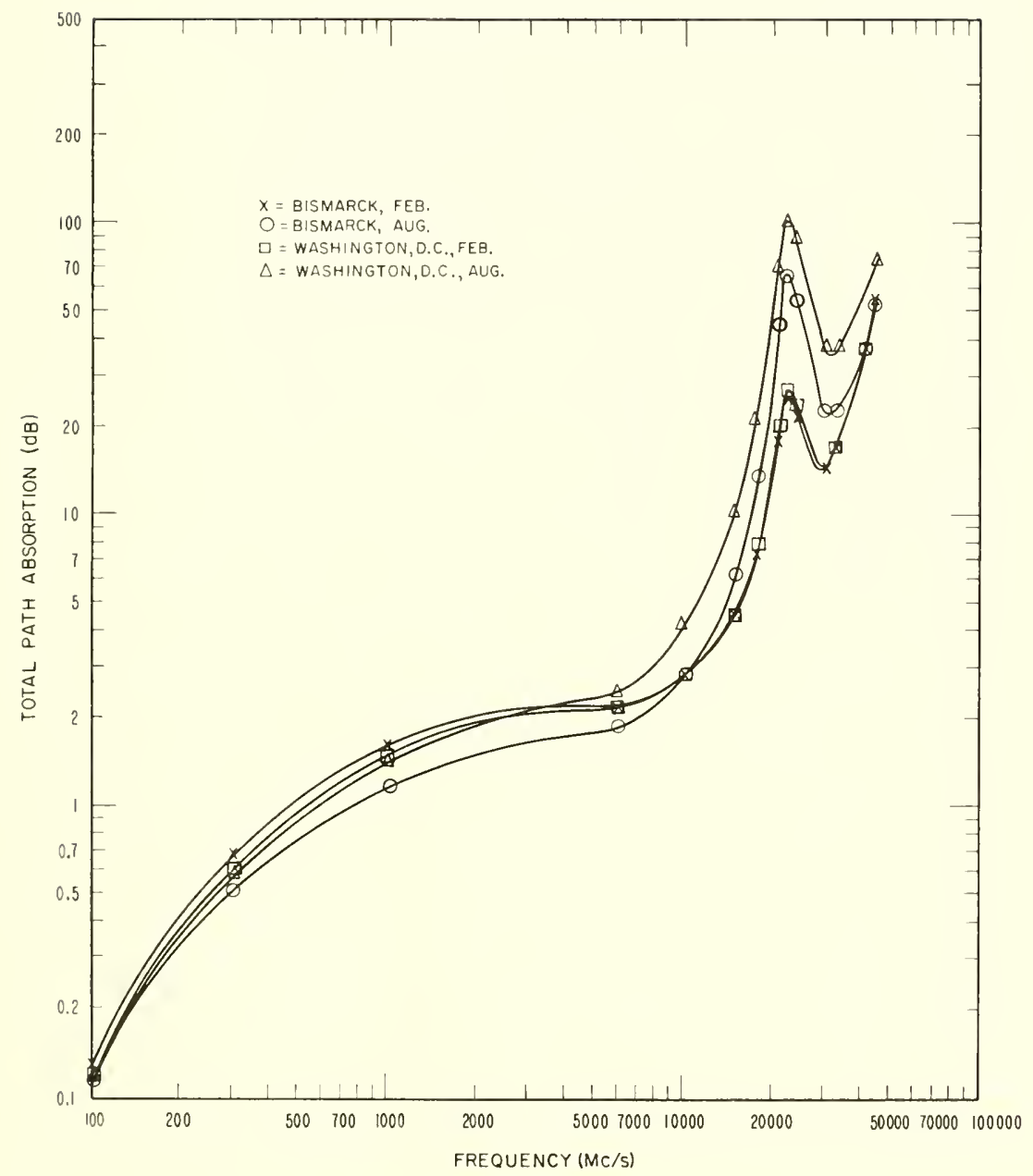

FIgURE 7.12. Total path absorption over a $1000 \mathrm{~km}$ propagation path with the climate of Bismarck, N. Dak. 


\subsection{Derivation of Absorption Estimate for Other Areas}

The values of total path absorption given above are for two specific locations. For this particular study to be of practical use, a means should be provided for arriving at estimates of geographic and annual variations of total path absorption for various surface distances and frequencies. The method chosen utilizes the correlation between total path absorption and the surface value of the absolute humidity, expressed in grams of water vapor per cubic meter, which appears explicity in Van Vleck's water vapor absorption formulas $[4,5]$. The basis for expecting a correlation to exist between the absolute humidity and the total path absorption is that the absorption at those frequencies for which water vapor absorption is dominant (approximately 10 to $32 \mathrm{Gc} / \mathrm{s}$ ) varies directly as the absolute humidity while, for those frequencies at which oxygen absorption is dominant, it varies inversely as the absolute humidity due to the inverse relationship of oxygen density and water vapor density. That is, during the warm seasons of the year the total atmospheric pressure tends towards its yearly minimum (as does the oxygen absorption), while the absolute humidity tends towards its yearly maximum (as does the water vapor absorption). Conversely, during the colder seasons of the year, the pressure tends towards its maximum value while the absolute humidity tends towards its minimum value [3].

As an example of the correlation method, the surface absorption was calculated at a water vapor-dominated frequency $(22.2 \mathrm{Gc} / \mathrm{s})$ and oxygen-dominated frequency $(50 \mathrm{Gc} / \mathrm{s})$ for each month throughout the year for both Washington and Bismarek. These values are plotted on figure 7.13. The term surface absorption is used for the values of absorption calculated from standard ground level weather observations. The water-dominated $22.2 \mathrm{Gc} / \mathrm{s}$ data fall on a smooth curve despite the pressure and temperature differences of the two stations. The oxygendominated $50 \mathrm{Gc} / \mathrm{s}$ data, however, display an interesting separation of points for each station, although the distribution of points at the two locations display similar slopes. The $50 \mathrm{Gc} / \mathrm{s}$ absorption is more sensitive to the atmospheric density difference between the stations. If the pressure differences were taken into account, the Bismarck data would increase about 12 percent and the two curves would be distributed along a common line with the same slope as the original two curves. This figure, then, indicates that the absorption is correlated with the absolute humidity. The above illustration is for surface values rather than for integrated propagation path values. Variations in the upper air meteorology that are not reflected in the surface values will tend to diminish the correlation. (For comparison of percentage absorption over a $300-\mathrm{km}$ path, the first few hundred feet contribute about 5 percent at $100 \mathrm{Mc} / \mathrm{s}$ increasing to 42 percent at $10 \mathrm{Gc} / \mathrm{s}$ and remaining constant to $50 \mathrm{Gc} / \mathrm{s}$.) Keeping these reservations in mind, one may utilize the method of least 


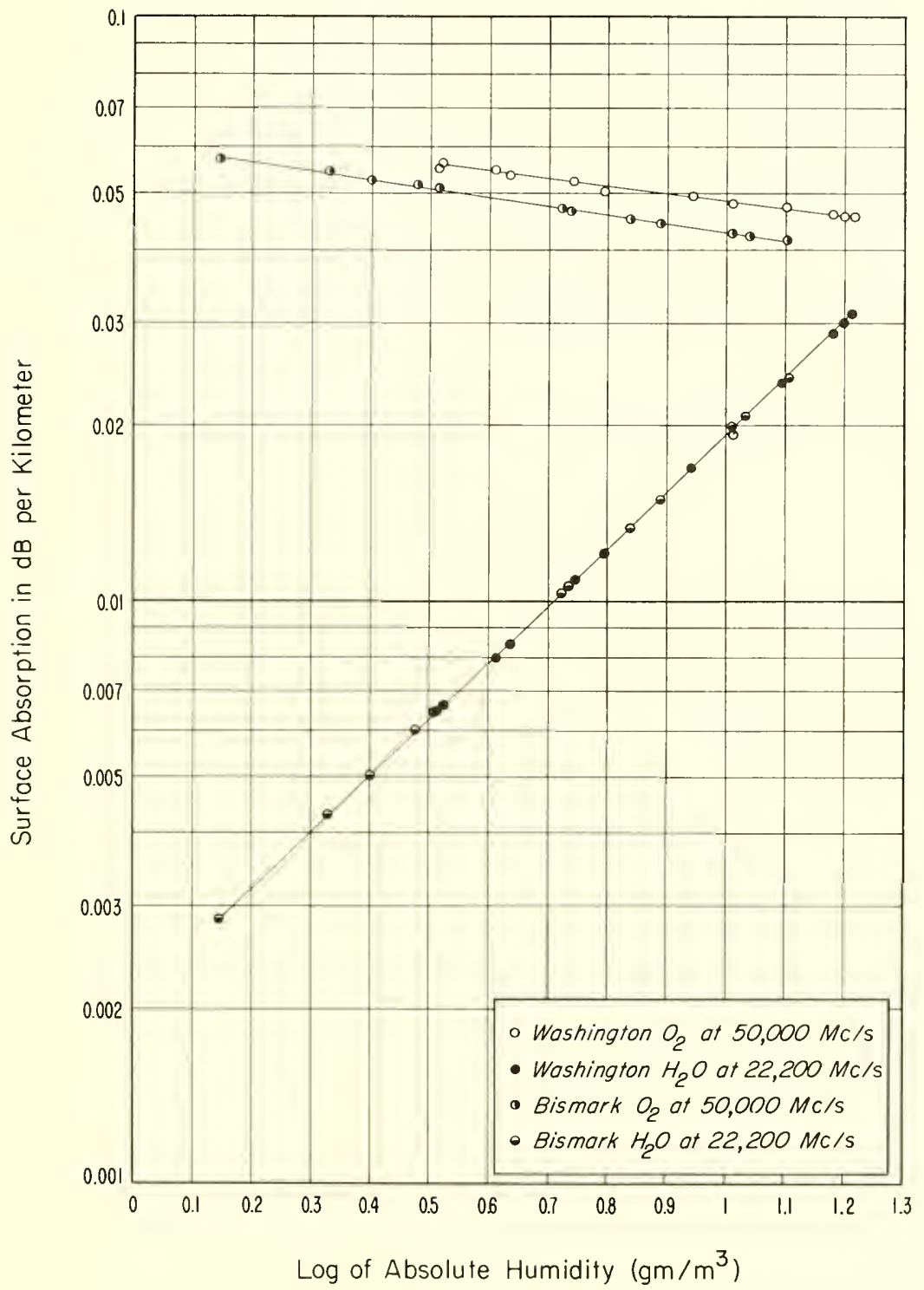

Figure 7.13. Surface value of 22.2 and $50 \mathrm{Gc} / \mathrm{s}$ absorption versus absolute humidity at Washington, D.C., and Bismarck, N.Dak. 
TABLE 7.5. Values of $\mathrm{m}$ and $\mathrm{b}$ in the regression line $\mathrm{y}=\mathrm{mx}+\mathrm{b}$, where $y$ is the logarithm of the total path attenuation, $\gamma, b$ is the value of $y$ when the absolute humidity is $1 \mathrm{~g} / \mathrm{m}^{3}$, and $x$ is the logarithm of the absolute humidity.

[After Bean and Abbott]

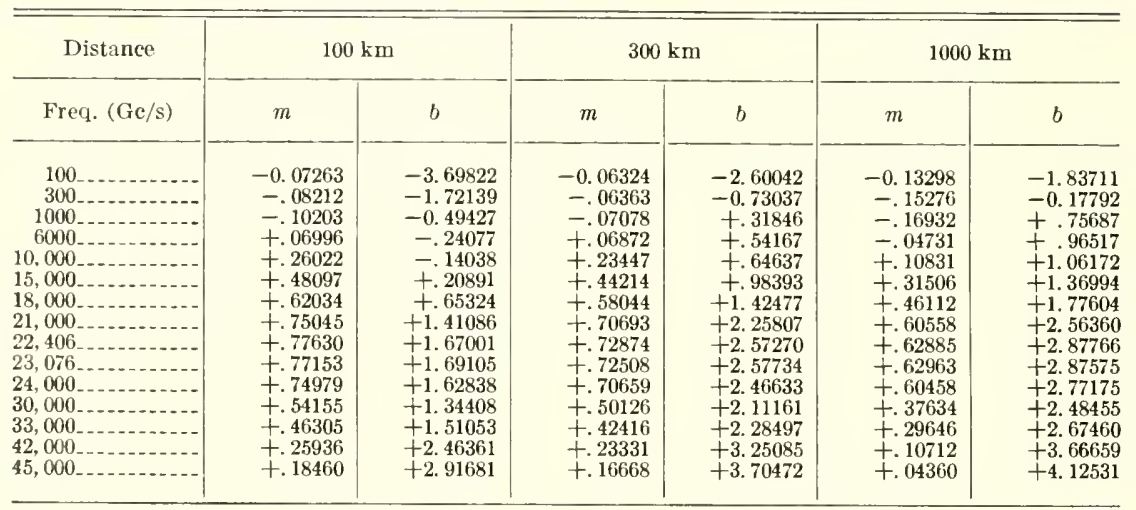
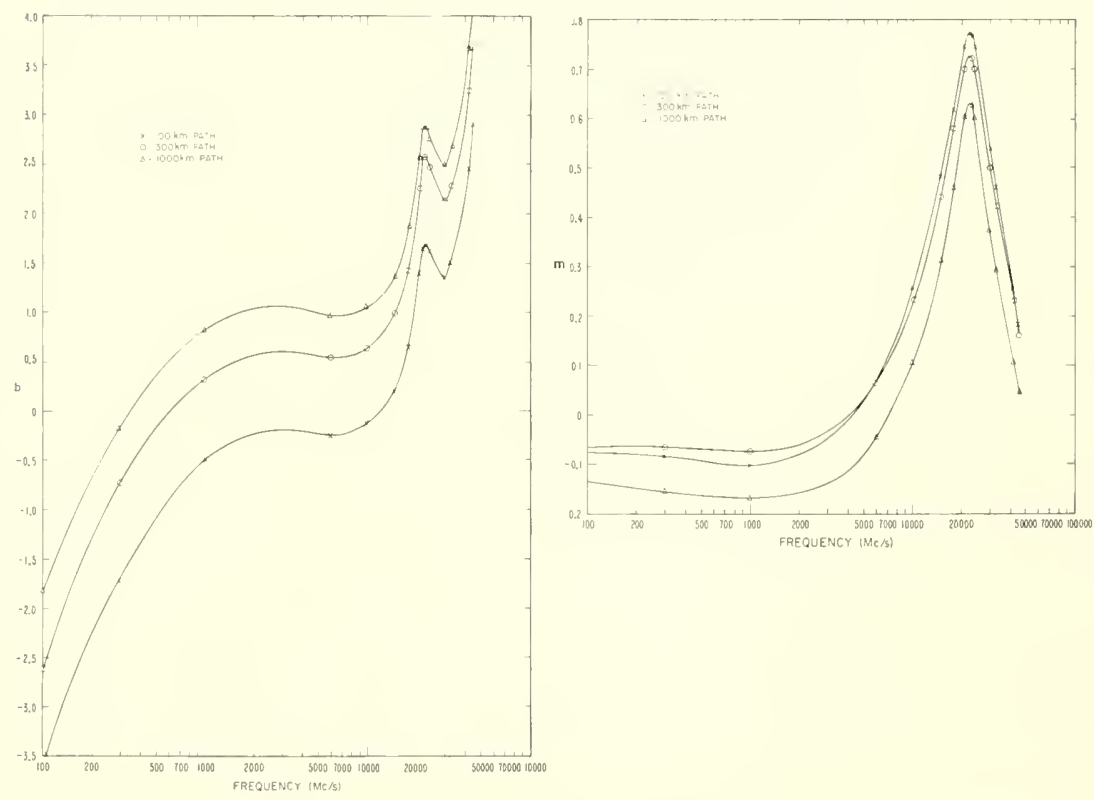

Figure 7.14. Values of $\mathrm{b}$ and $\mathrm{m}$ in the regression line $\mathrm{y}=\mathrm{mx}+\mathrm{b}$, where $\mathrm{y}$ is the logarithm of the total path absorption, $\mathrm{x}$ is the logarithm of the absolute humidity, $\mathrm{b}$ is the value of $\mathrm{y}$ when the absolute humidity is $1 \mathrm{~g} / \mathrm{m}^{3}$. 
squares to obtain the total path absorption as a linear function of the absolute humidity. The parameter is a statistical regression line at each frequency for path propagation distances of 100,300 , and $1,000 \mathrm{~km}$ as given in table 7.5, and also plotted on figure 7.14, allowing the reader to estimate the regression line for frequencies other than those of the present study [3].

Statistics for the variation of absolute humidity have already been given, and contours of the mean values of absolute humidity for the world are given on figures 7.15 and 7.16 for the months of February and August respectively. It is noted that the absolute humidity for either month is greater in the coastal regions than in the continental interiors and that the windward sides of continents have larger values than do the leeward sides.

By reference to figures 7.15 and 7.16 [16] for the United States, and figure 7.14, estimates of radio power loss due to absorption may be obtained. As an example, figure 7.17 gives the values expected to be exceeded 1 percent of the time over a $300 \mathrm{~km}$ propagation path at 10,000 $\mathrm{Mc} / \mathrm{s}$ for the United States during the month of August.

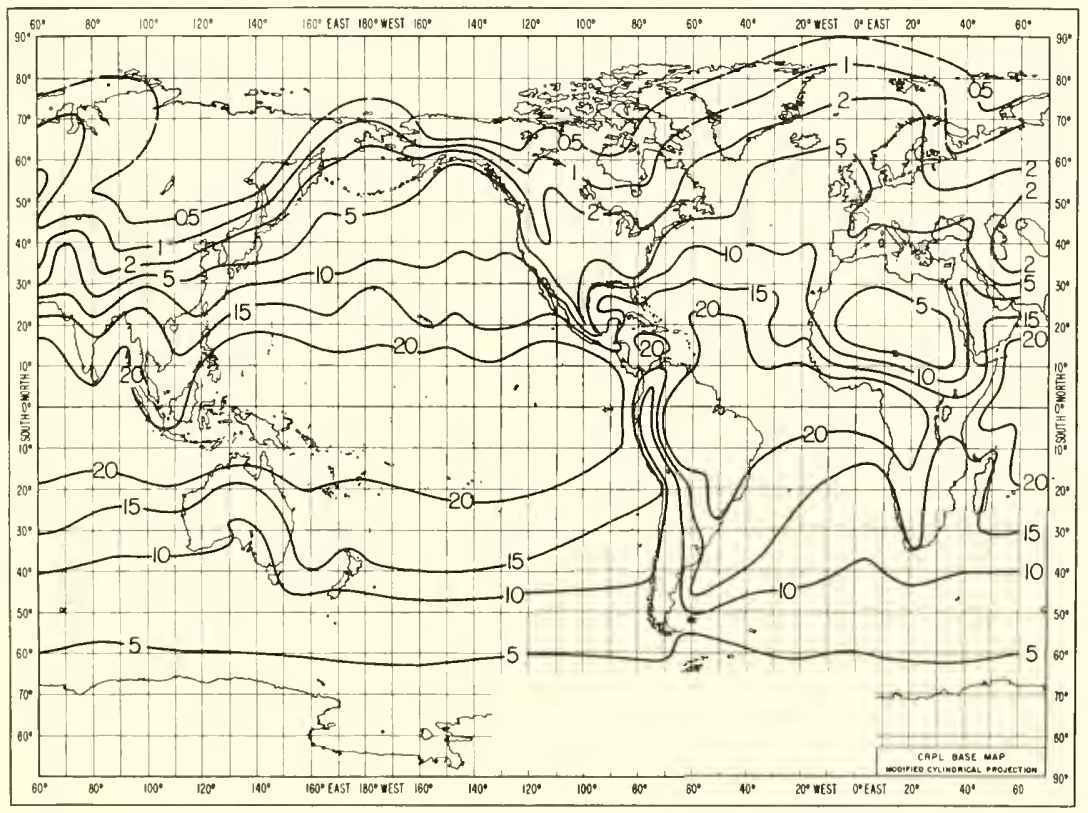

Figure 7.15. Average absolute humidity $\left(\mathrm{g} / \mathrm{m}^{3}\right)$, February. 


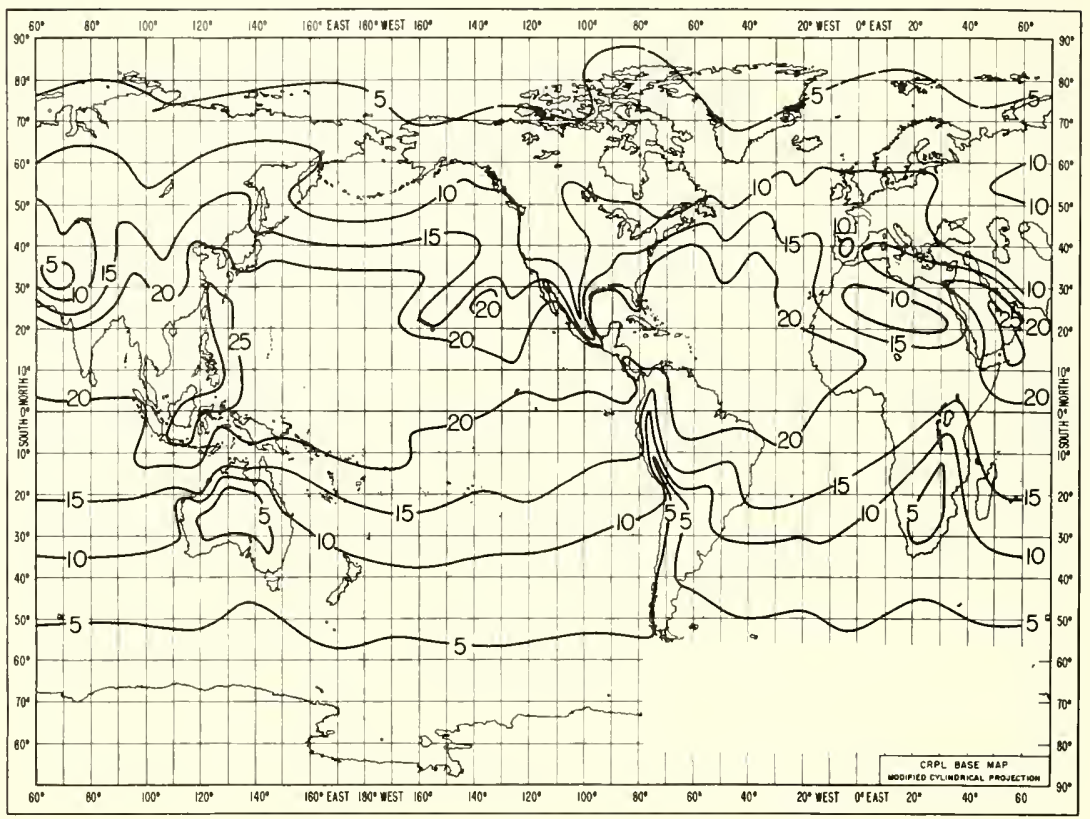

Figure 7.16. Average absolute humidity $\left(g / m^{3}\right)$, August.

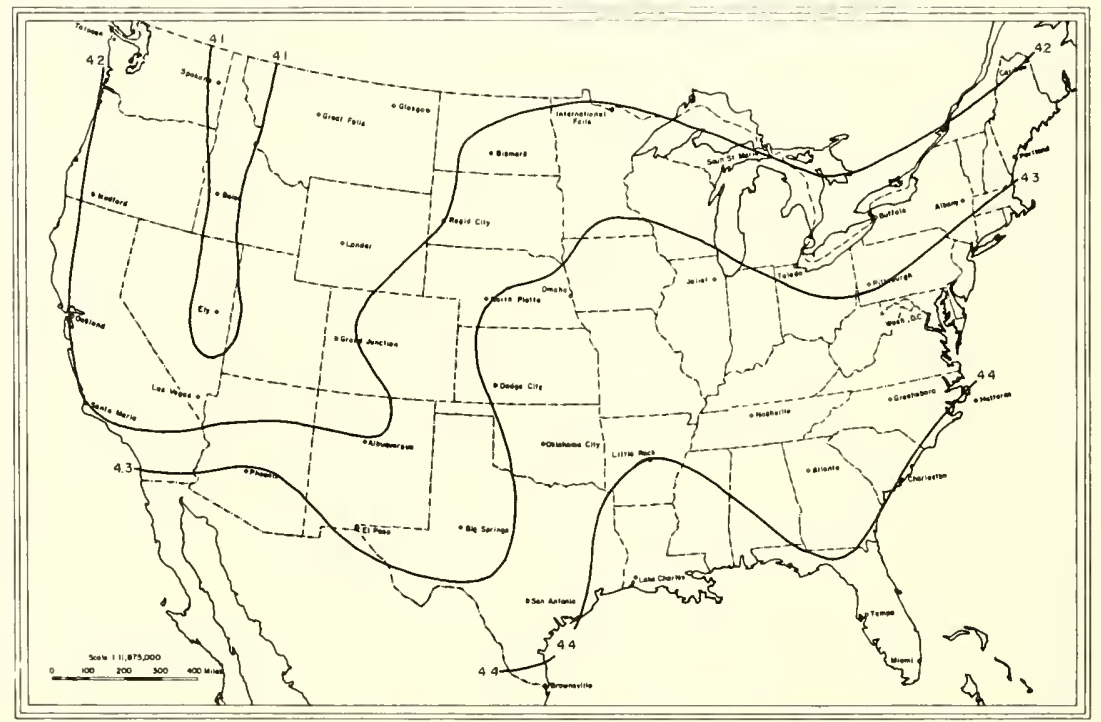

Figure 7.17. August values of 300-mi propagation path absorption loss (in $d B$ ) to be exceeded 1 percent of the time for a frequency of $10,000 \mathrm{Mc} / \mathrm{s}$. 


\subsection{Attenuation in Clouds}

Cloud droplets are regarded here as those water or ice particles having radii smaller than $100 \mu$ or $0.01 \mathrm{~cm}$. For wavelengths of incident radiation well in excess of $0.5 \mathrm{~cm}$, the attenuation becomes independent of the drop size distribution. The generally accepted equations for attenuation by clouds usually show the moisture component of the equations in the form of the liquid water content $\left(\mathrm{g} / \mathrm{m}^{3}\right)$. Observations indieate that the liquid water concentration in elouds generally ranges from 1 to $2.5 \mathrm{~g} / \mathrm{m}^{3}$ [18], although Weickmann and aufm Kampe [19] have reported isolated instances of cumulus congestus elouds with a reading of $4.0 \mathrm{~g} / \mathrm{m}^{3}$ in the upper levels. In ice clouds, it will rarely exceed 0.5 and is often less than $0.1 \mathrm{~g} / \mathrm{m}^{3}$. The attentuation of eloud drops may be written as:

$$
K=K_{1} M \text {, }
$$

where

$K=$ attenuation in $\mathrm{dB} / \mathrm{km}$,

$K_{1}=$ attenuation coefficient in $\mathrm{dB} / \mathrm{km} / \mathrm{g} / \mathrm{m}^{3}$, and

$M=$ liquid-water content in $\mathrm{g} / \mathrm{m}^{3}$.

Values of $K_{1}$ by ice and water clouds are given for various wavelengths and temperatures by Gunn and East in table 7.6.

TABLe 7.6. One-way attenuation coefficient, $K_{1}$, in clouds in $\mathrm{dB} / \mathrm{km} / \mathrm{gm} / \mathrm{m}^{3}$

\begin{tabular}{|c|c|c|c|c|c|}
\hline \multirow{2}{*}{\multicolumn{2}{|c|}{$\begin{array}{c}\text { Temperature } \\
\left({ }^{\circ} \mathbf{C}\right)\end{array}$}} & \multicolumn{4}{|c|}{ Wavelength (cm) } \\
\hline & & 0.9 & 1.24 & 1.8 & 3.2 \\
\hline Water cloud & $\begin{array}{r}20 \\
10 \\
0 \\
-8\end{array}$ & $\begin{array}{r}0.647 \\
.681 \\
.99 \\
1.25\end{array}$ & $\begin{array}{r}0.311 \\
.406 \\
.532 \\
.684\end{array}$ & $\begin{array}{r}0.128 \\
.179 \\
.267 \\
.34 \\
\text { (extrapolated) }\end{array}$ & $\begin{array}{r}0.0483 \\
.0630 \\
.0858 \\
.112 \\
\text { (extrapolated) }\end{array}$ \\
\hline Ice cloud & $\begin{array}{r}0 \\
-10 \\
-20\end{array}$ & $\begin{array}{l}\text { 8. } 74 \times 10^{-3} \\
2.93 \times 10^{-3} \\
2.0 \times 10^{-3}\end{array}$ & $\begin{array}{l}\text { 6. } 35 \times 10^{-3} \\
2.11 \times 10^{-3} \\
1.45 \times 10^{-3}\end{array}$ & $\begin{array}{l}4.36 \times 10^{-3} \\
1.46 \times 10^{-3} \\
1.0 \times 10^{-3}\end{array}$ & $\begin{array}{l}2.46 \times 10^{-3} \\
8.19 \times 10^{-3} \\
\text { 5. } 63 \times 10^{-4}\end{array}$ \\
\hline
\end{tabular}

Several important facts are demonstrated by table 7.6. The decrease in attenuation with increasing wavelength is elearly shown. The values ehange by about an order of magnitude for a change of $\lambda$ from 1 to $3 \mathrm{em}$. The data presented here also show that attenuation inereases with decreasing temperature. Ice clouds give attenuations about two orders of magnitude smaller than water elouds of the same water content. The attenuation of microwaves by ice clouds ean be neglected for all practical purposes [20]. The comprehensive works of Gunn and East [15] and Battan [20] on attenuation offer excellent sources of detailed information on this subject. 


\subsection{Attenuation by Rain}

Ryde and Ryde [21] caleulated the effects of rain on microwave propagation and showed that absorption and scattering effects of raindrops become more pronounced at the higher microwave frequencies where the wavelength and the raindrop diameters are more nearly comparable. In the $10 \mathrm{~cm}$ band and at lower wavelengths the effects are appreciable, but at wavelengths in excess of $10 \mathrm{~cm}$ the effects are greatly decreased. It is also known that suspended water droplets and rain have an absorption rate in excess of that of the combined oxygen and water vapor absorption [3].

In practice it has been convenient to express rain attenuation as a function of the precipitation rate, $R$, which depends on both the liquid water content and the fall velocity of the drops, the latter in turn depending on the size of the drops.

Ryde studied the attenuation of microwaves by rain and showed that this attenuation in $\mathrm{dB} / \mathrm{km}$ can be approximated by:

$$
K_{R}=K \int_{0}^{r} R(r)^{\alpha} d r
$$

where $\quad K_{R}=$ total attenuation in $\mathrm{dB}$

$K=$ constant

$R(r)=$ rainfall rate

$r \quad=$ length of propagation path in $\mathrm{km}$, and

$\alpha=$ constant.

Laws and Parsons [22] observed the distribution of drop sizes for various rates of fall on a horizontal surface. The higher the rainfall rate, the larger the drops, and also the greater the range in size of the drops. However, in order to derive the size distributions occurring while the drops are falling in the air, each rainfall rate must be divided by the particular velocity of fall appropriate to the corresponding drop diameter. Figure 7.18 shows the resulting distribution in air, expressed as the relative mass of drops of given diameter, for three chosen representative values of precipitation rate, $p$, namely, $2.5 \mathrm{~mm} / \mathrm{hr}, 25 \mathrm{~mm} / \mathrm{hr}$, and 100 $\mathrm{mm} / \mathrm{hr}$ [23]. The excess path loss per mile, according to Ryde, for the three carrier frequency bands of 4,6 , and $11 \mathrm{Gc} / \mathrm{s}$ is shown on figure 7.19 . Figure 7.20 [24] is a seatter diagram showing transmission loss versus rainfall rate. For comparison, Ryde's equation is plotted on figure 7.20.

The greatest uncertainty in predictions of attenuation due to rainfall, using theoretical formulae as a basis for calculation, is the extremely limited knowledge of drop size distribution in rains of varying rates of fall observed under differing climatic and current weather conditions. 


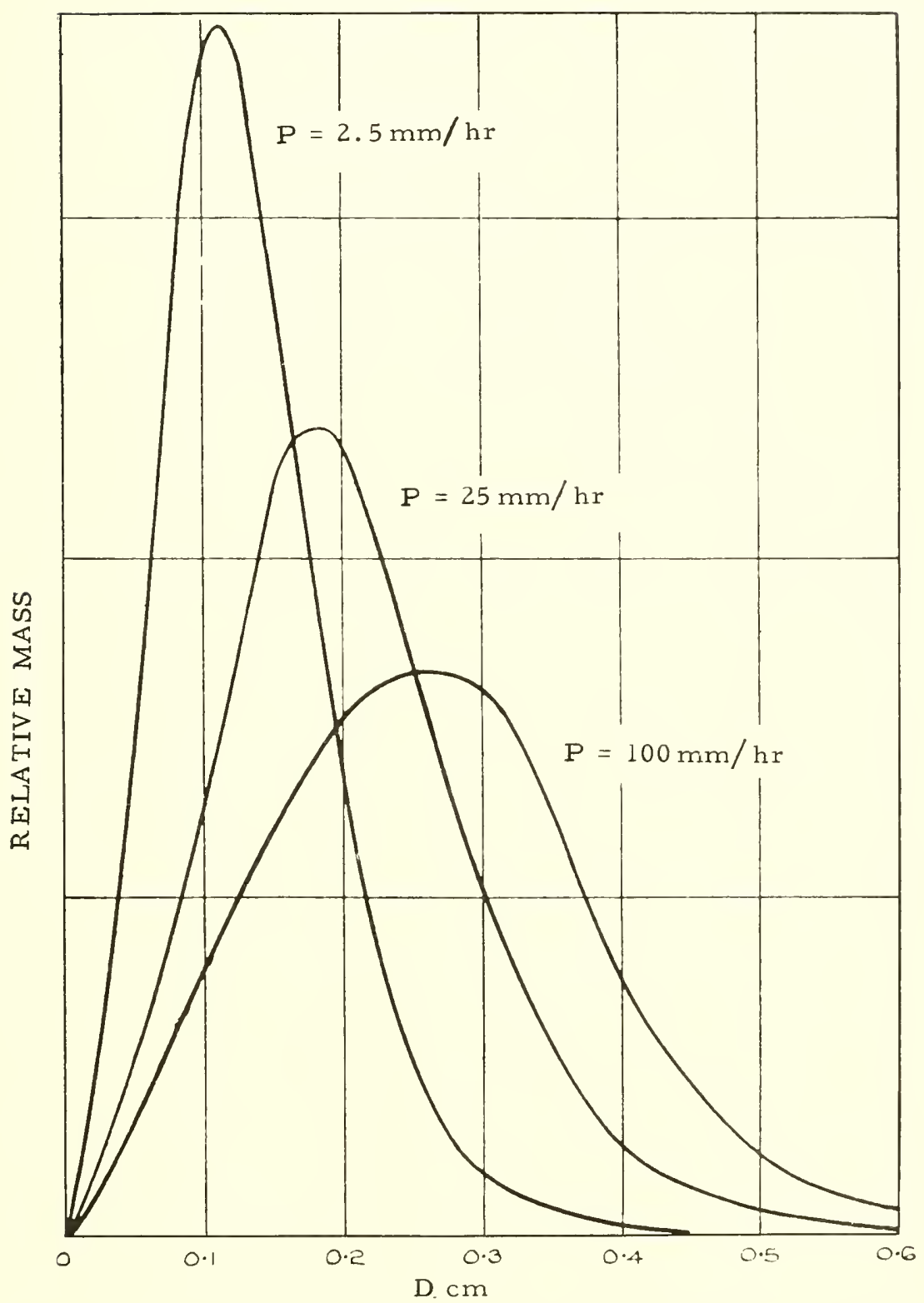

FIGURE 7.18. Relative total mass of liquid water in the air contributed by rain drops of diameter $\mathrm{D}$ for various precipitation rates.

(Derived from Laws and Parsons' distributions for a horizontal surface by dividing by the appropriate terminal vclocities.) 


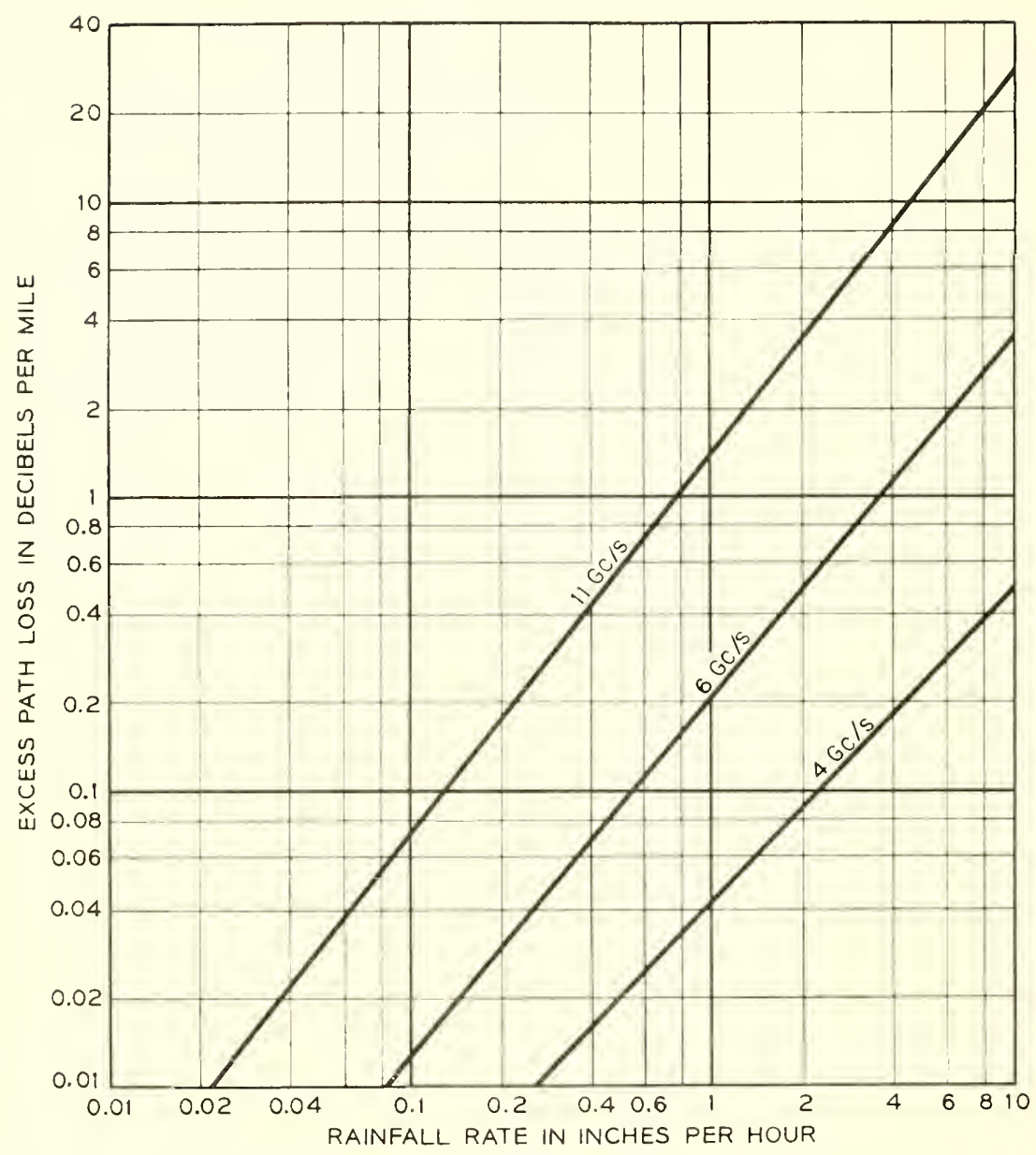

Figure 7.19. Rain attenuation versus rainfall rate.

(Theoretical, after Ryde and Ryde).

There is little evidence that a rain with a known rate of fall has a unique drop-size distribution though studies on this problem seen to indicate that a most probable drop size distribution can be attached to a rain of given rate of fall [22]. Table 7.8 shows the percentage of total volume of rainfall occupied by raindrops of different diameters $(\mathrm{cm})$ and varying rainfall rates $(\mathrm{mm} / \mathrm{hr})$. Tables 7.8 and 7.9 are offered as an aid to estimating, through a qualitative approach, the attenuation of radio waves by raindrops [25].

Table 7.8 gives the decibel attenuation per kilometer in rains of different rates of fall and radio wavelengths between 0.3 and $10 \mathrm{~cm}$. In table 7.9, 
similar to table 7.8 an additional set of results is contained for rains of measured drop size distribution.

Since the total attenuation cross section [26] depends on the temperat ure (because of its effects on the dielectric properties of water), it is important to evaluate the attenuation of rains whose drops are at different temperatures from those in the preceding tables. Table 7.10 contains the necessary data relative to the change of attenuation with temperature and is to be used with table 7.8. For example, in table 7.8, with a rrocipitation rate of $p=0.25 \mathrm{~mm} / \mathrm{hr}$, temperature of $18^{\circ} \mathrm{C}, \lambda=1.25 \mathrm{~cm}$, the attenuation is $0.0215 \mathrm{~dB} / \mathrm{km}$. Using the correction factors obtained from table 7.10 , for the same general conditions of precipitation and wavelength, for a temperature reading of $0^{\circ} \mathrm{C}$, the attenuation reads $0.02043 \mathrm{~dB} / \mathrm{km}$; for a value of $30^{\circ} \mathrm{C}$ an attenuation of $0.019350 \mathrm{~dB} / \mathrm{km}$ is noted; and for a temperature of $40^{\circ} \mathrm{C}$ the attenuation is $0.01742 \mathrm{~dB} / \mathrm{km}$.

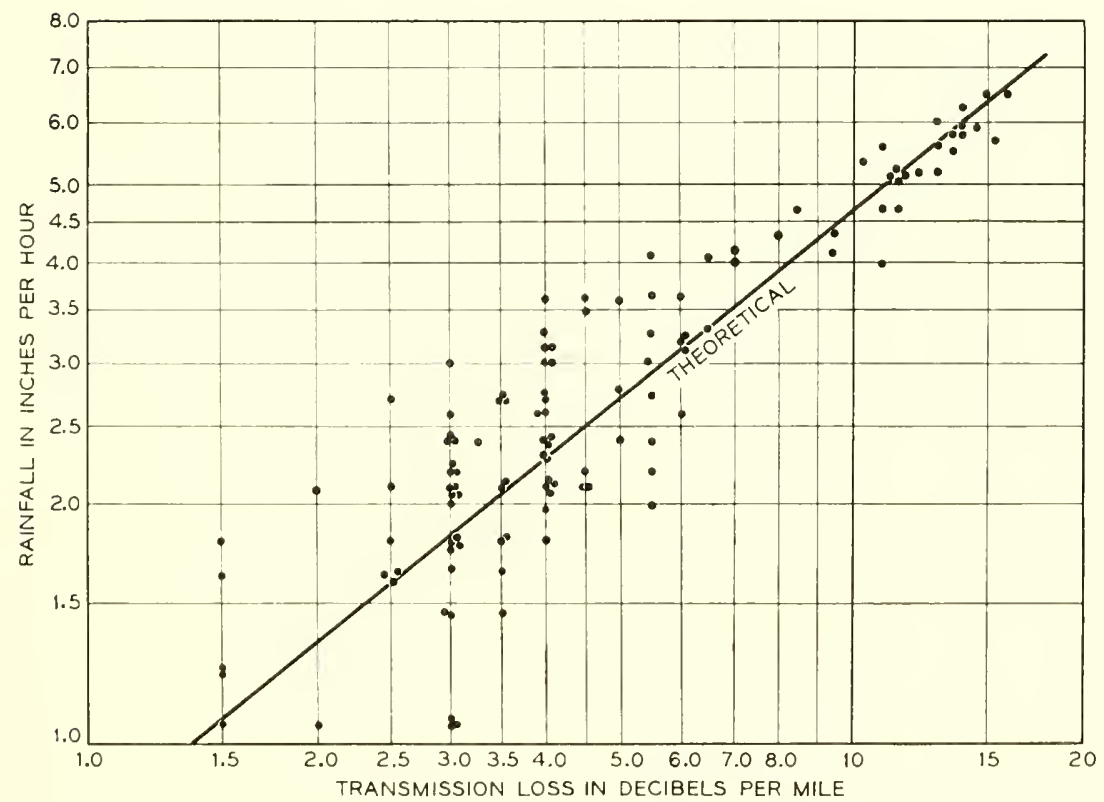

Figure 7.20. Transmission loss in decibels per mile versus rainfall in inches per hour. (After Iathaway and Evans, 1959). 
Table 7.7. Drop size distribution

(Burrows-Attwood)

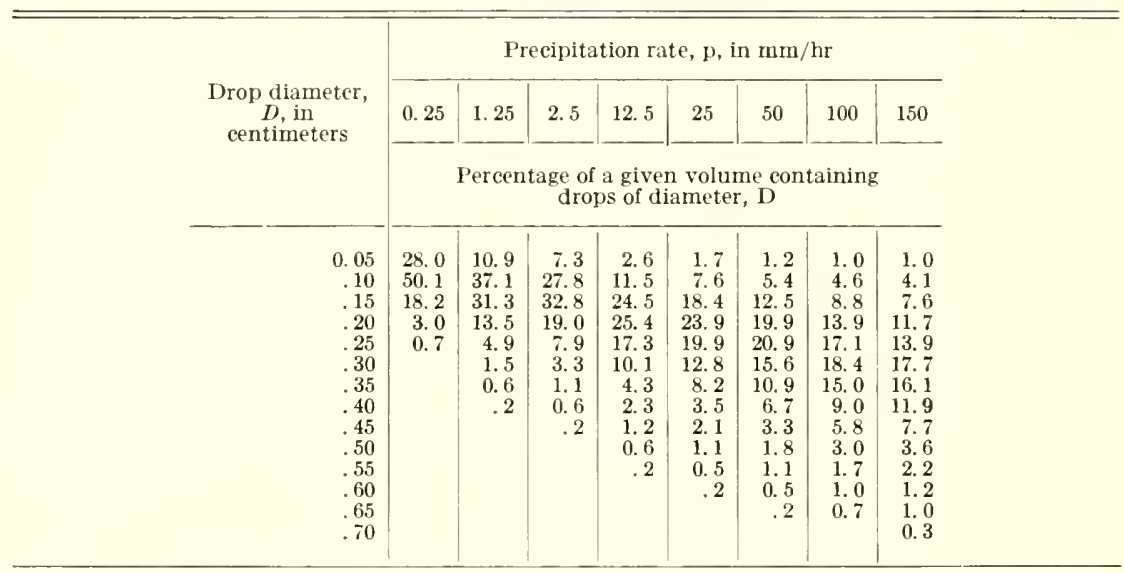

TABLE 7.8. Attenuation in decibels per hilometer for different rates of rain precipitation Temperature $18^{\circ} \mathrm{C}$

(Burrows-Attwood)

\begin{tabular}{|c|c|c|c|c|c|c|c|c|c|}
\hline \multirow{2}{*}{$\begin{array}{c}\text { Precipi- } \\
\text { tation } \\
\text { rate, } p, \\
\text { in } \mathrm{mm} / \mathrm{hr}\end{array}$} & \multicolumn{9}{|c|}{ Wavelength, $\lambda$ in $\mathrm{cm}$} \\
\hline & $\lambda=0.3$ & $\lambda=0.4$ & $\lambda=0.5$ & $\lambda=0.6$ & $\lambda=1.0$ & $\lambda=1.25$ & $\lambda=3.0$ & $\lambda=3.2$ & $\lambda=10$ \\
\hline $\begin{array}{l}0.25 \\
1.25 \\
2.5 \\
12.5 \\
25.0 \\
50 . \\
100 . \\
150 .\end{array}$ & $\begin{array}{l}0.305 \\
1.15 \\
1.98 \\
6.72 \\
11.3 \\
19.2 \\
33.3 \\
46.0\end{array}$ & $\begin{array}{l}0.230 \\
0.929 \\
1.66 \\
6.04 \\
10.4 \\
17.9 \\
31.1 \\
43.7\end{array}$ & $\begin{array}{l}0.160 \\
0.720 \\
1.34 \\
5.36 \\
9.49 \\
16.6 \\
29.0 \\
40.5\end{array}$ & $\begin{array}{l}0.106 \\
0.549 \\
1.08 \\
4.72 \\
8.59 \\
15.3 \\
27.0 \\
37.9\end{array}$ & $\begin{array}{l}0.037 \\
0.228 \\
0.492 \\
2.73 \\
5.47 \\
10.7 \\
20.0 \\
28.8\end{array}$ & $\begin{array}{l}0.0215 \\
0.136 \\
0.298 \\
1.77 \\
3.72 \\
7.67 \\
15.3 \\
22.8\end{array}$ & $\begin{array}{l}0.00224 \\
0.0161 \\
0.0388 \\
0.285 \\
0.656 \\
1.46 \\
3.24 \\
4.97\end{array}$ & $\begin{array}{l}0.0019 \\
0.0117 \\
0.0317 \\
0.238 \\
0.555 \\
1.26 \\
2.80 \\
4.39\end{array}$ & $\begin{array}{l}0.0000997 \\
0.000416 \\
0.000785 \\
0.00364 \\
0.00728 \\
0.0149 \\
0.0311 \\
0.0481\end{array}$ \\
\hline
\end{tabular}

TABLE 7.9. Attenuation in rains of known drop size distribution and rate of fall (decibels per kilometer)

(Burrows-Attwood)

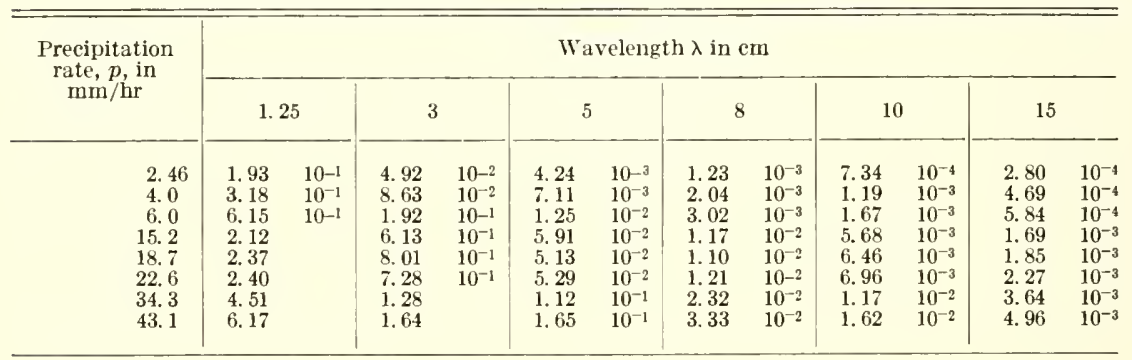


TABLE 7.10 Correction factor (multiplicative) for rainfall attenuation

(Burrows-Attwood)

\begin{tabular}{|c|c|c|c|c|c|c|}
\hline $\begin{array}{l}\text { Precipitation } \\
\text { rate, } p \text {, in } \\
\mathrm{mm} / \mathrm{hr}\end{array}$ & $\lambda \mathrm{cm}$ & $0^{\circ} \mathrm{C}$ & $10^{\circ} \mathrm{C}$ & $18^{\circ} \mathrm{C}$ & $30^{\circ} \mathrm{C}$ & $40^{\circ} \mathrm{C}$ \\
\hline \multirow[t]{4}{*}{0.25} & 0.5 & 0.85 & 0.95 & 1. 0 & 1.02 & 0.99 \\
\hline & 1. 25 & .95 & 1. 00 & 1.0 & 0.90 & .81 \\
\hline & 3.2 & 1. 21 & 1. 10 & 1. 0 & .79 & .55 \\
\hline & 10.0 & 2.01 & 1.40 & 1.0 & 70 & .59 \\
\hline \multirow{4}{*}{2.5} & 0.5 & 0.87 & 0.95 & 1.0 & 1.03 & 1.01 \\
\hline & 1. 25 & .85 & .99 & 1. 0 & 0.92 & 0.80 \\
\hline & 3. 2 & .82 & 1. 01 & 1.0 & .82 & .64 \\
\hline & 10.0 & 2.02 & 1. 40 & 1.0 & 70 & 59 \\
\hline \multirow[t]{4}{*}{12.5} & 0.5 & 0.90 & 0.96 & 1.0 & 1.02 & 1.00 \\
\hline & 1. 25 & .83 & .96 & 1.0 & 0.93 & 0.81 \\
\hline & 3.2 & .64 & .88 & 1.0 & .90 & .70 \\
\hline & 10.0 & 2.03 & 1.40 & 1.0 & 70 & 59 \\
\hline \multirow[t]{4}{*}{50.0} & 0.5 & 0.94 & 0.98 & 1.0 & 1.01 & 1. 00 \\
\hline & 1.25 & .84 & .95 & 1.0 & 0.95 & 0.83 \\
\hline & 3. 2 & .62 & .87 & 1.0 & .99 & .81 \\
\hline & 10.0 & 2.01 & 1.40 & 1.0 & 70 & 58 \\
\hline \multirow[t]{4}{*}{150.} & 0.5 & 0.96 & 0.98 & 1.0 & 1.01 & 1.00 \\
\hline & 1. 25 & 86 & .96 & 1.0 & 0.97 & 0.87 \\
\hline & 3.2 & .66 & .88 & 1.0 & 1.03 & .89 \\
\hline & 10.0 & 2.00 & 1.40 & 1.0 & 0.70 & .58 \\
\hline
\end{tabular}

\subsection{Rainfall Attenuation Climatology}

The above paragraphs have been concerned with a descriptive presentation of the theoretical and technical background of the problem of power loss due to attenuation by rain and atmospheric gases. In an attempt to circumvent the difficulties of the above methods of attenuation prediction it was considered important to try a climatological approach to this problem. However, the results of such a study were disappointing due to the fact that the problems of a systematic chimatological estimation of rainfall attenuation are many and varied. Answers are needed for such questions as; how often do various rainfall rates and drop sizes oceur in geographical areas and over how large an area do these rate and drop size statistics apply? Furthermore, to what height in the atmosphere do these data apply [1]?

Unfortunately, the present state of meteorological knowledge concerning these problems in such that no conclusions can be drawn on a systematic and climatological basis. In view of these facts, it appears prudent at this time to provide only engineering estimates of the combined gaseous and rain absorption.

In this regard, Bussey [1] has shown that the absorption due to rainfall exceeds that of gaseous constituents about 5 percent of the time for frequencies around $6,000 \mathrm{Mc} / \mathrm{s}$. The 5 percent figure was obtained by a study of the rainfall rate distribution for various locations in the United States. Figure 7.21 shows the combined rain and gaseous absorption to be exceeded 1 per cent of the time. 


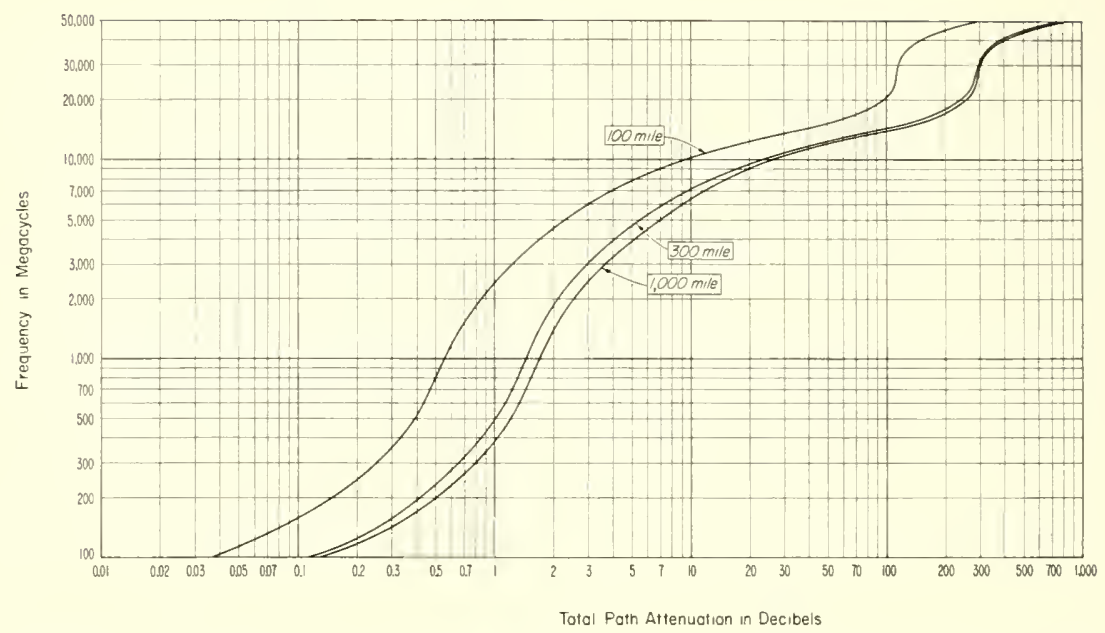

Figure 7.21. Combined rain and gaseous absorption to be exceeded 1 percent of the time.

\subsection{Rain Attenuation Effects on Radio Systems Engineering}

Attenuation due to rainfall is obviously a dominating factor in determining the reliability of a communications system, especially at frequencies in excess of $30 \mathrm{Gc} / \mathrm{s}$. Rain varies greatly in frequency of occurrence from one region to another, so it is important to have an effective method of predicting the performance of a radio system in any region in order that the communications engineer will be able to gain the widest possible application and degree of reliability, consistent with cost, in any system of his design.

This section will be concerned, in the main, with the results of the Bell System's [24] field experiment in the Mobile, Ala., area, which was designed to establish a relationship between excess path attenuation and instantaneous rate of rainfall and to seek out any relationship between the profile of rate of rainfall along a radio path and rainfall measured at a point.

The main problem concerns the ability to predict outage time due to rainfall (time the system noise exceeds the system objective) at $11 \mathrm{Gc} / \mathrm{s}$ in all areas of the country. This is obviously a difficult problem since, due to reasons of cost, it is not feasible to measure rainfall attenuation in all parts of the country. Therefore, it is desirable to be able to use what rainfall data are available and to couple the data, through what are thought to be reasonable assumptions, to the relationships between rainfall and attenuations. 
In approaching the problem of predicting the outage time due to rainfall, it has been assumed that the annual distribution of one-hour point rainfall rates is indicative of the instantaneous values over 30 -mi radio paths [1] and that the frequency of severe rainfall of the type measured in the Mobile area will be reduced in other parts of the country in proportion to the distribution of annual point rates of $1 \mathrm{in.}$ or more rainfall per hour.

Figure 7.22 indicates the expected outage time due to rainfall for various path lengths in different rain areas of the United States. The curves $A$ through $H$ correspond to the areas contoured in figure 7.23 which illustrates contours of constant path length for fixed outage times for different areas of the United States. The longer paths have been somewhat weighted to take into account the less severe rainfall covering larger geographical areas than the intense storms typical of the Gulf region.

In a complete 11-Gc/s Bell System point-to-point relay system, the rain outages of the individual hops must be added to obtain the performance of the system. It is desirable that the individual hops meet the same objective, but this is not always possible. Sometimes one or more

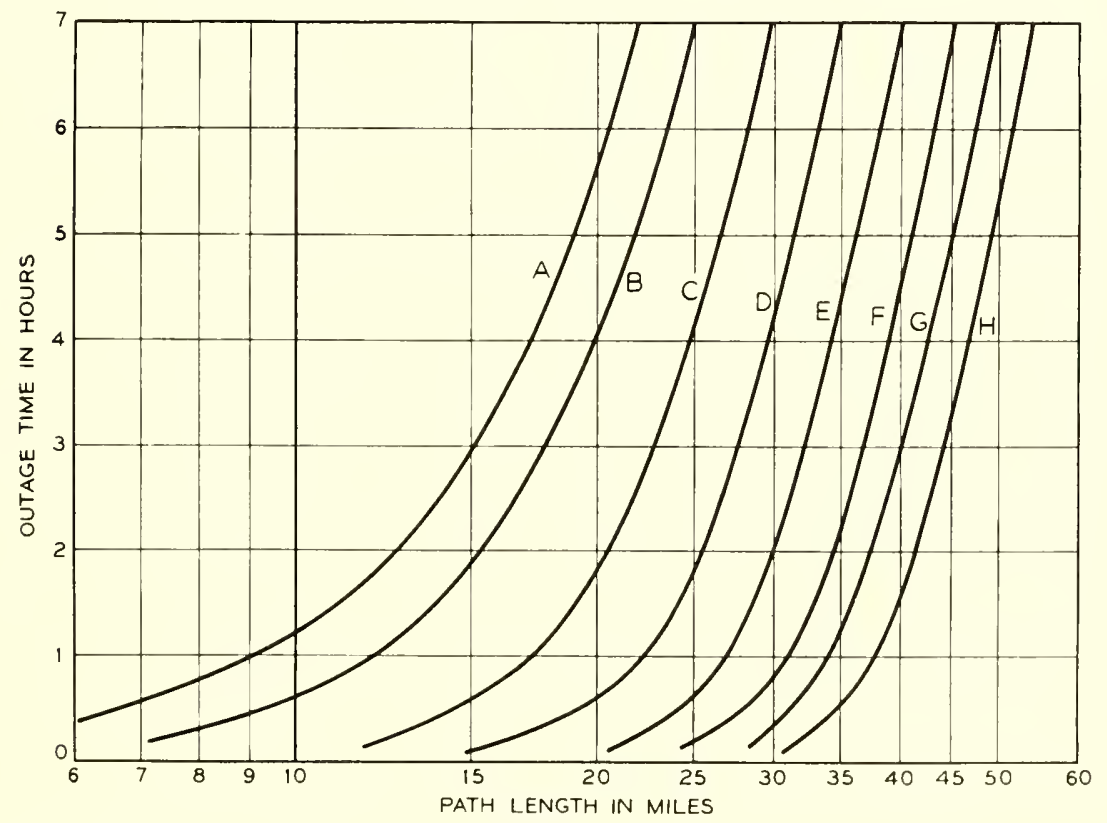

Figure 7.22. Expected outage time in hours per year versus path length in miles for various areas of the United States.

(After llathaway and Evans, 1959).

The eurves A through 11 correspond to the areas contoured in figure 7.23. 


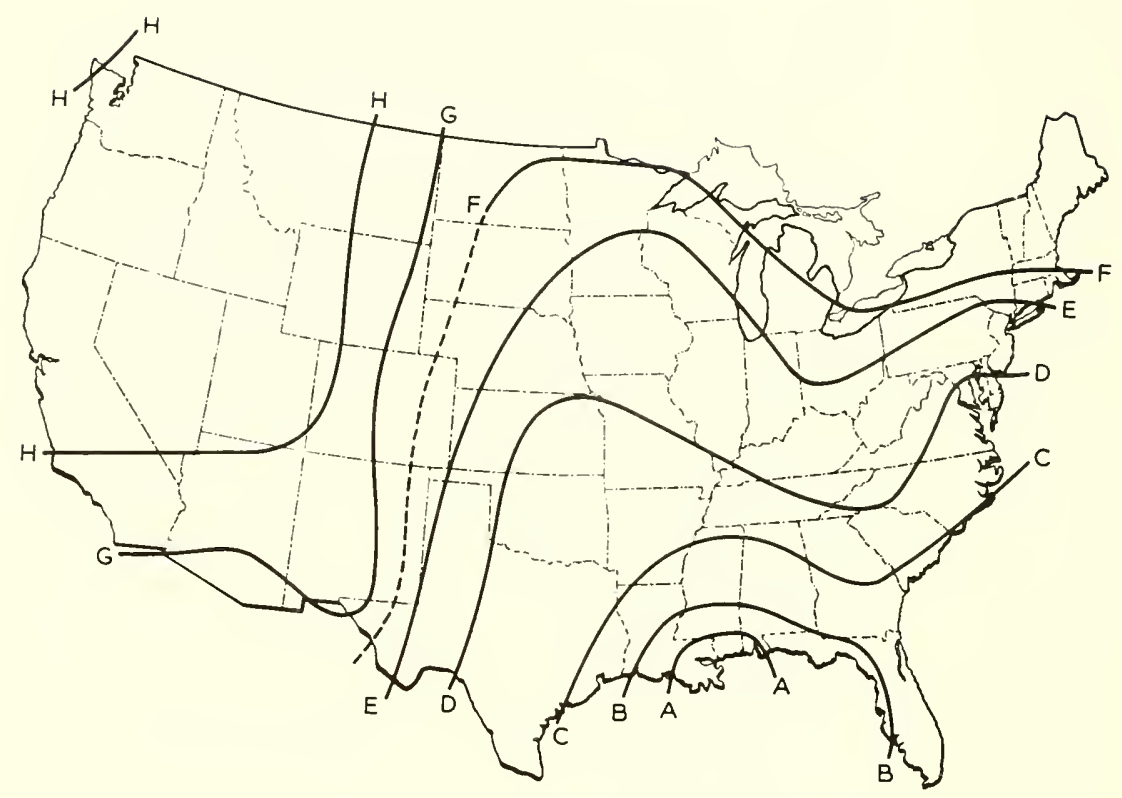

Figure 7.23. Contours of constant path length for fixed outage time. (After Hathaway and Evans, 1959).

hops of a system are electrically long; they will have insufficient fading margin (the number of $\mathrm{dB}$ the receiver input level can be reduced before the noise exceeds the system objective) and hence contribute more than their share of the outage time. So, this excess must be made up by imposing tighter requirements on the remaining hops. To meet the overall objective of the Bell System, it is necessary to know the contributions of the long hops-those having a fading margin less than $40 \mathrm{~dB}$. Figure 7.24 shows the excess path loss due to rain, versus hours per year for the Mobile area study. Since the shape of this curve is nearly identical to Bussey's curve of cumulative distribution for point rates in Washington, D.C. (if we assume the shape of this curve to be representative for other areas of the country), then the additional outage time for path lengths given by figure 7.23 can be estimated for hops having a fading margin less than $40 \mathrm{~dB}$. The data shown on figure 7.24 have been rationalized and are shown in figure 7.25 as an estimate of additional outage time.

Sometimes it is practical to shorten a proposed path to bring the fading margin up to $40 \mathrm{~dB}$. An approximation of the necessary reduction path length can be made if uniform rainfall rate is assumed over the path. 


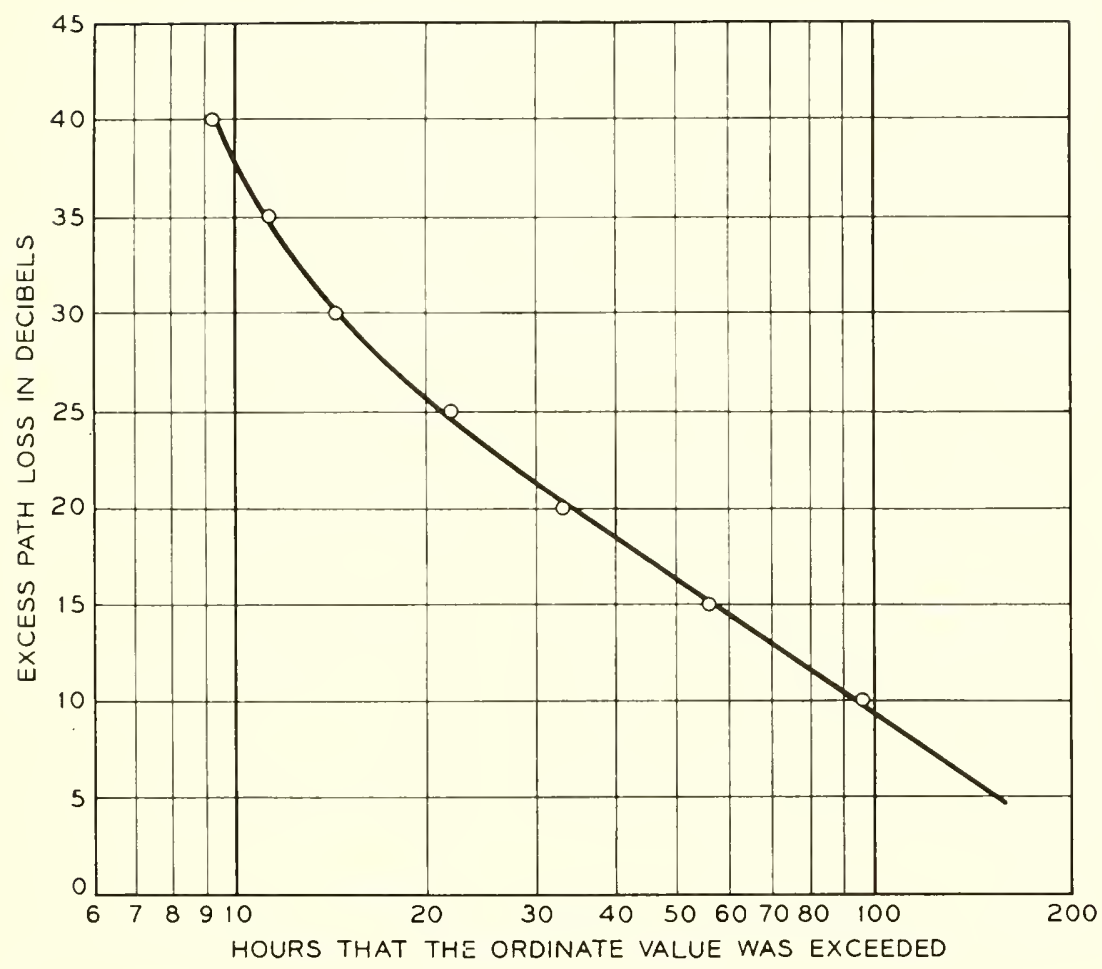

Figure 7.24. Excess path loss due to rainfall versus hours per year at Mobile, Ala. (After Hathaway and Evans, 1959).

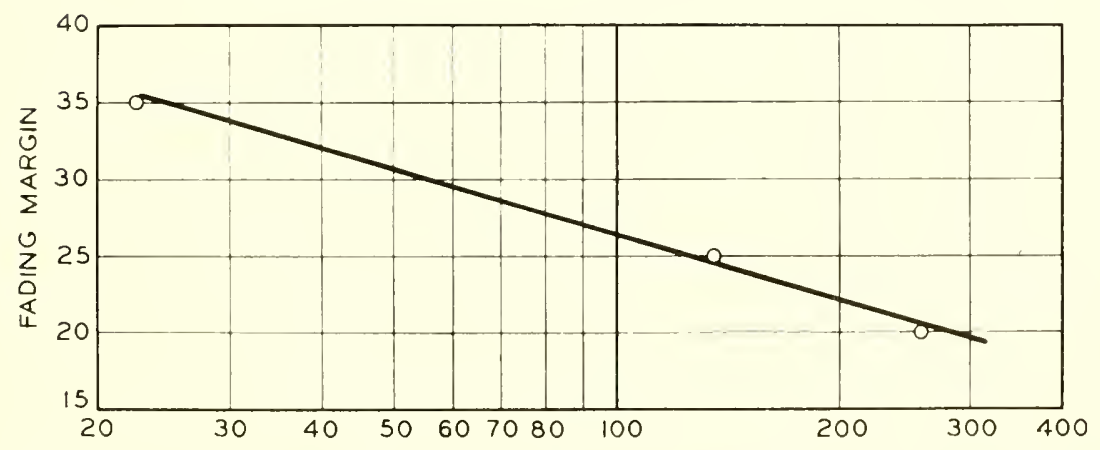

FIgURE 7.25. Additional outage time expected for $11 \mathrm{Gc} / \mathrm{s}$ systems having a fading margin less than $40 \mathrm{~dB}$. 
Under this condition the attenuation due to rainfall should be directly proportional to the path length. Thus the path length in figure 7.24 can be shortened to correct for insufficient fading margin.

Bell System results indicate that for their microwave relay links in the extreme southeastern region of the United States rainfall will limit 11 $\mathrm{Gc} / \mathrm{s}$ radio systems having a $40 \mathrm{~dB}$ fading margin to path lengths of approximately 10 to $15 \mathrm{mi}$, depending on the number of hops, if normal reliability objectives are to be met. Path lengths of 20 to $30 \mathrm{mi}$ should be acceptable in the central area and paths as long as $35 \mathrm{mi}$ should be acceptable in the northwestern part of the country. However, in their existing point-to-point radio relay systems, the paths average about 23 mi due to other considerations than those of propagation. It appears that the $11 \mathrm{Gc} / \mathrm{s}$ systems will not be penalized unduly except in the southeastern part of the United States.

An illustration of the correlation between the rainfall and path loss on March 15, 1956, of the Mobile study is presented in figure 7.26 in support of the above conclusions.

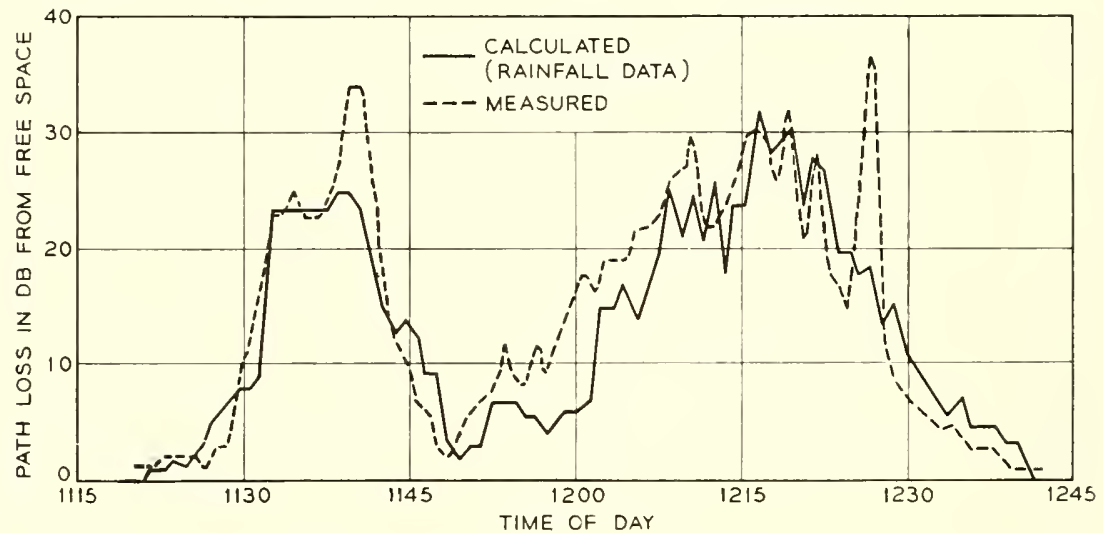

Figure 7.26. Correlation between rainfall and path loss, March 15, 1956. (After Hathaway and Evans, 1959).

\subsection{Attenuation by Hail}

Ryde concluded that the attenuation caused by hail is one-hundredth that caused by rain, that ice crystal clouds cause no sensible attenuations, and that snow produces very small attenuation even at the excessive rate of fall of $5 \mathrm{in}$. an hour. However, the scattering by spheres surrounded by a concentric film of different dielectric constant does not give the same effect that Ryde's results for dry particles would indicate [24, 27]. For example, when one-tenth of the radius of an ice sphere of radius $0.2 \mathrm{~cm}$ melts, the scattering of $10 \mathrm{~cm}$ radiation is approximately 90 percent of the value which would be scattered by an all-water drop. 
At wavelengths of 1 and 3 cm with $\gamma=0.126(\gamma=2 a / \lambda ; a=$ ladius of drop) Kerker, Langleben, and Gunn [27] found that particles attained total-attenuation cross sections corresponding to all-melted particles when less than 10 percent of the ice particles were melted. When the melted mass reached about 10 to 20 percent, the attenuation was about twice that of a completely melted particle. These calculations show that the attenuation in the melting of ice immediately under the $0{ }^{\circ} \mathrm{C}[28]$ isotherm can be substantially larger than in the snow region just above, and under some circumstances, greater than in the rain below the melting level. Further melting cannot lead to much further enhancement, apparently, and may lead to a lessening of the reflectivity of the particle by bringing it to sphericity or by breaking up of the particle. This effect, combined with the fact that hail has greater terminal velocities than rain, gives rise to the so-called "bright band" near the $0^{\circ}$ isotherm.

\subsection{Attenuation by Fog}

The characteristic feature of a fog is the reduction in visibility. Visibility is defined as the greatest distance in a given direction at which it is just possible to see and identify with the unaided eye (a) in the daytime, a prominent dark object against the sky at the horizon, and (b) at night, a known, preferably unfocused moderately intense light source [29].

Although the visibility depends upon both drop size and number of drops and not entirely upon the liquid-water content, yet, in practice, the visibility is an approximation of the liquid-water content, and therefore, may be used to estimate radio-wave attenuation [28]. On the basis of Ryde's work, Saxton and Hopkins [30] give the figures in table 7.11 for the attenuation in a fog or clouds at $0{ }^{\circ} \mathrm{C}$ temperature. The attenuation varies with the temperature because the dielectric constant of water varies with temperature; therefore, at 15 and $25^{\circ} \mathrm{C}$ the figures in table 7.11 should be multiplied by 0.6 and 0.4 respectively. It is immediately noted that cloud or fog attenuation is an order of magnitude greater at $3.2 \mathrm{~cm}$ than at $10 \mathrm{~cm}$. Nearly another order of magnitude increase occurs between $3.2 \mathrm{~cm}$ and $1.25 \mathrm{~cm}$.

TABLE 7.11. Attenuation caused by clouds or fog [30]

Temperature $=0^{\circ} \mathrm{C}$

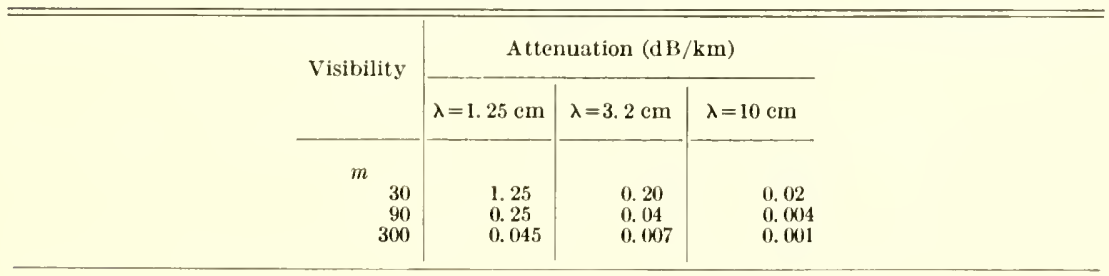




\subsection{Thermal Noise Emitted by the Atmosphere}

General laws of thermodynamics relate the absorption characteristics of a medium to those of emission. Good absorbers of radiation are also good emitters, and vice versa. Thus, in the microwave region, the atmosphere is also a good emitter, as well as a strong absorber, of radiation. We may, therefore, describe quantitatively both emission and absorption by the same parameter; namely, the absorption coefficient.

The emission characteristics of any real body at a fixed frequency may be compared to those of a blackbody at the same temperature. In the microwave region, the noise intensity emitted by a blackbody is given by the Rayleigh-Jeans law:

$$
\psi(\nu)=2 k T\left(\frac{\nu}{c}\right)^{2}
$$

where $\psi(\nu)=$ emitted blackbody flux density per unit frequency

$\nu \quad=$ frequency

$T=$ absolute temperature, ${ }^{\circ} \mathrm{K}$

$c \quad=$ the velocity of light, and

$k=$ Boltzmann constant $\left(1.38054 \times 10^{-16} \mathrm{erg} /{ }^{\circ} \mathrm{K}\right)$

The emission per unit length along an actual ray path may now be expressed as

$$
B(\nu)=\gamma(\nu) \psi(\nu)
$$

where $\gamma(\nu)=$ attenuation per unit length. Remembering that the fraction of energy absorbed in a path length, $d s$, is given by the optical depth, $d \tau=\gamma(\nu) d s$, we may obtain the differential equation for transmission of radiation through the atmosphere:

$$
\frac{d I(\nu)}{d \tau}=-I(\nu)+\psi(\nu)
$$

where $I(\nu)$ is the flux density per unit frequency.

The solution to this radiative transfer equation is

$$
\begin{aligned}
I(\nu)=\sum_{m} I_{m}(\nu) \exp & \left(-\int_{\tau_{s}}^{\tau_{r_{m}}} d \tau\right) \\
& +\int_{\tau_{s}}^{\tau_{\infty}} \psi(\nu) \exp \left(-\int_{\tau_{s}}^{\tau_{r}} d \tau\right) d \tau
\end{aligned}
$$


where the summation extends over all discrete noise sources which may be present, $I_{m}(\nu)$ is the unattenuated flux density transmitted from the $m$ th discrete source located at position $r_{m}, s$ is the point of reception of energy, and the other symbols have their previous meaning. It should be recognized that the above integrals extend over a ray path determined by the refractive properties of the medium and cannot be evaluated unless these refractive properties are known.

In analogy to the temperature dependence of the noise energy as by the Rayleigh-Jeans law, we may, in the microwave region, relate the intensity of radiation received from a particular direction, $I(\nu)$, to an equivalent temperature, $T_{n}(\nu)$, by the following relation

$$
I(\nu)=\frac{2 k T_{n}(\nu)}{\lambda^{2}}
$$

or, from $(7.8)$

$$
\begin{aligned}
T_{n}(\nu)=\sum_{m} T_{n, m}(\nu) \exp \left(-\int_{\tau_{s}}^{\tau_{r_{m}}} d \tau\right) & \\
& +\int_{\tau_{s}}^{\tau_{\infty}} T \exp \left(-\int_{\tau_{s}}^{\tau_{r}} d \tau\right) d \tau .
\end{aligned}
$$

This equivalent temperature is called the thermal noise temperature.

It is apparent that the thermal noise temperature of the atmosphere, as measured by an antenna, will depend explicitly upon the antenna angle and the frequency, and implicitly upon the atmospheric conditions along the ray path giving rise to attenuation and emission of energy. It seems plausible, therefore, that one could exploit this dependence of thermal noise on atmospheric conditions as a probe of atmospherie structure.

Thermal noise is equally important in communications receiving, since it represents the lowest possible noise level that ean be attained by an antenna immersed in the atmosphere. This minimum noise level will, of course, vary, depending on atmospheric conditions, the frequency, and the antenna orientation. For example, in the microwave region, the antenna noise temperature at vertical orientation may be as low as $1^{\circ} \mathrm{K}$, and in a horizontal position, where more of the lower layer's are "seen" the noise temperature may be of the same order as the actual temperature of the lower atmosphere; i.e., around $280^{\circ} \mathrm{K}$. Figure 7.27 shows sky temperature as frequency for various antenna angles for mean atmospheric conditions at Bismarek, N. Dak., during February 1940-43. 


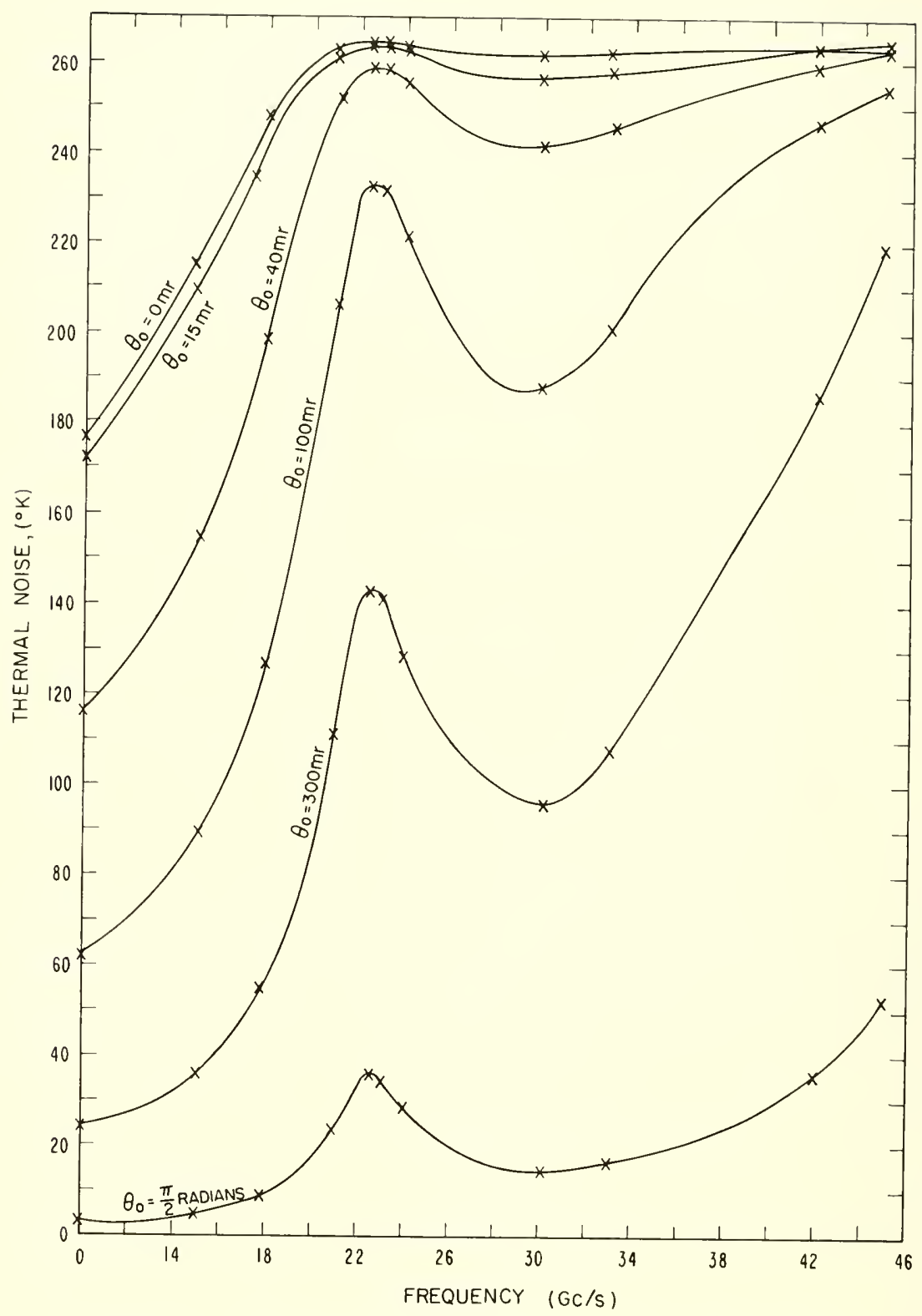

Figure 7.27. Thermal noise versus frequency for mean profile conditions at Bismarck, N.Dak. 
TABLE 7.12

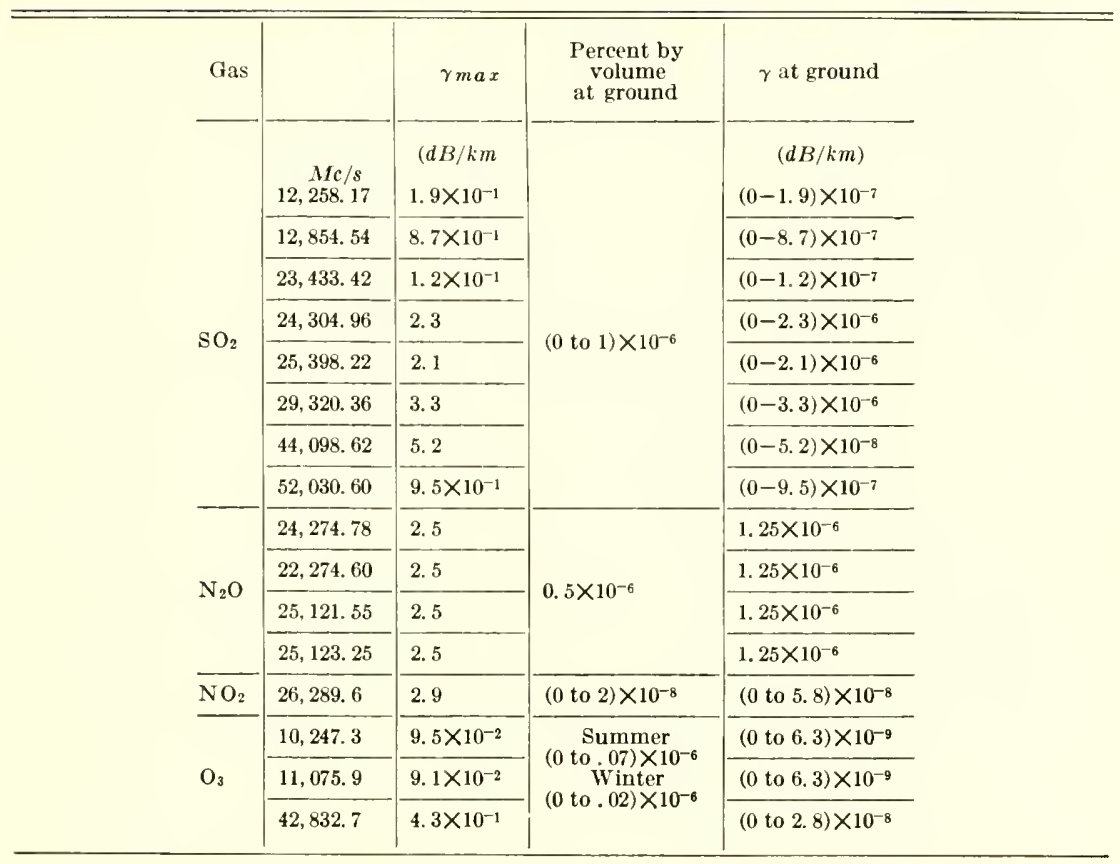

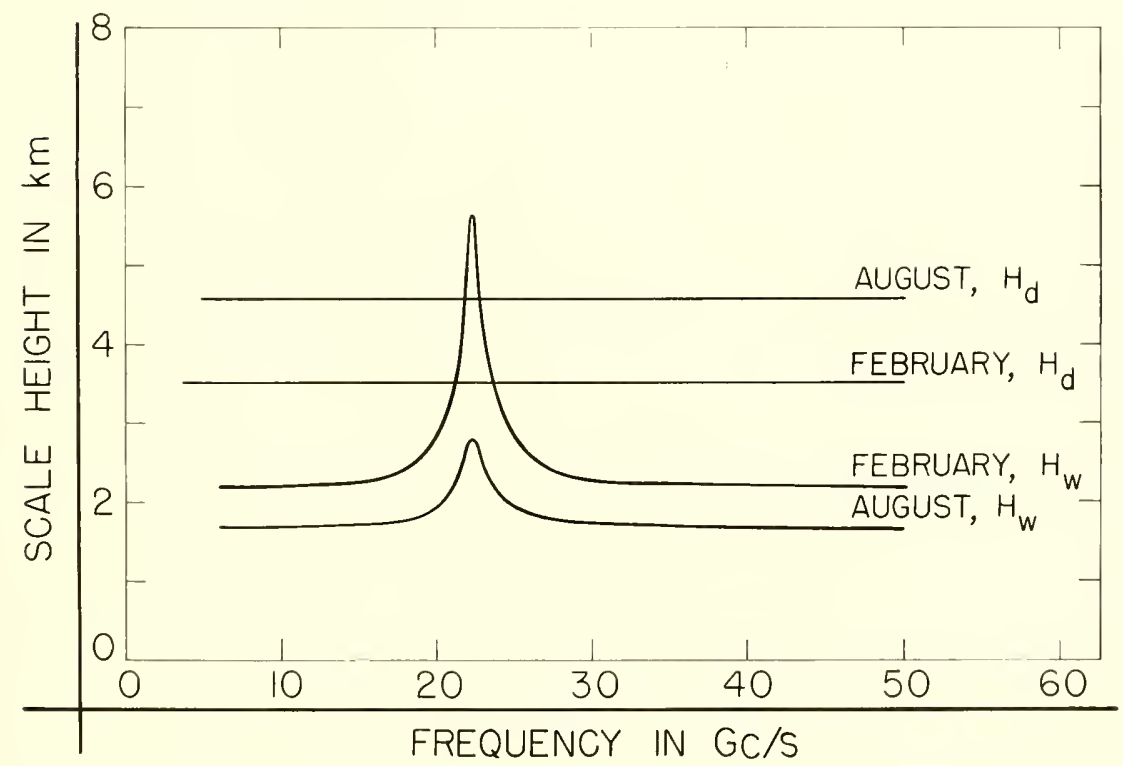

Figure 7.28. Variations with frequency of the scale heights of the bi-exponential absorption model at Verkhoyansk, U.S.S.R. 


\subsection{References}

[1] Bussey, H. E. (Aug. 1950), Microwave attenuation statistics estimated from rainfall and water vapor, Proc. IRE 38, No. 7, 781.

[2] Davidson, D., and A. Pote (Dec. 1955), Designing over-horizon communication links, Electronics, 28, 126.

[3] Bean, B. R., and R. Abbott (May-Aug. 1957), Oxygen and water vapor absorption of radio waves in the atmosphere, Geofis. Pura Appl. 37, 127-144.

[4] Van Vleck, J. H. (Apr. 1947), Absorption of microwaves by oxygen, Phys. Rev. 71, 413-424.

[5] Van Vleck, J. H. (Apr. 1947), The absorption of microwaves by uncondensed water vapor, Phys. Rev. 71, 425-433.

[6] Van Vleck, J. H. (1951), Theory of absorption by uncondensed gases, Book, Propagation of Short Radio Waves, pp. 646-664 (McGraw-Hill Book Co., Inc., New York, N.Y.).

[7] Becker, G. B., and S. H. Autler (Sept. 1, 15, 1946), Water vapor absorption of electromagnetic radiation in the centimeter wavelength range, Phys. Rev. 70, Nos. 5 and $6,300-307$.

[8] Birnbaum, G., and A. A. Maryott (Sept. 15, 1955), Microwave absorption in compressed oxygen, Phys. Rev. 99, 1886.

[9] Artman, J. O., and J. P. Gordon (Dec. 1954), Absorption of microwaves by oxygen in the millimeter wavelength region, Phys. Rev. 96, No. 5, 1237-1245.

[10] Tinkham, M., and M. W. P. Strandberg (July 15, 1955), Line breadths in the microwave magnetic resonance spectrum of oxygen, Phys. Rev. 99, No. 2, $537-539$.

[11] Hill, R. M., and W. Gordy (Mar. 1954), Zeeman effect and line breadth studies of the microwave lines of oxygen, Phys. Rev. 93, 1019.

[12] Artman, J. O. (1953), Absorption of microwaves by oxygen in the millimeter wavelength region, Columbia Radiation Lab. Rept. (Columbia Univ. Press, New York, N.Y.).

[13] Straiton, A. W., and C. W. Tolbert (May 1960), Anomalies in the absorption of radio waves by atmospheric gases, Proc. IRE 48, No. 5, 898-903.

[14] Tolbert, C. W., and A. W. Straiton (Apr. 1957), Experimental measurement of the absorption of millimeter radio waves over extended ranges, IRE Trans. Ant. Prop. AP-5, No. 2, 239-241.

[15] Gunn, K. L. S., and T. W. R. East (Oct.-Dec. 1954), The microwave properties of precipitation particles, Quart. J. Roy. Meteorol. Soc. 80, 522-545.

[16] Bean, B. R., and B. A. Cahoon (Sept. 1957), A note on the climate variation of absolute humidity, Bull. Am. Meteorol. Soc. 28, No. 7, 395-398.

[17] Ratner, B. (1945), Upper air average values of temperature, pressure, and relative humidity over the United States and Alaska (U.S. Weather Bureau).

[18] Donaldson, Ralph J., Jr. (June 1955), The measurement of cloud liquid-water content by radar, J. Meteorol. 12, No. 3, 238-244.

[19] Weickmann, H. K., and H. J. aufm Kampe (June 1953), Physical properties of cumulus clouds, J. Meteorol. 10 204-221.

[20] Battan, L. J. (1959), Radar Meteorology, Book, p. 43 (Univ. of Chicago Press, Chicago, Ill.).

[21] Ryde, J. W., and D. Ryde (1945), Attenuation of centimeter waves by rain, hail, fog, and clouds (General Electric, Wembly, England).

[22] Laws, J. O., and D. A. Parsons (Apr. 1943), The relationship of raindrop size to intensity, Trans. Am. Geophys. Union, 24th Annual Meeting, 452-460.

[23] Ryde, J. W. (1946), The attenuation and radar echoes produced at centimetre wave-lengths by various meteorological phenomena, Meteorological Factors in Radio Wave Propagation, pp. 169-188 (The Physical Society, London, England). 
[24] Hathaway, S. D., and H. W. Evans (Jan. 1959), Radio attenuation at $11 \mathrm{kMc}$ and some implications affecting relay systems engineering, Bell Syst. Tech. J. 38, No. 1.

[25] Burrows, C. R., and S. S. Atwood (1949), Radio wave propagation, Consolidated Summary Technical Report of the Committee on Propagation, NDRC, p. 219 (Academic Press, Inc., New York, N.Y.).

[26] Mie, G. (1908), Beitrage zur Optik trüber Medien, speziell Kolloidaler Metalläsungen, Ann. Physik, 25, 377.

[27] Kerker, M., M. P. Langleben, and R. L. S. Gunn (Dec. 1951), Seattering of microwaves by a melting spherical ice particle, J. Meteorol. 8, 424.

[28] Best, A. C. (1957), Physics in Meteorology (Pittman and Sons, London, England).

[29] Glossary of Meteorology (1959), Am. Meteorol. Soc. 3, 613.

[30] Saxton, J. A., and H. G. Hopkins (Jan.-Feb. 1951), Some adverse influences of meteorological factors on marine navigational radar, Proc. IRE 98, Pt. III, 26.

[31] Zhevankin, S. A., and V. S. Troitskii (1959), Absorption of centimetre waves in the atmosphere, Radioteknika i Elektronika 4, No. 1, 21-27.

[32] Dutton, E. J., and B. R. Bean (June 1965), The biexponential nature of tropospheric gaseous absorption of radio waves, Radio Sci. J. Res. NBS 69D, No. 6, 885-892.

[33] Ghosh, S. N., and H. D. Edwards (Mar. 1956), Rotational frequencies and absorption coefficients of atmospheric gases, Air Force Surveys in Geophysics (Air Force Cambridge Research Center, ARDC, USAF). 



\section{Chapter 8. Applications of Tropospheric Refraction and Refractive Index Models}

\subsection{Concerning the Bi-Exponential Nature of the Tropospheric Radio Refractive Index}

\subsubsection{Introduction and Background}

The recent explosive growth of space science and telecommunications has spurred the development of new models of the tropospheric radio refractive index to account for the systematic refraction of radio waves and the calculation of theoretical radio field strengths at satellite heights. The simple exponential model has been found to represent, to a first approximation, the average refractive index structure within the first few kilometers above the ground for the United States [1], ${ }^{1}$ France [2], and Japan [3]. All of the above investigations have reported varying degrees of departure of the atmosphere from this model and Misme [4] has endeavored to delineate the regions of the world where the exponential model is most applicable, although subsequent analysis of several types of data has shown this model to be more generally applicable than at first sight and not unreasonable for use even in arctic and tropic locations [5].

If one consider's that $N$ is composed of a dry term,

$$
D=\frac{77.6 P}{T}
$$

and a wet term,

$$
W=\frac{3.73 \times 10^{5} e}{T^{2}},
$$

then one may consider the height variation of each term separately. We shall examine the possible advantages of a model of the form

$$
N(z)=D_{0} \exp \left\{-\frac{z}{H_{d}}\right\}+W_{0} \exp \left\{-\frac{z}{H_{w}}\right\}
$$

to describe the average decrease of $N$ with height, where $D_{0}$ and $W_{0}$ are the values of the dry and wet components at the earth's surface and $H_{d}$

\footnotetext{
${ }^{1}$ Figures in brackets indicate the literature references on p. 373.
} 
and $H_{w}$ are the seale heights of $D$ and $W$, respectively. This particular form has been found useful by Katz [6], in his derivation of the potential refractive modulus, and by Zhevankin and Troitskii [7] in their treatment of atmospheric absorption. It would be well for the reader to recall that scale height, as used in this study, is merely the height at which the value of the atmospheric property has decreased to $1 / e$ of its surface value.

Typical values of $D_{0}, W_{0}$, and $N_{0}$ are listed for arctic, temperate, and tropical locations in table 8.1. It is seen that the contribution of $W$ to the total value of $N$ is nearly negligible in the arctic but becomes greater as one passes from temperate to tropical climates. There is, of course, generally an inverse correlation between the magnitude of $D$ and $W$ since, at sea level, where $P \sim 1,000 \mathrm{mbar}$, the low arctic temperature increases the $D$ term and, combined with low atmospheric water vapor capacity, decreases the wet term. Conversely, the higher temperatures of the temperate and tropical climates depress the $D$ term and provide a greater water vapor capacity with the result that $W$ may have a sizable contribution to the total $N$.

TABLE 8.1. Typical average values of the dry and wet components of $\mathrm{N}$

\begin{tabular}{|c|c|c|c|}
\hline Station and climate & $D_{0}$ & $3_{0}$ & $N_{0}$ \\
\hline $\begin{array}{l}\text { Isachsen }\left(78^{\circ} 50^{\prime} \mathrm{N}\right) \text {, aretic } \\
\text { Washington, D.C. }\left(38^{\circ} 50^{\prime} \mathrm{N}\right) \text {, temperate } \\
\text { Canton Island }\left(2^{\circ} 46^{\prime} \mathrm{S}\right) \text {, tropic }\end{array}$ & $\begin{array}{l}332.0 \\
266.1 \\
259.4\end{array}$ & $\begin{array}{r}0.8 \\
58.5 \\
111.9\end{array}$ & $\begin{array}{l}332.8 \\
324.6 \\
371.3\end{array}$ \\
\hline
\end{tabular}

\subsubsection{N Structure in the I.C.A.O. Atmosphere}

One may examine $N$ structure in a standard atmosphere as a guide to its general distribution in the free atmosphere. On this basis the I.C.A.O. standard atmosphere [8] (fig. 8.1) was examined. The conditions specified for this atmosphere are an approximately exponential pressure decrease with respect to height and a linear temperature decrease from ground level to the tropopause. It is evident, then, that in this atmosphere $D$ decreases in an exponential fashion with height.

When these data are converted into refractive index and piotted on semilogarithmic paper, as on figure 8.2. both $D$ and $W$ are seen to display an approximately exponential distribution from the surface to the tropopause. This conclusion is based upon the observation that the distribution is nearly linear as one would expect if one inverted the function

$$
y=A \exp (-h / c)
$$




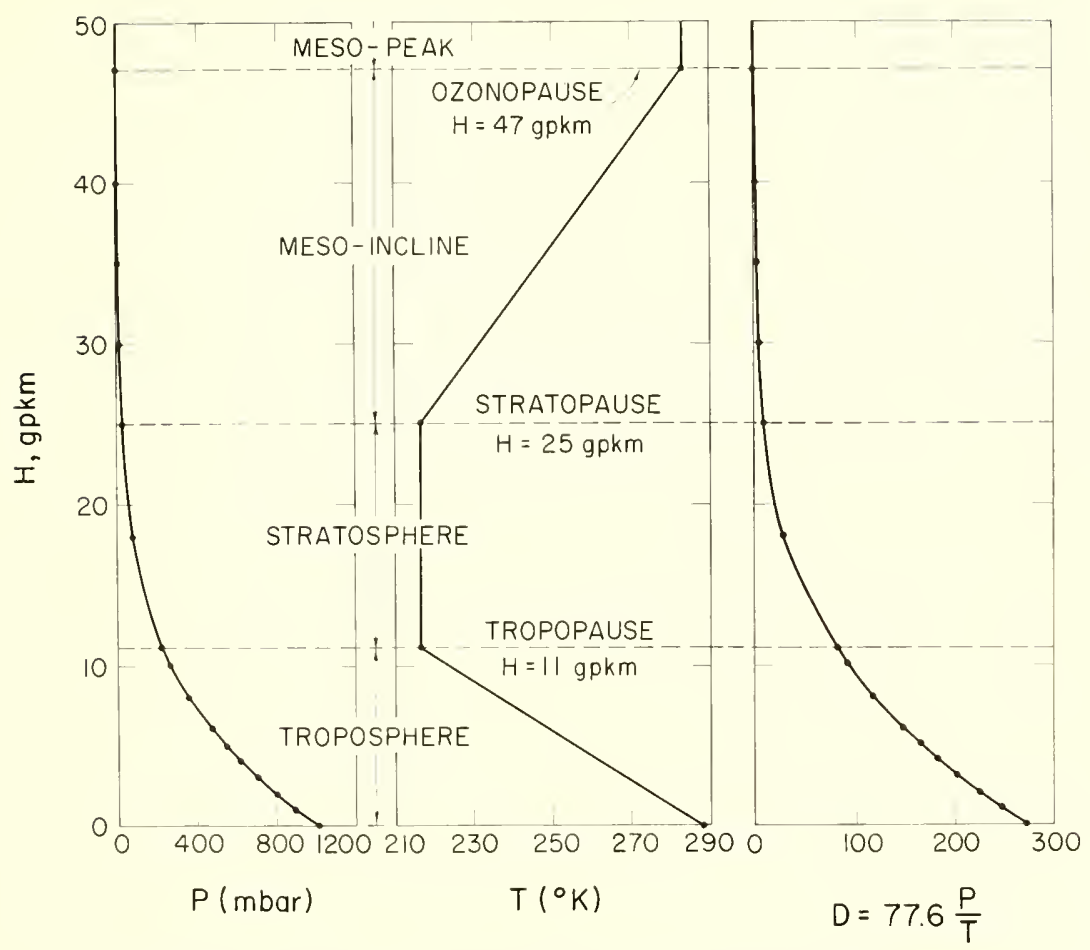

Figure 8.1. The U.S. extension to the I.C.A.O. Standard Atmosphere.

into

$$
\ln y=-h / c+\ln A \text {, }
$$

which is the equation of a straight line on semilogarithmic paper. The exponential distribution of $W$ with height in this atmosphere follows naturally from the definition of constant relative humidity, since the saturation vapor pressure, $e_{s}$, is itself, to a first approximation, an exponential function of temperature. It is evident that the value of $W$ can significantly affect the surface values of $N$ but has no appreciable effect upon the value of $N$ at the tropopause.

An examination of long term means from observations in the actual atmosphere shows that this same general bi-exponential trend is observed in practice, for temperate climates at least. Examples are given on figures 8.3 and 8.4 for Bismarck, N.Dak., and Brownsville, Tex.

Bismarck is typical of the high, dry great plains region of North America which is frequently subjected to strong intrusions of aretic air, while Brownsville represents the humid periphery of the (iulf of Mexico. Even in these very dissimilar climates one finds a strong tendency towards a bi-exponential distribution of $N$, particularly when $W$ is large. 


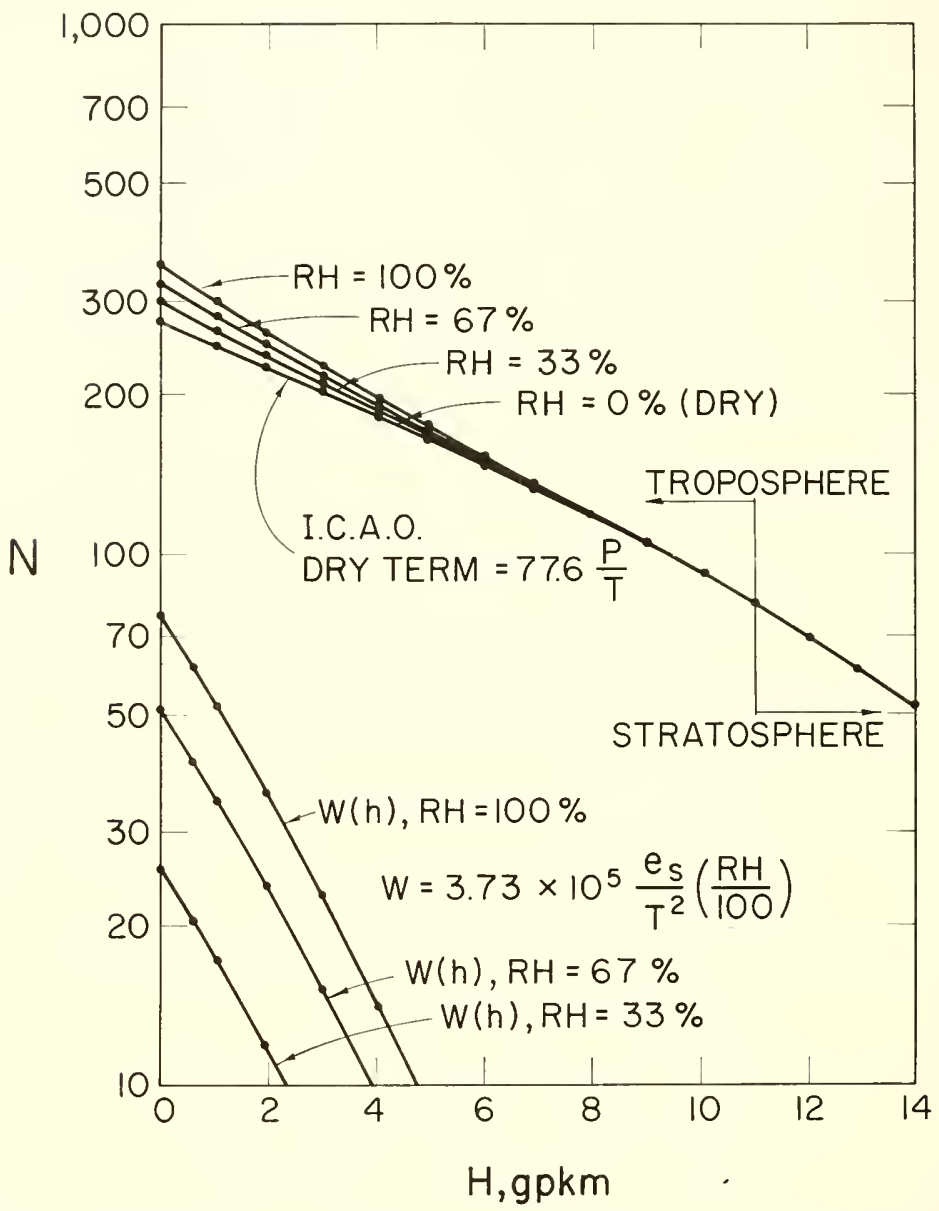

Figure 8.2. $\mathrm{N}$ distribution for the I.C.A.O. standard atmosphere.

\subsubsection{Properties of the Dry and Wet Term Scale Heights}

The average values of $D$ and $W$ versus height were determined for 22 U.S. radiosonde stations located about the country. The data used were the published values of mean pressures, temperatures, and humidities for the United States [9] which may be converted into mean values of the refractive index with negligible error [10]. The method of least squares was then used to determine the scale heights of the wet term, $H_{w}$, and of the dry term, $H_{d}$. Examination of these scale heights did not reveal any simple method of predicting their geographic and seasonal behavior, other than simply to map them. Such maps were prepared for the United States 
for both winter (February) and summer (August). The immediate conclusion that one reaches from these maps, figures 8.5 and 8.8 , is that $H_{w}$ has a year round, country-wide average value of perhaps $2.5 \mathrm{~km}$ while $H_{d}$ has an average value of about $9 \mathrm{~km}$.

Since the scale height is the height at which the value of an atmospheric property has decreased to $1 / e$ of its surface value it reflects the degree of stratification of the property. For example, cold aretic air is very stratified with very little vertical motion, with the result that its density scale height would be expected to be low. By contrast, tropical maritime air that has moved over land is characteristically unstable with convective activity thoroughly mixing the original moist surface air

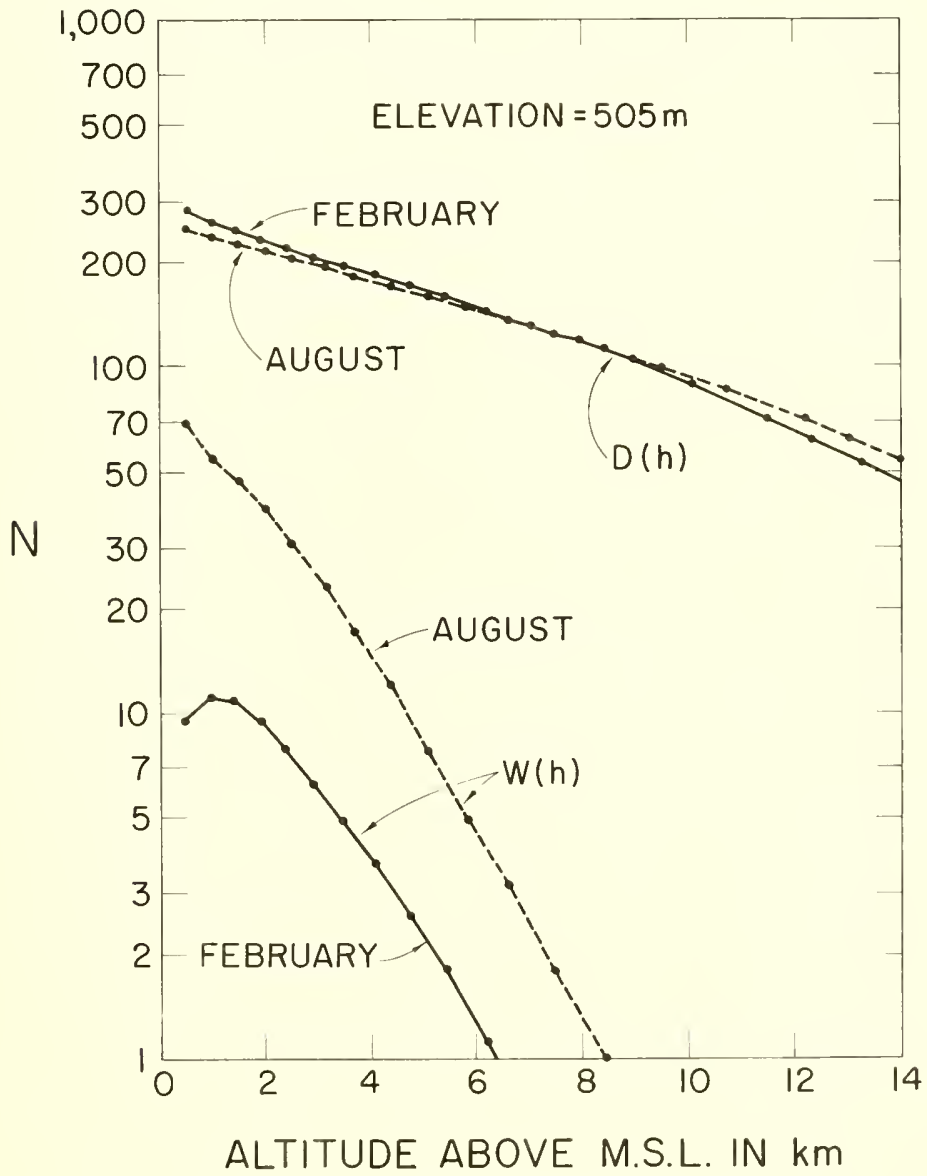

Figure 8.3. N distribution for Bismarck, N.Dak.

Note that altitude rather than geopotential height is used lere to facilitate the eventual calculation of radioray bending through aetual atmospherie layers. 


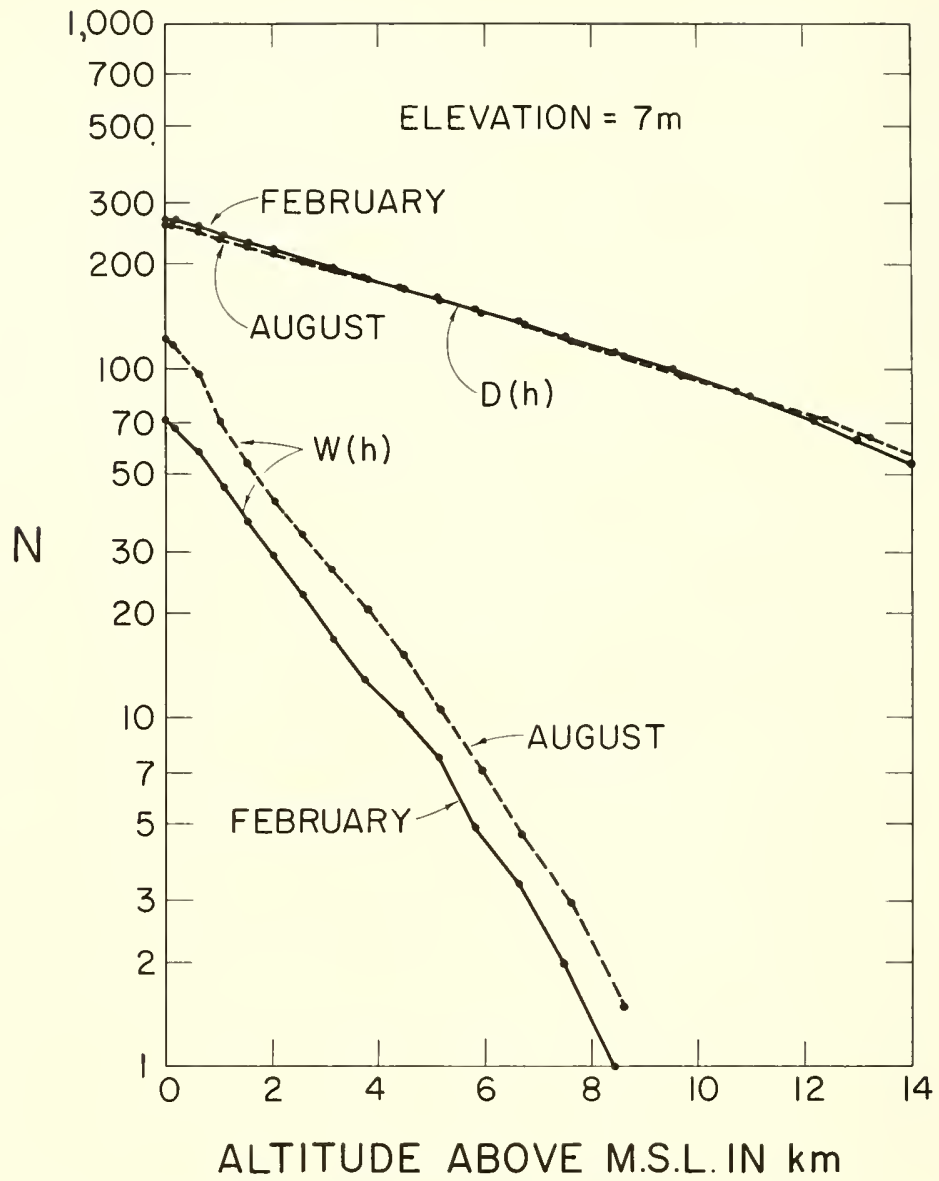

Figure 8.4. N distribution for Brownsville, Tex.

throughout the entire troposphere, with the result that the density scale height is relatively larger than in the case of arctic air. The dry term scale height on figures 8.5 and 8.7 show a distinct tendency to be larger during the warm summer months when the atmosphere is well mixed to great heights. Consequently $D$ decreases slowly with height.

A slight geographic pattern is observed in the $H_{d}$ maps: the coastal regions display somewhat higher values than the inland regions. The north-south direction of the isopleths along the west coast on the February $H_{d}$ map definitely reflects the uniform onshore advection of low-density maritime air. By contrast, the east coast shows an east-west isopleth pattern, the high values in Florida reflecting the well-mixed nature of subtropical air, and the lower values in New England indicating the presence 


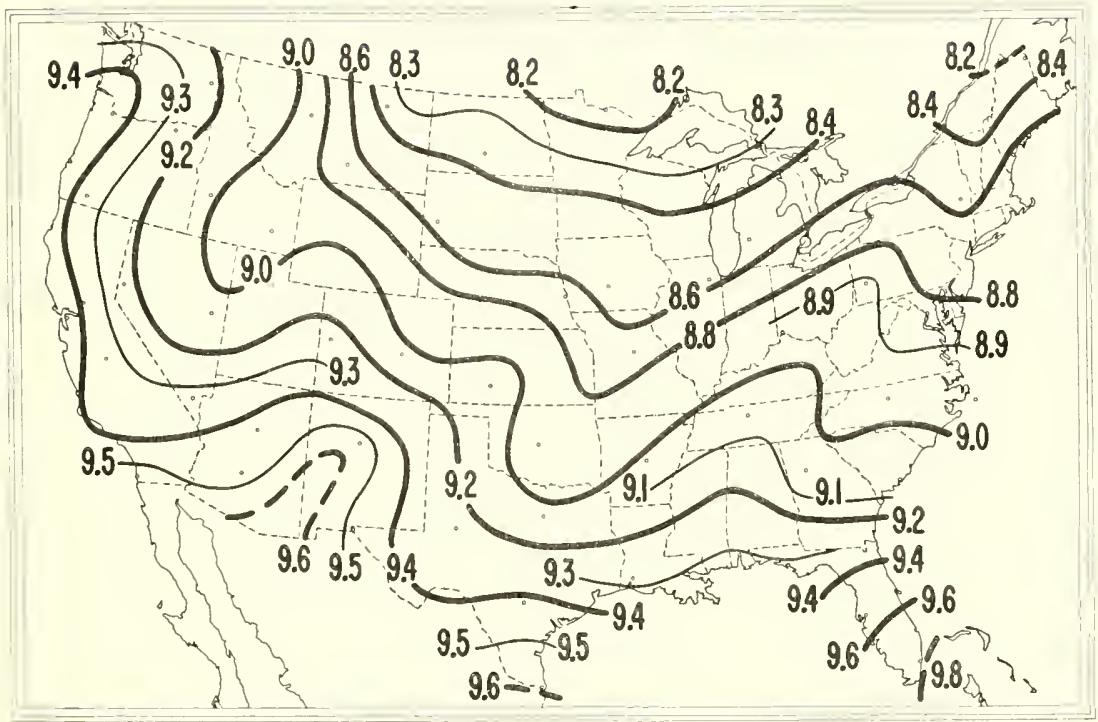

Figure 8.5. Dry term scale height, $\mathrm{H}_{\mathrm{d}}$, in kilometers for February.

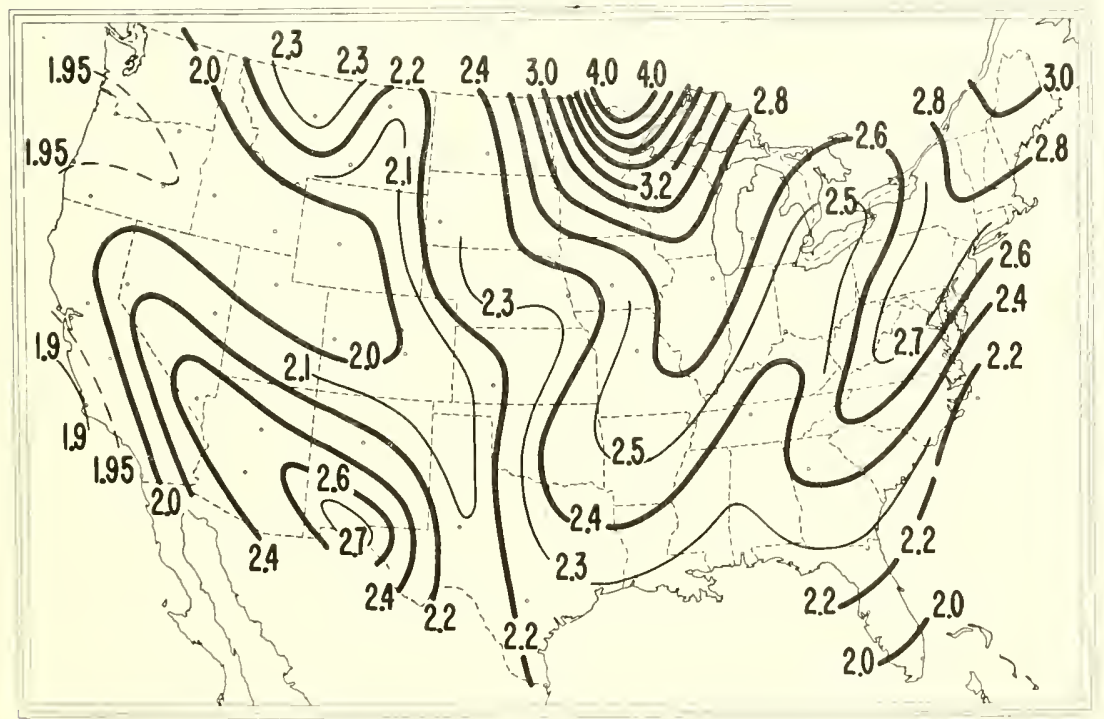

Figure 8.6. Wet term scale height, $\mathrm{H}_{\mathrm{w}}$, in kilometers for F'ebruary. 


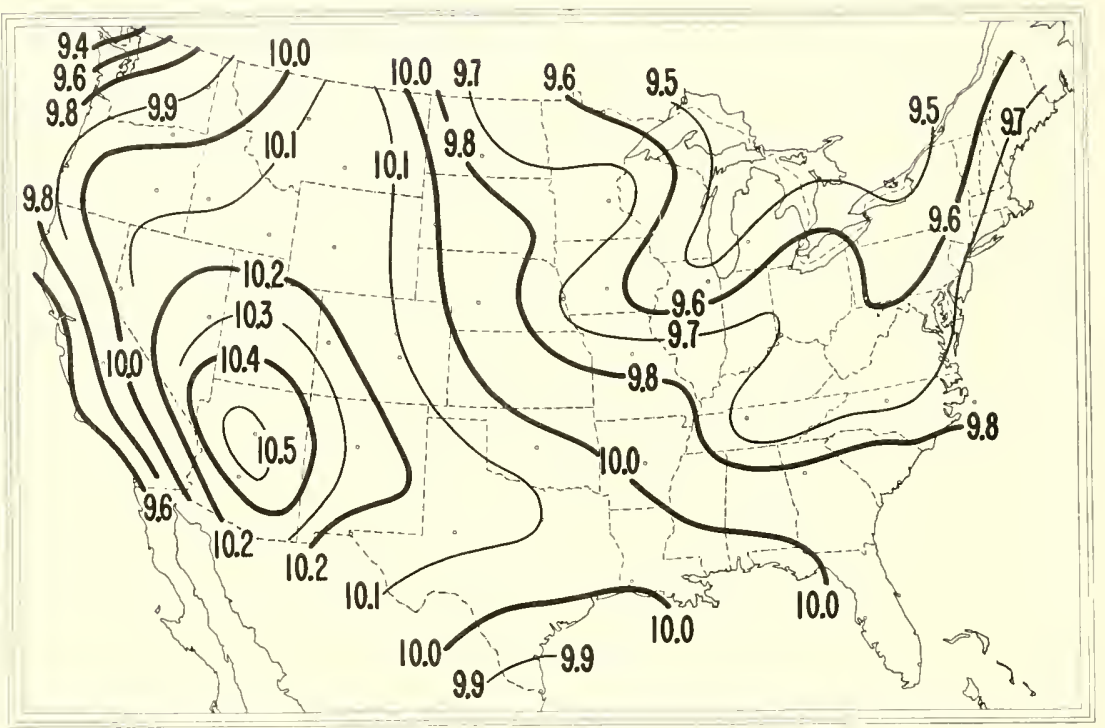

Figure 8.7. Dry term scale height, $\mathrm{H}_{\mathrm{d}}$, in kilometers for August.

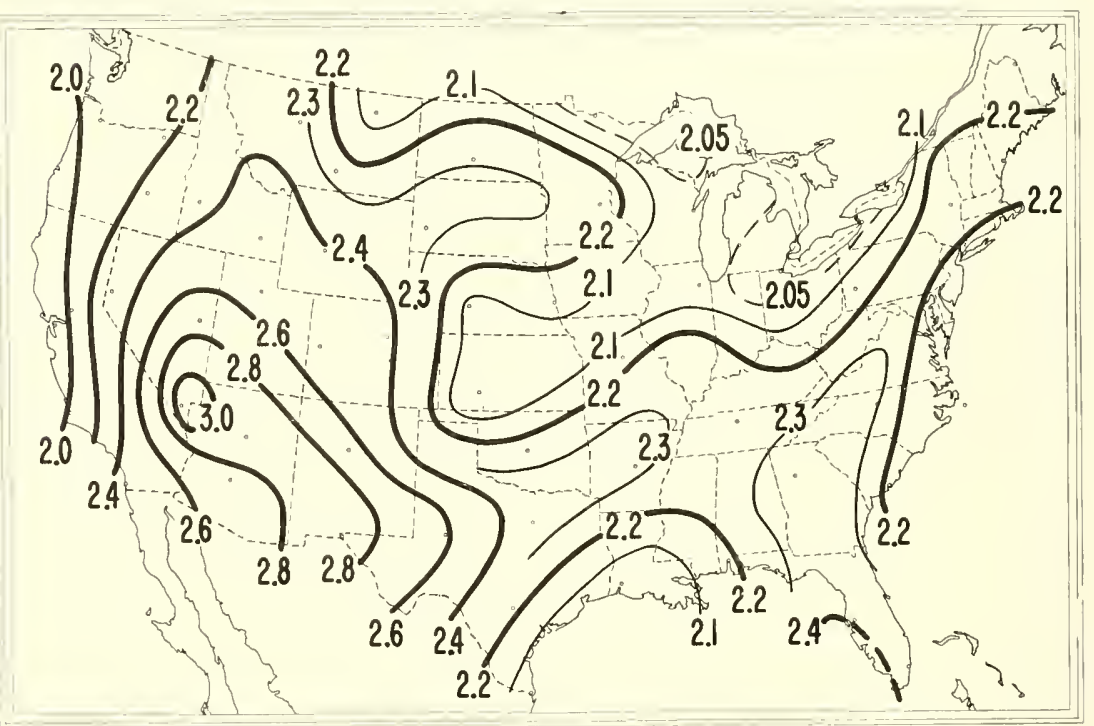

Figure 8.8. Wet term scale height, $\mathrm{H}_{\mathrm{w}}$, in kilometers for August. 
of the more dense and stratified continental air that customarily flows offshore during the winter months. The same pattern is repeated on the summertime map along the west coast but is less pronounced on the east coast due to the combination of more uniform heating and also onshore advection of maritime air produced by the circulation pattern of the Bermuda high-pressure area. The high value of $H_{d}=10.5 \mathrm{~km}$ observed in the southwest during the summer appears to be due to the intense heating with resultant convective mixing to great heights so common in that desert area. A somewhat opposite pattern is evidenced by the $H_{w}$ maps. For example, the coastal areas generally have the lowest values and thus reflect the characteristic strong humidity stratification of maritime air. The smaller humidity gradients of the inland regions produce somewhat larger scale heights for that area. The summer $H_{w}$ map is quite surprising in that very little variation is shown, perhaps indicating uniform vertical convection of the available moisture at all locations throughout the country. The strong convection indicated in the southwest on the summer $H_{d}$ map is again reflected by the high value of $H_{w}=3.0 \mathrm{~km}$ for that same area.

It is quite evident from figure 8.9 that within the troposphere, $h \leq 10$ $\mathrm{km}$, the bi-exponential model has a lower rms error for the common, near-zero angles of departure used in tropospheric propagation of radio waves. Both models yield about a 12 percent error in determining $\tau$ for $\theta_{0}=0$ and $h=11 \mathrm{~km}$. At $\theta_{0}=100 \mathrm{mrad}$, however, the percentage error decreases to 4 percent for the bi-exponential model and 7 percent for the exponential reference atmosphere. The rather marked errors of the single exponential model at $10 \mathrm{~km}$ simply reflect that that model is deliberately fitted to the average $N$ structure over the first few kilometers with the result that this model systematically departs from the average atmospheres in the vicinity of the tropopause. This is particularly apparent at the higher values of $\theta_{0}$ where the integral tends to become a function of the limits of integration. That is, using the theorem of the mean for integrals,

$\tau=-\int_{n_{s}}^{n} \cot \theta d n \sim-\cot \theta_{0} \int_{n_{s}}^{n} d n=\left[N_{s}-N\right] 10^{-6} \cot \theta_{0}$

under the assumption that $\cot \theta$ may be replaced by $\cot \theta_{0}$ over the interval of integration. For $\theta_{0} \leq 20 \mathrm{mrad}$ this assumption introduces less than a 10 percent error for the interval $0 \leq h \leq 10 \mathrm{~km}$. It is apparent from (8.9) that the error in predicting $\tau$ for large $\theta_{0}$ is then simply a mat ter of how closely the model approaches the true value of $n$ in the atmosphere. At $\theta_{0}$ near zero, however, the integral for $\tau$ is very heavily weighted towards the effect of $n$ gradients near the earth's surface [1]. Since the values of $\theta_{0}$ commonly used in tropospheric propagation are as near as 
practically possible to zero, and both models show comparable rms errors for $\theta_{0}=0$, one concludes that there is no clear advantage to the biexponential model for this application. This conclusion is furthered by the fact that charts of $H_{d}, H_{w}, D_{0}$, and $W_{0}$ are not available, while to use the single exponential model one need only to refer to existing regional or worldwide maps $[10,12]$.

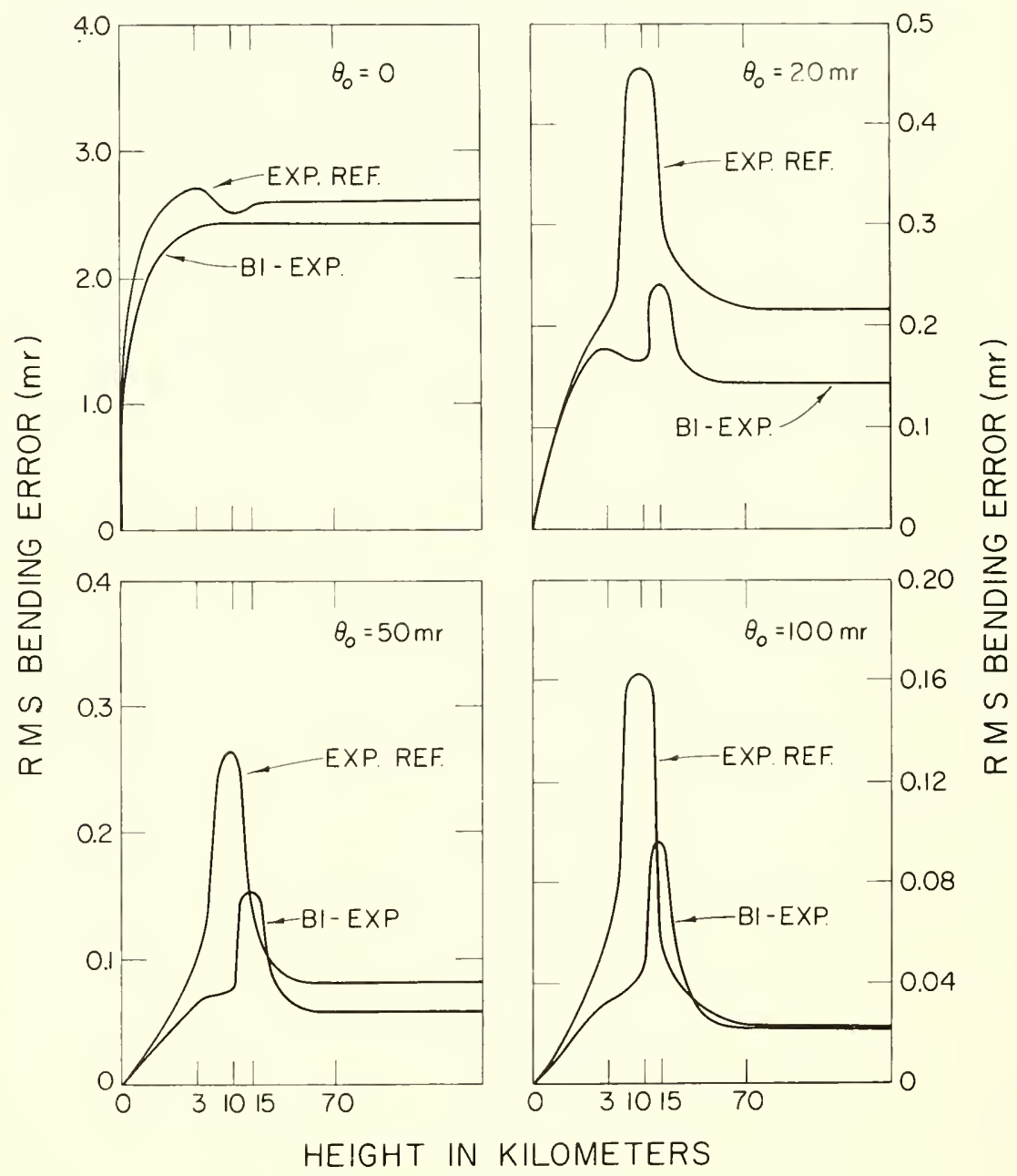

FIgURE 8.9. Root-mean-square errors of predicting bending for both the bi-exponential refractive index model and the C.R.P.L. (single) exponential atmosphere.

Height is used here to indicate the actual thickness of atmosphere traversed by the radio ray 


\subsubsection{Refraction in the Bi-Exponential Model}

The test of a model of atmospherie refractive index is the degree to which it represents the average $N$ strueture of the atmosphere. A further eritical test is the degree to which the refraction, or bending, of a radio ray is represented by this atmosphere. The bending is given as the angular change of a radio ray as it passes from $n_{1}$ to $n_{2}$ in a spherically stratified atmosphere and is adequately represented by

$$
\tau_{1,2}=-\int_{n_{1}}^{n_{2}} \cot \theta d n
$$

where $\theta$ is the local elevation angle and is determined at any point from Snell's law,

$$
n r \cos \theta=n_{0} r_{0} \cos \theta_{0}
$$

where $r$ is the radial distance from the center of the earth and zero subseript denotes the initial conditions. It is eustomary to evaluate (8.6) numerically [11] since the integral is intractable for all but the most simple models of $n$ versus $r$. The bending was obtained for the mean $N$ profiles for one-half of the 22 U.S. weather stations mentioned earlier. The values of $\tau$ predicted by the bi-exponential model for these same stations were obtained by preparing U.S. maps of $H_{d}$ and $H_{w}$ from the other half of our data, selecting values of $H_{d}$ and $H_{w}$ for the test stations, ealculating the bending and obtaining rms differences between these values and those obtained from the mean $N$ profiles. These rms differences are shown on figure 8.9. Also, for comparison, the rms errors obtained from the CRPL exponential atmosphere are shown. This atmosphere, based upon a single exponential curve passing from the surface value, $N_{s}$, to the value at $1 \mathrm{~km}, N_{1}$, is founded upon the expression

$$
N_{1}=N_{s}-7.32 \exp \left\{0.005577 N_{s}\right\}
$$

which has been found to be applicable in the United States [1].

An obvious advantage of the bi-exponential model is that the seale heights do reflect the physieal properties of the atmosphere in a much clearer way than does the single exponential model.

\subsubsection{Extension to Other Regions}

The present study is based upon data from the continental United States and one wonders if the same approach might be of utility in other regions. As a brief check, the $D$ and $W$ term scale heights were determined for conditions typical of the long aretic night (Isachsen, Northwest 
Territory, Canada, for February) and for humid tropical areas (Cant on Island, South Pacific, February) from 5-yr means of $N$ versus height and are listed in table 8.2.

The extreme meteorological differences of these two locations are quite evident. The $H_{d}=9.4 \mathrm{~km}$ and $H_{w}=2.0 \mathrm{~km}$ at Canton Island indicate a warm atmosphere with a strong humidity gradient, while the value of $H_{d}=6.3 \mathrm{~km}$ at Isachsen indicates very stratified air with high surface density and a strong density decrease with height. The value of $H_{w}=$ $6.5 \mathrm{~km}$ at Isachsen reflects a very low humidity gradient; in fact, at no point in the troposphere does $W$ exceed $3 N$ units for this location and season.

TABLE 8.2. $\mathrm{H}_{d}$ and $\mathrm{H}_{w}$ for arctic and tropical locations

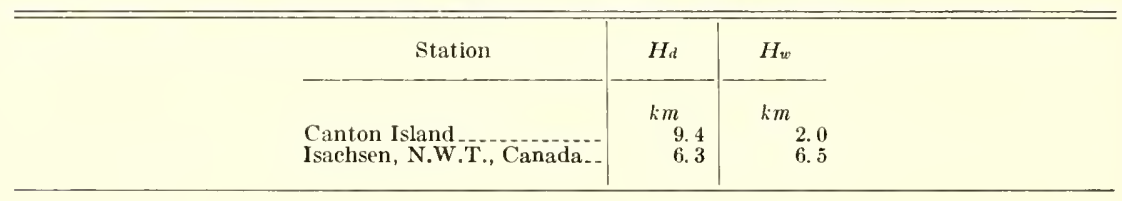

The value of $H_{w}=2.6 \mathrm{~km}$ reported for the characteristic altitude of water vapor for the middle belt of the U.S.S.R. [7] also appears to be in agreement with the conclusions of the present study.

\subsection{Effect of Atmospheric Horizontal Inhomogeneity Upon Ray Tracing}

\subsubsection{Introduction and Background}

It is common in ray tracing studies to assume that the refractive index of the atmosphere is spherically stratified with respect to the surface of the earth. Thus, the effect of refractive index changes in the horizontal direction is normally not considered, although Wong [13] has considered the effect of mathematically smooth horizontal changes in airborne propagation problems.

Neglecting the effect of horizontal gradients seems quite reasonable in the tropospheric because of the relatively slow horizontal change of refractive index in contrast to the rapid decrease with height. In fact, examination of climatic data indicates that one must compare sea-level stations located $500 \mathrm{~km}$ from each other on the earth's surface in order to observe a difference in refractive index values which would be comparable to that obtained by taking any one of these locations and comparing its surface value with the refractive index $1 \mathrm{~km}$ above the location. Although the assumption of small horizontal changes of the refractive index appears 
to be true in the average or climatic sense, there are many special cases, such as frontal zones and land-sea breeze effects, where one would expect the refractive index to change abruptly within the 80-odd kilometers of horizontal distanee traversed by a tangential ray passing through the first kilometer in height.

It is these latter variations that are investigated in this section. Two eases of marked horizontal change of refractive index conditions were studied, one which occurred over the Canterbury Plain in New Zealand, and the other at Cape Kennedy, Fla. Although these particular sites were chosen for severai reasons, such as land-to-sea paths and subtropical location (where marked changes in refraction conditions are common), the major eonsideration was that detailed aircraft and ground meteorological observations were available for prolonged periods.

These detailed measurements allow a quantitative evaluation of the error apt to be incurred by assuming that the refractive index is horizontally stratified. The procedure used was to determine the refractive index structure vertically over the transmitter and assume that this same structure vertically described the atmosphere everywhere. Rays were then traced through this horizontally laminated atmosphere. These ray paths were then compared with those obtained by the step-by-step ray tracing through the detailed convolutions of refractive index structure in the two cases under study.

In the sections that follow we will discuss the two cases chosen for study, the methods of calculation used to evaluate refraction effects, and the degree of confidence to which standard prediction methods may be used under conditions of horizontal inhomogeneity.

\subsubsection{Canterbury}

The Canterbury data were compiled by a radio-meteorological team working from September 1946, through November 1947, on the South Island of New Zealand under the leadership of R. S. Unwin [14]. This report proved invaluable in this investigation, as it was very carefully prepared, giving minute details of the experiment on a day-to-day basis. Anson aircraft and a trawler were used for meteorological measurements over the sea, and three mobile sounding trucks for observations on land. The trucks and the trawler carried wired sonde equipment, whereby elements for measuring temperature and humidity up to a height of from $150 \mathrm{~m}$ to $600 \mathrm{~m}$ (depending on wind eonditions) were elevated by means of balloons or kites. Standard meteorological instruments provided a continuous record of wind, surface pressure, temperature, and humidity at stations at the coast and $14 \mathrm{~km}$ and $38 \mathrm{~km}$ inland. The headquarters of the project were at Ashburton Aerodrome, and the observations extended out to sea on a line perpendicular to the coastline of Canterbury 
Plain. Aircraft were equipped with a wet-bulb and dry-bulb psychrometer, mounted on the portside above the wing. Readings were taken three or four times on each horizontal flight leg of 2 or 3 min duration. Special lag and airspeed corrections were applied, resulting in accuracy of $\pm 0.1{ }^{\circ} \mathrm{C}$. It was found that, under the variety of conditions in which observations were made, the aircraft flights were more or less parallel to the surface isobars; hence, the sea-level pressure as recorded at the beach site was considered to hold over the whole track covered by the aircraft. The relationship used for calculating the pressure, $P$, in millibars at a height $h$ in feet was:

$$
P(h)=P_{0}-h / 30
$$

where $P_{0}$ is the surface pressure. This approximation (determined by averaging the effect of the temperature and humidity distributions on pressure in a column of air) resulted in a maximum error in the refractivity of 0.5 percent at $900 \mathrm{~m}$. Radiosonde ascents at Hokitika on the west coast of South Island and Paraparaumuo and Auckland on North Island were used to supplement the aircraft measurements, particularly in the altitude levels above $1 \mathrm{~km}$.

The observations, diagrams, and meteorological records were studied, and a profile of unusually heterogeneous nature was chosen. The synoptic situation for the morning of November 5, 1947, was selected, as it revealed a surface-ducting gradient near the coast with an elevated layer about $100 \mathrm{~km}$ off shore. A cross section of the area from Ashburton to a point $200 \mathrm{~km}$ offshore was plotted with all available data, and isopleths of modified refractive index, $M$, were drawn to intervals of 2.5 units

$$
M=N+\left(K_{e} h\right) 10^{6},
$$

where $K_{e}=(15.70)\left(10^{-8}\right) / \mathrm{m}$, and $N$ is as defined in chapter $1,(1.20)$. A simplified version of the lower portion of this cross section with the corresponding $M$ curves is accompanied by a sketch of the general location of the experiment in figure 8.10. Some smoothing was necessary, particularly near the sea surface and in those areas where aircraft slant ascents and descents caused lag errors in altimeter readings and in temperature and humidity elements. Isopleths over land were plotted above surface rather than above sea level, with an additional adjustment in the scale ratios of height and distance in an attempt to simplify the reading of values from the diagram.

\subsubsection{Cape Kennedy}

The second area studied was the Cape Kennedy to Nassau path for the period of April 24 to May 8, 1957. This material was supplied by the Wave Propagation Branch of Naval Research Laboratories and the 

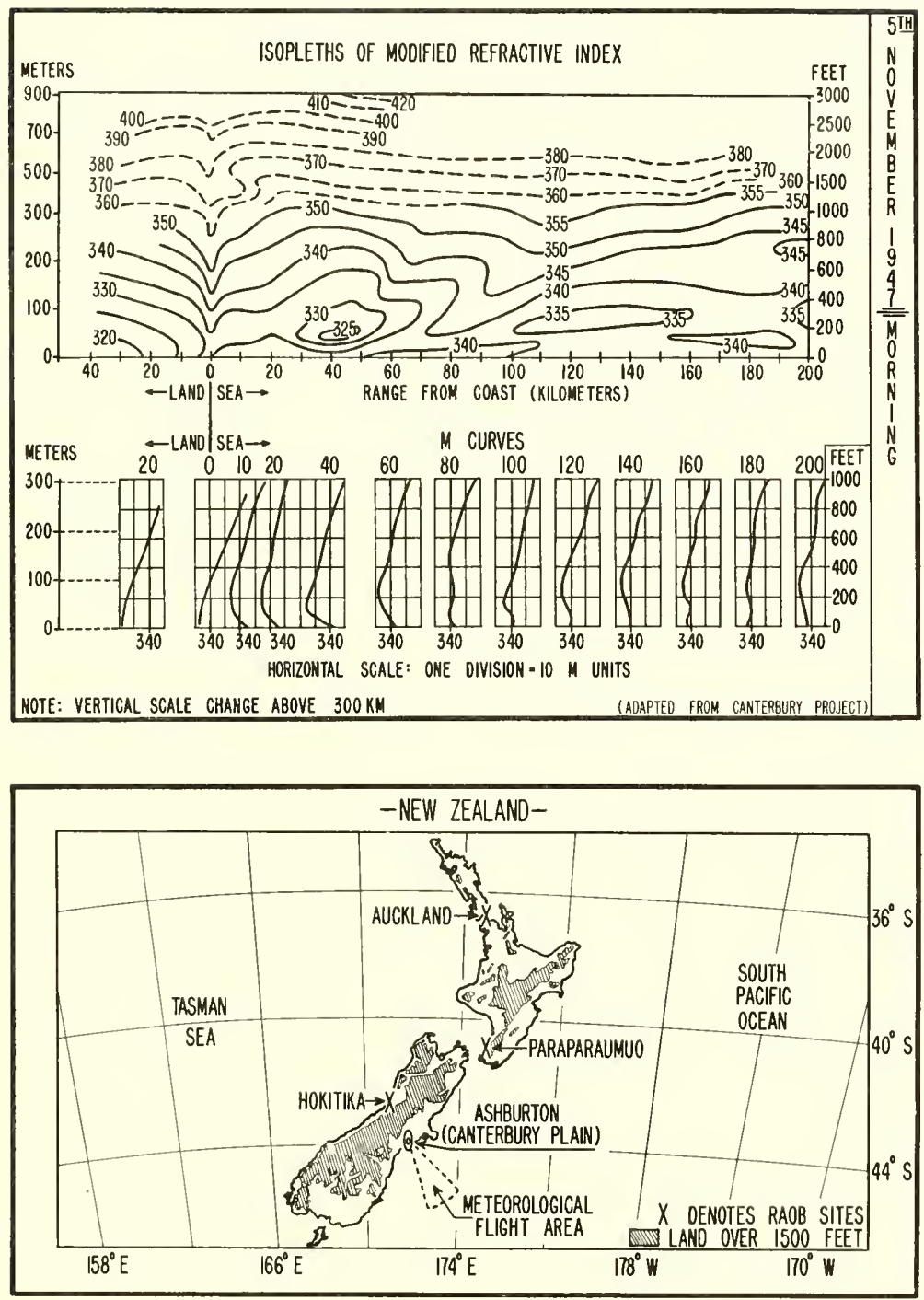

Figure 8.10. Isopleths and curves of refractive index for November 5, 1947, Canterbury, with a map locating sources of meteorological data.

(Courtesy of the Canterbury projeet). 
University of Florida. The particular case chosen for study was the meteorological profile of May 7, 1957 (2000 L.T.) due to its heterogeneous nature, showing a well-defined elevated layer at about 1,500 m. Fourteen refractometer soundings from aircraft measurements taken at various locations along the $487 \mathrm{~km}$ path (fig. 8.11) and six refractive index profiles (deduced from radiosonde ascents from Cape Kennedy, Grand Bahama Island, and Eleuthera Island) were read in order to plot a cross section of the atmosphere which would represent as closely as possible the actual refractive conditions at that time. Unfortunately, the data near the surface (up to $300 \mathrm{~m}$ ) were quite sparse compared to those recorded in the Canterbury Project, and calibration and lag errors had not been noted as carefully in this preliminary report; therefore, some interpolation and considerable smoothing of refractive index values were necessary when drawing isopleths.

\subsubsection{Ray Bending}

The classic expression for the angular change, $\tau$, or the bending of a ray passing from a point where the refractive index is $n_{1}$ to a second point where the refractive index is $n_{2}$ is given in chapter 3 as

$$
\tau_{1,2}=-\int_{n_{1}}^{n_{2}} \frac{d n}{n} \cot \theta
$$

where $\theta$ is the local elevation angle. Equation (8.11) was evaluated by use of

$$
\Delta \tau_{1,2}=\frac{\left(N_{1}-N_{2}\right)}{n_{1,2}} \times 10^{-6} \cot \bar{\theta}
$$

where

$$
\bar{\theta}=\frac{\theta_{1}+\theta_{2}}{2} .
$$

The value of $\theta$ at each point was determined from Snell's law:

$$
n_{1} r_{1} \cos \theta_{1}=n_{2} r_{2} \cos \theta_{2}=\text { constant, }
$$

where $r$ is the radial distance from the center of the earth and is given by $a+h$, where $a$ represents the radius of the earth and $h$ the altitude of the point under consideration. For simplicity one may rewrite (8.13) as

$$
\begin{aligned}
\left(1+N_{1} \times 10^{-6}\right)(a+ & \left.h_{1}\right) \cos \theta_{1} \\
& =\left(1+N_{2} \times 10^{-6}\right)\left(a+h_{2}\right) \cos \theta_{2} .
\end{aligned}
$$



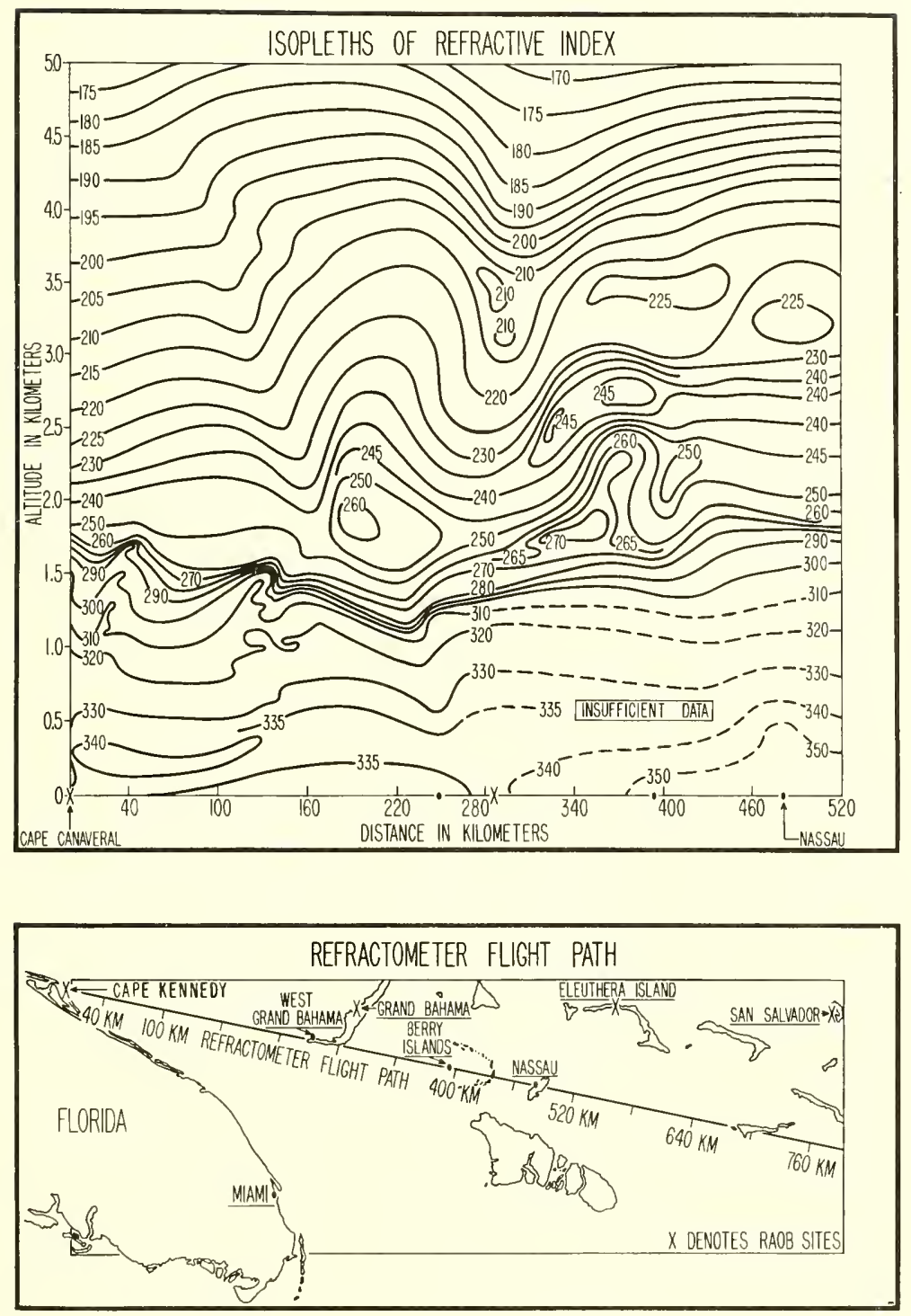

Figure 8.11. Isopleths of refractive index and map of refractometer fight path for May 7, 1957, Cape Kennedy to Nassau. 
Then, when $\theta$ is small, one may expand (8.14) [as in chapter 3, (3.50) to (3.58)] and obtain the convenient expression:

$$
\theta_{2}=\left\{\theta_{1}^{2}+\frac{2\left(h_{2}-h_{1}\right)}{a}-2\left(N_{1}-N_{2}\right) \times 10^{-6}\right\}^{1 / 2}
$$

where all values of $\theta$ are in milliradians.

After obtaining $\tau$ by use of (8.12) and (8.15), one may determine the distance, $d$, along the earth's surface that the ray has traveled from:

$$
d_{1,2}=a\left[\tau_{1,2}+\left(\theta_{2}-\theta_{1}\right)\right]
$$

Thus by successive application of the above formulas, one may trace the progress of the radio wave as it traverses its curved path through the atmosphere. Normally the use of these equations is quite straightforward. When considering horizontal changes in $n$, however, one must satisfy these equations by iterative methods. In the present application, since $n$ had to be determined by graphical methods, it was felt to be sufficient to assume a constant distance increment of 250 to $500 \mathrm{~m}$, solve for appropriate height increment from

$$
\Delta h=\Delta d \tan \theta_{1}\left[1+\frac{h}{a}\right],
$$

graphically determine $N$ for the point $d_{1}+\Delta d, h_{1}+\Delta h$, and then determine $\theta_{2}$ and $\tau_{1,2}$.

This latter type of ray tracing was done for various rays of initial elevation angles between $261.8 \mathrm{mrad}\left(15^{\circ}\right)$ and $10 \mathrm{mrad}\left(\sim 0.5^{\circ}\right)$. The calculations were not carried to smaller elevation angles, since this type of ray tracing is not valid within surface ducts for initial elevation angles below the angle of penetration [16].

\subsubsection{Comparisons}

Although both of the calculated ray paths consisted of an oversea itinerary with coastal transmission sites, they are quite different in other aspects. Canterbury Plain is located southeast of the $10,000-\mathrm{ft}$ chain of the Southern New Zealand Alps at a latitude of $44^{\circ} \mathrm{S}$ (the equatorward edge of the westerly belt of winds in November). Cape Kennedy is located on a sca-level peninsula at $28^{\circ} \mathrm{N}$ (the poleward edge of the northeast trade circulation in May). While the Canterbury profile showed superrefractive tendencies, the Cape Kennedy profile illustrated subrefraction at the surface counterbalanced by an elevated trade wind inversion layer, indicating that the total bending values of Canterbury 
would be higher than normal, while the Cape Kennedy example would have values near or lower than normal.

These differences are illustrated by figures 8.12 and 8.13 where the bending, $\tau$, in milliradians is plotted versus altitude in kilometers. The effect of horizontal changes is most pronounced for rays with initial elevation angles of 10 mrad. On these figures the term "vertical" ray is used to designate the ray path through the horizontally homogeneous $n$ structure determined from the refractive index vertically over the station. The term "horizontal" ray designates the ray path through the complex actual $n$ structure. It is quite evident that a consistent difference in bending of about $1 \mathrm{mrad}$ exists between the "vertical" and "horizontal" rays at Canterbury above $1 \mathrm{~km}$ for $\theta_{0}=10 \mathrm{mrad}$. This would be expected since the vertical $M$ profile (fig. 8.10) at the beach (our hypothetical transmitter site) is nearly normal in gradient while as little as $10 \mathrm{~km}$ to sea a duct exists, thus indicating a near maximum difference between the "horizontal" and the "vertical" rays at any initial elevation angle small enough to be affected by the duct. This is in contrast, however, with the case of Cape Kennedy where, except for the region of the elevated duct centered at about $1,500 \mathrm{~m}$, the "vertical" and "horizontal" rays are in quite close agreement. These two examples illustrate that horizontal variations must be near the surface to be most effective. The importance of the altitude of the variation is due to the fact that refraction effects are very heavily weighted toward the initial layers [16].

Also shown on figures 8.12 and 8.13 are the values of the bending which would be predieted from the Central Radio Propagation Laboratory corrected exponential reference atmosphere [1]. The values shown are obtained from the value of $N$ at the transmitter site as corrected by the vertical gradient over the first $100 \mathrm{~m}$. It is noted that, for $\theta_{0}=10 \mathrm{mrad}$ at Canterbury, the value of bending predicted by the model is in essential agreement with the "vertical ray" bending but under estimates the "horizontal ray" (which has the largest variation of $n$ with horizontal distanee) by about $1.25 \mathrm{mrad}$. For Cape Kennedy at $\theta_{0}=10 \mathrm{mrad}$, the model atmosphere overestimates the bending by about $1.25 \mathrm{mrad}$ for altitudes in excess of $2 \mathrm{~km}$. It should be emphasized that, although the model exponential atmosphere appears to represent the average of the two specific cases studied, the departure from this average arises from quite different causes in each case. The differences in the Canterbury case arise from the marked effect of horizontal variation of $n$ as is indicated by the agreement of the vertical ray bending with the model atmosphere. The disagreement in the Cape Kennedy case is due to the presence of a very shallow surface layer of nearly normal gradient topped by a strong subrefractive layer; therefore, it represents a shortcoming of the model rather than an effect of horizontal changes of $n$. 


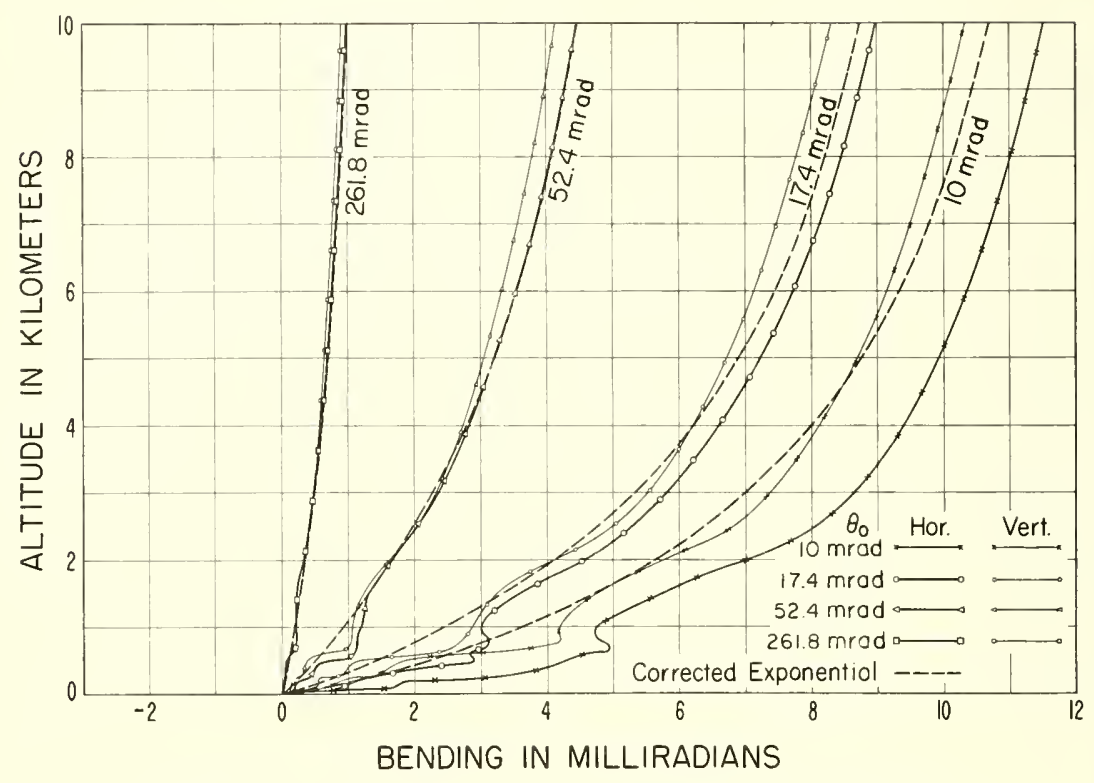

FIgURE 8.12. Canterbury, 0 to $10 \mathrm{~km}$, altitude versus ray bending.

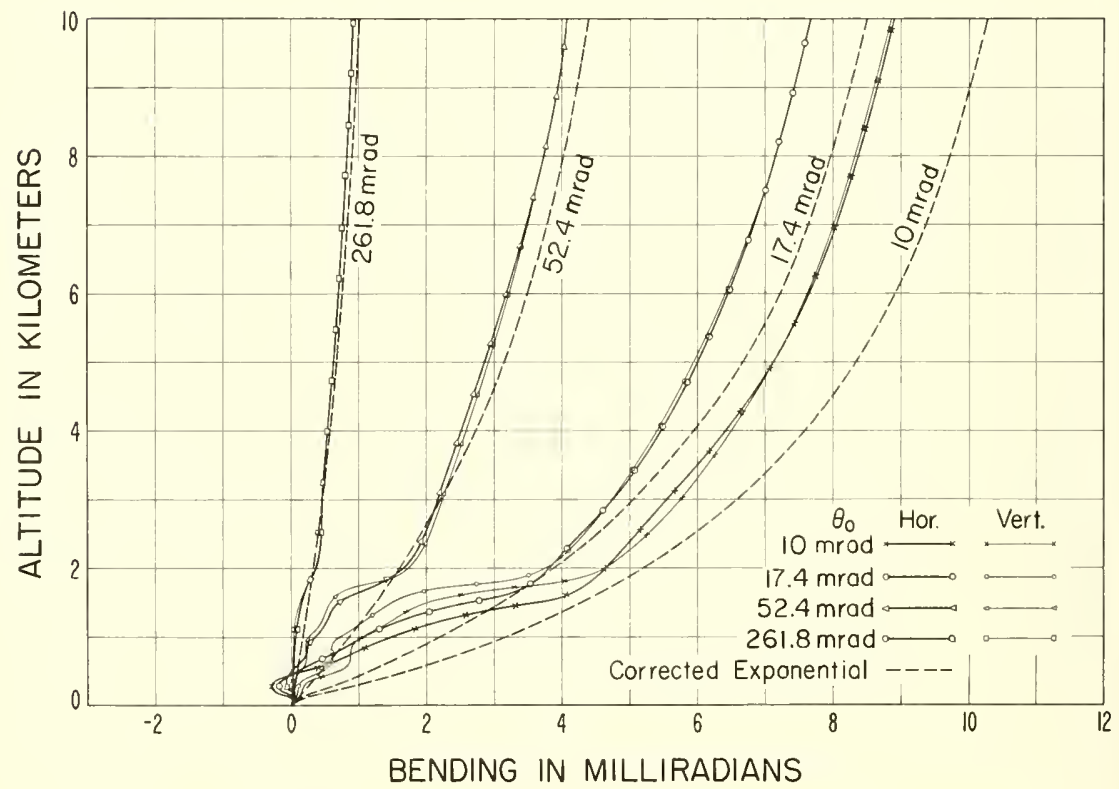

Figure 8.13. Cape Kennedy, 0 to $10 \mathrm{~km}$, altitude versus ray bending. 
The preceding analysis of bending throws the refractive differences in each case into sharp relief. The effect of refraction, of course, is to vary the ray path. Figures 8.14 and 8.15 show the ray paths corresponding to the bendings of figures 8.12 and 8.13. Note that for Canterbury at $\theta_{0}=\mathrm{mrad}$, the effect of the horizontal variation of $n$ is to produce a difference in estimation of about $1 \mathrm{~km}$ in height or $20 \mathrm{~km}$ in ground distance at $300 \mathrm{~km}$ from what would be obtained from considering the vertical $n$ profile as a representation of the entire path. The effect of the subrefractive layer at Cape Kennedy is not so large, but it does cause an overestimation of the ground distance by about $5 \mathrm{~km}$ and an underestimation of the height by less than one quarter of a kilometer at a ground distance of $300 \mathrm{~km}$ by assuming that the vertical profile may be used throughout the entire ray path.

\subsubsection{Extension to Other Regions}

It should be pointed out that the ducting case at Canterbury represents an extreme refraction condition and is not necessarily typical of conditions observed in other regions or, indeed, at Canterbury. The Canterbury project was purposely restricted to a study of ducting eonditions with the result that less than 20 percent of the total observations for the fifteen

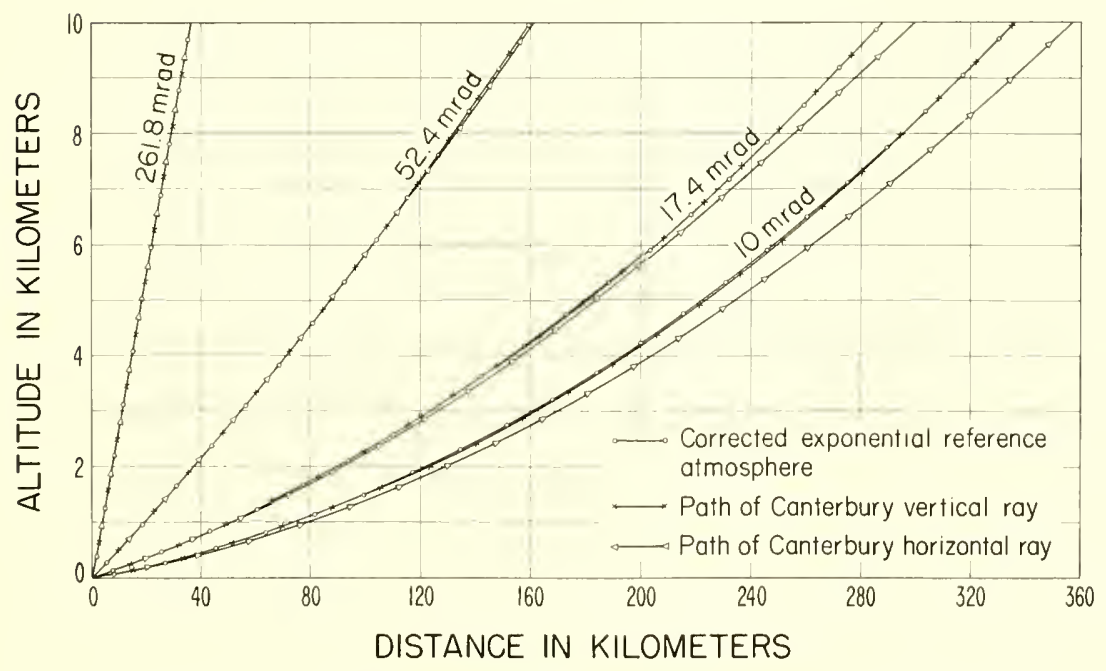

Figure 8.14. Canterbury, 0 to $10 \mathrm{~km}$, altitude of ray versus distance. 


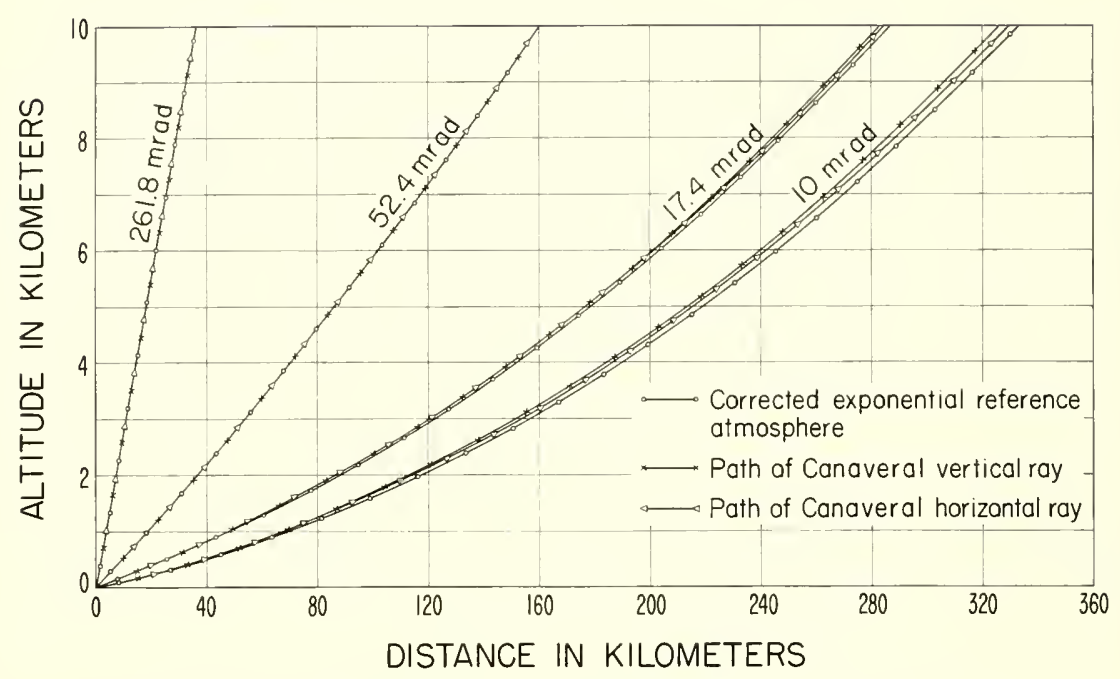

Figure 8.15. Cape Kennedy, o to $10 \mathrm{~km}$, altitude of ray versus distance.

months are reported. Therefore, because one of the more extreme cases is represented by the November 5 example, one might conclude that much less than 20 percent of the observations would show the same degree of horizontal $n$ change as the profile studied.

If one further hypothesizes that the greatest horizontal $n$ change would be associated with ducting conditions, then the percentage incidence of ducts as evaluated from radiosonde observations, listed for various stations in table 8.3 would indicate that the effects of horizontal changes of $n$ sufficient to cause variations in the ray path as large as those of the present study would be observed less than 15 percent of the time, regardless of geographic location.

The probable importance of subrefractive layers upon the prediction of refraction effects has emerged as a secondary result of the present study. Although subrefraction is normally neglected, it is potentially a very important refractive factor for distances of, say, less than $40 \mathrm{~km}$. Even though the percentage occurrence of subrefractive layers can be as large as 6 percent (see table 8.4), this effect is frequently offset by the concurrent occurrence of an adjacent superrefractive layer, as is illustrated by the Cape Kennedy example. 
TABLE 8.3. Percentage occurrence of surface ducts during the years 1952 to 1956

\begin{tabular}{|c|c|c|c|c|}
\hline \multirow{2}{*}{ Station } & \multicolumn{4}{|c|}{ Percent incidenee } \\
\hline & February & May & August & November \\
\hline $\begin{array}{l}\text { Fairbanks, Alaska. } \\
\text { Columbia, Mo } \\
\text { Washington, D.C. } \\
\text { Canton Island } \\
\text { Miami, Fla }\end{array}$ & $\begin{array}{r}9.4 \\
0.7 \\
0.7 \\
10.0 \\
0.7\end{array}$ & $\begin{array}{l}0.4 \\
2.5 \\
4.8 \\
9.2 \\
3.5\end{array}$ & $\begin{array}{r}0.4 \\
8.4 \\
4.3 \\
12.4 \\
8.5\end{array}$ & $\begin{array}{r}6.2 \\
1.3 \\
1.4 \\
11.5 \\
2.7\end{array}$ \\
\hline
\end{tabular}

TABLE 8.4. Percentage occurrence of surface subrefractive layers during the years 1952 to 1956

\begin{tabular}{|c|c|c|c|c|}
\hline \multirow{2}{*}{ Station } & \multicolumn{4}{|c|}{ Pereent ineidenee } \\
\hline & February & May & August & November \\
\hline $\begin{array}{l}\text { Fairbanks, Alaska } \\
\text { Columbia, Mo } \\
\text { Washington, D.C. } \\
\text { Canton Island } \\
\text { Miami, Fla }\end{array}$ & $\begin{array}{l}0.0 \\
0.0 \\
0.9 \\
0.0 \\
0.7\end{array}$ & $\begin{array}{l}0.0 \\
1.6 \\
2.2 \\
0.0 \\
0.3\end{array}$ & $\begin{array}{l}1.2 \\
0.6 \\
5.8 \\
0.0 \\
0.9\end{array}$ & $\begin{array}{l}0.4 \\
4.0 \\
2.7 \\
0.3 \\
0.7\end{array}$ \\
\hline
\end{tabular}

\subsubsection{Conclusions}

The conclusions of the present study could be considerably modified by the analysis of many more examples, although it is evident that horizontal variation of $n$ near the carth's surface produces the most marked deviations from the ray paths obtained by assuming horizontal stratification of $n$. The effect of horizontal changes occurring more than a kilometer above the surface appear from our present examples, to have little effect. Further, the effects of horizontal changes appear to be most pronounced in the presence of surface ducts and at small elevation angles. The tentative conclusion is reached that the effect of horizontal $n$ change is normally small, since ducting will occur less than 15 pereent of the time.

\subsection{Comparison of Observed Atmospheric Radio Re- fraction Effects With Values Predicted Through the Use of Surface Weather Observations}

\subsubsection{Introduction}

The atmospheric radio refraction effects considered in this section are of two general types: errors in measuring distance by means of timing the transit of radio signals between two points, known as radio range errors, and errors in estimating the elevation angle of a target by means of measuring the angle of arrival of radio signals from the target, known 
as elevation angle errors. Many methods have been proposed to take into account these refraction effects for the purpose of improving measurements by removing systematic bias. One of these involves the use of the surface value of the radio refractivity, $N_{s}$, a quantity which can be measured directly with a microwave refractometer, or calculated from the ordinary meteorological variables of temperature, pressure, and humidity, to predict values of either range error or elevation angle error; this method has been shown theoretically to be useful, with the accuracy increasing with increasing initial elevation angle [17, 18, 19]. It is the purpose of the present note to compare recent experimental determinations of atmospheric refractive effects with values estimated theoretically from surface meteorological conditions.

\subsubsection{Theory}

The operation of a radio tracking system depends on the measurement, in some manner, of radio signals received from the target. The radio signals are transmitted in the form of radio waves which travel from the target to the tracking system. The form of these radio waves is distorted by the presence of the earth's atmosphere. Since solutions of the wave equation are extremely difficult to obtain for the case of general atmospheric propagation over a spherical earth, it is common practice to evaluate refraction effects by means of ray tracing, a process which is based on the use of Snell's law.

One of the two types of refraction errors considered in this appendix is the elevation angle error, $\epsilon$, which is the difference between the apparent direction to a target, as indicated by the angle of arrival of a normal to the radio wave front, and the true direction. This error is primarily a function of the refraction, or bending, of the radio ray. For targets beyond the atmosphere, the two quantities are asymptotically equal (with increasing range). The values of $\epsilon$ and $\tau$ at any point on the ray path obey the following inequality:

$$
\tau / 2 \leqq \epsilon \leqq \tau .
$$

Recalling that (chapter 3 ) the bending of a radio ray may be expressed by an equation of the form

$$
\tau=a+b N_{s}
$$

where $a$ and $b$ would be functions of the initial elevation angle of the ray, $\theta_{0}$, and the height (or range) along the ray path at which the bending is to be calculated. Such an assumption can be checked by examining the behavior of values of $\tau$, ray traced for a number of observed height profiles of radio refractive index, plotted against the corresponding values of $N_{s}$. 
Such a plot is shown in figure 8.16 for a small initial elevation angle, $50 \mathrm{mrad}$ (about $3^{\circ}$ ), and a "target" height beyond the atmosphere, $70 \mathrm{~km}$. The family of $N$ profiles used in ray tracing this sample of bending values is referred to as the CRPL Standard Sample. ${ }^{2}$ It can be seen from inspection of figure $\$ .16$ that the assumption of linearity expressed in (8.1S) is justified for this case. A similar conclusion can be reached from examination of data for other cases, including low target heights and elevation angles down to zero degrees, although for these extremes the degree of eorrelation between $\epsilon$ and $N_{s}$ is not as marked as that shown in figure 8.16.

The other refraction variable treated in this section is the radio range error, $\Delta R_{e}$, which is here defined as being that error incurred in measuring the distance between two points by means of timing the transit of radio signals between the points, and assuming that the velocity of propagation is equal to that of free space. For the case of a radio ray, this error is eomposed of two parts: the difference between the curved length of the ray path, ealled the geometric range, $R_{\theta}$, and the true slant range, $R_{0}$; and the discrepancy eaused by the lowered veloeity of propagation in a refractive medium. The geometric range is given by

$$
R_{g}=\int_{0}^{h_{t}} \operatorname{cse} \theta d h
$$

and the apparent, or radio, range by

$$
R_{e}=\int_{0}^{h_{t}} n \csc \theta d h .
$$

Thus the total radio range error, $\Delta R_{e}=R_{e}-R_{0}$, is given by

$$
\Delta R_{e}=\int_{0}^{h_{t}} n \csc \theta d h-R_{0}
$$

or

$$
\Delta R_{e}=10^{-6} \int_{0}^{h_{\imath}} N \csc \theta d h+\int_{0}^{h_{\imath}} \csc \theta d h-R_{0} .
$$

${ }^{2}$ Meaning explained in section 8.3.3. 
TABLE 8.5. Typical and extreme values of range errors for targets beyond the atmosphere

\begin{tabular}{|c|c|c|c|c|c|c|c|}
\hline \multirow[b]{2}{*}{$\theta_{0}$} & \multicolumn{3}{|c|}{ Typical $N_{s}=320$} & \multicolumn{3}{|c|}{ Extreme $N_{s}=400$} & \multirow{2}{*}{$\frac{\begin{array}{c}\text { Maximum } \\
\text { percent }\end{array}}{\Delta R_{g} / \Delta R_{e}}$} \\
\hline & $\Delta R_{g}$ & $\Delta R_{N}$ & $\Delta R_{e}$ & $\Delta R_{g}$ & $\Delta R_{N}$ & $\Delta R_{e}$ & \\
\hline $\begin{array}{c}0 \\
20 \mathrm{mrad} \\
50 \mathrm{mrad} \\
100 \mathrm{mrad} \\
200 \mathrm{mrad} \\
500 \mathrm{mrad}\end{array}$ & $\begin{array}{l}\text { meters } \\
10 \\
2.5 \\
0.7 \\
0.14 \\
0.02 \\
0.001\end{array}$ & $\begin{array}{l}100 \\
62.5 \\
38.1 \\
22.26 \\
11.9 \\
5.01\end{array}$ & $\begin{array}{c}110 \\
65 \\
38.8 \\
22.4 \\
11.9 \\
5.01\end{array}$ & $\begin{array}{l}60 \\
4.5 \\
1.0 \\
0.2 \\
0.03 \\
0.002\end{array}$ & $\begin{array}{c}165 \\
73 \\
43 \\
24.8 \\
13.0 \\
5.50\end{array}$ & $\begin{array}{l}225 \\
77.5 \\
44 \\
25 \\
13.0 \\
5.50\end{array}$ & $\begin{array}{l}27 \\
6 \\
2.3 \\
0.8 \\
0.23 \\
0.04\end{array}$ \\
\hline
\end{tabular}

The first term on the right-hand side of (8.19) is the "velocity" or "refractivity" error, $\Delta R_{N}$; the last two terms represent the geometric range error, $\Delta R_{g}$, which is the difference in length between the straight path, $R_{0}$, and the curved ray path, $R_{g}$. Table 8.5 gives some typical and extreme values of range errors ray traced for observed $N$ profiles.

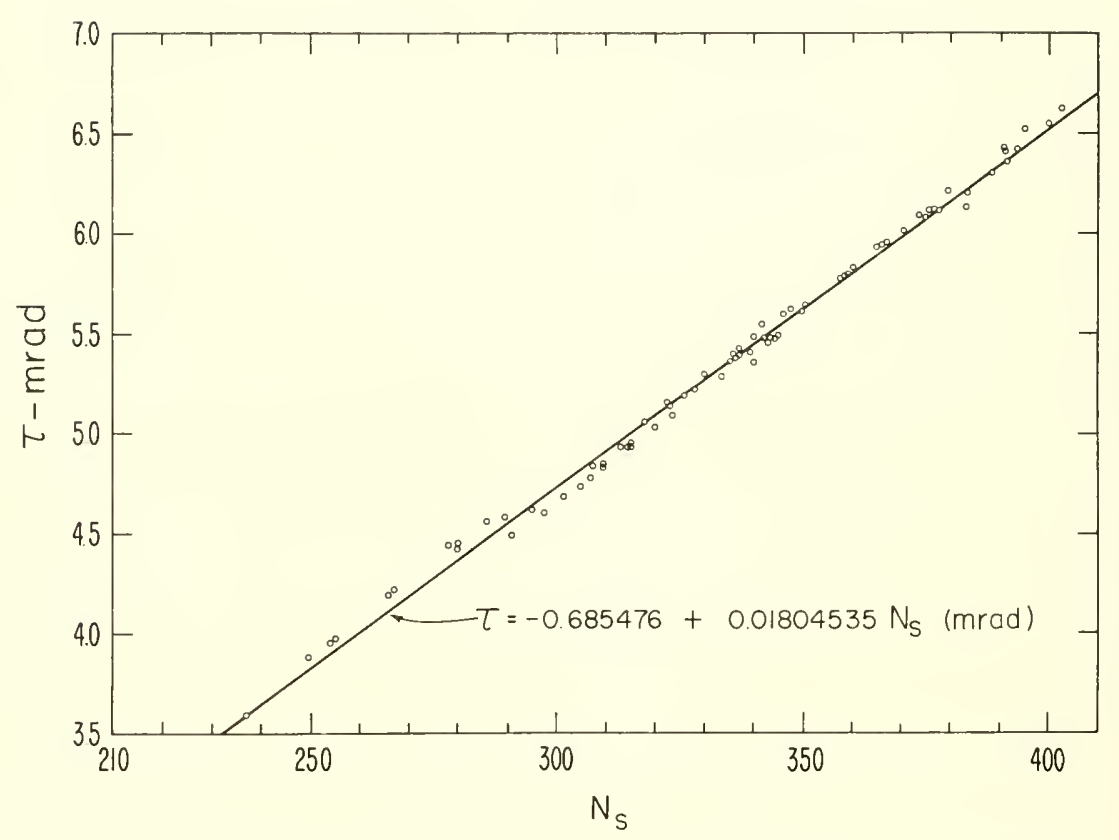

Figure 8.16. Total refraction at $\theta_{0}$ equals $50 \mathrm{mrad}$, h equals $70 \mathrm{~km}$, for the $C R P L$ standard sample. 
From table 8.5 it can be seen that the geometric range error, $\Delta R_{g}$, does not represent a significant portion of the total range error except at very small initial elevation angles, between zero and about $3^{\circ}$. This being so, the behavior of the total range error will be primarily a function of the first integral in (8.19) for elevation angles greater than about $3^{\circ}$. The integral expression

$$
\Delta R_{e} \cong 10^{-6} \int_{0}^{h_{t}} N \csc \theta d h
$$

may be rewritten as

$$
\Delta R_{e} \cong \csc \theta_{0} \times 10^{-6} \int_{0}^{h_{t}} \frac{N d h}{1-2 \sin ^{2}\left(\frac{\theta-\theta_{0}}{2}\right)+\cot \theta_{0} \sin \left(\theta-\theta_{0}\right)},
$$

or

$$
\begin{aligned}
\Delta R_{e} \cong \csc \theta_{0} & \int_{0}^{h_{t}} N d h \\
+ & \sum_{i=1}^{\infty}(-1)^{i+1} \int_{0}^{h_{t}} N\left[\cot \theta_{0} \sin \left(\theta-\theta_{0}\right)-2 \sin ^{2}\left(\frac{\theta-\theta_{0}}{2}\right)\right]^{i} d h,
\end{aligned}
$$

for $\sin \theta<2 \sin \theta_{0}, 0<\theta<\pi / 2$. This expression is analogous to that derived for the case of ray bending, (3.6) of chapter 3 , and, similarly, the integral series on the right-hand side of (8.20) contributed only 3 percent or less to the value of $\Delta R_{e}$ for $\theta_{0}$ larger than about $10^{\circ}$. From (8.20) one would thus suspect that the radio range error might be well estimated as a linear function of the integral of $N$ with respect to height. In treating this integral, it is informative to note that any given $N(h)$ profile may be "broken up" into three primary components:

$$
N(h)=N^{\prime}\left(N_{s}, h\right)+N^{\prime \prime}\left(h+h_{s}\right)+\delta N(h)
$$

where $N^{\prime}$ is that part of the profile which can best be expressed as a function of $N_{s}$ and height, $N^{\prime \prime}$ is a standard distribution of refractivity with respect to altitude above mean sea level $\left(h+h_{s}\right)$ which is independent of $N_{s}$, especially above the tropopause, and $\delta N$ represents a random component of the profile which cannot in general be accounted for a priori. 
The $N^{\prime}$ component is generally effective over the first few kilometers, while above 6 or $7 \mathrm{~km}$ altitude, the $N^{\prime \prime}$ component forms the bulk of the profile [1]. Thus the integral of the $N$ profile with respect to height may be written as:

$\int_{0}^{h_{t}} N d h=\int_{0}^{h_{t}} N^{\prime}\left(N_{s}, h\right) d h+\int_{h_{s}}^{h_{t}+h_{s}} N^{\prime \prime}\left(h+h_{s}\right) d h+\int_{0}^{h_{t}} \delta N(h) d h$,

or,

$$
\int_{0}^{h_{t}} N d h=F_{1}\left(N_{s}, h_{t}\right)+F_{2}\left(h_{s}, h_{t}\right)+\delta F\left(h_{t}\right)
$$

where $\delta F$ is the random contribution to the integral. For any particular $h_{t}$ then

$$
\int_{0}^{h_{t}} N d h=F_{1}\left(N_{\mathrm{s}}\right)+F_{2}\left(h_{s}\right)+\delta F
$$

or

$$
\int_{0}^{h_{t}} N d h=F_{2}\left(h_{s}=0\right)+F_{1}\left(N_{s}\right)-F_{3}\left(h_{s}\right)+\delta F
$$

where

$$
F_{3}=\int_{0}^{h_{s}} N^{\prime \prime}\left(h+h_{s}\right) d h, \text { and } F_{2}\left(h_{s}=0\right) \text { is a constant. }
$$

It was found empirically, from integrated $N(h)$ profiles, that

$$
\int_{0}^{h_{t}} N d h \cong a+b_{1} N_{s}-b_{2} h_{s} \simeq S . E .
$$

The analogy between (8.21) and (8.22) is plain (the standard error of estimate of (8.22), SE, represents the standard deviation, $\delta F$, of (8.21)). The results of such an empirical study are shown in figure 8.17 for the CRPL Standard $N$ Profile Sample, for $h_{t}$ beyond the atmosphere.

For any particular application of (8.22) at a single location, the term $b_{2} h_{s}$ will be absorbed into the constant $a$, since $h_{s}$ does not vary. However the introduction of this term is necessary to explain the station 


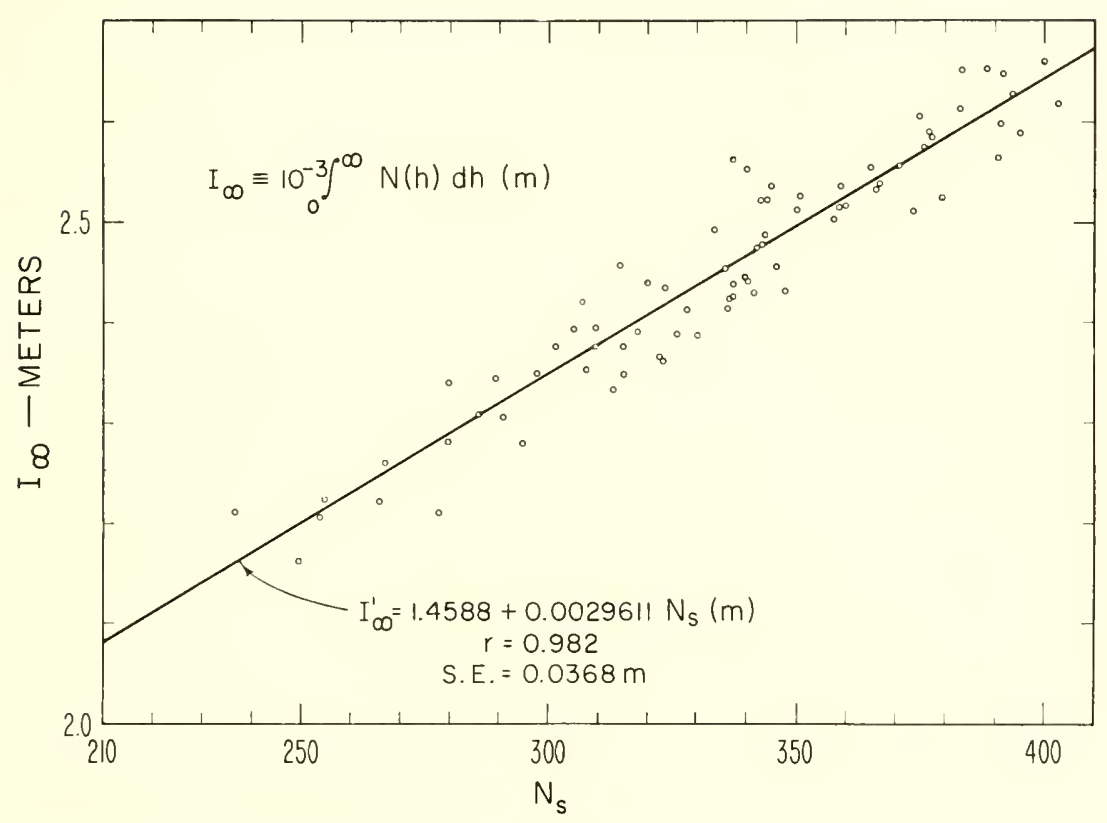

Figure 8.17. Inlegrated refractive index profiles for the CRPL Standard Sample.

elevation dependence of integrated $N(h)$ profiles when taken from a sample containing stations at widely differing elevations, such as the Standard Sample.

It is thus apparent that radio range errors, at least at the higher elevation angles, are primarily a linear function of $N_{s}$. That this is also true at comparatively low angles is shown in figure 8.18 , for $\theta_{0}=50 \mathrm{mrad}$ $\left(\right.$ about $3^{\circ}$ ) for the same profile sample. The reader should especially note the similarity of the distributions of the points about the regression lines between figures 8.17 and 8.18, showing that the range errors at about $3^{\circ}$ are still primarily a function of the integral of $N$ with respect to height, or the range error at $90^{\circ}$.

It has thus been demonstrated that, theoretically, it should be possible to estimate both the angle of refraction of radio rays and errors in radio range measurements from measurement of the refractive index at the surface of the earth. This should be true for targets in or beyond the atmosphere, at elevation angles down to, and possibly lower than, $3^{\circ}$. In addition, if the behavior of refractive index profiles is similar in different parts of the world, it should be possible to specify "universal" values 


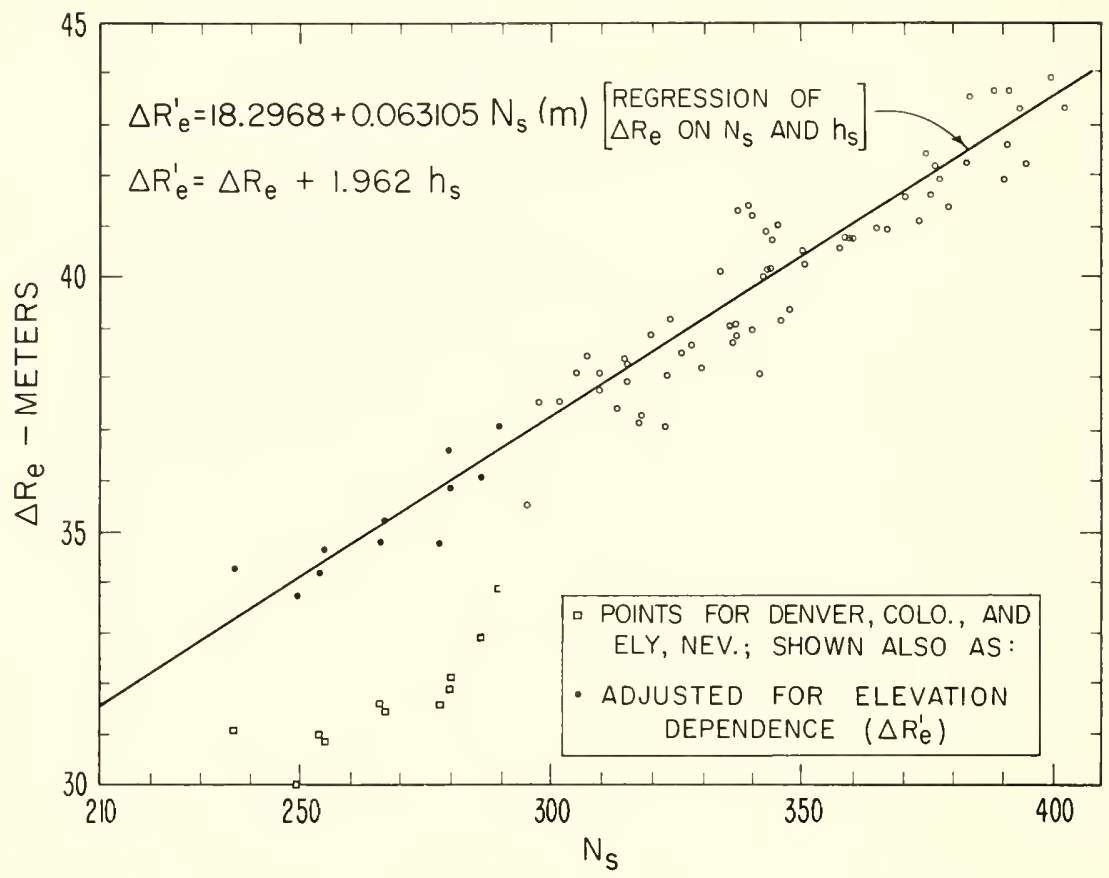

Figure 8.18. Total range error at $\theta_{0}$ equals $50 \mathrm{mrad}$, h equals $70 \mathrm{~km}$, for the CRPL standard sample.

of the coefficients in (8.18) and (8.22) and to predict these values in advance by analysis of a large heterogeneous sample of refractive index profiles. In the suceeeding subsections it will be shown how this has been done, and a comparison will be made between the results so derived and the results of some measurements over actual radio paths.

\subsubsection{The GRPL Standard Atmospheric Radio Refractive Index Profile Sample}

In the preceding section it was shown that, theoretically, it should be possible to estimate either radar elevation angle errors or radio range errors at any particular location by means of a system of linear equations in $N_{s}$, where the coefficients are functions of the target position. The target position can be specified by either the apparent elevation angle and target height, or the apparent range and target height (or as a third possibility, the apparent range and elevation angle), each having advantages in different situations [19]. The equations recommended are

$$
\boldsymbol{\epsilon}=a_{1}\left(\theta_{0}, h_{\imath}\right)+b_{1}\left(\theta_{0}, h_{t}\right) N_{s} \pm \text { S.E. }{ }_{1}\left(\theta_{0}, h_{t}\right),
$$


and

$$
\Delta R_{e}=a_{2}\left(R_{e}, h_{t}\right)+b_{2}\left(R_{e}, h_{t}\right) N_{s} \pm \mathrm{S} . \mathrm{E} .2\left(R_{e}, h_{t}\right),
$$

where $\epsilon$ is the elevation angle error, $\Delta R_{e}$ is the radio range error, $\theta_{0}$ is the apparent elevation angle, $h_{t}$ is the target height, $R_{e}$ is the apparent radio range, and S.E. is the standard error of estimate about the regression line of $\epsilon$ or $\Delta R_{e}$ on $N_{s}$. V'alues of the coefficients may be obtained by performing linear regressions of $\epsilon$ or $\Delta R_{e}$, as ray traced for an appropriate sample of radio refractive index profiles, upon $N_{s}$ for a large number of target positions. As a byproduct of these calculations, one also obtains, for each target position, a value of the residual error (the standard error of estimate) to be expected for the particular type of profile sample used.

In order to obtain a general set of equations to be useful under arbitrary conditions of location, climate, and weather, a large sample of $N$ profiles has been assembled which is believed to be representative of both mean climatic and geographic trends and the larger synoptic variations which may be encountered. This was done by choosing 13 radiosonde stations representative of the major geographic and climatic types of the world, and then choosing from each station six $N$ profiles of particular types, two of which are typical of the extremes of monthly mean conditions for that location, and the other four of which are typical of some of the variations which are found at that location [18]. The result is a sample of $77 \mathrm{~N}$ profiles, ${ }^{3}$ which has been found over a period of years to be a sound cross section of general refractive conditions and has thus been named the CRPL. Standard Atmospheric Radio Refractive Index Profile Sample, hereafter referred to as the CRPL Standard Sample. Although the locations chosen for this sample are heavily weighted towards the United States, it has been found that the general behavior of the refractive index structure as inferred from the standard sample is typical of conditions experienced in most parts of the world [5].

The remainder of this section will be devoted to some comparisons of observed radio refraction data with the predictions supplied by the CRPL Standard Sample, as derived from the linear regressions mentioned above.

Since the refraction measurements reported here consist of samples taken at particular locations over comparatively short periods of time, they should provide a test for the general set of coefficients derived from the Standard Sample; not only is the general theoretical approach tested against measured values, but the measurements coming from places of more or less homogeneous nature, they provide a check as to whether or not coefficients derived for a large heterogeneous sample of data are applicable also to individual places and times; i.e., they should reveal

${ }^{3}$ One of the types could not be found for one of the stations used. 
how much of the observed correlation of the heterogeneous sample is derived from correlation between "classes" of data (in the statistical sense). For a more thorough treatment of the CRPL Standard Sample and the associated regression coefficients for range error and elevation angle error, the reader is referred to Bean and Thayer [19].

For the Standard Sample, the standard error of estimate is equal to the standard prediction error within \pm 1 percent over the range of $N_{s}$ from 200 to 470 , and will be used interchangeably with the latter.

\subsubsection{Comparison With Independent Data}

Before turning to an examination of the experimental refraction data and the degree of success realized in applying the theoretical prediction model to those data, it seems appropriate to examine the accuracy of the prediction model when applied to some independent theoretical (i.e., raytraced) data. For this purpose, four check stations were selected which were not only independent in the sense of not having been included in the original 13-station Standard Sample, but were from locations widely differing from the region of selection of the original sample. It was decided to select one station representative of an arctic type climate, one temperate, one tropical, and one from a "problem" climate area.

Amundsen-Scott station at the South Pole (lat. $90^{\circ} \mathrm{S}$ ) was chosen as the aretic type; this station was expected to present the most rigorous test of the prediction model (as based on the Standard Sample) that could be obtained anywhere in the world. In the first place the extreme areticcontinental climate, with almost no water-vapor contribution to the refractive index and the nearly incessant temperature inversion, is more alien to the Standard Sample than any other type; in the second place the station elevation is $2800 \mathrm{~m}$, which is $900 \mathrm{~m}$ in excess of the highest station (Ely, Nev., $1908 \mathrm{~m}$ ) included in the Standard Sample. These two effects were expected to augment each other as regards refraction.

Dakar, Senegal, on the western coast of Africa, was selected as a "problem" climate station; an inverse relationship exists there between $N_{s}$ and $\Delta N$ (the $N$-gradient over the first kilometer above the surface). A Congo basis station, Bangui, in what was French Equatorial Africa, was selected as the tropical location, and Moscow, U.S.S.R., was selected as the temperate location.

In order to combine brevity with comprehensiveness, ray tracings were done of the total refraction (bending at $70 \mathrm{~km}$ target height) at two elevation angles, $20 \mathrm{mrad}$ and $100 \mathrm{mrad}$, for six profiles from each location. The six profiles were selected as representing roughly the range of $N_{s}$ in winter (February), summer (August), and spring-fall (May and November), two profiles being selected from February, two from August, and one from each of May and November. The 20 -mrad elevation angle 

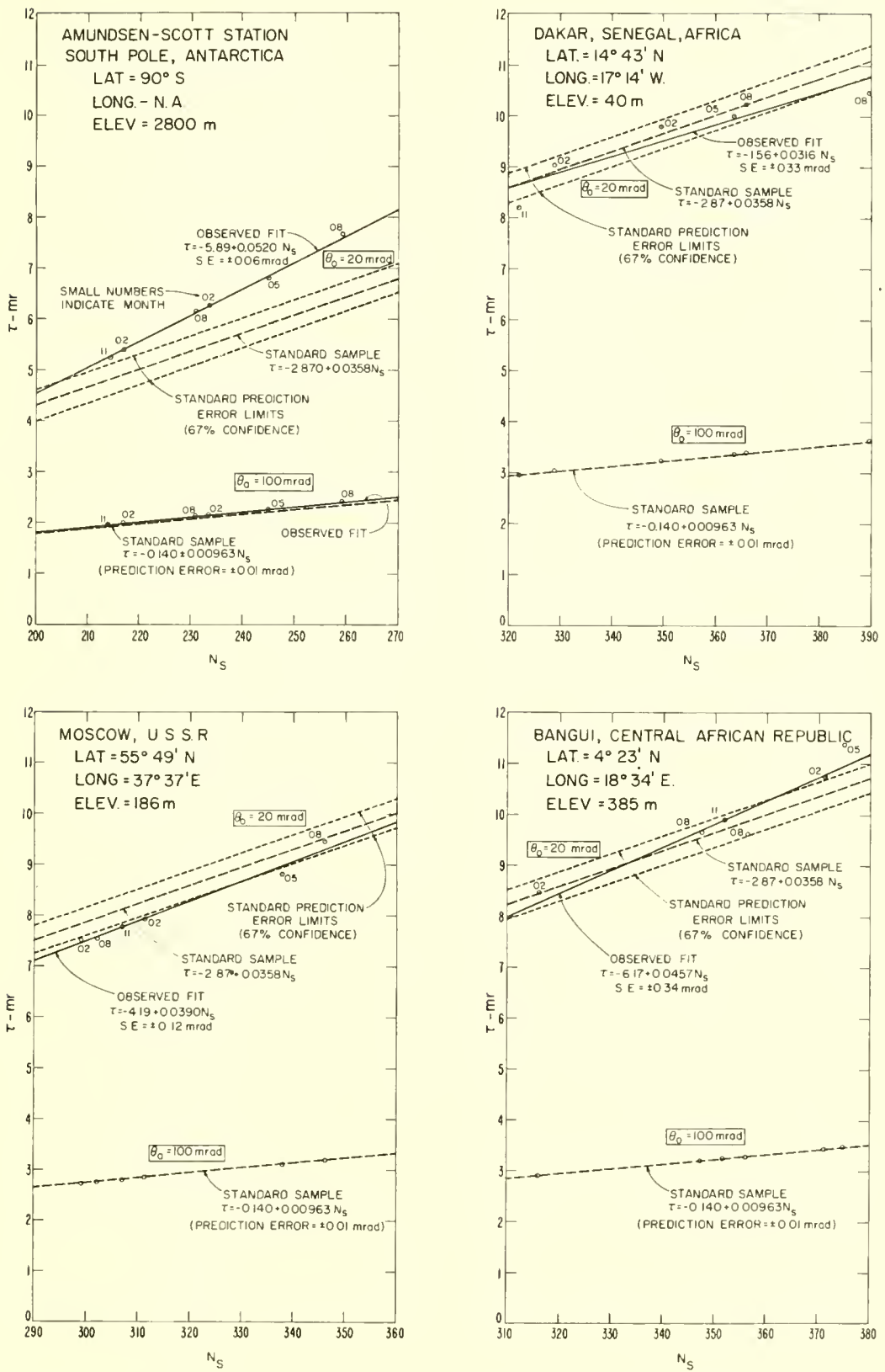

FiguRE 8.19. Comparison of predicted refraction at h equals $70 \mathrm{~km}$ for $\theta_{0}$ 's of $20 \mathrm{mrad}$ and 100 mrad from regressions of the CRPL standard sample and ray-traced values from four independent locations. 
was selected as representing roughly the lower limit of elevation angles for which the bending is expected to be strongly correlated with $N_{s}$ (say $r>0.9$ ), while at $100 \mathrm{mrad}$ (about $6^{\circ}$ ) the correlation is expected to be extremely high (say $r>0.99$ ) and the refraction should be reasonably free of random profile effects.

The results of the ray tracings and the comparison with predicted values are shown in figure 8.19. As expected, the results from the South Pole seem to depart significantly from the predicted values, at least for the 20-mrad elevation angle. At the 100-mrad elevation angle some of the calculated points lie more than one standard deviation from the predicted line (the theoretical prediction error is too small to show on the graph clearly); however, in all four cases the differences are less than 50 $\mu \mathrm{rad}$, a figure which as shall be seen may represent the limit of accuracy obtainable from the atmosphere in actual practice. At angles over 100 mrad the errors would be smaller; in fact they should tend to decrease in inverse proportion to the square of the initial elevation angle, as indeed they do between 20 and 100 mrad.

A conclusion which may be drawn from the above results is that any regions where the prediction model based on the Standard Sample would not be expected to provide the theoretical accuracy are probably regions of climatic extremes, and at least for the case of angular errors the effects will be negligible for elevation angles of a few degrees or more. As an interesting aside it can be noted that apparently the Antarctic may be a desirable area for tracking systems location, at least with respect to atmospheric refraction effects, since (most likely because of the lack of substantial water vapor and the relatively homogeneous conditions) the prediction error for $\theta_{0}=20 \mathrm{mrad}$ in figure 8.19 is only about one-fifth as large as for temperate climates, indicating a possibly more stable atmosphere (even 90 percent confidence limits for the SE in figure 8.19 yield a value less than half of the theoretical temperate value of $\pm 0.286 \mathrm{mrad}$ ).

\subsubsection{Comparison With Experimental Results}

Before comparing the theoretical and experimental results, it is appropriate at this point to examine what one would expect to observe on the basis of propagation theory. In the case of angular errors it is expected that propagation through the real, turbulent atmosphere will produce random variations in the shape of the incoming wavefront, so that measurements made with systems in which the receiving antenna is alined with the incoming signal will have random variations introduced in addition to the ordinary refraction effects. Since these variations will probably not be a function of elevation angle to any great extent, this implies that the residual variance in predicting the elevation angle errors will probably always be greater than predicted from theoretical (static) considerations, and that there will probably be some minimum value of this variance for 
very large elevation angles. Thus, in some cases, the residual crrors will probably not decrease steadily with increasing elevation angle, but will tend to flatten out at some point and assume a more or less constant value above that point. These effects will be complicated in comparing one set of data with another by such things as differences in the location or time of day or season in which data are taken, and instrumental effects such as aperture averaging.

The case of range errors is more straightforward. The effects of turbulent atmospheric inhomogeneities are expected to average out over regions of abnormally high or low density, when considering the transit time of particular points on the wave front. Hence the effect on the residual range errors is expected to be small, and the observed values are expected to compare rather well with the predicted (theoretical )values.

Turning first to the comparison of observed and predicted elevation angle errors, figure 8.20 shows some data on the mean refraction of 1.85 $\mathrm{cm}$ radio waves received from the sun, a target at essentially infinite range so that the elevation angle error is identical with the total angular bending of the radio ray, $\tau$. The data shown in figure 8.20 were obtained by tracking the sun with a precise radio sextant developed by the Collins Radio Company, and were collected in August through December of 1959 at Cedar Rapids, Iowa [20]. These data represent essentially instantaneous measurements. The mean of all observations at each elevation angle is plotted for elevation angles ranging from 2 to $65^{\circ}$, and the mean value of $N_{s}$ associated with each point is about 332; the curve for the mean bending of the CRPL Standard Sample corresponds to the mean value of $N_{s}$ of 334.6 for that sample and hence the data should be comparable. The standard deviation "wings" refer to the standard deviation of the individual "instantaneous" data, not to the standard error of estimate of the mean value. The close agreement observed for elevation angles between 2 and $35^{\circ}$ constitutes not only a confirmation of the usefulness of the Standard Sample, but also a verification of the accuracy of ray-tracing theory in estimating radio wave refraction in the actual, and thus heterogeneous, atmosphere. The standard deviation of the Collins data (shown on the lower part of fig. 8.20) is generally lower than for the standard sample, but this is to be expected in view of the larger range of climatic variation contained in the CRPL Standard Profile Sample. The apparent discrepancies in the measurements made at elevation angles over $40^{\circ}$ are apparently due to some slight inaccuracies in the calibration procedure used on the radio sextant during the period of data acquisition. ${ }^{4}$ In fact, the data shown in figure 8.20 are almost precisely

${ }^{4}$ The data for the highest elevation angles in figure 8.20 were necessarily collected during the early part of the period when the sun was higher in the sky. In a private communication, Anway states that the mean $N_{s}$ applicable to the data at $60^{\circ}$ to $65^{\circ}$ was 358 rather than 332 ; this difference would account for about one-third of the discrepancies noted, reducing the residual bias to a maximum of about $40 \mu \mathrm{rad}$. 


\section{ELEVATION ANGLE, $\theta_{0}$, DEGREES}

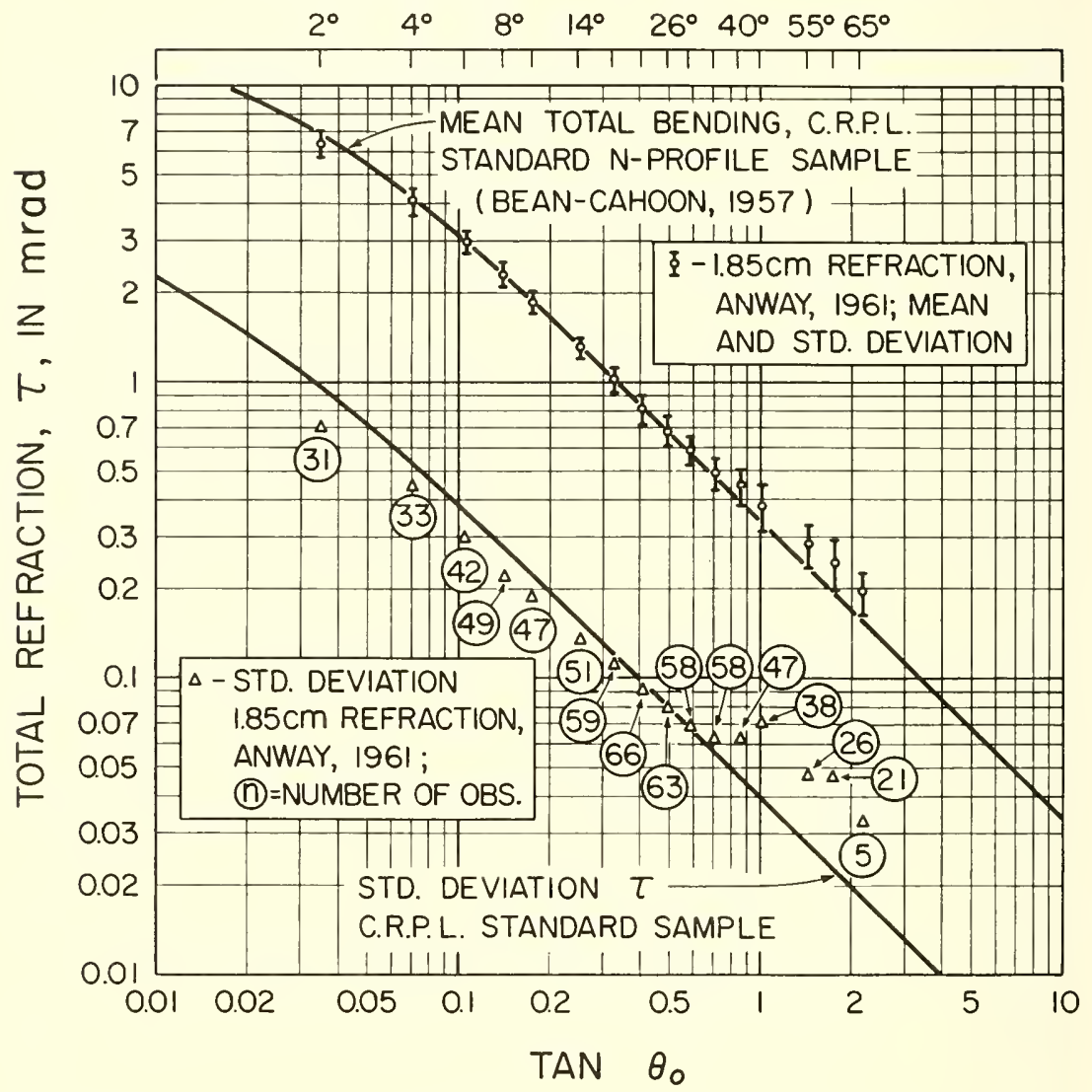

Figure 8.20. Comparison of measured total atmospheric refraction of $1.85 \mathrm{~cm}$ radio waves at Cedar Rapids, Iowa, with values predicted from $\mathrm{N}_{\mathrm{s}}$.

what one would expect to observe if all of the measured values of refraction were increased by a systematic calibration error of about $50 \mu \mathrm{rad}$ over their correct values. The standard deviations in figure 8.20 tend to flatten out at high elevation angles, an effect which is to be expected theoretically as pointed out previously. At any rate, the largest difference between the observed data and the predicted curve in figure 8.20 at elevation angles over $30^{\circ}$, is only about $50 \mu \mathrm{rad}$ or $10 \mathrm{sec}$ of are (the angular diameter of the planet Mars at its average distance from the earth is 10 sec of arc, an angle not discernible to the naked eye). Although this discrepancy might be significant militarily, it is only about 0.5 percent of the diameter of the target sun and is probably near the limit of accuracy of the equipment used. 
Figure 8.21 shows the results of the specific measurements reported by Anway for the radio sextant for all cases at an elevation angle of $8^{\circ} \pm$ $0.09^{\circ}$; each point represents an "instantaneous" reading. The solid line represents the linear regression of the measured refraction data on the values of $N_{s}$; the dashed line shows the predicted linear relationship derived from least squares fits to the CRPL Standard Sample ray traced refraction data. The mean bias between the two lines is about $40 \mu \mathrm{rad}$, interestingly close to, and in the same direction as, the apparent calibration error noted in the mean refraction data at high elevation angles. The standard error of estimate is considerably higher than predicted; however, the rms uncertainty of $\pm 0.052^{\circ}$, or $\pm 0.91 \mathrm{mrad}$, in the apparent elevation angle would be sufficient by itself to increase the standard error of estimate to about $\pm 0.017 \mathrm{mrad}$, which is 4 times larger than the predicted value. It is not known how much of the total standard error of $\pm 0.12 \mathrm{mrad}$ is due to measurement errors as opposed to unforeseen fluctuations in actual atmospheric refraction.

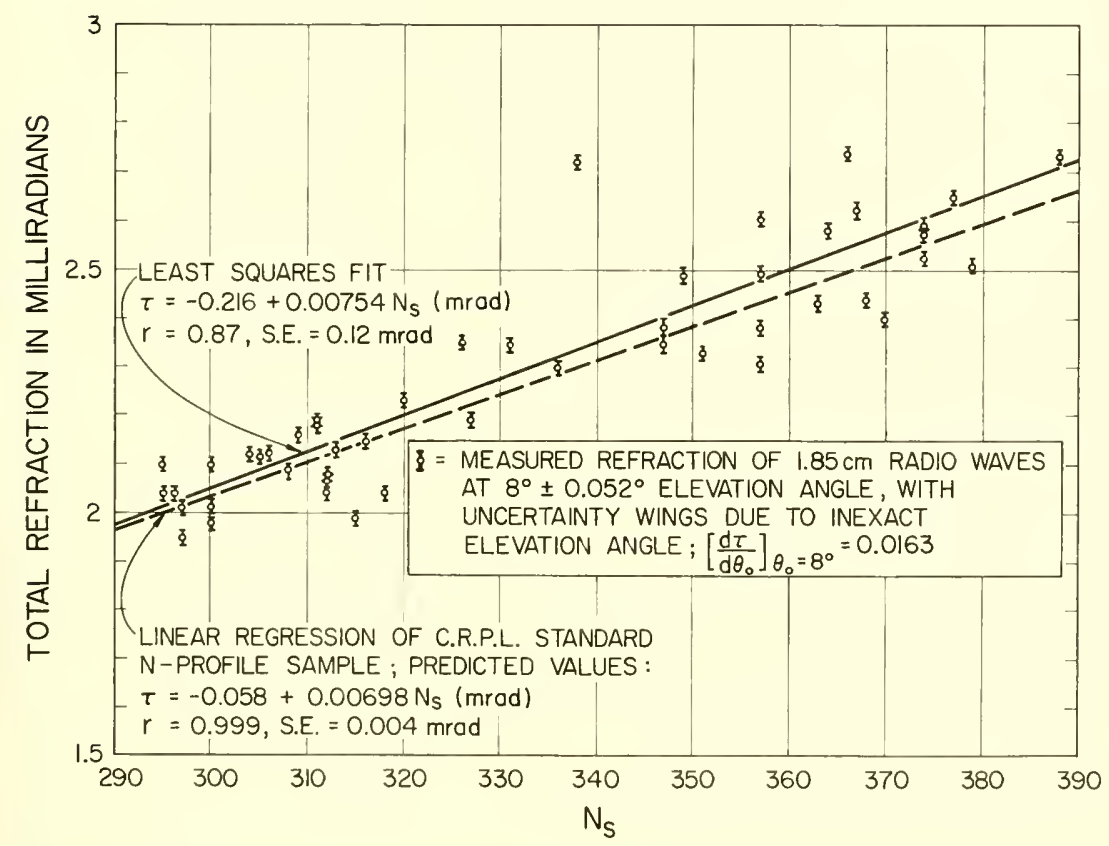

Figure 8.21. $1.85 \mathrm{~cm}$ radio refraction at an elevation angle of 8 degrees, at Cedar Rapids, Iowa.

(After Anway, 1961). 


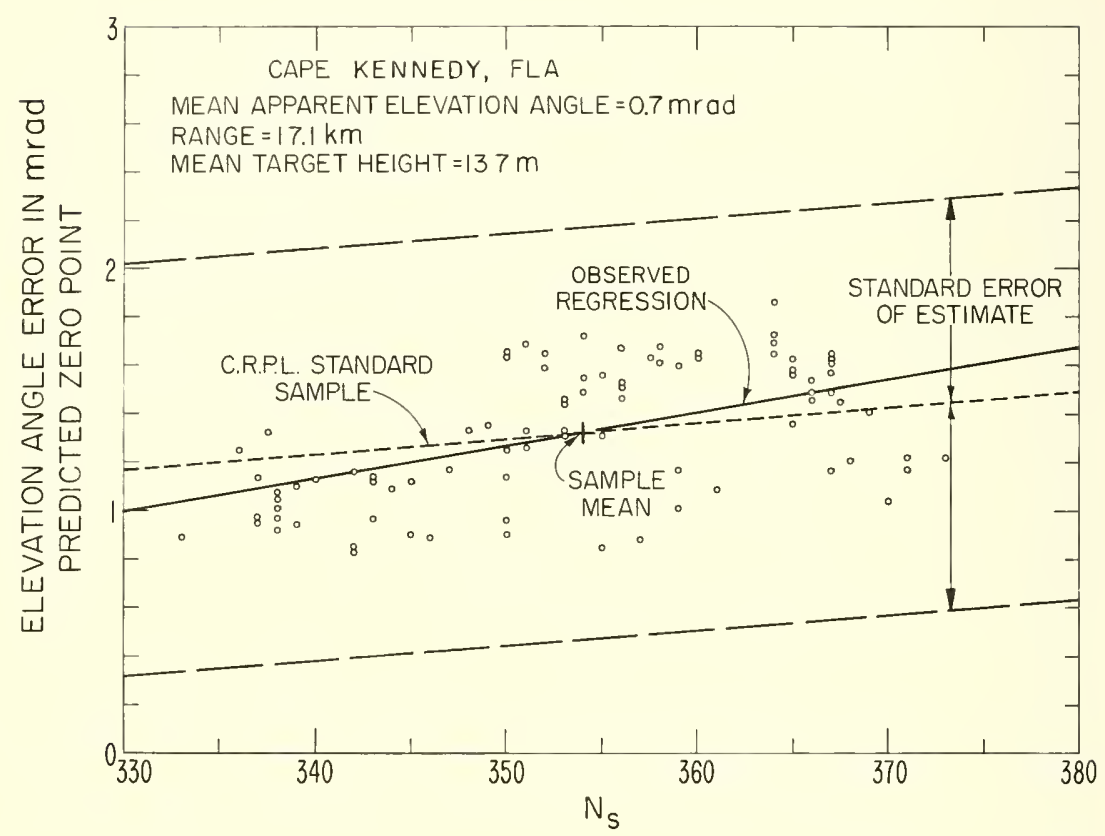

FIGURE 8.22. Elevation angle fluctuations from phase differences taken across a 24-ft vertical baseline, at Cape Kennedy, Fla.

Figure 8.22 shows some results of measurements taken at Cape Kennedy, Fla., on November 1-3, 1959, at a very low elevation angle, about $0.7 \mathrm{mrad}$ or $0.04^{\circ}$. These are "instantaneous" measurements, taken at half-hourly intervals, of the phase difference fluetuations between the signals from a beacon as they arrived at the upper and lower terminals of a vertical 24-ft baseline, thus being very elosely equivalent to a measurement of the fluctuations in the angle of arrival of the wave front at the centerpoint of the baseline (the altitude difference between this point and the target beacon is referred to as the "mean" target height). Since only the fluctuations and not the total phase differences were measured, only the slope and scatter of the elevation angle errors as a function of the observed $N_{s}$ data can be compared with the predicted values from the CRPL Standard Sample. The zero point on the graph is set by the predicted mean value for the sample. The correlation coefficient is, as expected, only 0.57. In this ease the scatter of the observed data is well inside the limits of the standard error of estimate of the regression for the standard sample, even at this very small elevation angle where horizontal changes in the $N$ profile can exert a large effect on elevation angle errors. 
Figure 8.23 shows the results of a comparison between predictions of elevation angle errors estimated from the CRPL Standard Sample and some measurements made with a 6 -cm radar at Tularosa Basin, N. Mex. [21]. Each point represents the mean of five "instantaneous" readings made at 1-min intervals over a period of $4 \mathrm{~min}$. The standard deviation of each five-reading group averaged $0.16 \mathrm{mrad}$, and the maximum range in any one group was $0.58 \mathrm{mrad}$. The radio energy was propagated over a 45-mi path at a mean apparent elevation angle of $18 \mathrm{mrad}$; the target was a beacon located on a mountain peak $5610.5 \mathrm{ft}$ higher than the desert floor where the radar was located. The data in figure 8.18 show that even for this rather extreme case, where the degree of correlation between $N_{s}$ and $\epsilon$ is expected to be only 0.4 , agreement is obtained between:

(a) the predicted and observed mean refraction,

(b) the observed and predicted slopes of the $\epsilon$ versus $N_{s}$ relation, and

(c) the observed and predicted residual errors of predicting $\epsilon$ from $N_{s}$ alone.

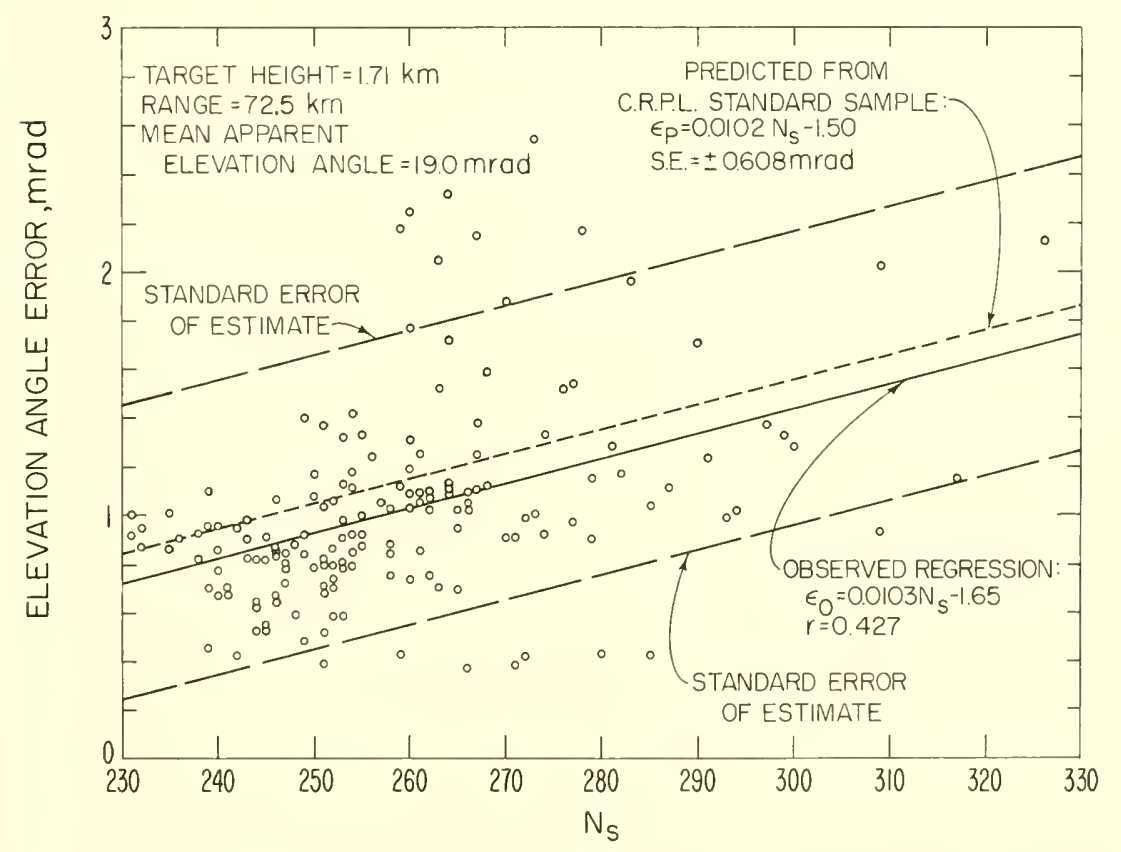

Figure 8.23. Measured refraction of C-band radar at Tularosa Basin, N.Mex. 


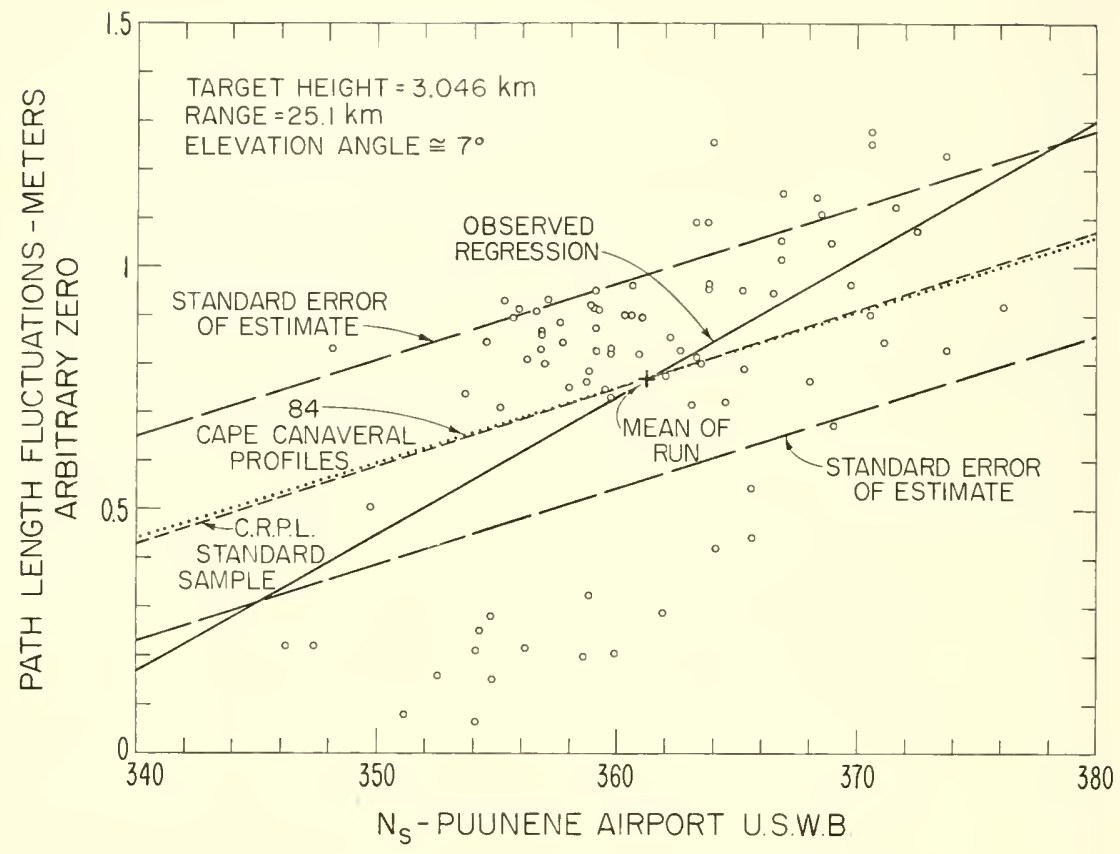

Figure 8.24. Range error fluctuations observed on Maui path.

The small discrepancy between the intercepts (i.e., between the mean refraction) of the observed and predicted $\epsilon$ versus $N_{s}$ lines may be perhaps attributed to, for example, antenna lobe pattern distortion caused by differential refraction, or defoeusing [22].

The remaining data which are examined were of necessity taken in such a manner as to have a rather high degree of autocorrelation (trends). Such data are not as suitable for confirming the accuracy of a regression prediction process as are independent data. A discussion of this is included at the end of this section.

Turning to examination of radio range errors, figure 8.24 shows the results of some measurements of apparent radio range fluctuations over a 25-km path on the island of Maui, Hawaii, on November 9-11, 1956 [23]. These measurements were made at 1-hr intervals, and are essentially "instantaneous" values. The target beacon was situated on the summit of Mount Haleakala at an elevation of $10,025 \mathrm{ft}$, while the "ground" station was near Puunene Airport at an elevation of $104 \mathrm{ft}$, thus yielding a target height of $3.046 \mathrm{~km}$, in a region of critical target heights for prediction of radio range errors in tropical climates [19]. The measured range fluctuations (absolute errors not measured) are plotted against values of $N_{s}$ taken at about the same time (mostly 15 to 20 min later) by 
U.S. Weather Bureau personnel at the Puunene Airport weather station. The agreement between observation and prediction is fairly good, especially when one considers that only 32 of the 86 points lie outside of the predicted standard error of estimate limits, while chance would indicate that 29 points would exceed these limits. Also, it should be kept in mind that in this case, as for all except the Collins data, the target beacon is located on the surface of the earth, whereas the predictions from the CRPL Standard Profile Sample are derived for targets in the free atmosphere; there is undoubtedly some bias introduced in this way.

As a part of a continuing investigation into the atmospheric limitations imposed on electronic distance measuring equipment, some measurements have been made recently by the Tropospheric Physics Section, NBS, of both range errors and range difference errors (across a phasedifferencing baseline) over a propagation path near Boulder, Colo. Figures 8.25 and 8.26 are based on some of the preliminary results of these measurements [24]. Figure 8.25 shows the results of measurements of the fluctuations in apparent range, made at half-hour intervals on May 9-11, 1961, over a $15.5-\mathrm{km}$ path between a transmitting beacon

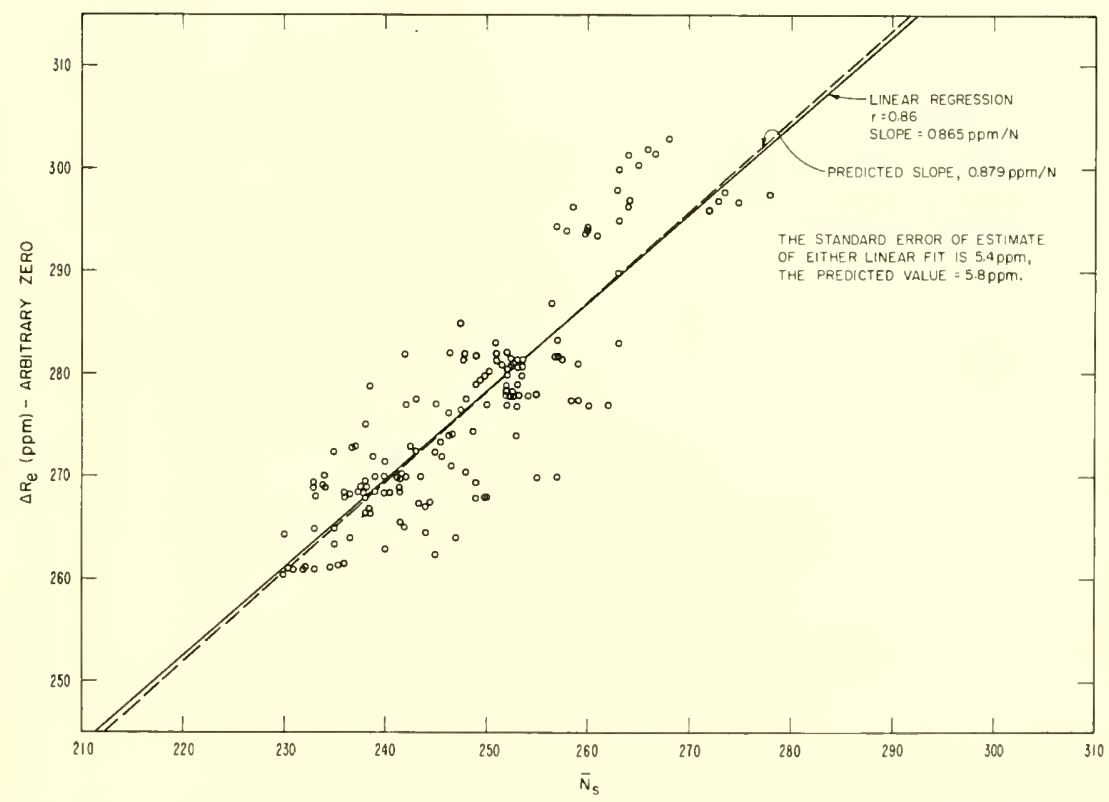

Figure 8.25. Range error fluctuations observed over the Boulder Creek-Green Mounlain path, Colo.

3-day run, half-hourly readings, range $=15.5 \mathrm{~km}$, target height $688 \mathrm{~m}$, approx elevation angle $26^{\circ}$ May $9-12,1961$ 


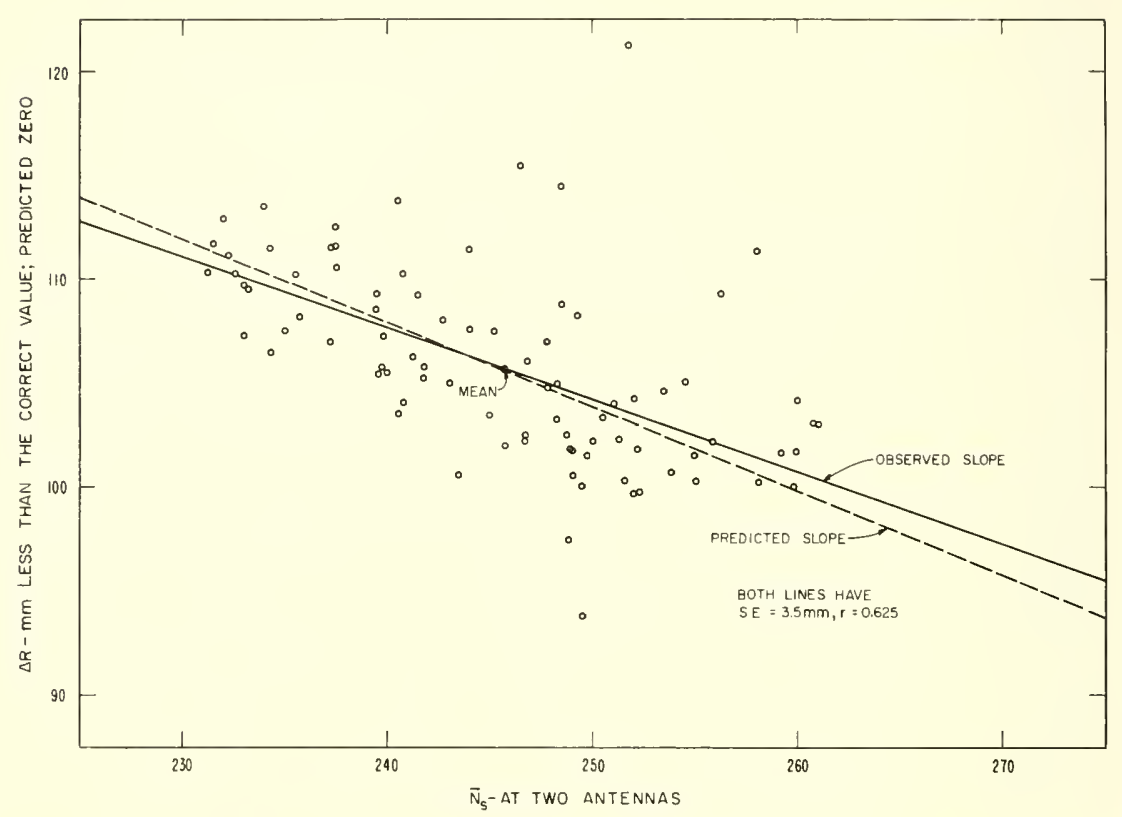

Figure 8.26. Range difference fluctuations observed over a 460-m in-line baseline, Boulder Creek-Green Mountain path, Colo.

4.15-hr run, 460 meter in-line baseline, target height $=688 \mathrm{~m}$ apparent range $15.5 \mathrm{~km}$, May 9-11, 1961

on Green Mountain at an elevation of $2242 \mathrm{mr}$ and a receiving antenna located near Boulder Creek at an elevation of $1554 \mathrm{~m}$, the true target height thus being $688 \mathrm{~m}$.

The apparent range fluctuations, expressed in parts per million of the $15.5-\mathrm{km}$ path length (with an arbitrary zero since the total range was not measured), are plotted as a function of the surface value of the refractive index taken at a point quite close to the lower terminal. Quite good agreement is seen between the simple linear regression of the observed $\Delta R_{e}$ values on $N_{s}$ and the predicted linear relationship obtained from the CRPL Standard Sample. Note that both lines have statistically equal standard errors of estimate with respect to the observed data.

Figure 8.26 shows the results of the range difference measurements made over a $460-\mathrm{m}$ baseline essentially in line with the transmission path, where the second antenna was farther from the target beacon than the primary antemna. Here the range difference fluctuations (again with an arbitrary zero) have been plotted as a function of the mean value of $N_{s}$ measured at each end of the baseline. The zero point on the graph is set by the predicted mean of the sample. In this case there seems to be some discrepancy between the regression of the data and the predicted slope; 
however, note that the standard errors of estimate for the two lines are, to two significant figures, equal, indieating that the difference in the slopes is probably statistically insignificant.

There are some data points in figure 8.26 having a rather large deviation from the regression lines. Statistical theory (using the "Student" $t$-distribution for $84 \mathrm{deg}$ of freedom) shows that, if the data points are drawn from a normally distributed population, there should be only one point having a deviation of more than $\pm 9 \mathrm{~mm}$ from the observed regression line. There are in fact five points in figure 8.26, four above and one below the line. If these five points are thrown out, on the grounds that they weight too heavily the extremes of the distribution of data points (this is especially true when using least squares regression), and the regression is then redone using the remaining 81 data points, the resulting value of the slope is $-0.385 \mathrm{~mm} / N$-unit with $r=0.77$ compared to the predicted slope of $-0.381 \mathrm{~mm} / N$-unit, a rather elose agreement.

\subsubsection{Discussion of Results}

As a summary of the results of the experimental versus theoretical comparisons given in the preceding section, a statistical analysis has been run on the significance of the differences between the slopes of the observed and predicted regression lines. In order to make the tests more stringent, it was assumed that the slopes derived from the Standard Sample should be taken to be the slopes of the population regression lines $(\beta)$, thus yielding an estimate of the significance of the departure of the observed slope from the assumed population value.

A value of $t$ was first calculated for each ease using the relation

$$
t_{j-2}=\frac{\left|b-\beta_{0}\right| \sqrt{\Sigma\left(x_{i}-\bar{x}\right)^{2}}}{S E}
$$

where $b$ is the observed slope, $\beta_{0}$ the assumed population, or theoretical, slope, $x$ refers to the independent variable in each regression, $N_{s}, \mathrm{SE}$ is the standard error of estimate, and $t_{j-2}$ is the value of $t$ for $j-2$ deg of freedom. From $t_{j-2}$, confidence limits for $\beta$ at the $100(1-a)$ percent level can be calculated from [25]

$$
b-\frac{t_{j-2, \alpha} S E}{\sqrt{\Sigma\left(x_{i}-\bar{x}\right)^{2}}}<\beta<b+\frac{t_{j-2, \alpha} S E}{\sqrt{\Sigma\left(x_{i}-\bar{x}\right)^{2}}}
$$

The probability that the observed value $b$ would have fallen out side of these limits by chance is $\alpha$. Many statisticians consider a value of $t_{j-2}$ 
falling below the $100 \alpha=5$ percent level to be not significant, between the 5 percent and 1 percent levels to be of questionable significance, and over the 1 percent level to be significant [25]. An observed slope $b$ falling

$$
\left|b-\beta_{0}\right|>\frac{t_{j-2,0.01} \mathrm{SE}}{\sqrt{\Sigma\left(x_{i}-\bar{x}\right)^{2}}}
$$

would thus be taken to represent a significant departure from the value $\beta_{0}$, and would thus imply the possibilities

(a) $\beta_{0}$ does not represent $\beta$, or

(b) $b$ represents the regression of data from a population different than that used in determining $\beta_{0}$, or

(c) both.

Before making the significance tests, however, the value of $j$, the number of independent observations going into the determination of $b$, must be known. In general, data of the type presented here are more or less highly autocorrelated, and hence not all independent. The data presented here, with the possible exception of the Collins data and the Tularosa Basin data for which the calculations could not be performed, have autocorrelation coefficients $r_{k}$, for lag $k(k=1,2,3$ units of time between successive measurements) that can be approximately described by

$$
r_{k} \cong\left(r^{\prime}\right)^{k},
$$

and for this type of data the effective number of pieces of independent data, $j$, is given by [26]

$$
j=n\left[\frac{1-r^{\prime}}{1+r^{\prime}}\right] \text {. }
$$

For the data treated here weighted mean values of $r^{\prime}$ were calculated from

$$
r^{\prime} \cong \frac{r_{1}+4 r_{2}^{1 / 2}+9 r_{3}^{1 / 3}+\cdots+k^{2} r_{k}^{1 / k}}{1+4+9+\cdots k^{2}}
$$

where $k$ was the largest lag for which the autocorrelation coefficient was calculated, usually 4 or 5 . No special justification is offered for the use of (8.28) other than the obvious fact that $r_{k}$ is to be approximated by the $k$ th power of $r^{\prime}$, and hence a function of $k$ would seem to be the most logical weighting function to use; the use of $k^{2}$ as a weighting function seemed to give the best overall fit to the series of $r_{k}$ encountered from these data. 
Table 8.6 shows the results of the significance tests on the slopes of the various experimental and theoretical (predicted) regression lines. The number of pieces of data is shown in the first column, the observed slope $b$ and theoretical slope $\beta$ in the second and third columns, the autocorrelation coefficient for lag of one time unit in the fourth column, and the weighted mean $r^{\prime}$ as defined in (8.28) in the fifth column. In column 6 the effective number of independent pieces of data, $j$, is shown, while in column 7 the value of $t_{j-2}$ is shown for the difference between $b$ and $\beta$. The next column shows the value of $t_{j-2,0.5}$, the value for the 50 percent significance level for $j-2$ degrees of freedom.

Only one of the $t$ values turns out to be significant at the 50 percent level, which means that there was a better than even chance that such differences would have occurred by chance in the other cases. In the case of the Collins data at $\theta_{0} \cong 8^{\circ}$, the value of $t=1.01$ would not be significant at the 25 percent level; the value $t_{46}=1.01$ corresponds to $\alpha=0.34$, or a 34 percent chance that the observed deviation $|b-\beta|$ is of a random nature, and thus not significant.

TABLE 8.6. Experimental versus theoretical slopes

\begin{tabular}{|c|c|c|c|c|c|c|c|c|c|}
\hline$\epsilon$ vs $N_{s}$ & $n$ & $b$ & $\beta$ & $r_{1}$ & $r^{\prime}$ & $j$ & $t_{j-2}$ & $\begin{array}{c}t(0.50 \\
j-2)\end{array}$ & $\begin{array}{c}\text { Is }[b-\beta] \text { sig- } \\
\text { nificant at } \\
\text { the } a=50 \\
\text { percent level? }\end{array}$ \\
\hline Collins data $8^{\circ} \ldots$ & 48 & 0.00754 & 0.00698 & - & - & (48) & 1.01 & 0.68 & Yes \\
\hline Tularosa Basin & 161 & 0.0103 & 0.0102 & - & - & $(161)$ & 0.031 & 0.676 & No \\
\hline $\begin{array}{l}\text { Cape Canaveral } \\
\Delta R_{e} \text { vs } N_{s}\end{array}$ & 86 & 0.01356 & 0.00648 & .870 & .860 & 6.5 & 0.708 & 0.73 & No \\
\hline Maui data....... & 86 & 0.02833 & 0.01610 & .974 & .950 & 2.2 & 3. 56 & 7.6 & No \\
\hline $\begin{array}{l}\text { Boulder Creek- } \\
\text { Green Mt.. } \\
\Delta(\Delta R) \text { vs } N_{s}\end{array}$ & 155 & 0.865 & 0.879 & .944 & .950 & 4.0 & 0.32 & 0.82 & No \\
\hline $\begin{array}{l}\text { Boulder Creek- } \\
\text { Green Mt.---- }\end{array}$ & 86 & -0.344 & -0.381 & .957 & .946 & 2.4 & 0.60 & 2.0 & No \\
\hline
\end{tabular}

From the point of view of a statistician, the results of these tests are such that no significance can be attached to any of the apparent discrepancies between theory and observation, and given reason to believe that the values of $\beta$ are theoretically sound, one could say that the results are significantly positive in nature. The significance of the differences between the predicted and observed slopes of the regression lines for $\theta_{0}=$ $20 \mathrm{mrad}$ for the independent data check of subsection 8.3.4 were tested using the same method as the preceding tests, except that the six observations in each case were assumed to be independent. The results are summarized in table 8.7 and confirm the general use of the Standard Sample for $\theta_{0}>20 \mathrm{mrad}$. 
From the experimental data which are available at the present time it may be concluded that:

(a) Radio range and elevation angle errors can be predicted from the surface value of the radio refractive index, and the accuracy obtained will be generally commensurate with the estimates of residual errors made from theoretical ray-tracing considerations.

(b) The functional dependence of either angular refraction or range errors on the surface value of the refractive index as derived from the CRPL standard $N$ profile sample may be applied to arbitrary locations or climates without noticeable decrease in accuracy over that obtained with a sample from the location under consideration.

(c) The effects of horizontal inhomogeneities of the refractive index, which certainly must have been prevalent over the transmission paths for which experimental data have been presented, do not appear to introduce any bias or additional residual variance into the values of observed refraction variables over those predicted from surface observations.

TABLE 8.7. Comparison of slopes for independent check

Predicted slope at $\theta_{0}=20 \mathrm{mrad}: 0.358 \mathrm{mrad} / N_{\mathrm{s}}$

\begin{tabular}{|c|c|c|c|c|c|c|}
\hline Station & $\begin{array}{l}\text { Observed } \\
\text { slope }\end{array}$ & $\begin{array}{c}\text { Difference } \\
\boldsymbol{b}-\boldsymbol{\beta}_{0}\end{array}$ & $\sqrt{\mathrm{S}\left(N_{s}-\vec{N}_{s}\right)^{2}}$ & $t$ & $100 \alpha$ & Significance \\
\hline $\begin{array}{l}\text { Amundsen-Scott } \ldots \\
\text { Dakar } \\
\text { Bangui } \\
\text { Moscow }\end{array}$ & $\begin{array}{l}0.0520 \\
.0316 \\
.0457 \\
.0390\end{array}$ & $\begin{array}{r}+0.0162 \\
-.0042 \\
+.0099 \\
+.0032\end{array}$ & $\begin{array}{l}37.9 \\
56.5 \\
47.2 \\
44.3\end{array}$ & $\begin{array}{r}10.2 \\
0.72 \\
1.37 \\
1.18\end{array}$ & $\begin{array}{r}<0.1 \text { percent } \\
52 \text { percent } \\
25 \text { percent } \\
31 \text { percent }\end{array}$ & $\begin{array}{l}\text { very high } \\
\text { none } \\
\text { very low } \\
\text { very low }\end{array}$ \\
\hline
\end{tabular}

$$
t_{4, \alpha} \alpha=\left[b-\beta_{0}\right] \frac{\sqrt{\Sigma\left(N_{s}-\bar{N}_{s}\right)^{2}}}{\text { S. E. }}
$$

\subsection{Correction of Atmospheric Refraction Errors in Radio Height Finding}

\subsubsection{Introduction}

As a radio ray passes through the atmosphere, the length and direction of its path varies with the radio refractive index. Uncorrected radar output determines the position of a target by a straight line path at constant velocity. The difference between the straight path and the actual path results in an error which becomes increasingly significant as the distance to the target increases. The height error (the component of the position error normal to the surface of the earth) constitutes over 95 percent of the total error. Until recently, the range of height finding equipment was sufficiently limited so that the refraction errors could be either neglected, or approximated by the effective (four-thirds) earth's radius correction [27]. 
Bauer, Mason, and Wilson [28] obtained an equation for accurately estimating radar target heights in a specific exponential atmosphere. Beckmann [29] presented a probability estimate of the height errors without meteorological measurements.

The purpose of the study is to investigate the correlation between available meteorological parameters and height errors for targets of interest in terminal air traffic control and to develop height error correction procedures using these parameters.

\subsubsection{Refractive Index}

The radio refractive index, $n$, of a propagation medium is the ratio of the free space velocity of light, $c$, to the velocity in the medium, $v$, (i.e., $n=c / v)$. Since the propagation velocity of the atmosphere is only slightly less than the free space velocity, it is often convenient to use the scaled up difference between the refractive index and unity. This quantity is the refractivity.

The refractivity is obtained from (1.20). Normally, the equation for $N$ is dominated by the first term so that the refractivity can be approximated by an exponential function of height as shown in section 3.8.

\subsubsection{Ray Theory}

If the refractive index is assumed to satisfy (for a spherically stratified atmosphere)

$$
\frac{d n}{d r}>-\frac{1}{r}
$$

then, for frequencies greater than $100 \mathrm{kc} / \mathrm{s}$, the path of a radio ray is determined by Snell's law for polar coordinates (3.1) of chapter 3 (see fig. 8.27) and the bending angle, $\tau$, is determined from (3.2). The distance, $d$, along the surface of the earth is obtained from (3.62).

The length of the path is called the geometric range and is obtained by

$$
R=\int_{r_{o}}^{r_{o}+h} \csc \theta d r
$$

and the apparent or radio range is found by

$$
R_{e}=\int_{r_{o}}^{r_{o}+h} n \csc \theta d r=R+\int_{r_{o}}^{r_{o}+h} N \times 10^{-6} \csc \theta d r .
$$




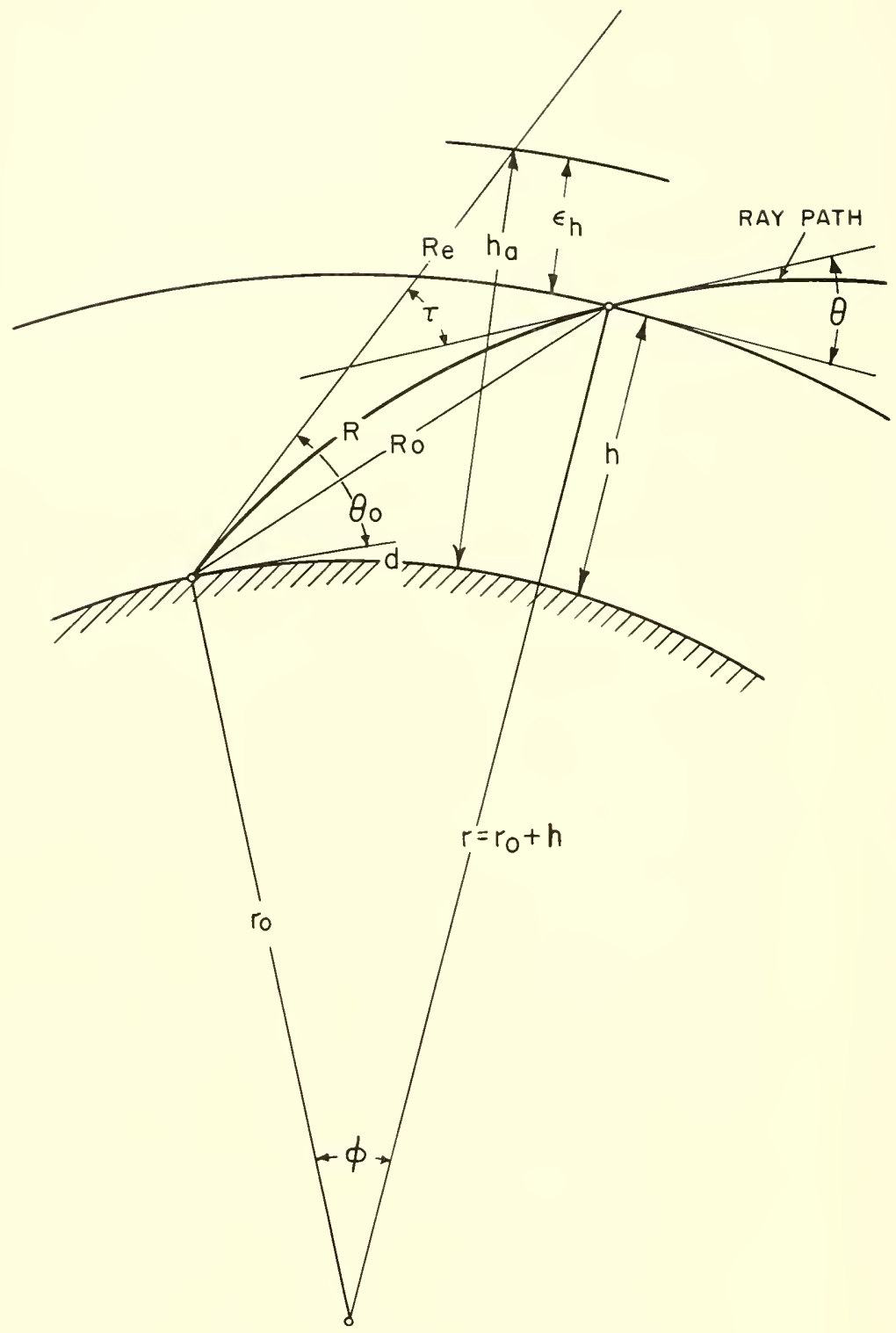

Figure 8.27. Geometry of radio ray refraction. 


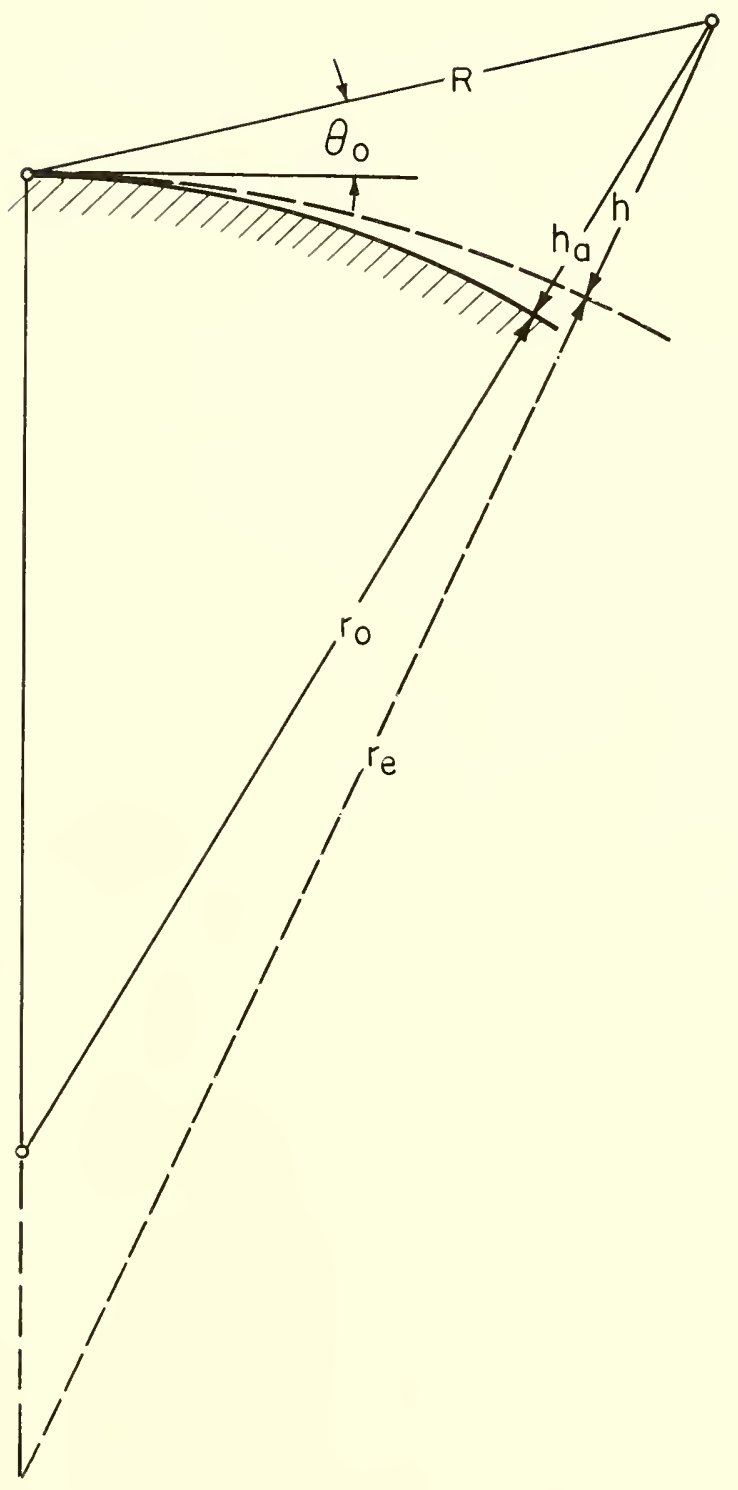

FIGURE 8.28. Effective carth's radius grometry. 
Because the difference between $R_{e}$ and the true slant range, $R_{0}$, is small compared to the height error, the slant range and radio range are assumed to be identical to the geometric range, $R$.

The apparent height of the target, in figure 8.28 , is obtained by solving

$$
\left(r_{0}+h_{a}\right)^{2}=r_{0}^{2}+R^{2}+2 r_{0} R \sin \theta_{0}
$$

for $h_{a}$. The following form is useful for numerical calculations:

$$
h_{a}=\frac{R\left(R+2 r_{0} \sin \theta_{0}\right)}{r_{0}+\sqrt{r_{0}^{2}+R\left(R+r_{0} \sin \theta_{0}\right)}}
$$

The height error for a target at height $h$ is found by

$$
\epsilon_{h}=h_{a}-h
$$

which will always be positive if $n$ decreases with height.

If the refractive index is known as a function of height the foregoing procedure is useful for determining the height error when the true height and the arrival angle of the ray are hypothesized. Unfortunately, it is not applicable for obtaining the height error from the apparent position of the target.

\subsubsection{Use of the Effective Earth's Radius}

The inaccuracy of the "four-thirds earth" correction stems mainly from the assumption that all radio rays have the same constant curvature. The accuracy would be greatly enhanced if an "average" effective radius could be determined for each ray path.

The following expression, with the effective earth's radius denoted by $r_{e}$ in figure 8.28 ,

$$
\left(r_{e}+h\right)^{2}=r_{e}^{2}+R^{2}+2 r_{e} R \sin \theta_{0},
$$

can be combined with (8.32) and (8.34) to obtain

$$
\epsilon_{h}+\frac{h_{a} \epsilon_{h}}{r_{e}}-\frac{\epsilon_{h}^{2}}{2 r_{e}}=\left(\frac{R^{2}-h_{a}^{2}}{2}\right)\left(\frac{1}{r_{0}}-\frac{1}{r_{e}}\right)
$$

The difference bet ween the curvature of the actual earth and the "average" effective earth for the ray path represents the "average" curvature of the ray. Thus, if the ray curvature can be determined as a function of the 
target position and the refractive index structure, (8.36) will provide a simple formula for approximating the height error,

$$
\epsilon_{h} \cong\left(\frac{R^{2}-h_{a}^{2}}{2}\right)\left(\frac{1}{r_{0}}-\frac{1}{r_{e}}\right)
$$

since for target heights $(h \leq 70,000 \mathrm{ft})$ at the maximum range $(R \simeq 150$ mi) to be considered

$$
\left|\frac{h_{a} \epsilon_{h}}{r_{e}}-\frac{\epsilon_{h}^{2}}{2 r_{e}}\right|<20 \mathrm{ft},
$$

or less than one percent of the total height error.

If the curvature of a ray, $1 / \rho$, at any point on the path is called $K$, then, from (8.29) and (3.20)

$$
K=-\frac{n_{0} \cos \theta_{0}}{n^{2}\left(1+h / r_{0}\right)} \frac{d n}{d h}
$$

From (8.32) ignoring terms of the order $1 / r_{0}{ }^{2}$, one obtains

$$
\cos ^{2} \theta_{0} \simeq\left(1+\frac{h_{a}}{r_{0}}\right)\left(\frac{R^{2}-h_{a}^{2}}{R^{2}}\right)
$$

so that $(8.38)$ becomes

$$
K \simeq-\frac{n_{0}}{n^{2}} \frac{\left(R^{2}-h_{a}^{2}\right)^{1 / 2}}{R} \frac{\left(1+\frac{h_{a}}{r_{0}}\right)^{1 / 2}}{\left(1+\frac{h}{r_{0}}\right)^{\frac{d n}{d h}} .}
$$

The refractive index usually decreases with height so that the quantity

$$
\frac{n_{0}}{n^{2}} \frac{\left(1+\frac{h_{a}}{r_{0}}\right)^{1 / 2}}{1+\frac{h}{r_{0}}} \simeq 1
$$

varies only slightly with height, and the eurvature at a point on the ray path can be approximated by

$$
K \simeq\left(\frac{R^{2}-h_{a}^{2}}{R}\right)^{1 / 2}\left|\frac{d n}{d h}\right|
$$


Therefore, (8.37) becomes

$$
\left.\epsilon_{h} \simeq \frac{\left(R^{2}-h_{a}^{2}\right)}{2 R}\right)^{3 / 2}
$$

where $g$ represents the "average" gradient on the ray path (i.e., $g=d n / d h$ at an intermediate point on the path).

Since $g$ depends upon the meteorological conditions along the path, the basic problem is to determine $g$ for a given target from the conditions at and/or near the surface.

\subsubsection{Meteorological Parameters}

Measurement of the refractivity at the radar site will provide an estimate of the gradient if a model of the refractive index structure is assumed. In the exponential model, for example,

$$
n(h)=1+N_{s} \exp (-c h) \times 10^{-6}
$$

where $N_{s}$ is the surface refractivity and $c$ is a constant, then

$$
\frac{d n}{d h}=-c N_{s} \exp (-c h) \times 10^{-6} .
$$

For a target at a height, $h_{t}$, the average gradient along the ray path is

$$
g=\frac{N_{s}}{h_{t}}\left[1-\exp \left(-c h_{t}\right)\right] \times 10^{-6},
$$

but since $h_{t}$ is not known, $g$ must be approximated as a function of the apparent height.

Additional meteorological measurements at a sufficient height above the surface to obtain values significantly different from the surface values can be used to determine the initial gradient,

$$
g_{0}=\left.\frac{d n}{d h}\right|_{h=0}=\frac{N_{s}}{H} \ln \left(\frac{N_{H}}{N_{s}}\right) \times 10^{-6}
$$

where $N_{I I}$ is the refractivity at the height, $H$, of the above surface measurements. The initial gradient provides a boundary condition for estimating $g$ as a function of the apparent height. For convenience the initial gradient of refractivity, $G_{0}=g_{0} \times 10^{6}$, with $H$ in kilometers, was used as a prediction parameter. 
For the purposes of this study, the average (per kilometer) gradient of the first kilometer of the atmosphere is the only prediction parameter used which will require upper air measurements. The average $1 \mathrm{~km}$ gradient,

$$
\Delta N=N_{1}-N_{s},
$$

where $N_{1}$ is refractivity at $1 \mathrm{~km}$ above the surface, was selected because climatological summaries, as in chapter 4 , can be used to estimate the height error when meteorological measurements are unobtainable.

\subsubsection{Calculation and Correlation of Height Errors}

Bean, Cahoon, and Thayer [18] selected refractive index profiles, determined from radiosonde observations at 13 climatically distinct locations, which represent a wide variety of mutually exclusive profile types. The ray paths at arrival angles varying from $0^{\circ}$ to near $90^{\circ}$ were determined for each profile by numerical evaluation of (8.29) through (8.33) using methods similar to those described by Bean and Thayer [1]. The height errors were calculated with (8.33) and (8.34) at selected height intervals of $70,000 \mathrm{ft}$ for each ray path. Newton's method of interpolation was used to determine height errors for fixed ground distances to $150 \mathrm{mi}$. The limits of height and distance were chosen to extend beyond the current needs in terminal air traffic control, but are sufficiently restricted to allow some of the previous assumptions.

The prediction parameters, $N_{s}, G_{0}$, and $\Delta N$, were obtained from each of the refractive index profiles. Linear and multiple regression analysis were employed to obtain least squares estimates of the height error at each height and distance for each prediction parameter and for various combinations of the parameters.

\subsubsection{Estimation of the Average Gradient}

Based on the correlations the following forms, suggested by (8.43), were selected for approximating $g$ :

$$
\begin{gathered}
g^{\prime}=\frac{N_{s}}{h_{a}} f_{11}\left(h_{a}\right), \\
g_{2}=\frac{N_{s}}{h_{a}} f_{21}\left(h_{a}\right)+\frac{G_{0}}{h_{a}} f_{22}\left(h_{a}\right)
\end{gathered}
$$

or

$$
g_{3}=\frac{N_{s}}{h_{a}} f_{31}\left(h_{a}\right)+\frac{G_{0}}{h_{a}} f_{32}\left(h_{a}\right)+\frac{\Delta N}{h_{a}} f_{33}\left(h_{a}\right)
$$

depending upon the availability of $G_{0}$ and $\Delta N$. 
To obtain a direct estimate of the height error, (8.46) through (8.48) were combined with (8.42) and the functions $f_{i j}(i \geq j=1,2,3)$ were determined as least squares polynomials.

\subsubsection{Regression Analysis}

The volume of data processed is of sufficient magnitude that it is impractical to include it all in this report. Therefore, certain information obtained from the regression analysis was selected as being the most significant.

The mean height error provides the best general estimate obtainable if meteorological data are not available. The standard deviation (about the mean) of the height errors determines the reliability of this estimate, since 68 percent of the observed height errors are within \pm 1 standard deviation of the mean height error if the observations are normally distributed. In figure 8.29, the mean height error was plotted for each target position, then contour lines were drawn to display the mean height error as a function of true height and distance. By similar construction the standard deviation of the height error as a function of target position is displayed in figure 8.30.

The standard error of estimate establishes the same confidence limits for prediction with a regression equation as the standard deviation does for the mean. Thus, comparison of the standard error to the standard deviation indicates the improvement in accuracy of prediction with

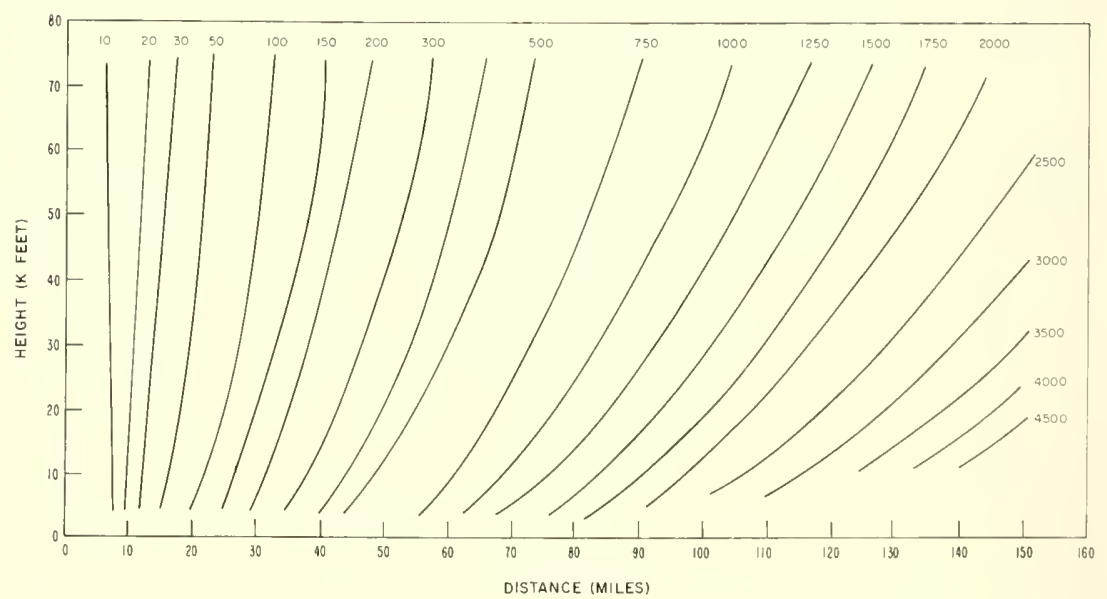

Figure 8.29. Mean height errors in feet. 


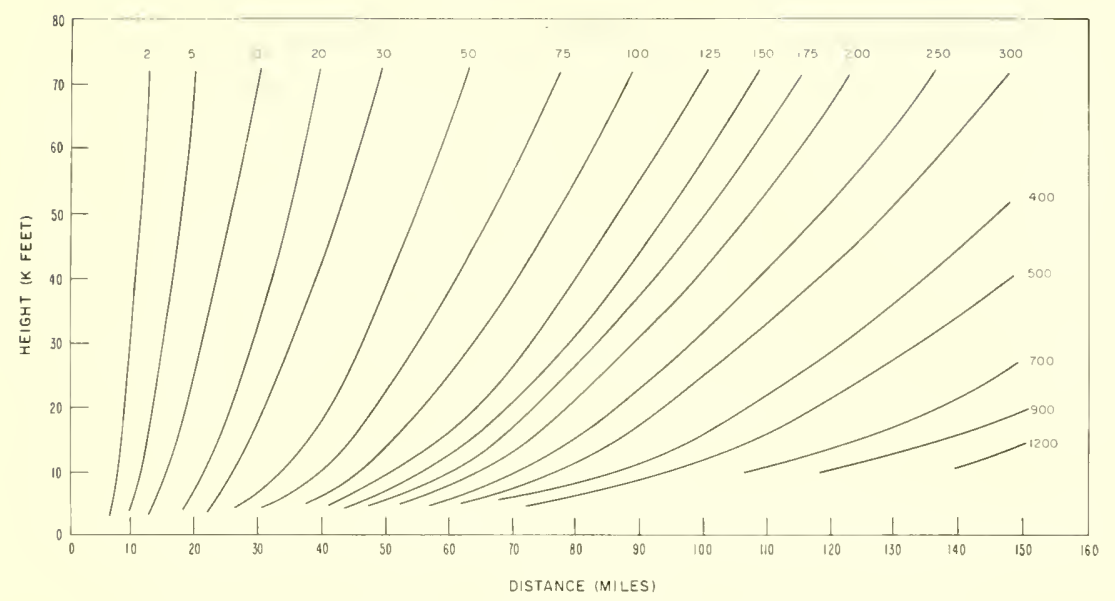

Figure 8.30. Standard deviation of height errors.

meteorological parameters. The standard error as a function of target position for

$$
\epsilon_{h}=b_{1} N_{s}+a
$$

is shown in figure 8.31 ; for

$$
\epsilon_{h}=b_{1} N_{s}+b_{2} G_{0}+a
$$

in figure 8.32 ; and for

$$
\epsilon_{h}=b_{1} N_{s}+b_{2} G_{0}+b_{3} \Delta N+a
$$

in figure 8.33 .

The regression equations, (8.49) through (8.51), were used because the respective figures demonstrate how each additional parameter enhances the accuracy of the estimate. The parameters $G_{0}$ and $\Delta N$ applied individually, that is,

$$
\epsilon_{h}=b G_{0}+a
$$

and

$$
\epsilon_{h}=b \Delta N+a,
$$

were of significant value only for targets at low heights ( $h \leq 10,000 \mathrm{ft}$ ). Examination of the figures shows that prediction of $\epsilon_{h}$ with $N_{s}$ provides significant improvement over the mean for target heights above $15,000 \mathrm{ft}$. The addition of $G_{0}$ improves the estimate for heights below $15,000 \mathrm{ft}$ and the addition of $\Delta N$ provides a slight overall improvement. 
In figures 8.29 through 8.33 the contours do not extend below $15,000 \mathrm{ft}$ for distances greater than $120 \mathrm{mi}$ and $10,000 \mathrm{ft}$ for distances greater than $80 \mathrm{mi}$. Correlations were not calculated for these target positions, because for certain refractive index profiles they are beyond the radio horizon and for certain other profiles the arrival angle is too low for the ray to penetrate a trapping layer. If conditions exist, without violating the assumptions of sections 8.4.2. through 8.4.4. such that a target at $5000 \mathrm{ft}$ height and $150 \mathrm{mi}$ distance would be visible to radar, the resulting height error would be about 10,000 ft.

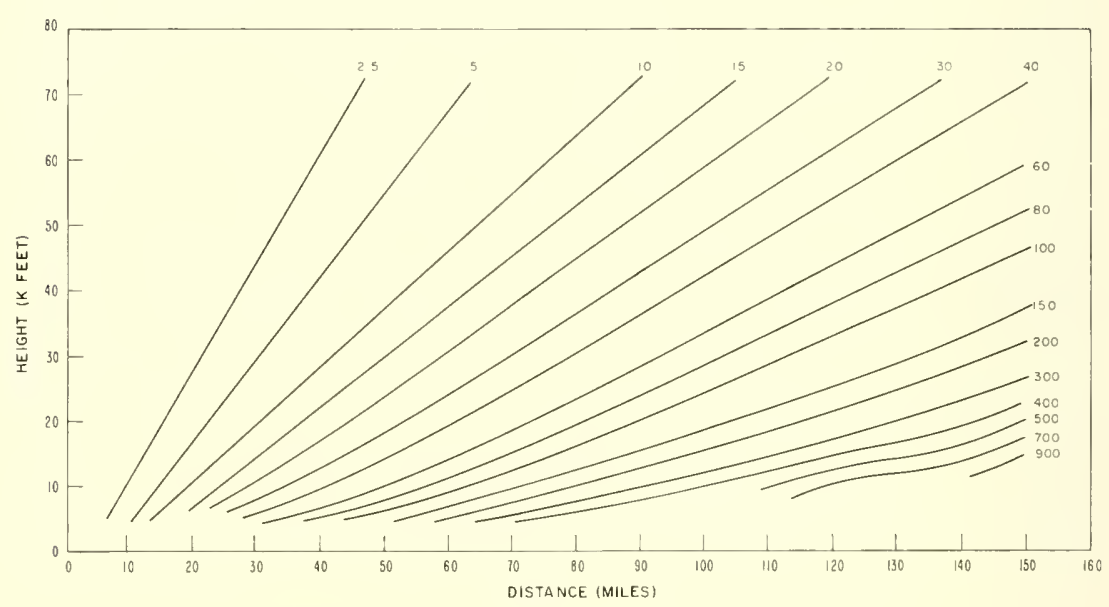

Figure 8.31. Standard error of $\epsilon_{\mathrm{L}}$ versus $N_{\mathrm{B}}$.

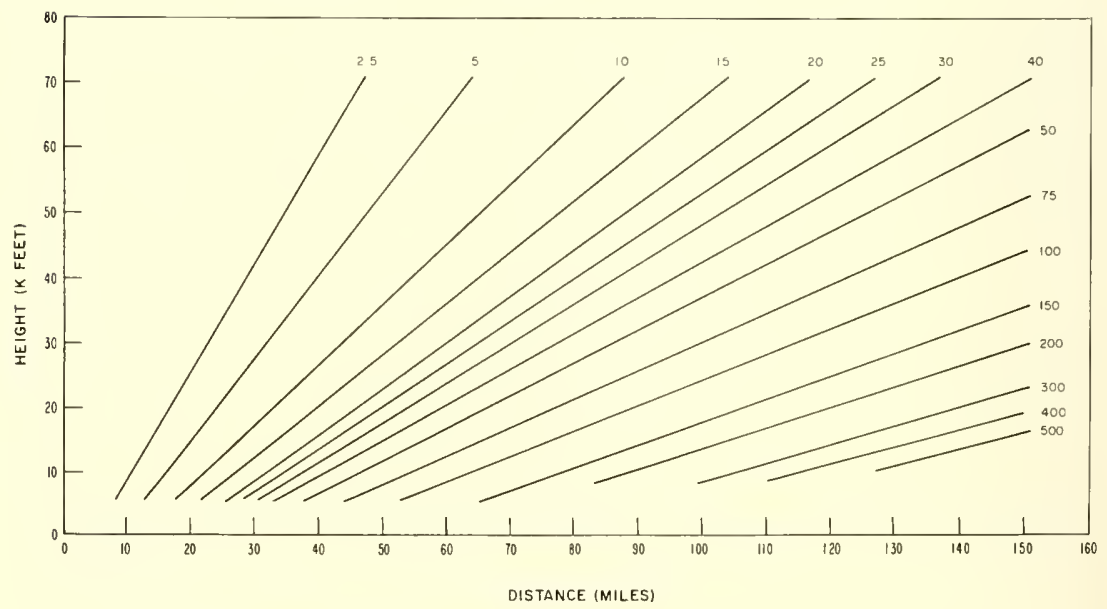

Figure 8.32. Standard error of $\epsilon_{\mathrm{h}}$ versus $\mathrm{N}_{\mathrm{B}}$ and $\mathrm{G}_{0}$. 


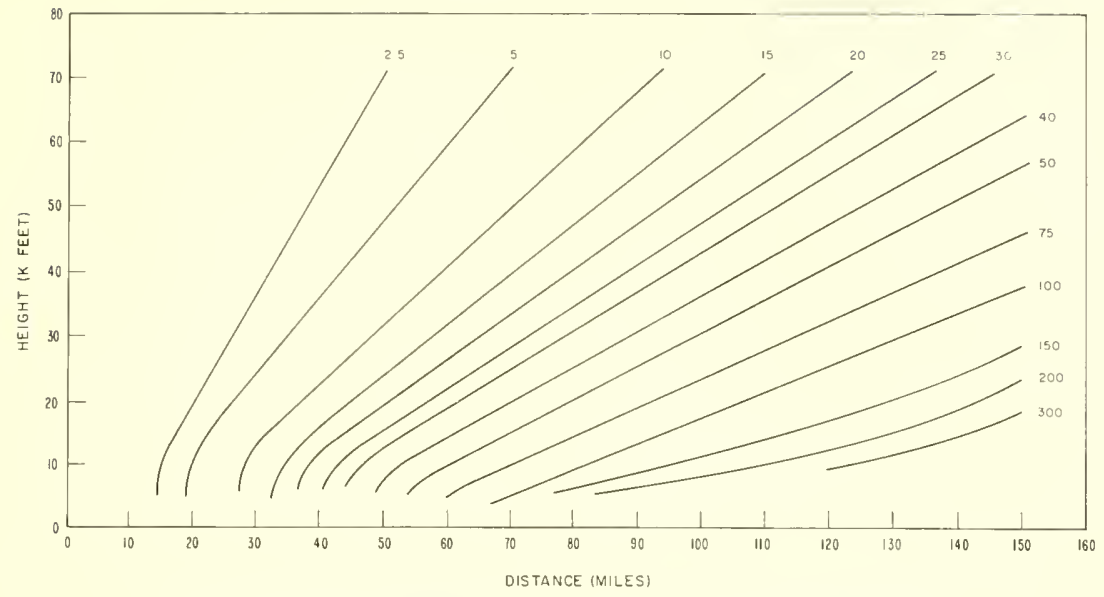

Figure 8.33. Standard error of $\epsilon_{\mathrm{h}}$ versus $\mathrm{N}_{\mathrm{s}}, \mathrm{G}_{0}$, and $\Delta \mathrm{N}$.

As an aid to further studies the coefficients for (8.49) through (8.51) are listed in tables 8.9 to 8.17 .

TABLE 8.8. The coefficients $\mathrm{a}_{\mathrm{ij}}$ for (8.52) through (8.57)

\begin{tabular}{|c|c|c|c|c|c|c|c|c|}
\hline$j$ & 0 & 1 & 2 & 3 & 4 & 5 & 6 & 7 \\
\hline $\begin{array}{l}1 \\
1 \ldots \\
3 \\
3\end{array}$ & $\begin{array}{l}-19.596 \\
-17.849 \\
-15.319\end{array}$ & $\begin{array}{l}.014096 \\
.011202 \\
.006388\end{array}$ & $\begin{array}{r}0.77906 \times 10^{-4} \\
.13665 \times 10^{-3} \\
.18549 \times 10^{-3}\end{array}$ & $\begin{array}{r}0.67545 \times 10^{-6} \\
.58925 \times 10^{-2} \\
.39074 \times 10^{-7}\end{array}$ & $\begin{array}{r}-0.64975 \times 10^{-2} \\
-.55818 \times 10^{-2}\end{array}$ & $\begin{array}{r}0.12340 \times 10^{-3} \\
.12671 \times 10^{-3}\end{array}$ & \begin{tabular}{|l|} 
\\
-0.023980
\end{tabular} & $-0.22547 \times 10^{-4}$ \\
\hline
\end{tabular}

TABLE 8.9. Constant term, a, in the regression equation (8.52)

\begin{tabular}{|c|c|c|c|c|c|c|c|c|c|c|c|}
\hline \multirow{2}{*}{$\underset{\mathrm{mi}}{\text { Distance, }}$} & \multicolumn{11}{|c|}{ Height, kft } \\
\hline & 5 & 10 & 15 & 20 & 25 & 30 & 35 & 40 & 50 & 60 & 70 \\
\hline $\begin{array}{l}5 \\
5 \\
10 \\
15 \\
20 \\
25\end{array}$ & $\begin{array}{l}-3 \\
-14 \\
-31 \\
-55 \\
-86\end{array}$ & $\begin{array}{l}-3 \\
-13 \\
-29 \\
-53 \\
-83\end{array}$ & $\begin{array}{l}-2 \\
-10 \\
-24 \\
-44 \\
-69\end{array}$ & $\begin{array}{l}-1 \\
-8 \\
-19 \\
-35 \\
-56\end{array}$ & $\begin{array}{l}-6 \\
-15 \\
-28 \\
-45\end{array}$ & $\begin{array}{l}-4 \\
-12 \\
-23 \\
-37\end{array}$ & $\begin{array}{r}1 \\
-3 \\
-9 \\
-18 \\
-29\end{array}$ & $\begin{array}{r}1 \\
-2 \\
-7 \\
-14 \\
-24\end{array}$ & $\begin{array}{r}2 \\
-4 \\
-9 \\
-15\end{array}$ & $\begin{array}{r}2 \\
1 \\
-2 \\
-5 \\
-10\end{array}$ & $\begin{array}{r}2 \\
1 \\
-1 \\
-3 \\
-7\end{array}$ \\
\hline $\begin{array}{l}30-\ldots \\
35 \\
40 \\
45 \\
50\end{array}$ & $\begin{array}{l}-123 \\
-166 \\
-215 \\
-269 \\
-327\end{array}$ & $\begin{array}{l}-119 \\
-162 \\
-211 \\
-267 \\
-329\end{array}$ & $\begin{array}{l}-100 \\
-136 \\
-178 \\
-226 \\
-280\end{array}$ & $\begin{array}{l}-81 \\
-111 \\
-146 \\
-186 \\
-231\end{array}$ & $\begin{array}{r}-66 \\
-91 \\
-120 \\
-153 \\
-190\end{array}$ & $\begin{array}{r}-54 \\
-74 \\
-98 \\
-125 \\
-156\end{array}$ & $\begin{array}{r}-44 \\
-60 \\
-80 \\
-102 \\
-127\end{array}$ & $\begin{array}{r}-35 \\
-49 \\
-65 \\
-83 \\
-104\end{array}$ & $\begin{array}{l}-23 \\
-32 \\
-43 \\
-55 \\
-70\end{array}$ & $\begin{array}{l}-15 \\
-22 \\
-30 \\
-39 \\
-49\end{array}$ & $\begin{array}{l}-11 \\
-16 \\
-22 \\
-29 \\
-36\end{array}$ \\
\hline $\begin{array}{l}60 \\
70 \\
80 \\
90 \\
100\end{array}$ & $\begin{array}{l}-454 \\
-586 \\
-709\end{array}$ & $\begin{array}{r}-470 \\
-633 \\
-812 \\
-1000 \\
-1186\end{array}$ & $\begin{array}{l}-404 \\
-552 \\
-722 \\
-913 \\
-1122\end{array}$ & $\begin{array}{l}-335 \\
-461 \\
-607 \\
-776 \\
-965\end{array}$ & $\begin{array}{l}-277 \\
-381 \\
-505 \\
-648 \\
-812\end{array}$ & $\begin{array}{l}-228 \\
-315 \\
-418 \\
-539 \\
-678\end{array}$ & $\begin{array}{l}-187 \\
-259 \\
-345 \\
-446 \\
-563\end{array}$ & $\begin{array}{l}-153 \\
-212 \\
-284 \\
-367 \\
-465\end{array}$ & $\begin{array}{l}-103 \\
-144 \\
-193 \\
-252 \\
-320\end{array}$ & $\begin{array}{l}-73 \\
-102 \\
-137 \\
-179 \\
-228\end{array}$ & $\begin{array}{r}-54 \\
-76 \\
-103 \\
-134 \\
-171\end{array}$ \\
\hline $\begin{array}{l}110 \\
120 \\
130 \\
140 \\
150\end{array}$ & & $\begin{array}{l}-1352 \\
-1473\end{array}$ & $\begin{array}{l}-1342 \\
-1565 \\
-1774 \\
-1946 \\
-2048\end{array}$ & $\begin{array}{l}-1176 \\
-1404 \\
-1644 \\
-1889 \\
-2123\end{array}$ & $\begin{array}{r}-997 \\
-1203 \\
-1429 \\
-1672 \\
-1928\end{array}$ & $\begin{array}{r}-836 \\
-1014 \\
-1215 \\
-1434 \\
-1674\end{array}$ & $\begin{array}{r}-697 \\
-850 \\
-1022 \\
-1214 \\
-1428\end{array}$ & $\begin{array}{r}-578 \\
-707 \\
-852 \\
-1019 \\
-1203\end{array}$ & $\begin{array}{l}-399 \\
-491 \\
-597 \\
-718 \\
-855\end{array}$ & $\begin{array}{l}-286 \\
-352 \\
-429 \\
-618 \\
-620\end{array}$ & $\begin{array}{l}-214 \\
-264 \\
-322 \\
-389 \\
-465\end{array}$ \\
\hline
\end{tabular}


TABLE 8.10. Constant term, a, in the regression equation (8.53)

\begin{tabular}{|c|c|c|c|c|c|c|c|c|c|c|c|}
\hline \multirow{2}{*}{$\begin{array}{c}\text { Distance, } \\
\mathrm{mi}\end{array}$} & \multicolumn{11}{|c|}{ Height, kft } \\
\hline & 5 & 10 & 15 & 20 & 25 & 30 & 35 & 40 & 50 & 60 & 70 \\
\hline $\begin{array}{l}5 \\
10 \\
15 \\
20 \\
25\end{array}$ & $\begin{array}{l}-3 \\
-12 \\
-28 \\
-50 \\
-77\end{array}$ & $\begin{array}{r}-3 \\
-12 \\
-29 \\
-51 \\
-80\end{array}$ & $\begin{array}{l}-2 \\
-10 \\
-24 \\
-43 \\
-67\end{array}$ & $\begin{array}{r}-1 \\
-8 \\
-19 \\
-35 \\
-55\end{array}$ & $\begin{array}{c}- \\
-6 \\
-15 \\
-28 \\
-45\end{array}$ & $\begin{array}{r}- \\
-4 \\
-12 \\
-22 \\
-36\end{array}$ & $\begin{array}{r}1 \\
-3 \\
-9 \\
-18 \\
-29\end{array}$ & $\begin{array}{r}1 \\
-2 \\
-7 \\
-14 \\
-23\end{array}$ & $\begin{array}{r}2 \\
-4 \\
-9 \\
-15\end{array}$ & $\begin{array}{r}2 \\
1 \\
-2 \\
-5 \\
-10\end{array}$ & $\begin{array}{r}2 \\
1 \\
-1 \\
-3 \\
-7\end{array}$ \\
\hline $\begin{array}{l}30 \\
35 \\
40 \\
45 \\
50\end{array}$ & $\begin{array}{l}-110 \\
-148 \\
-190 \\
-235 \\
-284\end{array}$ & $\begin{array}{l}-115 \\
-156 \\
-204 \\
-257 \\
-316\end{array}$ & $\begin{array}{r}-97 \\
-133 \\
-174 \\
-221 \\
-273\end{array}$ & $\begin{array}{l}-80 \\
-110 \\
-144 \\
-183 \\
-227\end{array}$ & $\begin{array}{r}-65 \\
-90 \\
-118 \\
-151 \\
-187\end{array}$ & $\begin{array}{r}-53 \\
-73 \\
-97 \\
-124 \\
-154\end{array}$ & $\begin{array}{r}-43 \\
-60 \\
-79 \\
-101 \\
-126\end{array}$ & $\begin{array}{r}-35 \\
-48 \\
-64 \\
-82 \\
-103\end{array}$ & $\begin{array}{l}-23 \\
-32 \\
-43 \\
-55 \\
-69\end{array}$ & $\begin{array}{l}-15 \\
-22 \\
-30 \\
-38 \\
-48\end{array}$ & $\begin{array}{l}-11 \\
-16 \\
-22 \\
-28 \\
-36\end{array}$ \\
\hline $\begin{array}{l}60 \\
70 \\
80 \\
90 \\
100\end{array}$ & $\begin{array}{l}-383 \\
-473 \\
-537\end{array}$ & $\begin{array}{r}-449 \\
-600 \\
-762 \\
-925 \\
-1076\end{array}$ & $\begin{array}{r}-394 \\
-536 \\
-700 \\
-880 \\
-1075\end{array}$ & $\begin{array}{l}-329 \\
-452 \\
-595 \\
-797 \\
-940\end{array}$ & $\begin{array}{l}-273 \\
-376 \\
-497 \\
-637 \\
-797\end{array}$ & $\begin{array}{l}-225 \\
-311 \\
-413 \\
-531 \\
-667\end{array}$ & $\begin{array}{l}-185 \\
-257 \\
-341 \\
-441 \\
-556\end{array}$ & $\begin{array}{l}-151 \\
-210 \\
-281 \\
-363 \\
-460\end{array}$ & $\begin{array}{l}-102 \\
-143 \\
-191 \\
-249 \\
-317\end{array}$ & $\begin{array}{l}-72 \\
-101 \\
-136 \\
-178 \\
-226\end{array}$ & $\begin{array}{l}-54 \\
-76 \\
-102 \\
-133 \\
-170\end{array}$ \\
\hline $\begin{array}{l}110 \\
120 \\
130 \\
140 \\
150\end{array}$ & & $\begin{array}{l}-1189 \\
-1236\end{array}$ & $\begin{array}{l}-1275 \\
-1468 \\
-1637 \\
-1748 \\
-1765\end{array}$ & $\begin{array}{l}-1141 \\
-1355 \\
-1576 \\
-1794 \\
-1989\end{array}$ & $\begin{array}{r}-976 \\
-1174 \\
-1390 \\
-1619 \\
-1855\end{array}$ & $\begin{array}{r}-822 \\
-996 \\
-1189 \\
-1400 \\
-1630\end{array}$ & $\begin{array}{r}-687 \\
-837 \\
-1005 \\
-1192 \\
-1399\end{array}$ & $\begin{array}{r}-571 \\
-697 \\
-840 \\
-1003 \\
-1183\end{array}$ & $\begin{array}{l}-395 \\
-486 \\
-590 \\
-708 \\
-843\end{array}$ & $\begin{array}{l}-283 \\
-349 \\
-425 \\
-512 \\
-612\end{array}$ & $\begin{array}{l}-212 \\
-262 \\
-319 \\
-385 \\
-460\end{array}$ \\
\hline
\end{tabular}

TABLE 8.11. Constant term, a, in the regression equation (8.54)

\begin{tabular}{|c|c|c|c|c|c|c|c|c|c|c|c|}
\hline \multirow{2}{*}{$\begin{array}{c}\text { Distance, } \\
\text { mi }\end{array}$} & \multicolumn{11}{|c|}{ Height, kft } \\
\hline & 5 & 10 & 15 & 20 & 25 & 30 & 35 & 40 & 50 & 60 & 70 \\
\hline $\begin{array}{l}5 \\
10 \\
15 \\
20 \\
25\end{array}$ & $\begin{array}{l}-2 \\
-2 \\
-4 \\
-9\end{array}$ & $\begin{array}{r}-2 \\
-8 \\
-18 \\
-33 \\
-51\end{array}$ & $\begin{array}{r}-1 \\
-8 \\
-18 \\
-33 \\
-51\end{array}$ & $\begin{array}{r}-1 \\
-6 \\
-15 \\
-28 \\
-44\end{array}$ & $\begin{array}{l}- \\
-5 \\
-12 \\
-23 \\
-37\end{array}$ & $\begin{array}{l}-3 \\
-10 \\
-19 \\
-31\end{array}$ & $\begin{array}{r}1 \\
-2 \\
-8 \\
-15 \\
-25\end{array}$ & $\begin{array}{r}1 \\
-2 \\
-6 \\
-12 \\
-20\end{array}$ & $\begin{array}{r}2 \\
-3 \\
-7 \\
-13\end{array}$ & $\begin{array}{l}\frac{2}{-2} \\
-5 \\
-9\end{array}$ & $\begin{array}{r}2 \\
1 \\
-1 \\
-3 \\
-6\end{array}$ \\
\hline $\begin{array}{l}30 \\
35 \\
40 \\
45 \\
50\end{array}$ & $\begin{array}{l}-12 \\
-13 \\
-13 \\
-10 \\
-5\end{array}$ & $\begin{array}{r}-73 \\
-98 \\
-125 \\
-156 \\
-187\end{array}$ & $\begin{array}{r}-74 \\
-100 \\
-130 \\
-164 \\
-201\end{array}$ & $\begin{array}{r}-64 \\
-87 \\
-114 \\
-144 \\
-178\end{array}$ & $\begin{array}{r}-54 \\
-74 \\
-97 \\
-123 \\
-152\end{array}$ & $\begin{array}{r}-45 \\
-62 \\
-81 \\
-104 \\
-129\end{array}$ & $\begin{array}{r}-37 \\
-51 \\
-67 \\
-86 \\
-107\end{array}$ & $\begin{array}{l}-30 \\
-42 \\
-55 \\
-71 \\
-88\end{array}$ & $\begin{array}{l}-20 \\
-28 \\
-37 \\
-48 \\
-60\end{array}$ & $\begin{array}{l}-13 \\
-19 \\
-26 \\
-33 \\
-42\end{array}$ & $\begin{array}{l}-10 \\
-14 \\
-19 \\
-25 \\
-31\end{array}$ \\
\hline $\begin{array}{l}60 \\
70 \\
80 \\
90 \\
100\end{array}$ & $\begin{array}{l}21 \\
70 \\
152\end{array}$ & $\begin{array}{l}-254 \\
-318 \\
-369 \\
-396 \\
-383\end{array}$ & $\begin{array}{l}-285 \\
-378 \\
-478 \\
-578 \\
-669\end{array}$ & $\begin{array}{l}-256 \\
-346 \\
-448 \\
-560 \\
-677\end{array}$ & $\begin{array}{l}-221 \\
-302 \\
-395 \\
-499 \\
-615\end{array}$ & $\begin{array}{l}-187 \\
-257 \\
-338 \\
-431 \\
-536\end{array}$ & $\begin{array}{l}-156 \\
-216 \\
-285 \\
-366 \\
-457\end{array}$ & $\begin{array}{l}-129 \\
-179 \\
-237 \\
-306 \\
-384\end{array}$ & $\begin{array}{l}-88 \\
-123 \\
-164 \\
-213 \\
-269\end{array}$ & $\begin{array}{r}-62 \\
-87 \\
-117 \\
-152 \\
-194\end{array}$ & $\begin{array}{r}-47 \\
-66 \\
-88 \\
-115 \\
-146\end{array}$ \\
\hline $\begin{array}{l}110 \\
120 \\
130 \\
140 \\
150\end{array}$ & & $\begin{array}{l}-310 \\
-163\end{array}$ & $\begin{array}{l}-741 \\
-776 \\
-754 \\
-649 \\
-436\end{array}$ & $\begin{array}{l}-795 \\
-906 \\
-998 \\
-1055 \\
-1057\end{array}$ & $\begin{array}{r}-739 \\
-867 \\
-996 \\
-1117 \\
-1217\end{array}$ & $\begin{array}{r}-652 \\
-776 \\
-909 \\
-1044 \\
-1179\end{array}$ & $\begin{array}{l}-560 \\
-673 \\
-797 \\
-929 \\
-1068\end{array}$ & $\begin{array}{l}-473 \\
-573 \\
-682 \\
-804 \\
-933\end{array}$ & $\begin{array}{l}-334 \\
-408 \\
-491 \\
-485 \\
-690\end{array}$ & $\begin{array}{l}-241 \\
-296 \\
-359 \\
-430 \\
-510\end{array}$ & $\begin{array}{l}-182 \\
-224 \\
-272 \\
-327 \\
-389\end{array}$ \\
\hline
\end{tabular}


TABLE 8.12. Coefficient of $\mathrm{N}_{\mathrm{s}}, \mathrm{b}_{1}$, in the regression equation (8.52)

\begin{tabular}{|c|c|c|c|c|c|c|c|c|c|c|c|}
\hline \multirow{2}{*}{$\begin{array}{c}\text { Distance, } \\
\mathrm{mi}\end{array}$} & \multicolumn{11}{|c|}{ Height, $\mathrm{kft}$} \\
\hline & 5 & 10 & 15 & 20 & 25 & 30 & 35 & 40 & 50 & 60 & 70 \\
\hline $\begin{array}{l}5 \\
10 \\
15 \\
20 \\
25\end{array}$ & $\begin{array}{r}0.0318 \\
.1153 \\
.2545 \\
.4495 \\
.7005\end{array}$ & $\begin{array}{r}0.0313 \\
.1059 \\
.2303 \\
.4046 \\
.6291\end{array}$ & $\begin{array}{r}0.0296 \\
.0944 \\
.2025 \\
.3541 \\
.5494\end{array}$ & $\begin{array}{r}0.0281 \\
.0848 \\
.1795 \\
.3123 \\
.4835\end{array}$ & $\begin{array}{r}0.0267 \\
.0770 \\
.1608 \\
.2784 \\
.4300\end{array}$ & $\begin{array}{r}0.0257 \\
.0705 \\
.1454 \\
.2504 \\
.3858\end{array}$ & $\begin{array}{r}0.0248 \\
.0652 \\
.1326 \\
.2271 \\
.3489\end{array}$ & $\begin{array}{r}0.0243 \\
.0608 \\
.1218 \\
.2074 \\
.3177\end{array}$ & $\begin{array}{r}0.0236 \\
.0543 \\
.1056 \\
.1776 \\
.2704\end{array}$ & $\begin{array}{r}0.0231 \\
.0498 \\
.0943 \\
.1568 \\
.2373\end{array}$ & $\begin{array}{r}0.0225 \\
.0462 \\
.0858 \\
.1413 \\
.2127\end{array}$ \\
\hline $\begin{array}{l}30 \\
35 \\
40 \\
45 \\
40\end{array}$ & $\begin{array}{l}\text { 1. } 0078 \\
\text { 1. } 3714 \\
\text { 1. } 7917 \\
2.2688 \\
2.8030\end{array}$ & $\begin{array}{l}.9040 \\
1.2295 \\
1.6060 \\
2.0336 \\
2.5129\end{array}$ & $\begin{array}{l}.7889 \\
1.0728 \\
1.4018 \\
1.7762 \\
2.1968\end{array}$ & $\begin{array}{r}.6934 \\
.9424 \\
1.2311 \\
1.5599 \\
1.9295\end{array}$ & $\begin{array}{r}.6159 \\
.8366 \\
1.0923 \\
1.3837 \\
1.7113\end{array}$ & $\begin{array}{r}.5519 \\
.7489 \\
.9774 \\
1.2377 \\
1.5305\end{array}$ & $\begin{array}{r}.4983 \\
.6756 \\
.8812 \\
1.1154 \\
1.3788\end{array}$ & $\begin{array}{r}.4531 \\
.6136 \\
.7998 \\
1.0119 \\
1.2503\end{array}$ & $\begin{array}{r}.3841 \\
.5190 \\
.6754 \\
.8536 \\
1.0539\end{array}$ & $\begin{array}{l}.3359 \\
.4529 \\
.5885 \\
.7429 \\
.9163\end{array}$ & $\begin{array}{l}.3003 \\
.4042 \\
.5244 \\
.6613 \\
.8150\end{array}$ \\
\hline $\begin{array}{l}60 \\
70 \\
80 \\
90 \\
100\end{array}$ & $\begin{array}{l}\text { 4. } 0426 \\
5.5119 \\
7.2127\end{array}$ & $\begin{array}{r}3.6268 \\
4.9494 \\
6.4794 \\
8.2133 \\
10.1439\end{array}$ & $\begin{array}{l}3.1787 \\
4.3513 \\
5.7236 \\
7.2966 \\
9.0736\end{array}$ & $\begin{array}{l}2.7938 \\
3.8300 \\
5.0453 \\
6.4464 \\
8.0412\end{array}$ & $\begin{array}{l}2.4780 \\
3.3981 \\
4.4788 \\
5.7273 \\
7.1529\end{array}$ & $\begin{array}{l}2.2155 \\
3.0380 \\
4.0046 \\
5.1225 \\
6.4005\end{array}$ & $\begin{array}{l}1.9952 \\
2.7355 \\
3.6053 \\
4.6119 \\
5.7634\end{array}$ & $\begin{array}{l}1.8085 \\
2.4788 \\
3.2666 \\
4.1779 \\
5.2207\end{array}$ & $\begin{array}{l}1.5223 \\
2.0845 \\
2.7442 \\
3.5078 \\
4.3804\end{array}$ & $\begin{array}{l}1.3216 \\
1.8072 \\
2.3769 \\
3.0342 \\
3.7846\end{array}$ & $\begin{array}{l}\text { 1. } 1738 \\
\text { 1. } 6032 \\
2.1060 \\
2.6854 \\
\text { 3. } 3456\end{array}$ \\
\hline $\begin{array}{l}110 \\
120 \\
130 \\
140 \\
150\end{array}$ & & $\begin{array}{l}12.2529 \\
14.5240\end{array}$ & $\begin{array}{l}11.0533 \\
13.2284 \\
15.5836 \\
18.0866 \\
20.7053\end{array}$ & $\begin{array}{r}9.8363 \\
11.8360 \\
14.0399 \\
16.4440 \\
19.0333\end{array}$ & $\begin{array}{r}8.7649 \\
10.5679 \\
12.5772 \\
14.7931 \\
17.2171\end{array}$ & $\begin{array}{r}7.8482 \\
9.4723 \\
11.2895 \\
13.3002 \\
15.5220\end{array}$ & $\begin{array}{r}7.0684 \\
8.5370 \\
10.1777 \\
12.0051 \\
14.0251\end{array}$ & $\begin{array}{r}6.4028 \\
7.7337 \\
9.2192 \\
10.8816 \\
12.7148\end{array}$ & $\begin{array}{r}5.3687 \\
6.4808 \\
7.7254 \\
9.1125 \\
10.6504\end{array}$ & $\begin{array}{l}4.6329 \\
5.5863 \\
6.6512 \\
7.8354 \\
9.1488\end{array}$ & $\begin{array}{l}4.0909 \\
\text { 4. } 9257 \\
5.8573 \\
6.8904 \\
8.0339\end{array}$ \\
\hline
\end{tabular}

TABLE 8.13. Coefficient of $\mathrm{N}_{\mathrm{B}}, \mathrm{b}_{1}$, in the regression equation (8.53)

\begin{tabular}{|c|c|c|c|c|c|c|c|c|c|c|c|}
\hline \multirow{2}{*}{$\begin{array}{c}\text { Distance, } \\
\text { mi }\end{array}$} & \multicolumn{11}{|c|}{ Height, kft } \\
\hline & 5 & 10 & 15 & 20 & 25 & 30 & 35 & 40 & 50 & 60 & 70 \\
\hline $\begin{array}{l}5 \ldots \\
10 \\
15 \\
20 \ldots \ldots \\
25\end{array}$ & $\begin{array}{r}0.0285 \\
.1014 \\
.2226 \\
.3915 \\
.6072\end{array}$ & $\begin{array}{r}0.0303 \\
.1015 \\
.2202 \\
.3862 \\
.5997\end{array}$ & $\begin{array}{r}0.0292 \\
.0922 \\
.1972 \\
.3444 \\
.5430\end{array}$ & $\begin{array}{r}0.0279 \\
.0835 \\
.1763 \\
.3065 \\
.4742\end{array}$ & $\begin{array}{r}0.0267 \\
.0762 \\
.1588 \\
.2746 \\
.4239\end{array}$ & $\begin{array}{r}0.0257 \\
.0701 \\
.1441 \\
.2479 \\
.3817\end{array}$ & $\begin{array}{r}0.0249 \\
.0649 \\
.1317 \\
.2253 \\
.3459\end{array}$ & $\begin{array}{r}0.0244 \\
.0607 \\
.1212 \\
.2061 \\
.3155\end{array}$ & $\begin{array}{r}0.0237 \\
.0543 \\
.1053 \\
.1768 \\
.2690\end{array}$ & $\begin{array}{r}0.0232 \\
.0498 \\
.0942 \\
.1563 \\
.2365\end{array}$ & $\begin{array}{r}0.0227 \\
.0463 \\
.0857 \\
.1410 \\
.2122\end{array}$ \\
\hline $\begin{array}{l}30 \ldots \ldots \\
35 \\
40 \\
45 \\
50\end{array}$ & $\begin{array}{l}.8686 \\
1.1741 \\
1.5217 \\
1.9089 \\
2.3325\end{array}$ & $\begin{array}{l}.8603 \\
1.1680 \\
1.5225 \\
1.9235 \\
\text { 2. } 3701\end{array}$ & $\begin{array}{l}.7661 \\
1.0411 \\
1.3591 \\
1.7204 \\
2.1254\end{array}$ & $\begin{array}{r}.6797 \\
.9234 \\
1.2056 \\
1.5268 \\
1.8873\end{array}$ & $\begin{array}{r}.6069 \\
.8240 \\
1.0755 \\
1.3619 \\
1.6836\end{array}$ & $\begin{array}{r}.5457 \\
.7403 \\
.9658 \\
\text { 1. } 2227 \\
\text { 1. } 5114\end{array}$ & $\begin{array}{r}.4939 \\
.6694 \\
.8729 \\
\text { 1. } 1046 \\
\text { 1. } 3651\end{array}$ & $\begin{array}{r}.4497 \\
.6089 \\
.7935 \\
1.0037 \\
1.2400\end{array}$ & $\begin{array}{r}.3821 \\
.5162 \\
.6716 \\
.8486 \\
1.0475\end{array}$ & $\begin{array}{l}.3346 \\
.4511 \\
.5860 \\
.7396 \\
.9121\end{array}$ & $\begin{array}{l}.2994 \\
.4028 \\
.5226 \\
.6589 \\
.8119\end{array}$ \\
\hline $\begin{array}{l}60 \ldots \\
70 \ldots \\
80 \\
90 \\
100\end{array}$ & $\begin{array}{l}3.2717 \\
4.2984 \\
5.3577\end{array}$ & $\begin{array}{l}3.3976 \\
4.5945 \\
5.9423 \\
7.4110 \\
8.9558\end{array}$ & $\begin{array}{l}3.0671 \\
4.1841 \\
5.4790 \\
6.9435 \\
8.5676\end{array}$ & $\begin{array}{l}\text { 2. } 7287 \\
3.7339 \\
4.9073 \\
6.2511 \\
7.7696\end{array}$ & $\begin{array}{l}2.4355 \\
3.3361 \\
4.3907 \\
5.6047 \\
6.9854\end{array}$ & $\begin{array}{l}2.1864 \\
2.9957 \\
3.9449 \\
5.0400 \\
6.2887\end{array}$ & $\begin{array}{l}1.9743 \\
2.7052 \\
3.5629 \\
4.5537 \\
5.6849\end{array}$ & $\begin{array}{l}1.7928 \\
2.4560 \\
3.2348 \\
4.1346 \\
5.1625\end{array}$ & $\begin{array}{l}1.5126 \\
2.0705 \\
2.7249 \\
3.4816 \\
4.3456\end{array}$ & $\begin{array}{l}1.3152 \\
1.7981 \\
2.3643 \\
3.0171 \\
3.7620\end{array}$ & $\begin{array}{l}1.1691 \\
1.5965 \\
2.0968 \\
2.6730 \\
3.3294\end{array}$ \\
\hline $\begin{array}{l}110 \ldots \ldots \\
120 \ldots \ldots \\
130 \ldots \ldots \\
140 \\
150 \ldots \ldots\end{array}$ & & $\begin{array}{l}10.5049 \\
11.9716\end{array}$ & $\begin{array}{l}10.3305 \\
12.1954 \\
14.1047 \\
15.9660 \\
17.6672\end{array}$ & $\begin{array}{r}9.4588 \\
11.3115 \\
13.3104 \\
15.4244 \\
17.6007\end{array}$ & $\begin{array}{r}8.5356 \\
10.2579 \\
12.1560 \\
14.2189 \\
16.4316\end{array}$ & $\begin{array}{r}7.6975 \\
9.2713 \\
11.0200 \\
12.9405 \\
15.0401\end{array}$ & $\begin{array}{r}6.9635 \\
8.3974 \\
9.9945 \\
11.7614 \\
13.7056\end{array}$ & $\begin{array}{r}6.3258 \\
7.6323 \\
9.0868 \\
10.7087 \\
12.4899\end{array}$ & $\begin{array}{r}5.3231 \\
6.4215 \\
7.6489 \\
9.0139 \\
10.5245\end{array}$ & $\begin{array}{l}4.6035 \\
5.5484 \\
6.6027 \\
7.7737 \\
9.0705\end{array}$ & $\begin{array}{l}\text { 4. } 0699 \\
\text { 4. } 8989 \\
5.8234 \\
6.8477 \\
7.9804\end{array}$ \\
\hline
\end{tabular}


TABLE 8.14. Coefficient of $\mathrm{N}_{\mathrm{B}}, \mathrm{b}_{1}$, in the regression equation (8.54)

\begin{tabular}{|c|c|c|c|c|c|c|c|c|c|c|c|}
\hline \multirow{2}{*}{$\begin{array}{c}\text { Distance, } \\
\mathrm{mi}\end{array}$} & \multicolumn{11}{|c|}{ Height, kft } \\
\hline & 5 & 10 & 15 & 20 & 25 & 30 & 35 & 40 & 50 & 60 & 70 \\
\hline $\begin{array}{l}5 \ldots \ldots \ldots \\
10 \ldots \ldots \ldots \\
15 \\
20 \ldots \ldots \ldots \\
25 \ldots \ldots \ldots\end{array}$ & $\begin{array}{r}0.0091 \\
.0217 \\
.0418 \\
.0682 \\
.0991\end{array}$ & $\begin{array}{r}0.0231 \\
.0689 \\
.1447 \\
.2497 \\
.3830\end{array}$ & $\begin{array}{r}0.0259 \\
.0744 \\
.1551 \\
.2677 \\
.4119\end{array}$ & $\begin{array}{r}0.0265 \\
.0720 \\
.1478 \\
.2539 \\
.3902\end{array}$ & $\begin{array}{r}0.0265 \\
.0687 \\
.1391 \\
.2378 \\
.3646\end{array}$ & $\begin{array}{r}0.0262 \\
.0652 \\
.1303 \\
.2214 \\
.3386\end{array}$ & $\begin{array}{r}0.0260 \\
.0619 \\
.1218 \\
.2058 \\
.3138\end{array}$ & $\begin{array}{r}0.0258 \\
.0589 \\
.1141 \\
.1915 \\
.2912\end{array}$ & $\begin{array}{r}0.0255 \\
.0540 \\
.1016 \\
.1683 \\
.2542\end{array}$ & $\begin{array}{r}0.0252 \\
.0504 \\
.0923 \\
.1511 \\
.2268\end{array}$ & $\begin{array}{r}0.0248 \\
.0474 \\
.0850 \\
.1378 \\
.2058\end{array}$ \\
\hline $\begin{array}{l}30 \ldots \ldots \\
35 \\
40 \\
45 \\
50\end{array}$ & $\begin{array}{l}1325 \\
.1660 \\
.1971 \\
.2232 \\
.2418\end{array}$ & $\begin{array}{r}.5430 \\
.7281 \\
.9358 \\
1.1641 \\
1.4090\end{array}$ & $\begin{array}{r}.5873 \\
.7933 \\
1.0291 \\
1.2939 \\
1.5863\end{array}$ & $\begin{array}{r}.5567 \\
.7532 \\
.9795 \\
\text { 1. } 2354 \\
\text { 1. } 5206\end{array}$ & $\begin{array}{r}.5198 \\
.7033 \\
.9151 \\
1.1553 \\
1.4239\end{array}$ & $\begin{array}{r}.4821 \\
.6520 \\
.8483 \\
1.0712 \\
1.3209\end{array}$ & $\begin{array}{r}.4462 \\
.6030 \\
.7843 \\
.9903 \\
1.2213\end{array}$ & $\begin{array}{r}.4133 \\
.5579 \\
.7253 \\
.9155 \\
1.1290\end{array}$ & $\begin{array}{r}.3595 \\
.4843 \\
.6287 \\
.7929 \\
.9772\end{array}$ & $\begin{array}{l}.3196 \\
.4295 \\
.5567 \\
.7014 \\
.8637\end{array}$ & $\begin{array}{l}.2891 \\
.3878 \\
.5020 \\
.6318 \\
.7775\end{array}$ \\
\hline $\begin{array}{l}60 \ldots \ldots \ldots \\
70 \ldots \ldots \\
80 \ldots \ldots \\
90 \ldots \ldots \ldots \\
100 \ldots \ldots\end{array}$ & $\begin{array}{r}.2501 \\
.2231 \\
.2005\end{array}$ & $\begin{array}{l}1.9361 \\
2.4816 \\
3.0016 \\
3.4452 \\
3.7639\end{array}$ & $\begin{array}{l}2.2485 \\
2.9993 \\
3.8207 \\
4.6780 \\
5.5316\end{array}$ & $\begin{array}{l}\text { 2. } 1771 \\
\text { 2. } 9439 \\
\text { 3. } 8126 \\
\text { 4. } 7689 \\
\text { 5. } 7994\end{array}$ & $\begin{array}{l}\text { 2. } 0459 \\
\text { 2. } 7804 \\
\text { 3. } 6248 \\
\text { 4. } 5741 \\
\text { 5. } 6251\end{array}$ & $\begin{array}{l}1.9009 \\
2.5892 \\
3.3863 \\
4.2912 \\
5.3042\end{array}$ & $\begin{array}{l}\text { 1. } 7588 \\
\text { 2. } 3986 \\
\text { 3. } 1423 \\
\text { 3. } 9913 \\
4.9474\end{array}$ & $\begin{array}{l}\text { 1. } 6263 \\
\text { 2. } 2192 \\
\text { 2. } 9101 \\
\text { 3. } 7012 \\
\text { 4. } 5952\end{array}$ & $\begin{array}{l}1.4070 \\
1.9202 \\
2.5191 \\
3.2075 \\
3.9877\end{array}$ & $\begin{array}{l}1.2423 \\
1.6944 \\
2.2224 \\
2.8288 \\
3.5170\end{array}$ & $\begin{array}{l}1.1170 \\
1.5223 \\
1.9953 \\
2.5384 \\
3.1545\end{array}$ \\
\hline $\begin{array}{l}110 \ldots \ldots \\
120 \ldots \ldots \\
130 \ldots \ldots \\
140 \ldots \ldots \\
150 \ldots \ldots\end{array}$ & & $\begin{array}{l}3.9201 \\
3.9312\end{array}$ & $\begin{array}{l}6.3291 \\
7.0067 \\
7.4937 \\
7.7293 \\
7.7099\end{array}$ & $\begin{array}{r}6.8710 \\
7.9475 \\
8.9769 \\
9.8930 \\
10.6160\end{array}$ & $\begin{array}{r}6.7588 \\
7.9627 \\
9.2092 \\
10.4569 \\
11.6542\end{array}$ & $\begin{array}{r}6.4182 \\
6.6279 \\
8.9193 \\
10.2723 \\
11.6633\end{array}$ & $\begin{array}{r}6.0094 \\
7.1738 \\
8.4423 \\
9.7952 \\
11.2325\end{array}$ & $\begin{array}{r}5.5936 \\
6.6971 \\
7.9028 \\
9.2175 \\
10.6218\end{array}$ & $\begin{array}{l}4.8629 \\
5.8369 \\
6.9123 \\
8.0909 \\
9.3770\end{array}$ & $\begin{array}{l}4.2900 \\
5.1516 \\
6.1053 \\
7.1551 \\
8.3049\end{array}$ & $\begin{array}{l}3.8464 \\
4.6173 \\
5.4713 \\
6.4118 \\
7.4432\end{array}$ \\
\hline
\end{tabular}

TABLE 8.15. Coefficient of $\mathrm{G}_{0}, \mathrm{~b}_{2}$, in the regression equation (8.53)

\begin{tabular}{r|r|r|r|r|r|r|r|r|r}
\hline \hline \\
\hline \\
Distance,
\end{tabular}


TABLE 8.16. Coefficient of $\mathrm{G}_{0}, \mathrm{~b}_{2}$, in the regression equation (8.54)

\begin{tabular}{|c|c|c|c|c|c|c|c|c|c|c|c|}
\hline \multirow{2}{*}{$\begin{array}{c}\text { Distance, } \\
\text { mi }\end{array}$} & \multicolumn{11}{|c|}{ Height, kft } \\
\hline & 5 & 10 & 15 & 20 & 25 & 30 & 35 & 40 & 50 & 60 & 70 \\
\hline & -0.0032 & -0.0060 & -0.0002 & -0.0001 & -0.0000 & 0.0000 & 0.0000 & 0.0000 & -0.0000 & -0.0000 & 0.0000 \\
\hline 10 & -.0133 & -.0029 & -.0013 & -.0005 & -.0002 & -.0001 & -.0000 & -.0000 & .0000 & .0000 & .0000 \\
\hline 15. & -.0309 & -.0067 & -.0031 & -.0012 & -.0005 & -.0002 & -.0001 & -.0000 & -.0000 & .0000 & 0000 \\
\hline 20 & -.0573 & -.0124 & -.0056 & -.0022 & -.0011 & -.0004 & -.0002 & -.0001 & -.0000 & .0000 & .0000 \\
\hline 25 & -.0942 & -.0202 & -.0091 & -.0036 & -.0017 & -.0008 & -.0003 & -.0001 & -.0000 & .0001 & .0000 \\
\hline $30_{-}$ & -.1442 & -.0306 & -.0136 & -.0055 & -.0027 & -.0012 & -.0005 & -.0002 & -.0001 & .0001 & -0000 \\
\hline 35 & -.2105 & -.0442 & -.0194 & -.0079 & -.0038 & -.0017 & -.0007 & -.0004 & -.0001 & .0001 & -.0000 \\
\hline 40 & -.2974 & -.0618 & -.0265 & -.0109 & -.0053 & -.0024 & -.0010 & -.0006 & -.0002 & .0001 & -.0000 \\
\hline 45 & -.4101 & -.0840 & -.0354 & -.0146 & -.0072 & -.0034 & -.0015 & -.0008 & -.0002 & .0002 & -.0001 \\
\hline $50 \ldots$ & -.5553 & -.1130 & -.0464 & -.0193 & -.0095 & -.0045 & -.0020 & -.0012 & -.0004 & .0002 & -.0001 \\
\hline 60. & -.9784 & -.1951 & -.0767 & -.0322 & -.0159 & -.0078 & -.0037 & -.0022 & -.0007 & .0001 & -.0002 \\
\hline 70 & -1.6559 & -.3276 & -.1222 & -.0516 & -.0256 & -.0129 & -.0065 & -.0038 & -.0014 & -.0000 & -.0003 \\
\hline 80 & -2.7138 & -.5396 & -.1916 & -.0809 & -.0401 & -.0206 & -.0107 & -.0063 & -.0024 & -.0004 & -.0006 \\
\hline $90 \ldots$ & & -.8786 & -.2981 & -.1261 & -.0622 & -.0323 & -.0171 & -.0102 & -.0040 & -.0010 & -.0010 \\
\hline $100_{----}$ & & -1.4169 & -.4631 & -.1907 & -.0929 & -.0488 & -.0266 & -.0160 & -.0065 & -.0020 & -.0017 \\
\hline 110. & & -2.2638 & -.7186 & -.2906 & -.1416 & -.0741 & -.0406 & -.0246 & -.0101 & -.0035 & -.0026 \\
\hline 120 & & -3.5736 & -1.1171 & -.4432 & -.2097 & -.1095 & -.0622 & -.0371 & -.0153 & -.0059 & -.0039 \\
\hline 130 & & & -1.7384 & -.6752 & -.3139 & -.1646 & -.0906 & -.0548 & -.0229 & -.0093 & -.0059 \\
\hline 140 & & & -2.7042 & -1.0330 & -.4716 & -.2424 & -.1372 & -.0810 & -.0339 & -.0142 & -.0085 \\
\hline 150 & & & -4.1878 & -1.5853 & -.7079 & -.3584 & -.1978 & -.1175 & -.0485 & -.0212 & -.0123 \\
\hline
\end{tabular}

TABLE 8.17. Coefficient of $\Delta \mathrm{N}, \mathrm{b}_{3}$, in the regression equation (8.54)

\begin{tabular}{|c|c|c|c|c|c|c|c|c|c|c|c|}
\hline \multirow{2}{*}{ 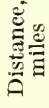 } & \multicolumn{11}{|c|}{ Height, kft } \\
\hline & 5 & 10 & 15 & 20 & 25 & 30 & 35 & 40 & 50 & 60 & 70 \\
\hline $\begin{array}{r}5 \\
10 \\
15 \\
20 \\
25\end{array}$ & $\begin{array}{r}-0.0806 \\
-.3321 \\
-.7533 \\
-1.3470 \\
-2.1168\end{array}$ & $\begin{array}{r}-0.0301 \\
-.1360 \\
-.3146 \\
-.5687 \\
-.9026\end{array}$ & $\begin{array}{r}-0.0136 \\
-.0739 \\
-.1754 \\
-.3196 \\
-.5084\end{array}$ & $\begin{array}{r}-0.0060 \\
-.0481 \\
-.1189 \\
-.2191 \\
-.3498\end{array}$ & $\begin{array}{r}-0.0011 \\
-.0313 \\
-.0819 \\
-.1535 \\
-.2468\end{array}$ & $\begin{array}{l}0.0021 \\
-.0201 \\
-.0575 \\
-.1104 \\
-.1793\end{array}$ & $\begin{array}{r}0.0043 \\
-.0127 \\
-.0411 \\
-.0813 \\
-.1336\end{array}$ & $\begin{array}{r}0.0057 \\
-.0075 \\
-.0296 \\
-.0608 \\
-.01015\end{array}$ & $\begin{array}{r}0.0073 \\
-.0012 \\
-.0154 \\
-.0355 \\
-.0616\end{array}$ & $\begin{array}{r}0.0083 \\
-.0024 \\
-.0077 \\
-.0218 \\
-.0401\end{array}$ & $\begin{array}{r}0.0087 \\
-.0044 \\
-.0029 \\
-.0132 \\
-.0265\end{array}$ \\
\hline $\begin{array}{l}30 \\
35 \\
40 \\
45 \\
50\end{array}$ & $\begin{array}{l}-3.0664 \\
-4.1993 \\
-5.5178 \\
-7.0221 \\
-8.7091\end{array}$ & $\begin{array}{l}-1.3217 \\
-1.8327 \\
-2.4440 \\
-3.1634 \\
-4.0036\end{array}$ & $\begin{array}{r}-.7448 \\
-1.0320 \\
-1.3744 \\
-1.7769 \\
-2.2458\end{array}$ & $\begin{array}{r}-.5125 \\
-.7091 \\
-.9818 \\
-1.2136 \\
-1.5276\end{array}$ & $\begin{array}{r}-.3628 \\
-.5028 \\
-.6680 \\
-.8604 \\
-1.0819\end{array}$ & $\begin{array}{l}-.2648 \\
-.3679 \\
-.4894 \\
-.6308 \\
-.7935\end{array}$ & $\begin{array}{r}-.1986 \\
-.2768 \\
-.3690 \\
-.4761 \\
-.5992\end{array}$ & $\begin{array}{l}-.1519 \\
-.2126 \\
-.2842 \\
-.3672 \\
-.4625\end{array}$ & $\begin{array}{l}-.0940 \\
-.1330 \\
-.1788 \\
-.2320 \\
-.2929\end{array}$ & $\begin{array}{r}-.0628 \\
-.0901 \\
-.1222 \\
-.1592 \\
-.2016\end{array}$ & $\begin{array}{l}-.0430 \\
-.0627 \\
-.0860 \\
-.1127 \\
-.1434\end{array}$ \\
\hline $\begin{array}{r}60 \\
70 \\
80 \\
90 \\
100\end{array}$ & $\begin{array}{l}-12.5871 \\
-16.9766 \\
-21.4838\end{array}$ & $\begin{array}{r}-6.0883 \\
-8.8019 \\
-12.2504 \\
-16.5206 \\
-21.6282\end{array}$ & $\begin{array}{r}-3.4103 \\
-4.9354 \\
-6.9080 \\
-9.4378 \\
-12.6475\end{array}$ & $\begin{array}{l}-2.2978 \\
-3.2912 \\
-4.5605 \\
-6.1745 \\
-8.2077\end{array}$ & $\begin{array}{l}-1.6230 \\
-2.3149 \\
-3.1905 \\
-4.2931 \\
-5.6667\end{array}$ & $\begin{array}{l}-1.1893 \\
-1.6931 \\
-2.3271 \\
-3.1194 \\
-4.1012\end{array}$ & $\begin{array}{r}-.8979 \\
-1.2773 \\
-1.7521 \\
-2.3426 \\
-3.0720\end{array}$ & $\begin{array}{r}-.6937 \\
-.9865 \\
-1.3526 \\
-1.8054 \\
-2.3636\end{array}$ & $\begin{array}{r}-.4402 \\
-.6263 \\
-.8573 \\
-1.1417 \\
-1.4910\end{array}$ & $\begin{array}{r}-.3038 \\
-.4320 \\
-.5909 \\
-.7843 \\
-1.0207\end{array}$ & $\begin{array}{l}-.2171 \\
-.3092 \\
-.4227 \\
-.5608 \\
-.7284\end{array}$ \\
\hline $\begin{array}{l}110 \\
120 \\
130 \\
140 \\
150\end{array}$ & & $\begin{array}{l}-27.4311 \\
-33.4941\end{array}$ & $\begin{array}{l}-16.6690 \\
-21.6151 \\
-27.5400 \\
-34.3121 \\
-41.4796\end{array}$ & $\begin{array}{l}-10.7799 \\
-14.0135 \\
-18.0521 \\
-23.0424 \\
-29.0964\end{array}$ & $\begin{array}{r}-7.4021 \\
-9.5614 \\
-12.2761 \\
-15.6717 \\
-19.9016\end{array}$ & $\left|\begin{array}{r}-5.329 \mathrm{I} \\
-6.8460 \\
-8.7510 \\
-11.1154 \\
-14.067 \mathrm{I}\end{array}\right|$ & $\begin{array}{r}-3.9747 \\
-5.0972 \\
-6.4662 \\
-8.1908 \\
-10.3024\end{array}$ & $\begin{array}{l}-3.0501 \\
-3.8960 \\
-4.9325 \\
-6.2123 \\
-7.7822\end{array}$ & $\begin{array}{l}-1.9168 \\
-2.4355 \\
-3.0686 \\
-3.8447 \\
-4.7800\end{array}$ & $\begin{array}{l}-1.3061 \\
-1.6531 \\
-2.0720 \\
-2.5769 \\
-3.1896\end{array}$ & $\begin{array}{r}-.9311 \\
-1.1731 \\
-1.4666 \\
-1.8159 \\
-2.2378\end{array}$ \\
\hline
\end{tabular}




\subsubsection{Height Error Equations}

The equations for approximating $\epsilon_{h}$ were determined as

$$
\begin{aligned}
& \epsilon_{h_{1}}=a_{10} \frac{D}{h_{a}{ }^{2}}+\frac{D^{3 / 2}}{R} g_{1}+k, \\
& \epsilon_{h_{2}}=a_{20} \frac{D}{h_{a}{ }^{2}}+\frac{D^{3 / 2}}{R} g_{2}+k,
\end{aligned}
$$

and

$$
\epsilon_{h_{3}}=a_{30} \frac{D}{h_{a}^{2}}+\frac{D^{3 / 2}}{R} g_{3}+k
$$

where

$$
D=R^{2}-0.03587 h_{a}^{2}
$$

Values for $g_{i}$ from (8.46) through (8.48) are found from

$$
\begin{aligned}
& f_{i 1}\left(h_{a}\right)=a_{i 1}+a_{i 2} h_{a}+a_{i 3} h_{a}{ }^{2},(i=1,2,3), \\
& f_{i 2}\left(h_{a}\right)=a_{i 4}+a_{i 5} h_{a} \quad,(i=2,3),
\end{aligned}
$$

and

$$
f_{33}\left(h_{a}\right)=a_{36}+a_{37} h_{a}
$$

for $R$ in miles and $h_{a}$ in thousands of feet. The term in $D / h_{a}{ }^{2}$ was introduced to account for, in part, a large negative constant term which tended to produce negative height errors for ranges less than $30 \mathrm{mi}$. Furthermore, the inclusion of this term increased the accuracy of the estimate of $\epsilon_{h_{i}}$ by about 2 percent. An additional term in $h_{a}{ }^{3}$ for (8.55) increased in the accuracy by about 1 percent but introduced a fictitious minimum near $60,000 \mathrm{ft}$, while a term in $h_{a}{ }^{2}$ for (8.56) and (8.57) increased the accuracy of $(8.53$ and $(8.54)$ by less than 0.1 percent. The relative improvement of (8.53) over (8.52) is about one percent. The coefficients $\alpha_{i j}$ are listed in table 8.8. The constant term, $k$, which would vanish if the equations were exact, is about -70 for a least squares approximation. 


\subsubsection{Conclusions}

Height-error correction can be significantly improved by accounting for the surface refractivity at the radar site. The use of the initial gradient, in addition to the surfare refractivity, yields a significant improvement only for targers beyond about $60 \mathrm{mi}$ and below $15,000 \mathrm{ft}$. In this case, $G_{0}$ is important not only to improve the accuracy but to determine if the assumption in section 2.2 has been violated, namely, if $G_{0} \leq-10^{6} / r_{0}$. The still further improvement obtained with the use of $\Delta N$ would not, in general, justify the trouble and expense of measuring this parameter.

If the distance to the target exceeds about $50 \mathrm{mi}$, the normal decrease with height of the gradient should be accounted for in a height error correction.

\subsection{References}

[1] Bean, B. R., and G. D. Thayer (May 1959), On models of the atmospheric refractive index, Proc. IRE 47, No. 5, 740-755.

[2] Misme, P. (Nov.-Dec. 1958), Essai de radioclimatologie d'altitude dans le nord de la France, Ann. Telecommun. 13, Nos. 11-12, 303-310.

[3] Tao, K., and K. Hirao (1960), Vertical distribution of radio refractive index in the medium height of the atmosphere, J. Radio Res. Lab. 7, No. 30, 85-93.

[4] Misme, P. (Nov.-Dec. 1960), Quelques aspects de la radio-climatologie, Ann. Telecommun. 15, 266.

[5] Bean, B. R., and G. D. 'Thayer (Aug. 1960), Rebuttal to P. Misme's comments on "Models of the Atmospheric Radio Refractive Index," Proc. IRE 48, No. 8, $1499-1501$.

[6] Craig, R. A., I. Katz, R. B. Montgomery, and P. J. Rubenstein (1951), Gradient of refractive modulus in homogeneous air, potential modulus, Book, Propagation of Short Radio Waves, ed. D. E. Kerr, pp. 198-199 (McGraw-Hill Book Co., Inc., New York, N.Y.).

[7] Zhevankin, S. A., and V. S. Troitskii (1959), Absorption of centimeter waves in the atmosphere, Radioteknika i Electronika 4, No. 1, 21-27.

[8] Minzner, R. A., W. S. Ripley, and T. P. Condron (1958, U.S. extension to the ICAO standard atmosphere, Tables and Data to 300 Standard geopotential kilometers, U.S. Government Printing Office, Washington, D.C. 20402.

[9] Ratner, B. (1945), Upper air average values of temperature, pressure, and relative humidity over the United States and Alaska (U.S. Weather Bureau).

[10] Bean, B. R., and J. D. Horn (Nov.-Dec. 1959), The radio refractive index near the ground, J. Res. NBS 63D (Radio Prop.), No. 3, 259-273.

[11] Schulkin, M. (May 1952), Average radio-ray refraction in the lower atmosphere, Proc. IRE 40, No. 5, 554-561.

[12] Bean, B. R., J. D. Horn, and A. M. Ozanich, Jr. (1960), Climatic Charts and Data of the Radio Refractive Index for the United States and the World, NBS Mono. 22.

[13] Wong, M. S. (Sept. 1958), Refraction anomalies in airborne propagation, Proc. IIRE 46, No. !), 1628-1639.

[14] Report of Factual Data from the Canterbury Project (1951), Vols. I-III, (1)ept. of Scientifie and Industrial Research, Wellington, New Zealind). 
[15] Freehafer, J. E. (1951), Tropospheric refraction, Book, Propagation of Short Radio Waves, ed. D. E. Kerr, pp. 9-22 (McGraw-Hill Book Co., Inc., New York, N.Y.).

[16] Bean, B. R. (July-Aug. 1959), Climatology of ground-based radio ducts, J. Res. NBS 63D (Radio Prop.), No. 1, 29.

[17] Bean, B. R., and B. A. Cahoon (Nov. 1957), The use of surface weather observations to predict the total atmospheric bending of radio waves at small elevation angles, Proc. IRE 45, No. 11, 1545-1546.

[18] Bean, B. R., B. A. Cahoon, and G. D. Thayer (1960), Tables for the statistical prediction of radio ray bending and elevation angle errors using surface values of the refractive index, NBS Tech. Note 44.

[19] Bean, B. R., and G. D. Thayer (May-June 1963), Comparison of observed atmospheric radio refraction effects with values predicted through the use of surface weather observations, J. Res. NBS 67D (Radio Prop.), No. 3, 273.

[20] Anway, A. C. (1961), Empirical determination of total atmospheric refraction at centimeter wavelengths by radiometric means, Collins Res. Rept. CRR-2425 (Collins Radio Company, Cedar Rapids, Iowa).

[21] Anderson, W. L., N. J. Beyers, and R. J. Rainey (Aug. 1960), Comparison of experimental and compeutd refraction, IRE Trans. Ant. Prop. AP-8, 456.

[22] Wilkerson, R. E. (July-Aug. 1962), Divergence of radio rays by the troposphere, J. Res. NBS 66D (Radio Prop.), No. 4, 479.

[23] Norton, K. A., J. W. Herbstreit, H. B. Janes, K. O. Hornberg, C. F. Peterson, A. F. Barghausen, W. E. Johnson, P. I. Wells, M. C. Thompson, Jr., M. J. Vetter, and A. W. Kirkpatrick (1961), An experimental study of phase variations in line-of-sight microwave transmissions, NBS Mono. 33.

[24] Thompson, M. C., Jr. (1962), Private communication.

[25] Bennett, C. A., and N. L. Franklin (1954), Book, Statistical Analysis in Chemistry and the Chemical Industry (John Wiley \& Sons, Inc., New York, N.Y.).

[26] Brooks, C. E. P., and N. Carruthers (1953), Handbook of Statistical Methods in Meteorology (Her Majesty's Stationery Office, London).

[27] Schelleng, J. C., C. R. Burrows, and E. B. Ferrell (Mar. 1933), Ultra-short-wave propagation, Proc. IRE 21, No. 3, 427-463.

[28] Bauer, J. R., W. C. Mason, and F. A. Wilson (1958), Radio Refraction in a Cool Exponential Atmosphere, Tech. Rept. 186, Lincoln Laboratory, Massachusetts Institute of Technology, Cambridge, Mass.

[29] Beckmann, P. (1958), Height errors in radar measurements due to propagation causes, Acta Technika, 3, No. 6, 471-488 


\section{Chapter 9. Radio-Meteorological Charts, Graphs, Tables, and Sample Computations}

\subsection{Sample Computations of Atmospheric Refraction}

The following problem will serve to illustrate the application of the various methods of calculating bending of a radio ray as described in chapter 3. A particular daily set of RAOB readings from Truk in the Caroline Islands yields the following data:

$\begin{array}{cc}\text { Height above the } & N \text { value } \\ \text { surface }(\mathrm{km}) & (N \text { units }) \\ 0.000 & 400.0=N_{s} \\ 0.340 & 365.0 \\ 0.950 & 333.5 \\ 3.060 & 237.0 \\ 4.340 & 196.5 \\ 5.090 & 173.0 \\ 5.300 & 172.0 \\ 5.940 & 155.0 \\ 6.250 & 152.0 \\ 7.180 & 134.0 \\ 7.617 & 125.5 \\ 9.660 & 98.0 \\ 10.870 & 85.0\end{array}$

What is the total bending up to the $3.270-\mathrm{km}$ level at initial elevation angles of $0^{\circ}, 10 \mathrm{mrad}, 52.4 \mathrm{mrad}\left(3^{\circ}\right)$, and $261.8 \mathrm{mrad}\left(15^{\circ}\right)$ by (a) Schulkin's approach, (b) the exponential model, (c) the initial gradient method, (d) the departures from normal method, (e) the use of regression lines, and (f) the graphical method of Weisbrod and Anderson? Since the gradient between the ground and the first layer is

$$
\frac{\Delta N}{\Delta h}=\frac{365.0-400.0}{0.340}=-102.9 \mathrm{~N} \text { units } / \mathrm{km},
$$

and this is a decrease of $N$ per kilometer that is less than the $-157 \mathrm{~N}$ units $/ \mathrm{km}$ required for ducting, no surface duct is present. However, 
should a surface duct have been present, it would have been necessary to calculate the angle of penetration,

$$
\theta_{p}=\sqrt{2\left[N_{s}-N_{h}-156.9(\Delta h)(\text { in } \mathrm{km})\right]},
$$

to find the smallest initial elevation angle that yields a non-trapped ray. Any initial elevation angle less than $\theta_{p}$ cannot be used in bending calculations.

Schulkin's approach (a) of (3.13) yields the results shown in table 9.19 for $0 \mathrm{mrad}$, table 9.20 for $10 \mathrm{mrad}$, table 9.21 for $52.4 \mathrm{mrad}$, and table 9.22 for $261.8 \mathrm{mrad}$, where $\theta_{k+1}$ is determined from (3.58) using $r_{k}=a+h_{k}$, and $a$ is the radius of the earth,

$$
a=6370 \mathrm{~km} \text {. }
$$

It should be remembered that $\theta_{k}=0,10,52.4$, or $261.8 \mathrm{mrad}$ only for the first-level calculation, and that thereafter $\theta_{k}$ is equal to the $\theta_{k+1}$ computed for the preceding layer; e.g., for the second layer of table 9.19 $\left(\theta_{0}=0 \mathrm{mrad}\right), \theta_{k}=6.15 \mathrm{mrad}$, which is the $\theta_{k+1}$ calculated for the first layer.

The exponential model solution (b) may be found by using tables 9.10 through 9.17. Interpolation will usually be necessary for $N_{s}, \theta_{0}$, and height; this interpolation may be done linearly. In practice, one of these three variables will often be close enough to a tabulated value that interpolation will not be necessary, thus reducing from 7 to 3 the number of interpolations necessary. Since in the problem for $N_{s}=404.9, h=10.0 \mathrm{~km}$ and $\theta_{0}=10 \mathrm{mrad}$

$$
\tau_{0,10.0(10 \mathrm{mrad})}=15.084 \mathrm{mrad}
$$

and at $h=20.00 \mathrm{~km}, \theta_{0}=10 \mathrm{mrad}$.

$$
\tau_{0,20.00(10 \mathrm{mrad})}=15.946 \mathrm{mrad},
$$

and thus by linear interpolation for $h=10.870 \mathrm{~km}, \theta_{0}=10 \mathrm{mrad}$,

$$
\begin{aligned}
\tau_{0,10.870,(10 \mathrm{mrad})} & =15.084+(15.946-15.084) \frac{10.870-10.00}{20.00-10.00} \\
& =15.159 \mathrm{mrad} .
\end{aligned}
$$


Similarly for $N_{s}=377.2$ in the exponential tables,

$$
\tau_{0,10.870(10 \mathrm{mrad})}=13.120 \mathrm{mrad} .
$$

Again using linear interpolation, but now between the $N_{s}=377.2$ and $N_{s}=344.5 \mathrm{~atm}$, the desired value of $\tau$ at $3.270 \mathrm{~km}$ for $N_{s}=360.0$ and $\theta_{0}=10 \mathrm{mrad}$ is obtained.

Thus

$$
\begin{aligned}
\tau_{0,10.870(10 \mathrm{mrad})} & =13.120+(15.159-13.120) \frac{400.0-377.2}{404.9-377.2} \\
& =14.798 \mathrm{mrad} .
\end{aligned}
$$

For the $\theta_{0}=0,52.4$, and $261.8 \mathrm{mrad}$ cases, by similar calculations, using linear interpolation:

$$
\begin{gathered}
\tau_{0,10.870,(0 \mathrm{mrad})}=21.386 \mathrm{mrad}, \\
\tau_{0,10.870,(52.4 \mathrm{mrad})}=5.816 \mathrm{mrad}, \\
\tau_{0,10.870,(261.8 \mathrm{mrad})}=1.270 \mathrm{mrad} .
\end{gathered}
$$

The initial gradient correction method (c) may be used if one determines the $N_{s}{ }^{*}$ which corresponds to the observed initial gradient and then applies (3.45). The initial $N$ gradient is $-102.9 N$ units $/ \mathrm{km}$, which, as can be seen from table 3.17 , corresponds to the $N_{s}=450.0$ exponential atmosphere. Therefore, using the exponential tables of Bean and Thayer [1] ${ }^{1}$ and (3.45) to determine the bending for the $\theta_{0}=0 \mathrm{mrad}$ case, one finds by linear interpolation

$$
\begin{aligned}
\tau_{10,000(0)}= & \tau_{10,000}(400.0,0 \mathrm{mrad})+\left[\tau_{100}(450.0,0 \mathrm{mrad})\right. \\
& \left.\quad-\tau_{100}(400.0,0 \mathrm{mrad})\right] \\
= & 21.309 \mathrm{mrad}+[5.908-3.657] \mathrm{mrad}=23.560 \mathrm{mrad} .
\end{aligned}
$$

The $\tau_{100}(400.0,0 \mathrm{mrad})$ is determined by linear interpolation between the 404.9 and $377.2 \mathrm{~atm}$. At $h=20.0 \mathrm{~km}$ as given in the tables,

$$
\begin{aligned}
\tau_{20,000(0 \mathrm{mrad})}= & \tau_{20,000}(400.0,0 \mathrm{mrad})+\left[\tau_{100}(450.0,0 \mathrm{mrad})\right. \\
& \left.-\tau_{100}(400.0,0 \mathrm{mrad})\right] \\
= & 22.191+[5.908-3.657] \mathrm{mrad} \\
= & 24.442 \mathrm{mrad} .
\end{aligned}
$$

${ }^{1}$ Figures in brackets indicate the literature references on p. 423 . 
Hence by linear interpolation,

$$
\begin{aligned}
\tau_{10.870(0 \mathrm{mrad})} & =23.560+[24.442-23.560] \frac{10,870-10,000}{20,000-10,000} \\
& =23.637 \mathrm{mrad} .
\end{aligned}
$$

The bendings for $\theta_{0}=10,52.4$, and $261.8 \mathrm{mrad}$ are as given below:

$$
\begin{aligned}
& \theta_{0}=10 ; \tau_{10,870(10)}=15.053 \mathrm{mrad} \\
& \theta_{0}=52.4 ; \tau_{10,870(52.4)}=5.864 \mathrm{mrad} \\
& \theta_{0}=261.8 ; \tau_{10,870(261.8)}=1.280 \mathrm{mrad}
\end{aligned}
$$

To use the departures-from-normal method (d) of determining bending, it is first necessary to know the atmosphere which must be used for the calculation. In the problem,

$$
-\left.\frac{d N}{d h}\right|_{\text {initial }}=102.9 \mathrm{~N} \text { units } / \mathrm{km}
$$

which is within the range of the $N_{s}=450.0$ exponential atmosphere, as can be seen from table 9.18. Thus one will use table 9.17 to determine the $\theta$ 's and the $\tau$ 's in the $N_{s}=450.0$ exponential atmosphere, or one can use the exponential atmosphere tables [1].

For an $N_{s}=450.0$ atmosphere to a height of $10.870 \mathrm{~km}$ :

$$
\begin{aligned}
& \tau_{N s(0 \mathrm{mrad})}=30.776 \mathrm{mrad}, \\
& \tau_{N s(10 \mathrm{mrad})}=19.414 \mathrm{mrad}, \\
& \tau_{N s(52.4 \mathrm{mrad})}=7.024 \mathrm{mrad}, \\
& \tau_{N s(261.8 \mathrm{mrad})}=1.506 \mathrm{mrad} .
\end{aligned}
$$

Equation (3.58) should be used for the $\theta$ interpolation in preference to linear interpolation, although, if no tables or other facilities are present at the engineering site for easy acquisition of square roots, linear interpolation will suffice. Proceeding in table 9.17 with (3.58) for the first layer at $h=0.340 \mathrm{~km}$ and the $\theta_{0}=0 \mathrm{mrad}$ case:

$$
\theta=\sqrt{\theta_{0}^{2}+\frac{2\left(r_{1}-r_{0}\right)}{r_{0}} \times 10^{6}-2\left(N_{s}-N_{1}\right)}
$$

$=6.388 \mathrm{mrad}$. The remaining $\theta$ 's for the various layers are shown in table 9.23. To determine the value of $A$ at the bottom and top of the 
layer, one makes use of (3.46) or figure 3.17. First, however, one must determine the value of $c$ in (3.46) to be used. Usually interpolation will be necessary in table 9.18 , but in the $N_{s}=450.0$ case it is not possible, and thus the straight $N_{s}=450.0$ exponential atmosphere values are used.

From (3.46)

$$
A\left(N_{s}, h\right)=N(h)+N_{s}[1-\exp (-c h)],
$$

and for the layer running from $h=0$ to $h=0.340 \mathrm{~km}$, figure 3.17 yields

$$
377.2(1-\exp (c 0)]=0.0
$$

and

$$
450.0[1-\exp (-c \times 0.340)]=32.8,
$$

and, therefore,

$$
A(450.0,0)=400.0+0=400.0
$$

and

$$
A(450.0 ., 0.340)=365.0+32.8=397.8,
$$

whence

$\Delta A=A(450.0,0.340)-A(450.0,0)=397.8-400.0=-2.2 N$ units. Therefore, the departure term of (3.23),

$$
\frac{-2}{\theta_{k}+\theta_{k+1}}\left[\Delta A\left(N_{s}\right)\right]_{N_{k}}^{N_{k+1}} \text {, }
$$

becomes

$$
-\frac{2}{0+6.388}[-2.2]_{400.0}^{365.0}=+0.689 \mathrm{mrad}
$$

The remaining calculations are tabulated in table 9.23 for the $\theta_{0}=0 \mathrm{mrad}$ case, in table 9.24 for the $\theta_{0}=10 \mathrm{mrad}$ case, in table 9.25 for the $\theta_{0}=52.4$ mrad case, and in table 9.26 for the $\theta_{0}=261.8 \mathrm{mrad}$ case. The sum of the departures for the 0 mrad case is

$$
\sum_{k=0}^{k_{n}} \frac{-2}{\theta_{k}+\theta_{k+1}}\left[\Delta A\left(N_{s}\right)\right]_{N_{k}}^{N_{k+1}}=-5.335 \mathrm{mrad} .
$$

Determination of the bending is required in part (e) of the problem by using regression lines. By (3.10), using table 9.7 and 9.8 , it is found for the $\theta_{0}=0 \mathrm{mrad}$ ease that at $10.0 \mathrm{~km}$ (from table 9.7)

$$
\begin{aligned}
\tau_{0,10.0} & =(0.1149)(400.0)-18.5627 \pm 7.5227 \\
& =27.3973 \pm 7.5227 \mathrm{mrad}
\end{aligned}
$$


and at $20.0 \mathrm{~km}$ (from table 9.8 )

$$
\begin{aligned}
\tau_{0,20.0} & =(0.1165)(400.0)-17.9573 \pm 7.5131 \\
& =28.6427 \pm 7.5131 \mathrm{mrad}
\end{aligned}
$$

Thus, by linear interpolation

$$
\begin{gathered}
\tau_{1,2}=\tau_{0,10.87}=27.3973+(28.6427-27.3973) \frac{10.87-10.00}{20.00-10.00} \pm 7.5227 \\
\mp(7.5227-7.5131) \frac{10.87-10.00}{20.00-10.00} \\
\tau_{1,2}=27.5056 \pm 7.5218 \mathrm{mrad}
\end{gathered}
$$

Similarly, for the remaining $\theta$ 's,

$$
\begin{array}{ll}
\tau_{1,2(10 \mathrm{mrad})} & =13.9548 \pm 0.9701 \mathrm{mrad} \\
\tau_{1,2(53.4 \mathrm{mrad})} & =5.2186 \pm 0.0817 \mathrm{mrad} \\
\tau_{1,2(261.8 \mathrm{mrad})} & =1.2695 \pm 0.0158 \mathrm{mrad}
\end{array}
$$

Determination of the bending by means of the graphical method (f) of Weisbrod and Anderson yields, from figure 3.18 for $500 \tan \theta$, for the first layer,

$$
\begin{array}{ccccc}
h(\mathrm{~m}) & \begin{array}{c}
\text { At } \theta_{0} \\
0 \mathrm{mrad}
\end{array} & \begin{array}{c}
\text { At } \theta_{0} \\
10 \mathrm{mrad}
\end{array} & \begin{array}{c}
\text { At } \theta_{0} \\
52.4 \mathrm{mrad}
\end{array} & \begin{array}{c}
\text { At } \theta_{0} \\
=261.8 \mathrm{mrad}
\end{array} \\
\hline 0.000 & 0.0 & 5.0 & 26.2 & 134.0 \\
0.340 & 3.0 & 5.8 & 27.0 & 134.0
\end{array}
$$

which yields for the bending in the first layer,

$$
\begin{aligned}
& \begin{array}{c}
\text { At } \theta_{0} \\
=0 \mathrm{mrad} \\
11.67
\end{array} \quad \frac{\begin{array}{c}
\text { At } \theta_{0} \\
3.24
\end{array}}{10 \mathrm{mrad}}
\end{aligned}=\begin{gathered}
\text { At } \theta_{0} \\
=52.4 \mathrm{mrad}
\end{gathered} \quad \begin{gathered}
\text { At } \theta_{0} \\
=261.8 \mathrm{mrad} .
\end{gathered}
$$

Similarly, the bending for the entire profile may be obtained, and shown to be

$$
\begin{aligned}
& \text { At } \theta_{0} \\
& \text { At } \theta_{0} \\
& \text { At } \theta_{0} \\
& \text { At } \theta_{0} \\
& =\frac{0 \mathrm{mrad}}{24.42} \\
& =\frac{10 \mathrm{mrad}}{14.00} \\
& \frac{=52.4 \mathrm{mrad}}{5.32} \\
& =\frac{261.8 \mathrm{mrad}}{1.17} \text {. }
\end{aligned}
$$


The answers to the several parts of the problem are summarized in the next table of this chapter (immediately preceding the main body of tables). Bending values for the assumed profile, from a method which exponentially interpolated layers between given layers and then integrated between resulting layers, assuming only a linear decrease of refractivity between interpolated layers, are included for the sake of comparison. The computations were performed on a digital computer.

The reason that the answers to part (e) vary so radically from the remaining answers for the $\theta_{0}=0 \mathrm{mrad}$ case and not so much for the $\theta_{0}=261.8 \mathrm{mrad}$ case is the fact that the accuracy of the regression line method increases with increasing initial elevation angle, $\theta_{0}$. It must be remembered that the statistical regression technique, like the exponential model, is an adequate solution to the bending problem for all $\theta_{0}$ 's larger than about $10 \mathrm{mrad}$, and all heights above $1 \mathrm{~km}$.

The reason that the answers in part (f) and part (a) agree more closely than with any other of the answers is because (3.49) is, as mentioned before, Schulkin's result with only the approximation, $\tan \theta_{k} \cong \theta_{k}$ for small angles, omitted. For this individual profile, the bending obtained from an exponential atmosphere does not give particularly accurate bendings; however, for 22 five-year mean refractivity profiles, figure 3.9 shows that exponential bending predicts accurately within 1 percent of the average bending for these five-year means. Figure 3.19 shows the rms error in predicting bending at various heights as a per cent of mean bending (not including superrefraction).

In summary, it is recommended that the communications engineer either use the statistical regression technique or the exponential tables [1] without interpolation (i.e., pick the values of height, $N_{s}$, and $\theta_{0}$ that are closest to the given parameters) for a quick and facile bending result, keeping in mind the restrictions on these methods. However, as mentioned before, use of Schulkin's method is recommended if accuracy is the primary incentive.

Summary of refraction results for the sample computation

\begin{tabular}{|c|c|c|c|c|c|}
\hline $\begin{array}{l}\text { Prob- } \\
\text { lem } \\
\text { part }\end{array}$ & Method used & $\begin{array}{l}\text { Bending } \\
\text { in mrad at } \\
\theta_{0}=0 \mathrm{mrad}\end{array}$ & $\begin{array}{c}\text { Bending } \\
\text { in mrad at } \\
\theta_{0}=10 \mathrm{mrad}\end{array}$ & $\begin{array}{c}\text { Bending } \\
\text { in mrad at } \\
\theta_{0}=52.4 \mathrm{mrad}\end{array}$ & $\begin{array}{c}\text { Bending } \\
\text { in mrad at } \\
\theta_{0}=261.8 \mathrm{mrad}\end{array}$ \\
\hline a. & Schulkin's method.................... & 24. 248 & 14.008 & 5.341 & 1.196 \\
\hline b. & Exponential model................... & 21.386 & 14.798 & 5.816 & 1. 270 \\
\hline c. & Initial gradient correction method_ & 23.637 & 15. 053 & 5.864 & 1. 280 \\
\hline d. & Departures from normal method - - & 25.441 & 14.858 & 5.350 & 1.143 \\
\hline e. & Statistical regression method...... & $\begin{array}{r}27.506 \\
\pm 7.522\end{array}$ & $\begin{array}{l}13.955 \\
\pm 0.9701\end{array}$ & $\begin{array}{r}5.2186 \\
\pm 0.0817\end{array}$ & $\begin{array}{r}1.2695 \\
\pm 0.0158\end{array}$ \\
\hline f. & Graphieal method.--. & 24.42 & 14.00 & 5. 32 & 1.168 \\
\hline \multicolumn{2}{|c|}{$\begin{array}{l}\text { Comparison (exponential layer } \\
\text { interpolation) bending }\end{array}$} & 24171 & 14. 104 & 5. 343 & 1. 168 \\
\hline
\end{tabular}


Tables 9.1 through 9.9 are tables of coefficients, $a$ and $b$, standard errors of estimate, SE, and correlation coefficients, $r$, for use in the statistical method of refraction prediction.

TABLE 9.1. Variables in the statistical method of refraction prediction for $\mathrm{h}-\mathrm{h}_{\mathrm{s}}=0.1 \mathrm{~km}$

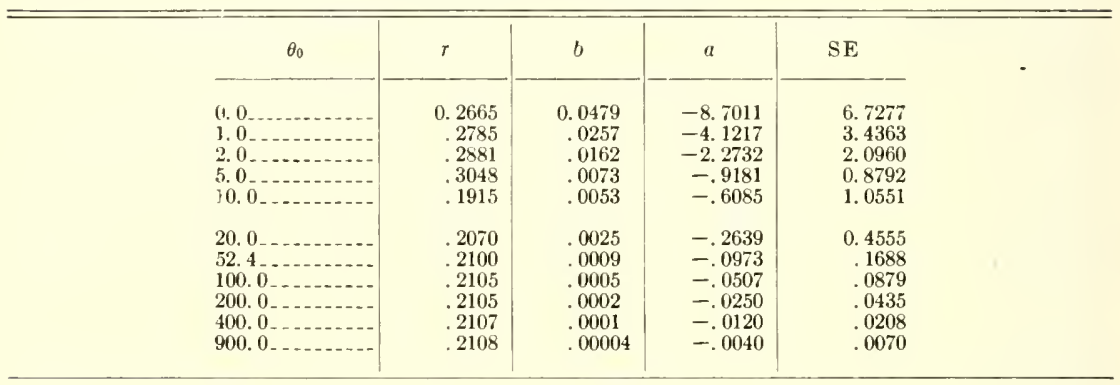

TABLE 9.2. Variables in the statistical method of refraction prediction for $\mathrm{h}-\mathrm{h}_{\mathrm{s}}=0.2 \mathrm{~km}$

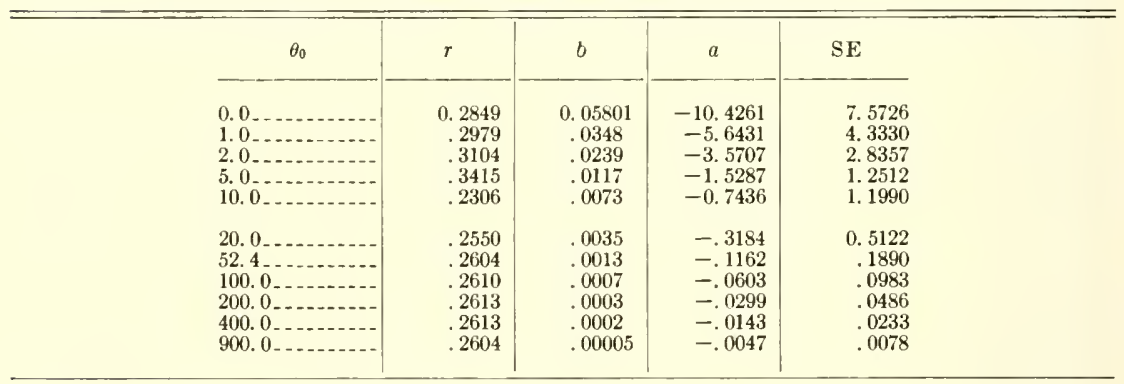

TABLE 9.3. Variables in the statistical method of refraction prediction for $\mathrm{h}-\mathrm{h}_{\mathrm{s}}=0.5 \mathrm{~km}$

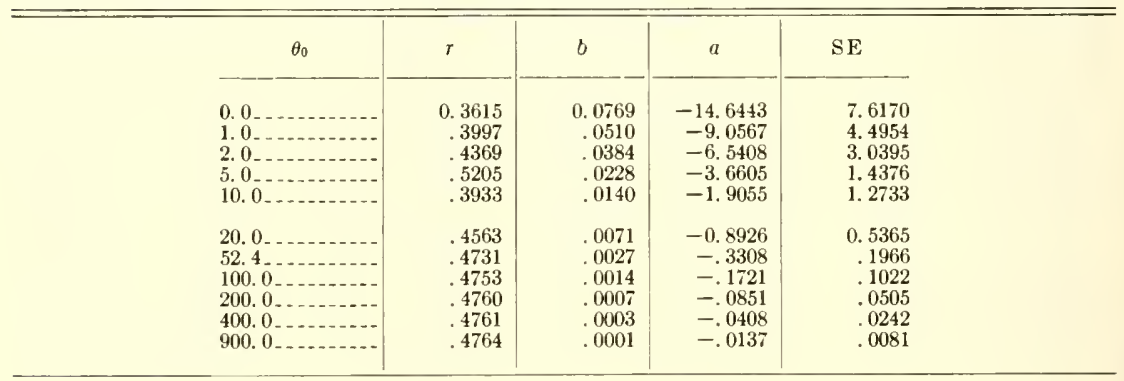


TABLE 9.4. Variables in the statistical method of refraction prediction for $\mathbf{h}-\mathrm{h}_{\mathrm{s}}=1.0 \mathrm{~km}$

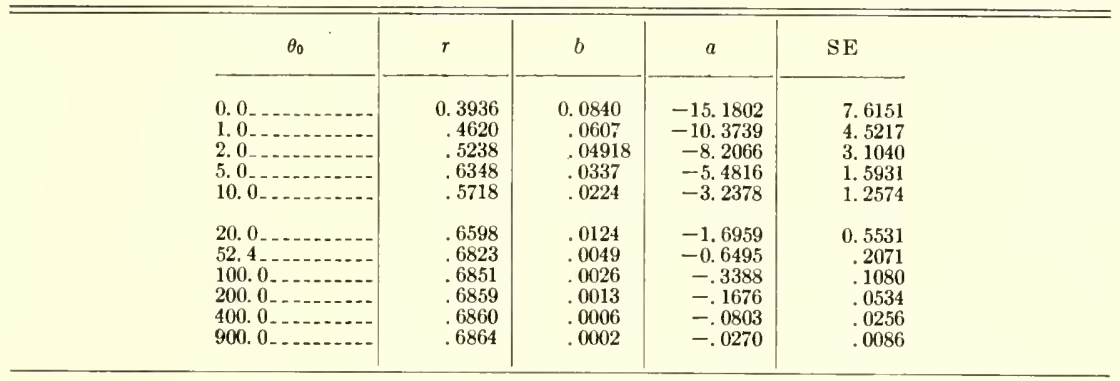

TABLE 9.5. Variables in the statistical method of refraction prediction for $\mathrm{h}-\mathrm{h}_{\mathrm{B}}=2.0 \mathrm{~km}$

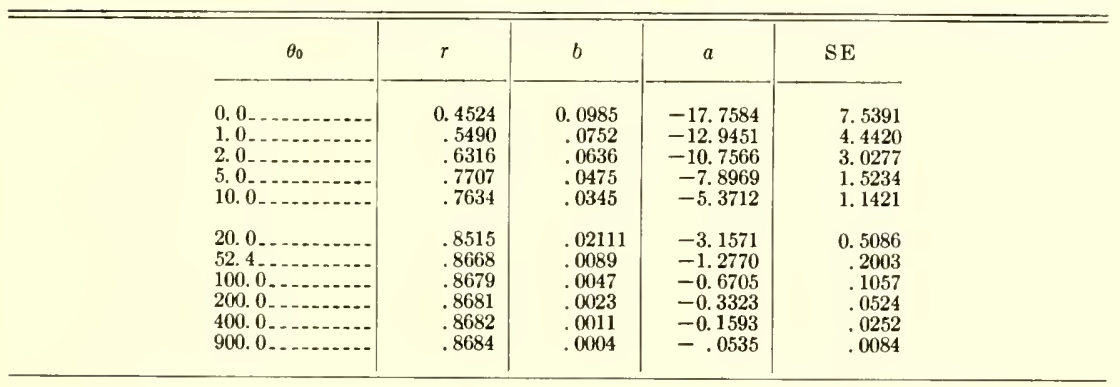

TABLE 9.6. Variables in the statistical method of refraction prediction for $\mathbf{h}-\mathbf{h}_{\mathbf{s}}=5.0 \mathrm{~km}$

\begin{tabular}{|c|c|c|c|c|}
\hline$\theta_{0}$ & $r$ & $b$ & $a$ & SE \\
\hline $\begin{array}{l}0.0 \ldots \\
1.0 \\
2.0 \\
5.0 \\
10.0 \ldots \ldots\end{array}$ & $\begin{array}{r}0.4962 \\
.6101 \\
.7030 \\
.8504 \\
.8674\end{array}$ & $\begin{array}{r}0.1115 \\
.0881 \\
.0764 \\
.0601 \\
.0464\end{array}$ & $\begin{array}{r}-19.1704 \\
-14.3543 \\
-12.1589 \\
-9.2514 \\
-6.6445\end{array}$ & $\begin{array}{l}7.5676 \\
4.4401 \\
3.0001 \\
1.4422 \\
1.0420\end{array}$ \\
\hline $\begin{array}{l}20.0 \\
52.4 \\
100.0 \\
200.0 \\
400.0 \\
900.0\end{array}$ & $\begin{array}{l}.9484 \\
.9674 \\
.9695 \\
.9701 \\
.9702 \\
.9703\end{array}$ & $\begin{array}{l}.0308 \\
.0139 \\
.0075 \\
.0037 \\
.0018 \\
.0006\end{array}$ & $\begin{array}{r}-4.0706 \\
-1.6236 \\
-0.8348 \\
-.4098 \\
-.1960 \\
-.0658\end{array}$ & $\begin{array}{r}0.4028 \\
.1426 \\
.0739 \\
.0365 \\
.0175 \\
.0059\end{array}$ \\
\hline
\end{tabular}


TABLE 9.7. Variables in the statistical method of refraction prediction for $\mathrm{h}-\mathrm{h}_{\mathrm{s}}=10.0 \mathrm{~km}$

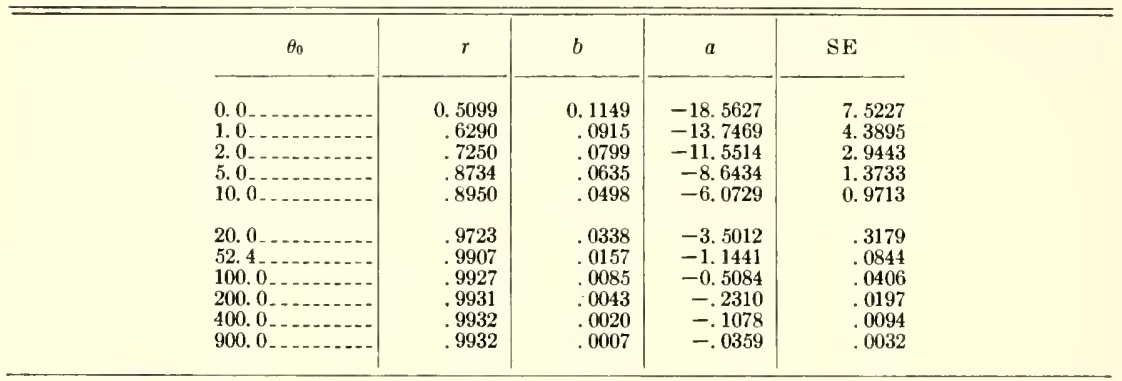

TABLE 9.8. Variables in the statistical method of refraction prediction for $\mathrm{h}-\mathrm{h}_{\mathrm{s}}=20.0 \mathrm{~km}$

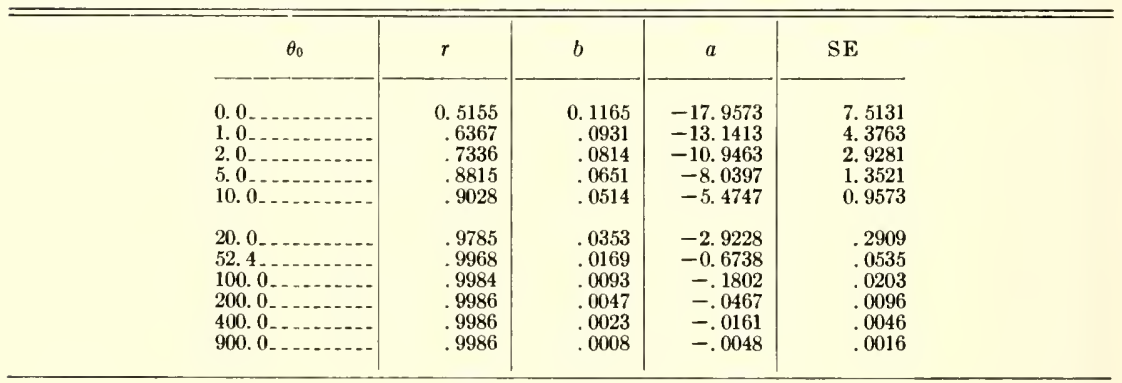

TABLE 9.9. Variables in the statistical method of refraction prediction for $\mathrm{h}-\mathrm{h}_{\mathrm{s}}=70.0 \mathrm{~km}$

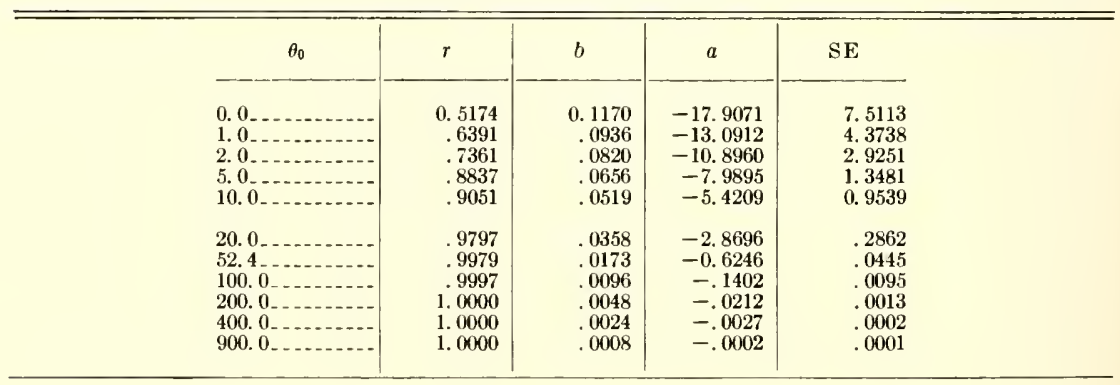



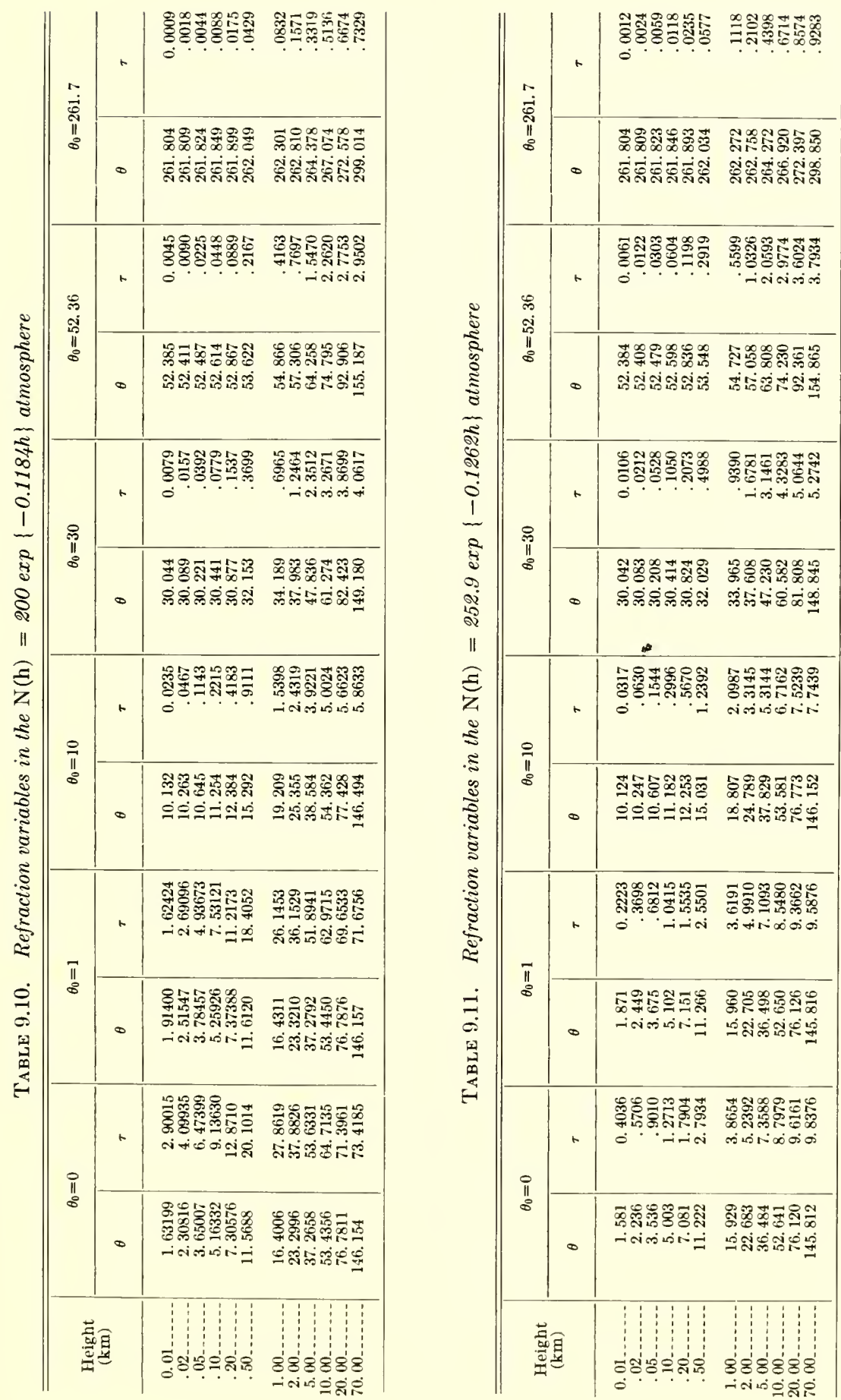

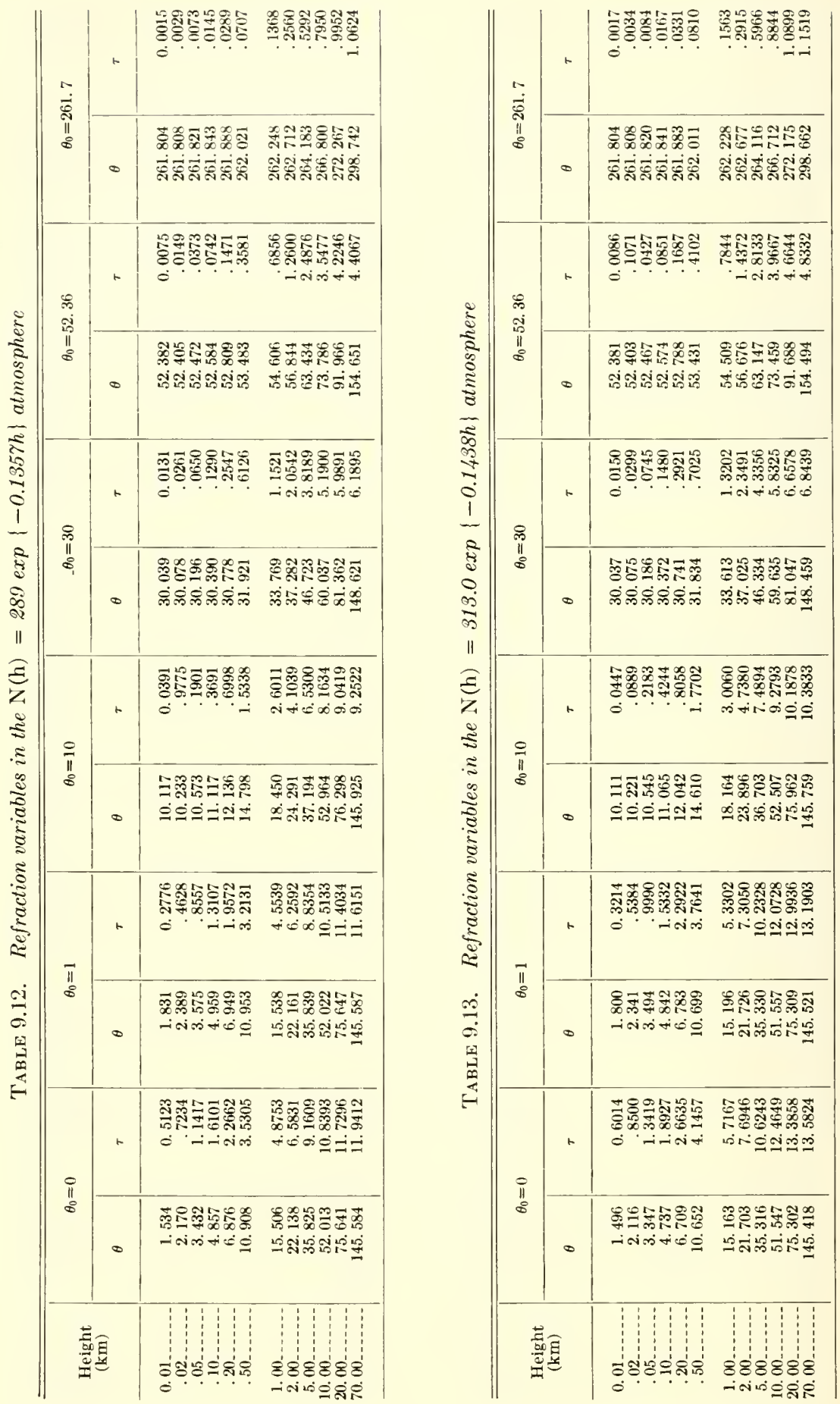

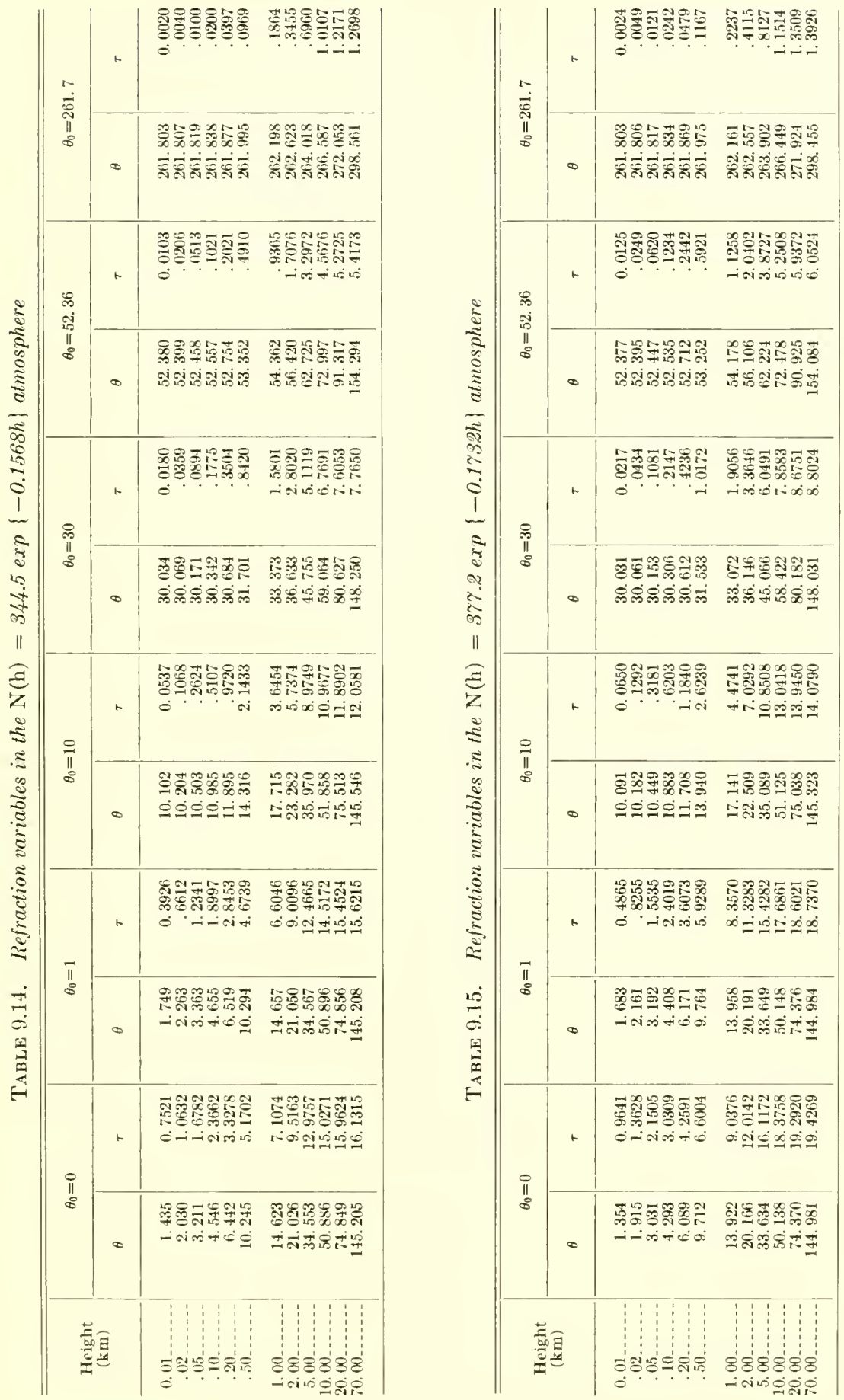

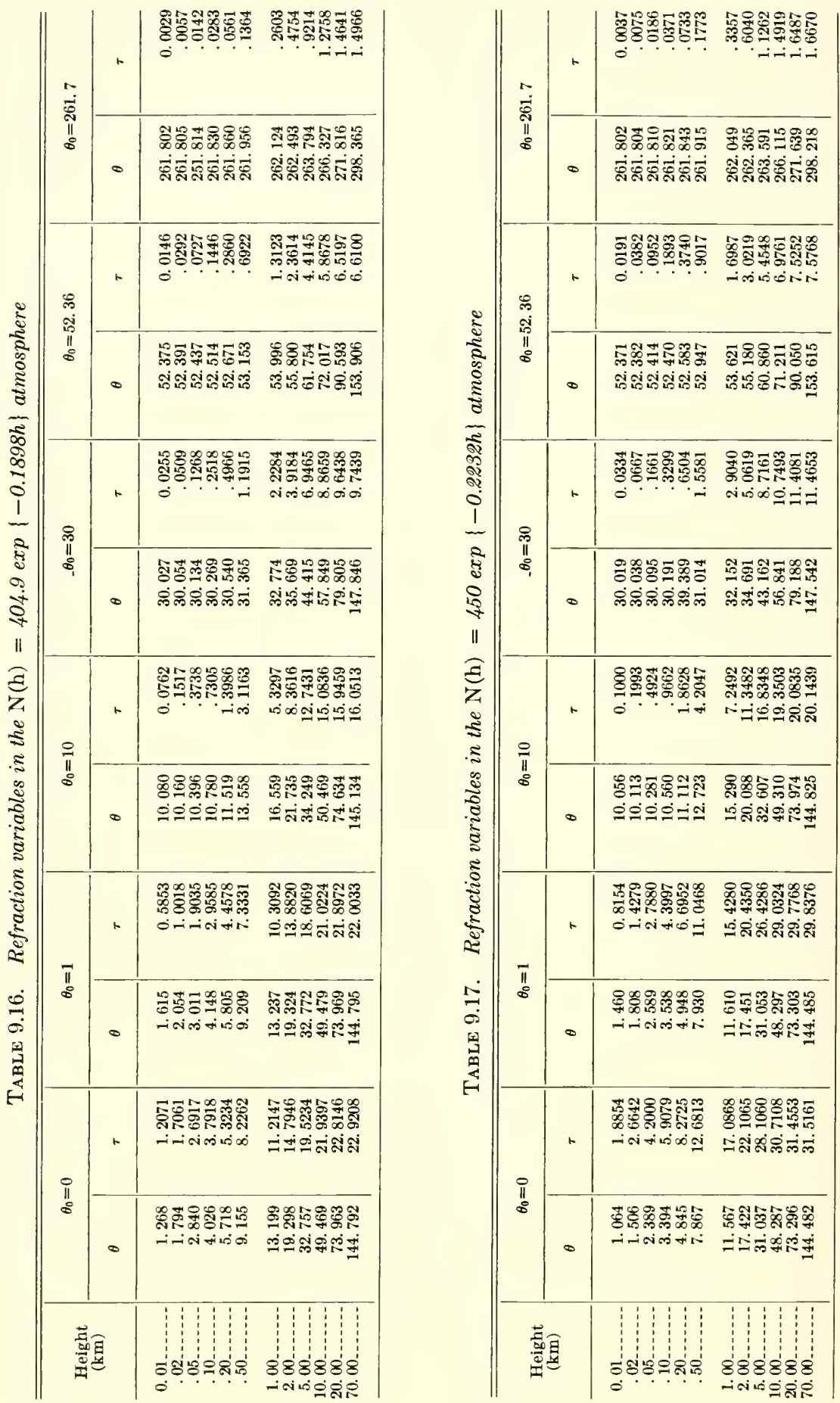
TABLE 9.18. Initial $\mathrm{N}$ gradients, $\triangle \mathrm{N}_{\epsilon}$, in the $C R P L$ Exponential Reference Atmosphere*

\begin{tabular}{|c|c|}
\hline & \\
\hline & \\
\hline $\begin{array}{l}\text { Range of } \Delta N \epsilon \\
(N \text { units } / \mathrm{km})\end{array}$ & $N(h)$ \\
\hline $\begin{array}{l}\quad \Delta N \epsilon \leq 27.55 \\
27.55<\Delta N \epsilon \leq 35.33 \\
35.33<\Delta N \epsilon \leq 47.13 \\
42.13<\Delta N \epsilon \leq 49.52 \\
49.52<\Delta N \epsilon \leq 59.68 \\
59.68<\Delta N \epsilon \leq 71.10 \\
71.10<\Delta N \epsilon \leq 88.65 \\
88.65<\Delta N \epsilon\end{array}$ & $\begin{array}{l}200 \exp (-0.1184 h) \\
252.9 \exp (-0.1262 h) \\
289 \exp (-0.1257 h) \\
313 \exp (-0.1428 h) \\
344.5 \exp (-0.1568 h) \\
377.2 \exp (-0.1732 h) \\
404.9 \exp (-0.1898 h) \\
450 \exp (-0.2232 h)\end{array}$ \\
\hline
\end{tabular}

*Note height, $h$, is in kilometers. 


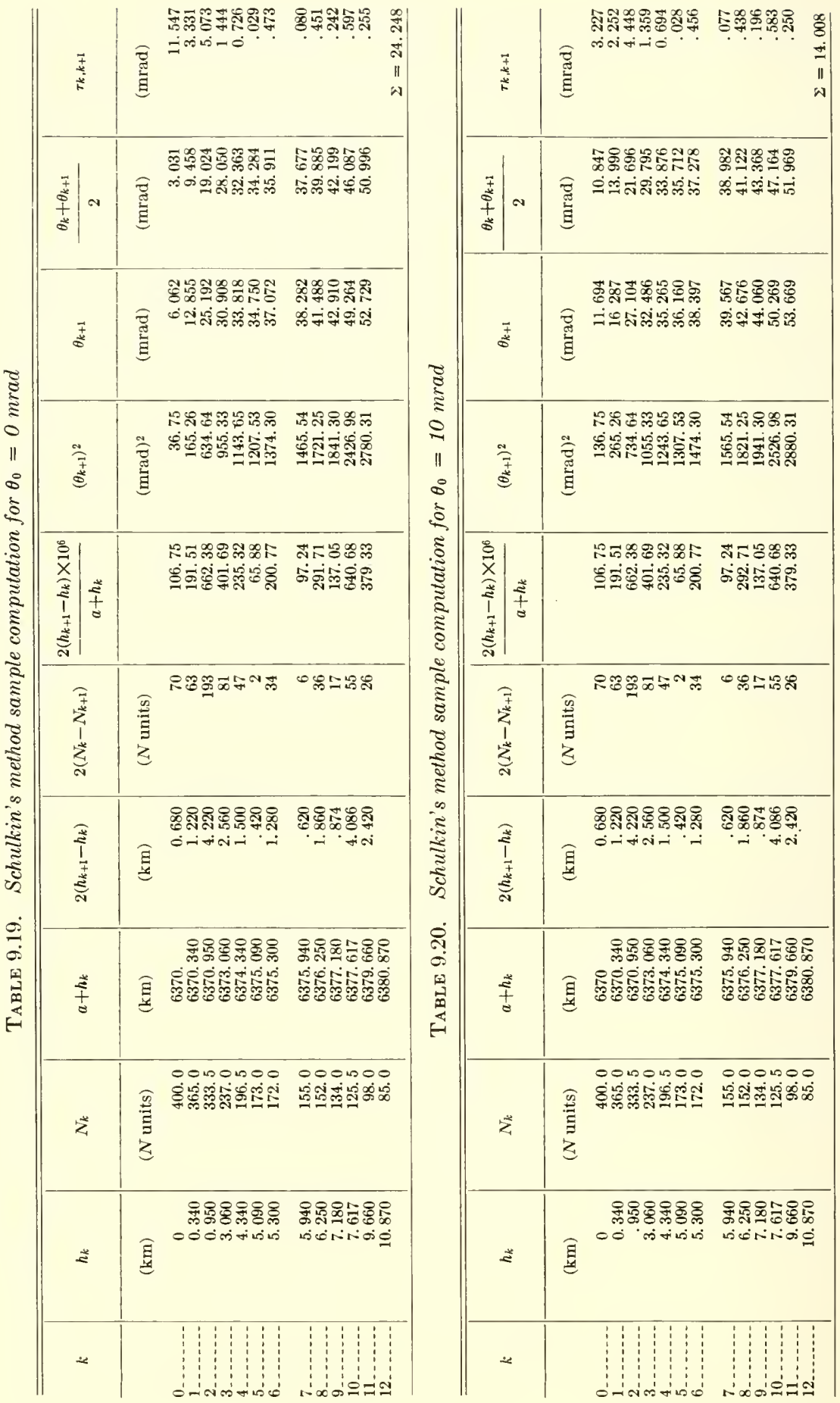




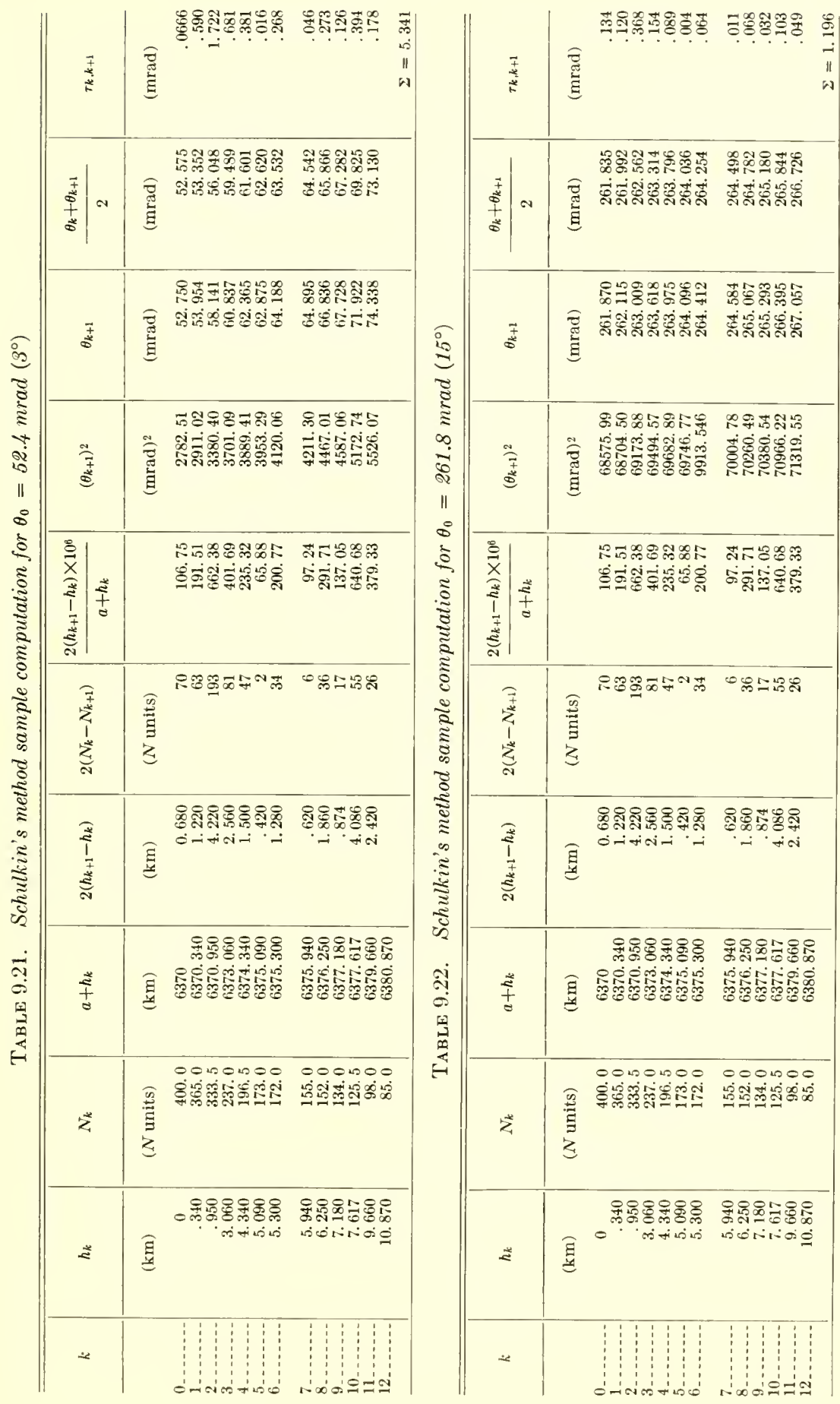


TABLE 9.23. Departures method sample computation for $\theta_{0}=10 \mathrm{mrad}$

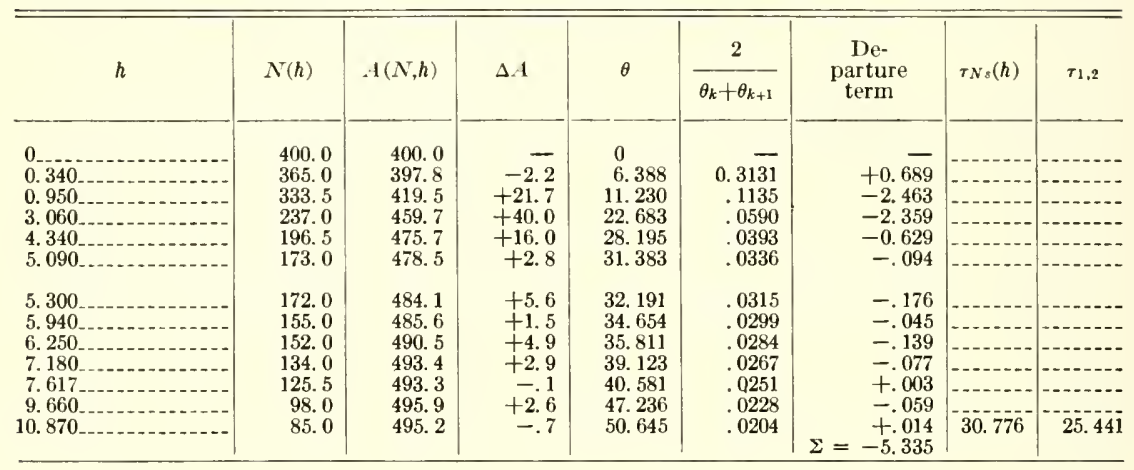

TABLE 9.24. Departures method sample computation for $\theta_{0}=10 \mathrm{mrad}$

\begin{tabular}{|c|c|c|c|c|c|c|c|c|}
\hline$h$ & $N(h)$ & $A(N, h)$ & $\Delta A$ & $\theta$ & $\frac{2}{\theta_{k}+\theta_{k+1}}$ & $\begin{array}{c}\text { De- } \\
\text { parture } \\
\text { term }\end{array}$ & $\tau_{N}(h)$ & $\tau_{1,2}$ \\
\hline 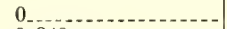 & 400.0 & 400.0 & - & 10.000 & - & - & & 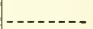 \\
\hline 0.340 & 365.0 & 397.8 & -2.2 & 11.873 & 0.0914 & +0.201 & - & \\
\hline $0.950 \ldots$ & 333.5 & 419.5 & +21.7 & 15.038 & .0743 & -1.613 & - & $\ldots$ \\
\hline 3. 060 & 237.0 & 459. 7 & +40.0 & 24.789 & .0502 & -2.008 & - & $-\ldots \ldots$ \\
\hline 4. 340 & 196.5 & 475. 7 & +16.0 & 29.947 & .0365 & -.585 & $-\cdots--$ & $---1-$ \\
\hline $5.090 \ldots$ & 173.0 & 478.5 & +2.8 & 32.938 & .0318 & -.089 & 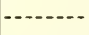 & - n..... \\
\hline $5.300 \ldots$ & 172.0 & 484.1 & +5.6 & 33.712 & .0300 & -.168 & - & - \\
\hline 5. $940 \ldots \ldots$ & 155. 0 & 485.6 & +1.5 & 36.069 & .0287 & -.043 & 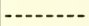 & 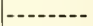 \\
\hline 6.250 & 152.0 & 490.5 & +4.9 & 37.181 & .0273 & -.134 & 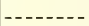 & $-\ldots-1$ \\
\hline $7.180 \ldots \ldots$ & 134.0 & 493. 4 & +2.9 & 40.383 & .0258 & -.075 & 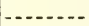 & $-\ldots \ldots$ \\
\hline 7.617 & 125.5 & 493.3 & -.1 & 41.801 & .0243 & +.002 & 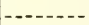 & 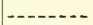 \\
\hline 9. 660 & 98.0 & 495.9 & +2.6 & 48. 282 & .0222 & -.058 & 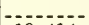 & $-\ldots-1$ \\
\hline $10.870 \ldots \ldots$ & 85.0 & 495.2 & -.7 & 51.630 & .0200 & $\Sigma=\begin{array}{r}+.014 \\
-4.556\end{array}$ & 19.414 & 14.858 \\
\hline
\end{tabular}

TABLE 9.25. Departures method sample computation for $\theta_{0}=52.4 \mathrm{mrad}\left(3^{\circ}\right)$

\begin{tabular}{|c|c|c|c|c|c|c|c|c|}
\hline$h$ & $N(h)$ & $A(N s, h)$ & $\Delta A$ & $\theta$ & $\frac{2}{\theta_{k}+\theta_{k+1}}$ & $\begin{array}{l}\text { De- } \\
\text { parture } \\
\text { term }\end{array}$ & $\tau_{N s}(h)$ & $\tau_{1,2}$ \\
\hline - & 400.0 & 400.0 & - & 52.4 & - & - & & \\
\hline 0.340 & 365.0 & 397.8 & -2.2 & 52.750 & 0.0190 & +0.042 & -.. & $\ldots$ \\
\hline 0.950 & 333.5 & 419.5 & +21.7 & 53. 550 & .0188 & -.408 & $\ldots$ & - \\
\hline 3.060 & 237.0 & 459.7 & +40.0 & 57.061 & .0181 & -.724 & - n. & $\ldots$ \\
\hline 4. 340 & 196.5 & 475.7 & +16.0 & 59.567 & .0171 & -.274 & $---n$ & $-\cdots$ \\
\hline $5.090 \ldots$ & 173.0 & 478.5 & +2.8 & 61.045 & .0166 & -.046 & -...... & $\ldots$ \\
\hline 5.300 & 172. 0 & 484.1 & +5.6 & 61.477 & .0163 & -.091 & & \\
\hline 5. 940 & 155.0 & 485.6 & +1.5 & 62.792 & .0161 & -.024 & $-\ldots$ & --- \\
\hline 6. 250 & 152.0 & 490.5 & +4.9 & 63.433 & .0158 & -.078 & $-\ldots$ & - . \\
\hline 7. 180 & 134.0 & 493. 4 & +2.9 & 65. 368 & .0155 & -.045 & - n.... & 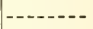 \\
\hline 7.617 & 125.5 & 493.3 & -.1 & 66.277 & .0152 & +.002 & - n. & $\ldots$ \\
\hline 9. 660 & 98. 0 & 495. 9 & +2.6 & 70.512 & .0146 & -.038 & $--\bar{y}-1$ & $-2-2-2$ \\
\hline $10.870 \ldots$ & 85. 0 & 495. 2 & -.7 & 72.920 & .0139 & $\Sigma=\begin{array}{r}+.010 \\
\Sigma=-1.674\end{array}$ & 7.024 & 5.350 \\
\hline
\end{tabular}


TABLE 9.26. Departures method sample computation for $\theta_{0}=261.8 \mathrm{mrad}\left(15^{\circ}\right)$

\begin{tabular}{|c|c|c|c|c|c|c|c|c|}
\hline$h$ & $N(h)$ & $A(N, h)$ & $\Delta A$ & $\theta$ & $\frac{2}{\theta_{k}+\theta_{k+1}}$ & $\begin{array}{c}\text { De- } \\
\text { parture } \\
\text { term }\end{array}$ & $\tau_{N} s(h)$ & $\tau_{1} .2$ \\
\hline 0 & 400.0 & 400.0 & - & 261.8 & - & - & & \\
\hline 0.340 & 365.0 & 397.8 & -2.2 & 261.880 & 0.0038 & +0.008 & & \\
\hline 0.950 & 333.5 & 419.5 & +21.7 & 262.035 & .0038 & -.083 & & \\
\hline 3. 060 & 237.0 & 459.7 & +40.0 & 262. 758 & .0038 & -.152 & & $-\cdots$ \\
\hline 4. 340 & 196.5 & 475. 7 & +16.0 & 263. 308 & .0038 & -.061 & & \\
\hline 5.090 & 173.0 & 478.5 & +2.8 & 263.633 & .0038 & -.011 & - & $\ldots \ldots$ \\
\hline 5. $300_{-}$ & 172.0 & 484.1 & +5.6 & 263. 733 & .0038 & -.021 & & \\
\hline 5. $940 \ldots$ & 155. 0 & 485. 6 & +1.5 & 264.034 & .0038 & -.006 & & - \\
\hline 6. 250 & 152.0 & 490.5 & +4.9 & 264. 184 & .0038 & -.019 & $-\ldots$ & - \\
\hline 7. 180 & 134. 0 & 493. 4 & +2.9 & 264.647 & .0038 & -.011 & $\ldots$ & - n- \\
\hline $7.617 \ldots$ & 125.5 & 493.3 & -.1 & 264.872 & .0038 & +.000 & -- & $\ldots \ldots$ \\
\hline 9.660 & 98.0 & 495. 9 & +2.6 & 265.934 & .0038 & -.010 & - & -..... \\
\hline 10.870 & 85.0 & 495.2 & -.7 & 266.592 & .0038 & $\Sigma=\begin{array}{r}+.003 \\
-0.363\end{array}$ & 1.506 & 1. 143 \\
\hline
\end{tabular}

\subsection{Tables of Refraction Variables for the Exponential Reference Atmosphere}

The following table of estimated maximum errors should serve as a guide to the accuracy of the tables.

Errors in elevation angle, $\theta$ :

$$
\left.\begin{array}{rl}
\theta_{0} & \leq 4 \mathrm{mrad} \pm 0.00005 \mathrm{mrad} \\
4 \mathrm{mrad}<\theta_{0} & <100 \mathrm{mrad} \pm 0.000005 \mathrm{mrad} \\
\theta_{0} & \leq 100 \mathrm{mrad} \pm 0.00004 \mathrm{mrad}
\end{array}\right\} \begin{aligned}
& \text { nearly } \\
& \text { independent } \\
& \text { of } N_{s} .
\end{aligned}
$$

Errors in $\tau, \epsilon$ (in milliradians):

\begin{tabular}{|c|c|c|c|c|c|}
\hline$N_{s}=450$ & 404.8 & 377.2 & 344.5 & 313 & 252.9 \\
\hline $0=0 \pm 5.0$ & 2.7 & 1.8 & 1.2 & 0.8 & 0.65 \\
\hline$\theta_{0}=1^{\circ}$ & 0.3 & 0.25 & 0.2 & 0.17 & 0.15 \\
\hline
\end{tabular}

$\begin{array}{rlllllll}N_{s}=450 & 404.8 & 377.2 & 344.5 & 313 & 252.9 & 200 \\ \theta_{0}=0 \pm 0.001 & 0.00065 & 0.0005 & 0.0004 & 0.0003 & 0.0002 & 0.0002 \\ \theta_{0}=1^{\circ} & 0.0003 & 0.00015 & 0.0001 & 0.00008 & 0.00006 & 0.00005 & 0.00005 \\ \theta_{0}=3^{\circ} & 0.00004 & 0.000025 & 0.00002 & 0.000017 & 0.000015 & 0.000013 & 0.000012\end{array}$

Errors in $R_{0}, R, R_{e}$, or $\Delta h$ (in meters):

Assume that the error in $\Delta R$ or $\Delta R_{e}$ is \pm 0.5 percent or $\pm 0.1 \mathrm{~m}$, whichever is larger. 
In tables 9.27 to 9.29 the following equations were used in determining the various profile variables:

$$
\begin{gathered}
-\Delta N=7.32 \exp \left(0.005577 N_{s}\right) \\
c_{e}=\ln \left(\frac{N_{s}}{N_{s}+\Delta N}\right) \\
-d N_{u}=c_{e} N_{s} \\
k=\frac{1}{1-\frac{r_{o}}{n_{s}}\left(c_{e} N_{s}\right) \times 10^{-6}}, \\
n_{s} \equiv 1+N_{s} \times 10^{-6}
\end{gathered}
$$

where $r_{0}$ is taken as $6373.024987 \mathrm{~km}$ for all values of $N_{s}$ in these tables.

\begin{tabular}{|c|c|c|c|c|}
\hline$N$. & $-\Delta N$ & $c_{e}$ & $-d N_{0}$ & $k$ \\
\hline $\begin{array}{l}200 \\
210 \ldots \\
220 \\
230 \\
240\end{array}$ & $\begin{array}{l}22.3317700 \\
23.6125966 \\
24.9668845 \\
26.3988468 \\
27.9129385\end{array}$ & $\begin{array}{r}0.118399435 \\
.119280212 \\
.120458179 \\
.121916361 \\
.123642065\end{array}$ & $\begin{array}{l}23.6798870 \\
25.0488444 \\
26.5007993 \\
28.0407631 \\
29.6740955\end{array}$ & $\begin{array}{l}\text { 1. } 17769275 \\
\text { 1. } 18991401 \\
\text { 1. } 20315637 \\
\text { 1. } 21752719 \\
\text { 1. } 23314913\end{array}$ \\
\hline $\begin{array}{l}250 \ldots \\
2600 \\
270 \\
280 \\
290\end{array}$ & $\begin{array}{l}29.5138701 \\
31.2066224 \\
32.9964614 \\
34.8889558 \\
36.8899932\end{array}$ & $\begin{array}{l}.125626129 \\
.127862319 \\
.130346887 \\
.133078254 \\
136056720\end{array}$ & $\begin{array}{l}31.4065323 \\
33.2442030 \\
35.1936594 \\
37.2619112 \\
39.4564487\end{array}$ & $\begin{array}{l}\text { 1. } 25016295 \\
\text { 1. } 26873080 \\
\text { 1. } 28904048 \\
\text { 1. } 31131073 \\
\text { 1. } 33579768\end{array}$ \\
\hline $\begin{array}{l}300 \\
310 \\
320 \\
330 \\
340\end{array}$ & $\begin{array}{l}\text { 39. } 0057990 \\
41.2429556 \\
43.6084233 \\
46.1095611 \\
48.7541501\end{array}$ & $\begin{array}{l}.139284287 \\
.142764507 \\
.146502381 \\
.150504269 \\
.154777865\end{array}$ & $\begin{array}{l}\text { 41. } 7852861 \\
44.2569972 \\
46.8807620 \\
49.6664087 \\
52.6244741\end{array}$ & $\begin{array}{l}\text { 1. } 36280330 \\
\text { 1. } 39268608 \\
\text { 1. } 42587494 \\
\text { 1. } 46288731 \\
\text { 1. } 50435338\end{array}$ \\
\hline $\begin{array}{l}350 \\
360 \\
370 \\
380 \\
390\end{array}$ & $\begin{array}{l}51.5504184 \\
54.5070651 \\
57.6332884 \\
60.9388149 \\
64.4339281\end{array}$ & $\begin{array}{l}.159332141 \\
.164177379 \\
.169325150 \\
.174788368 \\
.180581312\end{array}$ & $\begin{array}{l}55.7662495 \\
59.1038565 \\
62.6503054 \\
66.4195799 \\
70.4267116\end{array}$ & $\begin{array}{l}\text { 1. } 55104840 \\
\text { 1. } 60393724 \\
\text { 1. } 66423593 \\
1.73349938 \\
1.81374807\end{array}$ \\
\hline $\begin{array}{l}400 \ldots \\
410 \\
420 \\
430 \\
440\end{array}$ & $\begin{array}{l}68.1295015 \\
72.0370324 \\
76.1686780 \\
80.5372922 \\
85.1564647 \\
90.0405683\end{array}$ & $\begin{array}{l}.186719722 \\
.193220834 \\
.200103517 \\
.207388355 \\
.215097782 \\
.223256247\end{array}$ & $\begin{array}{r}74.6878887 \\
79.2205420 \\
84.0434770 \\
89.1769927 \\
94.6430240 \\
100.4653113\end{array}$ & $\begin{array}{l}1.90765687 \\
2.01884302 \\
2.15232187 \\
2.31525447 \\
2.51823286 \\
2.77761532\end{array}$ \\
\hline
\end{tabular}

Table 9.29 was prepared using an iterative method for solution of transcendental equations involved. In all these tables the accuracy may be taken as \pm 1 in the last digit listed.

TABLE 9.27. Parameters for exponential refraction with height for various values of $\mathrm{N}_{\mathrm{s}}$ 
TABLE 9.28. Parameters for exponential refraction with height for various values of $-\Delta N$

\begin{tabular}{|c|c|c|c|c|}
\hline$-\Delta N$ & $N_{s}$ & $c_{e}$ & $-d N_{0}$ & $k$ \\
\hline $\begin{array}{l}20 \ldots \\
22 \ldots \\
24 \ldots \\
26 \ldots \\
28 \ldots\end{array}$ & $\begin{array}{l}180.226277 \\
197.316142 \\
212.917967 \\
227.270255 \\
240.558398\end{array}$ & $\begin{array}{r}0.117626108 \\
.118216356 \\
.119594076 \\
.121491305 \\
.123746115\end{array}$ & $\begin{array}{l}21.1993155 \\
23.3259953 \\
25.4637276 \\
27.6113599 \\
29.7681671\end{array}$ & $\begin{array}{l}\text { 1. } 15617524 \\
\text { 1. } 17457412 \\
\text { 1. } 19366808 \\
\text { 1. } 21348565 \\
\text { 1. } 23406110\end{array}$ \\
\hline $\begin{array}{l}30 \ldots \\
32 \ldots \\
34 \ldots \\
36 \ldots \\
38 \\
\ldots\end{array}$ & $\begin{array}{l}252.929362 \\
264.501627 \\
275.372099 \\
285.621054 \\
295.315731\end{array}$ & $\begin{array}{l}.126255291 \\
.128950180 \\
.131783550 \\
.134721962 \\
.137741207\end{array}$ & $\begin{array}{l}31.9336703 \\
34.1075325 \\
36.2895127 \\
38.4794288 \\
40.6771452\end{array}$ & $\begin{array}{l}\text { 1. } 25543336 \\
\text { 1. } 27764560 \\
\text { 1. } 30074523 \\
\text { 1. } 32478398 \\
\text { 1. } 34981825\end{array}$ \\
\hline $\begin{array}{l}40 \ldots \ldots \\
42 \ldots \ldots \\
44 \ldots \\
46 \\
48 \ldots\end{array}$ & $\begin{array}{l}304.513148 \\
313.261483 \\
321.602888 \\
329.573439 \\
337.204713\end{array}$ & $\begin{array}{l}.140823306 \\
.143955014 \\
.147125889 \\
.150328075 \\
.153555418\end{array}$ & $\begin{array}{l}\text { 42. } 8825481 \\
\text { 45. } 0955611 \\
\text { 47. } 3161107 \\
\text { 49. } 5441405 \\
51.7796106\end{array}$ & $\begin{array}{l}\text { 1. } 37590934 \\
1.40312414 \\
1.43153539 \\
1.46122250 \\
1.49227226\end{array}$ \\
\hline $\begin{array}{l}50 \\
52 \\
54 \\
56 \\
58\end{array}$ & $\begin{array}{l}344.524418 \\
351.557000 \\
358.324138 \\
364.845143 \\
371.137293\end{array}$ & $\begin{array}{l}.156803056 \\
.160067149 \\
.163344614 \\
.166633002 \\
.169930326\end{array}$ & $\begin{array}{l}54.0224815 \\
56.2727266 \\
58.5303179 \\
60.7952415 \\
63.0674811\end{array}$ & $\begin{array}{l}1.52477960 \\
1.55884863 \\
1.59459364 \\
1.63214058 \\
1.67162830\end{array}$ \\
\hline $\begin{array}{l}60 \\
62 \\
64 \\
66 \\
68\end{array}$ & $\begin{array}{l}\text { 377. } 216108 \\
383.095581 \\
388.788373 \\
394.305974 \\
399.658845\end{array}$ & $\begin{array}{l}.173234984 \\
.176545680 \\
.179861358 \\
.183181171 \\
.186504431\end{array}$ & $\begin{array}{l}\text { 65. } 3470266 \\
67.6338699 \\
69.9280046 \\
72.2294300 \\
74.5381454\end{array}$ & $\begin{array}{l}1.71321044 \\
1.75705732 \\
1.80335830 \\
1.85232456 \\
1.90419225\end{array}$ \\
\hline $\begin{array}{l}70 \\
72 \\
74 \\
76 \\
78\end{array}$ & $\begin{array}{l}404.856538 \\
409.907798 \\
414.820650 \\
419.602477 \\
424.260086\end{array}$ & $\begin{array}{l}.189830583 \\
.193159183 \\
.196489873 \\
.199822385 \\
.203156494\end{array}$ & $\begin{array}{l}76.8541525 \\
79.1774555 \\
81.5080567 \\
83.8459677 \\
86.1911914\end{array}$ & $\begin{array}{l}\text { 1. } 95922635 \\
2.01772514 \\
2.08002556 \\
2.14650999 \\
2.21761358\end{array}$ \\
\hline $\begin{array}{l}80 \\
82 \\
84 \\
86 \\
88\end{array}$ & $\begin{array}{l}428.799768 \\
433.227348 \\
437.548229 \\
441.767432 \\
445.889634\end{array}$ & $\begin{array}{l}.206492043 \\
.209828917 \\
.213167031 \\
.216506335 \\
.219846812\end{array}$ & $\begin{array}{l}88.5437400 \\
90.9036251 \\
93.2708570 \\
95.6454475 \\
98.0274147\end{array}$ & $\begin{array}{l}\text { 2. } 29383429 \\
2.37574437 \\
2.46400458 \\
2.55938222 \\
2.66277367\end{array}$ \\
\hline $\begin{array}{l}90 \\
92 \\
94 \\
96 \\
98 \\
100\end{array}$ & $\begin{array}{l}449.919193 \\
453.860184 \\
457.716416 \\
461.491458 \\
465.188659 \\
468.811163\end{array}$ & $\begin{array}{l}.223188453 \\
.226531281 \\
.229875327 \\
.233220637 \\
.236567271 \\
.239915290\end{array}$ & $\begin{array}{l}100.4167688 \\
102.8135290 \\
105.2177108 \\
107.6293319 \\
110.0484114 \\
112.4749663\end{array}$ & $\begin{array}{l}\text { 2. } 77523207 \\
2.89800399 \\
3.03257531 \\
\text { 3. } 18073184 \\
\text { 3. } 34463902 \\
\text { 3. } 52694820\end{array}$ \\
\hline
\end{tabular}


TABLE 9.29. Parameters for exponential refraction with height for various values of $\mathrm{k}$

\begin{tabular}{|c|c|c|c|c|}
\hline$k$ & $N s$ & $-\Delta N$ & $c_{c}$ & $-d N_{0}$ \\
\hline $1.0_{\ldots}$ & 0.0 & 0.0 & 0.0 & 0.0 \\
\hline 1.2 & 217.689023 & 24. 6471681 & .120160519 & 26. 1576259 \\
\hline 1. 3 . & 275.037959 & 33. 9367000 & .131692114 & 36. 2203304 \\
\hline 1. 4. & 312.297111 & 41. 7747176 & .143600133 & 44.8459068 \\
\hline 1.5 & 339.003316 & 48. 4839018 & . 154339490 & 52. 3215987 \\
\hline 1.6 & 359. 298283 & 54.2941700 & .163827653 & 58.8629945 \\
\hline 1.7. & 375.341242 & 59. 3759008 & .172203063 & 64.6349115 \\
\hline 1.8 & 388. 391792 & 63.8586055 & . 179626805 & 69. 7655765 \\
\hline 1.9 & 399. 243407 & 67.8426334 & .186242834 & 74. 3562234 \\
\hline $2.0 \ldots$ & $\begin{array}{l}\text { 408. } 424907 \\
416.304322\end{array}$ & $\begin{array}{l}71.4070090 \\
74.6148487\end{array}$ & $\begin{array}{r}.192172034 \\
197514185\end{array}$ & 78. 4878451 \\
\hline & & & & 82.2200091 \\
\hline 2. $2 \ldots$ & 423. 146728 & 77.5171828 & . 202351472 & 85. 6243634 \\
\hline 2. $3 \ldots$ & $\begin{array}{r}429.148472 \\
\end{array}$ & 80. 1557288 & .206751820 & 88. 7272277 \\
\hline $\begin{array}{l}2.4- \\
2.5\end{array}$ & $\begin{array}{l}434.458411 \\
439.191718\end{array}$ & $\begin{array}{l}82.5649192 \\
84.7734613\end{array}$ & $\begin{array}{r}.210771674 \\
214458304\end{array}$ & $\begin{array}{l}91.5715264 \\
94.1883108\end{array}$ \\
\hline $2.6 \ldots$ & 443. 438906 & 86.8054237 & $\begin{array}{r}.214458304 \\
.217851443\end{array}$ & 96.6038056 \\
\hline 2. 7 - & 447. 272272 & 88. 6811886 & .220984823 & 98. 8403838 \\
\hline 2.8 & 450.750273 & 90.4181120 & .223887193 & 100.9172131 \\
\hline$\infty-$ & 523. 299600 & 135.5109159 & . 299693586 & 156. 829534 \\
\hline $4 / 3 \ldots$ & 289. 036274 & 36. 6922523 & .135758874 & 39. 2392391 \\
\hline
\end{tabular}

\subsection{Refractivity Tables}

Tables 9.30 through 9.35 have been prepared on a digital computer to assist the reader in making an easy determination of the refractivity, $N$, value from pressure, temperature, and relative humidity data. Standard pressure values of 700,850 , and 1000 mbar have been used for the tables. The tables are at every 10 percent relative humidity and for every degree centigrade ranging for -45 to $+45{ }^{\circ} \mathrm{C}$.

Interpolation for the $N$ tables is accomplished in the following manner. First, round the temperature to the nearest degree, then interpolate linearly for the values of pressure, $P$, and relative humidity, $\mathrm{RH}$, used. No distortion is introduced by this method as the wet term, $W$ (as described in chapter 4), is a linear function of $\mathrm{RH}$, and the dry term, $D$, is a linear function of $P$.

\section{Example:}

$$
\text { Given } \begin{gathered}
P=980 \text { mbar } \\
\mathrm{RH}=47 \text { percent } \\
T=22^{\circ} \mathrm{C}
\end{gathered}
$$

and knowing

$$
\begin{aligned}
D & =D(P, T) \\
N & =N(P, T, \mathrm{RH}) \\
W & =W(T, \mathrm{RH}),
\end{aligned}
$$

then

$$
\begin{aligned}
& D(850,22)=N(850,22,0)=223.6 N \text { units. } \\
& D(980,22)=N(980,22,0)=223.6(980 / 850)=257.8 N \text { units. }
\end{aligned}
$$


Now interpolating for humidity,

$$
N(850,22,50)=280.3 N \text { units. }
$$

Since

$$
N(850,22,50)-D(850,22)=W(22,50),
$$

thus

$$
\begin{aligned}
& W(22,50)=280.3-223.6=56.7 \mathrm{~N} \text { units, and } \\
& W(22,47)=56.7(47 / 50)=53.3 \mathrm{~N} \text { units. }
\end{aligned}
$$

Adding

$$
\begin{aligned}
& W(22,47) \text { to } D(980,22) \text { yields } \\
& \begin{aligned}
N(980,22,47) & =D(980,22)+W(22,47) \\
& =257.8+53.3=311.1 N \text { units. }
\end{aligned}
\end{aligned}
$$

Substitution directly into (1.20) and use of the Smithsonian Meteorological Tables [2] for vapor pressure yields the identical result to four significant figures.

$$
N(980,22,47)=311.1 N \text { units. }
$$

Ambient recording thermometers generally read to a tenth of a degree centigrade. Rounding the temperature to the nearest whole degree introduces an extraneous error into the interpolation process. At $45{ }^{\circ} \mathrm{C}$, $1000 \mathrm{mbar}$, and at a relative humidity of 100 percent, this error will have a maximum of $7.7 \mathrm{~N}$ units. However, these conditions are an extreme rarity climatologically speaking, and at normal temperature ranges an error of more than $2 \mathrm{~N}$ units is unlikely. 
TABLE 9.30. Refractivity for a pressure of 700 mbar

\begin{tabular}{|c|c|c|c|c|c|c|c|c|c|c|c|}
\hline \multirow{2}{*}{$\begin{array}{l}\text { Temper- } \\
\text { ature }\end{array}$} & \multicolumn{11}{|c|}{ Relative humidity } \\
\hline & 0 & 10 & 20 & 30 & 40 & 50 & 60 & 70 & 80 & 90 & 100 \\
\hline $\begin{array}{l}{ }^{\circ} \mathrm{C} \\
-45 \\
-44 \\
-43 \\
-42 \\
-41\end{array}$ & $\begin{array}{l}238.2 \\
237.2 \\
236.2 \\
235.2 \\
234.1\end{array}$ & $\begin{array}{l}238.3 \\
237.3 \\
236.3 \\
235.3 \\
234.3\end{array}$ & $\begin{array}{l}238.4 \\
237.4 \\
236.4 \\
235.4 \\
234.4\end{array}$ & $\begin{array}{l}238.5 \\
237.5 \\
236.5 \\
235.5 \\
234.5\end{array}$ & $\begin{array}{l}238.6 \\
237.6 \\
236.6 \\
235.6 \\
234.6\end{array}$ & $\begin{array}{l}238.6 \\
237.6 \\
236.7 \\
235.7 \\
234.7\end{array}$ & $\begin{array}{l}238.7 \\
237.7 \\
236.8 \\
235.8 \\
234.8\end{array}$ & $\begin{array}{l}238.8 \\
237.8 \\
236.9 \\
235.9 \\
235.0\end{array}$ & $\begin{array}{l}238.9 \\
2379 \\
237.0 \\
236.0 \\
235.1\end{array}$ & $\begin{array}{l}239.0 \\
238.0 \\
237.1 \\
236.1 \\
235.2\end{array}$ & $\begin{array}{l}239.0 \\
238.1 \\
237.1 \\
236.2 \\
235.3\end{array}$ \\
\hline $\begin{array}{l}-40 \\
-39 \\
-38 \\
-37 \\
-36\end{array}$ & $\begin{array}{l}233.1 \\
232.1 \\
231.1 \\
230.2 \\
229.2\end{array}$ & $\begin{array}{l}233.3 \\
232.3 \\
231.3 \\
230.3 \\
229.4\end{array}$ & $\begin{array}{l}233.4 \\
232.4 \\
231.5 \\
230.5 \\
229.6\end{array}$ & $\begin{array}{l}233.5 \\
232.6 \\
231.6 \\
230.7 \\
229.8\end{array}$ & $\begin{array}{l}233.7 \\
232.7 \\
231.8 \\
230.9 \\
230.0\end{array}$ & $\begin{array}{l}233.8 \\
232.9 \\
231.9 \\
231.0 \\
230.1\end{array}$ & $\begin{array}{l}233.9 \\
233.0 \\
232.1 \\
231.2 \\
230.3\end{array}$ & $\begin{array}{l}234.0 \\
233.1 \\
232.2 \\
231.4 \\
230.5\end{array}$ & $\begin{array}{l}234.2 \\
233.3 \\
232.4 \\
231.5 \\
230.7\end{array}$ & $\begin{array}{l}234.3 \\
233.4 \\
232.6 \\
231.7 \\
230.9\end{array}$ & $\begin{array}{l}234.4 \\
233.4 \\
232.7 \\
231.9 \\
231.1\end{array}$ \\
\hline $\begin{array}{l}-35 \\
-34 \\
-33 \\
-32 \\
-31\end{array}$ & $\begin{array}{l}228.2 \\
227.3 \\
226.3 \\
225.4 \\
224.5\end{array}$ & $\begin{array}{l}228.4 \\
227.5 \\
226.6 \\
225.7 \\
224.8\end{array}$ & $\begin{array}{l}228.6 \\
227.7 \\
226.8 \\
225.9 \\
225.1\end{array}$ & $\begin{array}{l}228.9 \\
228.0 \\
227.1 \\
226.2 \\
225.3\end{array}$ & $\begin{array}{l}229.1 \\
228.2 \\
227.3 \\
226.5 \\
225.6\end{array}$ & $\begin{array}{l}229.3 \\
228.4 \\
227.6 \\
226.7 \\
225.9\end{array}$ & $\begin{array}{l}229.5 \\
228.6 \\
227.8 \\
227.0 \\
226.2\end{array}$ & $\begin{array}{l}229.7 \\
228.9 \\
228.1 \\
227.3 \\
226.5\end{array}$ & $\begin{array}{l}229.9 \\
229.1 \\
228.3 \\
227.6 \\
226.8\end{array}$ & $\begin{array}{l}230.1 \\
229.3 \\
228.6 \\
227.8 \\
227.1\end{array}$ & $\begin{array}{l}230.3 \\
2295 \\
228.8 \\
228.1 \\
227.4\end{array}$ \\
\hline $\begin{array}{l}-30 \\
-29 \\
-28 \\
-27 \\
-26\end{array}$ & $\begin{array}{l}223.5 \\
222.6 \\
221.7 \\
220.8 \\
219.9\end{array}$ & $\begin{array}{l}223.9 \\
223.0 \\
222.1 \\
221.9 \\
220.4\end{array}$ & $\begin{array}{l}224.2 \\
223.3 \\
222.5 \\
221.6 \\
220.8\end{array}$ & $\begin{array}{l}224.5 \\
223.7 \\
222.9 \\
222.1 \\
221.3\end{array}$ & $\begin{array}{l}224.8 \\
224.0 \\
223.2 \\
222.5 \\
221.7\end{array}$ & $\begin{array}{l}225.1 \\
224.4 \\
223.6 \\
222.9 \\
222.2\end{array}$ & $\begin{array}{l}225.5 \\
224.7 \\
224.0 \\
223.3 \\
222.6\end{array}$ & $\begin{array}{l}225.8 \\
225.1 \\
224.4 \\
223.7 \\
223.1\end{array}$ & $\begin{array}{l}226.1 \\
225.4 \\
224.8 \\
224.1 \\
223.5\end{array}$ & $\begin{array}{l}226.4 \\
225.8 \\
225.1 \\
224.5 \\
224.0\end{array}$ & $\begin{array}{l}226.8 \\
226.1 \\
225.5 \\
225.0 \\
224.4\end{array}$ \\
\hline $\begin{array}{l}-25 \\
-24 \\
-23 \\
-22 \\
-21\end{array}$ & $\begin{array}{l}219.0 \\
218.2 \\
217.3 \\
216.4 \\
215.6\end{array}$ & $\begin{array}{l}219.5 \\
218.7 \\
217.9 \\
217.0 \\
216.2\end{array}$ & $\begin{array}{l}220.0 \\
219.2 \\
218.4 \\
217.7 \\
216.9\end{array}$ & $\begin{array}{l}220.5 \\
219.7 \\
219.0 \\
218.3 \\
217.6\end{array}$ & $\begin{array}{l}221.0 \\
220.3 \\
219.6 \\
218.9 \\
218.3\end{array}$ & $\begin{array}{l}221.5 \\
220.8 \\
220.2 \\
219.5 \\
218.9\end{array}$ & $\begin{array}{l}222.0 \\
221.3 \\
220.7 \\
220.2 \\
219.6\end{array}$ & $\begin{array}{l}222.5 \\
221.9 \\
221.3 \\
220.8 \\
220.3\end{array}$ & $\begin{array}{l}222.9 \\
222.4 \\
221.9 \\
221.4 \\
221.0\end{array}$ & $\begin{array}{l}223.4 \\
222.9 \\
222.5 \\
222.0 \\
221.6\end{array}$ & $\begin{array}{l}223.9 \\
223.5 \\
223.0 \\
222.7 \\
222.3\end{array}$ \\
\hline $\begin{array}{l}-20 \\
-19 \\
-18 \\
-17 \\
-16\end{array}$ & $\begin{array}{l}214.7 \\
213.9 \\
213.0 \\
212.2 \\
211.4\end{array}$ & $\begin{array}{l}215.4 \\
214.6 \\
213.9 \\
213.1 \\
212.4\end{array}$ & $\begin{array}{l}216.2 \\
215.4 \\
214.7 \\
214.0 \\
213.4\end{array}$ & $\begin{array}{l}216.9 \\
216.2 \\
215.6 \\
215.0 \\
214.3\end{array}$ & $\begin{array}{l}217.6 \\
217.0 \\
216.4 \\
215.9 \\
215.3\end{array}$ & $\begin{array}{l}218.4 \\
217.8 \\
217.3 \\
216.8 \\
216.3\end{array}$ & $\begin{array}{l}219.1 \\
218.6 \\
218.1 \\
217.7 \\
217.3\end{array}$ & $\begin{array}{l}219.8 \\
219.4 \\
219.0 \\
218.6 \\
218.3\end{array}$ & $\begin{array}{l}220.6 \\
220.2 \\
219.9 \\
219.6 \\
219.3\end{array}$ & $\begin{array}{l}221.3 \\
221.0 \\
220.7 \\
220.5 \\
220.3\end{array}$ & $\begin{array}{l}222.0 \\
221.8 \\
221.6 \\
221.4 \\
221.3\end{array}$ \\
\hline $\begin{array}{l}-15 \\
-14 \\
-13 \\
-12 \\
-11\end{array}$ & $\begin{array}{l}210.5 \\
209.7 \\
208.9 \\
208.1 \\
207.3\end{array}$ & $\begin{array}{l}211.6 \\
210.9 \\
210.2 \\
209.5 \\
208.8\end{array}$ & $\begin{array}{l}212.7 \\
212.0 \\
211.4 \\
210.8 \\
210.2\end{array}$ & $\begin{array}{l}213.8 \\
213.2 \\
212.7 \\
212.1 \\
211.6\end{array}$ & $\begin{array}{l}214.8 \\
214.3 \\
213.9 \\
213.5 \\
213.1\end{array}$ & $\begin{array}{l}215.9 \\
215.5 \\
215.1 \\
214.8 \\
214.5\end{array}$ & $\begin{array}{l}217.0 \\
216.7 \\
216.4 \\
216.1 \\
216.0\end{array}$ & $\begin{array}{l}218.0 \\
217.8 \\
217.6 \\
217.5 \\
217.4\end{array}$ & $\begin{array}{l}219.1 \\
219.0 \\
218.9 \\
218.8 \\
218.8\end{array}$ & $\begin{array}{l}220.2 \\
220.1 \\
220.1 \\
220.2 \\
220.3\end{array}$ & $\begin{array}{l}221.3 \\
221.3 \\
221.4 \\
221.5 \\
221.7\end{array}$ \\
\hline $\begin{array}{r}-10 \\
-9 \\
-8 \\
-7 \\
-6\end{array}$ & $\begin{array}{l}206.5 \\
205.8 \\
205.0 \\
204.2 \\
203.4\end{array}$ & $\begin{array}{l}208.1 \\
207.4 \\
206.8 \\
206.1 \\
205.5\end{array}$ & $\begin{array}{l}209.6 \\
209.1 \\
208.5 \\
208.0 \\
207.5\end{array}$ & $\begin{array}{r}211.2 \\
210.7 \\
210.3 \\
29.9 \\
29.6\end{array}$ & $\begin{array}{l}212.7 \\
212.4 \\
212.1 \\
211.8 \\
211.6\end{array}$ & $\begin{array}{l}214.3 \\
214.1 \\
213.9 \\
213.8 \\
213.7\end{array}$ & $\begin{array}{l}215.8 \\
215.7 \\
215.7 \\
215.7 \\
215.7\end{array}$ & $\begin{array}{l}217.4 \\
217.4 \\
217.4 \\
217.6 \\
217.8\end{array}$ & $\begin{array}{l}218.9 \\
219.0 \\
219.2 \\
219.5 \\
219.8\end{array}$ & $\begin{array}{l}220.4 \\
220.7 \\
221.0 \\
221.4 \\
221.9\end{array}$ & $\begin{array}{l}222.0 \\
222.3 \\
222.8 \\
223.3 \\
223.9\end{array}$ \\
\hline $\begin{array}{r}-5 \\
-4 \\
-3 \\
-2 \\
-1 \\
0\end{array}$ & $\begin{array}{l}202.7 \\
201.9 \\
201.2 \\
200.4 \\
199.7 \\
199.0\end{array}$ & $\begin{array}{l}204.9 \\
204.3 \\
203.7 \\
203.1 \\
202.6 \\
202.0\end{array}$ & $\begin{array}{l}207.1 \\
206.6 \\
206.2 \\
205.8 \\
205.4 \\
205.1\end{array}$ & $\begin{array}{l}209.3 \\
209.0 \\
208.7 \\
208.5 \\
208.3 \\
208.2\end{array}$ & $\begin{array}{l}211.4 \\
211.3 \\
211.2 \\
211.2 \\
211.2 \\
211.2\end{array}$ & $\begin{array}{l}213.6 \\
213.7 \\
213.7 \\
213.8 \\
214.0 \\
214.3\end{array}$ & $\begin{array}{l}215.8 \\
216.0 \\
216.2 \\
216.5 \\
216.9 \\
217.3\end{array}$ & $\begin{array}{l}218.0 \\
218.3 \\
218.7 \\
219.2 \\
219.8 \\
220.4\end{array}$ & $\begin{array}{l}220.2 \\
220.7 \\
221.2 \\
221.9 \\
222.6 \\
223.4\end{array}$ & $\begin{array}{l}222.4 \\
223.0 \\
223.8 \\
224.6 \\
225.5 \\
226.5\end{array}$ & $\begin{array}{l}224.6 \\
225.4 \\
226.3 \\
227.3 \\
228.4 \\
229.6\end{array}$ \\
\hline
\end{tabular}


TABLE 9.31. Refractivity for a pressure of 700 mbar.

\begin{tabular}{|c|c|c|c|c|c|c|c|c|c|c|c|}
\hline \multirow{2}{*}{$\begin{array}{l}\text { Temper- } \\
\text { ature }\end{array}$} & \multicolumn{11}{|c|}{ Relative humidity } \\
\hline & 0 & 10 & 20 & 30 & 40 & 50 & 60 & 70 & 80 & 90 & 100 \\
\hline${ }^{\circ} \mathrm{C}$ & & & & & & & & & & & \\
\hline 0 & 199.0 & 202. 0 & 205.1 & 208. 2 & 211. 2 & 214. 3 & 217.3 & 220.4 & 223. 4 & 226.5 & 229.0 \\
\hline 1 & 198.2 & 201.5 & 204.8 & 208.0 & 211.3 & 214. 6 & 217.8 & 221.1 & 224. 4 & 227.6 & 230.9 \\
\hline 2 & 197.5 & 201.0 & 204.5 & 208.0 & 211.5 & 214.9 & 218.4 & 221.9 & 225.4 & 228.9 & 232,3 \\
\hline 3 & 196.8 & 200.5 & 204.2 & 207.9 & 211.7 & 215.4 & 219.1 & 222.8 & 226.5 & 230.2 & 233.9 \\
\hline 4 & 196.1 & 200.1 & 204.0 & 208.0 & 211.9 & 215.9 & 219.8 & 223.8 & 227.7 & 231.7 & 235.6 \\
\hline 5 & 195.4 & 199.6 & 203.8 & 208.0 & 212. 2 & 216.5 & 220.7 & 224.9 & 229.1 & 233.3 & 237.5 \\
\hline 6 & 194.7 & 199. 2 & 203.7 & 208.1 & 212.6 & 217.1 & 221.6 & 226.1 & 230.5 & 235.0 & 239.5 \\
\hline 7 & 194.0 & 198.8 & 203.5 & 208. 3 & 213. 1 & 217.8 & 222.6 & 227.4 & 232.1 & 236.9 & 241.7 \\
\hline 8 & 193. 3 & 198.4 & 203.4 & 208.5 & 213.6 & 218.7 & 223.7 & 228.8 & 233.9 & 238.9 & 244.0 \\
\hline 9 & 192.6 & 198.0 & 203.4 & 208.8 & 214. 2 & 219.6 & 224.9 & 230.3 & 235.7 & 241.1 & 246.5 \\
\hline 10 & 191. 9 & 197.7 & 203.4 & 209.1 & 214.8 & 220.5 & 226.3 & 232.0 & 237.7 & 243.4 & 249.1 \\
\hline 11 & 191.3 & 197.3 & 203.4 & 209.5 & 215.6 & 221.6 & 227.7 & 233.8 & 239.8 & 245.9 & 252.0 \\
\hline 12 & 190.6 & 197.0 & 203.5 & 209.9 & 216.4 & 222.8 & 229.2 & 235.7 & 242.1 & 248.6 & 255.0 \\
\hline 13 & 189.9 & 196.8 & 203.6 & 210.4 & 217.3 & 224.1 & 230.9 & 237.7 & 244.6 & 251.4 & 258.2 \\
\hline 14 & 189.3 & 196.5 & 203.7 & 211.0 & 218.2 & 225.5 & 232.7 & 239.9 & 247.2 & 254.4 & 261.7 \\
\hline 15 & 188.6 & 196.3 & 204.0 & 211.6 & 219.3 & 227.0 & 234.6 & 242.3 & 250.0 & 257.6 & 265.3 \\
\hline 16 & 188.0 & 196.1 & 204.2 & 212.3 & 220.4 & 228.6 & 236.7 & 244.8 & 252.9 & 261.1 & 269.2 \\
\hline 17 & 187.3 & 195.9 & 204.5 & 213.1 & 221.7 & 230.3 & 238.9 & 247.5 & 256.1 & 264.7 & 273.3 \\
\hline 18 & 186.7 & 195.8 & 204. 9 & 213. 9 & 223.0 & 232.1 & 241.2 & 250.3 & 259.4 & 268.5 & 277.6 \\
\hline 19 & 186.0 & 195. 6 & 205.3 & 214.9 & 224.5 & 234.1 & 243.7 & 253.3 & 262.9 & 272.6 & 282.2 \\
\hline 20 & 185.4 & 195.6 & 205.7 & 215.9 & 226.0 & 236.2 & 246.4 & 256.5 & 266.7 & 276.9 & 287.0 \\
\hline 20 & 184.8 & 195.5 & 206.2 & 217.0 & 227.7 & 238.4 & 249.2 & 259.9 & 270.6 & 281.4 & 292. 1 \\
\hline 22 & 184.1 & 195.5 & 206.8 & 218.1 & 229.5 & 240.8 & 252.2 & 263.5 & 274.8 & 286.2 & 297.5 \\
\hline 23 & 183.5 & 195.5 & 207.4 & 219.4 & 231.4 & 243.3 & 255.3 & 267.3 & 279.2 & 291. 2 & 303.2 \\
\hline 24 & 182.9 & 195.5 & 208.1 & 220.8 & 233.4 & 246.0 & 258.6 & 271.3 & 283.9 & 296.5 & 309.1 \\
\hline 25 & 182.3 & 195.6 & 208.9 & 222. 2 & 235.5 & 248.8 & 262.2 & 275.5 & 288.8 & 302.1 & 315.4 \\
\hline 26 & 181. 7 & 195. 7 & 209.7 & 223.8 & 237.8 & 251.8 & 265.9 & 279.9 & 293.9 & 308.0 & 322.0 \\
\hline 27 & 181.1 & 195.9 & 210.6 & 225.4 & 240.2 & 255.0 & 269.8 & 284.6 & 299.3 & 314.1 & 328.9 \\
\hline 28 & 180.5 & 196.0 & 211.6 & 227.2 & 242.7 & 258.3 & 273.9 & 289.5 & 305.0 & 320.6 & 336.2 \\
\hline 29 & 179.9 & 196.3 & 212.7 & 229.0 & 245.4 & 261.8 & 278.2 & 294.6 & 311.0 & 327.4 & 343.8 \\
\hline 30 & 179.3 & 196.5 & 213.8 & 231. 0 & 248.3 & 265.5 & 282.8 & 300.0 & 317.3 & 334.5 & 351.8 \\
\hline 31 & 178.7 & 196.8 & 215.0 & 233.1 & 251. 3 & 269.4 & 287.6 & 305.7 & 323.8 & 342.0 & 360.1 \\
\hline 32 & 178.1 & 197.2 & 216.3 & 235.3 & 254.4 & 273.5 & 292.6 & 311.7 & 330.7 & 349.8 & 368.9 \\
\hline 33 & 177.5 & 197.6 & 217.6 & 237.7 & 257.7 & 277.8 & 297.8 & 317.9 & 337.9 & 258.0 & 378.1 \\
\hline 34 & 176.9 & 198.0 & 219.1 & 240.1 & 261. 2 & 282.3 & 303.4 & 324.4 & 345.5 & 366.6 & 387.6 \\
\hline 35 & 176.4 & 198.5 & 220.6 & 242.7 & 264.9 & 287.0 & 309.1 & 331.3 & 353.4 & 375.5 & 397.6 \\
\hline 36 & 175.8 & 199.0 & 222.3 & 245.5 & 268.7 & 291.9 & 315.2 & 338.4 & 361.6 & 384.9 & 408.1 \\
\hline 37 & 175.2 & 199.6 & 224.0 & 248.4 & 272.7 & 297.1 & 321.5 & 345.9 & 370.2 & 394.6 & 419.0 \\
\hline 38 & 174.7 & 200.2 & 225.8 & 251.4 & 277.0 & 302.5 & 328.1 & 353.7 & 379.2 & 404.8 & 430.4 \\
\hline 39 & 174.1 & 200.9 & 227.7 & 254.5 & 281.4 & 308.2 & 335.0 & 361.8 & 388.6 & 415. 4 & 442.3 \\
\hline 40 & 173.5 & 201.7 & 229.8 & 257.9 & 286.0 & 314.1 & 342.2 & 370.3 & 398.4 & 426.5 & 454.6 \\
\hline 41 & 173.0 & 202.4 & 231.9 & 261. 4 & 290.8 & 320.3 & 349.7 & 379.2 & 408.6 & 438. 1 & 467.5 \\
\hline 42 & 172.4 & 203.3 & 234.1 & 265.0 & 295.9 & 326.7 & 357.6 & 388.4 & 419.3 & 450.1 & 481.0 \\
\hline 43 & 171.9 & 204.2 & 236.5 & 268.8 & 301.1 & 333.4 & 365.7 & 398.0 & 430.3 & 462.6 & 494.9 \\
\hline 44 & 171.4 & 205.2 & 239.0 & 272.8 & 306.6 & 340.4 & 374.2 & 408.1 & 441.9 & 475.7 & 509.5 \\
\hline 45 & 170.8 & 206.2 & 241.6 & 277.0 & 312.3 & 347.7 & 383.1 & 418.5 & 453.9 & 489.2 & 524.6 \\
\hline
\end{tabular}


TABLE 9.32. Refractivity for a pressure of 850 mbar

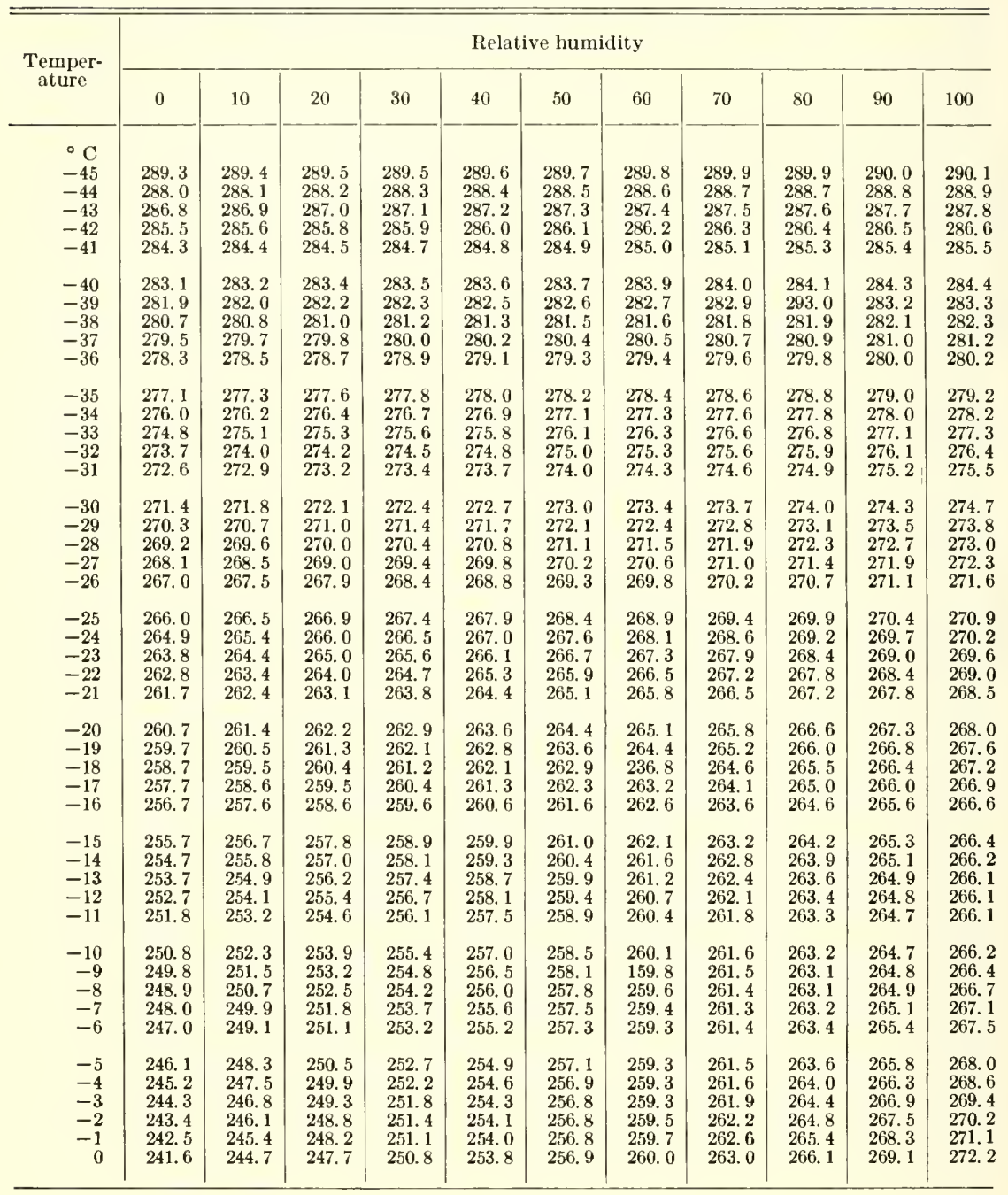


TABLE 9.33. Refractivity for a pressure of 850 mbar

\begin{tabular}{|c|c|c|c|c|c|c|c|c|c|c|c|}
\hline \multirow{2}{*}{$\begin{array}{l}\text { Temper- } \\
\text { ature }\end{array}$} & \multicolumn{11}{|c|}{ Relative humidity } \\
\hline & 0 & 10 & 20 & 30 & 40 & 50 & 60 & 70 & 80 & 90 & 100 \\
\hline${ }^{\circ} \mathrm{C}$ & & & & & & & & & & & \\
\hline 0 & 241.6 & 244.7 & 247.7 & 250.8 & 253.8 & 256.9 & 260.0 & 263.0 & 266.1 & 269.1 & 272.2 \\
\hline 1 & 240.7 & 244.0 & 247.3 & 250.5 & 253.8 & 257.1 & 260.3 & 263.6 & 266.8 & 270.1 & 273.4 \\
\hline 2 & 239.9 & 243.3 & 246.8 & 250.3 & 253.8 & 257.3 & 260.7 & 264.2 & 267.7 & 271. 2 & 274.7 \\
\hline 3 & 239.0 & 242.7 & 246.4 & 250.1 & 253.8 & 257.5 & 261. 3 & 265.0 & 268.7 & 272.4 & 276.1 \\
\hline 4 & 238.1 & 242.1 & 246.0 & 250.0 & 253.9 & 257. 9 & 261. 9 & 265. 8 & 269.8 & 273.7 & 277.7 \\
\hline 5 & 237.3 & 241.5 & 245. 7 & 249.9 & 254. 1 & 258.3 & 262.5 & 266.7 & 271.0 & 275.2 & 279.4 \\
\hline 6 & 236.4 & 240.9 & 245.4 & 249.9 & 254. 3 & 258.8 & 363.3 & 267.8 & 272. 3 & 276.8 & 281.2 \\
\hline 7 & 235.6 & 240.3 & 245.1 & 249.9 & 254.6 & 259.4 & 264. 2 & 268.9 & 273.7 & 278.5 & 283. 2 \\
\hline 8 & 234. 7 & 239.8 & 244. 9 & 249.9 & 255.0 & 260.1 & 265.1 & 270.2 & 275.3 & 280.3 & 285.4 \\
\hline 9 & 233.9 & 239.3 & 244.7 & 250.1 & 255.4 & 260.8 & 266. 2 & 271.6 & 277.0 & 282.4 & 287.8 \\
\hline 10 & 233.1 & 238.8 & 244.5 & 250.2 & 256.0 & 261.7 & 267.4 & 273.1 & 278.8 & 284.5 & 280.3 \\
\hline 11 & 232.3 & 238.3 & 244.4 & 250.5 & 256.5 & 262.6 & 268.7 & 274.8 & 280.8 & 286.9 & 293.0 \\
\hline 12 & 231.4 & 237. 9 & 244.3 & 250.8 & 257.2 & 263. 6 & 270.1 & 276.5 & 283.0 & 289.4 & 295.9 \\
\hline 13 & 230.6 & 237.5 & 244.3 & 251. 1 & 258.0 & 264.8 & 271.6 & 278.4 & 285.3 & 292.1 & 298.9 \\
\hline 14 & 229.8 & 237.1 & 244.3 & 251.5 & 258.8 & 266.0 & 283.3 & 280.5 & 287.7 & 295. 0 & 302.2 \\
\hline 15 & 229.0 & 236.7 & 244.4 & 252.0 & 259.7 & 267.4 & 275.0 & 282.7 & 290.4 & 298. 1 & 305.7 \\
\hline 16 & 228.2 & 236.4 & 244.5 & 252.6 & 260.7 & 268.8 & 277.0 & 28.5 .1 & 293.2 & 301.3 & 309.5 \\
\hline 17 & 227.4 & 236.0 & 244.6 & 253.2 & 261.8 & 270.4 & 279.0 & 287.6 & 296.2 & 304.8 & 313.4 \\
\hline 18 & 226.7 & 235.8 & 244. 9 & 253.9 & 263.0 & 272.1 & 281. 2 & 290.3 & 299. 4 & 308. 5 & 317.6 \\
\hline 19 & 225.9 & 235.5 & 245.1 & 254. 7 & 264.4 & 274.0 & 283.6 & 293. 2 & 302.8 & 212. 4 & 322.0 \\
\hline 20 & 225.1 & 235.3 & 245.4 & 255.6 & 265.8 & 275.9 & 286.1 & 296.3 & 306.4 & 316.6 & 326.7 \\
\hline 21 & 224.4 & 235.1 & 245.8 & 256.6 & 267.3 & 278.0 & 288.8 & 299.5 & 310.2 & 321.0 & 331.7 \\
\hline 22 & 223.6 & 234. 9 & 246.3 & 257.9 & 268.9 & 280.3 & 291.6 & 302.9 & 314.3 & 325.6 & 337.0 \\
\hline 23 & 222.8 & 234.8 & 246.8 & 258.7 & 270.7 & 272.7 & 294.6 & 306.6 & 318.6 & 330.5 & 242.5 \\
\hline 24 & 222.1 & 234.7 & 247.3 & 260.0 & 272.6 & 285.2 & 297.8 & 310.4 & 323.1 & 335.7 & 348. 3 \\
\hline 25 & 221.3 & 234.7 & 248.0 & 261. 3 & 274.6 & 287.9 & 301.2 & 314.5 & 327.8 & 341.1 & 354.5 \\
\hline 26 & 220.6 & 234.6 & 248.7 & 262.7 & 276.7 & 280.9 & 304.8 & 318.8 & 332.9 & 346.9 & 360.9 \\
\hline 27 & 219.9 & 234. 7 & 249.4 & 264. 2 & 279.0 & 293.8 & 308. 6 & 323.4 & 338.1 & 352.9 & 367.7 \\
\hline 28 & 219. 1 & 234.7 & 250.3 & 265.8 & 281.4 & 297.0 & 312.6 & 328.1 & 343.7 & 359.3 & 374.8 \\
\hline 28 & 218.4 & 234.8 & 251.2 & 267.6 & 284. 0 & 300.4 & 316.8 & 333.2 & 349.6 & 365.9 & 382.3 \\
\hline 30 & 217.7 & 234.9 & 252.2 & 269.4 & 286.7 & 303.9 & 321.2 & 338.4 & 355.7 & 372.9 & 390.2 \\
\hline 31 & 217.0 & 235.1 & 253.3 & 271.4 & 289.6 & 307.7 & 325.8 & 344.0 & 362.1 & 380.3 & 398.4 \\
\hline 32 & 216. 3 & 235.3 & 254.4 & 273.5 & 292.6 & 311.7 & 330.7 & 249.8 & 368.9 & 388.0 & 407.1 \\
\hline $3 \overline{3}$ & 215. 6 & 235.6 & 255. 7 & 275.7 & 295.8 & 315.8 & 335.9 & 355.9 & 376.0 & 296.0 & 416.1 \\
\hline 34 & 214.9 & 235.9 & 257.0 & 278.1 & 299.1 & 320.2 & 341.2 & 362.3 & 383.4 & 404.5 & 425.5 \\
\hline 35 & 214.2 & 236.3 & 258.4 & 280.5 & 302.7 & 324.8 & 346.9 & 369.0 & 391.2 & 413.3 & 435.4 \\
\hline 36 & 213.5 & 236.7 & 259. 9 & 283.2 & 305.4 & 329.6 & 352.8 & 376.1 & 399. 3 & 422.5 & 445.8 \\
\hline 37 & 212.8 & 237.2 & 261. 5 & 285.9 & 310.3 & 334.7 & 359.0 & 383.4 & 407.8 & 432.2 & 456.5 \\
\hline 38 & 212.1 & 237.7 & 263. 2 & 288.8 & 314.4 & 340.0 & 365.5 & 391.1 & 416.7 & 422.2 & 467.8 \\
\hline 39 & 211.4 & 238.2 & 265.0 & 291. 9 & 318.7 & 345.5 & 372.2 & 399.1 & 425.9 & 452.7 & 479.6 \\
\hline 40 & 210.7 & 238.8 & 267.0 & 295.1 & 323.2 & 351.3 & 379.4 & 407.5 & 435.6 & 463.7 & 491.8 \\
\hline 41 & 210.1 & 239.5 & 269.0 & 298.4 & 327.9 & 357.3 & 386.8 & 416. 2 & 445.7 & 475.1 & 504,6 \\
\hline 42 & 209.4 & 240.2 & 271.1 & 302.0 & 332.8 & 363.7 & 394.5 & 425. 4 & 456.2 & 487.1 & 517.9 \\
\hline 43 & 208.7 & 241.0 & 283. 3 & 305.6 & 338.0 & 370.3 & 402.6 & 434.9 & 467.2 & 499.5 & 531.8 \\
\hline 44 & 208.1 & 241.9 & 275.7 & 309.5 & 343.3 & 377.1 & 411. 0 & 444.8 & 478.6 & 512.4 & 546. 2 \\
\hline 45 & 207.4 & 242.8 & 278.2 & 313.6 & 348.9 & 384.3 & 419.7 & 455.1 & 490.5 & 525.8 & 561.2 \\
\hline
\end{tabular}


TABLE 9.34. Refractivity for a pressure of 1,000 mbar

\begin{tabular}{|c|c|c|c|c|c|c|c|c|c|c|c|}
\hline \multirow{2}{*}{$\begin{array}{l}\text { Temper- } \\
\text { ature }\end{array}$} & \multicolumn{11}{|c|}{ Relative humidity } \\
\hline & 0 & 10 & 20 & 30 & 40 & 50 & 60 & 70 & 80 & 90 & 100 \\
\hline${ }^{\circ} \mathrm{C}$ & & & & & & & & & & & \\
\hline-45 & 340.4 & 340.4 & 340.5 & 340.6 & 340.7 & 340.8 & 340.8 & 340.9 & 341.0 & 241.1 & 341.1 \\
\hline-44 & 338.9 & 339.0 & 339.0 & 339.1 & 339.2 & 339.2 & 339.4 & 339.5 & 339.6 & 339.7 & 339.7 \\
\hline-43 & 337.4 & 337.5 & 337.6 & 337.7 & 337.8 & 337.9 & 338.0 & 338.1 & 338.2 & 338.3 & 338.4 \\
\hline-42 & 335.9 & 336.0 & 336.1 & 336.3 & 336.4 & 336.5 & 336.6 & 336.7 & 336.8 & 336.9 & 337.0 \\
\hline-41 & 334.5 & 334.6 & 334.7 & 334.8 & 335.0 & 335.1 & 335.2 & 335.3 & 335.4 & 335.5 & 335.7 \\
\hline-40 & 333.0 & 333.2 & 333.3 & 333.4 & 333.6 & 333.7 & 333.8 & 334.0 & 334.1 & 334.2 & 334.3 \\
\hline-39 & 331.6 & 331.8 & 331.9 & 332.1 & 332.2 & 332.3 & 332.5 & 332.6 & 332.8 & 332.9 & 333.1 \\
\hline-38 & 330.2 & 330.4 & 330.5 & 330.7 & 330.8 & 331.0 & 331.2 & 331.3 & 331.5 & 331.6 & 331.8 \\
\hline-37 & 328.8 & 329.0 & 329.2 & 329.3 & 329.5 & 329.7 & 329.8 & 330.0 & 330.2 & 330.4 & 330.5 \\
\hline-36 & 327.4 & 327.6 & 327.8 & 328.0 & 328.2 & 328.4 & 328.6 & 328.7 & 328.9 & 329.1 & 329.3 \\
\hline-35 & 326.1 & 326. 3 & 326.5 & 326.7 & 326.9 & 327.1 & 327,3 & 327.5 & 327.7 & 327.9 & 328.1 \\
\hline-34 & 324.7 & 324.9 & 325.1 & 325.4 & 325.6 & 325.8 & 326.0 & 326.3 & 326.5 & 326.7 & 326.9 \\
\hline-33 & 323.3 & 323.6 & 323.8 & 234.1 & 324.3 & 324.6 & 324.8 & 235. 1 & 325.3 & 325.6 & 325.8 \\
\hline-32 & 322.0 & 322.3 & 322.6 & 322.8 & 323.1 & 323. 3 & 323.6 & 323.9 & 324.2 & 324.4 & 324.7 \\
\hline-31 & 320.7 & 321.0 & 321.3 & 321.5 & 321.8 & 322.1 & 322.4 & 322.7 & 323.0 & 323.3 & 323.6 \\
\hline-30 & 319.3 & 319.7 & 320.0 & 320.3 & 320.6 & 320.9 & 321.3 & 321.6 & 321.9 & 322.2 & 322.6 \\
\hline-29 & 318.0 & 318.4 & 318.7 & 319.1 & 319.4 & 319.8 & 320.1 & 320.5 & 320.8 & 321.2 & 321.5 \\
\hline-28 & 316.7 & 317.1 & 317.5 & 317.9 & 318.3 & 318.6 & 319.0 & 319.4 & 319 & 320.2 & 320.5 \\
\hline-27 & 315.4 & 315.9 & 316.3 & 316.7 & 317.1 & 317.5 & 317.9 & 318.4 & 318.8 & 319.2 & 319.6 \\
\hline-26 & 314.2 & 314.6 & 315.1 & 315.5 & 316. 0 & 316.4 & 316.9 & 317.3 & 317.8 & 318.2 & 318.7 \\
\hline-25 & 312.9 & 313.4 & 313.9 & 314.4 & 314.9 & 315.4 & 315.8 & 316.3 & 316.8 & 316.3 & 317.8 \\
\hline-24 & 311.6 & 312.2 & 312.7 & 313. 2 & 313.8 & 314.3 & 31 & 315.4 & 31 & 316.4 & 317.0 \\
\hline-23 & 310.4 & 311.0 & 311.6 & 312.1 & 312.7 & 313. 3 & 313. 9 & 314.4 & 315.0 & 415. 6 & 316.2 \\
\hline-22 & 309. 2 & 309.8 & 310.4 & 311.0 & 311.7 & 312.9 & 313.5 & 314.2 & 314.2 & 314.8 & 315.4 \\
\hline-21 & 307.9 & 308.6 & 309.3 & 310.0 & 310.6 & 311.3 & 312.0 & 312.7 & 313.3 & 314.0 & 314.7 \\
\hline-20 & 306.7 & 307.6 & 308. 2 & 308.9 & 309.6 & 310.4 & 311.1 & 311.8 & 312.6 & 313. 3 & 314.0 \\
\hline-19 & 305.5 & 306.3 & 307.1 & 307.9 & 308.7 & 309.5 & 310.3 & 311.0 & 311.8 & 312.6 & 313.4 \\
\hline-18 & 304.3 & 305.2 & 306. 0 & 306.9 & 307.7 & 308.6 & 309.4 & 310.3 & 311.1 & 312.0 & 312.9 \\
\hline-17 & 303.1 & 304.0 & 305. 0 & 305.9 & 206.8 & 307.7 & 308.7 & 309.6 & 310.5 & 311.4 & 312.3 \\
\hline-16 & 301.9 & 302.9 & 303. 9 & 304.9 & 305.9 & 306.9 & 307.9 & 308.9 & 309.9 & 310.9 & 311.9 \\
\hline-15 & 300.8 & 301.8 & 312.9 & 403. 0 & 305.1 & 306.1 & 307 & 30 & 309.4 & 310.4 & 311.5 \\
\hline-14 & 299.6 & 300.8 & 301.9 & 303.1 & 304.2 & 305.4 & $30 \mathrm{f}$ & 30 & & 310.0 & 311.2 \\
\hline-13 & 298.5 & 299.7 & 300.9 & 302.2 & 303. 4 & 304.7 & 305.9 & 307.2 & 308.4 & 309. 7 & 310.9 \\
\hline-12 & 297.3 & 298.7 & 300.0 & 301.3 & 302.7 & 304.0 & 305.3 & 306.7 & 308. 0 & 309.4 & 310.7 \\
\hline-11 & 296.2 & 297.6 & 299.1 & 300.5 & 301.9 & 303.4 & 304.8 & 306. 2 & 307.7 & 309.1 & 310.6 \\
\hline-10 & 295.1 & 296.6 & 298. 1 & 299.7 & 301.2 & 302.8 & 304.3 & 305.9 & 307.4 & 309.0 & 310.5 \\
\hline-9 & 203.9 & 295. 6 & 297. 3 & 298. 9 & 300.6 & 302.2 & 303.9 & 305.5 & & 308.9 & \\
\hline-8 & 292.8 & 294.6 & 296.4 & 298.2 & 299.9 & 301.7 & 303.5 & 305.3 & 307.1 & 308.8 & 310.6 \\
\hline-7 & 291.7 & 293.6 & 296.5 & 297.5 & 299.4 & 301.3 & 303. 2 & 305.1 & 307.0 & 308.9 & 310.8 \\
\hline-6 & 290.6 & 292.7 & 294.7 & 296.8 & 298.8 & 300.9 & 302.9 & 305.0 & 307.0 & 309.0 & 311.1 \\
\hline-5 & 289.6 & 291.7 & 293.9 & 296.1 & 298. 3 & 300.5 & 302.7 & 304.9 & 307.1 & 309.3 & 311.5 \\
\hline-4 & 288.5 & 290.8 & 293.2 & 295.5 & 297.9 & 300.2 & 302.5 & 304.9 & 307.2 & 309.6 & 311.9 \\
\hline-3 & 287.4 & 289.9 & 292.4 & 294.9 & 297.4 & 299.9 & 302.5 & 305.0 & 307.5 & 310.0 & 312.5 \\
\hline-2 & 296.3 & 289.0 & 291.7 & 294.4 & 297.1 & 299.8 & 302.4 & 305.1 & 307.8 & 310.5 & 313.2 \\
\hline-1 & 285.3 & 288. 2 & 291.0 & 293.9 & 296.8 & 299. 6 & 302.5 & 305. 3 & 308.2 & 311.1 & 313.8 \\
\hline 0 & 284.2 & 287. 3 & 290.4 & 293.4 & 296.5 & 299.5 & 302,6 & 305.7 & 308.7 & 311.8 & 314.8 \\
\hline
\end{tabular}


TABLE 9.35. Refractivity for a pressure of 1,000 mbar

\begin{tabular}{|c|c|c|c|c|c|c|c|c|c|c|c|}
\hline \multirow{2}{*}{$\begin{array}{l}\text { Temper- } \\
\text { ature }\end{array}$} & \multicolumn{11}{|c|}{ Relative humidity } \\
\hline & 0 & 10 & 20 & 30 & 40 & 50 & 60 & 70 & 80 & 90 & 100 \\
\hline \multicolumn{12}{|l|}{${ }^{\circ} \mathrm{C}$} \\
\hline 0 & 284.2 & 287.3 & 290.4 & 293.4 & 296.5 & 299.5 & 302.6 & 305.7 & 308.7 & 311.8 & 314.8 \\
\hline 1 & 283.2 & 286.5 & 289.7 & 293.0 & 296.3 & 299.6 & 302.8 & 306.1 & 309.3 & 312,6 & 315.9 \\
\hline 2 & 282.2 & 285.7 & 289.1 & 292.6 & 269.1 & 299.6 & 303.1 & 306.6 & 310.0 & 313.5 & 317.0 \\
\hline 3 & 281.2 & 284.9 & 288.6 & 292.3 & 296.0 & 299.7 & 303.4 & 307.1 & 310.9 & 314.6 & 318.3 \\
\hline 4 & 280.1 & 284.1 & 288.1 & 292.0 & 296.0 & 299.9 & 303.9 & 307.8 & 311.8 & 315.7 & 319.7 \\
\hline 5 & 279.1 & 283.3 & 287.6 & 291.8 & 296.0 & 300.2 & 304.4 & 308.6 & 213.8 & 317.0 & 321.2 \\
\hline 6 & 278.1 & 282.6 & 287.1 & 291.6 & 296. 1 & 300.5 & 305.0 & 309.5 & 314.0 & 318.5 & 323.0 \\
\hline 7 & 277.1 & 281. 9 & 286.7 & 291. 4 & 296. 2 & 301.0 & 305. 7 & 310.5 & 315.3 & 320.0 & 324.8 \\
\hline 8 & 276.2 & 281. 2 & 286.3 & 291.4 & 296.7 & 301.5 & 306.6 & 311.6 & 316.7 & 321.8 & 326.8 \\
\hline 9 & 275. 2 & 280.6 & 285. 9 & 291.3 & 296. 7 & 302.1 & 307.5 & 213. 9 & 318.3 & 323.6 & 329.0 \\
\hline 10 & 274.2 & 279.9 & 285.6 & 291.4 & 297.1 & 302.8 & 308.5 & 314. 2 & 320.0 & 325.7 & 331.4 \\
\hline 11 & 273.2 & 279.3 & 285.4 & 291.5 & 297.5 & 303.6 & 309.7 & 315.7 & 321.8 & 327.9 & 334.0 \\
\hline 12 & 272.3 & 278.7 & 285,2 & 291.6 & 298.0 & 304.5 & 310.9 & 317.4 & 323.8 & 330.3 & 336.7 \\
\hline 13 & 271.3 & 278. 2 & 285.0 & 291.8 & 298. 7 & 305.5 & 312.3 & 319.1 & 326.0 & 332.8 & 339.6 \\
\hline 14 & 270.4 & 277.6 & 284.9 & 292.1 & 299.3 & 306.6 & 313.8 & 321.1 & 328.3 & 335.5 & 342.8 \\
\hline 15 & 269.4 & 277.1 & 274.7 & 292.5 & 300.1 & 307.8 & 315.5 & 323.1 & 330.8 & 338.5 & 346.1 \\
\hline 16 & 268.5 & 276.6 & 284.8 & 292.9 & 301.0 & 309.1 & 317. 2 & 325.4 & 333.5 & 341.6 & $\begin{array}{l}349.7 \\
349 .\end{array}$ \\
\hline 17 & 267.6 & 276.2 & 284.8 & 293,4 & 302.0 & 310.6 & 319.2 & 327.8 & 336,4 & 344.9 & 353.5 \\
\hline 18 & 266.7 & 275.8 & 284.9 & 293. 9 & 303.0 & 312.1 & 321.2 & 330.3 & 339.4 & 348.5 & 357.6 \\
\hline 19 & 265.8 & 275.4 & 285.0 & 294.6 & 304. 2 & 313.8 & 323.4 & 333.1 & 342.7 & 352.3 & 361.9 \\
\hline 20 & 264.8 & 275.0 & 285.2 & 295. 3 & 305.5 & 315.8 & 325,8 & 336.0 & 346.1 & 356.3 & 366.5 \\
\hline 21 & 363. 9 & 274. 7 & 285.4 & 296.2 & 306.9 & 317.6 & 328.4 & 339.1 & 349.8 & 360.6 & 371.3 \\
\hline 22 & 263.1 & 274.4 & 285.7 & 297.1 & 308.4 & 319.7 & 331.1 & 342.4 & 353.7 & 365.1 & 376.4 \\
\hline 23 & 262. 2 & 274.1 & 286.1 & 298.1 & 310.0 & 322.0 & 334.0 & 345.9 & 357. 9 & 369.8 & 381.8 \\
\hline 24 & 261. 3 & 273.9 & 286.5 & 299.1 & 311.8 & 324.4 & 337.0 & 349.6 & 362.3 & 374.9 & 387.5 \\
\hline 25 & 260.4 & 273.7 & 287.0 & 300.3 & 313.6 & 327.0 & 340.3 & 353.6 & 366.9 & 380.2 & 393.5 \\
\hline 26 & 259.5 & 273.6 & 287.6 & 301.6 & 315.7 & 329.7 & 343.7 & 357.8 & 371.8 & 385.8 & 399.8 \\
\hline 27 & 258.7 & 273.5 & 288. 2 & 303.0 & 317.8 & 332.6 & 347.4 & 362.2 & 276.9 & 391.7 & 306.5 \\
\hline 28 & 257.8 & 273.4 & 288.9 & 304.5 & 320.1 & 335.8 & 351.2 & 366.8 & 382.4 & 397.9 & 413.5 \\
\hline 29 & 257.0 & 273.3 & 289.7 & 306.1 & 322.5 & 338.9 & 355.3 & 371.7 & 388.1 & 404.5 & 420.9 \\
\hline 30 & 256.1 & 273.4 & 290.6 & 307.9 & 325.1 & 342.4 & 359.6 & 276.9 & 384.1 & 411.4 & 428,6 \\
\hline 31 & 255.3 & 273.4 & 291.6 & 309.7 & 327.8 & 346.0 & 364.1 & 382.3 & 400.4 & 418.6 & 436.7 \\
\hline 32 & 254.4 & 273.5 & 292.6 & 311.7 & 330.7 & 349.8 & 368.9 & 388.0 & 407.1 & 426.1 & 445. 2 \\
\hline 33 & 253. 6 & 273. 6 & 293.7 & 313.8 & 333.8 & 363.9 & 373.9 & 394.0 & 414.0 & 434.1 & 454.1 \\
\hline 34 & 252.8 & 273.8 & 294.9 & 316,0 & 337.0 & 358.1 & 379.2 & 444. 3 & 421. 3 & 442.4 & 463.5 \\
\hline 35 & 251.9 & 274. 1 & 296.2 & 318.3 & 340.5 & 362.6 & 384.7 & 406.8 & 429.0 & 451. 1 & 473.2 \\
\hline 36 & 251. 1 & 274.4 & 297.6 & 320.8 & 344.1 & 367.3 & 390.5 & 413.7 & 437.0 & 460.2 & 384.4 \\
\hline 37 & 250.3 & 274.7 & 299.1 & 323.5 & 347.8 & 372.2 & 396.6 & 421.0 & 445.3 & 469.7 & 494.1 \\
\hline 38 & 249.5 & 275.1 & 300.7 & 326.2 & 351.8 & 377.4 & 403.0 & 429.5 & 454.1 & 479.7 & 505. 2 \\
\hline 39 & 248.7 & 275.5 & 302.3 & 329.2 & 256.0 & 382.8 & 409.6 & 436.4 & 463. 2 & 490.1 & 516.9 \\
\hline 40 & 247.9 & 276.0 & 304.1 & 332.2 & 360.4 & 388.5 & 416.6 & 444.7 & 472.8 & 500.9 & 529.0 \\
\hline 41 & 247.1 & 276.6 & 306.0 & 335.5 & 364.9 & 394. 4 & 423.9 & 453.3 & 482.8 & 512.2 & 541.7 \\
\hline 42 & 246.3 & 277.2 & 308.1 & 338.9 & 369.8 & 400,6 & 431.5 & 462.3 & 493. 2 & 524.0 & 544.9 \\
\hline 43 & 245.6 & 277.9 & 310.2 & 342.5 & 374.8 & 407.1 & 439.4 & 471.7 & 504.0 & 536.3 & 568.6 \\
\hline 44 & 244.8 & 278.6 & 312.4 & 246.2 & 380.0 & 413.9 & 447.7 & 481.5 & 515. 3 & 549.1 & 582.9 \\
\hline 45 & 244.0 & 279.4 & 314.8 & 350.2 & 385.5 & 420.9 & 456.3 & 491.7 & 527.1 & 562.5 & 597.8 \\
\hline
\end{tabular}




\subsection{Climatological Data of the Refractive Index for the United States}

Charts of 8-year mean $N_{0}$ values for 0200 and 1400 local time are given for the four seasons of the year on figures 9.1 to 9.8 .

Selected stations illustrate the range of seasonal and diumal variations of $N_{s}$ in differing climatic regions across the country. These are plotted on figures 9.9 to 9.14 . Note the large annual and diurnal ranges of means for humid subtropical Washington, D.C. (fig. 9.9), compared with the modest variations exhibited by maritime-dominated Tatoosh Island (fig. 9.14). The results of these analyses are consistent with those obtained from climatic studies of the refractive index structure over North America, described in section 4 of chapter 4 . That is, large seasonal and diurnal ranges are exhibited by continental-climate stations (Colorado Springs) and small ranges by maritime-climate stations (Tatoosh Island).

Cumulative distribution curves for $N_{s}$, useful in radio ray bending predictions, are given in figures 9.15 to 9.20 for the example stations above.

Figures 9.21 and 9.22, standard deviation of $N_{s}$, provide a measure of the accuracy of the $N_{0}$ charts of figures 9.1 to 9.8 . As has been noted in chapter 4, a number of climatic features are apparent on the standard deviation maps. The climatic stability of various regions of the country, for example, is reflected in these charts. Small standard deviations characterize the maritime climate of the west coast. By comparison, the strong air mass changes of wintertime synoptic patterns sweeping across the southeastern United States are indicated by large standard deviations in that region (fig. 9.21).

\subsection{Statistical Prediction of Elevation Angle Error}

The elevation angle error, $\epsilon$, as defined in chapter 3 , can, like bending, be predicted from surface refractivity, $N_{s}$, by an equation of the form

$$
\epsilon=m N_{s}+\ell
$$

In tables 9.36 to 9.44 for a given height $h$, the coefficients $m$ and $\ell$ have been determined by a statistical regression performed on the Standard CRPL Sample, each having a unique $N_{s}$.

This process is analogous to the statistical bending prediction method described in chapter 3 . 


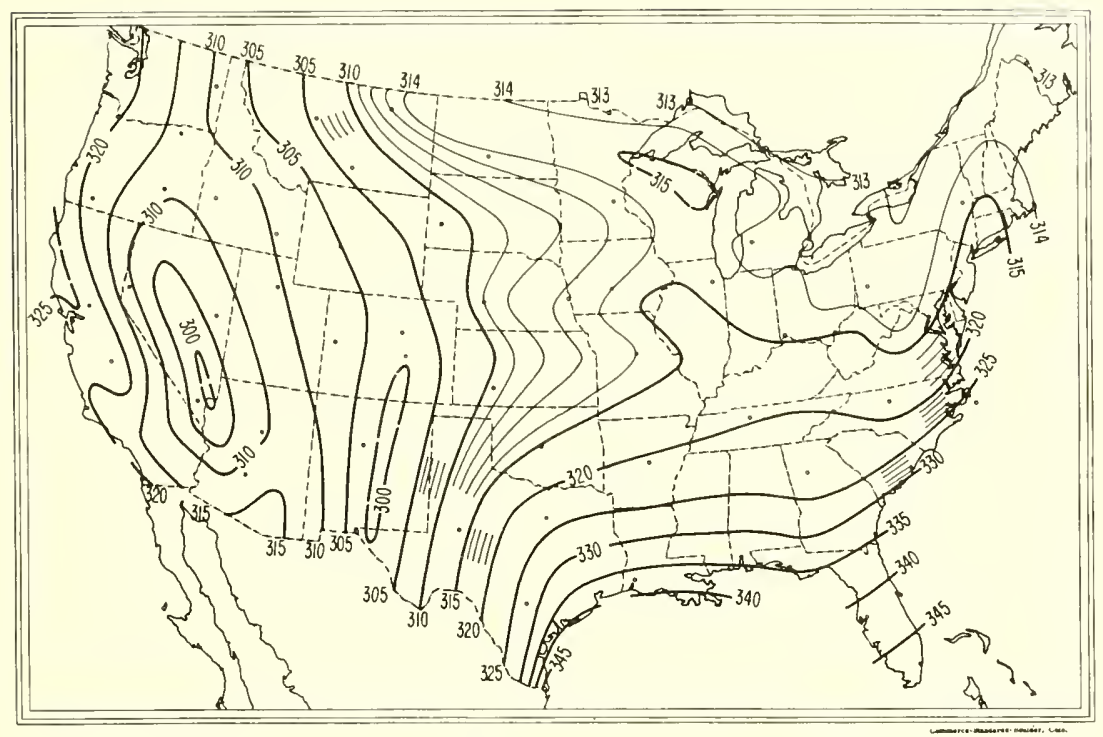

Figure 9.1. Mean $\mathrm{N}_{0}$, February 0200.

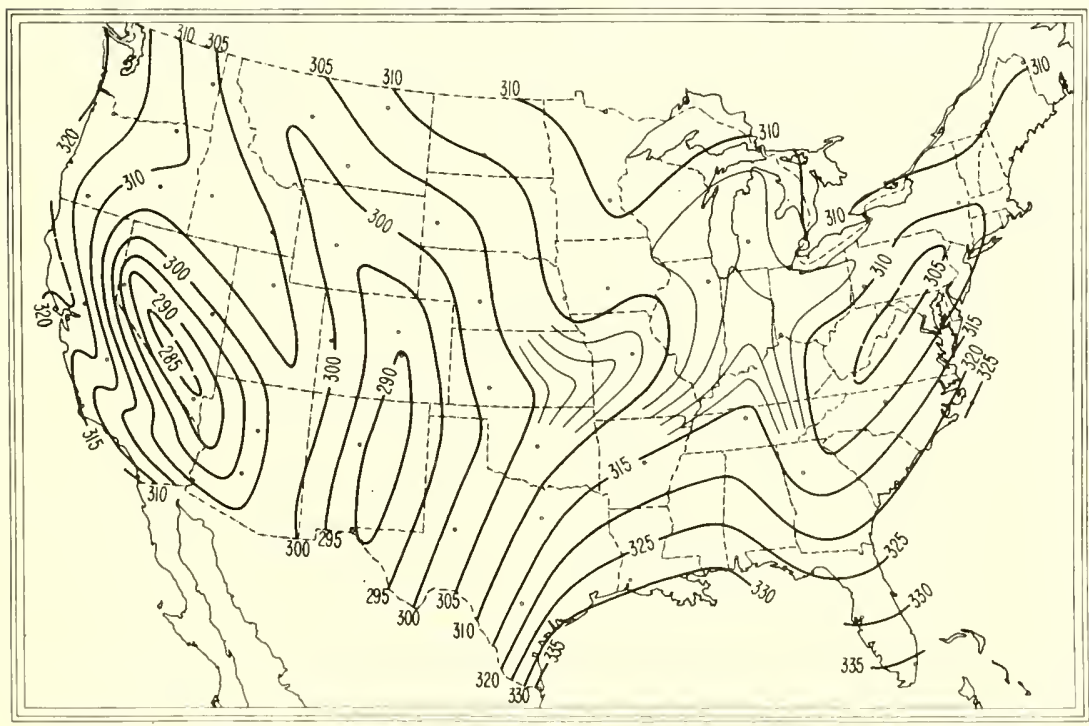

Figure 9.2. Mean $\mathrm{N}_{0}$, February 1400. 


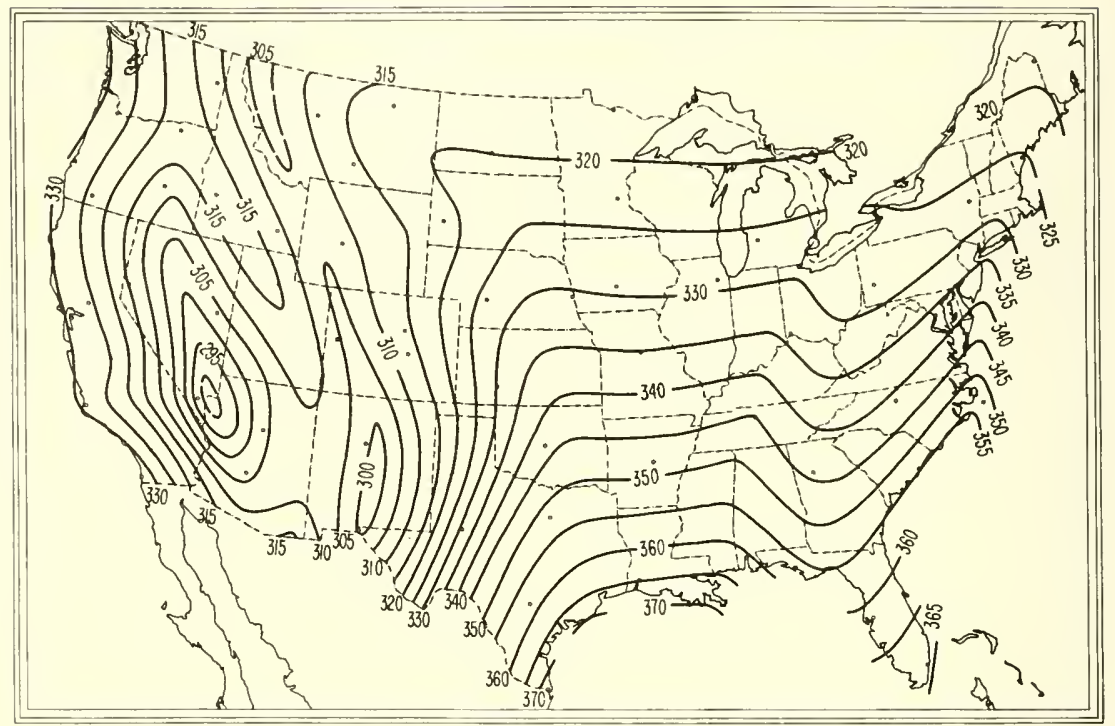

Figure 9.3. Mean $\mathrm{N}_{0}$, May 0200.

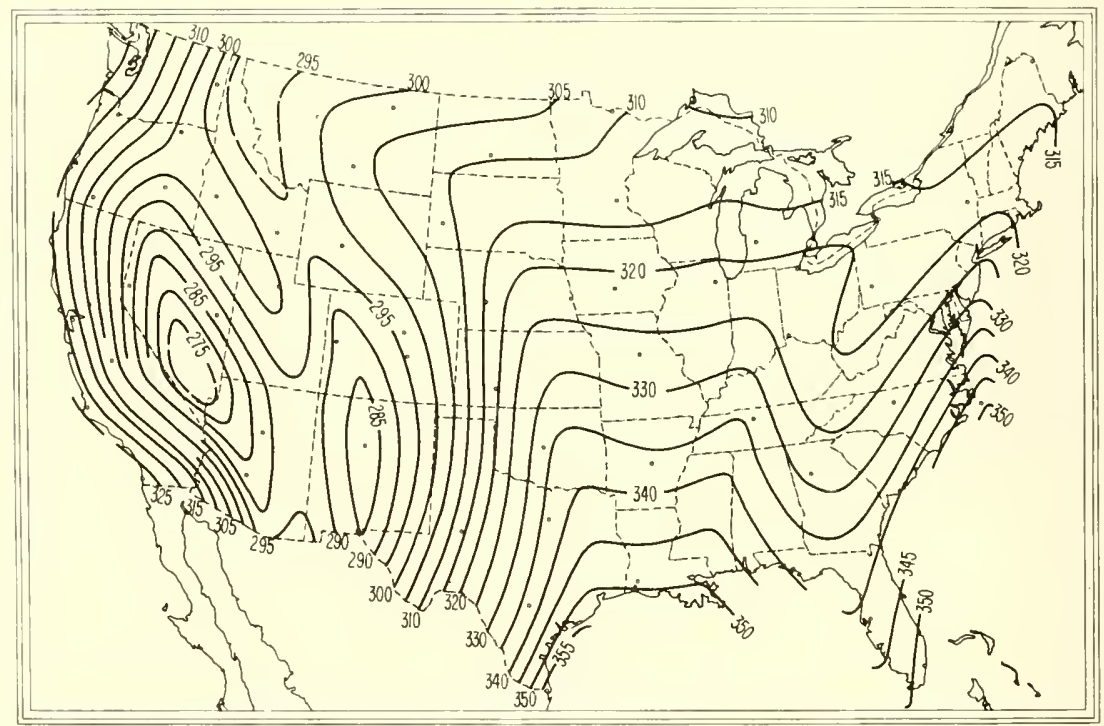

Figure 9.4. Mean $\mathrm{N}_{0}$, May 1400. 


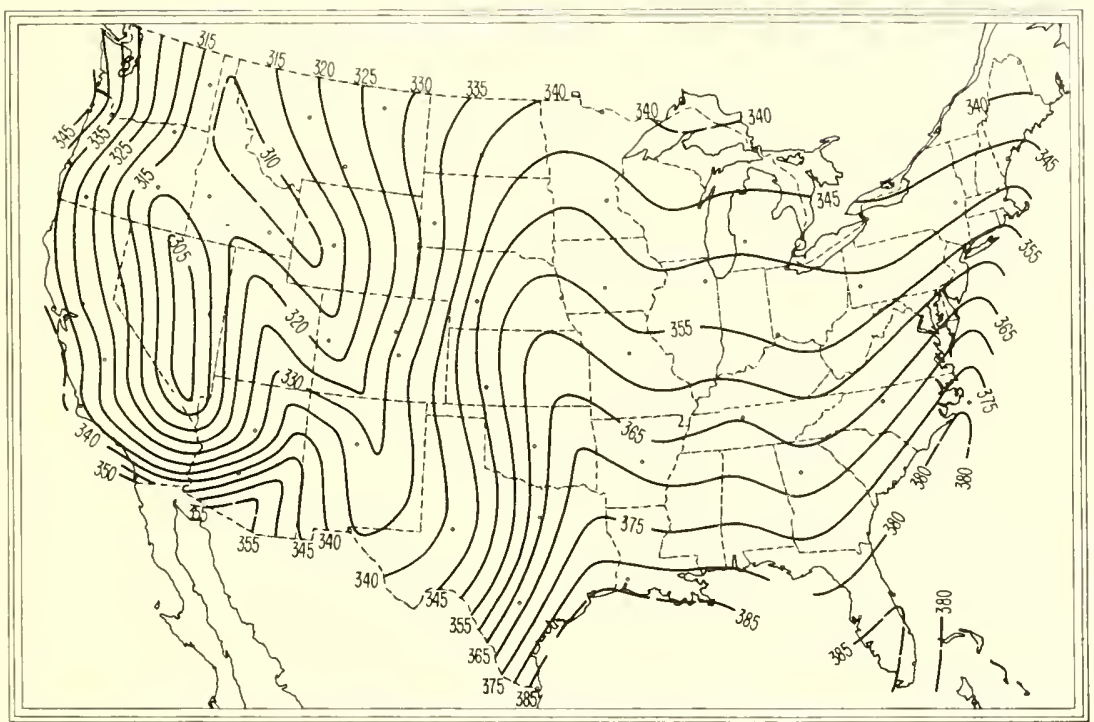

Figure 9.5. Mean No, August 0200.

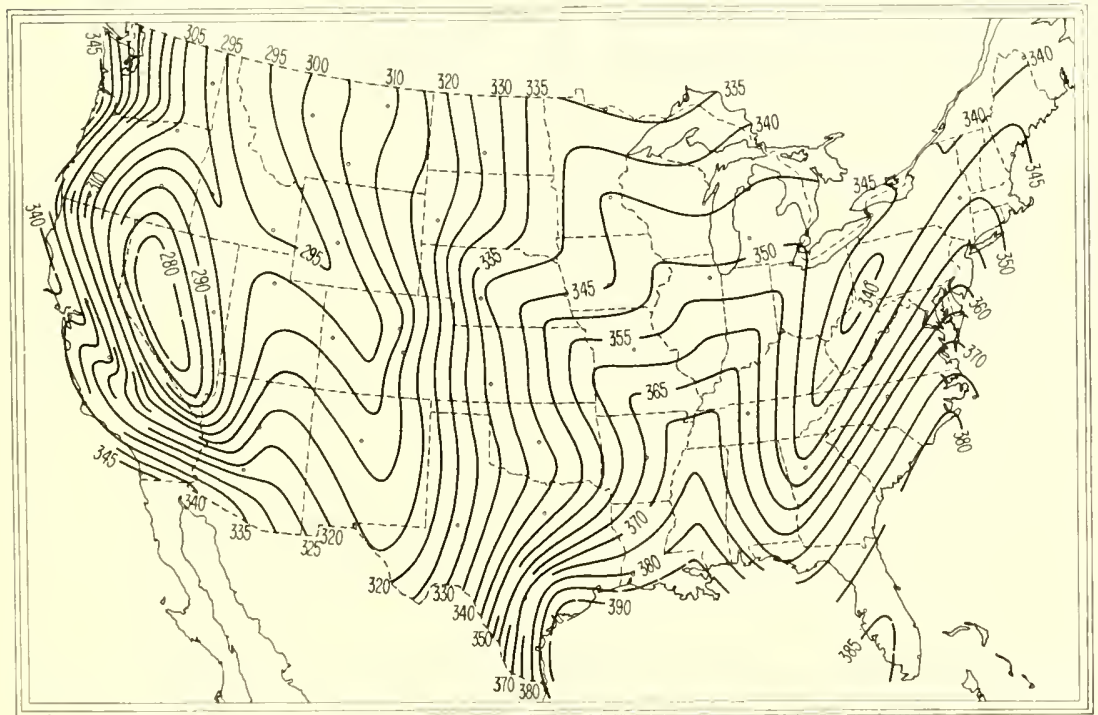

Figure 9.6. Mean $\mathrm{N}_{0}$, August 1400. 


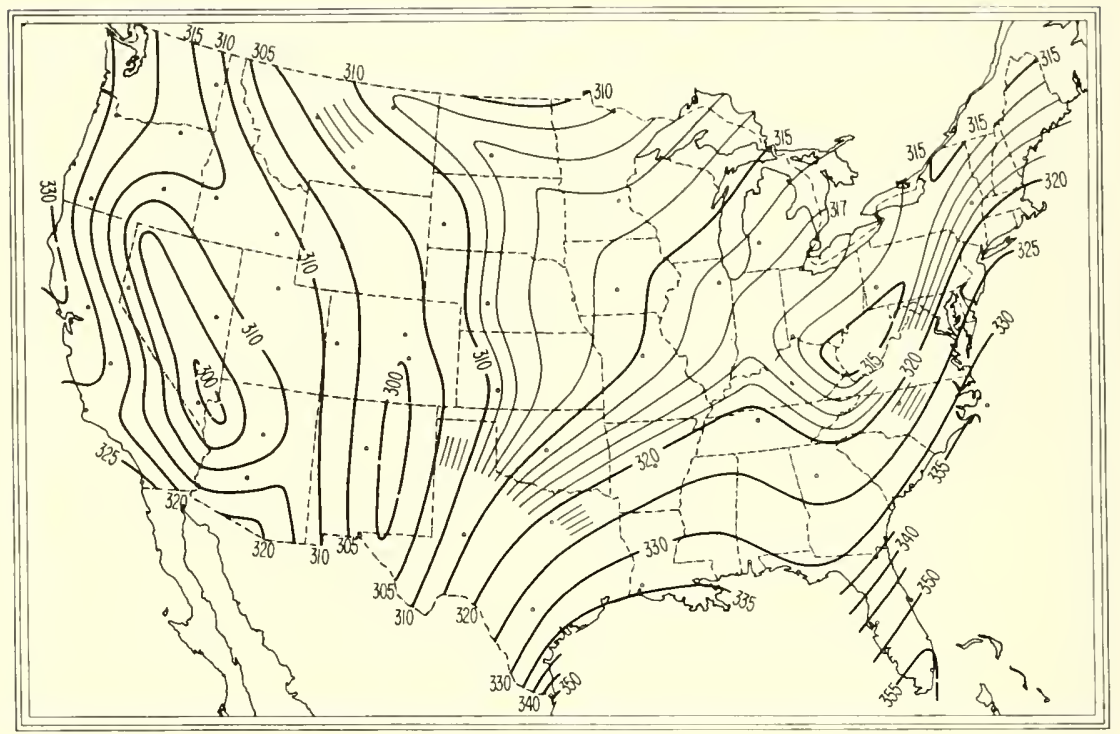

Figure 9.7. Mean $\mathrm{N}_{0}$, November 0200.

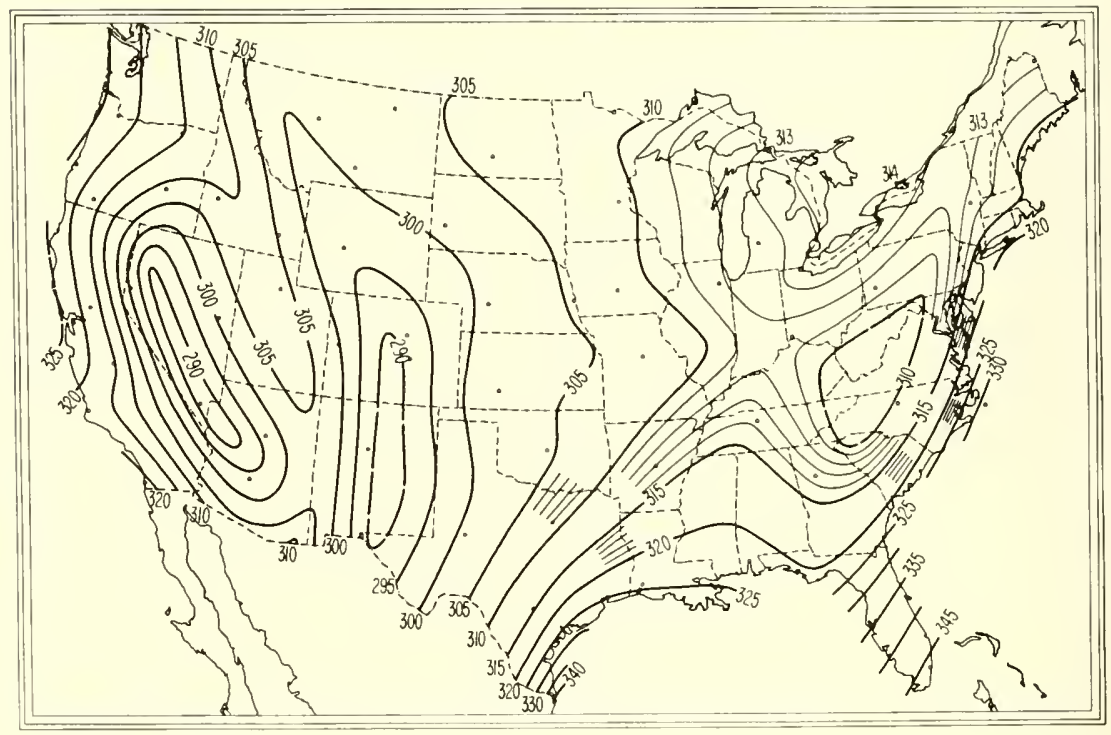

Figure 9.8. Mean $\mathrm{N}_{0}$, November 1400 . 

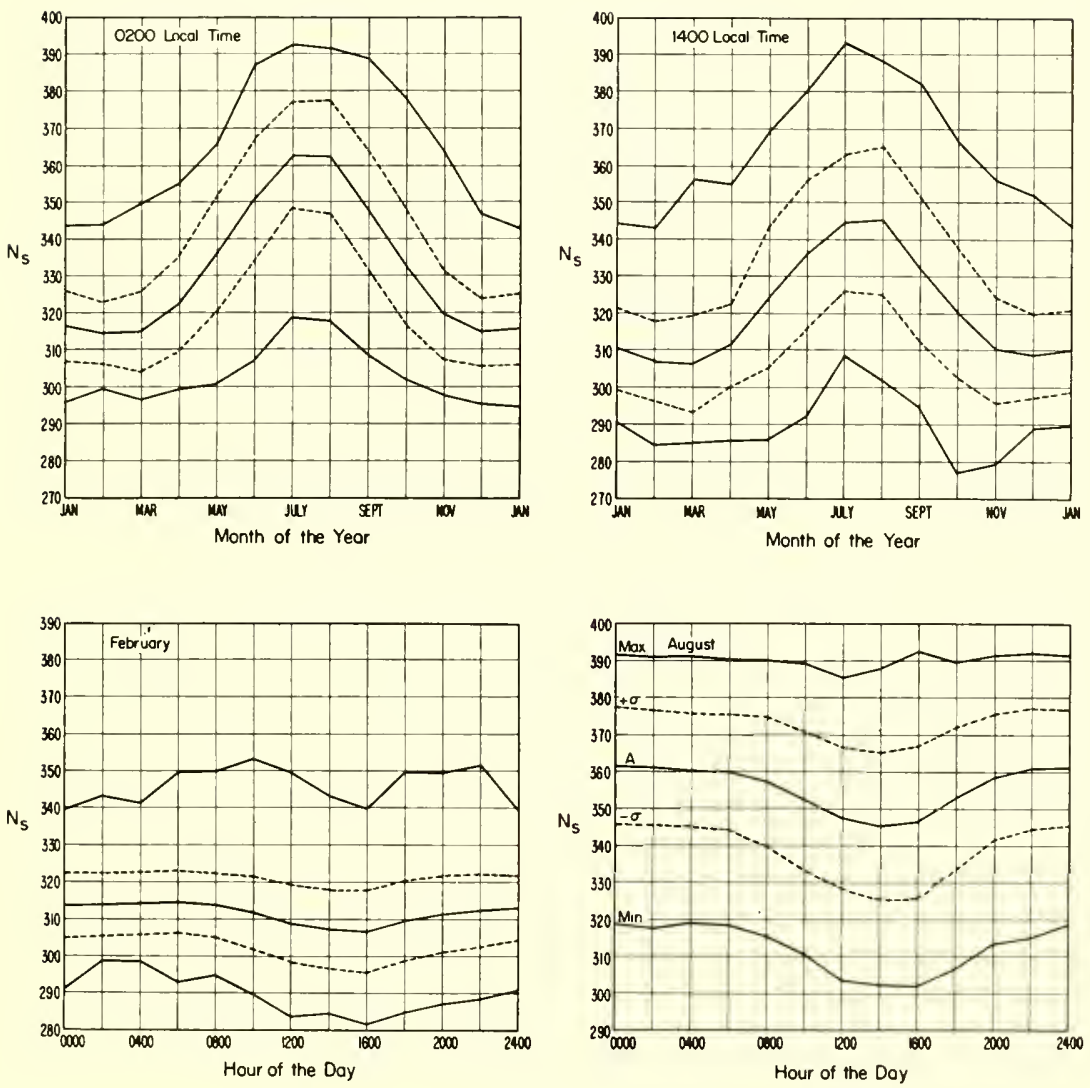

Figure 9.9. Annual and diurnal cycles of $\mathrm{N}_{\mathrm{B}}$ for Washington, D.C. 

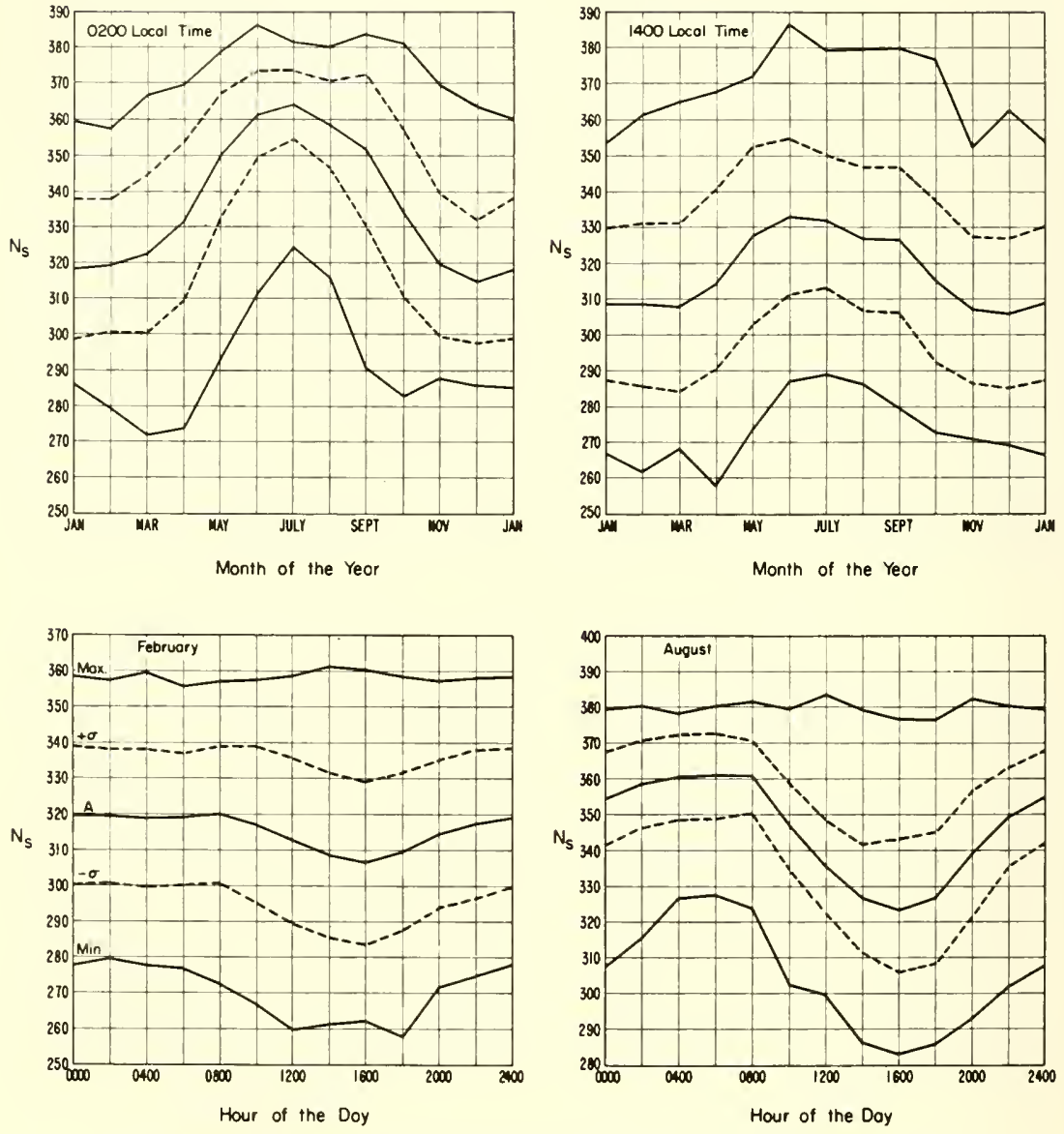

Figure 9.10. Annual and diurnal cycles of $\mathrm{N}_{\mathrm{s}}$ for San Antonio, Tex. 

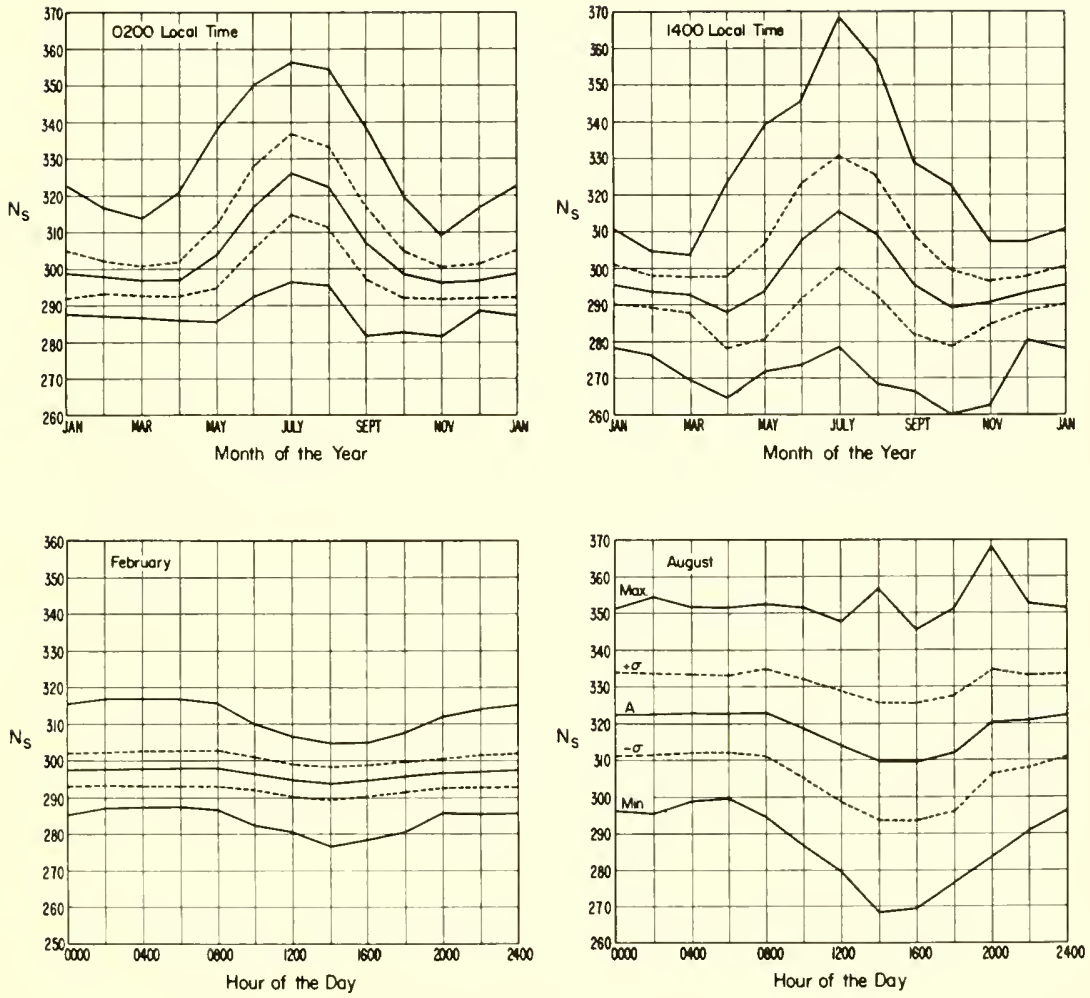

Figure 9.11. Annual and diurnal cycles of $\mathrm{N}_{\mathrm{s}}$ for Bismarck, N.Dak. 

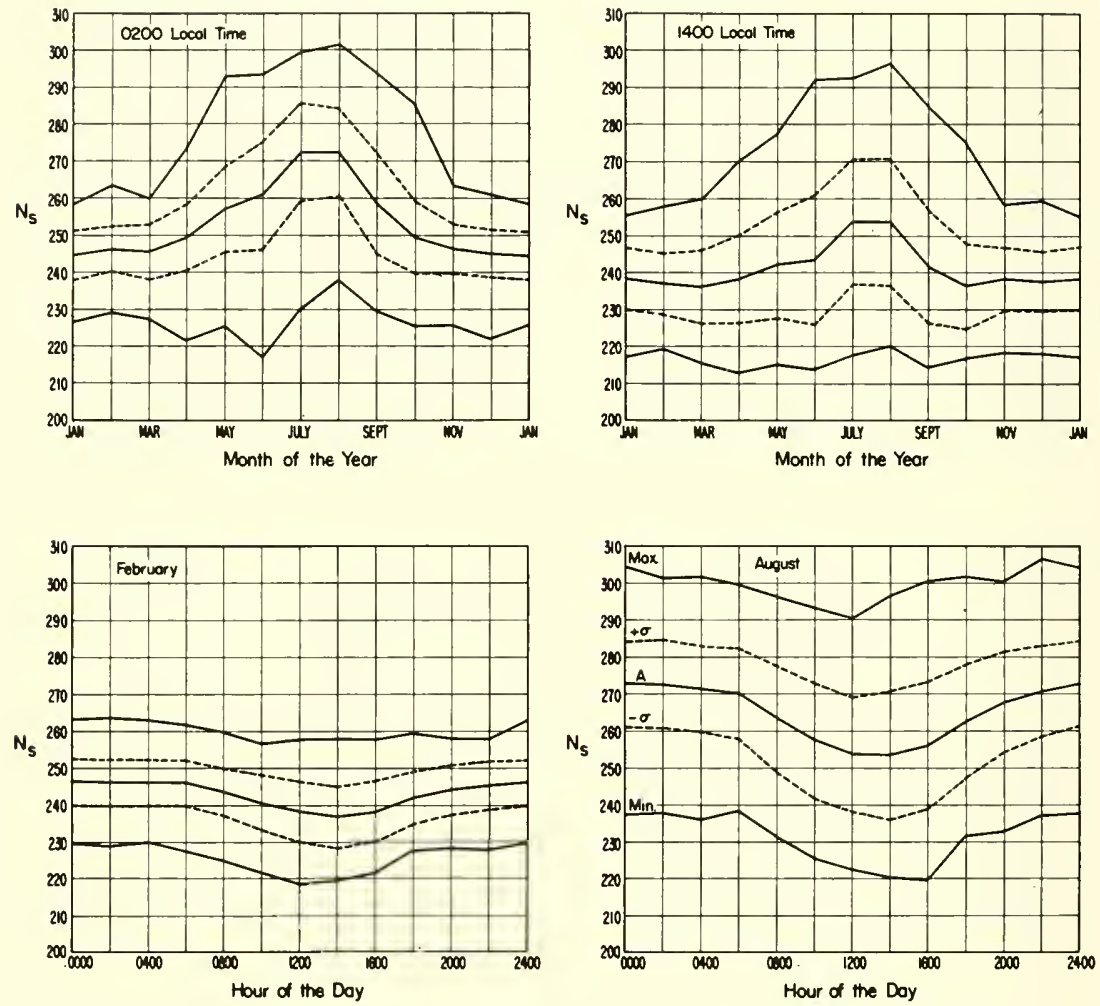

Figure 9.12. Annual and diurnal cycles of $\mathrm{N}_{\mathrm{B}}$ for Colorado Springs, Colo. 


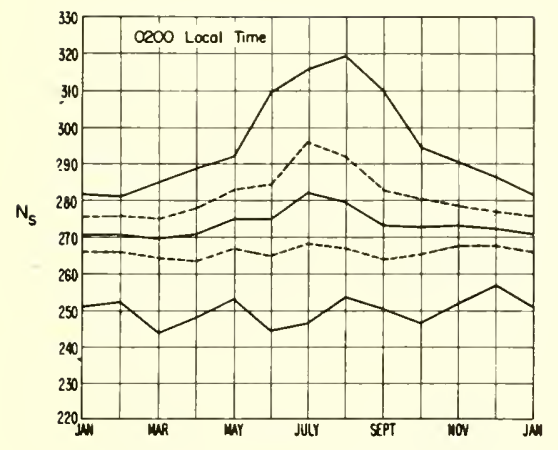

Month of the Year

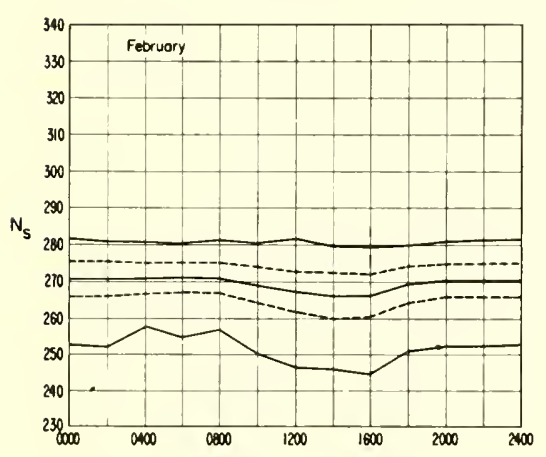

Hour of the Doy

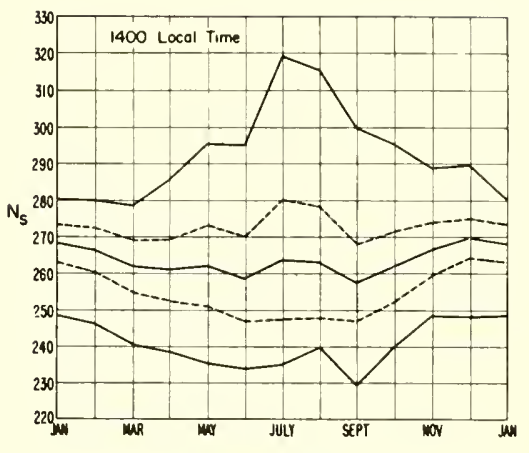

Month of the Year

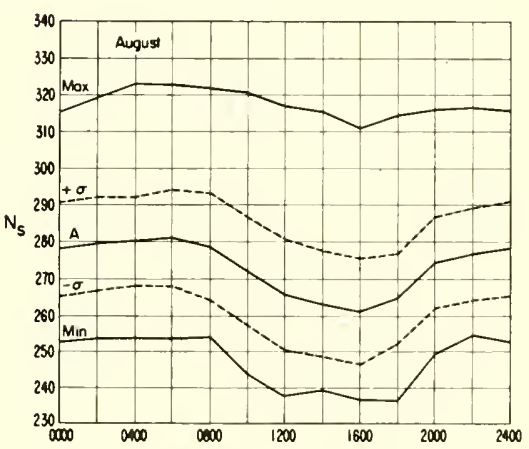

Hour of the Day

Figure 9.13. Annual and diurnal cycles of $\mathrm{N}_{\mathrm{s}}$ for Salt Lake City, Utah. 

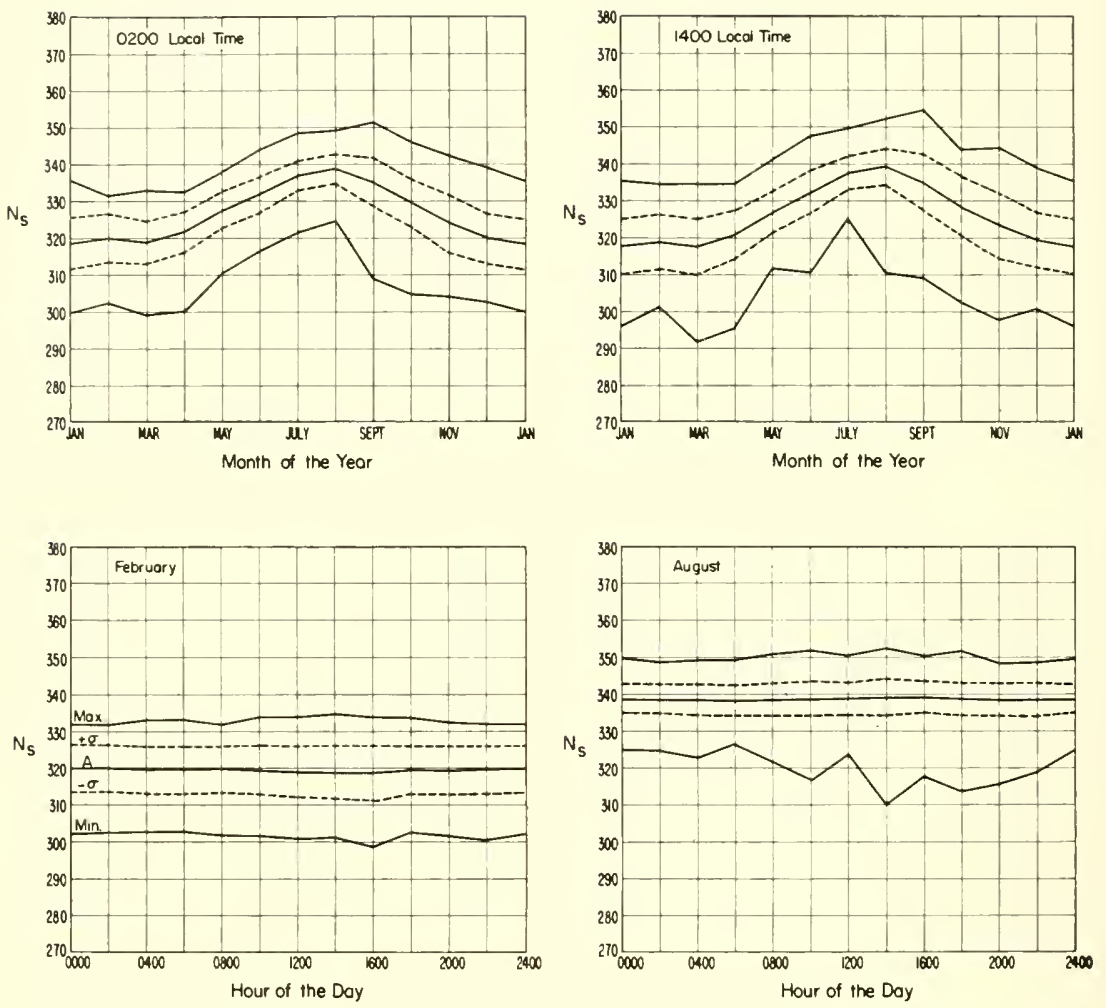

Figure 9.14. Annual and diurnal cycles of $\mathrm{N}_{\mathrm{s}}$ for Tatoosh Island, Wash. 

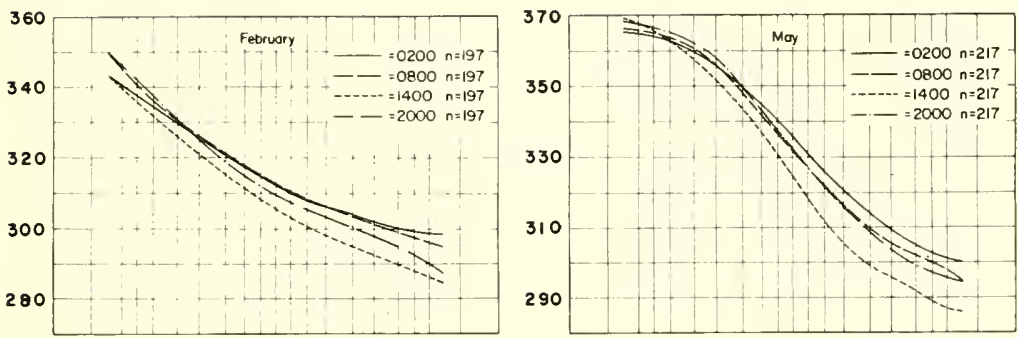

$\mathrm{N}_{\mathrm{s}}$
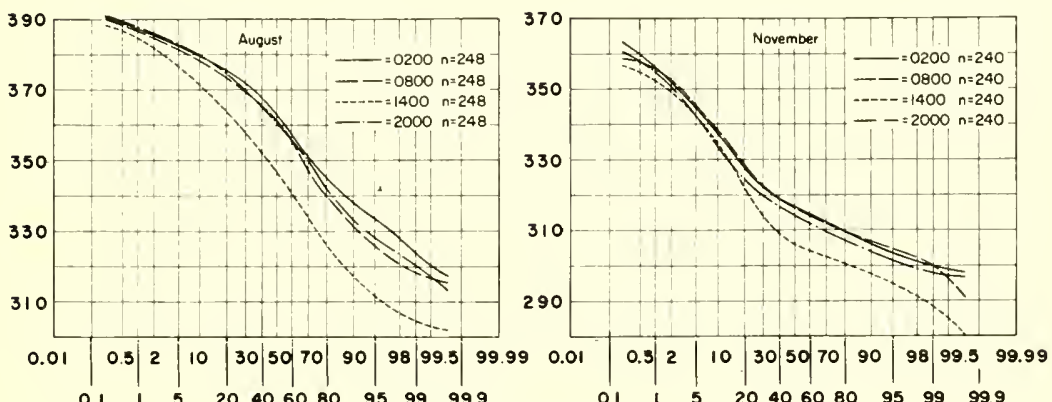

P OF $\mathrm{N}_{3} \geq$ ORDINATE VALUE

FIgURE 9.15. Cumulative probability distribution of $\mathrm{N}_{\mathrm{s}}$, Washington, D.C. 

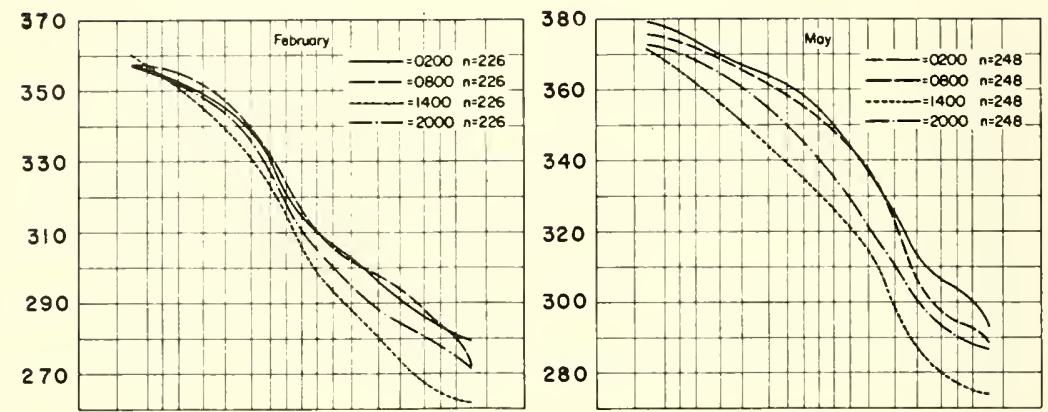

$\mathrm{N}_{\mathrm{S}}$

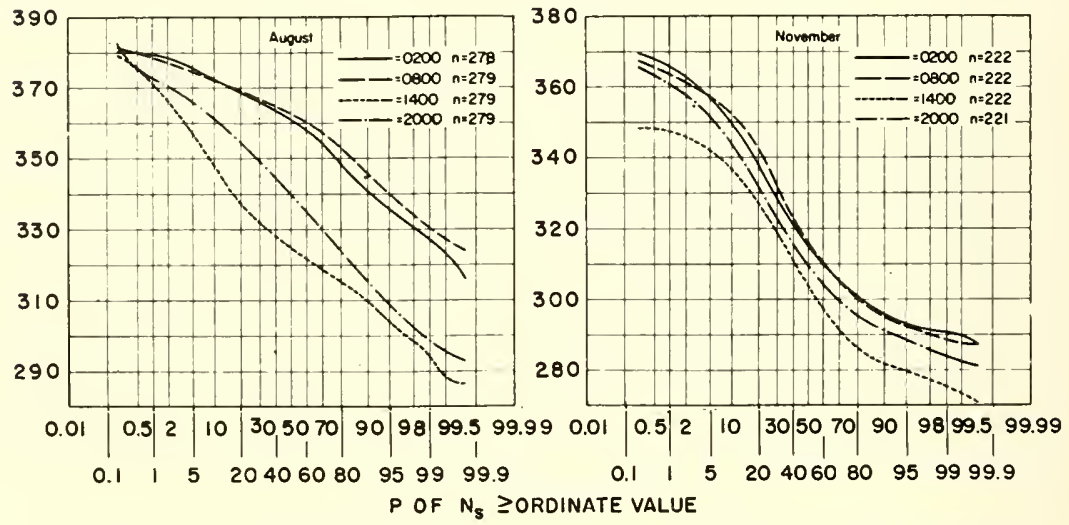

Figure 9.16. Cumulative probability distribution of $\mathrm{N}_{\mathrm{s}}$, San Antonio, Tex. 

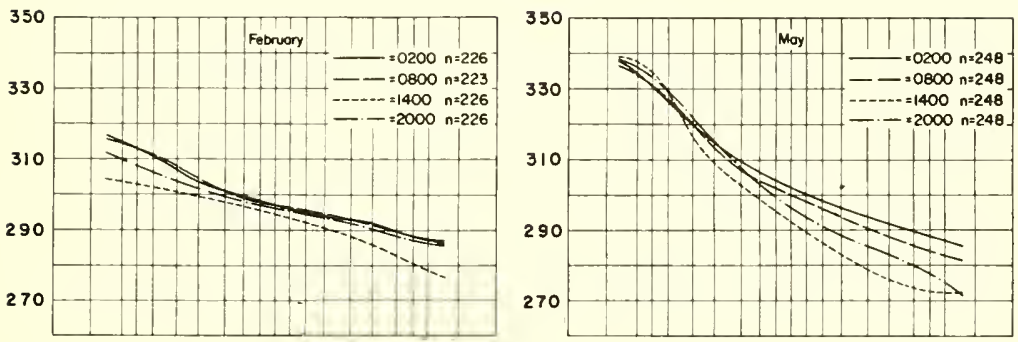

$N_{8}$

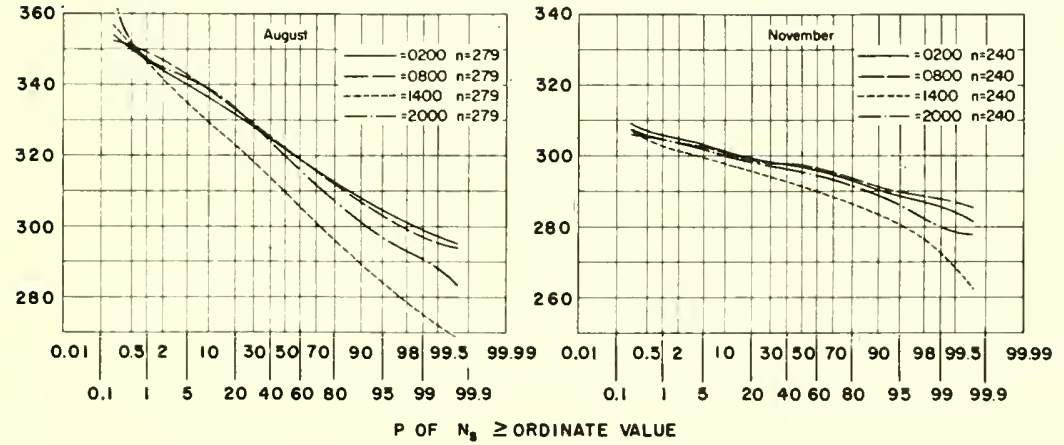

Figure 9.17. Cumulative probability distribution of $\mathrm{N}_{8}$, Bismarck, N.Dak. 

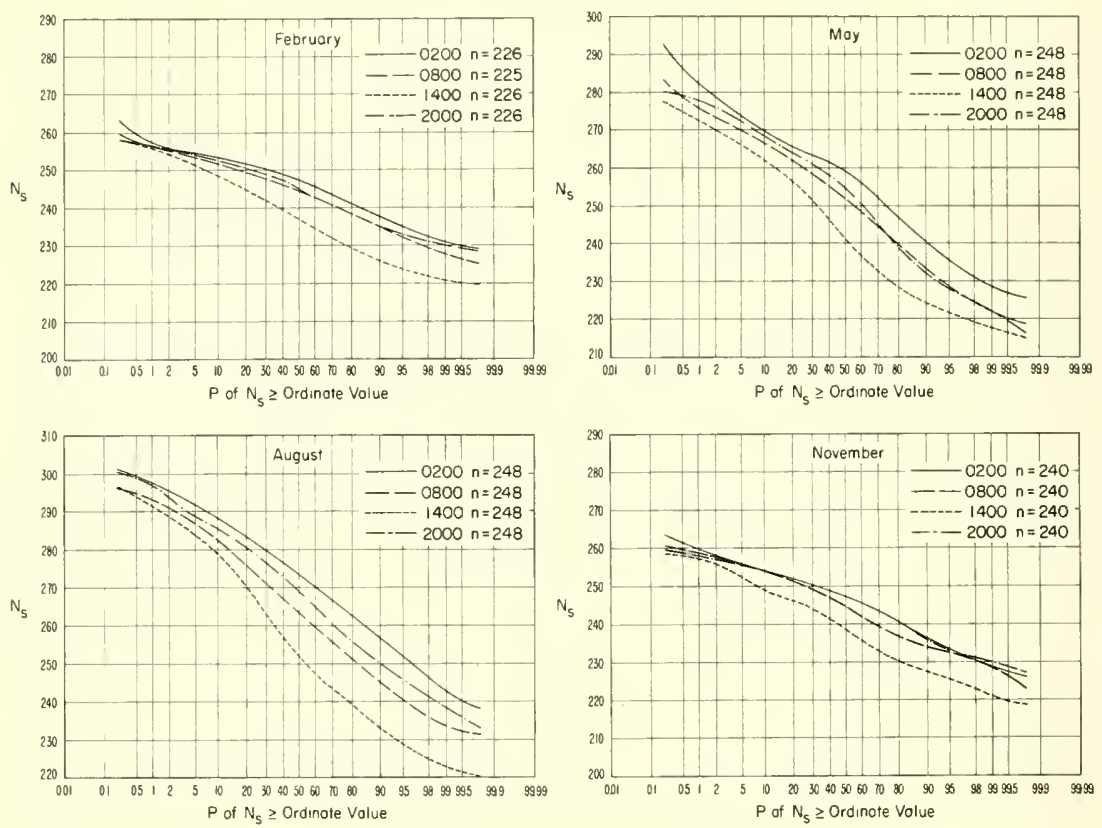

Figure 9.18. Cumulative probability distribution of $\mathrm{N}_{\mathrm{s}}$, Colorado Springs, Colo.
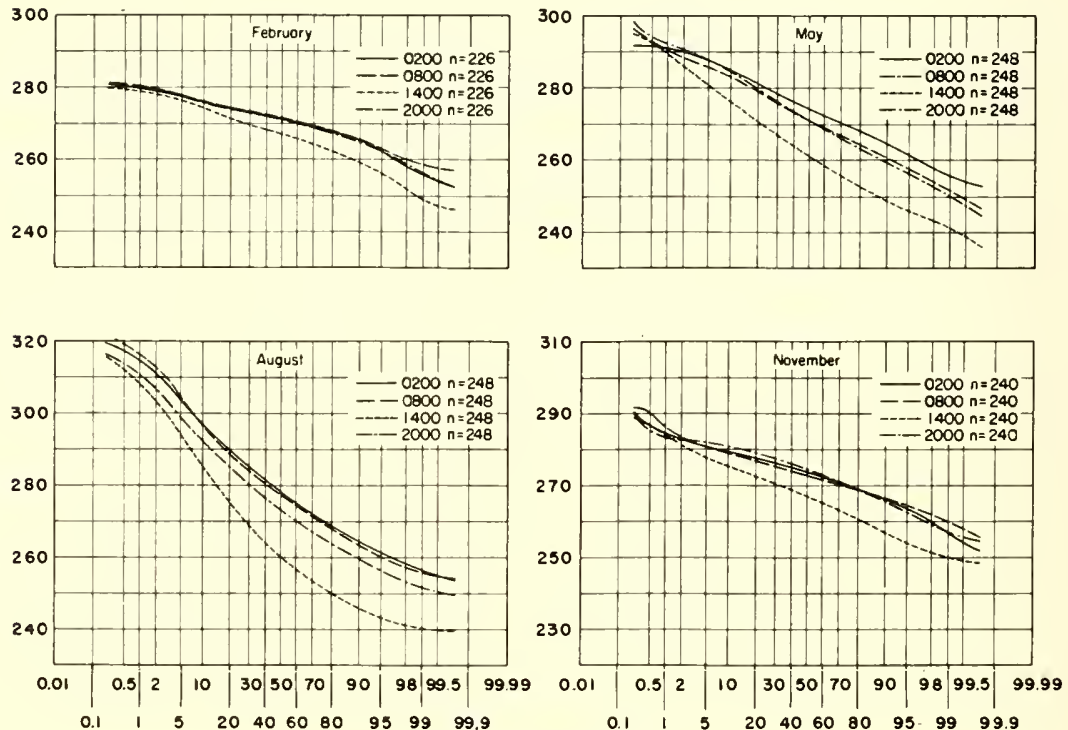

P OF $\mathrm{N}_{\mathrm{s}} \geq$ OROINATE VALUE

FIgURe 9.19. Cumulative probability distribution of $\mathrm{N}_{\mathrm{s}}$, Salt Lake City, Utah. 

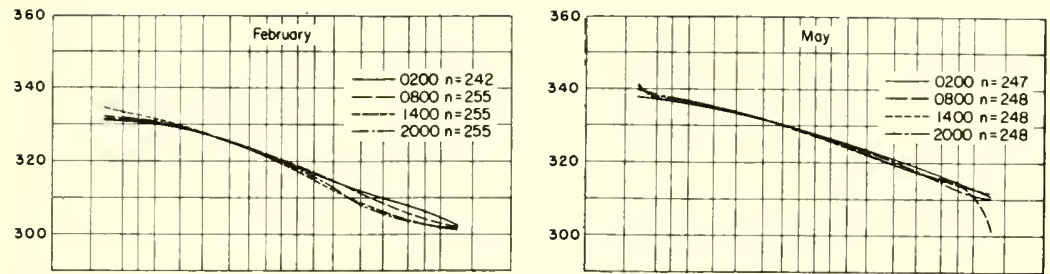

$\mathrm{N}_{2}$
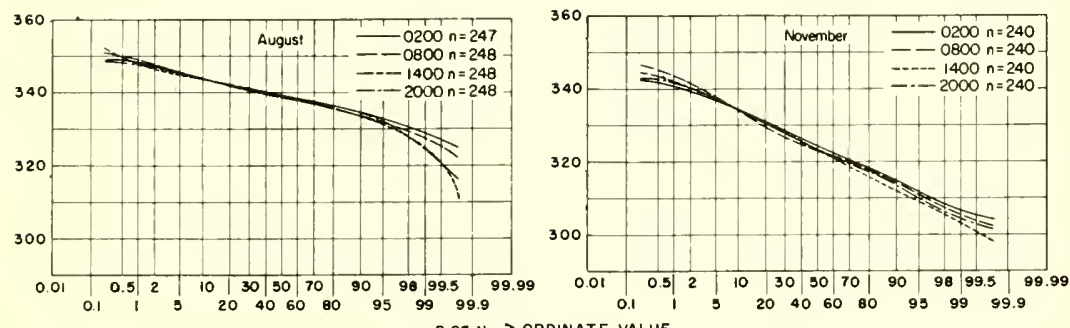

Figure 9.20. Cumulative probability distribution of $\mathrm{N}_{\mathrm{B}}$, Tatoosh Island, Wash.

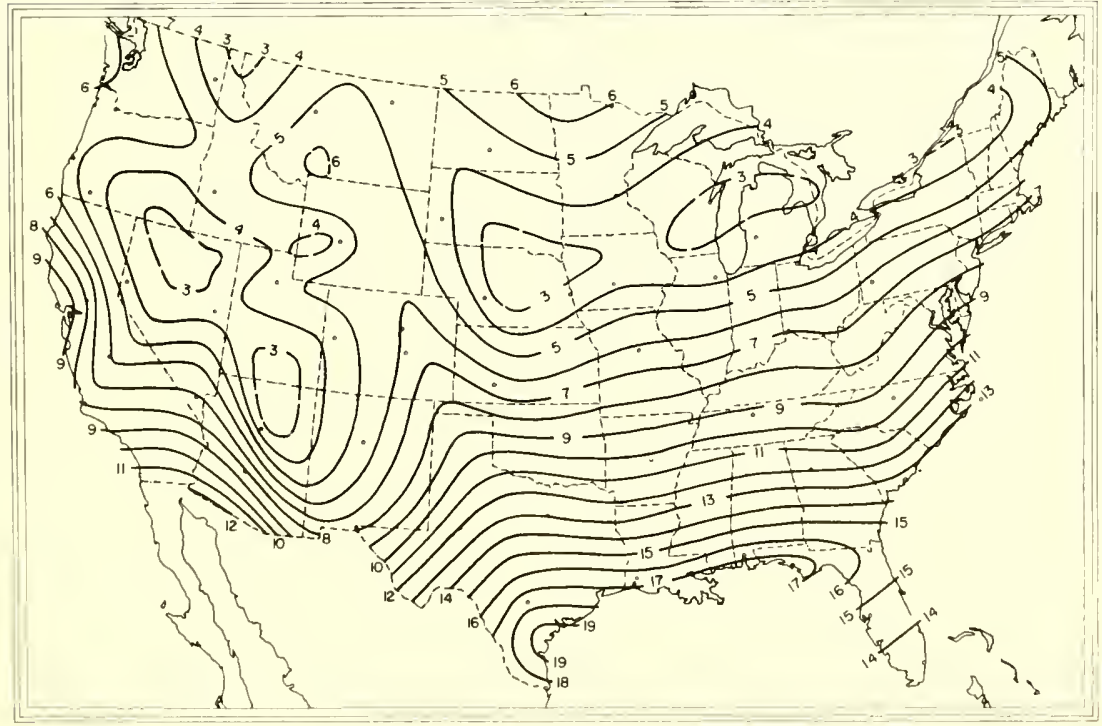

Figure 9.21. Standard deviation of $\mathrm{N}_{\mathrm{B}}$, February 0200. 


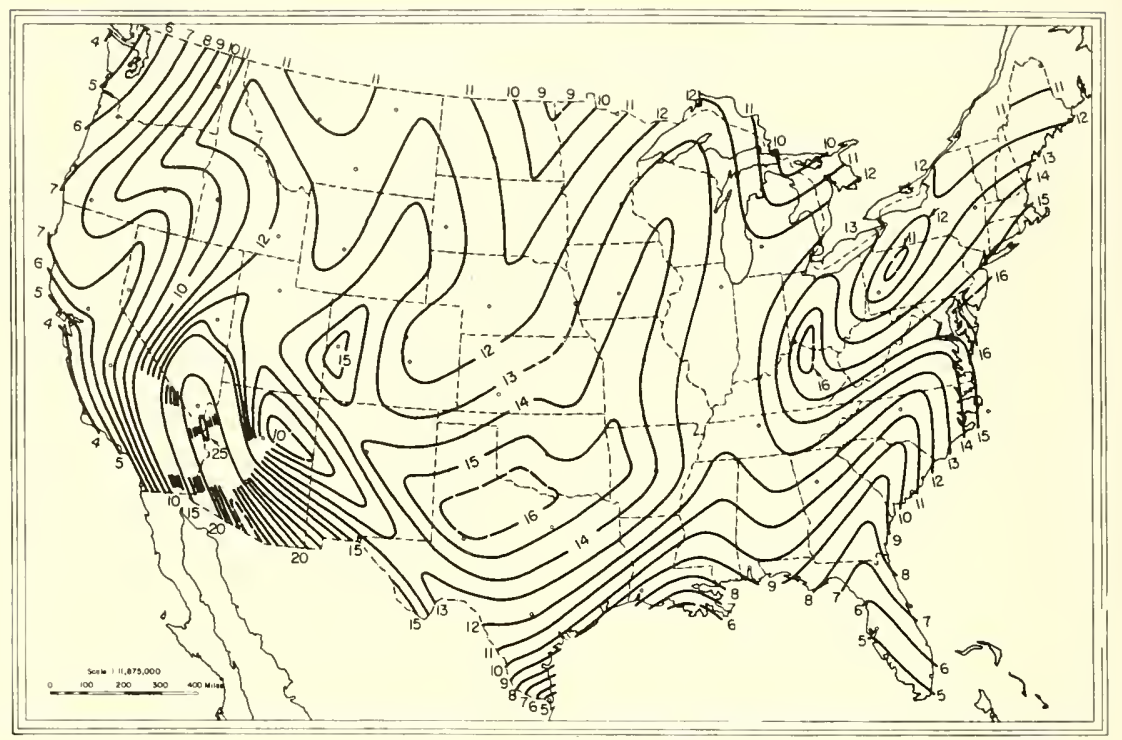

Figure 9.22. Standard deviation of $\mathrm{N}_{\mathrm{s}}$, August 0200.

\section{Tables 9.36 to 9.44}

These tables show values of $m$ and $\ell$ in the regression equation $\epsilon=m N_{s}+\ell$, where:

$\epsilon$ is the elevation angle error in milliradians to the height $h$, $N_{s}$ is the value of the radio refractivity at the earth's surface, $m$ the change in elevation angle error per unit change in $N_{s}$, $\ell$ is the zero intercept,

$r$ is the correlation coefficient,

$\mathrm{SE}$ is the standard error of prediction using the regression line, $\theta_{0}$ is the initial elevation angle in milliradians.

TABLE 9.36. Variables in the statistical method of elevation angle error for $\mathrm{h}=0.1 \mathrm{~km}$

\begin{tabular}{|c|c|c|c|c|}
\hline$\theta_{0}$ & $r$ & $m$ & $l$ & $\mathrm{SE}$ \\
\hline $\begin{array}{l}0.0 \\
1.0 \\
2.0 \\
5.0 \\
10.0 \\
20.0 \\
52.4 \\
100.0 \\
200.0 \\
400.0\end{array}$ & $\begin{array}{l}0.2637 \\
.2760 \\
.2855 \\
.3017 \\
.1609 \\
.1777 \\
.1805 \\
.1824 \\
.1815 \\
.1844\end{array}$ & $\begin{array}{l}0.0243 \\
.0131 \\
.0083 \\
.0037 \\
.0027 \\
.0012 \\
.0005 \\
.0002 \\
.0001 \\
.0001\end{array}$ & $\begin{array}{l}-4.3954 \\
-2.1055 \\
-1.2185 \\
-.4741 \\
-.2834 \\
-.1175 \\
-.0426 \\
-.0227 \\
-.0110 \\
-.0055\end{array}$ & $\begin{array}{l}3.4429 \\
1.7712 \\
1.0837 \\
0.4561 \\
.6501 \\
.2706 \\
.0998 \\
.0518 \\
.0256 \\
.0123\end{array}$ \\
\hline
\end{tabular}


TABLE 9.37. Variables in the statistical method of elevation angle error for $\mathrm{h}=0.2 \mathrm{~km}$

\begin{tabular}{|c|c|c|c|c|}
\hline$\theta_{0}$ & $r$ & $m$ & $l$ & $\mathrm{SE}$ \\
\hline $\begin{array}{l}0.0 \\
1.0 \\
2.0 \\
5.0 \\
10.0 \\
20.0 \\
52.4 \\
100.0 \\
200.0 \\
400.0\end{array}$ & $\begin{array}{l}0.2739 \\
.2838 \\
.2927 \\
.3146 \\
.1919 \\
.2125 \\
.2168 \\
.2175 \\
.2170 \\
.2170\end{array}$ & $\begin{array}{r}0.0310 \\
.0191 \\
.0132 \\
.0065 \\
.0044 \\
.0021 \\
.0008 \\
.0004 \\
.0002 \\
.0001\end{array}$ & $\begin{array}{l}-5.5364 \\
-3.0776 \\
-1.9685 \\
-.8506 \\
-.4623 \\
-.1941 \\
-.0705 \\
-.0367 \\
-.0179 \\
-.0085\end{array}$ & $\begin{array}{l}4.2249 \\
2.4984 \\
1.6702 \\
.7628 \\
.8906 \\
.3757 \\
.1385 \\
.0721 \\
.0356 \\
.0170\end{array}$ \\
\hline
\end{tabular}

TABLE 9.38. Variables in the statistical method of elevation angle error for $\mathrm{h}=0.5 \mathrm{~km}$

\begin{tabular}{|c|c|c|c|c|}
\hline$\theta_{0}$ & $r$ & $m$ & $l$ & $\mathrm{SE}$ \\
\hline $\begin{array}{l}0.0 \\
1.0 \\
2.0 \\
5.0 \\
10.0 \\
20.0 \\
52.4 \\
100.0 \\
200.0 \\
400.0\end{array}$ & $\begin{array}{r}0.3255 \\
.3457 \\
.3661 \\
.4197 \\
.2887 \\
.3304 \\
.3418 \\
.3430 \\
.3439 \\
.3408\end{array}$ & $\begin{array}{l}0.0430 \\
.0289 \\
.0216 \\
.0125 \\
.0083 \\
.0041 \\
.0016 \\
.0008 \\
.0004 \\
.0002\end{array}$ & $\begin{array}{r}-8.0534 \\
-5.0198 \\
-3.5807 \\
-1.9306 \\
-1.0741 \\
-.4899 \\
-.1803 \\
-.0934 \\
-.0463 \\
-.0218\end{array}$ & $\begin{array}{l}4.8023 \\
3.0131 \\
2.1131 \\
1.0427 \\
1.0693 \\
.4568 \\
.1679 \\
.0872 \\
.0431 \\
.0208\end{array}$ \\
\hline
\end{tabular}

TABLE 9.39. Variables in the statistical method of elevation angle error for $\mathrm{h}=1.0 \mathrm{~km}$

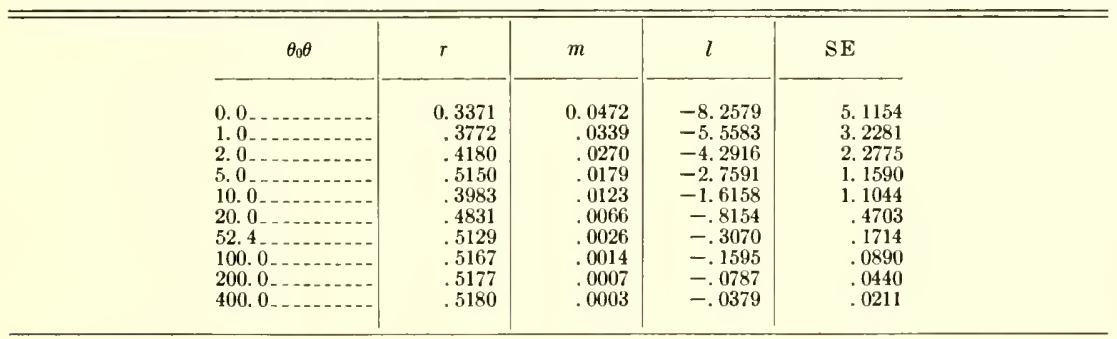

TABLE 9.40. Variables in the statistical method of elevation angle error for $\mathrm{h}=2.0 \mathrm{~km}$

\begin{tabular}{|c|c|c|c|c|}
\hline$\theta_{0}$ & $r$ & $m$ & $l$ & SE \\
\hline $\begin{array}{l}0.0 \\
1.0 \\
2.0 \\
5.0 \\
10.0 \\
20.0 \\
52.4 \\
100.0 \\
200.0 \\
400.0\end{array}$ & $\begin{array}{r}0.3795 \\
.4413 \\
.5020 \\
.6351 \\
.5642 \\
.7007 \\
.7531 \\
.7608 \\
.7633 \\
.7643\end{array}$ & $\begin{array}{r}0.0567 \\
.0427 \\
.0355 \\
.0258 \\
.0188 \\
.0113 \\
.0047 \\
.0025 \\
.0012 \\
.0006\end{array}$ & $\begin{array}{l}-9.8908 \\
-7.0696 \\
-5.7509 \\
-4.1251 \\
-2.7184 \\
-1.5650 \\
-.625 f i \\
-.3279 \\
-.1627 \\
-.0784\end{array}$ & $\begin{array}{r}\text { 5. } 3659 \\
3.3704 \\
2.3735 \\
1.2198 \\
\text { 1. } 0773 \\
.4499 \\
.1615 \\
.0835 \\
.0412 \\
.0198\end{array}$ \\
\hline
\end{tabular}


TABLE 9.41. Variables in the statistical method of elevation angle error for $\mathrm{h}=5.0 \mathrm{~km}$

\begin{tabular}{|c|c|c|c|c|}
\hline$\theta_{0}$ & $r$ & $m$ & $l$ & $\mathrm{SE}$ \\
\hline $\begin{array}{l}0.0 \\
1.0 \\
2.0 \\
5.0 \\
10.0 \\
20.00 \\
52.4 \\
100.0 \\
200.0 \\
400.0\end{array}$ & $\begin{array}{l}0.4324 \\
.5186 \\
.5983 \\
.7543 \\
.7424 \\
.8789 \\
.9248 \\
.9316 \\
.9336 \\
.9339\end{array}$ & $\begin{array}{l}0.0704 \\
.0554 \\
.0477 \\
.0372 \\
.0290 \\
.0194 \\
.0089 \\
.0048 \\
.0024 \\
.0012\end{array}$ & $\begin{array}{r}-12.0857 \\
-9.0682 \\
-7.6679 \\
-5.9085 \\
-4.3100 \\
-2.7570 \\
-1.1721 \\
-.6154 \\
-.3043 \\
-.1460\end{array}$ & $\begin{array}{l}5.6994 \\
3.5425 \\
2.4766 \\
1.2570 \\
1.0255 \\
.4118 \\
.1431 \\
.0733 \\
.0360 \\
.0173\end{array}$ \\
\hline
\end{tabular}

TABLE 9.42. Variables in the statistical method of elevation angle error for $\mathrm{h}=10.0 \mathrm{~km}$

\begin{tabular}{|c|c|c|c|c|}
\hline$\theta_{0}$ & $r$ & $m$ & $l$ & $\mathbf{S E}$ \\
\hline $\begin{array}{l}0.0 \\
1.0 \\
2.0 \\
5.0 \\
10.0 \\
20.0 \\
52.4 \\
100.0 \\
200.0 \\
400.0\end{array}$ & $\begin{array}{l}0.4617 \\
.5602 \\
.6475 \\
.8057 \\
.8125 \\
.9301 \\
.9679 \\
.9742 \\
.9763 \\
.9766\end{array}$ & $\begin{array}{l}0.0797 \\
.0637 \\
.0555 \\
.0443 \\
.0353 \\
.0245 \\
.0118 \\
.0065 \\
.0032 \\
.0016\end{array}$ & $\begin{array}{r}-13.2953 \\
-10.0839 \\
-8.5935 \\
-6.6912 \\
-4.9338 \\
-3.1637 \\
-1.3002 \\
-.6607 \\
-.3208 \\
-.1533\end{array}$ & $\begin{array}{r}5.9448 \\
3.6560 \\
2.5360 \\
1.2652 \\
.9913 \\
.3779 \\
.1195 \\
.0584 \\
.0281 \\
.0135\end{array}$ \\
\hline
\end{tabular}

TABLE 9.43. Variables in the statistical method of elevation angle error for $\mathrm{h}=20.0 \mathrm{~km}$

\begin{tabular}{|c|c|c|c|c|}
\hline$\theta_{0}$ & $r$ & $m$ & $l$ & SE \\
\hline $\begin{array}{l}0.0 \ldots \\
1.0 \\
2.0 \\
5.0 \\
10.0 \\
20.0 \\
52.4 \ldots \\
100.0 \ldots \\
200.0 \ldots \\
400.0\end{array}$ & $\begin{array}{r}0.4805 \\
.5866 \\
.6780 \\
.8351 \\
.8497 \\
.9538 \\
.9861 \\
.9917 \\
.9934 \\
.9939\end{array}$ & $\begin{array}{r}0.0874 \\
.0703 \\
.0616 \\
.0495 \\
.0397 \\
.0278 \\
.0136 \\
.0076 \\
.0038 \\
.0018\end{array}$ & $\begin{array}{r}-14.0318 \\
-10.5942 \\
-8.9972 \\
-6.9273 \\
-5.0189 \\
-3.0650 \\
-1.0682 \\
-.4672 \\
-.2067 \\
-.0947\end{array}$ & $\begin{array}{r}6.1895 \\
3.7658 \\
2.5905 \\
1.2665 \\
.9637 \\
.3423 \\
.0899 \\
.0384 \\
.0172 \\
.0080\end{array}$ \\
\hline
\end{tabular}

TABLE 9.44. Variables in the statistical method of elevation angle error for $\mathrm{h}=70.0 \mathrm{~km}$

\begin{tabular}{|c|c|c|c|c|}
\hline$\theta_{0}$ & $r$ & $m$ & $l$ & SE \\
\hline $\begin{array}{l}0.0 \\
1.0 \\
2.0 \\
5.0 \\
10.0 \\
20.0 \\
52.4 \\
100.0 \\
200.0 \\
400.0\end{array}$ & $\begin{array}{r}0.4999 \\
.6137 \\
.0783 \\
.8617 \\
.8811 \\
.9701 \\
.9948 \\
.9983 \\
.9992 \\
.9995\end{array}$ & $\begin{array}{r}0.0985 \\
.0794 \\
.0698 \\
.0563 \\
.0451 \\
.0317 \\
.0158 \\
.0089 \\
.0045 \\
.0022\end{array}$ & $\begin{array}{r}-15.2202 \\
-11.3649 \\
-9.5814 \\
-7.2363 \\
-5.0975 \\
-2.9121 \\
-.8032 \\
-.2595 \\
-.0822 \\
-.0319\end{array}$ & $\begin{array}{l}6.6214 \\
3.9661 \\
2.6984 \\
1.2867 \\
.9476 \\
.3102 \\
.0630 \\
.0202 \\
.0070 \\
.0028\end{array}$ \\
\hline
\end{tabular}




\subsection{References}

[1] Bean, B. R., and G. D. Thayer (1959), On models of the atmospheric radio refractive index, Proc. IRE 47, No. 5, 740-755.

[2] Smithsonian Meteorological Tables (1951), Table 63, Sixth Revised Ed. (Washington, D.C.). 



\section{Subject Index}

\section{A}

A-unit (potential refractivity), 16-20, 78-79, 166-170, 189-194, 197, 211, 213-227, 379

Absolute humidity, 270, 272, 275, 277-280, 286-290

Absorption coefficient (see also Van Vleck's absorption formulas), 272

Adiabatic refractivity decrease, 258-262

dry, 261

wet, 262

Adiabatic temperature lapse rate, 133, 258

Advection, 132, 201, 205, 213, 319

definition, 132

relative to duct formation, 133, 135

Air masses, 79, 102, 114, 119, 123, 163-164, 167-169, 173-176, 179-181, 191, 195$197,201,213,221-222$

refractive characteristics of, $163,169-170,174-175,211$

Angle of penetration (critical angle), 137, 140, 142, 376

Apparent height, 360

Apparent range, 51, 335, 357-360

ARDC Standard Atmosphere, 64

Attenuation of radio waves, 269-309

by fog, 303

by gases, $270-290$

by hail, $302-303$

by rain (see also Climatology), 292-297

in clouds, 291

B-unit, 14-15, 17-20, 189-191, 195

Barium fluoride strip, 26

Bending of a radio ray (see also Refraction)

definition, 49

methods of computing and predicting, 53-81, 375-393

Bi-exponential model

for absorption, 283, 307

for refractivity, 311-322

Birnbaum refractometer, 31, 33-34

Bright band, 303

Caustic, 148

Climatic types, relation to $N_{s, 102-104}$

Climatology, 89-172

of ground based radio ducts, 42-43, 132-145

of refractivity, $N$, near the ground $\left(N_{o}\right), 89-109,111-128,404-420$

of rainfall attenuation, 297-302

Clouds, attenuation in, 291

Cold front (see Frontal zone)

Composite parameter, 238

Continental cross sections of $N_{o}, 122-128$

Continental arctic air mass (see also Continental polar air mass), 102, 316

Continental polar air mass, 79, 123, 163-164, 169, 174-176, 179-180, 196-197, $221-222,319$

Continental tropical air mass, 79, 167-169, 175, 179

Convergence

centers of, $182,240-241$

influencing atmospheric refractivity, 182-185, 240

Core climate, 129 
Correlation of field strength with meteorological parameters, 105, 174, 229, 233-266 Crain refractometer, $31-33$

Cross-over height, 248-249, 263-264

CRPL Reference Atmosphere (see Exponential atmosphere model for refractivity)

CRPL Standard $N$-profile Sample, 335, 338-353, 355-356

Curvature of radio ray, $13,42,56,264,360-361$

Deam refractometer, $36-37$

Delta- $N(\Delta N)$ (see also Refractivity, vertical gradient), 15-16, 61, 63-66, 73-76, $106,129-131,139,229-230,342,363,365,371,373,389,394-396$ as a field strength predictor, $234-238,240,243-244,246,264-266$ usage for 24-hour horizontal changes, 201-204, 209-211

Departures-from-normal method of computing refraction, 77-79, 167-168, 181, 190 $191,375,378-379,381,392-393$

Dielectric constant of air (see also Refractive index), 2-8, 12

Dispersion, 5, 8

Diurnal index (diurnal ratio), 129-130

Diurnal range of field strength, 105, 235-236 of $N_{o}, 111-121,129-130,204$

Divergence centers of, 182, 240-241

influencing atmospheric refractivity, 182-185, 240

Drop size distribution, 292, 294-297

Dropsonde, 36

Dry term of atmospheric absorption, 283

of the Smith-Weintraub equation, 30, 91, 93-94, 136, 174-175, 311-322 scale heights of (see Scale height) surface values of, 93-94, 311-312, 320

Ducting, 42-45, 52, 89, 132-163, 241-246, 248, 324, 328, 331-333, 376

\section{E}

Effective earth, 13-15, 51, 56-62, 64-65, 106, 189, 195, 250, 264, 359-362

curvature, $13,56-57,62,106,195,361$

method of calculating refraction, 56-59

radius, $13-15,51,56-57,59-62,64-65,106,189,195,250,264,359-362$

Elevated layer, 241, 244-258, 324-329, 331

reflection coefficient of, $252-257$

Elevation angle (see also Refraction) approximation for, 82-83

definition, 49-50

errors, 49-50, 178, 333-335, 340-342, 344-345, 348-350, 356, 404, 420-422 prediction of, $174,334,340-342,344-345,348-350,356,404,420-422$

Epoch chart, 221-227

Equation of continuity, 183

Equivalent gradient, 236-239, 243, 246, 264-266

as a field strength predictor, $236-239,243,246,265$

method of calculation, 264

Exponential atmosphere models

for absorption, 283

for refractivity, 15-20, 56, 61, 63-80, 92-93, 106, 110, 149, 153-156, 164, 166$167,174,176-177,186,189-190,192-194,244-245,261-263,311-322$, 329-332, 357, 362, 375-378, 385-389, 393-396

in radiosonde correction process, 40-41

method of computing refraction, $375-377,385-388$

Eta $(\eta)$ parameter, 238-239

F

Fading (see also Field Strength), 146-163, 223

caused by ducting, 146-163

definitions, 147

due to rainfall attenuation, 298-302

Field strength prediction from radio meteorological parameters, 89, 105-106, 109, 174, 177-180, 229-236, 238-251, 253-258, 263-266 
Fog attenuation, 303

Four-thirds earth (see also Effective earth), 14-15, 20, 51, 57, 59-60, 62, 66-75, 247,360

Fresnel discontinuity value, 252,257

Frontal zone, influence on refractivity, 176-177, 187-193, 195-224, 323

\section{G}

Geometric range, 335,357

Gradient of refractive index (see Refractive index)

Gradient of refractivity (see Refractivity) regression expressions for, $363-364,372$

Graphical method of computing refraction, 80-81, 375, 380-381

Ground-based radio ducts (see also Ducting), 42-44, 132-163, 324, 328, 333, 375 frequency of occurrence, 140-141 thickness of, 140, 143-145

Ground-based superrefractive layers, 250

$H$-parameters, 265

Hail attenuation, 302-303

Half-length, 150-154, 160

Hay refractometer, $36-37$

Height errors, 356-357, 360-373

definition, 356,360

regression equations for, 364-372

High angle approximation for computing refraction, 53-54

Horizontal homogeniety, assumption of, $49,52,163,322$

Horizontal inhomogenieties, $52,322-333,356$

Horizontal ray, 329

I

ICAO Standard Atmosphere, 9, 11, 312-314

Initial gradient of refractivity (see Deita- $N$ and Refractivity)

Intensity factor, 273-274

Inversions (see Ducting and Elevated layers)

$\mathbf{K}$

K-unit, 180, 182, 239-240

$\mathbf{L}$

Lag times, 29, 38-45

in electrical thermometers, 26

of relative humidity sensor in radiosondes, 39, 41-45

of temperature sensor in radiosondes, $39,41-45$

Land breeze, relative to duct formation, 134-135, 323

Level of nondivergence, 183

Lifting condensation level, 260

Line widths of atmospheric gaseous absorption (see also Attenuation), 272-275

Lithium chloride humidity strip, 26, 28-29, 39, 45

\section{M}

$M$-parameter (see Thermal stability)

Macroscale fluctuation of refractive index, 173, 223

Maritime polar air mass, 79, 114, 169, 179, 197

Maritime tropical air mass, $79,102,119,125-126,164,167,169,174,177,179-181$, $201,213,221-222,319$

Maximum wavelengths trapped by a duct, 141-143

Mesocale fluctuation of refractive index, 173, 185, 223

Microscale fluctuation of refractive index, 173

Mixing ratio, 260

Models

of atmospheric absorption structure, 283

of atmospheres, $9,11,16-17,64,92-93,180,186,312-314$

of refractivity structure, $13-20,40-41,51,56-80,92-93,106,110,149,153-156$, $164,166-170,174,176-177,180,186,189-195,197,213-227,244-245,250$, 261-264, 311-322, 329, 331-332, 357, 360-362, 375-378, 381, 385-389, $393-396$ 
Modified effective earth's radius method of computing refraction, 59-65

Modified index of refraction ( $M$-unit), 14-15, 17-20, 193, 195, 324

$\mathbf{N}$

$N$-unit (see Refractivity)

NACA Standard Atmosphere, 16-17, 92-93, 186

Nocturnal duct formation (see also Ground-based radio ducts), 133, 135

Nonpolar gases, dielectric constant of, 2-3

O

Optical depth, 304

Outage time, 298-301

Oxygen

absorption properties of (see Attenuation by gases)

refractivity at STP, 9

$\mathbf{P}$

Permeability of air, 3-4

Pi-factors (turbulence parameters), 262-266

Polar air mass (see Continental polar air mass)

Polar front (see also Frontal zone), 195-197, 213

Polar wave, 193, 195-197, 213

Polar gases, dielectric constant of, $2-3$

Potential refractive modulus ( $\varphi$-unit), 17, 19-20, 166, 193

Pound oscillator (see Deam refractometer)

Precipitation rate, 292

Profile (gradient) classification (see also Air masses and Refractivity), 248-250

Profiles of refractive index (see Refractive index, vertical profile of)

Profiles of refractivity (see Refractivity, vertical profile of)

Psychrometric equation, 12, 24

\section{$\mathbf{R}$}

Radar

applications involving (see also Rain attenuation), 49, 51, 56, 59, 61, 77, 340, 357 anomalous propagation in (see Ducting)

Radiation, relative to duct formation, 133

Radiative transfer equation, 304

Radio

duct (see Ducting)

holes (see Ducting)

horizon

equation for, 59

in ducting, $146-148,152,155,157$

refractive index (see Refractive index)

Radiosonde (RAOB), 10-11, 26-30, 36, 38-43, 53-55, 59, 61, 70-72, 74-75, 79, 110-

$111,135,138-140,144,179,181,194,205,211,213,243,246,247-249 ; 251,253$, $255,258,264,280,283,326,363,375$

sensors in, 26-27, 29-30, 39-45

Rain attenuation, 292-297

Range

errors (radio), 12, 174, 335-342, 345, 350-353, 356

definitions, $335-336$

prediction of, $341,345,350-353$

total, $335-337$

of a radio ray, $51,335-336,357-361$

Ray tracing (see also Refraction), 52, 132, 135-136, 148, 322-323

limitations of, 52,132

Rayleigh-Jeans law, 304-305

Reflection coefficient (of an elevated layer), 185, 252-257 models of, $252-257$

Refraction (angular) of a radio ray, 14-15, 19-20, 49-87, 163-170, 178-181, 190, 192, $236,266,311,319-323,326-337,339,341-350,357,375-396$

definition, 49

derivation in terms of refractive index, 87 
Refractive index, atmospheric radio (see also Refractivity)

applications of , $12-14,31-34,36,49-87,136-138,150-152,165-166,174,223$,

$231-266,311,319-323,326-329,335-336,357,359-362,394$

definition, 23

dispersion, 5,8

expressions for, $3-7$

general discussion of $1-8,16-17,19-20,49,89-90,92,103,109-110,125,129$, $133,135,163-166,169-170,173-175,178,185-195,211,222,311,314,332-$ $333,342-343,356,404$

vertical gradient of (see also Delta- $N$ ), 13-14, 19, 36, 38, 52, 56-57, 64, 77, 106-107, $132,138-140,142,180,182,189,195,236,240-241,248,264,319,357,361-364$

vertical profile of, $14-15,40,42-45,52,59,62,135,173-176,179-180,182,187$, $211,222,229,240,247-252,254,257-258,331-332,334,339-342,363,366$

Refractivity (see also Refractive index)

applications of, 12-13, 19-20, 49-87, 105-107, 136-170, 178-181, 190, 229-266,

$319-373,375-396$

as a function of height (see Models)

definition, 4, 90, 357

diurnal range (see Diurnal range)

expressions for, $4-7$

constants in, 4-11

errors in, 9-12

measurement of, $23-45$

accuracy and errors in, $24-32,34-36,38-45$

near the ground (surface values), 15-20, 36, 42-43, 53-54, 58, 61, 63-80, 89-163, 176-182, 186-187, 192, 194, 197-205, 223, 229-230, 233-237, 239, 243-246, $258,261-266,311-312,319-321,333-356,361-363,365-367,369-370,373$, $375-388,392-396,404-422$

of atmospheric constituents

carbon dioxide, 5

dry air (see also Dry term), 9

oxygen, 9

water vapor (see also Wet term), 9

potential (see A-unit)

scale heights of (see Scale height)

synoptic variation of (see Frontal zone)

vertical gradient of (see also Delta- $N$ ) $, 9-12,15,20,33,36,42-43,61,63-66$, $73-77,79,106,114,123,129-131,136,143,145,147-148,150,152,166$, $168,229-230,234-241,243-244,246,248,250,264-266,342,362-363$, $365-367,370-371,373,375,378,389,394-396$

vertical profile of (see also CRPL Standard N-profile Sample), 15, 17-19, 42-45, $54-55,57,59-68,70-71,73,75,77-79,111,115,117,122,129,135,138-$ $139,146,162-164,167-170,174-177,194,211-227,235,244-245,263,266$, $321,329,336-339,341,344,375,381$

Refractometer, 8, 23, 31-38, 334

Resonant frequencies (absorption lines)

of oxygen, $270-275,280$

of water vapor, $270-275,280$

Rocket Panel data, 64

Satellite telecommunications, 65, 175

Saturation vapor pressure, exponential assumption for, 260

Scale height

of absorption, 283

of refrartivity, 15-17, 92-93, 108, 186, 261-263, 311-312, 314-322

of dry term, $93,261,263,311-312,314-322$

of wet term, 93, 262-263, 311-312, 314-322

Scattering by atmospheric constituents (see also Attenuation) 185, 247-248, 255, $269-270,292$

Schulkin's method of computing refraction, 54-55, 80, 179, 375-376, 390-391

Sea breeze, relative to duct formation, 134-135, 323

Seasonal index (seasonal ratio), 129-130 
Seasonal range

of field strength, 174

of $N_{o}$ (see also Climatology), 111-121, 129-130

Shadow zones, $148-150,152-155,157,159-160$

Slant range, 335,360

Snell's law in polar co-ordinates, 49, 84-87, 136-137, 165, 326

derivation, $84-87$

Snow attenuation, 302

Space cross sections of refractive index, 211-217, 221

Sprung's formula, 12

Stability

atmospheric, 238-241, 258, 260, 262-263, 266

thermal (see Thermal stability)

Standard atmosphere, 9, 11, 16-17, 64, 92-93, 180, 186, 312-314

Statistical method of computing refraction, $54,335,342-350,379-380,382-384$

Stratopause, appoximate location, 313

Stratosphere approximate extent, 313

meteorological characteristics of, 313

Subrefraction, 133, 248-249, 329, 332-333

Subsidence, relative to duct formation, 133-135, 180, 182, 185

Superior air mass, 79,169

Superrefraction (see also Ducting), 133-135, 146-148, 156, 169, 182, 248, 250, 328-329, $332-333$

Surface duct (see Ground-based radio ducts)

Synoptic illustration of $N$ change (see Frontal zone)

$\mathrm{T}$

Temperature dependence

of gaseous absorption (see Van Vleck's absorption formulas)

of refractivity, 1-8

Thermal noise temperature, 304-307

definition, 305

Thermal stability, 239-241, 262, 265

Tilted elevated layer, 249-251

Time cross sections of refractive index, 213, 217-221

Tracking system, radio, 334

Transhorizon propagation mechanisms, 248

Transition zone (see Frontal zone)

Trapping of a radio ray (see Ducting)

Tropopause, approximate location, 313

Troposphere approximate extent, 313

meteorological characteristics of, 313

Turbulence

atmospheric, 260

parameter (see Pi-factors)

Unstratified layer, 248, 250-251

Van Vleck's absorption formulas, 270, 272-275

Velocity of radio propagation, 56

Vertical

gradient of $N$ (see Refractivity)

profile of $N$ (see Refractivity)

ray, 329

Vetter refractometer, 31, 34-35

Warm front (see Frontal zone)

Water vapor

absorption properties of (see Attenuation by gases)

an average refractivity value of, 9

density (see absolute humidity)

dependence of refractivity, 1-8 
Weather influence on refractivity (see Frontal zone)

Wet term

of atmospheric absorption, 283

of the Smith-Weintraub equation, 30, 91, 93-94, 136, 174-175, 311-322 scale heights of (see Scale height)

surface values of, 93-94, 311-312, 320

Wind velocity

influence on atmospheric stability, 240-241

vertical component of, $182-184$

Wiresonde, 326 



\section{Author Index}

\section{A}

Abbott, R. 270, 274, 288, 308

Abild, V. B. 268

Adey, A. W. 46

Airy, G. B. 148, 172

Akita, K. I. 30, 45

Anderson, L. J. 22, 55, 80, 88, 171, 227, 268

Anderson, W. L. 374

Anway, A. C. 347, 374

Armstrong, H. L. 21

Arnold, E. 268

Artman, J. O. 273, 308

Arvola, W. A. 180, 182, 226, 227, 240, 266

Atwood, S. S. 8, 22, 296-297, 309

aufm Kampe, H. J. 291, 308

Autler, S. H. 273, 275, 308

Averbach, B. L. 46

\section{B}

Badcoe, S. R. 268

Barghausen, A. F. 374

Barrell, H. 5, 21

Barsis, A. P. 267

Battaglia, A. 8, 21

Battan, L. J. 291, 308

Bauer, J. R. 22, 255, 268, 357, 374

Bean, B. R. 22, 29, 46, 54, 87-88, 170$172,180,225-228,243,266-268$, $270,274,288,308-309,342,363$, $373-374,377,423$

Becker, G. B. 273, 275, 308

Beckmann, P. 357, 374

Bennett, C. A. 374

Best, A. C. 309

Beyers, N. J. 374

Birnbaum, G. 5-7, 21-22, 31, 33-34, 46$47,273,308$

Boithias, L. 236, 267

Bonavoglia, L. 230, 266

Booker, H. G. 87, 171, 227, 267

Born, M. 21

Boudouris, G. 8, 21

Brekhovskikh, I. M. 254, 268

Bremmer, H. 136, 171

Brooks, C. E. P. 374

Buckley, F. 5, 21

Bunker, A. F. 39, 47

Burrows, C. R. 8, 22, 56, 88, 106, 17I, $175,225,296-297,309,374$

Bussey, H. F. 46, 29)7, 3(12, 308

Byers, H. R. 167,172
C

Cahoon, B. A. 54, 88, 243, 266, 308, 363, 374

Carruthers, N. 374

Chatterjee, S. K. 6-7, 21

Clarke, L. C. 39, 47

Cline, D. E. 46

Clinger, A. H. 30, 46

Cole, C. F., Jr. 36, 47

Condron, T. D. 373

Cowan, L. W. 171

Craig, R. A. 8, 22, 267, 373

Crain, C. M. 6, 8, 21-22, 31-33, 46-47

Crawford, A. B. $8,22,225,238,256,258$, 267

Crozier, A. L. 30,46

\section{D}

Davidson, D. 308

Debye, P. 2, 4, 21

Deam, A. P. 36-37, 46-47

Doherty, L. H. 148, 172

Donaldson, R. J., Jr. 308

Dubin, M. 64, 88

du Castel, F. 171, 253, 267-268

Dunmore, F. W. 47

Dutton, E. J. 29, 46, 88, 172, 228, 309

\section{E}

East, T. W. R. 274, 276, 291, 308

Edwards, H. D. 280, 309

Englund, C. R. 8, 22, 225

Essen, L. 5-6, 8, 10-12, 21-22

Evans, H. W. 295, 299-302, 309

\section{F}

Fannin, B. M. 172, 179, 226

Fehlhaber, L. 22, 171, 268

Ferrell, E. B. 8, 21, 56, 88, 106, 171, 225, 374

Flavell, R. G. 180, 182, 227, 240, 267

Franklin, N. L. 374

Fraser, 1). W. 46

Freehafer, J. E. 87, 374

Freethey, F. E. 46

Friis, H. T. 238, 256, 258, 267

Froome, K. I). 5-6, 8, 10-12, 21

\section{G}

(iallet, R. M. 22, 171

(iarfinkel, S. 47

(ierson, N. C. 170, 174-176, 224

(ihosh, s. N. 280, 309

(iordon, J. P. 273, 308

Gordon, IV. E. 227, 267 
Gordy, W. 308

Gossard, E. E. 227, 268

Gozzini, A. 8, 21

Gray, R. E. 105, 171, 178, 226, 267

Grosskopf, J. F. 22, 171, 268

Groves, L. G. 6

Gunn, K. L. S. 274, 276, 291, 303, 308-309

\section{H}

Hasegawa, S. 47

Hathaway, S. D. 295, 299-302, 309

Hay, D. R. 36-37, 47, 171, 175, 224

Herbstreit, J. W. 31, 46, 148, 172, 374

Hewson, E. W. 268

Hill, R. M. 308

Hirai, M. 170, 225, 267

Hirao, K. 30, 45, 171, 373

Hogg, D. C. 238, 256, 258, 267

Holden, D. B. 182-183, 185, 227, 240, 266

Holmes, E. G. 46

Hopkins, H. G. 303, 309

Horn, J. D. 22, 171, 180, 226, 228, 267, 373

Hornberg, K. O. 374

Hughs, J. V. 21

Hull, R. A. 174, 225

Humphreys, W. J. 61, 88

Hurdis, E. C. 6, 21

\section{I}

Ikegami, F. 148, 172

\section{J}

Janes, H. B. 267,374

Jehn, K. H. 19, 22, 171-172, 179-180, $193,226-227,239-240,267$

Johnson, W. E. 45, 170, 374

Jones, F. E. 45,47

Jung, P. 47

\section{K}

Katz, I. 17, 22, 166, 171, 180, 193, 226, $239,267,312,373$

Kerker, M. 303, 309

Kerr, D. E. 141, 144, 171

Kirby, R. S. 148,172

Kirkpatrick, A. W. 374

Kitchen, G. F. 268

Krinsky, A. 47

Kryder, S. J. 5, 22

L

Lane, J. A. 16, 22, 182, 227, 240, 266-267

Langleben, M. P. 303, 309

Laws, J. O. 292-293, 308

Lement, B. S. 46

List, R. J. 24, 45

Longley, A. 266

Longley, R. W. 268

Lukes, G. D. 166, 172, 180, 226

Lyons, H. 5, 22

\section{M}

Macready, P. B., Jr. 45

Magee, J. B. 8, 42

Martin, H. C. 47

Maryott, A. A. 5, 21, 273, 308

Mason, W. C. $22,357,374$

Meadows, R. W. 268

Meaney, F. M. 266

Megaw, E. C. S. 227

Meyer, J. H. 255, 268

Middleton, W. E. K. 40, 47

Mie, G. 309

Millington, G. 13, 21

Minzner, R. A. 373

Misme, P. 30, 46, 147, 170-172, 175, 224$225,236,238-240,246,265-267,311$, 373

Moler, W. F. 182-183, 185, 223, 227, 240, 266

Montgomery, R. B. 22, 267, 373

Mumford, WV. W. 8, 22, 225

Murray, G. 47

\section{$\mathbf{N}$}

Niwa, S. $170,225,267$

Northover, F. H. 268

Norton, K. A. $62,88,105,148,171-172$, $250,266-267,374$

\section{O}

Onoe, M. 170, 225, 267

Ozanich, A. M., Jr. 171, 227, 373

\section{$\mathbf{P}$}

Palmer, C. E. 201, 228

Parsons, D. A. 292-293, 308

Perlat, A. 224

Peterson, C. F. 374

Phillips, W. C. 6, 21

Pickard, G. W. 105, 170, 224, 230, 232, 235,266

Pote, A. 308

Pound, R. V. 32, 36-37, 46

Price, W. L. 148,172

\section{$\mathbf{R}$}

Rainey, R. J. 374

Randall, D. L. 176-177, 224

Ratner, B. 168, 172, 280, 308, 373

Rice, P. L. $88,92,250,266-267$

Riggs, L. P. 22, 171, 180, 226, 228, 267

Ripley, W. S. 373

Roberts, C. S. 46

Rubenstein, P. J. 22, 267, 373

Ryde, D. 292, 294, 308

Ryde, J. W. 292, 294, 302-303, 308

\section{$\mathrm{S}$}

Saito, S. 8, 21

Sargent, J. A. $31,34,46$

Saxton, J. A. 1-2, 21, 182, 227, 238, 267$268,303,309$

Schelleng, J. C. 8, 21, 56, 88, 106, 171, $175,225,374$

Schilkorski, W. 268 
Schulkin, M. 88, 150, 164, 170, 172, 179, 227,373

Sheppard, P. A. 224

Silsbee, R. H. 47

Sion, E. 47

Smart, W. M. 49, 87, 172

Smith, E. K. 4, 6-8, 10-12, 17, 21

Smith, M. J. A. 47

Smith-Rose, R. L. 8, 22

Smyth, C. D. 21

Smyth, J. B. 6, 21, 227, 267

Snedecor, G. W. 171

Spilhaus, A. F. 40, 47

Spizzichino, A. 267

Sprung, A. 12, 21, 24, 45

Starkey, B. J. 268

Stetson, H. T. 105, 170, 224, 230, 232, 235,266

Stickland, A. C. 8, 22, 61, 88

Stover, C. M. 45

Straiton, A. W. 30, 46, 274, 308

Stranathan, J. J. 6, 21

Strandberg, M. W. P. 308

Sugden, S. 6

\section{$\mathbf{T}$}

Tao, K. 171, 373

Thayer, G. D. 22, 54, 87, 170, 225-226, $228,267,342,363,373-374,377,423$

Thiesen, J. F. 30, 46

Thompson, M. C., Jr. 46, 374

Tinkham, M. 308

Tolbert, C. W. 274, 308

Trewartha, G. T. 172

Troitskii, V. S. $309,312,373$

Trolese, I. G. 21, 227, 267

Turner, H. E. 47

Turner, W. R. 268
$\mathbf{U}$

Unwin, R. S. 323

\section{V}

Van Vleck, J. H. 270, 273-275, 308

Vetter, M. J. 31, 34-36, 46, 374

Villars, F. 267

Voge, J. 238-239, 267

Vogler, L. E. 62, 88

\section{W}

Wadley, T. L. 8, 12-13, 21

Wagner, N. K. 38-39, 42, 47, 253, 268

Walkinshaw, W. 87, 171, 267

Waters, D. M. 46

Watkins, T. B. 47

Waynick, A. H. 8, 22

Weickmann, H. K. 291, 308

Weintraub, S. 4, 6-8, 10-12, 17, 21

Weisbrod, S. 80,88

Weisskopf, V. F. 267

Wells, P. I. 374

Wensien, H. 268

Wexler, A. 28-29, 39, 41, 45-47

Wilkerson, R. E. 374

Willett, H. C. 172

Williams, C. E. 46

Wilson, F. A. 22, 357, 374

Wolf, E. 21

Wong, M. S. 322, 373

Y

Yerg, D. G. $45,47,174,225$

Z

Zhevankin, S. A. 309, 312, 373 






